\title{
THE NEXT GENERATION SPACE TELESCOPE
}

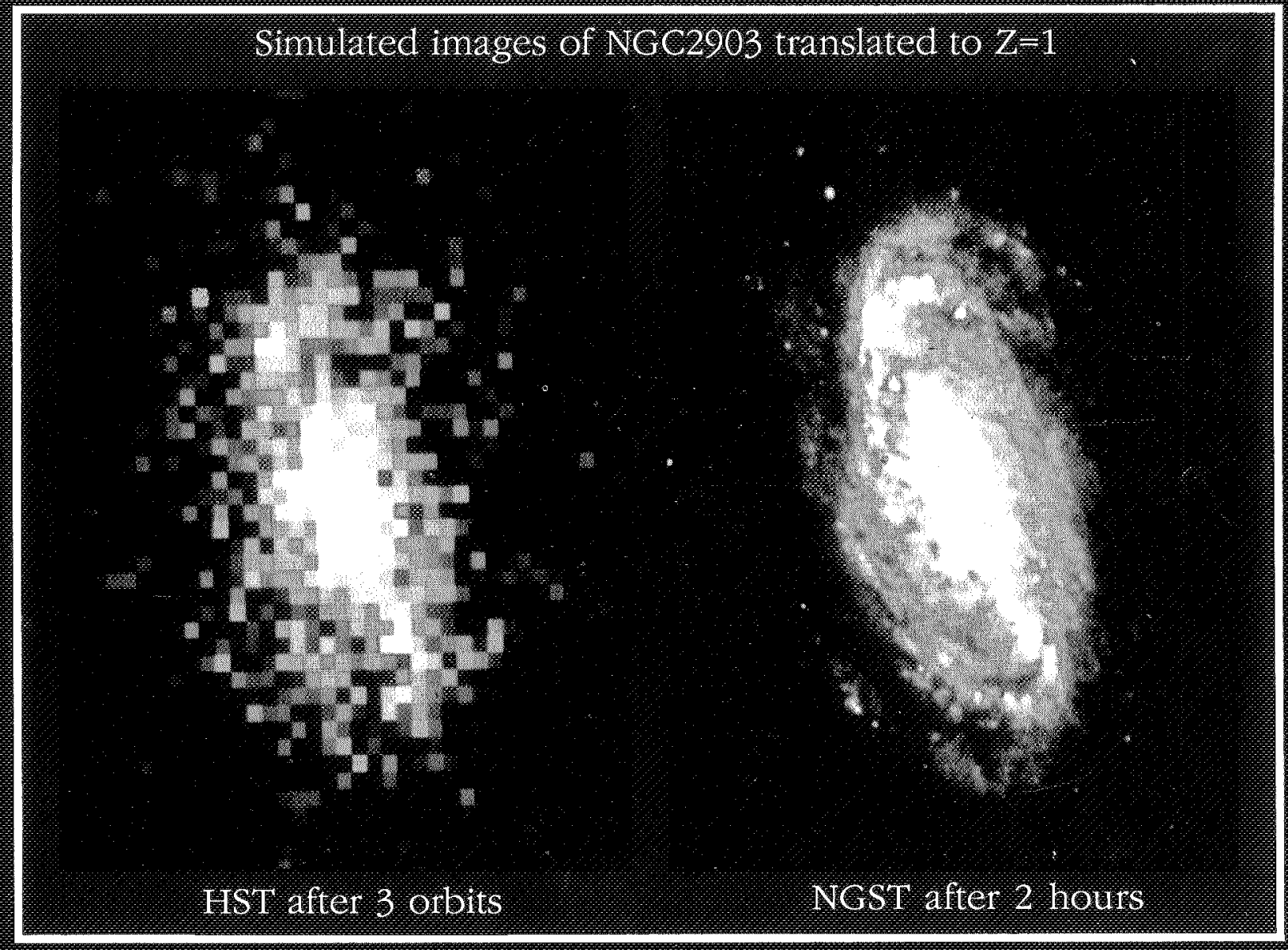

Proceedings of a Workshop held at the Space Telescope Science Institute

Baltimore, Maryland, 13-15 September 1989

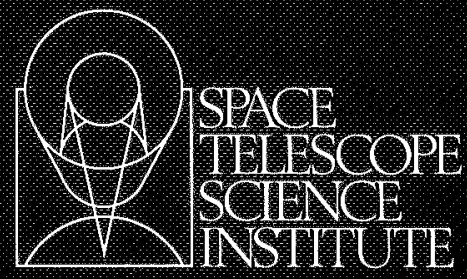


Cover: Two simulated images by James Gunn illustrate the improvements in resolution and sensitivity that the NGST would give when compared to the Hubble Space Telescope. 


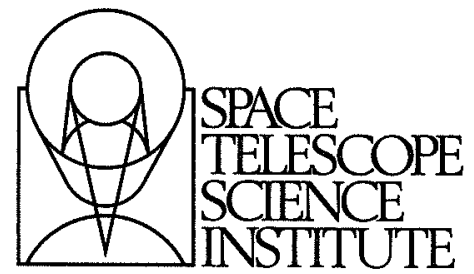

\section{THE NEXT GENERATION SPACE TELESCOPE}

Proceedings of a Workshop jointly sponsored

by the

National Aeronautics and Space Admnistration and the

Space Telescope Science Institute and held at the

Space Telescope Science Institute

Baltimore, Maryland,

13-15 September 1989

\section{Editors:}

Pierre-Yves Bely and Christopher J. Burrows

Space Telescope Science Institute

Garth D. Illingworth

University of California, Santa Cruz

Published and distributed by the Space Telescope Science Institute

3700 San Martin Drive, Baltimore, MD, 21218 


\section{Scientific Organisation Committee:}

Garth D. Illingworth (Chair), University of California

James Roger Angel, University of Arizona

Jacques M. Beckers, European Southern Observatory

James E. Gunn, Princeton University

Donald N.B. Hall, University of Hawaii

Malcolm Longair, Royal Observatory, Edinburgh

Hervey S. (Peter) Stockman, Space Telescope Science Institute

Edward J. Weiler, NASA Headquarters

\section{Local Organisation Committee:}

Pierre Y. Bely (Chair)

Christopher Burrows

Barbara Eller

Hervey S. (Peter) Stockman 
TABLE OF CONTENTS

Foreword . . . . . . . . . . ......... 1

Conclusions of the Workshop . . . . . . . . . . . . . . 5

Sage Advice . . . . . . . . . . . . . . . . . . . . . . 7

SESSION 1. INTRODUCTION AND PLANS

Summary of Space Science Board 1995-2015 Study, G. Field, CFA . . . . . 11-

Status and Future of NASA Astrophysics, E. Weiler, NASA Headquarters . . 16

ESA Long Term Plans and Status, F. Macchetto, STScI . . . . . . . . . . 27-2

The Next Generation UV-Visible-IR Space Telescope G. Illingworth, UCSC . 31- 3

SESSION 2. SCIENTIFIC POTENTIAL

NGST and Distant Galaxies, J. Gunn, Princeton . . . . . . . . . . . . . 39-4

Planetary Astronomy with a Large Space Telescope, R. Brown, STScI . . . $44-5$

Star Formation Studies with a Large Space Telescope, L. Blitz, U of Maryland $49^{-6}$

Quasi-stellar Objects and Active Galactic Nuclei: Prospects for

a 10 meter Space Telescope, J. Miller, Lick Obs. . . . . . . . . . . . . 54 -

Stellar Populations in Galaxies: the Scientific Potential for a

10-16 m Space Telescope, J. Gallagher, AURA . . . . . . . . . . . . 617

Quasar Absorption-line Studies with HST Successor, R. Green, NOAO . . . $72-8$

Use of 16m Telescope to Detect Earthlike Planets, R. Angel, Steward Obs. . 81

SESSION 3. LARGE OPTICS: GROUND-BASED DEVELOPMENTS

The Keck Telescope Project, J. Nelson, Lawrence Berkeley Lab., UCB ～. . $99^{-16}$

Stressed-lap Polishing, H.M. Martin and J.R.P Angel, Steward Obs. . . $101-/ /$

Large Telescope O-IR Astronomy from the Ground,

N. Woolf, Steward Obs.

\section{SESSION 4. CURRENT PROPOSALS AND STUDIES}

Considerations for a Next Generation UV-Optical Space Telescope,

M. Nein and S. H. Morgan, NASA MSFC . . . . . . . . . . . . . . . $117 \%$

IBIS: An Interferometer-Based Imaging System for Detecting Extrasolar

Planets with a Next Generation Space Telescope, D. Diner, JPL . . . . 133

The Large Deployable Reflector, P. Swanson, JPL ～. . . . . . . . . . . 142

PAMELA: High Density Segmentation for Large, Jltra-light,

High Performance Mirrors, J. Rather et al, Kaman Aerospace Corp. . . . 147 -15

Optical Interferometry from Space, R. Stachnik, NASA Headquarters . . . . 157

Direct IR Interferometric Detection of Extrasolar Planets, M. Shao, JPL . . 160 -// 
Design Concepts for Very Large Aperture, High-performance Telescopes,

D. Korsch, Korsch Optics . . . . . . . . . . . . . . . . . . . . . . $171-17$

The Lunar Configurable Array Telescope (ICAT), A. Meinel/M. Meinel, JPL $177-18$

Large Optical Pabrication Considerations and Speculation about

the Overall NGST Configuration, M. Krim, Perkin Elmer . . . . . . . . 182 -om

Large Segmented Optics Fabrication and Wavefront Control, R. Vyce, Itek $198-/ \%$

Fabrication of Large and Fast Mirrors with Extroardinary Smooth Surfaces,

D. Knohl, Carl Zeiss . . . . . . . . . . . . . . . . . . . . . . . . 209-26

Economical Production of Large Optics, D. Pileri and W. Prensky, Kodak . . 217-

Super Smooth Optics for Extrasolar Planet Detection,

R.J. Terrile, JPL, and C. Ftaclas, Hughes . . . . . . . . . . . . . . . $225-22$

Sensing and Control for Large Optical Systems, A. Wissinger, Perkin Elmer . 232- -3

Requirements for Diffraction Limited Optics, C. Burrows, STScI . . . . . . 241

Precision Segmented Reflectors (PSR), R. Lin, JPL . . . . . . . . . . . 252

ASCIE: An Integrated Experiment to Study Control Structure Interaction in

Large Segmented Optical Systems, J-N Aubrun and K. Lorell, LMSC . . . 256

Control Structure Interaction (CSI) Technology, W. Layman, JPL . . . . . . 263

Pointing Control System for a 10 meter Space Telescope, D. Tenerelli, LMSC 270

Moving Target Tracking for a Next Generation Space Telescope,

D. Skillman, NASA GSFC . . . . . . . . . . . . . . . . . . . . . . 274

Passive Cooling of a 10 meter Space Telescope, P. Tulkoff, Swales . . . . . 279

Cryogenics for Space Observatories: Technology, Requirements, Issues,

H. Schember, JPL . . . . . . . . . . . . . . . . . . . . . . . . . 285

Visible and UV Detectors for High Earth Orbit and Lunar Observatories,

B. Woodgate, NASA GSFC . . . . . . . . . . . . . . . . . . . . . 296

Infrared Detectors for a $10 \mathrm{~m}$ Space or Lunar Telescope,

R. Thompson, Steward Obs.

\section{SESSION 6. SPACE LOGISTICS}

Advantages of High vs. Low Earth Orbit for SIRTF,

P. Eisenhardt and M. Werner, NASA Ames

Orbital Sites Tradeoff Study,

D. Neill, P. Bely, G. Miller, and A. Spigler, STScI . . . . . . . . . . . . 333

The Moon as a Site for Astronomical Observatories,

J. Burns, New Mexico State U.

Required Technologies for a 10-16 m UV-Visible-IR Telescope on the Moon,

S. Johnson and J. Wetzel, BDM Corp. . . . . . . . . . . . . . . . . . 348

Space Logistics: Launching Capabilities, R. Furnas, NASA Headquarters . . . 360

On-orbit Assembly and Maintenance, D. Soderblom, STScI . . . . . . . . 365

Technological Spinoff from the Next Generation, V. Trimble, UMD/UCI . 367

List of Participants . . . . . . . . . . . . . . . . . . . . . . . . . . . . 369 


\section{FOREWORD}

In Space Science in the Twenty-First Century, the Space Science Board of the National Research Council identified high-resolution interferometry and high-throughput instruments as the imperative new initiatives for NASA in astronomy for the two decades spanning 1995 to 2015 . In the optical range, the study recommended an 8 to 16-meter space telescope, destined to be the successor of the Hubble Space Telescope (HST), and to complement the ground-based 8 to 10-meter-class telescopes presently under construction.

It might seem too early to start planning for a successor to HST. In fact, we are late. The lead time for such major missions is typically 25 years, and HST has been in the making even longer with its inception dating back to the early 1960s. The maturity of space technology and a more substantial technological base may lead to a shorter time scale for the development of the Next Generation Space Telescope (NGST). Optimistically, one could therefore anticipate that NGST be flown as early as 2010 . On the other hand, the planned lifetime of HST is 15 years. So, even under the best circumstances, there will be a five year gap between the end of HST and the start of NGST.

The purpose of this first workshop dedicated to NGST was to survey its scientific potential and technical challenges. The three-day meeting brought together 130 astronomers and engineers from government, industry and universities. Participants explored the technologies needed for building and operating the observatory, reviewed the current status and future prospects for astronomical instrumentation, and discussed the launch and space support capabilities likely to be available in the next decade. To focus discussion, the invited speakers were asked to base their presentations on two nominal concepts, a 10-meter telescope in space in high earth orbit, and a 16-meter telescope on the moon. Artist's view of these two concepts are shown in Figures 1 and 2, and their specifications are summarized in Table 1.

The workshop closed with a panel discussion focused mainly on the scientific case, siting, and the programmatic approach needed to bring NGST into being. The essential points of this panel discussion have been incorporated into a series of recommendations that represent the conclusions of the workshop.

Speakers were asked to provide manuscripts of their presentation. Those received were reproduced here with only minor editorial changes. The few missing papers have been replaced by the presentation viewgraphs. The discussion that follows each speaker's paper was derived from the question and answer sheets, or if unavailable, from the tapes of the meeting. In the latter case, the editors have made every effort to faithfully represent the discussion.

We are most thankful to all the speakers for their very thoughtful and valuable contributions. Their vast experience in science and engineering will be essential for the successful completion of a project of this scale. Thanks are due to Roger Angel, Jack Burns, Don Hall, Duccio Macchetto, Joe Miller, Jean Olivier, Peter Stockman, Dominick Tenerelli, and Rodger Thompson for chairing the various sessions or for their participation in the panel. We would also like to thank John Bahcall who introduced the workshop by sharing some of his experiences with the HST project. His pertinent remarks about the dedication of those involved in the development of HST emphasized the deep and widespread commitment needed to bring about its successor.

We would particularly like to thank Riccardo Giacconi for his keen interest and support of the workshop. He had urged us for some time to think of the long-term needs of the 
astrophysics community and to explore the scientific potential and technical challenges of a successor to HST. We also greatly appreciate the support given by Peter Stockman. He contributed invaluable advice and assistance throughout, ensured that the appropriate Institute resources were available, and gave an excellent summary of the meeting.

We would also like to extend our gratitude to Charles Pellerin for personally supporting the meeting and providing NASA funding for the publication of these proceedings.

This workshop is but an early step on the long journey to the completion of NGST. As evidenced by these proceedings, however, the spectacular views of the heavens to be provided by this telescope and a deeper understanding of our universe and its origin are a worthy destination for this complex and challenging journey.

The Editors 
Table 1. Nominal Next Generation Space Telescope (NGST)

\begin{tabular}{|c|c|c|}
\hline & Space-based & Lunar-based \\
\hline Aperture diameter & $10 \mathrm{~m}$ & $16 \mathrm{~m}$ \\
\hline Spectral Coverage & 0.12 to $10 \mu \mathrm{m}$ & 0.12 to $10 \mu \mathrm{m}$ \\
\hline Optics quality & diff. limtd $\lambda / 40$ & diff. $\operatorname{limtd} \lambda / 40$ \\
\hline Primary & monolithic or segmented & segmented \\
\hline Primary mirror f/ratio & $f / 1.2$ & $f / 1.5$ \\
\hline Optical combination & Ritchtey-Chretien & Ritchtey-Chretien \\
\hline Cassegrain focal ratio & $\mathbf{f} / 15$ & $f / 15$ \\
\hline \multicolumn{3}{|l|}{ Angular resolution: } \\
\hline UV & 3 mas & 2 mas \\
\hline $\mathbf{V}$ & 14 mas & 9 mas \\
\hline IR $(3 \mu)$ & 73 mas & 46 mas \\
\hline Guiding field diameter & $2.5^{\prime}$ & 2' \\
\hline Guide star magnitude & $17^{\text {th }}$ & $18^{\text {th }}$ \\
\hline Fine guidance - pitch/yaw & field star & field star \\
\hline Fine guidance - zoll & star tracker & calculation \\
\hline Tracking accrcy. - coarse & $0.1 ”$ & $0.1^{\prime \prime}$ \\
\hline Tracking accrcy. - fine (beam steering) & $0.3 \mathrm{mas}$ & 0.2 mas \\
\hline Operating temperature & $80^{\circ} \mathrm{K}$ & $100^{\circ} \mathrm{K}$ (night) \\
\hline Site & High Earth Orbit & See note 1 \\
\hline \multicolumn{3}{|l|}{ Bright object avoidance: } \\
\hline sun & $90^{\circ}$ & See note 2 \\
\hline moon & $40^{\circ}$ & - \\
\hline earth & $40^{\circ}$ & shielded \\
\hline Power & $5 \mathrm{~kW}$ & $5 \mathrm{~kW}$ \\
\hline Mass & $20 \mathrm{t}$ (goal) & $40 t$ \\
\hline Data rate & $10 \mathrm{Mbit} / \mathrm{s}$ & $10 \mathrm{Mbit} / \mathrm{s}$ \\
\hline
\end{tabular}

Notes:

1. Three types of lunar sites are considered:

- Near the equator and near the limb of the near side. Such sites have good sky coverage, direct communication with the Earth, and may be naturaly protected against earthshine if located in a crater. Their drawbacks are a limited to impractical observation during lunar day and large day/night temperature change $\left(300^{\circ} \mathrm{C}\right)$.

- Near the equator on the far side. Such sites have good sky coverage and do not require baffling against earthshine. Their drawbacks are a limited to impractical observation during lunar day and large day/night temperature change $\left(300^{\circ} \mathrm{C}\right)$. In addition they need relays for communication with the Earth.

- At one of the poles in a permanently shadowed location. Continuous shadowing is possible at the poles because the Moon's polar axis is nearly perpendicular to ecliptic. The advantages are lower operation temperature $\left(70^{\circ} \mathrm{K}\right.$ or less), no day/night thermal cycling, and possibly direct communication with earth. Their drawback is that only half of the sky is observable.

2. Daytime observation on the moon is difficult because of the thermal load and the high level of straylight. However, a limited class of programs may be possible with proper shielding and cooling. 


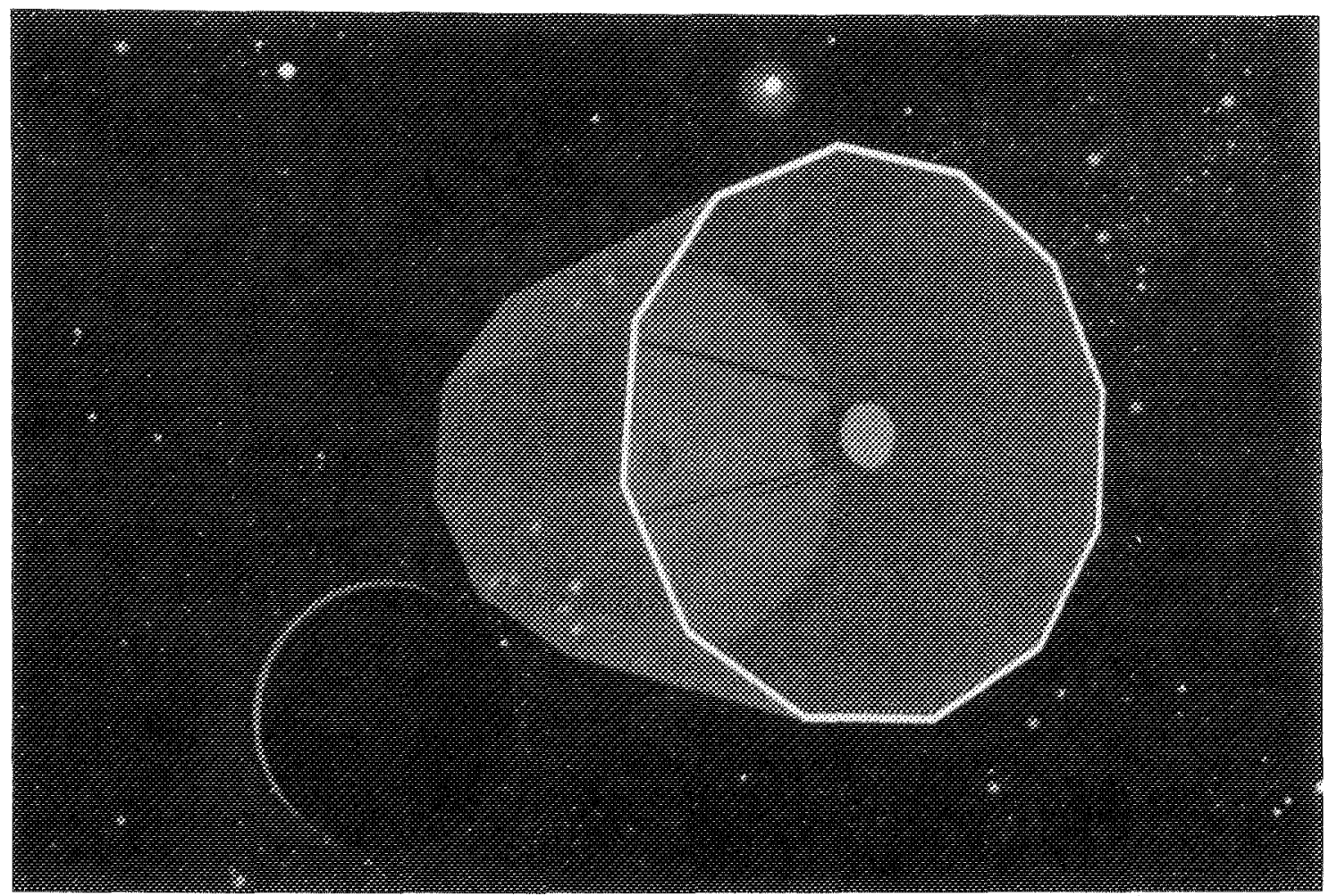

Tigure 1. Artist's concept of a 10 meter telescope in high earth orbit. The telescope is very compact thanks to a fast primary and the short baffle that a relaxation of sun, moon and bright earth avoidance angles in high orbit permits. Solar panels are fixed on the rear of the spacecraft to minimize mechanical disturbances.

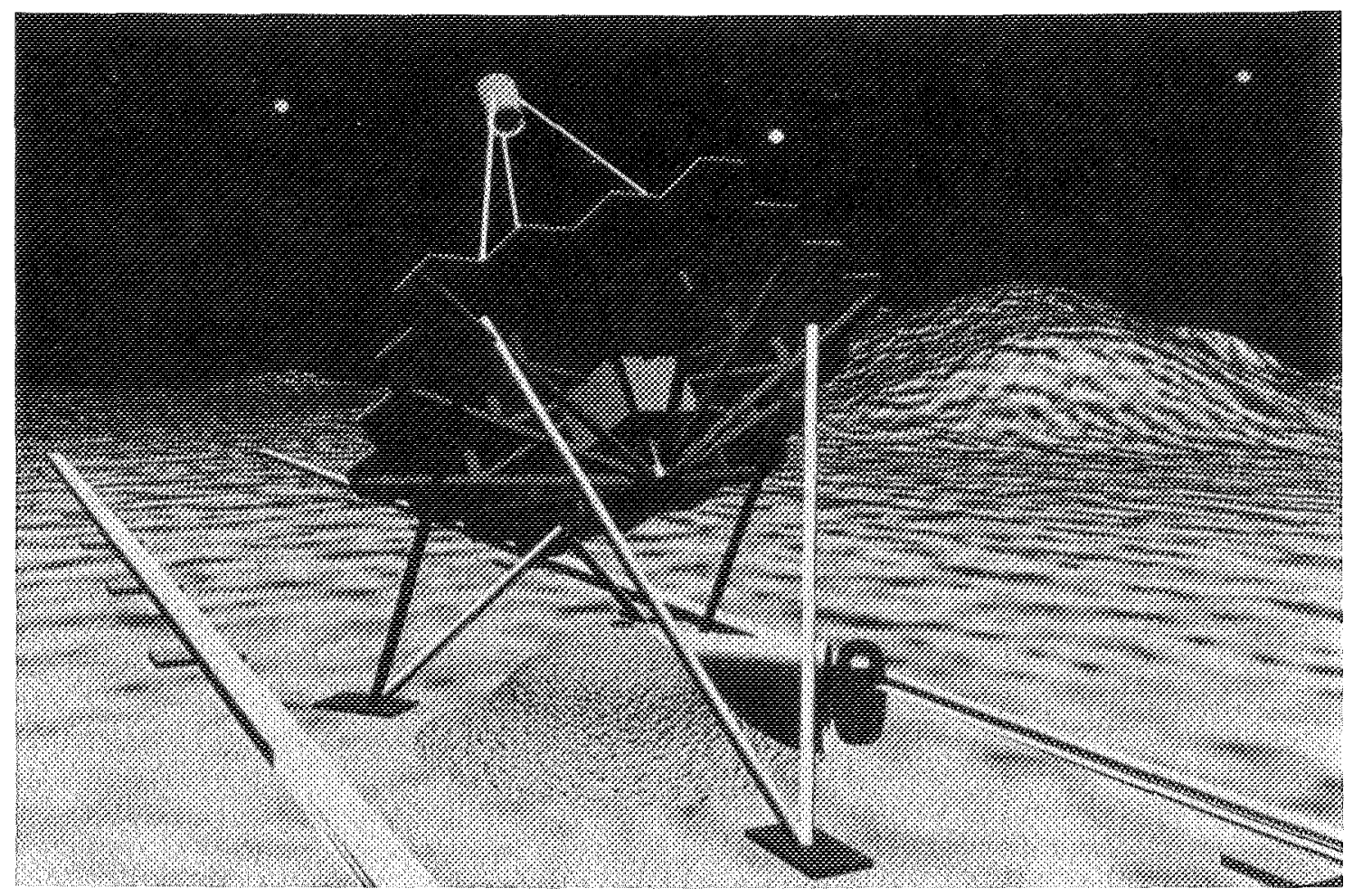

Tigure 2. Artist's concept of a 16 meter telescope on the moon. The segmented primary mirror is supported by a hexapod mount, the legs of which are extendable for pointing and tracking. A coudé-like arrangement is used to feed the scientific instruments which are located underground for radiation and meteorite protection. The rails are for a hangar-type shield which is rolled over the telescope during lunar day. 


\section{CONCLUSIONS OF THE WORKSHOP}

Formal conclusions were not drawn explicitly before adjournment of the Workshop. However, a consensus clearly emerged, especially during the panel discussion. The following statements and recommendations which were developed after the workshop, based on tapes of the panel discussion, are believed to reflect the spirit of this collective opinion.

\section{Scientific objectives:}

There will be a definitive need to continue and extend the observational capability offered by HST beyond its predicted lifetime. A gap of more than 5 years would be a blow to the vitality of forefront astronomical research.

The scientific potential of an HST follow-up mission with enhanced flux collecting power and spatial resolution, and with spectral coverage extended through the nearinfrared is enormous. It is viewed as complementary to large-baseline space interferometry missions which emphasize high spatial resolution imagery. An observatory providing high sensitivity and high-throughput spectroscopic capability at diffraction-limited spatial resolution from the UV to beyond 10 microns is vital for the study of the most fundamental questions of astrophysics. These include the formation and evolution of galaxies, stars and planets, and the nature of the young universe.

\section{Technological readiness:}

A telescope in the 10-16 m class is not an unrealistically large step beyond the current state of technical development. While development and demonstration programs are clearly needed, many of the core technologies are maturing to the point where the required goals appear to be within a very reasonable extrapolation of the current state-of-the-art. In particular, advances in the fabrication of lightweight optics and new techniques for polishing have the potential for very substantial weight savings and hence cost savings, while offering optical performance beyond what was possible in the past.

\section{Siting:}

Both the moon and high earth orbits are suitable sites for a next generation space telescope. Low earth orbits are undesirable because of high disturbance levels, insufficient passive cooling and low observing efficiency. Compared to a high earth orbit which would likely not be serviceable, a lunar site would permit maintenance and upgrading and thus longer amortization for any major scientific and financial investment. In addition, it would provide a very stable platform for the demanding pointing and tracking requirements, as well as having advantages for the shielding of detectors. Although the moon appears very attractive in view of the current Lunar Outpost initiative, an immediate commitment to that program or other NASA infrastructures is not required at this time. Space-based and lunar-based designs should be pursued in parallel for the next few years to clarify the observational, technical, space logistical and cost tradeoffs.

\section{Programmatic approach:}

A $10-16 \mathrm{~m}$ (space-based) to $16 \mathrm{~m}$ (lunar-based) aperture is considered a realistic goal. Future workshops should concentrate on further definition of the scientific objectives, 
review of preliminary studies and the identification of critical technologies. Strawman designs should be prepared to refine the various concepts and ideas and focus discussion.

A "Telescope Design Group" should be formed to guide and oversee the preliminary design work. This group, comprised of astronomers and technologists, should address the scientific goals and technical requirements together, paying particular attention to system engineering. In projects of this complexity, efficient design is the result of many compromises that can only be developed by successive iterations and by system-level analyses. The importance of this iterative process involving astronomers, physicists and engineers in the science-engineering tradeoffs and in defining the requirements was emphasized by many participants. The involvement of these different groups needs to occur during all phases of the project, from concept development, through technology development and fabrication, and finally during system-level testing.

Once clearly identified by the preliminary design process, the development of the key enabling technologies should be integrated with the appropriate long-term program of the national and international Space Agencies (e.g., NASA's Technology 21). This will ensure both that the technological requirements are addressed at the proper level, and that benefits are obtained from interaction with other programs.

\section{International cooperation:}

Like HST, the next generation space telescope project should be carried out cooperatively as an international program. Cost sharing renders such major missions more affordable for each participating country, and international collaboration often enhances quality and performance. Complex and pioneering space missions also benefit from the exchange of ideas and variety of approaches afforded by multicultural collaborations. 


\section{SAGE ADVICE}

"International cooperation may be critical for such a major project". Bahcall

"It's not often that we have a chance to participate in history". Danielson (as quoted by Bahcall)

"Astronomers will become addicted to HST observing". Ilingworth

"In comparison to ground-based uses, adaptive optics in space should be easy". Angel

"In space, only a filled aperture telescope makes sense". Swanson

"Don't underestimate the difficulty of achieving the required surface error". Swanson

"We should not simply accept a traditional R-C design for a 10 meter telescope". Korsch

"The more money that we put up front, the cheaper the next telescope will be". Breckinridge

"One of the things which should come from this workshop is a better understanding of the merits of filled apertures and interferometers". Breckinridge

"Time is the great enemy". Krim

"I don't want you to think that $100^{\circ} \mathrm{K}$ is guaranteed". Schember

"I recommend that you think beyond Shuttle C for the next ST". Furnas

"On M\&R, my rule of thumb is don't do it!". Soderblom

Collected during the Workshop by Pete Stockman 


\section{Page intentionally left blank}


Session 1

Introduction and Plans 


\section{Page intentionally left blank}


George Field

Center for Astrophysics

\section{Introduction}

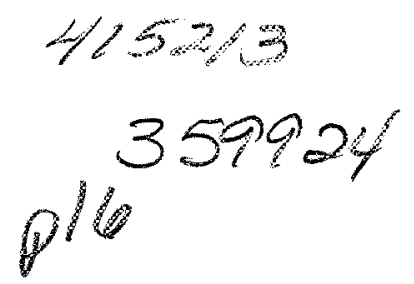

In 1984, NASA requested the Space Science Board to undertake a study to determine the principal scientific issues that the disciplines of space science would face during the period from about 1995 to $2015 .^{1}$ Six Task Groups were established, including one on Astronomy and Astrophysics, chaired by Bernie Burke. As Bernie could not be here, and I had served on both the Astronomy and Astrophysics Task Group and the Steering Group of the Study, I agreed to report on its activities in his absence here today. Roger Angel, a participant in this Workshop, was also a member of the Astronomy and Astrophysics Task Group.

\section{The Space Science Board Report: Space Science in the Twenty-First Century}

On page 46 of Space Science in the Twenty-First Century - Overview ${ }^{1}$ the recommended program for astronomy and astrophysics is given under three headings: (1) imaging interferometry, (2) large area and high-throughput telescopes, and (3) AstroMag, a massive cosmic-ray analyzer in space. Item $(2 \mathrm{~b})$ is an 8-16 meter optical space telescope, the subject of this Workshop. The same Report, in the volume on Astronomy and Astrophysics, ${ }^{2}$ p. 48 , states that,

"A large-aperture space telescope for the ultraviolet, optical, and infrared regions has immense scientific potential. The need for such a telescope will be very high after 10 to 20 years of use of HST and ground-based 8- to 10-m-class telescopes. Even now we see that some of the most fundamental of all astronomical questions will require the power of a filled-aperture telescope of 8- to 16-m diameter designed to cover a wavelength range of $912 \AA$ to $30 \mu \mathrm{m}$, with ambient cooling to $100^{\circ} \mathrm{K}$ to maximize the infrared performance.

"With the HST and SIRTF still to be launched, and the anticipated wealth of data not yet analyzed, it is difficult but not premature to formulate a detailed concept of such a large-scale telescope for the ultraviolet, optical, and infrared regions. This telescope will also complement a space interferometer. The diffraction limited resolution of a $15-\mathrm{m}$ telescope is six times sharper than that of the HST; it would be far more sensitive, both because of its greater collecting area and because of the small size of its diffraction-limited image. The image is $1 / 100$ arcsec in diameter for a 15 -m telescope in visible light. At this resolution the reflected zodiacal background is reached at magnitude 33, about 11 magnitudes fainter than ground-based telescopes, and four magnitudes fainter than HST.

"The large telescope would not simply be a scaled-up HST. Its infrared performance would be optimized by cooling the optics to at least the lower limit of passive radiation methods, about $100^{\circ} \mathrm{K}$. Similarly, the optical performance would be greatly enhanced by a wide-field optical design suitable for larger, high-resolution detector mosaics and multiple-image spectroscopy. Images with $10^{10}$ picture elements are possible with present optical designs, and the technology for charged coupled device (CCD) mosaics this large is advancing. 
"A large range of scientific problems could be undertaken only by a telescope of this type. The combination of light-gathering power and resolution offered by such a telescope, equipped with advanced spectrographs and detectors, would lead to a quantum leap in our understanding of some of the most fundamental questions in astronomy."

There follow six pages of dicussion of scientific objectives for an 8- to 16-m telescope.

On p. 55 of the same Report ${ }^{2}$ there is a section on technological developments, including a discussion of methods for fabricating the telescope. In particular, it is stated that,

"Two possible avenues are available for orbiting the large telescope. It seems likely that in the time frame under consideration large vehicles could launch a prefabricated telescope up to $8 \mathrm{~m}$ in diameter. Alternatively, for a 16-m diameter telescope, construction in orbit is probably the best route. Mirror segments would be polished and tested on the ground and assembled onto a frame structure built in space."

"Large telescopes designed to operate in a zero-g environment, but which do not have to withstand launch, are an exciting challenge to designers and engineers. Given a well-directed technology development program, the task group anticipates that an 8to 16 -meter telescope will be within closer reach than a simple extrapolation from HST would suggest."

Thus, the assessment of an 8- to 16-m space telescope by the Task Group on Astronomy and Astrophysics is relatively optimistic. It should be noted that as explained above, the large telescope is only one of the recommended elements of the program for astronomy and astrophysics in the 1995-2015 time frame. There are four other large-area telescopes for submillimeter, X-ray, and gamma-ray astronomy, and three facilities for imaging interferometry at optical and radio wavelengths, including one, the Large Space Telescope Array of nine 1.5-meter optical telescopes mounted on a tetrahedral structure $100 \mathrm{~m}$ or so in diameter, aimed for milliarcsecond resolution with about $20 \mathrm{~m}^{2}$ collecting area, about $1 / 4$ that of the large telescope.

The Task Group briefly addressed the question of cost, and assumed (p.68) that for each wavelength regime only one observatory class facility will operate at a time, that new facilities will be developed soon enough to prevent the occurence of substantial gaps in observational capability, and that new facilites will cost about twice as much as the ones they replace. On this basis, the Task Group concluded that the base program (Great Observatories and their replacements) can be carried out if the annual real-dollar NASA astrophysics budget increases over FY 85 at $2.3 \%$ per year. If the large telescope is taken to be the replacement for HST, one infers that the Task Group believes that it can be accomplished for about $\$ 4 \mathrm{~B}$ in 1986 dollars. I will retum to the question of cost in the last section, below.

The Astronomy and Astrophysics Task Group wrote an Appendix entitled, "Astrophysics at a Lunar Base," which, however, does not appear as part of its Report.

In its Overview volume, ${ }^{1}$ the Steering Group accepted the main outlines of the Task Group Repon, including the $8-\mathrm{m}$ to $16-\mathrm{m}$ telescope. In chapter 9 of the Overview there is a discussion of Human Presence in Space in which it is argued that if human presence extends further into the solar system, brief forays of human beings to remote places are not very useful for science. On the other hand, much useful science can be done by humans located at various stations (earth orbit, Moon, Mars) for long periods. It concludes ( $p$. 77) that 
"space science experiments, tended in space by human beings, may provide the most important rationale for the staging, assembly, maintenance, repair, and operation of major space facilities (e.g., space astronomical telescopes, earth science experiment payloads/platforms, launch vehicles for planetary missions)."

The Space Science Board Study was concluded in June, 1986, and the Report was published in 1988 .

\section{The Report of the National Commission on Space: Pioneering the Space Frontier}

In 1985 Congress chartered and the President appointed a National Commission on Space of 15 members, chaired by Dr. Torm Paine, former Administrator of NASA. As a member of the commission, I wrote the first draft of the section on space science in its Report, Pioneering the Space Frontier, published in $1986^{3}$. I drew heavily on the Space Science Board 1995-2015 Study ${ }^{1,2}$ discussed above. The National Commission on Space was charged with "formulating a bold agenda to carry the civilian space program into the 21st century." Told that the Space Station should be treated as a given in its deliberations, the Commission laid out a trillion-dollar plan that would result in a permanent Moon base in $2005 \mathrm{AD}$ and a Mars base in $2027 \mathrm{AD}$. Considerable space was given to science generally and to astronomy in particular. On p. 9, the Report3 recommends "a sustained program to understand the evolution of the Universe, through astronomical facilities of increasing power, precision, and sophistication at locations in more distant Earth orbits and at eventual locations on the Moon."

On p. 50, it states that

"Astronomical instruments can be located in low Earth orbit, geostationary orbit, solar orbit, or on the surface of the Moon. With a few notable exceptions, most of them have been in low Earth orbit, and in the near future will continue to be located there because of lower cost and direct support from the Space Station. As a lunar base is developed, astronomers will take advantage of the ability to build rigid telescope mounts, of the freedom from contamination by propellants, of the longer and colder nights, and of the shielding from Earth light and radio interference which are available on the far side of the Moon."

The recommendations of the Space Science Board 1995-2015 Study were transmuted (p. 52) into one for

"a large space telescope array composed of 25-foot $(8-\mathrm{m})$ diameter telescopes [to] operate in the ultraviolet, visible, and infrared. The combination of large diameter telescopes with a large number of telescopes would make this instrument 100 times more sensitive than Hubble Space Telescope. Because the image would be three times sharper, the limiting faintness for long exposures would increase more than 100 times."

This recommendation is broadly consistent with the Space Science Board recommendations for both the 8 - to $10-\mathrm{m}$ optical space telescope and the large space telescope array.

\section{The Ride Report: Leadership and America's Future in Space}

NASA Administrator Dr. James Fletcher formed a task group to define potential U.S. space initiatives, and to evaluate them in the light of the current space program and the nation's desire to regain and retain space leadership. Its report, Leadership and America's Future in Space, ${ }^{4}$ published in August 1987, can be viewed as a NASA response to the 
Report of the Rogers Commission to investigate the Challenger accident and the Paine Commission on the future of the space program. It narrowed the field of new initiatives beyond the current base program to four options:

(1) Mission to Planet Earth

(2) Exploration of the Solar System

(3) Outpost on the Moon

(4) Humans to Mars.

Briefly, Mission to Planet Earth would use the perspective afforded from space to characterize the Earth on a global scale; Exploration of the Solar System would retain U.S. leadership in the (unmanned) exploration of the outer solar system and regain U.S. leadership in exploration of comets, asteroids, and Mars; the Outpost on the Moon would return Americans to the Moon to continue exploration, to establish a permanent scientific outpost, and to begin prospecting for resources; and Humans to Mars would send astronauts on three round trips to land on the surface of Mars, leading to the eventual establishment of a permanent base. In view of the fact that astronomy is not mentioned in the Ride Report under options (1) or (2), one may surmise that the space astronomy program would not be highlighted if they are adopted. Astronomy is mentioned under Outpost on the Moon (p. 30), where it is stated that, "The Moon's unique environment provides the opportunity for significant scientific advances; the prospects for gains in lunar and planetary science is abundantly clear. Additionally, since the Moon is seismically stable and has no atmosphere, and since its far side is shielded from radio noise from Earth, it is a very attractive spot for experiments and observations in astrophysics, gravity-wave physics, and neutrino physics, to name a few." Astronomy is not mentioned under Humans to Mars. In concluding chapters, the Ride Report recommends that NASA "should embrace the Mission to Planet Earth," that "although not necessarily at the pace suggested in this initiative, planetary exploration must be solidly supported." The Ride Report is not enthusiastic about an early effort to land humans on Mars, but is favorable toward the timely establishment of an Outpost on the Moon.

Apparently the National Space Council is moving toward a decision as to which, if any, of the Ride Report initiatives to recommend to the President. This would have profound implications for the 10- to 16-m telescope being discussed in this Workshop. If the choice is to retum to the Moon, one may anticipate substantial increases in the NASA budget. Although Figures 14 and 15 on p. 46 of the Ride Report are not labelled in dollar amounts, one can surmise that starting in the mid 90's, NASA's budget would have to triple for either the Outpost on the Moon or the Humans to Mars programs to be carried out. On the other hand, Mission to Planet Earth or Exploration of the Solar System would apparently require only about a $30 \%$ increase in the NASA budget. I conclude that in the latter case, funds available for the $8-\mathrm{m}$ to $16-\mathrm{m}$ telescope will be little more than for the telescope it replaces, the HST. What might a telescope in Earth orbit actually cost? In connection with a study of the cost of space-based laser ballistic missile defense I carried out with David Spergel, ${ }^{5}$ I had occasion to look into the cost one might estimate for diffraction-limited optical systems in low earth orbit, including the associated systems for target acquisition and tracking (but not launch or maintenance). Our best estimate for the cost of a system of aperture $D$ (meters) was $\$ 3.6(\mathrm{D} / 10)^{1.7}$ billion in 1984 dollars, or $\$ 3.8(\mathrm{D} / 10)^{1.7}$ billion in 1986 dollars, consistent with the $\$ 4 \mathrm{~B}$ implied by the Space Science Board Study (see above). Hence I agree that a 10-meter in low earth orbit seems 
reasonable on fiscal grounds.

For a $16-\mathrm{m}$ on the Moon the scaling law indicated above would suggest an increase by a factor of 2.2 , up to $\$ 8.4 \mathrm{~B}$ in 1986 dollars, which might be acceptable under a much expanded (about three-fold) NASA program driven by an Outpost on the Moon. However, there will be major cost differentials between basing in earth orbit and on the Moon. Clearly the question of cost will be vital to address as planning proceeds.

\section{References}

1. Space Science in the Twenty-First Century: Imperatives for the Decades 1995-2015. In seven volumes; Volume I, Overview. Report of the Study Steering Group, Space Science Board, Commission of Physical Sciences, Mathematics, and Resources, National Research Council (Washington, D.C.: National Academy Press), 1988.

2. Ibid., Volume II, Astronomy and Astrophysics. Report of the Task Group on Astronomy and Astrophysics, Space Science Board, Commission on Physical Sciences, Mathematics, and Resources, National Research Council. (Washington, D.C.: National Academy Press), 1988.

3. Pioneering the Space Frontier: The Report of the National Commission on Space (New York: Bantam Books), 1986.

4. Leadership and America's Future in Space, by Sally K. Ride (Report to the Administrator of NASA, August 1987).

5. "Cost of Space-Based Laser Ballistic Missile Defense," by George Field and David Spergel, Science, 231, 1387-1393 (1986).

\section{DISCUSSION}

Pilcher: There was another NASA report following the Ride Report entitled Beyond Earth's Boundaries, the first annual report of the Office of Exploration (OESP). OEXP was formed in 1987 at Sally Ride's recommendation to provide an institutional home within NASA for human exploration of the solar system. In Beyond Earth's Boundaries, OEXP reported on a group of case studies including one on a lunar observatory. That case study was based in part on papers presented at a series of workshops including Future Astronomical Observatories on the Moon (held in 1986 at JSC, proceedings published as orange covered NASA Conference Proceedings); Lunar Bases and Space Activities of the 21st Century (held in 1984, proceedings published in 1985 by the Lunar and Planetary Institute, Houston); and A Lunar Farside Very Low Frequency Array (held at Univ. New Mexico, Albuquerque; Proceedings in Press). 
Status and Future of NASA Astrophysics

Edward J. Weiler

NASA Headquarters

\section{PART 1}

\section{STATUS OF CURRENT PROGRAM}

\section{HUBBLE SPACE TELESCOPE (HST)}

- FIRST OF FOUR GREAT OBSERVATORIES

- AMONG NASA'S hIGHEST PRIORITY PROgRAMS

- 2.4 METER APERTURE DIFFRACTION-LIMITED "OPTICAL" TELESCOPE

- SIX INITIAL INSTRUMENTS

- FOC

- HRS

- WF/PC

- HSP

- FOS

- FGS

- 1150 A TO 1.1 MICRON MEASURING RANGE

- 15 YEAR LIFETIME ACHIEVABLE WITH ON-ORBIT SERVICING

CURRENT STATUS: TELESCOPE SHIPMENT TO KSC IN OCTOBER 1989; LAUNCH IN SPRING 1990. 


\section{GAMMA RAY OBSERVATORY (GRO)

- gamma RAY ElEMENT Of GREAT OBSERVATORY PROGRAM

- FOUR INSTRUMENTS

- EGRET - OSSE

- COMPTEL $\quad$ BATSE

- WILL EXAMINE WAVELENGTH RANGE FROM 0.05 TO 30,000 MILLION ELECTRON VOLTS

- 2 YEAR LIFETIME THAT CAN BE EXTENDED TO 10 YEARS THROUGH OCCASIONAL ALTITUDE REBOOST WITH PROPULSION SUBSYSTEM

\section{ADVANCED X-RAY ASTROPHYICS FACILITY (AXAF)}

- X-RAY ElEMENT OF GREAT OBSERVATORY PROGRAM

- INCLUDES SET OF 6 GRAZING INCIDENCE MIRRORS; OVERALL APERTURE OF 1.2 METERS

- DESIGNED FOR ENERGY RANGE OF $0.1 \mathrm{KEV}$ TO $10 \mathrm{KEV}$

- UP TO 4 FOCAL PLANE SCIENCE INSTRUMENTS

- 1700 SQUARE CM TELESCOPE COLLECTING AREA; 0.5 ARC SECOND ANGULAR RESOLUTION; PROVIDES 100-FOLD INCREASE OVER ITS PREDECESSOR (HEAO-2)

- HAS goAL OF 15 YEAR OPERATIONAL LIFETIME

CURRENT STATUS: EARLY IN DEVELOPMENT; REQUESTED FLIGHT - LATE 1995 


\section{SPACE INFRARED TELESCOPE FACILITY (SIRTF)}

- $\quad$ LAST OF the gREAT observatories

- CRYO COOLED TELESCOPE WILL EXCEED SENSITIVITY OF IRAS BY 3 ORDERS OF MAGNITUDE

- $\quad$ THREE SCIENCE INSTRUMENTS

- INFRARED SPECTROMETER (IRS)

- INFRARED ARRAY CAMERA (IRAC)

- MULTIBAND IMAGING PHOTOMETER (MIPS)

- PRIMARILY GUEST OBSERVER PROgRAM

- $\quad$ PLANNED LIFETIME Of AT LEAST 5 YEARS IN 100,000 KM ORBIT

CURRENT STATUS: PHASE B RFP UNDER EVALUATION; IN OSSA

STRATEGIC PLAN FOR 1993 NEW START

\section{ASTRO - 1,2}

- SPACELAB PaYload WITH Four SEPARATE COMPLEMENTARY OPTICAL TELESCOPES

- THREE CO-POINTED FAR UV TELESCOPES: HUT, UIT, WUPPE

- BBXRT ON SEPARATE POINTER (TAPS)

- TOGETHER, OBTAIN MEASUREMENTS AT WAVELENGTHS BETWEEN 0.12 AND $3500 \AA$

- 200 - 300 INDEPENDENT OBSERVATIONS DURING TYPICAL 9-10 DAY MISSION

- ASTRO-2 TO INCLUDE GUEST OBSERVER PROGRAM

CURRENT STATUS: BBXRT INSTRUMENT SHIPPED TO KSC; ASTRO-1 LAUNCH PLANNED FOR SPRING 1990; ASTRO-2 LAUNCH PLANNED FOR 1991 


\section{EXTREME ULTRAVIOLET EXPLORER}

(EUVE)

- WILL PRODUCE AN ALL SKY SURVEY COVERING 80 TO 900 A IN 4 BANDS; SPATIAL RESOLUTION OF 30 ARC SECONDS

- DEEP SURVEY WILL SCAN REGION TWO DEGREES WIDE BY 180 DEGREES LONG ALONG THE ECLIPTIC; COVERS 100 TO 500 Å RANGE IN 2 BANDS

- DETAILED SPECTROSCOPY FOLLOWS

- PAYLOAD CONSISTS OF FOUR GRAZING INCIDENCE TELESCOPES AND A VARIETY OF OPTICAL FILTERS

- LAUNCHED ON EXPLORER PLATFORM SPACECRAFT USING DELTA II ROCKET

- MISSION DURATION AT LEAST 3 YEARS; EXCHANGED WITH XTE PAYLOAD VIA ON-ORBIT SERVICING FROM SHUTTLE

CURRENT STATUS: INSTRUMENT DELIVERY TO GSFC IN EARLY 1990;

LAUNCH IN AUGUST 1991

\section{ORBITING AND RETRIEVABLE FAR AND EXTREME ULTRAVIOLET SPECTROMETER (ORFEUS)}

- ONE-METER CLASS German ULTRAVIOLET TELESCOPE; ONE U.S. AND ONE GERMAN SPECTROMETER - IMAPS ULTRA HIGH RESOLUTION SPECTROMETER

- CARAIED IN SPACE SHUTtLE ON ASTRO-SPAS CARRIER

- DEPLOYED FROM CARGO BAY DURING 7 DAY MISSION

- PERFORMS SPECTROSCOPIC INVESTIGATIONS OF FAR AND EXTREME U $V$ EMISSIONS FROM CELESTIAL OBJECTS IN THE 40 TO 120 NM WAVELENGTH RANGE INCLUDING 1987 A SUPERNOVA REMNANT

- RETRIEVED BY SHUTTLE AND RETURNED TO EARTH AT CONCLUSION OF MISSION CURRENT STATUS: APPROVED PROGRAM; LAUNCH IN 1992 


\section{HUBBLE SPACE TELESCOPE 2ND GENERATION INSTRUMENTS (HST 2ND GENERATION)}

- THREE NEW INSTRUMENTS

- NEAR INFRARED CAMERA AND MULTI-OBJECT SPECTROMETER (NICMOS)

- 0.8 TO 2.5 MICRON IMAGING AND SPECTROSCOPY

- ADVANCED DETECTORS; FULLY DIFFRACTION - LIMITED OPERATION

- WIDE FIELD/PLANETARY CAMERA CLONE (WF/PC II)

- REPLICATION OF WF/PC WITH UPGRADED FOCAL PLANE ARRAY

- SPACE TELESCOPE IMAGING SPECTROgRaPH (STIS)

- 2-D SPECTROGRAPH OPERATING AT 1150 TO 11,000 A

- 30 TO 100 TIMES BETTER EFFICIENCY THAN FOS/HRS

- FULL HST SPATIAL RESOlUTION; 1000 TIMES MORE ELEMENTS THAN FOS

- 40 TIMES MORE SPECTRUM AND 20 TIMES MORE ELEMENTS THAN HRS

- THIRD GENERATION INSTRUMENT(S) in 1998 - 1999

CURRENT STATUS: NICMOS IN PHASE B; PLANNED FOR FIRST SERVICING IN 1995 WF/PC II TO BE AVAILABLE IN EARLY 1993

STIS IN PHASE A; FLIGHT READY BY 1996

\section{FAR ULTRAVIOLET SPECTROMETER EXPLORER} (FUSE)

- SPECTROSCOPY IN 900 TO 1200 Å REGION AT HIGH RESOLUTION $(\approx 30,000)$

- LIMITING MAG $\approx 17 \mathrm{~m}_{\mathrm{v}}$

- SPECTROSCOPY IN 100 TO $900 \AA$ REGION AT MODERATE RESOLUTION $(\approx 500)$

- 10 - 100 TIMES MORE SENSITIVE THAN EUVE

CURRENT STATUS: FINISHED PHASE A; CANDIDATE FOR EXPLORER PLATFORM PAYLOAD AFTER XTE MISSION (AWAITING DR. FISK SELECTION FOR FLIGHT IN $\approx 1996$ ) 


\section{PART 2}

\section{TECHNOLOGY 21}

\section{OVERVIEW OF "TECH 21"}

- PURPOSE OF PROGRAM: TO IDENTIFY AND DEVELOP CRITICAL PACING TECHNOLOGIES FOR FUTURE ASTROPHYSICS MISSIONS BEYOND THE GREAT OBSERVATORIES

- PROGRAM FUNDING TO BE COORDINATED BETWEEN ASTROPHYSICS DIVISION AND OFFICE OF AERONAUTICS AND SPACE TECHNOLOGY (OAST)

- ASTROPHYSICS DIVISION DEVELOPS MISSION-SPECIFIC TECHNOLOGIES

- OAST DEVELOPS GENERIC TECHNOLOGIES (I.E., TECHNOLOGIES REQUIRED BY MORE THAN ONE MISSION) 


\section{METHOD OF IDENTIFYING CRITICAL TECHNOLOGIES}

THE "LDR" METHOD HAS BEEN ADOPTED BY THE ASTROPHYSICS DIVISION AS THE BEST WAY TO IDENTIFY CRITICAL TECHNOLOGIES. THIS INVOLVES:

- IDENTIFICATION OF ONE OR MORE "STRAWMAN" MISSIONS WHICH ARE TYPICAL OF (BUT NOT NECESSARY IDENTICAL TO) THE PLANNED MISSIONS

- NASA CENTER SELECTED AS "LEAD" FOR EACH STRAWMAN MISSION

- SERIES OF 2-3 WORKSHOPS/MISSIONS, AT $\approx 1$ YEAR INTERVALS, TO DEFINE MISSION SCIENCE OBJECTIVES, METHOD OF IMPLEMENTATION, PACING TECHNOLOGIES, AND TO IDENTIFY SCIENCE/TECHNOLOGY TRADES

- MEETINGS CO-SPONSORED AND ATTENDED BY BOTH OAST AND OSSA

- FOLLOW- ON TECHNOLOGY STUDIES COORDINATED BETWEEN OFFICES, WITH RESULTS FEEDING INTO NEXT WORKSHOP

\section{TECHNOLOGY 21 PROGRAM STATUS}

- WORKSHOP SERIES COMPLETED FOR LDR

- WORKSHOPS CURRENTLY UNDERWAY FOR INTERFEROMETRY, GRAVITY PHYSICS, AND 10 METER TELESCOPE

- ADDITIONAL WORKSHOP SERIES CONTEMPLATED

- TECHNOLOGY 21 PROGRAM ELEMENTS SELECTED BY "POPULAR DEMAND"

- SIGNIFICANT FUNDING STARTS FY 92 AND RAMPS UP RAPIDLY

- LUNAR PROGRAM MAY RESULT IN AUGMENTATION (TO BE USED FOR LUNAR PROGRAM CANDIDATES) 


\section{LUNAR PLAN}

\section{EARLY LUNAR-BASED SKY SURVEY: THE CRATER TELESCOPE}

DESCRIPTION:

- EXTREMELY WIDE BAND, VERY DEEP, UV/OPTICAL IMAGING SURVEY

- SURVEY ADJACENT STRIPS (OR SAME STRIP) OF SKY AT 1 MONTH INTERVALS

- A WIDE-FIELD ZENITH-POINTED TELESCOPE OF $\approx 2$ M APERTURE

- Focal plane READ OUt AT APPARENT Sideral RATE

- SEVERAL DETECTORS IN FOCAL PLANE. SENSITIVITY: 0.1 TO 10 MICRONS SCIENTIEIC RATIONALE:

- SIMULTANEOUS UV/VIS/IR "PALOMAR SKY SURVEY"

- 100 TIMES MORE SENSITIVITY (V>25) AND 20 TIMES MORE RESOLUTION $(\approx 0.1$ ARC SECONDS AT $0.5 \mu$ ) THAN PALOMAR SURVEY

- 1000 SQUARE DEGREE FIELD OF VIEW, SAMPLED ANNUALLY

- SPECIAL ADVANTAGES OF LUNAR SITING:

- LONG INTEGRATION TIME (HIGH SENSITIVITY) AFFORDED BY LUNAR ORBIT

- NO POINTING REQUIRED

- TELESCOPE CAN BE PASSIVELY COOLED TO CRYOGENIC TEMPERATURES FOR OPERATION OF THE DETECTORS

- TELESCOPE CAN BE SHIELDED NATURALLY WITH CRATER OR EXCAVATION FOR PROTECTION FROM HIGH COSMIC RAY EVENT RATE

- PROgRAMMATIC RATIONALE:

- UNIQUE CAPABILITY TO SURVEY LARGE AREA OVER WIDE BANDPASS

- WILL SUPPORT OTHER LUNAR-BASED ASTRONOMY FACILITIES

- TECHNOLOGY WELL DEVELOPED

- LOW COST, EASY TO INSTALL AND OPERATE 


\section{METER UV/VIS/IR TELESCOPE}

- DESCRIPTION:

- DIFFRACTION-LIMITED 16 M APERTURE SEGMENTED TELESCOPE

- RESOLUTION: 6 MAS AT 0.3-0.4 $\mu \mathrm{M}, 60$ MAS AT 3-4 $\mu \mathrm{M}$

- WIDE BAND SYSTEM, WAVELENGTH RANGE $0.1 \mu \mathrm{M}-\sim 10 \mu \mathrm{M}$

- PASSIVE COOLING OF DETECTORS TO < 100 DEG K

- HIGH SENSITIVITY - VISIBLE BACKGROUND 33RD MAG/PIXEL

- STABLE TRACKING TO 1 MAS, POINTING TO APPROX. 10 MAS

- WIDE FIELD OF VIEW (>1 ARC MINUTE) REQUIRES DETECTOR MOSAICS

- SCIENTIFIC RATIONALE:

- UNPRECEDENTED POWER FOR TACKLING A WIDE RANGE OF FUNDAMENTAL PROBLEMS

- SPECIALADVANTAGES OF LUNAR SITING:

- STABLE POINTING AND TRACKING, PLUS VIBRATION ISOLATION

- ABLE TO SUPPORT LARGE INSTRUMENTS AND INSTRUMENT PLATFORMS

- COSMIC RAY SHIELDING

- IN-SITU ASSEMBLY

- PROgRAMMATIC RATIONALE:

- BUILDS ON SCIENTIFIC FOUNDATION DEVELOPED BY HST

- CAN BE USED AS AN ADDITIONAL ELEMENT OF THE INTERFEROMETER, INCREASING THE EFFECTIVE AREA OF THE LATTER TO THE GEOMETRIC MEAN AREA AND, HENCE, SIGNIFICANTLY INCREASING ITS SENSITIVITY 


\section{LUNAR-BASED SYNTHETIC APERTURE INTERFEROMETER}

- DESCRIPTION:

- optical equivalent to radio Vlbi

- 10 KM BASELINE INTERFEROMETER WITH MULTIPLE 1 - 3M APERTURES

- 10 MICRO ARC SECOND IMAGING, 10 - 100 NANO ARC SECOND ASTROMETRY

- EVOlUTIONARY DESIGN (START WITH 2 ELEMENTS, END WITH 20-30)

- total eVentual Collecting area 50 - 100 SQUare meters

- 30 MAG/PIXEL POINT SOURCE SENSITIVITY

- SCIENTIFIC RATIONALE:

- RESOLVE BROAD LINE REGION AND NARROW LINE REGION IN AGN'S

- ISOTROPY AND UNIFORMITY OF HUBBLE FLOW TO 1\%, PARALLAX OF OBJECTS TO 10'S OF MEGAPARSECS

- IMAGE WHITE DWARFS TO $10 \times 10$ PIXEL

- IMAGE ACCRETION DISKS AROUND STELLAR OBJECTS, NEUTRON STARS, BLACK HOLES

- DETECT AND CHARACTERIZE PLANETS

- SPECIAL ADVANTAGES OF LUNAR SITING:

- ULTRA-ACCURATE BASELINE DETERMINATION ON AN ATMOSPHERE - FREE BODY

- LOW NIGHTTIME TEMPERATURE (PASSIVE COOLING OF OPTICS)

- PROgRAMMATIC RATIONALE:

- eARLY SCI/TECH DEMONSTRATION POSSIBLE WITH SHORTER BASELINE (<100M) ORBITING INTERFEROMETER

- EVOLUTIONARY GROWTH OF LUNAR-BASED INTERFEROMETER

- A 16 METER TELESCOPE CAN BE A POWERFUL INTERFEROMETER ELEMENT 


\section{DISCUSSION}

Mlingworth: Can you comment on how the science goals in NASA's response to the President's July 20 initiative will be iterated with the science community following the submission of the report to the NSC.

Pilcher: NASA's 90 day study (in response to the President's July 20 initiative) must be broad, but its scope and brevity limits its depth. The process of defining the science content of human exploration missions must be a continuing one. Mike Duke has been detailed to the OSSA front office to work with all the science Divisions and the external communities on defining science for the 90 day report. That process must continue beyond the 90 day study to provide scientific input of increasing maturity to the Office of Exploration mission studies.

Weiler: We have agreed within Astrophysics that we will iterate and that we will use the advisory structure (the MOWG's), but we will also get help from the Astronomy and Astrophysics Survey Committee - from a broader community.

Breckinridge: Is the "crater" telescope considered to be a "suitcase" telescope mission to the moon? A 1 meter telescope is more like a large steamer trunk than a "suitcase."

Weiler: Yes, "suitcase" means requiring little or no assembly from the astronaut. Deployment and pointing (rough) are acceptable. Larger than a suitcase is ok. 


\title{
ESA Long Term Plans and Status
}

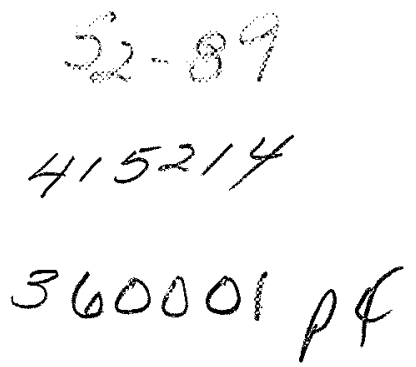

\section{Introduction}

\author{
Duccio Macchetto \\ European Space Agency
}

The European Space Research Organization, the predecessor of ESA, was established some 25 years ago, The main goal then was to coordinate the work of European astronomers and scientists to design and build a telescope to be placed in Earth's orbit. As it turned out, the first satellite that was built was not an astronomical satellite, it was a space plasma physics satellite. Since those early days, ESA has conducted a very active, although budget limited, research program in astronomy. The first astronomical satellite that was launched was TD-1A, which carried an ultraviolet telescope. It was followed by COS-B, which conducted an all sky survey of gamma-ray sources. That was followed by a joint mission with NASA, the International Ultraviolet Explorer satellite (IUE), well known to all of you, and then lately the EXOSAT, which explored the X-ray region.

\section{The Horizon 2000 Program}

Following these early successful missions, a study was conducted five years ago by ESA to define with European scientists the long range priorities in space research for the following decade. There were extensive studies within the European community to define the mission of interest to each discipline. This was further refined over a two-year period, and the result of that program is what is now called the European Space Agency's "Horizon 2000", represented in Figure 1.

The program is built around what are called the four "corner stone" missions. These typically cost upwards of 500 million dollars, while ESA's budget for scientific research is of the order of 300 million dollars per year. What that means is that ESA can build only one of these satellites at a time. While one mission is winding down a start will be made on the next. The first cornerstone to be built is the SOHO/CLUSTER mission, again a collaboration with NASA. It will be followed later by the X-ray mission XMM.

The smaller boxes in Figure 1 are missions of the 200 million dollar type. Such missions include satellites such as Hipparcos and the ESA's contribution to the Hubble Space Telescope. There are also a few empty boxes, and these are opportunities for future missions which I will briefly describe little later on. The circular central region in the center represent low-cost missions, on the order of 100 million dollars or less. These are either throw away type missions, assorted missions, or joint missions with NASA or other agencies where ESA's contribution would be less than 100 million dollars. The outer rectangular areas indicate missions which will be ready at a time well beyond Horizon 2000. In addition, the plan identifies a number of missions that have considerable scientific interest in the European community but are of a complexity, or cost or require technological advances of such a magnitude that it is impossible to carry them out in this century.

\section{ISO/XMM}

If we turn to astronomy, the mission that is currently being built is the Infrared Space Observatory, ISO. It is a 60 centimeter infrared telescope which is surrounded by a large 


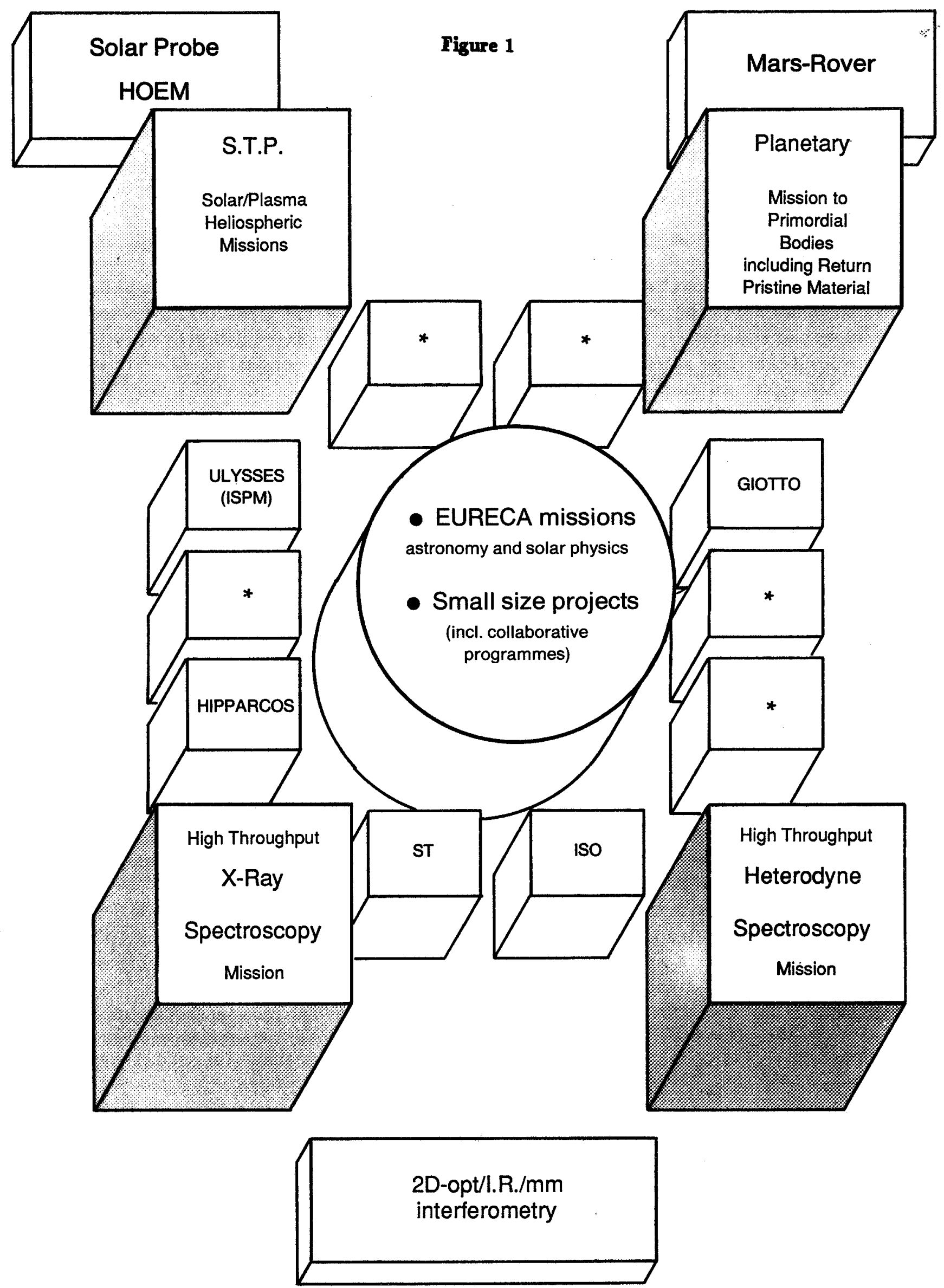

The European Long Term Programme

[*e.g. : - Solar Heliospheric (Soho), Multipoint Probes (Cluster), Auroral Multiprobes, etc., in Solar/Heliospheric/Plasma Physics; - Venus (Venture), Mars (Kepler), Lunar (Selene) Orbiters, etc., in Planetary Science; - Space VLBI, UV Spectroscopy, Stellar Sei smology, etc., in Astronomy.] 
Dewar which keeps the temperature of the telescope and the detectors at about 4 degrees Kelvin. It will carry about 2,000 liters of helium. The total mass is about 5,000 kilos and it is expected to be launched in about 1996.

Following ISO, other approved programs, which are not being built yet but are in a detailed definition phase, are: Cassini, a joint mission with NASA, that will explore the atmosphere and the surface of Titan which is scheduled for launch in 1996; XMM, which is a ligh X-ray spectroscopy mission to be launched in 1998 with the Ariane launcher; and the Columbus polar platform mission, which is mainly devoted to study solar terrestial physics.

Of particular relevance to this conference, XMM consists of a series of nested telescopes and a number of spectrometers and imagers. The imaging quality is only about 30 arc seconds, the emphasis being to build a light bucket to carry out intermediate resolution spectroscopy.

\section{FIRST}

The next cornerstone astronomy mission is the Far Infrared Space Telescope (FIRST). It is a heterodyne spectroscopy mission. It will cover the wavelength range between a hundred microns and one millimeter. This is an important range because of the availability of both continum radiation, and a number of atomic and molecular lines. The baseline is to have a passively cooled antenna about 8 meters in diameter, able to carry out high spatial resolution observations. The FIRST antenna can be either a deployable antenna or a segmented antenna, and that will depend on what type of technology is the best suited at the time of launch. The Dewar at the focal plane is of the same type of technology that has been developed for ISO and will keep the experiments at about 4 degrees Kelvin. The launcher should be an Ariane 5, and the orbit is a 24 hour type orbit. The pointing accuracy will be one arc second with a stability of about half an arcsecond. The design of the antenna is a Cassegrain with a prime focal ratio of F/1.35. It will not have a chopping secondary, because of the great added complexity that this will require. The material of the antenna will be carbon fiber and it will have a very high surface accuracy of about 6 microns. It will have an error of about 100 microns, and a random error of about 20 microns. The model payload detectors includes heterodyne spectroscopy with SIS mixers, spectrophotometry with a grating combination, a far infrared spectrometer to observe the wavelength range between 100 and 250 microns, and a far infrared photometer. The FIRST mission is currently planned for year 2010 .

\section{Interferometry}

ESA has established a space interferometry study team to discuss aperture synthesis in space. The terms of reference of that team were to define the main science goals, to establish a strategy for ESA involvement in the project, to identify and discuss different mission concepts, to identify science trade-offs and advise on the technical research program that is needed to carry out this long-term program. This team met a number of times and they have recently issued a report. They compare a number of different concepts from rigid structures with a number of free flying elements to floppy structures of the VLA type. The final two concepts that have emerged are Oasis and Float. Oasis has a large coherent field of view, while Float is a fiber-linked optical array. The problem 
here is of course to develop fiber-optics which have good transmission in the ultraviolet. The two studies, one was a Michelson-type arrangement, and the other is a Fizeau-type arrangement for beam recombination. In one case, the structure is floppy, in the Fizeau case is semi-rigid with active optics. The array shape in the case of Michelson would be a VLA type - a wide type array, with non-redundant spacing and Fizeau again would be a non-redundant space ring with about 12 different telescopes. In one case the structure is a space inflatable structure with optical elements connected through rigid tubes, the other an inflatable structure. These two types of structures are being developed by industry in Europe for other applications at this time. So there is some hope that it will be useful for this project. In the Michelson case the ultraviolet is clearly excluded from the arrangement, whereas in the Fizeau there is a possibility to go down to below 0.1 micron.

The recommendations and the conclusions of this team were to build a 30 meter optical interferometer with as many as 12 different telescopes in a single structure in a stationary orbit. It is important to reach visual magnitudes below 14, and actually a 30 meter array will go down to about magnitude 23 . It was felt that it was important to have a large coherent field of view to include reference stars within the same field of the object for stabilization and calibration purposes. It was essential to cover a large optical range, and particularly to cover the ultraviolet domain. The study team indicated that they felt that the infrared was better studied from the ground, at least in this kind of configuration. It was important to have imaging capability and high spectral resolution. An additional study will be carried out starting this year with the aim to define technologies and begin a development program in those that are the most demanding.

\section{Europe and NGST}

One final point I want to make is as you have seen, many of the ESA programs have been carried out and are going to be carried out in collaboration with NASA. I do hope that discussions are continued across the Atlantic in the mission that you are now studying. There is a large interest in the astronomical community in Europe for HST and also in a future HST. It is therefore important to include the European astronomical scientific community in a possible joint venture in a 10 to 16 meter next generation space telescope. 


\section{The Next Generation UV-Visible-IR Space Telescope}

\author{
Garth Mllingworth \\ University of California Observatories/Lick Observatory \\ Board of Studies in Astronomy and Astrophysics
}

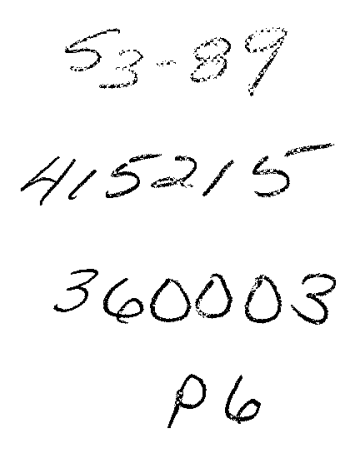

Synopsis

A large 10-16 m passively-cooled, diffraction-limited, filled-aperture space telescope would have unprecedented power for tackling a wide range of the most fundamental astrophysical problems, from the detection of earth-like planets to the structure of galaxies and protogalaxies at redshifts $z>1$. The telescope would have a lightweight, segmented primary with active wavefront sensing and control for diffraction-limited performance into the UV. The structure and optics would be passively-cooled to $100^{\circ} \mathrm{K}$, lowering the background in the 3-4 $\mu \mathrm{m}$ zodiacal "window" to less than $10^{-6}$ of that from the ground. Stateof-the-art mosaics of detectors would give diffraction-limited imaging and spectroscopy over a field of $>2$ arcmin from $0.3 \mu \mathrm{m}$ to beyond $10 \mu \mathrm{m}$, and nearly 1 arcmin in the UV. The observatory would combine remarkable imaging performance, with resolutions ranging from a few mas in the UV to some 40-60 mas in the zodiacal "window" at $3 \mu \mathrm{m}$, and with even greater capability for spectrographic observations of faint and/or low surface brightness objects at the highest spatial resolution.

The rationale for such an observatory is discussed in the light of HST and the other Great Observatories, and of expected gains in ground-based telescopes and computing capability. The importance of moving into concept development, and to technology development and evaluation programs, is highlighted within the context of the very long lead times for such missions to come to fruition. The importance of the new optics and structures technologies in breaking away from our current cost curve for large missions, and the potential gains from an enhanced national commitment to space are noted.

\section{Context}

With the launch of HST we will be entering a new era in astronomy. Even with the major discoveries made in space over the last two decades, we have only touched upon the true potential of astronomical observations from space. The broad-ranging capabilities, the sensitivity and the long lives of the Great Observatories will demonstrate the importance of space telescopes for addressing a wide range of fundamental issues in all areas of astrophysics.

I believe that the coming decade will come to be seen as the dawn of the golden age of astronomy. While the theoretical and observational base has matured to the point where significant inroads are possible into many fundamental problems, four developments will play a critical role in the coming decades. These key elements are:

- The Great Observatories - the new generation of long-lived, versatile, and highlycapable space observatories;

- Large ground-based telescopes - the light gathering power of this new generation of telescopes will provide essential complementary capability for the Great Observatories (primarily in spectroscopy), as well as bringing unique capabilities in their own right; 
- Detectors - the promise of CCDs is about to become reality with the likely near-term availability of arrays of close-packed large format $\left(2048^{2}\right)$ detectors and with further developments in energy-sensing CCDs, while the technology for near- and mid-IR detectors is leading to progressively larger arrays $\left(512^{2}\right.$ and larger);

- Computing power - with new generations of supercomputers and increasingly powerful workstations, the availability of cost effective access to computing and display power for theory, modelling, and data processing opens up broad areas that were impractical in the 1980s.

\section{Rationale}

With these tremendous gains, one can ask what sort of facilities and capabilities are needed for the decades beyond the coming one. Do we need substantial gains in capability, or will we be in a consolidation phase, using existing facilities to carry out long-term programs? The answer is "both of the above". Some problems will best be tackled with long-term effort with existing capability, while others will need substantially improvements beyond HST and the other Great Observatories. Furthermore, such long-term programs can only continue for the life of the Great Observatories, and these are projected to be in the range 5-15 years. Their limited lifetime alone forces the astrophysical community to look beyond, to the missions that will succeed the Great Observatories.

The long lead times for such major missions gives a sense of urgency to this process. The direction in which we need to move will be governed by the scientific goals that will develop as a result of using the facilities and capabilities listed above. While it would be presumptuous, and essentially impossible, to generate a comprehensive list of those problems that will be at the forefront, it is clear already that substantial gains in capability will be needed to tackle some of the questions that lie at the heart of astronomy. For example, how stars form, and how galaxies form and evolve are problems that must be tackled on a broad observational and theoretical front. They involve very complex processes, and demand telescopes with large light-gathering power, high resolution, broad spectral coverage, the lowest possible background, and efficient spectroscopic capability. They need versatile telescopes, because the path to understanding will depend upon previous results. The questions to be answered are still quite uncertain. These fundamental issues will not be solved by "physics-like" experiments.

This is, however, an approach that is still questioned. Great concern about the cost of major facilities has lead to many scientists asking if it wouldn't be cheaper and even more effective for the community to press for more specialized projects. These may have the collecting area of HST, or even of the class of the telescopes being discussed here, but be instrumented more simply, less capably, and be limited in wavelength coverage. I remain skeptical about the real cost savings to be made with this approach. For example, by asking for a diffraction-limited large optical system, surely a minimum goal for a space observatory, one already has set a substantial floor to the cost of the program.

Furthermore, I think such an approach is ill-advised for observatory-class missions. By their very nature they will, alone and synergistically with other space observatories and large ground-based telescopes, make substantial discoveries that will change the direction of science programs, even if the overall goal remains the same. There is no doubt that the formation of stars and the formation of galaxies will remain high priority programs through the decades to come. Yet I defy anyone to map out a strategy for solving these 
problems that they are confident will survive the coming decade or two of observations. These problems are not amenable to defining an "experiment" that will lead to their solution. Broad ranging capability is needed that will allow us to build upon a growing base of understanding - to branch out to follow the leads shown by observations.

Interestingly, I think that we have a consensus by example within the astrophysical community that is consistent with this view. There are large numbers of universities around the country that are planning telescopes with their hard-won private money. Almost invariably, when the funds allow, they choose large versatile telescopes, and are the envy of their colleagues for so doing. Astronomers are "voting with their feet" for large, versatile telescopes.

I think that this view will be strengthened as the community experiences the Great Observatories and the new 8-10+ $m$ large ground-based telescopes.

This is not to say that such telescopes are all that are needed. Problems such as surveys in wavebands inaccessible from the ground, the structure on the smallest scales in AGNs and quasars, defining the fundamental distance and reference systems, high energy events, etc. are going to require astrometric systems, interferometers, survey telescopes, specialized $x$-ray and $\gamma$-ray telescopes and so on. COBE is a current example of a very impressive and valuable experiment. These facilities provide an essential complementary element to the large versatile telescopes. The large telescopes can be thought of as providing the heart of an organic program which is supported by a wide variety of more directed, more focused capabilities.

Over the next 15 years or so of HST's lifetime, it is clear that the astrophysical community will become "addicted" to HST's capabilities - namely, its:

- resolving power,

- UV coverage,

- near-IR capability,

- and the predictability of its performance.

It will not only have a major impact on what we do, but also on how we do it.

Yet, for all the wonderful new capabilities offered by HST, it is clear that there will be a component of great frustration. It is simply too small to do spectroscopy at the level demanded by many of the outstanding problems, and almost certainly too small for followup of many of its discoveries with its powerful imaging systems. The new, large ground-based telescopes will be able to provide the necessary spectroscopic followup in many cases, but their poor imaging capabilities by comparison with those on HST, their lack of UV coverage, and their high IR background will be a limiting factor, and a substantial one.

\section{Next Generation Space Telescope}

While these general considerations provide a broad rationale for a next generation large UV-Visible-IR telescope, it is the scientific case itself which leads one to great enthusiasm for such an observatory. As can be seen in the subsequent science papers, a particularly compelling scientific case can be made for a 10-16 m class space telecope with the proposed performance capabilities noted in the introduction to the Workshop. Words such as "awesome" and "astonishing" have been used by more than one scientist upon thinking about or being shown the capabilities of such a telescope. 
The goal is for a $16 \mathrm{~m}$ class passively-cooled, diffraction-limited, wide-band telescope. Its instrument complement would be sensitive from the UV at $\sim 0.1 \mu \mathrm{m}$ to beyond 10 $\mu \mathrm{m}$. With passive cooling of the structure and optics to less than $100^{\circ} \mathrm{K}$, the background would be lowered in the 3-4 $\mu \mathrm{m}$ zodiacal "window" and at longer wavelengths to less than $10^{-6}$ of that from the ground. The diffraction-limited images would be 7 milliarcsec at 0.5 $\mu \mathrm{m}$. It could reach $32 \mathrm{mag}$ in the visible at $10: 1 \mathrm{~S} / \mathrm{N}$ in less than $10^{4} \mathrm{~s}$. State-of-the-art mosaics of detectors would give diffraction-limited imaging and spectroscopy over a field of $>2$ arcmin from $0.3 \mu \mathrm{m}$ to beyond $10 \mu \mathrm{m}$, and to nearly an arcmin in the UV.

The combination of high resolution, low background, wide bandwidth, and wide field are what give this telescope its unique capabilities. For example, the low background across the wavelength region covered by this telescope, $\sim 0.1-20 \mu \mathrm{m}$, allows for measurements to be made of much, if not most, of the baryonic matter in the universe, assuming the dark matter to be non-baryonic. Furthermore, the resolution provides an excellent match to some natural length scales in the universe. For example, the telescope is particularly well-matched to direct observations of the structure in galaxies and proto-galaxies at high redshift. Structures in galaxies (e.g., star forming regions, spiral arms, disk/bulge length scales, merger "arms and tails") have characteristic scales of $100 \mathrm{pc}-1 \mathrm{kpc}$. With 10 mas resolution we can resolve structures in galaxies at any redshift with the resolution that we now study the nearest cluster of galaxies, the Virgo cluster, from the ground. This is an astonishing capability and is shown very explicitly in the paper by Jim Gunn where he simulates images at a redshift $z=1$ of a spiral galaxy with HST and with a 15 m telescope.

The question of the detection of earth-like planets in stellar systems within $10 \mathrm{pc}$ of the sun and the subsequent spectroscopic observations of those planets with the goal of detecting ozone and other molecules indicative of life is addressed by Roger Angel in this volume. It is an extremely challenging observational program, and one which requires a $16 \mathrm{~m}$ telescope with all the capabilities summarised here. Yet it is an immensely exciting goal and one which captures the imagination not only of astronomers and life scientists but of people at large.

\section{Timescales}

HST has a nominal life of 15 years. It is now approaching 20 years since the start of Phase B for HST. It is clear that the pre-Phase A conceptual and technology development needs to start very soon to minimize the gap between HST and its successor. The maturity of astronomy, and the resultant difficulty and complexity of the scientific issues that are now at the forefront of the field, requires long-term observational capability. A substantial gap, greater than 5 years, for example, would have a very deleterious effort on the productivity in the field and would be a waste of the scientific talent and resources that will build up around HST and the Great Observatories.

An added concern, of course, is the tough environment in which spacecraft operate, especially low earth orbit spacecraft with the attendent thermal cycling. If HST, an immensely complex instrument, degrades faster than expected in a way which cannot be accommodated by the M\&R (Maintenance and Refurbishment) program, we could potentially be facing a large gap before the next mission. This would also impact the productivity of AXAF and SIRTF, because the multiwaveband synergy would be lost, undercutting one of the pillars of the Great Observatory program. A gap in capability in 
the central UV-Visible-IR wavelength region of 10 years would be far too long. The goal should be for a gap of less than 5 years between HST and its successor.

\section{Cost}

One scaling "law" that has been used in the past for telescopes has cost rising as the 2.7 power of the diameter, i.e., $\left(D_{1} / D_{2}\right)^{2.7}$. Applying such a factor for a $10-16 \mathrm{~m}$ class telescope based on HST's cost leaves one gasping. However, such an approach is inappropriate given changes in technology and the gains that can be accomplished by experience and attention to detail. Recent ground-based large telescopes have broken the cost-curve for 1950-70's telescopes by a factor of four, and further gains are in the pipeline. The German science community is seriously looking at a structure, the Hexapod structure like that shown in Figure 2 in the Introduction, that is based on experience with flight simulators. This offers large weight savings, and hence cost savings, even beyond the space frame designs currently being used for the Keck Telescope. For a $12 \mathrm{~m}$ diameter telescope, they project total weights comparable to previous-generation $4 \mathrm{~m}$ telescopes.

Another area where major gains in performance with attendent weight savings, and hence cost savings, can be made is in the area of lightweight optics. As we can see from the discussions at this meeting, this is an area where major improvements in fabrication and polishing technology are occuring. The combination of improved performance and lower weight for the optical segments will directly and dramatically affect the final cost of the NGST.

We are clearly very early on the learning curve for observatory-class missions, and should have every expectation for substantial gains in lowering the cost of such missions. HST can be an extremely valuable experience base for such gains. I hope that as we pass launch, as HST becomes a powerful, productive observatory, that we can revisit the construction of HST in a very objective, non-accusatory way with the goal of improving our ability to do such missions faster and cheaper. The computer industry and the Japanese automobile industry have made remarkable strides by learning from experience, by attention to details, and by maximizing the product per dollar. There is no reason why we cannot do the same.

Turning to the revenue side of the equation, I think that we are ripe for a renewed and expanded committment to space, and to space science. With the remarkable political changes that are taking place in the world, I see a renewed emphasis on space as the focus of our high-technology effort. Even modest real (after inflation) annual increases can very substantially increase the total budget over a multi-year period. For example, just by matching the GNP growth, say $3 \%$ per year, plus adding another $5 \%$ above inflation, for a period of fifteen years through to 2005 , the annual budget for space activities as carried out through NASA could grow from $\$ 13$ billion to $\$ 41$ billion in 1990 dollars. The space science program that could be carried out with the usual fraction of such a budget, combined with the increased capabilities that could be obtained per dollar through the technology and potential fabrication improvements noted above, is exciting, to say the least.

The goal of building a $16 \mathrm{~m}$ class telescope early in the next century is an ambitious one, but one that is closer at hand and more realistic than one might initially expect. This sentiment was echoed in a a study of the long-term goals for space science that was performed under the auspices of the National Academies of Science and of Engineering, 
and of the Institute of Medicine. The study group was tasked with developing a program for Space Science in the 21st century. The reports of the study groups and an overview volume have recently been published (1988) under the title Space Science in the TwentyFirst Century: Imperatives for the Decades 1995 to 2015.

The report contains a recommendation for an 8-16 m, passively-cooled, UV-VisibleIR telescope. The report's concluding remark (from the section discussing an 8-16 m telescope in space) notes that: Given a well-directed technology development program, the task group anticipates that an 8 to $16 \mathrm{~m}$ telescope will prove to be within closer reach than a simple extrapolation from HST would suggest.

While this report recognizes the scientific value of such a telescope, and I think realistically appraises the situation vis-a-vis the needed technology developments, it is but a start to a wide-ranging program leading to the development of a $16 \mathrm{~m}$ class telescope within a broad space science program. This workshop takes the next critical step. 


$$
\begin{gathered}
\text { MnT MOS } \\
\text { Pab }
\end{gathered}
$$

Session 2

\section{Scientific Potential}




\section{Page intentionally left blank}


NGST and Distant Galaxies

\author{
James Gunn \\ Princeton University
}
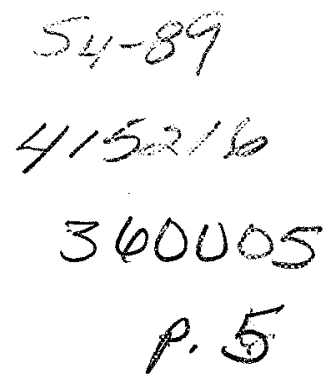

One of the most-heralded abilities which HST will bring us is the capability to study the morphologies of quite distant galaxies, distant enough that the look-back time is a substantial fraction of the age of the universe. It appears that with substantial expenditure of observing resources, crude morphological information can be obtained out to redshifts approaching unity, with quite good images at, say, $\mathrm{z}=0.5$.

Most theories of galaxy formation, and indeed the growing body of ground-based spectroscopic evidence, put the epoch of galaxy formation earlier, though I think the weight of modern evidence is that it is not much earlier, and the redshift range 2-3 promises to be of enormous interest. HST, by reason of its small collecting area and, to some extent, limited resolution, cannot address this problem directly. The awesome capability of a fifteen-meter diffraction-limited telescope in high orbit, however, would allow us to see what is going on at these epochs directly and with considerable ease-if still requiring a great deal of observing time.

It is perhaps worth a few words to say why one expects to see something interesting, and to discuss what one might see. On the theoretical front, the popular Cold Dark Matter scenario requires that galaxy formation be late, and furthermore in general quite chaotic. The model of Baron and White and the newer models of Katz(1989), the latter incorporating real hydrodynamics and an attempt at a physically reasonable star formation description, produce objects which at the present epoch look very much like the galaxies we see (the former an ellipical galaxy, the latter both ellipticals and convincing disk spirals). The gross morphology of these systems changes little past $z=1$, but between $z=2$ and $z=3$ the objects look very different from galaxies today, and in fact in most surveys, if they could be seen, would be counted as multiple objects, there being several more-or-less isolated islands of star formation in merging, still mostly gaseous, blobs. If one takes the predicted brightnesses of these objects seriously (which one does, of course, at one's peril- the star formation rates are are, well, very uncertain), they should be visible in the very deep ground-based surveys of Tyson and others, and if so, could well explain the mystery of the counts of galaxies. The counts are several times too high fainter than about 25 th magnitude to be explained by conventional models, and extreme evolutionary models which do fit them fail spectacularly to fit the observed redshift distributions at brighter levels. If, in fact, one were observing not galaxies at very faint levels, but pieces of galaxies putting themselves together to make galaxies, the problems with the counts would be much less severe. It is interesting (but again, regard with caution) that the visual brightness of these objects is predicted to change little between $z=2$ and $z=1$, the integrated brightness corresponding to about $\mathrm{V}=23-24$ for the progenitor of a big $(\mathrm{Mv}=$ -21) spiral at the present epoch. But that brightness is not lumped in a single concentration until about $z=1.5$, and prior to that is quite complex, with several separated regions of star formation contributing comparably to the total luminosity. 
What manner of instrumentation would it require to investigate this birth process? First of all, the lumps in which stars first form are very small, so one needs high resolution, ideally about $100 \mathrm{pc}$ at $\mathrm{z}=1-2$, or about 15 milliarcseconds. Secondly, one needs high sensitivity; in the 'turn-on' phase when the object is quite chaotic, the total brightness may correspond to $V=26$ but might consist of several lumps spread over a few arcseconds.

It becomes clear on a little reflection that a space-based telescope several times the aperture of HST is needed, and furthermore that such an instrument would do very nicely indeed. I will end this discussion by presenting some simple simulations of observations done with an instrument with the following properties:

- 16-meter aperture

- $\mathrm{f} / 20$ modified Ritchey optical system; the scale is 1.45 microns/mas

- 5 arcmin diffraction-limited field; this can be easily achieved with a single-element refractive astigmatism corrector

- FWHM = 10 mas or about 15 microns at $\mathrm{V}$

- Camera with 3 reflections and 1 transmissive element (astig. corr.)

- 8192x8192 CCD with 7.5 micron pixels; the focal plane might be paved with 25 of these devices; each has a field 40 " square

- Read noise of 2.5 electrons RMS for the CCD.

- For the near IR, a $1024 \times 1024 \mathrm{HgCdTe}$ device with 40 micron pixels would give a field of 30 arcseconds with 25 mas pixels; it seems likely that a read noise of 10 electrons will be attainable.

A comment about the assumed technology is perhaps in order...the tallest order in the list is almost certainly the diffraction-limited 15-meter primary, which others in this conference will address with much more expertise than I possess. The optical layout is straightforward, and the detector properties probably a much-too-conservative extrapolation of the current art. I have assumed a high-earth-orbit environment, which is operationally very much simpler than the low-orbit environment of HST. It will allow passive cooling of the optical detectors (though the IR imagers and other IR instrumentation which will doubtless be aboard will certainly require cryogens or other active cooling.)

The radiation environment is relatively benign. With moderate shielding, such as is used in the Galileo detectors, the proton rates are of order 3 per square centimeter per second and the electron rates much lower. The proton flux can rise by four orders of magnitude during a very energetic solar flare event, but the dose and duty cycle of such events is very much lower than the South Atlantic anomaly which one has to deal with in low orbit. The typical rates are higher than the WF/PC rates, for instance, but not by much. With the 7.5 micron pixels assumed for the optical sensors, the mean time for a given pixel to be hit is about $6 \times 10^{5}$ seconds, while the detected sky photon rate is of the order of $.015 /$ second in the visible and near IR with broad-band filters. Thus exposures of the order of 1000 seconds will have sky levels of order 15 electrons and will be well into the shot-noise limited regime while of order 1 pixel in 600 will have suffered a cosmic-ray hit; it seems straightforward that a three-exposure sequence might be standard, with a simple voting scheme to eliminate hits. The situation in the IR is much trickier; the assumed 40-micron pixels are 28 times larger than the assumed optical ones, the read noise worse, and the background fluxes lower. Dividing the exposure into roughly 200-500 second segments will still put one close to the shot-noise limit and have only one or two percent of the pixels hit during any one sub-exposure, but one would be much more comfortable 
with a lower noise floor (which may well be possible by the time this instrument is built.)

The capabilities of such an instrument are awesome for a very large range of problems. For example, if one imagines a set of broad (20\%) filters spanning the range $2900 A A$ to 2.2 microns, a 2-hour exposure would yield 10:1 signal-to-noise photometry at the following visual magnitudes:

$\begin{array}{lccccc}\text { Wavelength: } & 2900 \AA & 4300 \AA & 6200 \AA & 9300 \AA & 2.2 \mu \\ \text { Magnitude: } & 30.5 & 32.5 & 32.5 & 31.0 & 32.0\end{array}$

for an object with an energy distribution like an F0 star. The sun could be detected with 10:1 S/N at 3.5 Mpc, and a 10-day Cepheid at $200 \mathrm{Mpc}$. $\mathrm{A} z=2, \mathrm{Mv}=-21.5$ normal galaxy containing an 18th magnitude QSO could be detected at 5:1 S/N. (All the above for 2-hour exposures).

The simulations I have done are for the nearby galaxy NGC2903, an ScI-II spiral at about 10 Mpc distance moved to $\mathrm{z}=1$ with the assumption of no luminosity evolution. The image from which the simulations were made was obtained with the 4-shooter CCD camera on the Hale telescope in 0.8 arcsecond FWHM seeing with a Thuan-Gunn $g$ filter; the $\mathrm{S} / \mathrm{N}$ in the image is high enough that noise must be added to it to make the simulations. Recall that forming galaxies at $z=2-3$ are expected to have fluxes in the visible not too different from this, since they will have much more active star formation. They are all simulations of pictures taken in the 9300-Angstrom 20 per cent filter used in the above sensitivity calculations; the protogalaxy images will be better at somewhat shorter wavlengths because of the young stellar populations, though if there are normal galaxies at $\mathrm{z}=1$ the assumed band is probably optimal (we would be better off at 2.2 microns from the point of view of $S / N$, but the assumed sampling is not as good there.)

The first pictures are simulations of Keck telescope images of this galaxy, with exposures of 3 hours and 30 hours, with a CCD with 0.1 arcsecond pixels and 0.5 arcsecond FWHM seeing. Although the signal-to-noise in the long exposure is exquisite, the resolution is inadequate for any serious morphological study, and realizable gains in resolution by processing will be insufficient to change the situation significantly.

The situation is better, as might be expected, with HST. We show simulated images with superpositions of WFC images from three and thirty (!) orbits; the thirty-orbit picture begins to show some detail, and quantities like color gradients can be extracted with some accuracy, but the (undersampled) resolution of a little less than a kiloparsec is inadequate to make a clear picture of what is going on in the arms, and one would expect that a more chaotic, just forming galaxy, while just as bright, would present an even more confused picture at this level of image quality.

Finally, the 16-meter images for exposure times of $2 \mathrm{hr}$ and $24 \mathrm{hr}$ are shown; while 24 hours may seem excessive, remember that the focal plane of the telescope can cover 25 of the assumed detectors which will cover an entire protocluster with one pointing, so in principle several hundred galaxies like this one can be studied with one such long exposure. The resolution and $\mathrm{S} / \mathrm{N}$ in the long exposure is similar to the ground-based picture of $290310 \mathrm{Mpc}$ distant, and, in fact, had the seeing been significantly worse in the 2903 image, we would not have been able to simulate the 16-meter image of the galaxy at $\mathrm{z}=1$ at all realistically! 


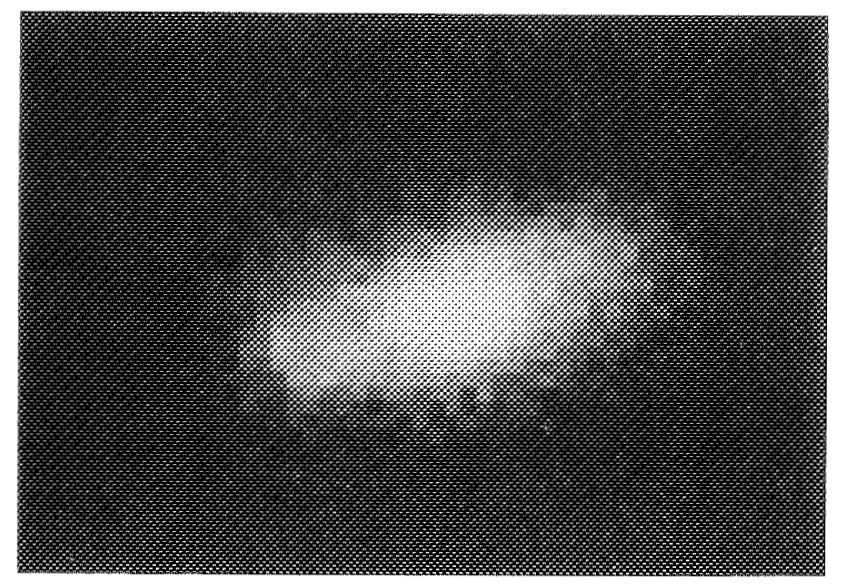

Keck after o hrs

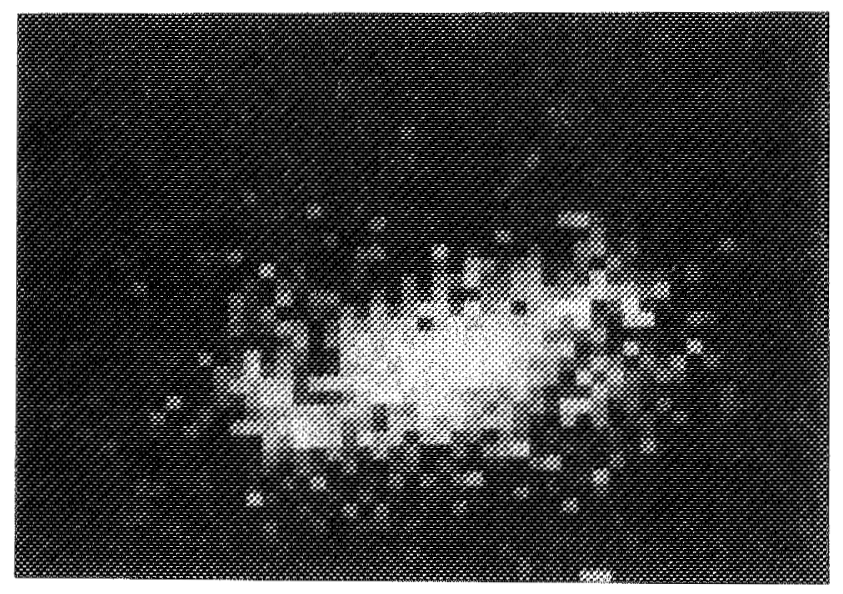

XIT after orbits

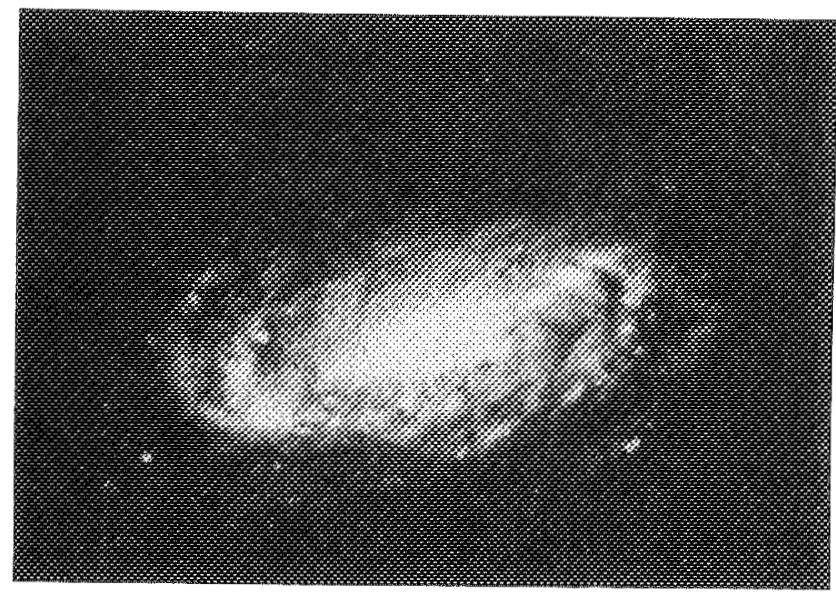

NGST after 2 hrs

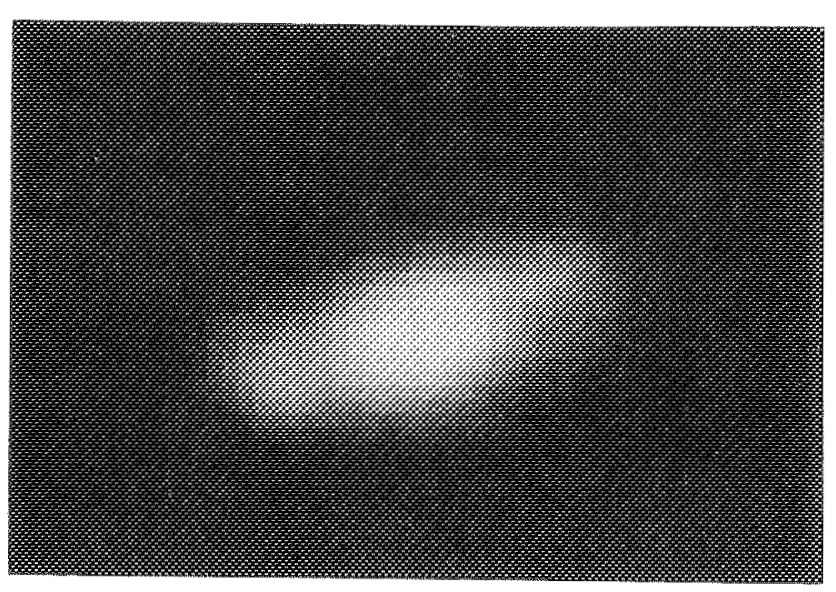

Keck after 30 hrs

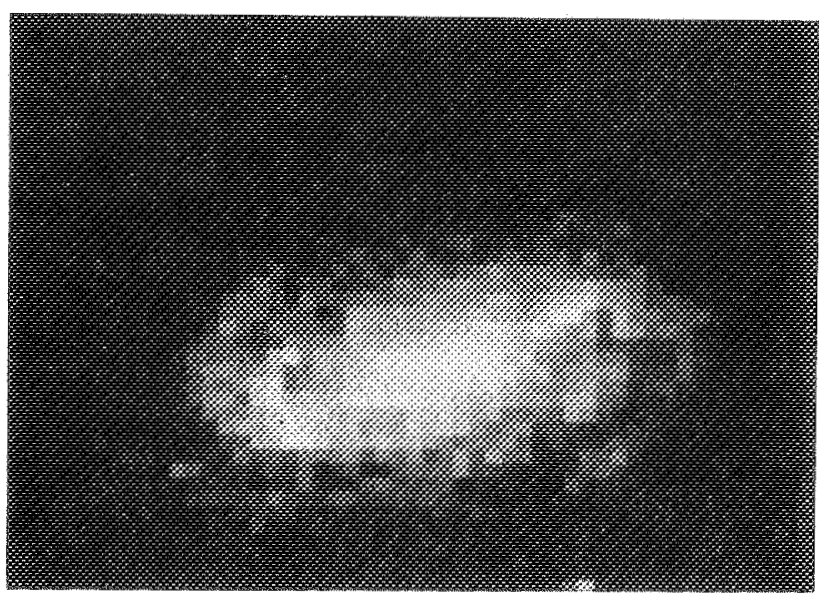

YIST after 30 orbits

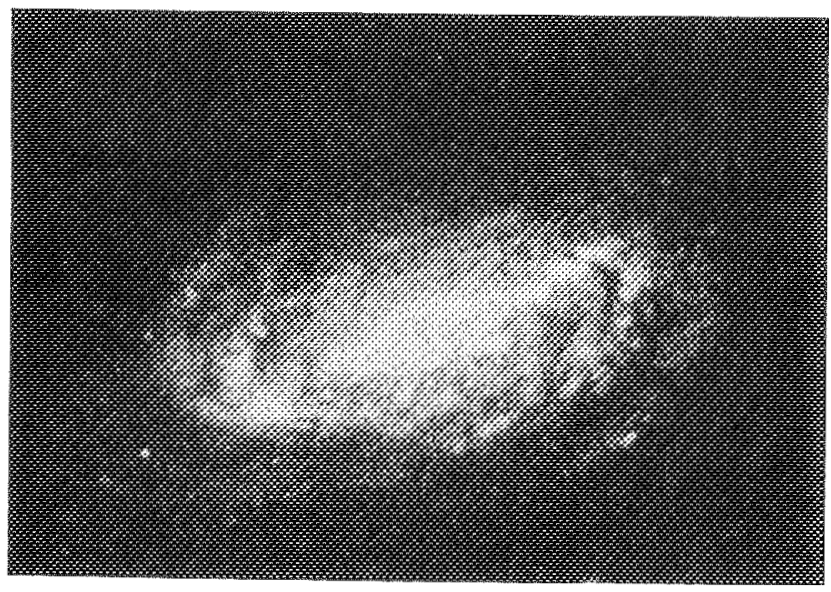

NGST after 24 hrs 
Similar spectacular gains are to be had with spectroscopy. It is easily possible to do dynamics on this galaxy at $\mathrm{z}=1$ with a spectrograph optimized for this sort of problem, which is an instrument much more like low-resolution spectrographs on large ground-based telescopes than the instruments typically used in space; one would hope that the dynamics of distant galaxies is an important enough problem that such an instrument might be part of its focal-plane complement. A spectrograph with a 0.1 arcsecond-wide slit critically sampled onto a CCD of the same sort as the imagers (i.e, with an $f / 2$ camera) would have a resolving power of 2000. A 24-hour exposure would result in an exposure level in the sky of about 3500 counts per pixel, with the galaxy at $10 \mathrm{kpc}$ radius contributing a signal of 300 counts. If one binned 4 pixels along the slit for an effective spatial resolution of $2 \mathrm{kpc}$, the resulting $\mathrm{S} / \mathrm{N}$ per resolution element is $12: 1$; farther in, at $2.5 \mathrm{kpc}$ radius, the $\mathrm{S} / \mathrm{N}$ in a single pixel row is 25:1 per resolution element, either sufficient to measure velocities to a few tens of $\mathrm{km} / \mathrm{sec}$ at this resolution (and, of course, to explore the stellar population and HII region chemistry in some detail.). The galaxy uses some 40 pixels (4 arcseconds) along the slit, so if, for instance, the spectrograph were fed by fiber ribbons, one could look at of order 50 galaxies at once if the field of the spectrograph could be made big enough, or using a fiber hydra, one could completely map a 4-arcsecond square region, essentially the whole galaxy. The latter possibility I find quite incredibly exciting; the dynamics of protogalaxies is certain to be complex, and 2-dimensional spectral mapping at relatively high spectral resolution would be a wonderful tool to study them, as well as a vast other suite of problems in astrophysics.

\section{DIsCUSSION}

Gallagher: How bad will the cosmic ray environment be in HEO?

Gunn: The cosmic ray environment is a problem for HST because of the high readout noise of the detectors - with the much lower noise expected for future detectors, repeated exposures can be made and cosmic rays identified and rejected.

Bahcall: I am surprised at the conservatism of these discussions regarding detectors and the field of view. In 20 years will we not do much better than a factor of 2 ?

Gnnn: We are looking at mosaics with a large number of pixels to cover the 5 arcmin field so it is a large gain.

Jackson: Can we support the data rates form these very large pixel sizes?

Gunn: Computer power increases so switfly that I do not see this being an issue in the future. 


\title{
Singeresent

$$
5 s-85
$$ \\ 360016 \\ 415217 \\ Planetary Astronomy with a Large Space Telescope
}

\author{
Robert A. Brown \\ Space Telescope Science Institute
}

$\mathrm{M}$ $\mathrm{y}$ topic is not well described in terms of objects; it is better viewed as a cluster of activities. The first is exploration. Voyager 2's recent fly-by of Neptune exemplifies the robotic thrust of the first thirty years of solar system exploration, which will culminate in human expansion into space. The second is planetary science, which is the investigation of the underlying processes that govern the planets or that brought them to their current state. The third is planetary astronomy, which is the gathering and analysis of light with planetary interest. Astronomy is one of several research pathways for advancing both solar system exploration and planetary science.

I am personally convinced that a 10-meter-or larger-telescope on the Moon or in Earth orbit would make major new contributions to both the exploratory and the scientific objectives related to planets and planetary systems. Nevertheless, we learned during the development of the Hubble Space Telescope (HST) that the potential contributions of a new system will remain just thatpotential-unless the investments are made to address the complexity of forefront planetary research. In this talk, I have selected some exciting projects in planetary astronomy, but my list is by no means exhausive. My purpose, simply, is to demonstrate both the latent scientific power and the associated special requirements of planetary studies using a large space telescope.

Solar System Inventory. The variety between and the relationships among the small bodies of the solar system is an arterial theme in planetary science. The study of these objects is a great research bridge between interstellar material and the meteorites. A large space telescope could extend significantly the inventory of small objects in the solar system.

Assuming detections are limited by background light, an aperture four times larger than HST would improve the faintest object that could be studied by a factor of 16 or 3 stellar magnitudes.

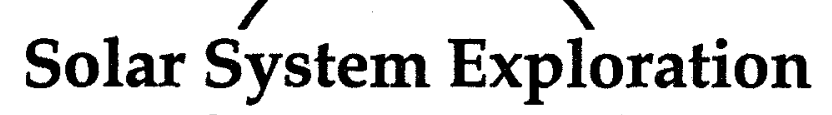
This gain would allow us to investigate the asteroid distribution to four-times smaller size or twotimes greater distance. Also, it could uncover entirely new classes of objects in the outer solar system.

An exhaustive search for faint solar system objects would require immediate access to all deep images taken with a large space telescope. Images of galactic and extra-galactic fields are, in effect, double exposures: the universe is seen through the solar system, which can introduce unexpected and interesting foreground images. Only if those objects are rapidly identified and recovered by follow-up observations, can the orbits are determined. Only then are the objects "known to science." To accomplish this, and so to derive maximum scientific benefit from the deep-space images, the proprietary data rights must be structured so as to permit dual access to their double information content.

Jupiter Atmosphere. Groundbased images of Jupiter at 5 micrometers wavelength indicate that openings exist in the Jupiter clouds through which telescopes can probe to warmer depths in the atmosphere. A correlation has been found between regions of higher brightness temperature and certain features seen at higher resolution in visible light. A diffraction limited 10-meter telescope could exploit this effect to study the vertical structure and distribution of minor constituents 
in the deep atmosphere by looking through the clouds. These observations-combining visible imagery and infrared spectroscopy-would benefit greatly from the small infrared diffractionlimited footprint, which would be about 400 kilometers at 4 AU distance.

Spectrometers with higher resolving power would be needed to perform critical studies of planetary atmospheres using a large space telescope. In the infrared, where the molecular constituents are spectroscopically active, the resolving power should be greater than about $10,000-a$ value somewhat higher than that discussed by Garth Ilingworth in his introduction to this workshop. In general, planetary studies require optimized instrumentation to derive full value from advances in the basic telescope itself.

Comet Nuclei. We believe that comets consist of interstellar grains and condensed gases conglomerated in the protosolar nebula. While the comet nucleus is thus an archaeological record, the coma and tail are not, because the gasses released from the nucleus are quickly modified by chemical reactions. Therefore, gathering the information locked in the nucleus requires addressing it or its immediate environment directly.

The acuity of large space telescope would allow studies of freshly produced parent molecules near a comet nucleus. For a 10-meter aperture, for example, one Nyquist-sampling resolution element at 0.6 micrometers would correspond to the apparent size of a typical 10 kilometer nucleus at a distance of $1 \mathrm{AU}$, and many comets come much closer to the Earth. Imagery obtained at this spatial resolution could discover how the morphology and rotational characteristics of the nucleus are related to its activity. One could, for example, place a spectrograph slit at various distances from the nucleus, measure parent molecules, and document their evolution in distance, which, in free flight, is time.

A critical requirement for observing comet nuclei-one shared by most planetary studies-is accurate target acquisition and tracking. The apparent motion of the target is complex, because it is a superposition of three components. One component is due to heliocentric orbiting, another is parallax caused by the telescope orbiting the Earth, and a third is possible planetocentric motions of the target-rotation or satellite orbiting, for example. The capabilities, first, to put the footprint of the telescope down on a definite place on the changing field, and, second, to hold it there, are prerequisites to interpreting the observational data with confidence. HST has taught us how detailed and challenging are the special pointing requirements for moving targets.

Mars Habitability. The President has declared human exploration of Mars to be a national objective, and that will require a deeper understanding of the geology, climate, and weather of Mars. One can envision major research programs on each of those topics.

A 10-meter space telescope would have approximately 6 kilometer resolution on the face of Mars at visible wavelengths. Using that, one could, for example, map geological units at a higher detail than has been possible to now. This would establish a basis to extend horizontally what robotic missions learn from in situ samples. Another application would be studies of martian dust storms, which regularly veil the entire planet. They originate in very small regions and become global in a matter of days. How do dust storms arise and grow? Can we understand them well enough to be confident about human habitation of Mars? A large space telescope could study Mars dust storms in an early growth phase with about 6 kilometer resolution-a powerful improvement in documenting their evolution.

Dust storm studies would require very rapid rescheduling performance by the operational system for a large telescope. The onset of a dust storm could be learned from groundbased observations. In a matter of hours, the large space telescope would need to acquire the planet and follow the storm's development continuously for a few days. This ability to respond rapidly to ephemeral events would benefit a wide range of planetary studies, and it could be designed into the planning and scheduling system of the large space telescope.

Io \& Jupiter's Magnetosphere. My next example uses the largest structure in the solar system, namely the magnetosphere of Jupiter. We have learned from four spacecraft flyby's and from groundbased observations that the jovian magnetosphere is dominated by heavy ions that originate in the volcanoes of the innermost major satellite, Io. We know many details about Io and its 
environment, but our understanding of its interactions are minimal. The phenomenology is extremely complex and variable-as in any great geophysical system. If we better understood the processes at Io, we would better understand the entire Jupiter magnetosphere.

A 10-meter telescope could grasp Io and its phenomena in detail. For example, with approximately 45 kilometer resolution at visible wavelengths, it could resolve the volcanoes and track its evolution. One could address such questions as: How exactly do the volcanoes develop? How does the volcanic material escape into the magnetosphere?

Complex, dynamic objects like Io call for complicated and flexible observing programs. It makes no sense to study just one facet of a complex problem, then to study some other aspect at a different time. An integrated campaign can study various aspects of the problem in one time frame. For Io, a campaign could follow the appearance of its surface, take spectra of its atmosphere, and coordinate with thermal ir and visible observations from the ground. This coordinated mode of research is used by the planetary community on a planetary missions. Synergistic investigations give a more complete picture of a complex problem. The \#1 recommendation of the planetary panel on the STAC - the Space Telescope Advisory Committee-was to develop this ability to integrate multiple, separately-justified observations in one observing suite. It requires additional integrative capability in the scheduling system, which HST does not currently possess. With forward planning, it could be designed into the operation of a future large telescope in space.

Extra-Solar Planetary Science. My final topic relates to a current intellectual revolution in planetary science prompted by the new ability to study other planetary systems-forming and already formed-around other stars. This new research area is based on generalizing the traditional questions about how the solar system formed. Now we can now ask: What is the prevalence of systems around other stars. Is the Kant-Laplace paradigm of planetary system formation valid? The answers are increasingly accessible in the astronomical record. For example, much of the Kant-Laplace evolutionary sequence of planetary systems has been exemplified by observations of collapsing molecular clouds, protostellar nebulae in Keplerian rotation, and disk structures around young stellar objects. Furthermore, there is every expectation that groundbased telescopes and HST will find self-luminous planets-young Jupiters and "superplanets" - around other stars. We will soon know how common and how various other planetary systems are.

A large space telescope could contribute two key things to extra-solar planetary studies. One, it could view the structures around stars that relate to planet forming. Two, it could detect mature planets like those in the solar sytem. I want to discuss those two topics separately.

Viewed from the nearest star-forming regions, about 150 pc distance, the Nyquist pixel at 0.6 micrometers for a 10-meter telescope subtends approximately $1 \mathrm{AU}$. This resolution would be powerful for studying, as examples, the evolution of protoplanetary disks and wind-disk interactions in T Tauri stars, and the nature of mature disks like $\beta$ Pictoris. In the near infrared, this telescope system could study hot, young planets.

Based on what we know about HST, could a large space telescope discover extra-solar planets using only reflected star-light? The graph shows HST's performance for an optimistic test case: the Sun-Jupiter system as viewed from 5 parsecs. At its greatest elongation of about 1", Jupiter would be 26 th visual magnitude, and the Jupiter/Sun flux ratio would be about $10^{-9}$. The central surface brightness of Jupiter is about 4 orders of magnitude lower than the wing of the Sun image, which would be the dominant background. Simply from the standpoint of information theory, to detect Jupiter under these conditions with a signal-to-noise ratio of five would require a 58 day exposure time. That is longer than the expected stability time of HST and its instruments.

For HST at visible wavelengths, the wing will consist of approximately equal contributions from aperture diffraction and micro-roughness scattering. This means that apodization cannot improve the contrast between the planet and star. (The diffracted component can be lowered, but the light scattered by surface errors cannot be removed.)

Using apodization, a future large space telescope can be made to operate with roughness scattering dominating the background. In that case, the contrast ratio of the planet to the stellar wing 


\section{HST \& Extra-Solar Planets}

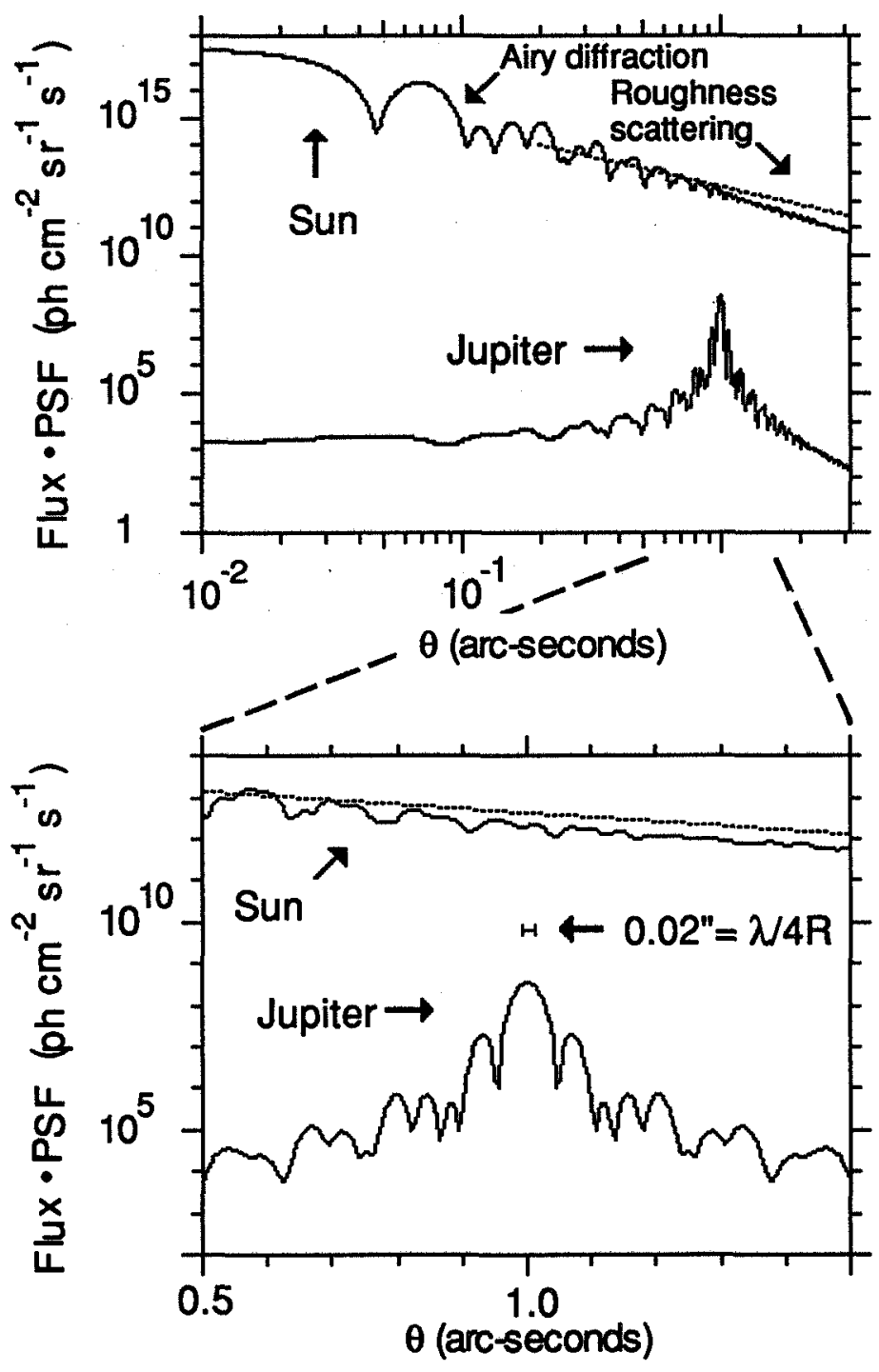

varies directly with the square of the aperture and inversely as the mean squared mirror roughness in the band of spatial frequencies on the telescope mirrors that scatter light of the given wavelength into the angular zone around the star being searched for planets. Therefore, the contrast ratio improves approximately a factor of 16 going from HST to a 10-meter telescope. Further, if the large space telescope has 25 times smoother optics, the contrast ratio would improve to unity-very favorable for searching a variety of nearby stars for planes.

At the time the HST mirrors were polished, the roughness power spectrum was not explicitly constrained by formal requirements. This was because no analysis existed of the scientific benefits associated with ultra-low scattering. With a future large space telescope, this could be corrected. The resultsbetter light management in the telescope and lower scatteringwould help many types of planetary observations. Indeed, lowering the veil of unwanted light around bright objects-quasars, stars, and planetswill open up one of the last unexplored regions of astronomical discovery.

Acknowledgements. Thanks to $\mathrm{Hal}$ Weaver, Marc Buie, and Ben Zellner for their contributions to this paper. Support for this work was provided by NASA under Contract NAS526555 through the Space Telescope Science Institute, which is operated by Aura, Inc.

\section{Summary}

Solar System Inventory Immediate access to all deep images Jupiter Atmosphere Special Instrumentation

Comet Nuclei Accurate pointing and tracking

Mars Habitability Rapid response to ephemeral events

Io/Jupiter Magnetosphere Integrated observing campaigns Extra-Solar Planetary Science Low-scattering optics 


\section{DISCUSSION}

Danly: Would you like to comment on the tradeoffs between separate telescopes dedicated to particular problems versus a very versatile telescope?

Brown: I don't believe we could justify both a special purpose planetary $10 \mathrm{~m}$ telescope and a special purpose "deep space" 10 meter telescope. The special requirements of various communities could be incorporated into the telescope at the beginning with little extra cost. It is expensive to add new requirements in the middle of a development project.

Diner: An exciting use of a 10 meter telescope would be the high resolution study of Venus at 2 microns which would have implications for the Greenhouse effect - as well as the study at 10 microns of high latitude clouds for dynamical processes.

Brown: There is certainly no end to the planetary problems awaiting investigation. Discussing the inner planets also raises another requirement, and that is the need to point the telescope closer than 40-50 degrees to the sun - which has implications for baffling. 


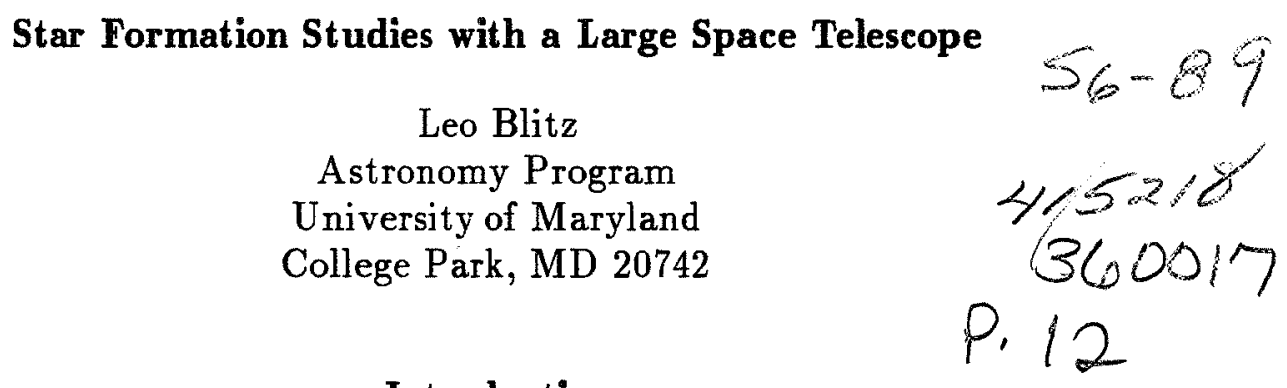

\section{Introduction}

Star formation is not a single topic. It encompasses virtually all of astronomy and virtually all interesting astronomical scales. This short paper can therefore be only the most cursory overview of the science that can be done with the next generation successor to the Hubble Space Telescope. Nevertheless, the broad range of science that can be addressed with this proposed instrument (which I will refer to as the NGST) in attacking questions of star formation is absolutely overwhelming. Furthermore, it is clear from other chapters in this report that many of the most interesting questions really devolve into questions of how stars form on various scales.

The first point that must be emphasized is that the actual process of star formation is hidden from view at optical wavelengths. The dust that veils the evolution from protostars into stars makes it impossible for any telescope working at optical wavelengths to probe into the placental environment to view the actual workings of the birth of a star. It is for this reason that much of the most important work that has been done in the last twenty years has occurred at millimeter and optical wavelengths. It is therefore largely as an infrared telescope that the NGST will obtain its most important observations for teaching us how stars form. There are nevertheless important questions that can be addressed only at optical wavelengths. The primary emphasis, then will be on the infrared and secondarily the optical capabilities of the NGST.

In what follows, I first discuss the general areas that make up the topic of star formation, and then address specific questions that astronomers are currently struggling with. The last part will be a discussion of the detailed capapbilities of the NGST in addressing some of these questions.

\section{The Questions of Star Formation}

The basic questions that are generally asked on the topic of star formation can be outlined as follows;

\section{1) How do new stars form?}

This is the basic question of star formation. What are the detailed physical processes that control the formation of stars? What controls the formation of duplicity and multiplicity in stars? Why do some stars form in clusters? What controls the low end of the initial mass function? What, in fact does the low end of the initial mass funtion look like? Why has it been so hard to detect the collapse of the interstellar medium into protostars?

2) How do planetary systems form? 
How common are planetary systems around other stars? Do planetary systems form at the same rate as do stars themselves, or are they much less common? What governs the formation of planets around new stars? Is there hope of detecting other planetary systems through direct imaging?

3) How do Stars form from Giant Molecular Clouds?

All stars currently form from molecular clouds, most form from Giant Molecular Clouds (GMCs). How do GMCs form? What controls the formation of stars within a GMC? What determines whether some stars are born in bound clusters and others in unbound associations? Does the initial mass function form all at once within a given GMC or is it the product of different modes of star formation (i.e. is star formation bimodal?)? Why are there apparently no globular clusters forming at the present epoch in the Milky Way, while at the same time there appear to be many forming in the Large Magellanic Cloud, one of the two satellite galaxies of the Milky Way? How do globular clusters form in the first place?

4) How do stars form in the disks of galaxies?

What role do the spiral arms have in the formation of stars in a galaxy? What determines the efficiency of star formation as a function of radius in the disk of a spiral galaxy? How does the process of star formation differ in the galaxies that have well defined spiral arms and those that have flocculent spiral structure? Similarly, what controls the star formation in $\mathrm{S} 0$ and $\mathrm{Sa}$ galaxies?

A related question regarding star formation in normal galaxies are is, for example, whether star formation is episodic in dwarf or other galaxies? Does star formation continue at a reduced rate in elliptical galaxies, especially now that we know that there are molecular clouds in such galaxies? If so, how does the star formation proceed in elipticals?

5) How do stars form in active galactic nuclei?

Stars are known to form with particular ferocity in starburst nuclei, and some active galactic nuclei such as Seyfert galaxies are also known to be starbursts. How do the conditions differ in the centers of starbursts from those in ordinary galaxies? Do the stars form differently in the nuclei of starburst galaxies? Is there an evolutionary connection between Seyfert nuclei and starbursts? What feeds the starburst? Are the ultraluminous galaxies simply this same phenomenon at a higher rate? Is the formation of quasars related to the starburst and Seyfert phenomenon?

\section{6) How did the first stars form?}

Are molecular clouds a necessary condition for the formation of stars? The conditions of star formation in the present epoch appear to require heavy elements to obtain the necessary cooling, which according to current ideas must have been formed from stars. How did the first generation of stars form, then, without these coolants (or alternatively, do we need to revise early cosmology to account for the first generation of stars?)? Was the initial mass function of the first generation of stars different from the present initial mass function?

\section{What to Look for}

The above questions by no means exhausts the set of important areas of investigation 
in the general area of star formation, but simply outlines some of the more obvious questions as I see them. To answer some of these questions we need to have specific methods of attack, particular observations that will answer as many of the questions as possible, and others that will illuminate the correct answers if a direct approach is not feasable. Let us consider some of the observations that can be made with the NGST to address a few of the questions outlined above. I shall indicate whether these appear to be best accomplished in the infrared or optical portion of the spectrum.

1) Direct detection of individual protostellar and neostellar systems. In this case, protostellar refers to stars that have not yet initiated nuclear burning, neostellar stars that have at least begun to generate internal energy by deuterium burning. Astronomers have not yet found unambiguous observational evidence for the collapse of the interstellar medium around an individual star. It may be that such evidence will require observations of the near infrared lines of hydrogen and carbon monoxide. Furthermore, the holy grail of star formation studies still eludes us as of this writing: the identification of a true protostar, as opposed to a deeply embedded neostallar object.

2) Direct and indirect detection of protoplanetary disks, and other planetary systems. The protoplanetary disks will be best observed (and possibly imaged) in the near infrared, while other planetary systems will be best observed in the optical through special imaging techniques and accurate astrometry.

3) Resolution of embedded stars and star clusters in galactic and extragalactic molecular clouds. Work on infrared arrays, especially the NICMOS system promise improvements of orders of magnitude from what is currently possible, so that imaging of embedded stellar populations can be carried out even in other galaxies with unprecedented sensitivity, resolution, and spatial coverage.

4) Work with these arrays, as well as the unprecedented sensitivity and resolution in the optical portion of the spectrum should make it possible to determine how the initial mass function behaves even below the hydrogen burning limit in both optically visible and embedded star clusters.

5) The detection of brown dwarf stars. As is shown below, the NGST is an ideal instrument for the detection of brown dwarf stars. Since brown dwarfs are also the prime candidates for baryonic dark matter, if the dark matter exists in that form, the NGST should, by being uniquely capable of doing important star formation studies, also provide important evidence for dealing with one of the most intractable conundrums in cosmology.

Other possible projects related to star formation studies with the NGST, most of which are not currently possible at any wavelength, are the following:

1) Resolution of $\mathrm{H}_{2}$ shock fronts in protostellar regions. This is not currently posible in the millimeter region, and furthermore, because the $\mathrm{H}_{2}$ can be observed directly, information on the primary molecular constituent is directly obtainable with the NGST.

2) Observation of the proper motions of protostellar and neostellar jets in 1-2 years.

3) Determination of the rotation curves of protoplanetary nebulae.

4) Direct imaging of rings, voids, and clearing of disks similar to the Kirkwood gaps in protoplanetary disks.

5) The near infrared spectroscopy of cocoon stars will be possible to distances $10^{4}$ times farther than is currently attainable.

6) Resolve star forming regions in nearby galaxies. 
7) Determination of the initial mass function of clusters down to A stars in galaxies as distant as M51.

8) Observations of $\mathrm{CO}$ lines at $2.3 \mu \mathrm{m}$ for stellar typing. It will be possible then to obtain the velocity dispersions of embedded star clusters.

9) Observations of the star cluster at the galactic center. It will be possible to resolve essentially all of the stars, determine the velocity dispersion of the stars as a function of galactic radius and confirm or refute the presence of a black hole at the galactic center.

\section{Capabilities of the NGST}

Having set the stage for what astronomers might like to observe with the NGST, we now turn our attention to the capabilities of the instrument. The science and the projects outlined above are necessarily an extrapolation from the interests and activities taking place at present. By the time the NGST is actually in operation, the science will have no doubt undergone a considerable change, and some of the questions posed above will have been answered. However, the representative capabilites discussed below should be expected to change very little, as long as the design parameters of the instrument do not change significantly.

In what follows, the design parameters outlined in the technical sections of this report are used.

\section{Optical detection of Jupiter-like planets}

At opposition, Jupiter has an absolute magnitude of $\sim-2$ at a distance of 4 AU. The NGST will be capable of detecting objects in the optical to a limiting magnitude of $\sim 31$. Thus it will be possible to detect Jupiter to a distance of $4 \times 10^{6} \times(4 \mathrm{AU})$ or $80 \mathrm{pc}$. That is, Jupiter itself could be detected with the NGST to a distance within which there are 100,000 stars! The major technical problem in such an observational undertaking would be to separate the image of a Jupiter-like planet from the central star. This problem is discussed in some detail in the contribution from Roger Angel.

\section{Protojupiters and Brown Dwarf Stars}

At a distance of $100 \mathrm{pc}$, Jupiter would be observed to be 50 milliarcseconds from the Sun. Observations of protojupiters would likely be done at $3 \mu \mathrm{m}$. The nearest rich molecular clouds are the Taurus and Ophiuchus star forming regions at a distance of 150 pc. Thus bright protojupiters could be marginally resolved in these clouds from their central stars. If Jupiter has a temperature of $\sim 100 \mathrm{~K}$, the peak of its blackbody spectrum occurs near $30 \mu \mathrm{m}$. In the 3 to $10 \mu \mathrm{m}$ region, an instrument has the greatest sensitivity to objects with surace temperatures of $300-1000 \mathrm{~K}$. Since this is the likely temperature range for brown dwarf stars, the NGST would be the best instrument for detecting these objects because of the excellent combination of large aperture and low infrared background.

\section{Protoplanetary Disks}

Known protoplanetary disks have a scale size of $\sim 1000$ AU. Again, one would wish to observe these disks in the near infrared. A $1000 \mathrm{AU}$ disk would subtend 50 milliarcsec at a distance of $20 \mathrm{kpc}$. Thus one could observe and detect protoplanetary disks virtually 
anywhere in the Milky Way! For the more nearby objects, one could detect structure in the disks, and possible see the early stages of clearing in Kirkwood gaps caused by a large Jupiter-like planet. Furtheremore, at distances within one kpc or so, it will be possible to obtain the detailed temperature structure of protoplanetary disks which would in turn provide important data to aid in understanding the chemical and physical evolution of the early solar system.

\section{Protostellar Outflowing Jets}

Protostellar jets have been the subject of much recent study because they seem inevitably to be a by-product of the star formation process and because understanding the physics of these objects may help to clarify the nature of extragalactic jets. These objects would primarily be observed in the optical portion of the spectrum. A typical size scale is $1 \mathrm{pc}$, which subtends 10 milliarcsec at a distance of $20 \mathrm{Mpc}$. Optical protostellar jets could thus be observed in the star forming regions in all of the galaxies in the local supercluster!

\section{Indirect Detection of Planets by Stellar Wobble}

The wobble induced by the Jupiter on the Sun is $\sim 0.005$ AU. At 1 pc, this subtends an angle of 5 milliarcsec. For astrometry, the NGST is expected to be able to measure stellar centroids to 0.1 milliarcsec. Thus one could detect the motion of the centroid of a star to $\sim 50$ pc. For more massive planets, or for those at larger distances from the central star than Jupiter is from the Sun, the limiting distances are correspondingly larger. If it were possible simultaneously to image the planet for which the stellar wobble were detected, good planetary mass estimates are possible for the nearer objects, as well as the possibility of inferring the presence of additional planets too faint to be imaged, but nonetheless massive enough to induce a wobble of the stellar centroid.

\section{Low End of the Stellar Initial Mass Function}

An interesting range to determine the low end of the stellar initial mass function consists of stars with absolute magnitudes in the range $M_{v}=15-20$ mag. With the NGST, one could have a complete volume limited sample of stars to distances of 100 pc, a volume containing $\sim 10^{6}$ stars. Thus the initial mass function could be completely determined to $M_{v}=20$ mag to this distance. Furthermore, since a number of well known clusters lie within or near $100 \mathrm{pc}$, it will be possible to determine whether the initial mass function for the field stars is the same as that for clusters at the lowest stellar masses.

In summary, the NGST would provide a unique capability to obtain observations of a host of questions central to understanding how stars form and how the star formation process affects and is affected by various environments in the Milky Way, in normal galaxies and in the nuclei of active galaxies and quasars.

\section{DISCUSSION}

Bender: What can one hope to say about the earliest star formation from 10 meter telescope data?

Blits: There are a number of observational approaches to the problem. The most direct is to try to determine the metal content of galaxies at the highest $\mathrm{z}$. No instrument will be better than the proposed telescope for this purpose. Other approaches are to look for stars in what appear to be otherwise primordial gas clouds, and imaging of starbursts at the highest redshifts attainable. 


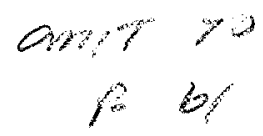

\title{
QUASI-STELLAR OBJECTS AND ACTIVE GALACTIC NUCLEI: PROSPECTS FOR A 10 METER SPACE TELESCOPE
}

\author{
Joseph S. Miller \\ Lick Observatory, Board of Studies in Astronomy and Astrophysics \\ University of California, Santa Cruz, California 95064
}

\begin{abstract}
I. Introduction
It is sobering to realize that, in spite of the fact that nearly 30 years have passed since the discovery of quasars, we still know very little about them. In consideration of what could be learned about them with a very large space telescope (VLST), I will start with an examination of the major issues associated with QSOs and active galactic nuclei (AGNs) as they appear at present. This will be followed by a short listing of the assumed performance of the VLST. I will conclude with an attempt to outline the major areas in which I would expect the VLST to make a large contribution.
\end{abstract}

\section{Major Issues}

\section{A. The central object}

We still do not know what the central source of energy in QSOs and AGNs is or how it works. It is not even clear that they all have the same kinds of central objects, though considerable uniformity in observed characteristics over the various classes of objects suggests that something basically similar is going on over a large range in luminosity. Accretion onto a massive black hole is the generally accepted hypothesis for the energy source, but direct observational evidence for this picture is slim at best; it has achieved prominance largely through elimination of other possibilities. Furthermore, even if we adopt the massive black hole hypothesis, how it works in detail is poorly understood. Mass estimates, generally based on theoretical considerations, range over three orders of magnitude. The source of fuel supply is unclear, as are details of the energy release mechanism.

We still do not understand the mechanisms that produce the continuum from $\mathrm{x}$-rays to the infrared, even at the level of deciding between thermal and non-thermal processes. The only exceptions for this are the blazars, where the observations strongly support a synchrotron emission process. We do not understand the origin of the emission-line clouds or their motions. In fact, the only thing that appears to be well established are that these 
clouds are photoionized by a flat spectrum source, and that the clouds come in both high density and low density versions (or perhaps a continuous range of densities).

The radio sources associated with quasars are also poorly understood. What produces them, and how is energy supplied? Why are most QSOs radio-quiet, while a small percentage are radio-loud?

\section{B. Evolution}

We know very little about the lifetimes of QSOs or AGNs. Was there an epoch of maximum QSO-activity, for which there is some evidence, and why? We do not know. Are low-luminosity AGNs the descendents of luminous QSOs? Do nearby galaxies contain dead QSOs, perhaps rather commonly? What do the luminous IRAS sources have to do with QSOs? Are they QSOs enshrouded in an envelope of dust? Does a "young" QSO differ from "mature" and "old" QSOs? Our ignorance in this general area is considerable.

\section{Relationship of AGNs to host galaxies}

While AGNs are clearly located in the centers of galaxies and there is a fair amount of direct evidence that QSOs are too, the relationship between the active nucleus and the surrounding galaxy is unclear. Are QSOs and AGNs a natural result of galactic evolution? That is, if we clearly understood the formation and evolution of galaxies, would we conclude that QSOs arise naturally and expectedly out of galactic processes, or are they pathological accidents resulting from some other influence, e.g., interactions between galaxies? We don't know.

Turning the question around, what is the influence of an active nucleus on the surrounding galaxy? Does it affect galactic structure or evolution? We don't know.

D. The bigger picture

Since QSOs can have very large luminosities and can produce large amounts of ultraviolet radiation, they may be important in affecting the ionization level of the intergalactic medium, but our information about this is rather limited. It is even possible that QSOs have played a role in galaxy formation. In any event, we can use distant QSOs as probes of the intergalactic medium and intervening galaxies, a field of research that continues to be very active and rewarding, even if we learn nothing about QSOs themselves from these studies.

Finally, as very distant objects with obviously extreme physical conditions, QSOs have the possibility of revealing new physics. While I don't consider this a likely possibility, it is still something that should be acknowledged. 
In the end we are not even clear about whether or not QSOs and AGNs are important. By important I mean that they play some vital role in the universe, such as, for example, important and necessary components in galaxy evolution or formation. Alternatively, they may be fascinating objects with bizarre properties whose real impact on the universe beyond them is of little consequence.

Anticipating the discussion in the last section, I expect that a VLST would have its major impact on A above, although some contribution to the remaining issues would also be expected.

\section{Performance}

For the discussion in Section IV, I will adopt the following performance characteristics for the VLST:

$\begin{array}{cl}\lambda & \text { Resolution } \\ 1600 \AA & 4 \text { mas (milliarcseconds) } \\ 5000 \AA & 14 \text { mas } \\ 3 \mu & 75 \text { mas } \\ 10 \mu & 0 ! 25 \\ 100 \mu & 2 ! ' 5\end{array}$

I feel that it is important that the VLST be a filled-aperture telescope. Synthesized large apertures can provide high resolution, but for objects like QSOs and AGNs, which are likely to have complex structures, I believe a filled-aperture telescope would make life vastly simpler. In addition, for a number of studies as will be indicated, the full light gathering power of a 10 meter aperture is extremely important. 


\section{Observational Prospects}

\section{A. Structure of the central object}

Even in the nearest AGN the expected size of the black hole and inner luminous accretion disk (if they exist) would be unresolved (sizes $\sim 10^{-5}-10^{-3} \mathrm{pc}$ ). Therefore we could not expect to see resolved the source of the central continuum, unless of course our current picture of what is going on is wrong. However, the broadline region (BLR) could be resolved in the nearest AGNs such as NGC 1068. Current models suggest that the BLR extends over a region roughly covering from $10^{-2}$ to of order a few parsecs, depending on the object. For NGC 1068, 4 mas corresponds to about $0.3 \mathrm{pc}$, and the VLST should at least partilaly resolve the BLR. Information about the geometrical organization of the clouds would be of enormous value, especially if some spatially-resolved velocity structure could be obtained for the BLR.

Though the BLR would be resolved only partially in the nearest objects, the VLST would be expected to resolve fully the narrow line region (NLR) out to redshifts of about 0.1 . Even for 3C 273 at $z=0.16$ one would expect to resolve the NLR partially. This could be of great potential value. For example information on the NLR ionization conditions as a function of position could give direct information on the properties of the central ionizing continuum source. Velocity structure within the NLR could direct information on the origin of the clouds and the nature of the central gravitational potential.

Our studies of NGC 1068 at Lick with a spectropolarimeter have revealed the presence of an obscured Seyfert type 1 region in this type 2 Seyfert galaxy. We have interpreted these results as indicating that the nucleus of NGC 1068 contains an obscuring torus that blocks our direct view of the type 1 region. Scattering of light from this region by electrons located outside of the torus allows us to see the hidden region, the scattered light being highly polarized. The VLST would resolve the scattering region and perhaps parts of the obscuring torus, giving us direct, detailed information about the structure of this object if spatially-resolved polarimetric observations were made. For example, if the ionizing radiation emerging from the central region is anisotropic in flux and hardness, as some models predict, this could be studied directly. Observations of the blue bump as seen reflected in a resolved scattering region could provide information on the origin of this feature, but this would be a difficult observation even with a VLST. It is possible that the IR emission region could be resolved at some wavelengths, which would provide us with an understanding of the mechanisms which produce the IR radiation.

In summary, I believe a VLST would provide crucial information otherwise unobtainable about the nature of the central objects in nearby AGNs. The interaction of observation and theory could take place at a much deeper level than it has in the past. 
B. The central regions of normal galaxies and those with AGNs

Recently evidence has been accumulating that some nearby galaxies (e.g., M31) contain massive compact objects in their centers, raising the possibiltiy that black holes may be common features in the nuclei of galaxies. This could also be interpreted as support for the view that many or even most massive galaxies go through an AGN phase, and in these nearby objects we are seeing the relic black holes of the dead QSOs. Two kinds of data are important for the investigation of this problem: high spatial resolution velocity profiles of the nuclear stars and accurate high-resolution light distributions. Clearly the Hubble Space Telescope (HST) will make very important contributions to this study, but principally only for relatively nearby objects. It is also important to carry out similar studies of AGNs and QSOs. For these the higher spatial resolution of a VLST is crucial, as one must overcome a large by large brightness contrast between the AGN and the stellar light.

As part of this type of study, there should also be a search for evidence for mergers. While it is well-established that quite a few active galaxies are involved in interactions or mergers with nearby. galaxies, it is also the case that many active galaxies appear to be quite isolated. Thus it remains unclear how important interactions are in producing AGNs. This question could be addressed at some level with VLST observations of the nuclear stars in AGNs and normal galaxies.

Recently work by Sargent, Filippenko, and others has shown that the AGN phenomenon extends to very low luminosity active nuclei, nuclei that would be thought to be "normal" or non-active without a careful study. How much can this result be extended? Do most or perhaps all galaxies contain a mini or micro-mini AGN? Once again HST will make important contributions to this question, but mainly for nearby galaxies. To reach out to more distant objects, important for evolutionary studies, will require the VLST.

\section{The galaxies associated with AGNs and QSOs}

For nearby AGNs such as Seyfert galaxies, it is fairly easy to study the general properties of the surrounding galaxies. But for more distant, luminous QSOs the nature of the associated galaxies remains unclear. This is an area of research where the HST will obviously make important new contributions, but again mainly for the nearer objects. Current evidence suggests that AGN activity increases with redshift, but nothing is known about the galaxies associated with distant luminous quasars and radio-quiet QSOs. From the limited studies by Boronson, Oke, Stockton, and others that are possible with ground-based telescopes, we have learned that the galaxies associated with QSOs often show distinct peculiarities. A good example, is $3 \mathrm{C} 48$, with its very high luminosity from early-type stars. I believe we will find that the galaxies associated with luminous 
quasars are in fact often abnormal; that is, if the AGNs were removed, we would still find the surrounding galaxy peculiar. An investigation of this requires observations of QSOs to at least a redshift of about 1-1.5, which will require both the high resolution and light-gathering power of a VLST.

\section{Radio source interactions}

Currently there is available some evidence from the studies of van Breugel, Miley, and others that radio jets can interact in important ways with the interstellar media of the galaxies in which they are embedded. Visible manifestations of this are emission regions, perhaps excited by shocks, and regions of enhanced star formation. The VLST could make unique contributions to the study of radio-source-galaxy interactions. Out to a redshift of $\sim 0.1$ it would be able to resolve the regions studied with VLBI; even for 3C 273 some resolution of the inner jet region could be achieved. This range of distance includes several superluminal sources such as $3 \mathrm{C} 84$. At present we know nothing about the optical characteristics of these galaxies at similar scales to those observed with VLBI, and HST will not have enough resolution to do much on this problem.

Further out in the galaxies ( $\mathrm{d} \sim 100-100,000 \mathrm{pc}$ ), the jets studied with VLA resolution could be studied with HST, but the limited light-gathering power of that telescope would be a limitation. Similarly, searches for optical radiation from radio lobes requires large apertures more for light gathering than for resolution, and the brightness of sky is a major handicap for ground-based telescopes.

There is currently a rapidly developing area of study concerning distant luminous radio galaxies. Much can still be done with ground-based telescopes, and the HST will also make important contributions in this area. I am sure a VLST would be needed to push this study to very distant objects, where available observations indicate strange things are going on.

E. High redshift QSOs and the epoch of galaxy formation

Several QSOs with $z>4$ have already been identified. In the context of current thinking about galaxy formation, these QSOs must be associated with quite young galaxies or galaxies still in formation. This prospect makes these objects all the more interesting. Careful studies with a VLST operating at the limit of its performance in light-gathering power and resolution could provide unique and extremely valuable data on the earliest stages of galaxy evolution, data which would be unavailable by any other means. 


\section{Concluding Remarks}

I feel I have hit only a few highpoints in this discussion of the impact of a VLST on AGN and QSO research. As I pointed out, for many studies the large light gathering power compared to the HST is as important as or more important than the resolution. I believe the greatest impact would be on studies of the structure of central objects themselves, about which we know embarrassingly little when one considers the effort put into their study. However, as is generally the case, the greatest discoveries will likely come in areas which cannot be anticipated at present.

\section{DISCUSSION}

Stockman: You mentioned the need for a full aperture - can you expand upon that and can you comment on the dynamic range that you need?

Miller: The dynamic range will be very large, virtually unlimited. It is hard to be quantitative but dynamic ranges of $10^{4}-10^{5}$, maybe $10^{\circ}$ will be very common. For the other part, the filled aperture, the structure in AGNs will be rather complicated and it is the details that you care about, not the overall structure. For example, if it is elongated or two blobs instead of one blob would be interesting, but the details could be even more interesting than a simple model. I see an interferometer and a filled aperture as actually working in parallel to one another.

Stockman: Is there a specific dynamic range that is required by a VLST to study the surrounding regions of QSOs?

Miller: I would expect that a dynamic range of about $10^{6}$ would be very useful if one wanted to study regions within $0.1^{\prime \prime}$ (or even less) of an active nucleus, but $I$ have not done any detailed calculations, so this a rough guess.

Thompson: Could you comment on the relative roles of polarization and infrared imaging in studying embedded sources?

Miller: We really do not know enough about the potentials of infrared imaging. I expect that polarization and infrared imaging data could be quite complementary, as they have been for star formation regions.

mingworth: Can one see directly into the nucleus in the infrared? That is, at a wavelength of order 10 microns, is the optical depth low enough?

Miller: Once again, we do not enough to answer that question in general, b ut from what little we do know I expect some or many will be optically thin at that wavelength. 
STEITAR FOFULATIONS IN GATAXIES: THE SCIFNITIC POIFNITAL

FOR A 10-16 in SPACE THI ESCOPE

$57-89$

J. S. CALTAGHER

AURA, Inc. and Space Telescope Science Institute<smiles>[Y]#[As][As]=C</smiles>

\section{INIRODUCIION}

$$
\text { p.11 } 3600 / 8
$$

With the advent of multi-wavelength data from advanced ground-based and space observatories, we are beginning to construct a consistent model of how the universe reached its present state. Despite some formidable difficulties, the big bang hypothesis has thus far provided a useful framework for interpreting many observable phenomena, including the abundances of expected primordial nucleosynthesis properties such as ${ }^{4} \mathrm{He}$ and the presence of the cosmic background radiation. There are also a number of remaining puzzles; e.g. how large scale structure forms and how most baryonic matter appears to become concentrated into galaxies. The situation is therefore encouraging but it is premature to say that we have solved the mystery of the origin of galaxies and other large structures that populate the current universe.

Some examples of the types of questions that we would like to answer are:

* When and how did galaxies form? Was this process relatively coeval as outlined in older models, or do galaxies form over a wide range of times?

* What fraction of the total baryonic and dark matter is gravitationally bound into galaxies? What is the dark matter? How does the dark and baryonic matter interact as galaxies form and evolve?

* How are properties of galaxies connected to large scale properties of their enviromment? For example, it has been known since the pioneering work of Hubble in the 1930s that galaxy morphologies systematically vary between low density regions of the universe and the very dense cores of rich clusters of galaxies, but the origin of this correlation is still not understood.

* How did the chemical elements form and disperse?

* Is there a physical connection between nuclear activity in galaxies and the formation or evolution of their "normal" stellar and gaseous components?

* What factors control the rate of evolution of galaxies; e.g. what determines the star formation rates and gas exhaustion time scales within galaxies? 
Having set the stage on which extragalactic astronomy currently must play, let me turn to the importance of studies of stellar populations in galaxies for further progress on fundamental astrophysical issues. As stars form they produce an analogy to a geological record by trapping some of the conditions at the time of their formation, such as elemental abundances or sometimes the state of the galactic gravitational component. The evolutionary histories of galaxies and therefore of the universe can in principle be recovered by making appropriate observations of stars within galaxies (see Norman, Renzini, and Tosi 1986 for an overview on stellar populations).

\section{THE STELTAR STRATA OF GAIAXIES}

\section{Overview}

Although a great deal of progress has been made in interpreting the integrated light from galactic stellar populations, the most unambiguous results are obtained when stars are studied individually. Unfortunately, at the present time investigations of the details of stellar populations through measurements of individual stellar parameters are limited primarily to our own Milky Way galaxy and its satellites (the seven dwarf spheroidal systems and the Magellanic Clouds; see van den Bergh and de Boer 1984). More general information on evolved low mass, and intermediate mass stars is just now becoming available from $C \mathrm{CD}$ observations of Iocal Group galaxies (e.g. Freedman 1989; Rich et al. 1989), but beyond the Iocal Group (distances greater than about $1 \mathrm{Mpc}$ ) data on resolved stellar populations are still sparse (see Hudon et al. 1989).

The current limitations impose severe constraints. For example, the only precise ages for very old stellar populations are for Milky Way globular star clusters. We must apply a kind of generalized cosmological principle when we think of star clusters (or abundances of radioactive elements and their decay products) as giving an "age" of the universe. Other galaxies could well be older than the Milky Way if galaxy formation is not coeval, which would increase the difficulty in reconciling ages from nuclear stellar clocks with those found from the expansion rate of the universe. An irreconcilable difference in these two time scales would obviously have profound intellectual consequences.

All astronomers are therefore eagerly anticipating the gains in angular resolution (factors of 3-10 over the best and typical ground-based data) that the Hubble Space Telescope (HST) will provide. These will enable us to really resolve and measure colors and magnitudes of a wide range of stellar types in nearby galaxies. We will then see if the Milky Way and its gravitationally bound neighbors are indeed the typical members of the Local Group of galaxies that we have always assumed.

Looking beyond the HST, higher angular resolution and more collecting area are desirable to push such investigations much more deeply into the stellar strata of Local Group galaxies as well as to rich stellar populations well beyond the Local Group. In justifying such a major effort it is useful to 
recall the basic advantages gained by observing the individual members of stellar populations:

* Mature Theory. The theoretical understanding and sophistication of numerical models for the structure, evolution, and atmospheres of stars equal or exceed those of any other class of astrophysical object. Empirical data are therefore readily interpreted in terms of stellar physical and evolutionary characteristics.

* Good Empirical Data. Galactic astronomy and its extensions to the Magellanic Clouds have provided an excellent framework for the interpretation of many other features of stellar populations that are yet to be fully incorporated in theory. Examples include calibration of pulsation period - luminosity relationships that allow precise distance determinations, defining standard ranges of mass loss rates for various classes of stars, or defining the spectroscopic and photometric signatures of interacting binary star systems.

* Historical Recond. Table 1 summarizes characteristic ages of the stellar strata that are seen in the Milky Way. The application of stellar populations to the ages of galaxies is one of many "historical" applications of stellar population studies. Counts of numbers of short lived $O B$ stars allow us to measure current star formation rates (Gallagher and Hunter 1987), while numbers of stellar remnants (e.g. white dwarfs; Iben and Laughlin 1989), long lived stars and elemental abundances give us a clue to the past history of the star formation process. Comparisons between the evolutionary histories of nearby galaxies and that deduced from in situ abservations of younger galaxies at high redshifts will provide a key check on our still rudimentary theoretical models for galaxy evolution. As these models improve, we will make detailed tests of our ideas about galaxy formation and evolution by establishing that they are in agreement with observable characteristics of galaxies at the present epoch.

* Superb Test Particles. Stars are good approximations to point masses. They also have the advantage of sharp spectral line features which allow velocities to be determined at the $\mathrm{km} / \mathrm{s}$ level even for apparently very faint stars. Furthermore, since stars have tiny angular sizes they can be used to define precise coordinate frames that are useful for proper motion studies that in combination with radial velocities to yield information on the full three dimensional dynamical structures of astrophysical systems.

Although not all of these issues can be addressed through observations of galactic stellar populations, an understanding of stellar populations will improve our ability to model evolutionary processes in galaxies. Two brief examples of this type of program which include illustrative recent references to ongoing work follow. 
TABIE 1

Stellar Ages

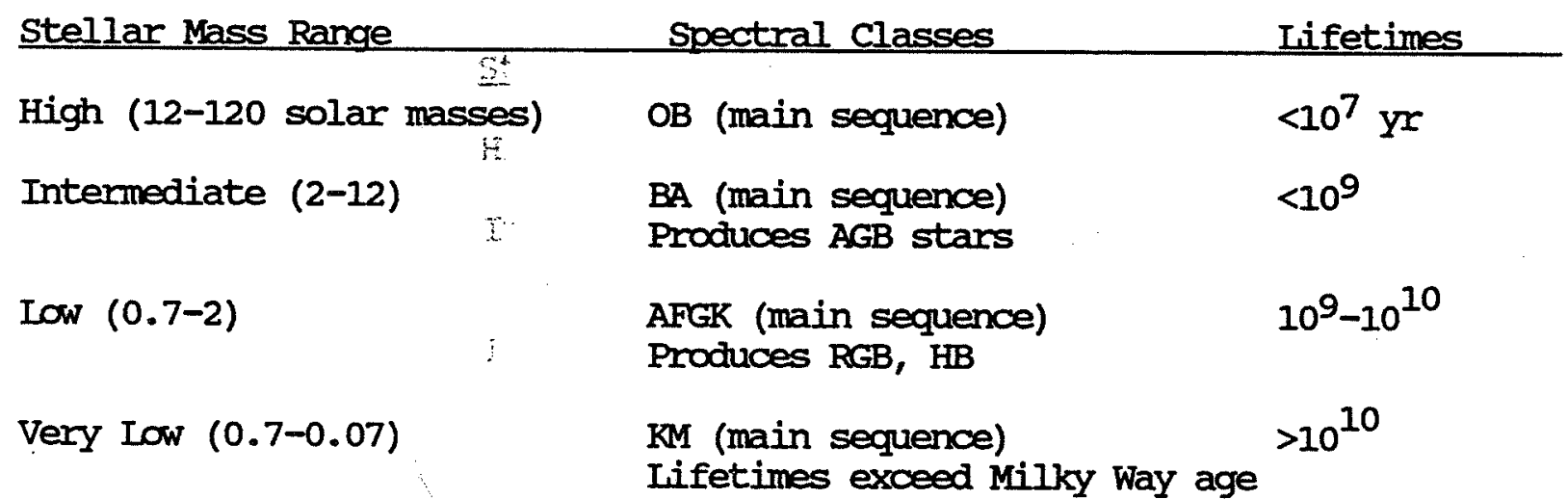

\section{Example: Evolution of old Stellar Populations}

The color magnitude diagrams for star clusters where more than about 5 Gyr has elapsed since stars last formed have well defined properties. The main features on such observers' Hertzsprung-Russell (H-R) diagrams are as follows:

* Main sequence turnoff. The luminosities and colors of low mass stars leaving the core hydrogen burning main sequence evolutionary phase are to first order functions of stellar age and metallicity. Observational determinations of the main sequence turnoff in combination with abundance estimates is the primary age dating tool for the oldest star clusters. Such observations require reaching to at least $\mathrm{M}_{V}+6$ for good colors for high quality age estimates. If the data go fainter than this, then information is also obtained on the initial mass function (see Hurley et al. 1989 and references therein).

* Red giant branch. After stars have left the main sequence evolutionary phase, they relatively rapidly change structure to have compact cores and distended convective envelopes. Stars in the red giant phase of their lives are primary optical luminosity sources in older stellar populations. The location of the red giant branch on color magnitude diagrams is a function of metal and helium abundances, but for masses below those of the sun, is less sensitive to stellar mass. Observations of the structure of the giant branch are therefore particularly useful as probes of element abundances in galaxies with old stellar populations.

* Horizontal branch. The location of stars on the horizontal branch of the globular cluster H-R diagram depends mainly on degree of mass loss from the hydrogen envelope and an unknown "second 
parameter". Some horizontal branch stars pulsate and are seen as RR Lyrae variables that are useful but intrinsically faint standard candles that can be used to measure distances.

Asymptotic giant branch. These are more luminous evolved stars than the red giants and represent stars in their last major nuclear burning phases. Properties of asymptotic giants depend on the age of the stellar population and degree of earlier mass loss.

Compact remants. Dying stars will leave behind a fraction of their mass in the form of a black hole, neutron star, or white dwarf, the type of remnant depending upon initial mass and details of evolutionary histories. Compact remnants are extremely faint (unless energized by mass accretion in close binary star systems, where they can become luminous broad band radiators), but provide unique information on properties of a star cluster at the time of their formation. The white dwarfs are especially useful for this type of investigation since they have well-defined cooling tracks as a function of mass.

Many of the key characteristics of old galactic stellar populations can therefore be determined through measurements of apparent magnitudes (fluxes) and colors for ensembles of stars. When combined with distances these lead to locations of stars on the color-luminosity plane that can be compared with well known local objects (e.g. globular star clusters in the Milky Way) and with theoretical predictions. In making such observations full wavelength coverage of normal stellar energy distributions (0.1-20 microns) are invaluable in minimizing errors due to transformations between observable and theoretical characteristics (e.g. bolometric luminosities and effective temperatures are primary theoretical quantities).

With present day ground-based telescopes, studies of resolved stellar populations can only extend as far as the Magellanic clouds for lower main sequence stars (e.g. Mateo 1988; Elson et al. 1989) and the Local Group for the red giant branch (c.f. Heasly et al. 1988; Rich et al. 1989). The proposed 10-16 m large space telescope would fantastically increase the power of these techniques and thereby enable us to firmly establish the evolutionary states of the galaxies that populate our local corner of the universe.

\section{Example: Young Stellar Populations - the Stellar Birth Process}

H-R diagrams for young stellar populations are in some ways less clean than those for very old stellar populations. While the core hydrogen burning main sequence is well defined, stars may cross regions of lower surface temperature more than once during their lifetimes in response to the effects of mass loss. Because main sequence stars radiate most of their flux in the ultraviolet, young stellar complexes are dominated at optical wavelengths by the cooler and intrinsically more luminous blue supergiants that evolve from massive main sequence stars. Yellow and red supergiants are rare among very massive stars, and can be mixed up with asymptotic giant branch stars at all but the highest red supergiant luminosities. Variables are conmon among evolved massive stars, and include the classical cepheid variables that are primary standard candles in determining extragalactic distances. 
Because main sequence 0 stars that photoionize hydrogen to produce highly visible HII regions are short lived, they are very useful as probes of galactic star formation rates. The technique is in principle simple (e.g., Gallagher and Hunter 1987). One measures the luminosity from an $H$ recombination emission line and uses this information to calculate the number of 'typical' $O$ stars that must be present. If one can guess at the total stellar mass produced each time a typical o star is formed, then one has measured the mass of stars produced during the lifetime of the typical 0 star and the star formation rate immediately follows. Similar methodology can be applied to any short-lived evolutionary phase for stars of known lifetime; e.g. for red supergiants or Type II supernovae. This technique has been applied to nearby galaxies using resolved stellar populations (e.g. Hoessel and Anderson 1986) and to more distant galaxies by measuring HBalmer emission line luminosities (Hunter et al. 1982; Kennicutt 1983).

There is, however, a key assumption: that the distribution function for initial stellar masses (initial mass function), which sets the relative mass fraction in massive stars, remains roughly constant in all types of galaxies. While there is reasonable evidence that this holds true in low density disks of galaxies, two widely discussed problem areas remain: (1) Low mass stars may form independently of massive stars, thus even if the initial mass function is invariant, the stellar birth rate derived from 0 stars will be too low. (2) In extreme circumstances such as ocour in star bursts, the initial mass function may be subject to major variations.

Thus to understand the current rate at which galaxies are turning their gas into stars, we must check the validity of the assumptions about the initial mass function. This will be done to rather faint magnitude limits in the Magellanic Clouds $\left(M_{v}>9\right)$ with the HST but stars whose main sequence lifetimes exceed galactic ages cannot be reached even elsewhere in the Local Group of galaxies. Similarly, in the nearest star burst galaxies the HST angular resolution corresponds to 1-2 pc, which is insufficient to over come crowding effects and allow us to directly study stellar content within active starbursts.

\section{APPIICATIONS OF A IARGE SPACE TEELSCOPE}

A 10-16 $\mathrm{m}$ space telescope has several attributes which make it well suited to studies of stellar populations: high angular resolution, large collecting area, and multi-wavelength coverage. In considering sample programs for such a facility, I chose to emphasize photometric observations (where the gains of such a telescope over ground based facilities are huge) that could be made in a survey mode. Other powerful capabilities for stellar population studies include UV/IR spectroscopy, especially in multi-object modes (for measurements of stellar kinematics, chemical abundances, mass loss rates, etc.). These areas will all be pioneered by the HST. To be conservative, I have based the projects on a $10 \mathrm{~m}$ aperture; even so the results would be incredible!

The performance of a $10 \mathrm{~m}$ space telescope in terms of galactic stellar population studies are sumarized in Tables 2 and 3. These capabilities will allow us to extend studies from the few nearest galaxies that are now 
accessible to hundreds of galaxies in the nearby universe thereby testing assumption that the Milky way is a typical galaxy. The methods that now can be used only to probe galaxies in the local universe will similarly be extended to high redshifts and thus to younger galaxies. A few programs are listed below to whet the observer's appetite:

* Ages of the Oldest Stars. New generation ground-based telescopes and the HST will complete the inventory of globular star clusters in the local supercluster and its surroundings. With a $10 \mathrm{~m}$ space telescope we will: (1) Determine precise ages of globular star clusters in the Local Group using data that will be of equivalent resolution and superior photometric quality (in terms of precision and wavelength coverage) to the best current observations of globular star clusters in the Milky Way. (2) Obiain the approximate ages for elliptical galaxies through cruder studies of globular star clusters in the nearest field elliptical galaxies. (3) Probe the Virgo cluster with the same types of techniques that are now being used in the Andromeda galaxy, which are sufficient to reveal dramatic differences between cluster and field galaxy early evolutionary histories. Note that in many models for origins of galaxies, cluster galaxies form first because of the higher densities of the cluster enviromment. These stellar ages will be compared with expansion ages (which will also be known to umprecedented precision due to new data on distances to galaxies based on primary stellar calibrators out to $\mathrm{D}=50-100 \mathrm{MpC}$ from the large space telescope). A fundamental cosmological test is then to see if these two types clocks in fact yield the same age for the universe, as they must in the standard cosmological model.

Universality of the Stellar Birth Process. A description of the stellar birth process is necessary for understanding the evolution of galaxies. With a $10 \mathrm{~m}$ class space telescope we can explore new aspects of this issue: (1) Measure the stellar luminosity function and infer initial mass functions for a range of stellar ages down to near the lower limit for stellar masses in the Magellanic Clouds. This will test the universality of the initial mass function in galactic disks, and as a side benefit will reveal the evolutionary histories of the Magellanic Clouds in great detail (e.g. Elston et al. 1989). (2) Directly observe the kinds of stars make up stellar populations in poorly explored dense stellar environments; e.g. the nuclei of the Milky Way, M31, and M33. (3) Young stellar populations in star burst galaxies will also be resolvable. Nearer examples of such galaxies include luminous blue galaxies (e.g., NGC 3310), M82, and extragalactic HII regions such as II ZW 40. Multi-wavelength observations of individual stars will provide the basis for a understanding the products of the star formation process when it is operating at high activity levels that are currently rare. 
Table 2

10. melescope Resolution Footprints in Galaxies

Location

Band

Size

Corments

Magellanic Clouds

optical

$0.004 \mathrm{pc}$

see most main sequence stars in MC disk \& clusters.

Local Group

optical

$0.05-0.1$

Resolve disks, bulges, star clusters to level of most evolved stars \& main sequence OBAFG.

\begin{tabular}{|c|c|c|c|}
\hline & Infrared & $0.1-1$ & Many protostars. \\
\hline Virgo Cluster & Optical & $1-2$ & $\begin{array}{l}\text { Like M31 with current } \\
\text { g } r \text { o u n d - b a s e d } \\
\text { telescopes. }\end{array}$ \\
\hline
\end{tabular}

'Nearby Universe'

Optical

10

UV

3

2-3 microns

10 microns
40

100
Unresolved galactic backgrounds set most performance limits.

Table 3

Stellar Sensitivity Benchmarks

Stellar Type Band Maximum Distance Objects

Sun, G2 V

M dwarf

K giant

M supergiant

O main sequence
Optical

Optical/ Infrared

Optical

Infrared

UV
$2 \mathrm{Mpc}$

$.1 \mathrm{Mpc}$

20 Mpc

$40 \mathrm{MpC}$

200 Mpc
Iocal Group

Magellanic Clouds

Virgo \& Formax Clusters

Iocal universe 
Chemical Abundances and the Evolution of Elliptical Galaxies. This program would involve both spectroscopy and photometry. Photometry would be done on resolved the $K$ giant populations in the outer regions of Virgo and Fornax cluster ellipticals. These data will enable us to examine the range and levels of heavy element abundances from even relatively mundane photometric measurements. Such information will constrain models for the formation of elliptical galaxies. Photometry would be followed up with spectroscopy to confirm specifics of the chemical abundance patterns, which provide further information on how nearby $\mathrm{E}$ galaxies formed and evolved during their active star forming phases billions of years ago.

Supernovae. Supernovae will be detectable to cosmological distances, even at near infrared wavelengths (cf. Van Buren and Norman 1989). The implications of such capabilities range from determination of key cosmolgical parameters through use of supernovae as standard candles to observing the creation of metals as supernovae explode in young, distant galaxies.

In summary, a large space telescope would place our understanding of the evolution of the nearby universe on a sound quantitative basis. There is no alternative to resolving individual stars if we wish to obtain an unambiguous understanding of the natures of galactic stellar populations. This goal is critical to understanding evolutionary processes in galaxies and to the effective use of galaxies as cosmological "markers". Telescopes with large collecting areas that are capable of high angular resolution (and therefore must be in space!) are required to do this job.

\section{ISSUES FOR THE DESIGNERS}

1. Stellar population studies in galaxies require imaging of fields with maximally complex structures (e.g. spatial structures with significant modulation on all scales down to the instrumental resolution). Often the scene will also cover a very wide range in surface brightness. Work on this problem therefore requires careful attention to scattered light and superior detector dynamic range, including resistance to poor behavior from saturated pixels.

2. High photometric precision is very desirable. Therefore careful attention needs to be paid to stability of the instrumental profile over time and with position across the field of view. High quality flat fielding will also be necessary.

3. Many stellar population projects require multi-wavelength observations of targets for maximal science return. Multiplexing detectors for simultaneous multi-wavelength (e.g. UV, optical, IR) observations can therefore greatly enhance scientific efficiency of the large space telescope for stellar population studies. 
4. Science trade-offs between angular resolution and collecting area should be thoroughly studied. Many sources suggested for study in this paper are likely to be confusion-limited for photometric studies with a $10 \mathrm{~m}$ class space telescope. The same collecting area configured as a compact interfercmeter could prove more powerful than a monolithic aperture for work in this field.

5. A $10 \mathrm{~m}$ telescope in space will be photon-starved for spectroscopy. The difference of 100 in bandwidth between "spectroscopic" and "photometric" observations combined with the factor of 2 better throughput of "photometric" systems lead to a 6 magnitude difference in limiting magnitude between the spectrosoopic and photometric operational modes. This can only be made up through long integration times in the spectroscopic mode, which will require superior detectors for low flux level, long exposure performance.

I would like to thank the Flagstaff members and associates on the Hubble Space Telescope WF/PC team, Bill Baum, Hugh Harris, Deidre Hunter, and Dave Monet, for many stimulating discussions about observations of galactic stellar populations with high angular resolution from space observatories.

\section{REFERENCAS}

Elson, R. A. W., Fall, S. M., and Freeman, K. C. 1989, Ap.J., 336, 734.

Freedman, W. L. 1989, A.J., 98, 1285.

Gallagher, J. S. and Hunter, D. A. 1987, in Star Formation in Galaxies, (NASA Conf. Pub. 2466), p.259.

Heasly, J. N., Christian, C. A., Friel, E. D., and Janes, K. A. 1988, A.J., 96, 1312 .

Hoessel, J. and Anderson, N. 1986, Ap.J. Suppl., 60, 507.

Hudon, J. D., Richer, H. B., Pritchet, C. J., Crabtree, D., Christian, C. A., and Jones, J. 1989, A.J., 98, 1265.

Hunter, D. A., Gallagher, J. S., and Rautenkranz, D. 1982, Ap.J. Suppl., 49, 53.

Hurley, D. J. C., Richer, H. B., and Fahlman, G. G. 1989, A.J., 98, 2124.

Iben, I., Jr. and Laughlin, G. 1989, Ap.J., 341, 312.

Kennicutt, R. C. 1983, Ap.J., 272, 54. 
Massey, P., Garmany, C. D., Sikley, M., and DeGioia-Fastwood, K. 1989, A.J., 97, 107.

Mateo, M. 1988, Ap.J., 331, 261.

Norman, C. A., Renzini, A., and Tosi, M. 1986, Stellar Populations in Galaxies, (Cambridge University Press: Cambridge).

Rich, R. M., Mould, J. R., Picard, A., Frogel, J. A., and Davies, R. 1989, Ap.J. Lett., 341, L51.

Van Buren, D. and Norman, C. A. 1989, Ap.J. Lett., 336, L67.

van den Bergh, S. and de Boer, K., eds. 1984, IAU Symposium No. 108. Structure and Evolution of the Magellanic Clouds, (Reidel, Dordrecht).

\section{DISCUSSION}

Thompson: Could you clarify your comment about the angular resolution of ground-based telescopes at 10 microns.

Gallagher: Even though the seeing on the ground at 10 microns is good enough that a large telescope can be diffraction-limited, the high background will limit the problems that can be studied compared to a cooled large space telescope. 

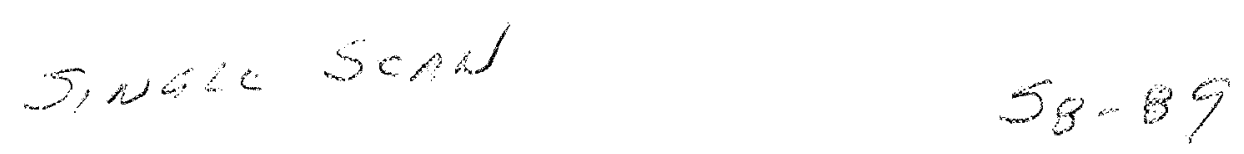

$4 / 5228$

Quasar Absorption-Line Studies with HST Successor

Richard F. Green

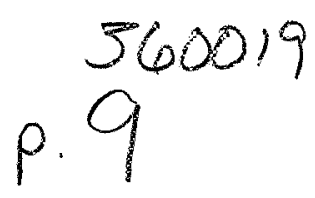

\section{Kitt Peak National Observatory \\ National Optical Astronomy Observatories}

The study of quasar absorption lines has occupied substantial amounts of time on large-aperture telescopes in the last 20 years. The importance of the topic is reflected in its being an accepted Key Project for HST and a major scientific driver in proposals for 8 - 11 meter ground-based telescopes.

Contained in the investigation of quasar absorption lines are fundamental astrophysical questions: What is the history of chemical enrichment in various galaxy environments? What is the major ionizing energy input to the clouds? Competing sources are the ultraviolet flux and shock energy from stellar winds and supernovae-in local regions of active star formation, as contrasted to the diffuse UV background from quasars and proto-galaxies. How are the clouds distributed with respect to the large-scale structure traced out by luminous material? The spatial distribution of the absorbers at higher redshifts may hold the clue to determining the evolution of large structures with cosmic time.

The current state of knowledge has absorption systems classified on the basis of the visibility of metal-line features and apparent velocity with respect to the quasar systemic redshift. Those systems with velocities within $-10,000 \mathrm{~km} / \mathrm{s}$ of the quasar redshift are the associated systems. The Broad Absorption Line systems are interpreted as arising in energetic outflows in the vicinity of the quasar, typically manifested in broad troughs of absorption in highly ionized species such as $\mathrm{N} \mathrm{V}$ and $\mathrm{O}$ VI with blueward extensions up to 0.1c. Associated narrow lines have been detected, particularly in C IV in radio quasars (Foltz et al. 1986, Sargent et al. 1988a). The existence of such systems could be attributable to halos of galaxies in clusters associated with the quasars, consistent with the finding of Yee and Green (1987) of an increasing tendency at higher redshift for radio quasars to be situated in moderately rich clusters. An alternative is that the interaction of the radio source with the intracluster medium produces a number of ionized clouds distributed in velocity along the line of sight.

Absorption systems with redshifts less than those of the quasars by velocities exceeding $10,000 \mathrm{~km} / \mathrm{s}$ are generally considered to be intervening. For typical abundances and ionizations, metal lines become visible when the optical depth at the 
hydrogen Lyman limit approaches unity. The detected systems are about evenly split among those with C II stronger than C IV (found only among the systems with the highest H I columns), those with C IV stronger than C II, and those with the two transitions about equal. Strong line features detected at moderate spectral dispersion are created by a number of individual components with a complex velocity structure and ionization varying strongly among components. These complex systems are observed too frequently to be attributed to lines of sight through the centers of rich galaxy clusters, intercepting multiple galaxy halos. In a photoionization model, the diffuse cosmic UV background is insufficient to produce the strengths of features with ionization potentials lower than that of hydrogen; a local source of additional energy is required, such as hot, young stars. As shown by several statistical studies (e.g., Lanzetta et al. 1987), the mean free path to absorption in both C IV and Mg II implies a very high absorption cross-section at moderate to high redshifts. As found by Tytler (1982) and Bechtold et al. (1984), and later confirmed by Sargent et al. (1988a), the distribution in redshift of the Lyman limit absorption systems is consistent with a uniform space density (in comoving coordinates) with a constant cross-section. All these lines of evidence are consistent with associating the optically thick absorbers with galaxies.

If that association is indeed true, then the absorption line systems contain valuable information about the evolution of physical conditions in the gas phase of galaxies with cosmic time. Those systems selected in surveys for the C IV doublet were shown by Sargent et al. (1988b) and Khare, York and Green (1989) to increase in numbers more rapidly than a uniform distribution back to $z=2$, then decrease toward earlier times. Although the evidence is not yet conclusive, Sargent et al. argue that the early epoch represents the build-up of the total $\mathrm{C}$ abundance. Trace elements in the most optically thick systems such as chromium and zinc give a measure of the depletion onto grains and net heavy element abundance $([\mathrm{Fe} / \mathrm{H}])$ in the range of 0.1 to 0.01 solar. The dust to gas ratio inferred from spectral reddening of quasars by Fall et al. (1989) is about 10\% of the Galactic value, while Chaffee et al. find molecular to atomic gas ratios below $10^{-4}$ of the Galactic value.

A paradigm for interpreting some of the metal-line systems was offered by York et al. (1986), in comparing the complex velocity and ionization structure to a line of sight through a region of active star formation (see Walborn et al. 1984). Turbulence and ionizing energy input comes from supernova shocks and strong winds from massive stars, which also provide an intense near-ultraviolet radiation field. The large crosssection to absorption is then produced by an aggregate of star-forming regions, such as dwarf galaxies, in the gravitational potential of what will become a more condensed 
system through dissipation and collapse.

The plethora of absorption lines observed at wavelengths less than that of the Lyman $\alpha$ emission-line of the quasar cannot all be associated with metal lines from the few strong systems, and are attributed to Ly $\alpha$. The connection with the metal-line systems is not clear: at the high column density end, about $20 \%$ show weak C III and $\mathrm{Si}$ III absorption. The extension of the distribution of neutral hydrogen column densities from the Ly $\alpha$ "forest" clouds to the metal-line clouds is also not clear, especially given the difficulties of component blending and profile fitting. There is a strong observed evolution in the number density of absorbers with redshift, and no evidence for a break in that trend to the highest redshifts observed ( $z=4.7$ by Schneider et al. 1989). There is no strong signal for velocity clustering in the Ly $\alpha$ only systems as there is for the metal-line systems. Study of absorption in quasar pairs with small angular separation shows some evidence for the existence of voids (Crotts 1989), although the results for single sight-lines are more controversial. There is also a well-documented "proximity effect", in which the density of Ly $\alpha$ clouds drops near the quasar redshift. This effect is interpreted in analogy to a Stromgren sphere, in which the quasar ionizes the surrounding intergalactic clouds; the radius (inferred from redshift) out to which the quasar has measurable influence allows a determination of the diffuse intergalactic radiation field.

Several theoretical models for the Ly $\alpha$ forest clouds are consistent with the existing data. It is possible that the clouds are composed of primordial material and are not associated with galaxies. They could be in pressure equilibrium with a hot, diffuse intergalactic medium. That equilibrium could depend on size and shape; some systems could be collapsing under self-gravity, while others could be expanding and "evaporating". The relative influence of shocks and photoionization must be determined.

What will HST (and FUSE) teach us about these different absorbing clouds? The general answer is that the space observations will allow direct comparison of physical conditions in low-redshift absorbers with those in high redshift absorbers as inferred from ground-based data. The low-redshift systems are identified by $\mathrm{Mg}$ II absorption; access to C IV, Ly $\alpha$ and other strong UV transitions require vacuum observations. A C IV survey will allow a stronger test for evolution of the number density of such systems, which can be interpreted in terms of a changing extragalactic radiation field and changing galaxy cross-section. HST spectra can be searched to build statistics on the low-redshift Ly $\alpha$ forest systems. Their distribution can then be compared with the galaxy distribution in the same volume to see if those clouds populate voids, or trace the galaxy density. The proximity effect can be derived statistically to determine a 
low-z value of the diffuse extragalactic background radiation density. From observing lines of sight through the Milky Way halo and local group galaxies, the depletion pattern can be determined as a function of metallicity. Because the low- $z$ absorbers can be associated directly with host galaxies, the problem of current gas-phase metallicity as it relates to the history of star formation can begin to be addressed.

Ultraviolet spectroscopy with HST (and FUSE) will have great value because of the large number of line diagnostics found at wavelengths shortward of $1200 \AA$ that yield densities and effective temperatures. The problem with working in the same spectral region redshifted to optical wavelengths is the extreme difficulty in de-blending and making unambiguous identifications, because the line density of metal lines and Ly $\alpha$ forest lines is so high. Key physical diagnostics such as C III $\lambda \lambda 977,1175$, can be nearly impossible to disentangle from the large number of Lyman series and other lines from lower redshift systems. With the smaller number of systems, and sharply decreased number of Ly $\alpha$ forest lines at low redshift, HST has a distinct advantage.

The key to relating quasar absorption lines to galaxies lies in the redshift range in which both can be well studied $(z<1)$. HST cannot adequately address this region because of photon limitations.

As a benchmark, take a 16th mag, low-redshift quasar. Then the flux in the UV is $4 \times 10^{-4}$ photons $\mathrm{cm}^{-2} \mathrm{~s}^{-1} \AA^{-1}$. The second generation spectrograph, STIS, will have an effective area of about $300 \mathrm{~cm}^{2}$ for a spectral resolution of 10,000 , yielding a count rate for the nominal quasar of 0.02 counts/s/resolution element. To detect a $10 \mathrm{~m} \AA$ line at 1 $\sigma$ significance requires a $S / N=25$, or an exposure of 30,000 seconds. Now, at 16 th mag, there are -200 quasars with $1 \delta 1>30^{\circ}$; the median $\mathrm{z}=0.15$ !

To study evolution in the physical properties of absorbers, it will be necessary to probe redshifts beyond 0.15 , probably to $z>0.5$. Yee and Green (1987) have argued that by $z=0.6$, there has been a significant brightening in the $4000 \AA$ radiation of the typical galaxy, which would certainly correspond to a change in the local component of ionizing flux above the Lyman limit. That redshift also marks a change in the dynamical properties of clusters that host radio galaxies and quasars.

To derive physical conditions and make detailed velocity comparisons with intervening galaxies requires the high dispersion spectroscopy, which then limits HST to objects around 16th mag. However, the expectation is that only 1 quasar in 65 will have a line of sight intercepting an absorber with $z<0.15$ (implying 3 systems for our sample) and only 1 in 15 will catch an absorber with $0.15<z<0.75$, or some 5 systems amenable to detailed study. The steepness of the quasar surface density as a 
function of magnitude gives a strong advantage to larger aperture for these programs. For throughput comparable to that of HST, a 10-m telescope would allow highdispersion spectroscopy of 19 th mag objects, with $2 /$ square degree; a 16-m would reach to 20 th mag at $12 /$ sq. deg.

Several gains will be realized with larger aperture. The physical conditions, such as temperature, density, abundances, and ionization in the absorbing clouds can be determined in direct relation to the abundance gradients and local radiation fields inferred from composite spectra and $\mathrm{H}$ II regions of the host galaxies. Increased aperture will allow access to other probes; for example, unreddened late B stars will be observable beyond the distance of M 33. The interstellar medium of local group galaxies can then be examined for velocity structures in star-forming regions, for metallicity as a function of star formation rate and history, and for depletion as a function of local metallicity. High quality telescope optics that yield high spatial resolution would provide an added bonus. With the capability of resolving "spectroscopic" binaries, we would have continuous spatial probing of adjacent sight lines as the orbital separation changes. It would be possible to separate turbulent, systematic and thermal motions along Galactic and Local Group sight lines at resolutions up to 100,000. The specified performance would yield $\sim 10$ A.U. separations at $1 \mathrm{kpc}$. Similarly, quasar lensed images can be used to probe the interstellar medium in the lensing object. At $\mathrm{z}=0.2,20$ mas separation corresponds to $100 \mathrm{pc}$, sufficient to probe individual clouds and complexes.

A strong scientific motivation for a large-aperture telescope with high throughput and the capability for high-dispersion ultraviolet spectroscopy is to raise quasar absorption line studies to the level of determining the chemical and dynamical evolution of interstellar and halo gas with cosmic time. 


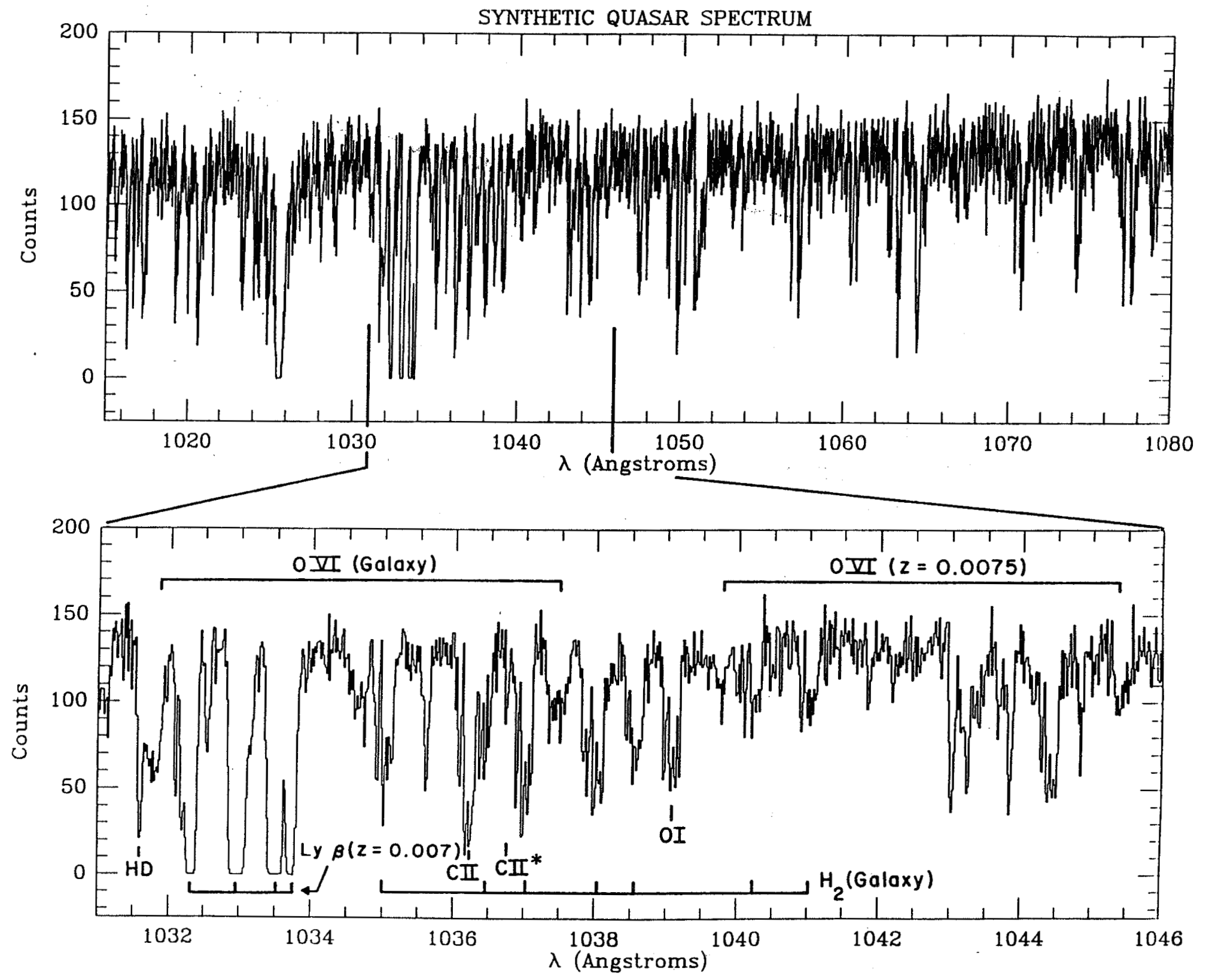

Synthetic quasar spectrum from Jenkins and Green showing the complex of spectral features anticipated near the O VI line for the Galaxy and a possible low red-shift absorbtion complex. The resoltuion is 30000 . 

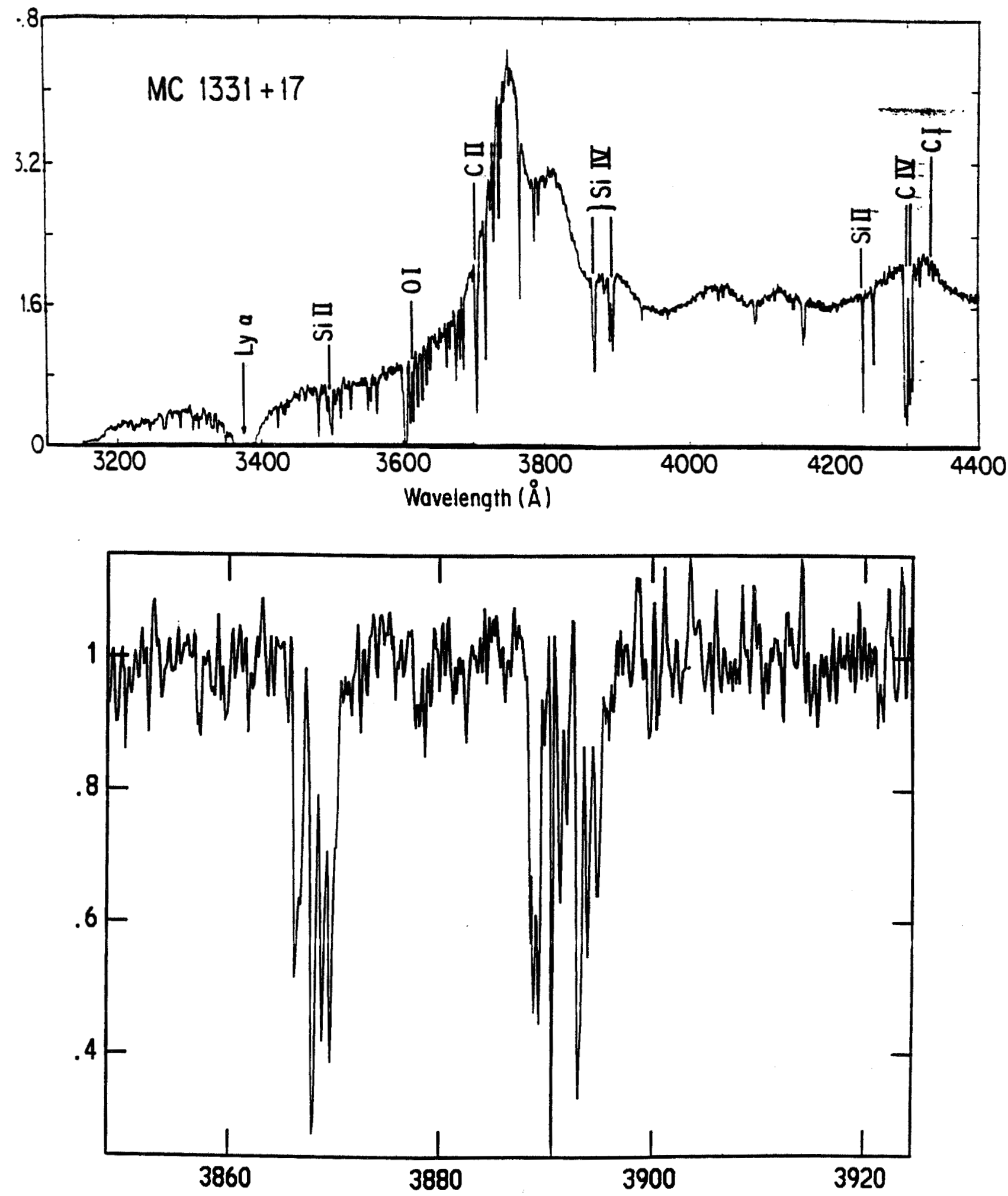

An example showing the necessity for high dispersion. The upper plot is from Chaffee et al., while the high-dispersion $(R=20,000)$ observation from Green and collaborators shows the complex velocity structure and blending of the Si IV line. 


\section{References}

Bechtold, J., Green, R.F., Weymann, R.J., Schmidt, M., Estabrook, F.B., Sherman, R.D., Wahlquist, H.D. and Heckman, T.M. 1984, Ap.J., 281, 76.

Crotts, A.P.S. 1989, Ap.J., 336, 550.

Fall, S.M., Pei, Y.C. and McMahon, R.G. 1989, Ap.J.(Lett.), 341, L5.

Foltz, C.B., Weymann, R.J., Peterson, B.M., Sun, L., Malkan, M.A. and Chaffee, Jr., F.H. 1986, Ap.J., 307, 504.

Khare, P., York, D.G. and Green, R.F. 1989, Ap.J., 347, 627.

Lanzetta, K.M., Turnshek, D.A. and Wolfe, A.M. 1987, Ap.J., 322, 739.

Sargent, W.L.W., Boksenberg, A. and Steidel, C.C. 1988a, Ap.J.Suppl., 68, 639.

Sargent, W.L.W., Steidel, C.C. and Boksenberg, A. 1988b, Ap.J., 334, 22.

Schneider, D., Schmidt, M., and Gunn, J.E. 1990, Ap.J.(Lett.), in press.

Tytler, D. 1982, Nature, 298, 427.

Walborn, N.R., Heckathorn, J.N. and Hesser, J.E. 1984, Ap.J., 276, 524.

Yee, H.K.C. and Green, R.F. 1987, Ap.J., 319, 28.

York, D.G., Dopita, M., Green, R.F. and Bechtold, J. 1986, Ap.J., 311, 610. 


\section{DISCUSSION}

Miller: What is the advantage over the ground of a large space telescope for absorption line studies? Is it working in the UV on low redshift objects?

Green: Yes, it is the ability to use the diagnostics we know (CIV, SiIV, OVI) in the redshift range where we can also study the galaxy $(z=0.25-0.75)$. Then we need access to the UV with the same light-gathering power.

Gunn: The calculations I described about galaxies around quasars indicate that if the absorbers are galaxies, and dwarf galaxies, they can be seen to redshifts of about 2 with the NGST.

Green: These observations will be key to closing the loop in establishing the relationship between the absorbers and distant galaxies. I would still emphasize the lower redshift systems, because we would probably be able to study the galaxies in more detail spectroscopically.

Stockman: Couldn't you use HST or NGST to study the actual clouds seen at redshifts of $z \simeq 2-3$ ? This would be the complement of your desire to study clouds at redshifts where the galaxies/clouds can be observed from the ground.

Green: The high redshift clouds can be related to NGST galaxy imaging, as pointed out by Jim Gunn. In order to understand the metallicity and velocity structure of a cloud in the context of its location and local history of star formation, we require detailed spectroscopic study of the associated galaxy; lower redshift galaxies will be much more amenable to spectroscopy with high spatial resolution. 


\title{
USE OF A 16 M TELESCOPE TO DETECT EARTHLIKE PLANETS
}

\author{
Roger Angel \\ Steward Observatory \\ University of Arizona \\ Tucson, AZ
}

\begin{abstract}
If there are other planets similar to the Earth, in orbit around nearby stars like the sun, they could be detected through their thermal emission, and their atmospheric compositions measured. A large telescope in Earth orbit is necessary, cooled to around $80 \mathrm{~K}$. In order to overcome the limitations set by diffraction and zodiacal background emission, a primary mirror area of some $200 \mathrm{~m}^{2}$ is required, and an overall dimension of some $20 \mathrm{~m}$. The $16 \mathrm{~m}$ telescope under consideration at this conference is ideally suited to the task. It could be configured as a filled round aperture, and the required very high contrast ratio could be obtained with focal plane instruments that block or interfere sections of the wavefront. Alternatively, the primary surface could be made from four slightly separated $8 \mathrm{~m}$ monoliths. The latter configuration yields the highest resolution and contrast when operated as an interferometer, with little negative impact on performance for direct imaging. A properly optimized telescope should yield spectra of good quality $(\lambda / \Delta \lambda=100$, signal/noise ratio of 50) of earthlike planets as far away as 10 pc, given integrations of a week or so.
\end{abstract}

\section{Introduction}

A popular T-shirt bears the now familiar image of the Earth from space and the caption, "Good planets are hard to find". It is the sad fact that the exploration of the solar system has found the Earth to be unique. The differences are perhaps no more clearly evident than in the beautiful spectra of thermal emission obtained by Hanel and his colleagues and collected together in figure 1. Mars atmosphere shows only carbon dioxide, Venus has also absorption by sulfuric acid. Only in the Earth's spectrum are found the features of water and oxygen in the form of ozone. The strong features of methane and steeply rising spectra of the outer planets reflect their very cold and inhospitable temperatures.

Today we seem to be on the brink of finding planets, albeit of the most massive type, around nearby stars. Astrometric and spectroscopic techniques with modern detectors have the capability to detect the motion of stars that would indicate massive orbiting planets. However, direct imaging that could give more information than simply mass and orbital motion, even of Jupiter sized planets of the nearest stars, is at the very limit of sensitivity of the Hubble space telescope. In this paper we will 


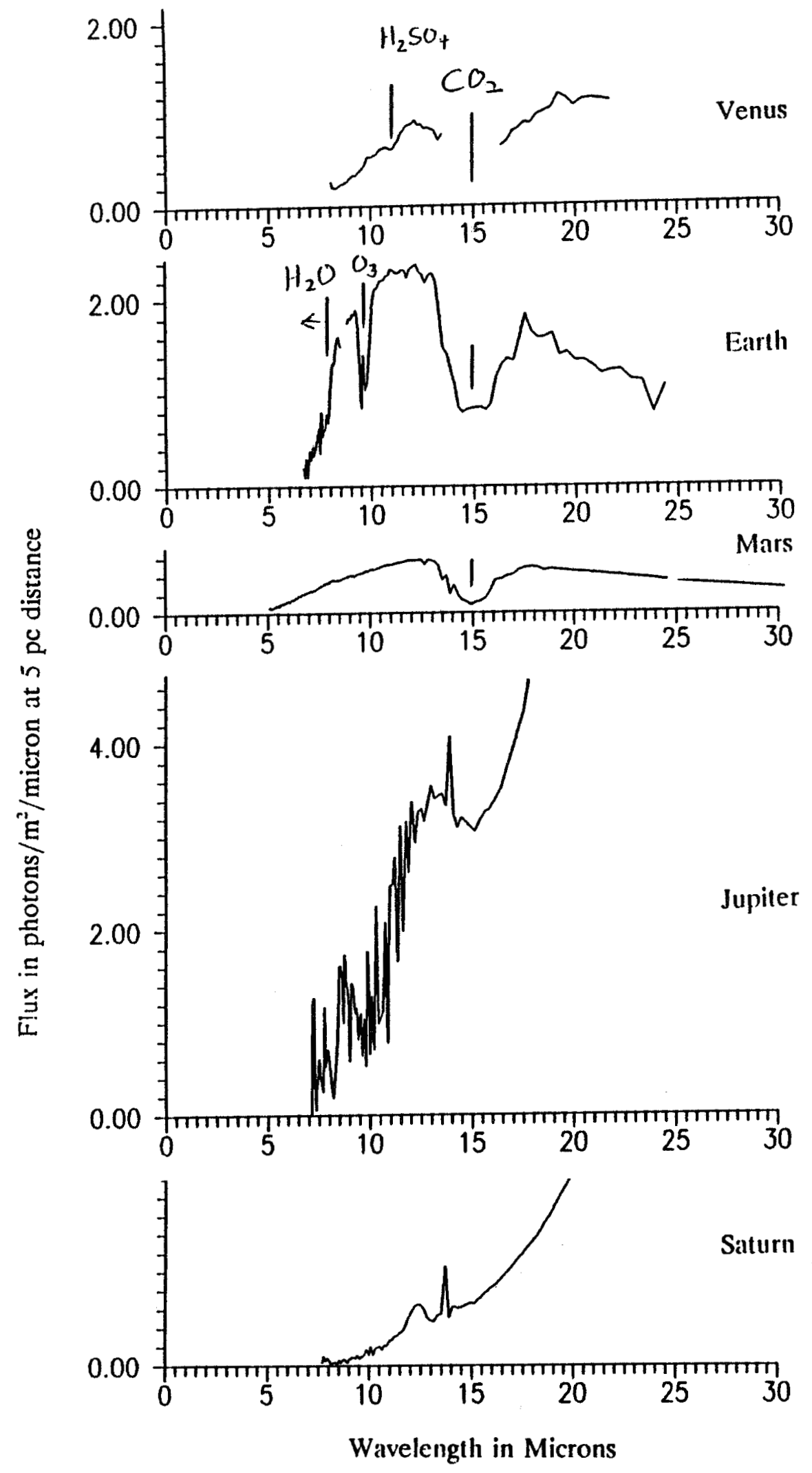

Figure 1 Spectra of thermal emission from planets in the solar system adapted from Hanel, (1983) and Kunde et al (1977). All are plotted to the same scale. 
consider the far more difficult task of searching for planets similar to the Earth in size and temperature, and orbiting solar type stars. Such earthlike planets, with mass one millionth that of the sun, will be completely undetectable by their gravitational influence, and can only be found by direct imaging. The difficulty arises because of their extreme faintness, and their very close proximity to their bright host star. The nearest stars that are like the sun and not in binary systems lie at a distance of about $4 \mathrm{pc}$, and a survey to include a dozen or so excellent candidates would need to extend to $8 \mathrm{pc}$. It follows that the angular separation of planets with radius of one a.u. will be $1 / 8$ to $1 / 4$ of an arc second.

The reward of detecting radiation from earthlike planets will be quite extraordinary. If there were a planet with Earth's characteristic atmospheric spectral features, these could be measured. This is a quite remarkable prospect, because the Earth's atmosphere has been strongly modified by the presence of life. Thus there is the possibility that infrared spectroscopy could reveal life on other planets, if its chemistry produces similar chemical imbalance. John Bahcall has raised the question at the meeting as to how certain one could be of a biological origin, if an earthlike spectrum with ozone were detected. This is a question for atmospheric chemists, and I hope will be given serious study. We do know at least that among the various atmospheres in our own solar system, ozone is produced in spectroscopically detectable quantity only on the Earth. Even if there were some ambiguity as to its origin, the discovery of a planet of Earth's temperature and an atmosphere with abundant water and oxygen would be truly momentous. The $16 \mathrm{~m}$ telescope should not be limited to detecting only the broad features of the infrared spectrum. Methane in the Earth atmosphere is sustained only because of metabolic activities of micro organisms (Lippincott et al 1967) and it results in a weak absorption feature at $7.7 \mu$ (Conrath et al 1970). As we shall show below, even this feature should be detectable given integration of days or weeks.

Hanel's spectra came from small telescopes carried by interplanetary spacecraft. We will find that to obtain similar spectra of planets beyond the solar system, a cooled $16 \mathrm{~m}$ telescope in Earth orbit is needed. Indeed, this was in large measure the original rationale for such a large telescope (Space Science Board, 1988). The sensitivity advantage of searching for planets by their thermal emission rather than their reflected light was first recognized by Bracewell and MacPhie (1979). Although originally proposed for giant planet detection, the advantage holds also for terrestrial planets, and is illustrated by plots of the solar and terrestrial fluxes in figure 2 . The Earth's photon flux at 10 microns wavelength is thirty times greater than at visible light, while the solar flux is 30 times weaker. This increase in contrast is critically important, given the very small angular separation and a flux ratio in visible light of nearly ten billion. Burke (1988) first took seriously the design of telescopes to search for life in earthlike planets via the oxygen feature in the optical spectra. The advantage of infrared measurements for not only detecting but analyzing the atmospheric composition was then recognized by Angel, Cheng and Woolf (1986), hereafter referred to as ACW, and is further discussed by Angel (1988). 


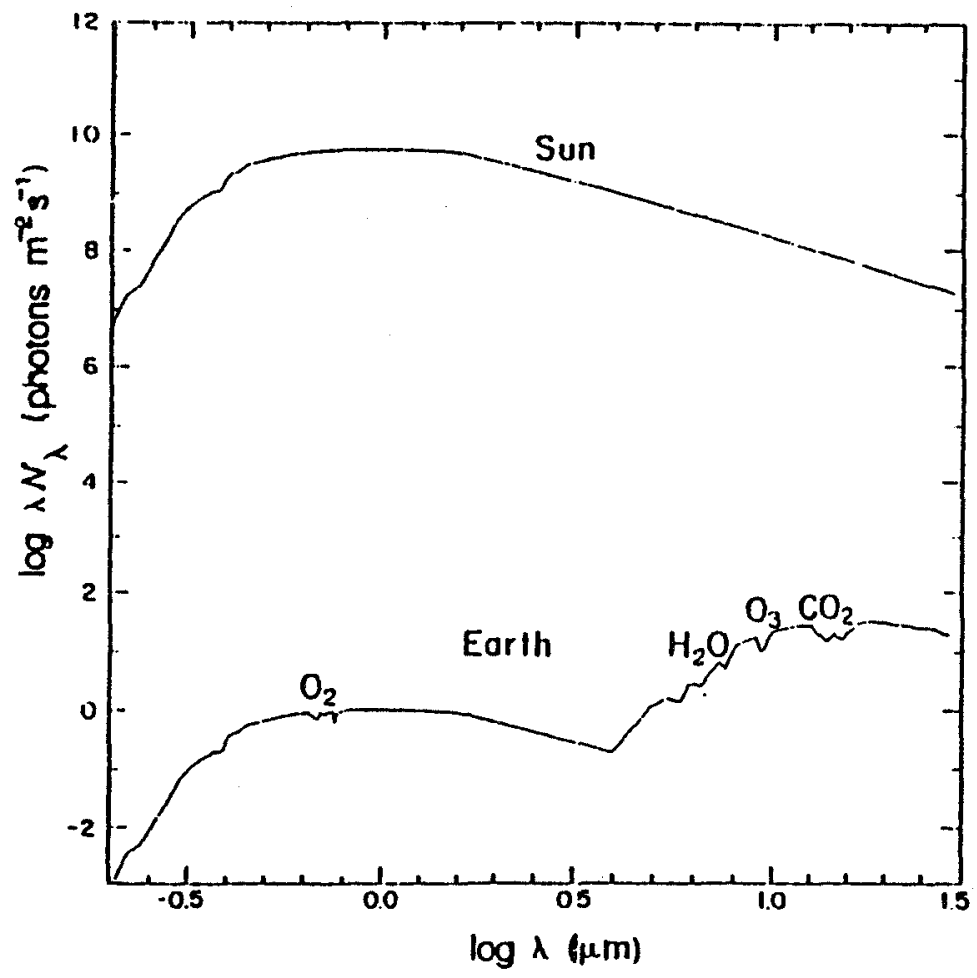

Figure 2 Fluxes of the sun and Earth as seen from 4 pc distance. From Angel, Cheng and Woolf, 1986.

\section{General Considerations of Telescope Design}

A large baseline is essential to resolve the planet's thermal emission from the star in the presence of diffraction. Single apertures significantly less than $16 \mathrm{~m}$ diameter lack the necessary resolution. In principle two smaller mirrors separated by $10 \mathrm{~m}$ could also yield the required resolution and contrast. However, there is a strong need also for the collecting area of a filled aperture. The contrast of the planet against diffuse zodiacal thermal background depends only on primary mirror area and the planet is faint enough that photon noise of this background is the fundamental limitation. In this limit, the integration time to reach a given quality spectrum depends on the inverse 4th power of mirror diameter. Another essential requirement is to cool the telescope. The background thermal noise even for an Antarctic telescope is much too strong. In space, cooling to $80 \mathrm{~K}$ will reduce telescope background to a negligible level.

Even given a cold $16 \mathrm{~m}$ space telescope, it will still be necessary to pay very careful attention to telescope design. The natural diffraction limited resolution for a circular aperture is $1.2 \mathrm{\lambda} / \mathrm{d}$, where $\mathrm{d}$ is the diameter. The limit is 0.15 arc seconds for a circular $16 \mathrm{~m}$ aperture at 10 microns wavelength, the angular separations of interest are right at this limit. However the planet will be typically ten million times (17.5 magnitudes) fainter than the star. The optical design must include a trick to 
strongly suppress the diffracted light from the star, if there is to be any hope of finding a planet. It is also necessary to pay very careful attention to the accuracy of the mirror surfaces, for tiny figure errors will produce weak features in the diffraction pattern that masquerade as planetary images. In the following sections we consider in more detail these technical aspects.

\section{Two Ways to Control Diffraction in a Filled Circular 16 m Telescope}

Focal plane instruments are needed to alter the normal Airy pattern of a 16 $\mathrm{m}$ telescope. Two options are available. The transmission or phase across the pupil can be modified to produce very dark regions in the diffraction pattern (apodization). Alternatively an interferometric beam-splitter can be arranged to cancel the starlight at one of a pair of foci.

Apodization is generally used to suppress the far wings of the diffraction pattern. What is wanted in the present case is strong suppression of the diffracted energy in a region as close as possible to the central maximum. ACW proposed a new method to this. All that is needed is to mask out the pupil in an annular region from radius .66 to .9 of the full aperture. This has the effect of making the first dark ring of the diffraction pattern extremely dark over a considerable width. The effect has been demonstrated recently in the laboratory by Peter Wizinowich, and is shown in figure 3. A spherical mirror of $2.4 \mathrm{~m}$ radius of curvature was masked with a full aperture of $16 \mathrm{~mm}$. Two point sources at .633 microns wavelength, one a thousand times brighter than the other, were placed near the center of curvature, and the nearby image recorded directly with a CCD camera. Figure $3 a$ shows the image with the two sources well separated. Over most of the field the apodization causes stronger diffraction rings than are in the Airy pattern, but the first dark ring is wider and much deeper. In figure $3 \mathrm{~b}$ the weaker source lias been moved close in, to an angular separation of $2 \lambda / \mathrm{d}$. It is clearly visible at this separation of only 1.7 times the Rayleigh criterion, despite the very large intensity ratio. The analysis by $\mathrm{ACW}$ of this apodization shows that suppression of the diffracted energy in the dark ring to $3 \times 10^{-6}$ of the peak intensity is realized, over an area several times larger than the image core.

The apodization method is simple and very powerful, particularly in allowing a search of a complete annular region in one image. However, it will not allow detection of planets closer in angular separation than about $1.6 \lambda / \mathrm{d}$, or 0.2 arc seconds at 10 microns wavelength. Its capability to find planets with 1 au orbits will be limited to only a few solar type stars. It is possible at least in principle to obtain nearly as strong star suppression still closer, by using interferometric instrumentation at the focal plane of the $16 \mathrm{~m}$ telescope. Suppose that the full aperture is masked into two elliptical apertures, as shown in figure 4a. The diffraction pattern then has a central narrow bright fringe, with the first dark fringe lying only $0.9 \lambda / \mathrm{d}$ from the central peak ( 0.11 arc seconds). The resolution is higher, but the dark fringe is too narrow to be useful. In this case, as in the classic Michaelson stellar interferometer. the interference fringes between the two apertures are formed in the focal plane. 
Much more efficient suppression of the stellar flux is obtained without loss of resolution if the two wavefronts are exactly superposed with the aid of a semitransparent beam splitter. In this case the fringes are projected onto the plane of the sky, and a point stellar source centered on a dark fringe gives no light at all at the focus where the interference is destructive. All the energy appears from constructive interference in the other beam. The situation is teversed for a planet located at angle $0.9 \lambda / \mathrm{d}$ from the star. This is the principle of the interferometer proposed by Bracewell and MacPhie (1979).

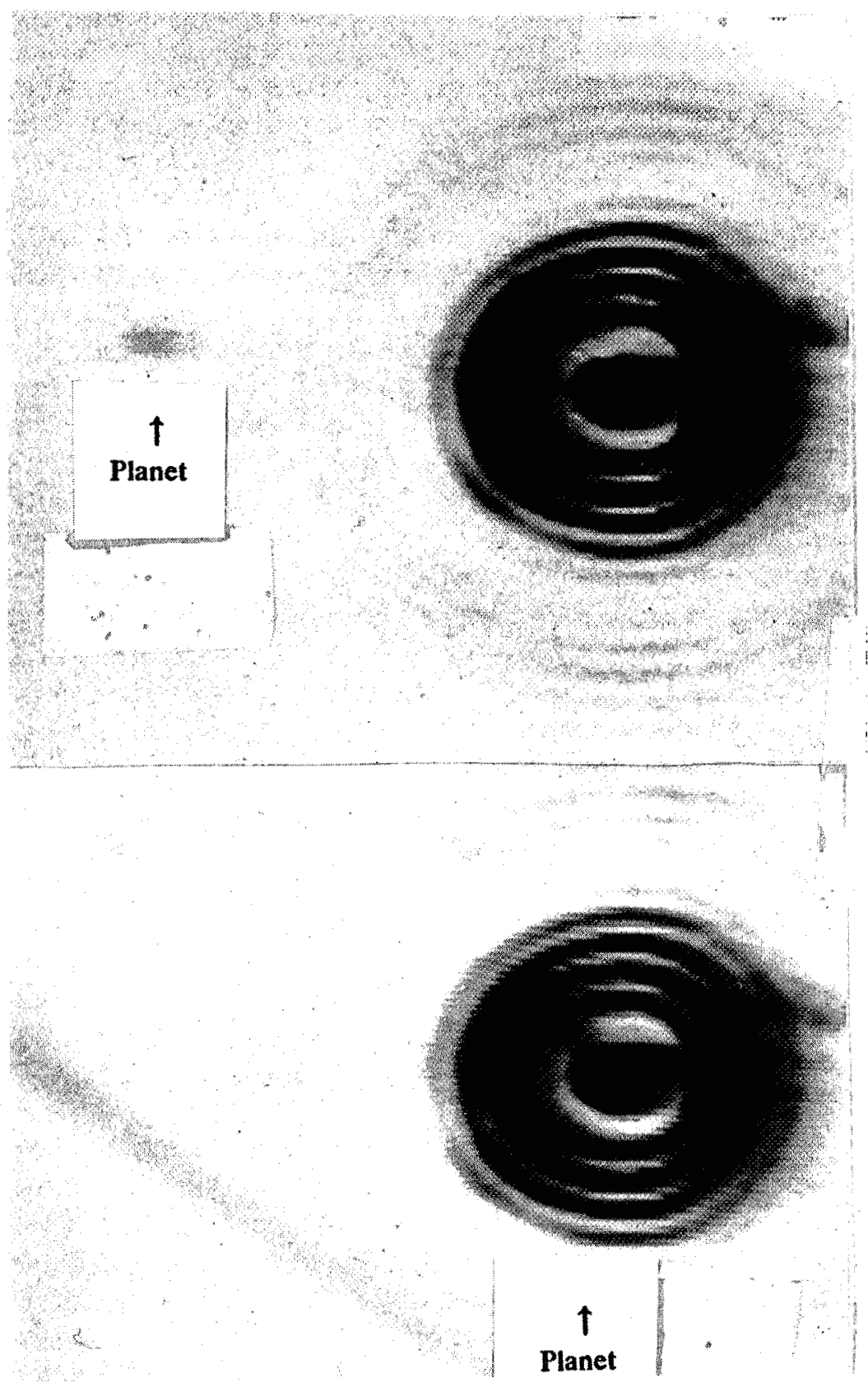

Figure $3 a$ and $3 b$ Laboratory images of sources differing by $10^{3}$ in intensity, obtained with a mirror apodized as described in the text. The weaker source is marked "planet". 

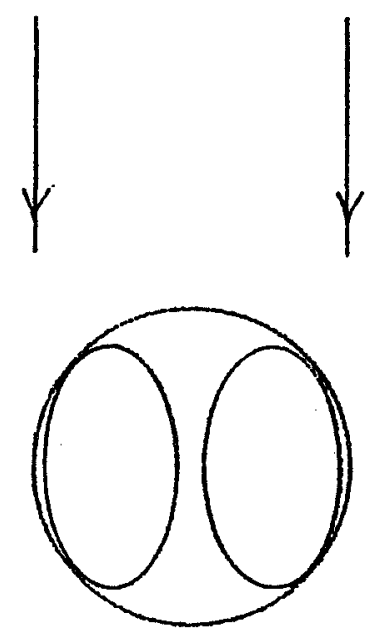

Figure 4a Apertures in the pupil of a $16 \mathrm{~m}$ telescope that define the two elements of an interferometer.

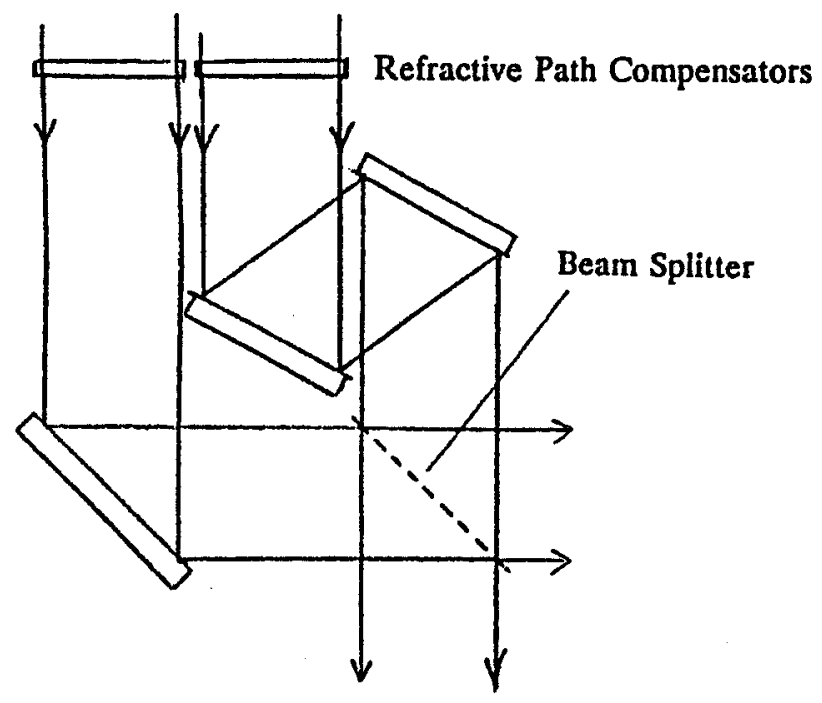

Figure 4b

Interferometer to combine the two adjacent beams.

The theoretical limit of starlight suppression for this type of two element interferometer of spacing $s$ is set by the finite angular size of the parent star. The response at angle $\theta$ off the null axis varies $\operatorname{as}^{2} \sin ^{2}(\pi \theta \mathrm{s} / \lambda)$ where $s$ is the spacing between centers of the interferometer elements. Suppose the planet is located at a maximum, and the center of the star is on the null axis. If the star has the same diameter as the sun, and the planet is at the furthest separation of a 1 a.u.radius orbit, then the stellar light will be reduced by a factor $1.3 \times 10^{-5}$, compared to the inphase signal. This background is about 4 times worse than in the apodization case. Realization of the interferometric null in practice imposes a severe constraint on pointing. If the telescope points off by one stellar diameter, about 1 milliarcsecond, the uncancelled image will brighten by an order of magnitude, and be about 1000 times brighter than the planet. In practice it will not be possible to point the $16 \mathrm{~m}$ telescope to the required accuracy, and it will be necessary to servo control the null via optical starlight, adjusting the interferometer path lengths to the required accuracy of a few nanometers. 
Some care is needed to devise a two beam interferometer for the present case, in which the individual diffraction patterns are not much larger than the fringe spacing. In the scheme of Bracewell and MacPhie, for example, the individual images of an off-axis object do not overlap, but separate on either side of the residual star image. This is of little consequence for widely spaced interferometer elements. In the present configuration, it prevents full constructive interference of the planet's flux, and increases the image area and hence sky background noise. Not only is the signal to noise ratio degraded, but it may be impossible in practice to distinguish a planet from the much brighter zodiacal light local to the star, which will be symmetrically distributed on either side of the star. The same type of problem arises in the rotating shearing interferometer, as described by Diner (1989) at this conference.

An interferometer optimized for two very close elements as would be derived from a single filled aperture is shown in figure $4 \mathrm{~b}$. It would be used in conjunction with an afocal secondary. The off-axis images are exactly superposed in this design, and are given simply by the single elliptical aperture diffraction pattern whose overall intensity is determined by the $\sin ^{2}$ fringes. The design has pathlength compensators incorporating refractive materials of different dispersion. These would be chosen so that the destructive interference at one focus is achromatic over a useful range of wavelengths. The phase change of $\pi$ on reflection by a metal could perhaps be used for this purpose, but we note that the phase difference at a metallic beamsplitter used in the Bracewell and Macphie interferometer is not $\pi$, as was desired.

\section{What is the Optimum Optical Configuration for the $16 \mathbf{m}$ Telescope?}

We have so far considered designs based on a filled circular aperture. A 16 $\mathrm{m}$ telescope is too big to be built and launched in one piece. It would have to be assembled from mirror segments. Diffraction spikes would be produced by the segment edges as well as the spider vanes supporting the secondary. Is it possible to redeploy the same collecting area to do a better job? Insofar as zodiacal background is the limiting factor, there is no advantage to be gained. Resolution can be improved, for example, by arranging the aperture as a two element array. With two separated $11.3 \mathrm{~m}$ mirrors, it would be possible to make narrower fringe spacing, and thus have the resolution to search more distant stars. However, there is a large number of candidate systems closer than $10 \mathrm{pc}$, and the very faint signal from earthlike planets beyond this distance would lead to extremely long and difficult observations, even if the interferometer gave adequate starlight suppression. For the nearer systems, there is a distinct disadvantage in using two element interferometer baselines longer that are needed to give more than half a fringe spacing between star and planet. If there were one and a half fringes, for example, then the star disc gives nine times more uncancelled background emission.

An arrangement that eliminates diffraction spikes, and gives excellent star rejection down to planet separations of 0.1 arc second (at $10 \mu$ wavelength) is shown in figure $5 \mathrm{~b}$. The telescope is made from four $8 \mathrm{~m}$ circular monolithic mirrors, arranged in a diamond pattern. These mirrors would be figured as off-axis segments 
of a single paraboloidal surface. A single paraboloidal secondary would be used to make an afocal telescope, which would directly feed an interferometric beam combiner for planetary work. This arrangement is preferred over an MMT style telescope, because there is no obscuration by individual secondaries and the field of view is larger. The
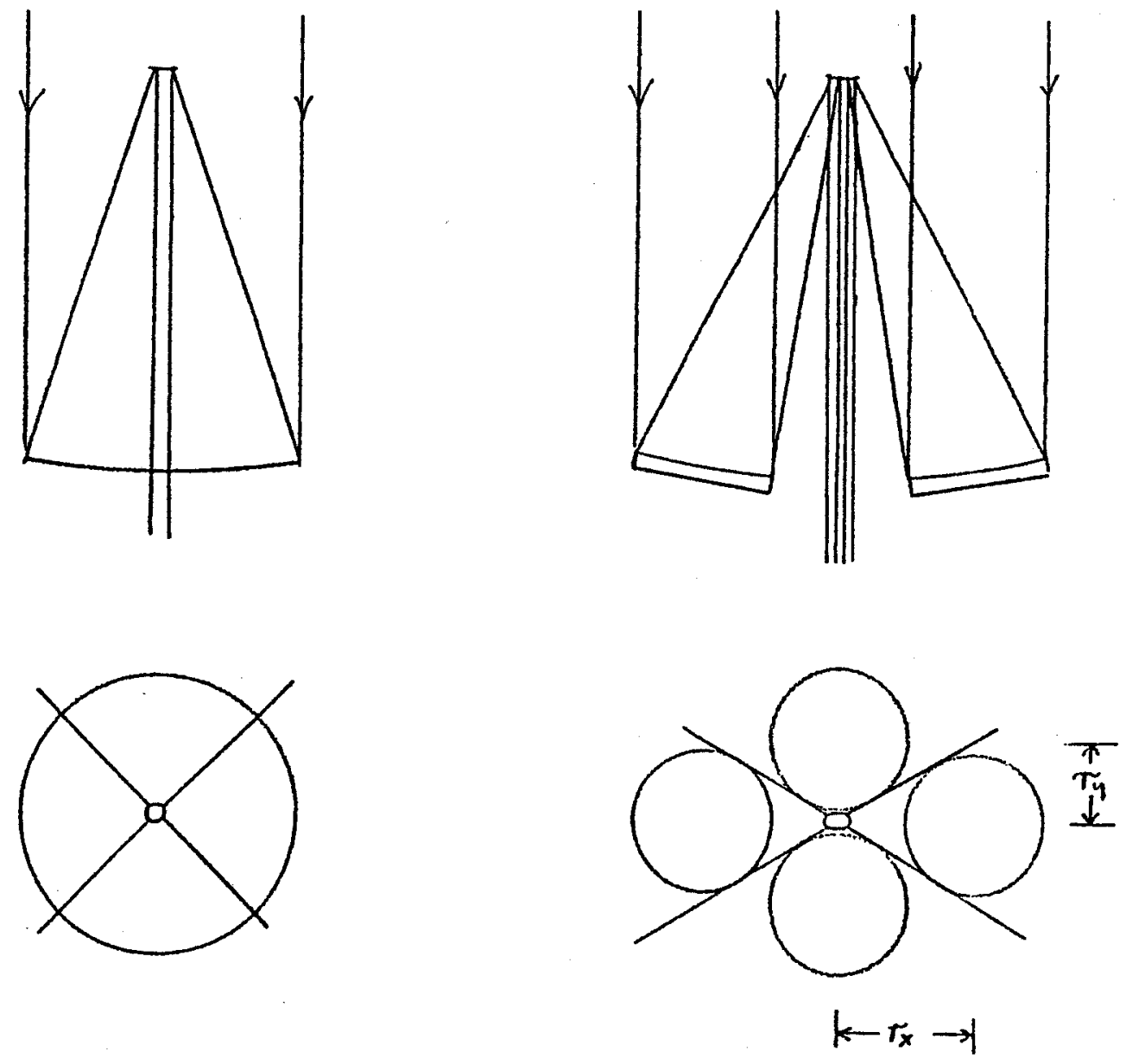

Figure 5a Filled $16 \mathrm{~m}$ circular

Figure $5 \mathrm{~b}$ Diamond configurations aperture. with four $8 \mathrm{~m}$ circular elements.

wavefronts from the two pairs of diagonal elements would be superposed in phase with interferometers like that shown in figure $4 \mathrm{~b}$, and the resulting wavefronts then combined out of phase with a third interferometer. The effect is to superpose on the sky two sets of sine-squared fringes at oblique angles.

If the mirror centers are at distances $r_{x}$ and $r_{y}$ from the telescope axis, then the intensity of the image of a point source at angle $\theta$ to the axis and position angle $\phi$ is given by

$$
I=1 / 4\left\{\cos \left(2 \pi r_{x} \theta \cos \phi / \lambda\right)-\cos \left(2 \pi r_{y} \theta \sin \phi / \lambda\right)\right\}^{2}
$$


This reduces near the axis to

$$
I=1 / 4(\pi \theta / \lambda)^{4}\left(r_{x}^{2} \cos ^{2} \phi-r_{y}^{2} \sin ^{2} \phi\right)^{2}, \quad[\theta<<\lambda / r]
$$

The dependence of $I$ on $\theta^{4}$ results is very efficient suppression of the star. Thus for $\mathrm{r} \sim 8 \mathrm{~m}$, and $\lambda \sim 10 \mu \mathrm{m}$, $\mathrm{I}$ is less than $10^{-6}$ for $\theta \leq 4$ mas and $\leq 10^{-8}$ for $\theta<1$ mas.

The ratio $r_{x} / r_{y}$ in diamond pattern is chosen so that no matter what the angular separation between planet and star, as long as it is above some minimum, the telescope can be oriented so that the planet lies close to an interferometric maximum when the star lies on the null axis. We find that with $r_{x}=8 \mathrm{~m}$ and $r_{y}=4.85 \mathrm{~m}$, points in the pattern with $\mathrm{I}>0.6$ can be found for all angular radi $\geq 0.1$ arc second (for $\lambda=10 \mu \mathrm{m}$ ), with I being typically $>0.9$. (Complete constructive interference of all four wavefronts corresponds to $I=1$ ).

\section{Performance Estimates}

The effect of mirror imperfections and misalignment will be to give systematic errors. Imperfectly cancelled starlight can easily mimic the appearance of a planet. It will be necessary to repeat measurements to check for and cancel these errors, which cannot be estimated without detailed system modelling. We can estimate here the errors due to photon noise in the thermal background, which are quite predictable, and set an absolute lower limit to the integration times that will be needed to get reliable data.

In the interferometric configurations, if the beamsplitters are ideal with $50 \%$ reflected and transmitted energy, both the signal from the planet and the photon noise from a diffuse zodiacal background remain the same as for a single dish with the same total area. All the flux collected from the planet appears in a single image at the focal plane where there is constructive interference. The interferometric image has the larger angular size of the single element diffraction pattern, but the zodiacal background does not interfere constructively, and is no stronger than it would be for one interferometer element. Thus in the case of the diamond array of four $8 \mathrm{~m}$ elements the image has four times the angular area of that for a $16 \mathrm{~m}$ filled aperture, but the background brightness is four times fainter, because each beam has passed twice through a semi-transparent beamsplitter.

Because zodiacal background is diffuse, it cannot be avoided by interferometry or apodization. We must take into account the emission both from within our own solar system, which is accurately known, and from the system under investigation. The latter is unknown, but for purposes of estimating the photon noise background we follow ACW in assuming a system just like the solar system. The total background is then 3 times the local level, integrating along the line of sight out of our system, 
into the new one and out again. The zodiacal emission in our own solar system is well represented by a grey body of temperature $275 \mathrm{~K}$ and optical depth $7 \times 10^{-8}$.

Under these assumptions, the flux incident from the total zodiacal background on a $16 \mathrm{~m}$ telescope in a beam large enough to capture the central bright spot of the Airy pattern is 23,000 photons/sec in a $1 \mu$ bandwidth at $10 \mu$ wavelength. The photon flux from a planet the same as the Earth incident on the telescope is about 500 photons $/ \mathrm{sec}$ if it is at $4 \mathrm{pc}$ distance, 80 photons $/ \mathrm{sec}$ if it is at $10 \mathrm{pc}$.

Suppose that a planet has been detected, and a spectrum of quality adequate to detect features like those in figure 1 is to be obtained. We will assume that a resolution of $\lambda / \Delta \lambda=100$ and a signal to noise ratio of 50 is required. An ideal 16 $\mathrm{m}$ system with no losses, detecting of the central Airy maximum with $100 \%$ quantum efficiency, would require an integration time of an hour for the planet at $4 \mathrm{pc}, 6$ hours at $10 \mathrm{pc}$. In reality, the combined effects of optical losses and optical imperfections that result in imperfect star cancellation will probably increase the necessary integration time by an order of magnitude. Furthermore, if the exact phase relationships for star cancellation cannot be maintained over a broad wavelength range, then a full spectrum must be built up from separate integrations of smaller spectral regions. Even so, a respectable spectrum of an earthlike planet as far away as $10 \mathrm{pc}$ could be obtained in an integration period of a week or two.

Over a period of years the telescope could complete a spectroscopic survey of tens of planetary systems like the solar system, if they are present. If a planet were found with a spectrum resembling Earth's, longer exposures could be used to search for weak features such as methane. The methane feature at 7.7 microns wavelength in the Earth's spectrum is about 0.03 microns in width and $10 \%$ deep. Integrations some five times longer than used for the routine survey spectra would be able to detect even this feature.

It is on the basis of these performance estimates that we believe the collecting area of a $16 \mathrm{~m}$ telescope, or close to it, is required. If the effective diameter were halved, for instance in an array of four $4 \mathrm{~m}$ elements, the integration time would be increased by a factor 16 , and become prohibitively long. A telescope at this size could detect earthlike planets, but would not be able to carry out spectroscopic analysis of good quality. Under any circumstance, a telescope to overcome the limits of diffraction must be based on a large and extremely rigid structure. Given this, it would not save much money to cover it only with small mirror area, but it would severely compromise performance.

\section{Impact on Normal Telescope Operation}

A $16 \mathrm{~m}$ telescope has enormous value for many aspects of astronomical research. It is fortunate that the requirements imposed to make the telescope suitable for earthlike planet studies enhance rather than compromise the performance for 
other observations. To get the nearly perfect diffraction limited performance at $10 \mu$ wavelength, the surface quality (on large spatial scales) is such as to realize normal diffraction limited imaging at $0.1 \mu(\mathrm{ACW})$.

For normal imaging and spectroscopy, the afocal beam from the telescope would be brought to a direct focus with an auxiliary telescope in place of the interferometric beam combiners. The diffraction pattern of the diamond array has higher resolution than that of a $16 \mathrm{~m}$ circular aperture, but has significant side lobes. A comparison is given in figure 6. The fraction of the total energy in the central maximum is $84 \%$ for the Airy pattern compared to $60 \%$ for the diamond array. Four of the six prominent side lobes each contain $5 \%$ of the total energy, with $2 \%$ in each of the remaining two. For imaging and background limited spectroscopy, the advantage of higher resolution offsets the disadvantage of lower concentration. Only a modest degree of image cleaning would be required to remove to sidelobe patterns. The full width at half maximum power in visible light (5000A) is 7.7 mas for the 16 $\mathrm{m}$ filled aperture, and 5.2 mas for the diamond array. Interferometric methods would allow a doubling of this resolution, as would halving the wavelength of observation.

\section{New Technology}

The major advance in optical technology required to build the telescope is in mirror fabrication. Off-axis segments need to be polished to very high accuracy. The stressed lap method (Martin et al. 1988) is extremely promising, for $8 \mathrm{~m}$ or smaller segments, but needs to be demonstrated explicitly in the off-axis case. Glass honeycomb substrates with zero thermal expansion at $70-80 \mathrm{~K}$ need to be developed. Borosilicate glass of suitably chosen composition has this property. The facilities for fabrication into $8 \mathrm{~m}$ substrates are now being developed (Angel 1989) and could be upgraded to handle the more refractory compositions needed for this application.

Work is needed to design an interferometric beam combiner in which phase shifts are controlled to the required very high accuracy, and on mechanical structure and active alignment techniques. The telescope with its thermal baffling is a large structure that will require assembly in orbit or on the Moon. If monolithic $8 \mathrm{~m}$ segments are used, then rockets large enough to contain them will be needed.

Given the present state of technology, none of these tasks is beyond our reach. The search for a planet like our own is a project of immense appeal, and could be the central goal of an international space telescope project, as envisaged by Bahcall et al. (1989). 


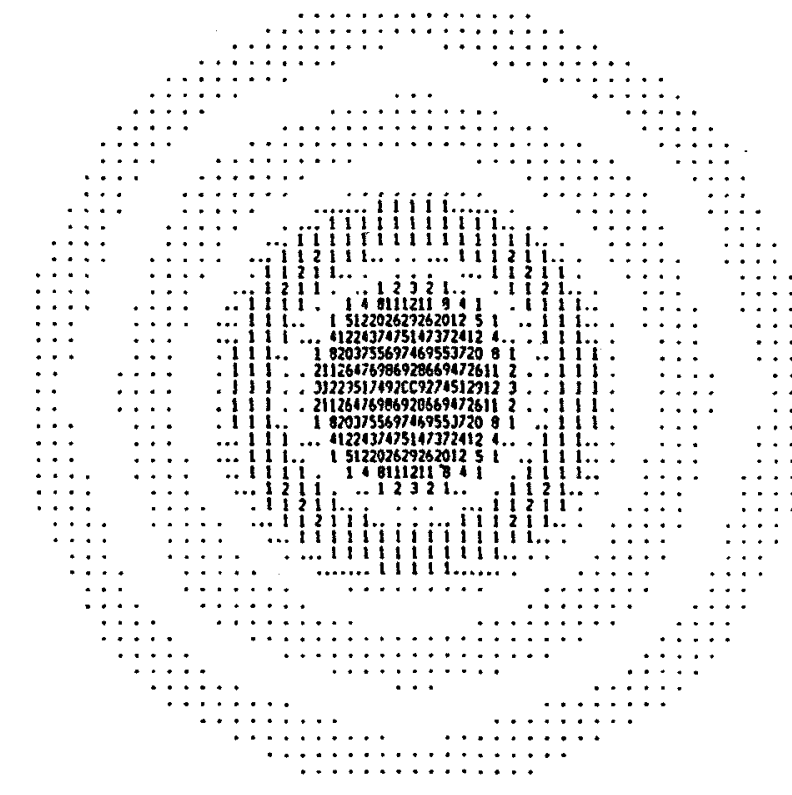

(a)

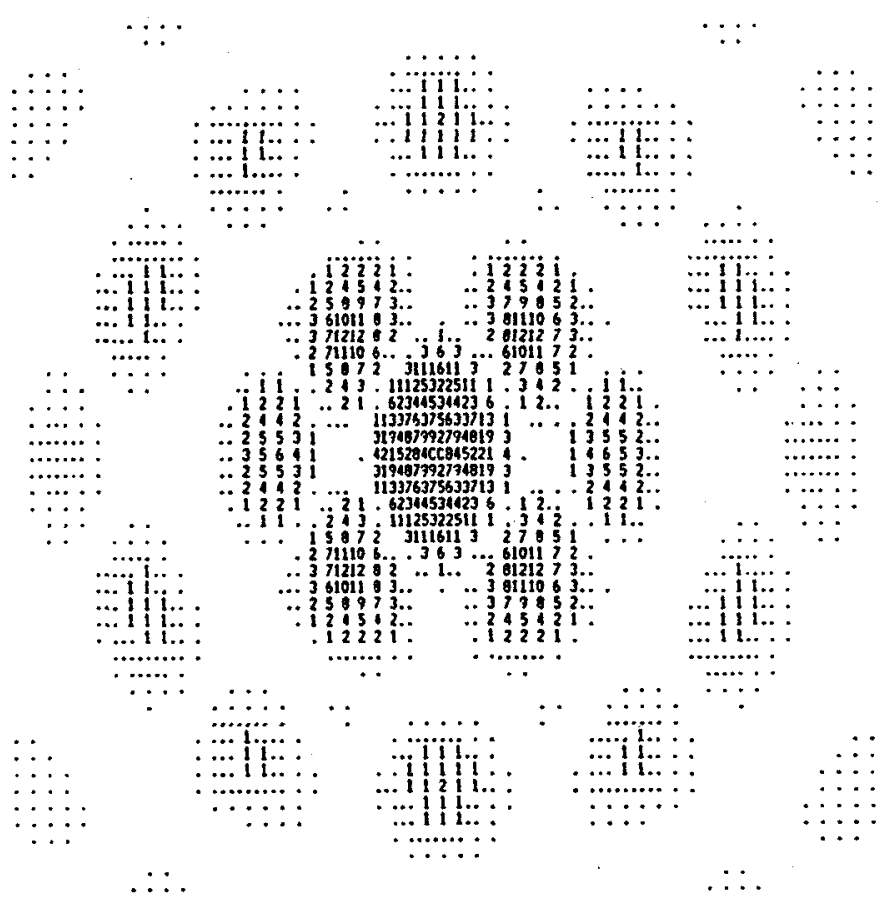

(b)

Figure 6 Diffraction patterns for $16 \mathrm{~m}$ telescope configurations operated as normal telescopes. The numbers give intensity as a percentage of the peak (marked cc). Intensities from $0.5-1 \%$ are marked with two dots, $0.1-0.5 \%$ with one dot. (a) is 
the Airy pattern for a filled $16 \mathrm{~m}$ aperture, (b) is the pattern with the same angular scale for a diamond array of $8 \mathrm{~m}$ telescopes with $r_{x}=8 m$ and $r_{y}=4.8 \mathrm{~m}$.

Acknowledgments

I am grateful to Neville J. Woolf for valuable discussions of this topic, to Peter Wizinowich for obtaining the laboratory apodized images, and to Michael Hart for assistance in preparing the montage of planetary spectra.

\section{References}

Angel, J.R.P., 1988, in "Bioastronomy -- The Next Steps," ed. G. Marx, Kluwer, 153.

Angel, J.R.P., Quarterly Journal Royal Astronomical Society, in press.

Angel, J.R.P., Cheng, A.Y.S. and Woolf N.J., 1986, Nature, 232, 341.

Bahcall, J.N., Giacconi, R., Rees, M., Sagdeev, R. and Sunyaev, R., 1989, Nature $\underline{339}, 574$.

Bracewell, R.N. and MacPhie, R.H., 1979, Icarus, $\underline{38}, 136$.

Burke, B. F. 1986, Nature, $\underline{322,} 340$.

Conrath, B.J., Hanel, R.A., Kunde, V.G. and Prabhakara, C., 1970, J. Geophys. Res. 75, 5831.

Diver, D.J., 1989, These Proceedings.

Hanel, R.A., 1983, in "Spectrometric Techniques", Volume 3, p. 43, G.A. Vanasse ed., Academic Press.

Lippincott, E.R., Eck, R.V., Dayhoff, M.O. and Sagan, C., 1966, Ap. J., 147, 753.

Martin, H.M., Angel, J.R.P. and Cheng, A.Y.S., 1988, Proceedings of the ESO Conference on Very Large Telescopes and Their Instrumentation, ed. M.H. Ulrich, Garching, Federal Republic of Germany, 353.

Space Science Board, 1988, in "Space Science in the 21st Century: Imperatives for the Decades 1995-2015". National Academy Press, Washington, D.C. 


\section{DISCUSSION}

Bahcall: How do you know that ozone is only the result of life.

Angel: The oxygen in our own atmosphere is now taken to be due to the presence of life. However, it would be good to look more closely at this approach.

Pilcher: It might also be good to look at other molecules (e.g., methane) that appear to be in chemical disequilibrium in our oxidizing atmosphere. These molecules in disequilibrium may also be useful diagnostics of life. Methane, for example, is in disequilibrium by 7 orders of magnitude while for oxygen it is about a factor of 20 .

Nelson: You stated that the gaps are not important. Can you expand on that?

Angel: The energy from the gaps spreads out over a large area - and so with narrow gaps (say $2 \%$ of the area) the level of the scattered light at the 0.25 " ring should be small, even compared to the highly attenuated starlight.

Unidentified: Doesn't working in the visible where the Airy disk is so much smaller offset the contrast gain in the Infrared? That is, doesn't the $400 \mathrm{X}$ gain in the Airy disk area offset the $600 \mathrm{x}$ gain for the IR in contrast?

Angel: The contrast is not only better in the IR, but the photon flux is much higher, and this observation is photon-flux limited. The 10 micron $\mathrm{O}_{3}$ line is also much stronger than the visible $\mathrm{O}_{2}$ line.

Mlingworth: One would also be concerned about the need for a surface that is significantly better than that required for the 10 micron observations - and that is already tough.

Fall: Could you do better by looking at brighter (earlier-type) stars at larger distances from the stars.

Angel: The problem is that the number of available stars is extremely small, and that it gets very difficult with bright stars.

Rehfield: Has scatter from the structure been considered in the telescope design?

Angel: Most of the complex structure is outside of the aperture and field of view. Only simple secondary supports contribute scatter.

Korsch: You need to consider the scattering properties of the additional optics to make the pupil plane for the apodizer.

Angel: It is really quite straightforward because a pupil plane can be obtained very simply most anywhere with such a small field of view $(0.25$ ”). 


\section{Page intentionally left blank}


Session 3

Large Optics: Ground-based Developments 


\section{Page intentionally left blank}




\title{
The Keck Telescope Project
}

\author{
Jerry Nelson \\ 50-351 Lawrence Berkeley Laboratory \\ Berkeley, CA 94720
}

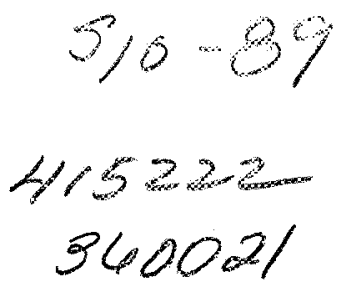

Introduction

P. 2

The Keck Observatory is a project to build a 10-m optical and infrared telescope for ground based astronomy. The primary is composed of 36 hexagonal segments that together form an $\mathrm{f} / 1.75$ hyperbola. The segment positions are actively controlled to remove the adverse effects of gravitational and thermal deformations on the supporting steel structure.

Details of the project are described in a number of internal reports and memos and periodic status are given in recent SPIE proceedings. A review of the characteristics of the Project with emphasis on the segment fabrication and the active control system was presented at this conference. Since most of this information is available in the recent SPIE proceedings information of a general nature is not repeated here. We only report here some interesting aspects of segment fabrication that may be of interest for large high accuracy telescopes in space.

\section{Segment Fabrication}

Our mirrors are being polished at Itek Optical Systems in Lexington, MA. They are polished as circular mirrors using stressed mirror polishing (SMP). With this technique, spheres are polished into an elastically deformed mirror so that when the externally applied deforming forces are removed, the sphere relaxes into the desired off axis section of a hyperboloid. Our hyperboloids vary up to about $100 \mu \mathrm{m}$ from a sphere.

After SMP the mirrors are cut into hexagons. Our experience is that the mirrors warp a significant amount from this cutting $(0.5 \mu \mathrm{m})$ and this error along with the imperfections in the polishing itself makes the mirror unacceptable optically. Our specification is that the mirrors should concentrate $80 \%$ of the light into 0.24 arcsecond diameter. After cutting the mirrors are typically about five times worse than this.

Originally our plans were to test these hexagons and make final correction of the surface using a technique called Computer Controlled Optical Surfacing (CCOS) where a small $(10 \mathrm{~cm})$ tool is driven over the surface with a dwell time controlled by computer to remove the high spots. We tried this technique with two mirrors and found the technique to be wanting for our application.

There were several problems with CCOS. First, the method only converged slowly to the desired surface, so many $(\sim 10)$ polish-test cycles were necessary to produce the final mirror. This of course makes the process relatively expensive. Second, the polishing process generally roughened the edges of the mirror up rather severely. Edges here mean a region about the diameter of the tool $(10 \mathrm{~cm})$ wide going around the entire segment perimeter. This is about $25 \%$ of the segment surface area, so it is important. The edge surfaces had high spatial frequency errors with amplitudes in the $50 \mathrm{~nm} \mathrm{rms} \mathrm{range.} \mathrm{This} \mathrm{led}$ to substantial broadening of the image size. Finally, even in the interior there were apparent grooves or lines caused by the tool motion pattern. These could be easily seen in 
interferograms of the surface. Their amplitude was relatively small $(\sim 10 \mathrm{~nm})$ so they were not a concern for observations in the red end of the spectrum, but in the blue-ultraviolet, it became a concern.

As a result of these difficulties we have chosen to make improvements to our mirror surface by deforming them gently with a set of corrective forces applied to the back of the mirror through our passive support system. This approach has some merit because the largest part of the surface error is in the form of very low spatial frequency errors that can be removed with these corrective forces. We call this set of springs that applies the corrective forces "warping harnesses". Our experience is that warping harnesses are very inexpensive and stable and they correct most of the errors of interest. At the moment, having made six mirrors, we still are about a factor of two away from our final polishing goals. We hope that as our fabrication experience grows our image quality will improve to the desired level.

\section{DISCUSSION}

Johnson: What is the reliability and performance life of the actuators on the segments of the Keck Telescope?

Nelson: The most significant problem noted is the possibility of leaking hydraulic fluid. Initially, many leaks were noted. Tightening up eliminated the leaks for now. In operation, it may be necessary to replace some actuators periodically. This replacement (and leakage) would be a problem in space, much more than on Earth.

Breckinridge: How much force do you need on your warping harness?

Nelson: We can safely apply forces up to about $300 \mathrm{~N}$ per support point. Typically we warp our mirrors with forces a factor of 5 less than this.

Manhart: Do you warp each mirror as it is being tested in its own harness, then mount it in the telescope and hope that it doesn't change? How often do you check its figure?

Nelson: Each mirror is tested after cutting at Itek. Our experience with warping harnesses is that their performance is predictable to within measurement errors. We can (and will) also test each segment in the telescope using a Hartmann-Shack camera with 240 points/segment.

Swanson: Is the Hartman test sufficient to phase the primary mirror in an absolute sense? You are measuring relative edge developments rather than absolute piston errors.

Nelson: Yes, it is adequate. The Hartman test uses four colors determined by filters to eliminate fringe ambiguity.

Korsch: Are the segments cut slightly different according to their position on the surface, as regular hexagons do not ideally fill a parabolic surface?

Nelson: The segments appear as regular hexagons as seen by a star. Viewed normal to the optical surface, one sees the segments are actually non-regular by typically few millimeters.

Kahan: Can you provide ball park segment production costs for generating/polishing and coating boules?

Nelson: The boules would go for about $\$ 100 \mathrm{k}$ each, fabrication would be about \$50k, and coating (aluminum) would be minor. All this presumes successful continued use of the warping harness. 


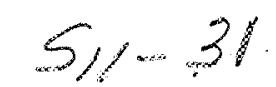

\title{
Stressed-lap Polishing
}

H. M. Martin and J. R. P. Angel

Steward Observatory

University of Arizona

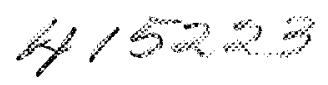

Tucson, AZ 85721

\begin{abstract}
We present a method for polishing fast aspheric mirrors to high accuracy. It is based on an actively stressed lap whose shape is changed continuously make it fit the mirror surface as it moves across that surface and rotates. A stressed lap $600 \mathrm{~mm}$ in diameter has been built to polish a 1.8-m $\mathrm{f} / 1$ paraboloid. It consists of a rigid aluminum disk which changes shape continuously under the influence of 12 moment-generating actuators. These actuators are programmed to produce the shape changes necessary to give the correct offaxis parabolic surface at all times. In this paper we describe the principles and design of the lap, and progress to date in polishing the $1.8-\mathrm{m}$ mirror.
\end{abstract}

\section{Introduction}

At the meeting Angel gave a discussion of stressed lap polishing as part of a more general description of the Columbus project. The Columbus telescope is the largest planned by the US community, to be located at $3200 \mathrm{~m}$ elevation on Mt. Graham in Arizona. It will have two $8 \mathrm{~m}$ mirrors on a common mount, each having a focal ratio of 1.2 , and an image quality of 0.125 arcsec FWHM. First light (for the first mirror) is projected for 1994. This report focuses only on the mirror polishing method to be used for the Columbus telescope, which is also of direct relevance to future space telescopes. A recent description of the Columbus and other ground-based telescopes is given by Angel et al. (1989).

Looking beyond the Hubble telescope, it is clear that telescopes in space will need mirrors that are not only larger, but also smoother and more accurate. The natural short wavelength limit for normal-incidence telescopes is at $91 \mathrm{~nm}$. Diffraction-limited performance at this wavelength, with a Strehl ratio of $90 \%$, requires a surface accuracy of $2 \mathrm{~nm}$ rms. Very much higher Strehl ratios, $>1-10^{-5}$, are needed to avoid scattered light for the detection of Earthlike planets at $10 \mu \mathrm{m}$ wavelength. In this case a surface accuracy of $2.5 \mathrm{~nm}$ rms must be maintained over the largest spatial scales. To avoid diffraction spikes, the mirror surfaces should ideally be off-axis segments of an aspheric parent surface. For compact telescopes with such optics, the mirror surface will also be strongly aspheric.

Mirrors are polished to high accuracy in an iterative process of measurement followed by corrective removal of material. Progress in optical metrology recently has been very great, and a moderate extension of present practice will allow even large surfaces to be measured more accurately than the above specifications. There is a problem, however, in removing material, even if we know where it needs to come off. The desired combinations 
of size, smoothness, accuracy, asphericity and lack of axisymmetry are well beyond the present state of the art in polishing, and will remain impossible with almost all existing polishing techniques.

The method of stressed-lap polishing holds the promise of solving all these problems. It is currently being tested for the first time, in the manufacture at the Steward Observatory Mirror Laboratory of the primary mirror for the Lennon Telescope being built by the Vatican Observatory and the University of Arizona. This mirror is $1.83 \mathrm{~m}$ (72 in) in diameter with a vertex radius of curvature equal to $3.66 \mathrm{~m}$ (144 in) and conic constant equal to -0.996 . Thus the mirror is slightly ellipsoidal, for use in an aplanatic Gregorian telescope, but is virtually an $\mathrm{f} / 1$ paraboloid. The surface has a peak-to-valley deviation from the best fitting sphere of $445 \mu \mathrm{m}$. The Vatican mirror forms a stringent test for the Columbus (and Magellan) telescope mirrors, which at $8 \mathrm{~m}$ and $\mathrm{f} / 1.2$ have not much larger absolute asphericity ( $1.1 \mathrm{~mm}$ peak to valley deviation from the best fitting sphere). For comparison, the fastest 4-m class mirror polished to date has about 5 times less asphericity than the Vatican mirror.

\section{Mirror specifications}

Telescopes on the ground are limited in performance by distortion caused by thermally induced variations of the refractive index of air. It might at first sight seem that the specification for ground-based telescopes would be much more relaxed than for space optics, and that the technologies for polishing would be quite different. In fact, the atmosphere under the best conditions causes little degradation on small spatial scales, up to about 0.5 $m$. It follows that ground-based mirrors must be very smooth in this domain if they are not to degrade performance. Smoothness on these scales is the most demanding task for mirror polishing, and thus the new ground-based and space-based mirrors drive the same critical new technology development.

The specification for the figure of the asphere for the Vatican mirror is given in terms of the structure function, or mean square phase difference between point pairs in the aperture as a function of their separation (Brown 1983). We choose a structure function of the same form as the atmospherically induced phase distortions according to the standard Kolmogoroff model of turbulence (Tatarski 1961), with a scale factor corresponding to an image FWHM of 0.125 arcsec at $633 \mathrm{~nm}$ wavelength. Since the atmospheric wavefront distortion approaches 0 toward small spatial scales, the relevant requirement for the mirror is that little light is lost due to diffraction by small-scale irregularities, as discussed by Angel (1987). The specification chosen allows a loss of $20 \%$ of light at $350 \mathrm{~nm}$ wavelength due to small-scale irregularities ( $6 \%$ loss at $633 \mathrm{~nm}$ ), with virtually no effect on the image size.

There are no definite plans yet for use of very aspheric mirrors in space astronomy, but it is worth noting that the mirror needed for the SOFIA airborne telescope is very similar in size and figure to the Vatican mirror. Working at infrared wavelengths, it does not need to have such an accurate surface, and could be finished easily by the stressed lap method. 


\section{Rationale for the stressed lap}

A number of innovative polishing techniques have been proposed for the 8-m-class mirrors and used on smaller and slower mirrors. The general problem that any viable polishing technique must overcome involves conflicting requirements for the polishing tool. The tool should be large and rigid, and must fit the mirror surface to an accuracy of order several microns. While a small rigid tool can maintain a good fit to the mirror surface even as it translates and rotates, it has to be so small $(15 \mathrm{~cm}$ in diameter to fit the 8-m $\mathrm{f} / 1.2$ mirror to within $3 \mu \mathrm{m}$ ) that polishing becomes unacceptably time-consuming. Furthermore, the tool's natural smoothing action works only on scales smaller than its diameter, so one has to actively control the figure over a large range of scales. The opposite extreme, a large flexible tool, must be sufficiently flexible that it rides over roughly $1 \mathrm{~mm}$ of asphericity. Such a tool will also ride over large errors in the mirror figure without exerting a significant corrective action.

We have developed a way to combine the advantages of large tools and rigid tools, by building a large rigid lap that is bent actively as it translates and rotates, so that it continuously matches the changing aspheric surface with which it is in contact. Actuators mounted on the lap apply edge moments to change its shape, based on commands from a computer that monitors the lap's position and orientation with respect to the mirror and evaluates the required forces. In this way the stressed lap removes the difficulties associated with asphericity from the polishing operation, in much the same way that a null corrector removes them from testing. The asphericity is, in a sense, transparent to the optician, who polishes the mirror as though he were polishing a sphere with a passive lap.

\section{A lap for a 1.8-m $\mathrm{f} / 1$ asphere}

The stressed lap consists of a metal disk with actuators attached to the upper face, and coated on the lower face with the traditional layer of pitch. The lap is pressed against the mirror prior to polishing, allowing the pitch surface to take on the correct absolute shape for one particular position and orientation of the lap. When the polishing run starts, the actuators must induce the correct changes in shape as the lap moves relative to the mirror.

One of the key aspects of making the stressed lap work was to find a reasonably simple arrangement of actuators that would accurately induce the required shape changes. These shape changes can be expressed as a sum of Zernike polynomials. For the 1/3-diameter lap that we use for the $\mathrm{f} / 1$ mirror, they are given to an accuracy of $0.3 \%$ by the first three polynomials that involve bending, namely defocus, astigmatism and coma. In their work on stressed-mirror polishing, Lubliner and Nelson (1980) showed that these bending modes can be produced in a thin plate by a distribution of bending moments and shear forces acting on the edge of the plate. We use the same principle for the stressed lap. 
The actuators that bend the lap work independently of the polishing machine, which drives the lap in translation and rotation. Since these actuators apply no net force or moment to the lap, they can be mounted and connected internally so that both applied forces and reaction forces are taken by the lap. As Lubliner and Nelson pointed out, the shear forces can be replaced by an equivalent distribution of twisting moments, which are simpler to apply with the internal system. Such bending and twisting moments are applied by lever arms attached to the perimeter of the disk and extending out of the plane of the disk. The ends of these lever arms are connected in groups by elements in tension, and the actuators vary the tension of these connecting elements. Angel (1984) and Martin, Angel and Cheng (1988) describe the general system and an early version of the actuator design. The actuators have been modified in the light of further analysis and experiment, and the current design being used for the 1.8-m mirror is shown in figure 1 .

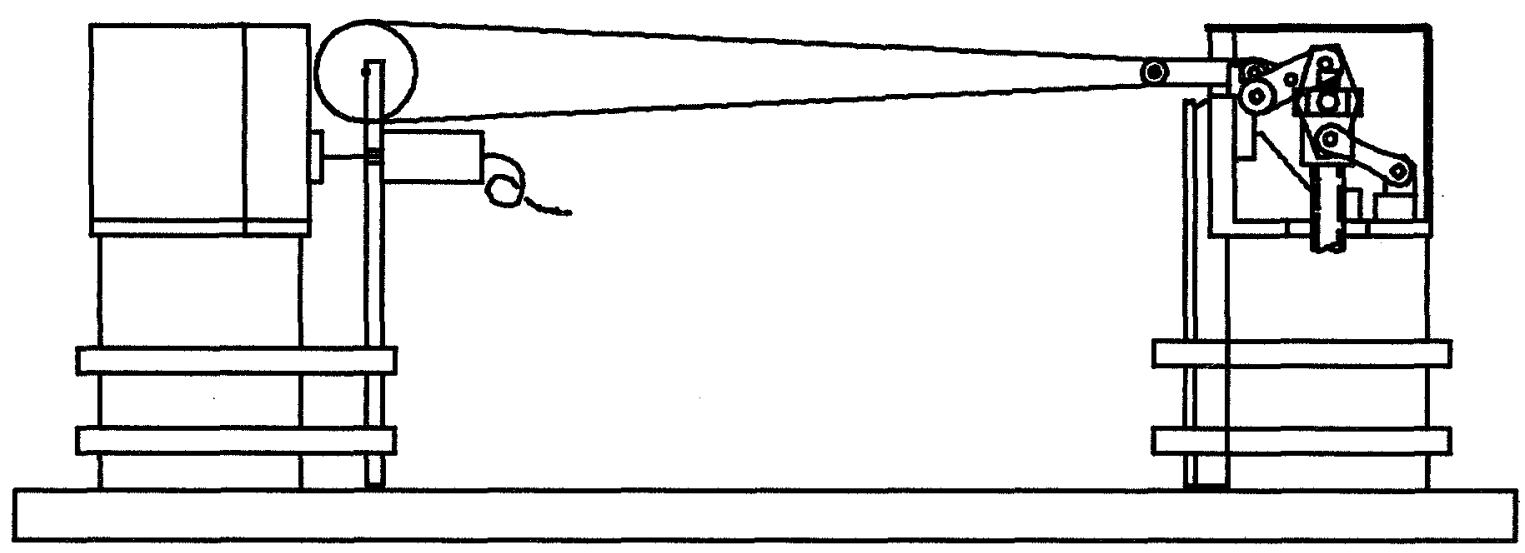

Figure 1. Side view of a stressed lap actuator. The actuator column serves as a lever arm which transmits bending and twisting moments to the lap plate. The actuator consists of a servomotor mounted inside the lever arm, a lead screw, and a linkage that converts the vertical force of the lead screw to a horizontal tension on a double band of steel. The applied force is measured and controlled through the deflection of a steel leaf spring to which the steel band is attached at its opposite end.

The lap is a solid aluminum plate $750 \mathrm{~mm}$ in diameter and $22 \mathrm{~mm}$ thick. Around the perimeter, twelve lever arms rise to a force-application point $250 \mathrm{~mm}$ above the plate. Only the inner $600 \mathrm{~mm}$ diameter of the lap is used for polishing, since ripples occur at the edge due to the finite number of actuators. The lap diameter was chosen to maximize the advantages of smoothing over large scales and instantaneous coverage of a large fraction of the mirror surface, while still allowing us to control the mirror figure by polishing preferentially on selected areas. The thickness was chosen to make the lap stiff enough 
not to conform to errors in the mirror figure that are outside the specification. The worst situation occurs when the lap overhangs the edge of the mirror by at most $1 / 4$ its diameter, in which case one can show that the lap distorts to give a maximum slope error of about 0.1 arcsec.

The stressed lap control system provides real-time, closed-loop control of the actuator forces. These forces are related to the lap deformations through an off-line calibration procedure that is performed as often as necessary to maintain accuracy, typically once per month. During operation, the control computer issues command signals which pass through a slipring onto the rotating lap. These signals update the command positions for analog servocontrol circuits controlling the actuators. The update rate is $1.8 \mathrm{kHz}$ for the entire set of twelve actuators, so the force variations are smooth and the instantaneous forces within $0.1 \%$ of the required values.

\section{Polishing machine and technique}

We are polishing the 1.8-m mirror on a computer-controlled machine designed to exploit the capabilities of the stressed lap. The position of the lap on the mirror is described in cylindrical coordinates, with one motor controlling radial motion of the lap and a second motor controlling rotation of the mirror. A third motor drives the lap in rotation. The three axes are controlled independently by a $386 \mathrm{PC}$, and arbitrary polishing strokes can be programmed. The motors produce accelerations up to $1 \mathrm{~m} / \mathrm{s}^{2}$, allowing large variations in removal rate as a function of position, and coverage of the entire surface in less than one minute. Because of the rapid coverage of the surface, uncontrolled variations in removal rate due to changes in abrasive concentration and properties, which typically occur on a timescale of several minutes, are averaged over the entire surface. Such rapid coverage of the surface is possible only because of the active control of the shape of the lap.

Our intention is to polish the mirror from start to finish with a single stressed lap, as opposed to several laps of different diameters. In order to develop techniques that would allow us to figure the mirror with this subdiameter lap, we initially polished the mirror as a sphere using a passive lap of the same size and stiffness as the stressed lap. This exercise also gave us the opportunity to solve the problems of mirror support and thermal control without the added complication of debugging the stressed lap.

The mirror was not generated prior to being finished as a sphere, but was lapped with loose abrasives from the spin-cast paraboloid, which had local surface irregularities of roughly $1 \mathrm{~mm}$ and overall astigmatism of about $50 \mu \mathrm{m}$. The 1/3-diameter lap has little automatic tendency to remove large-scale errors such as astigmatism; these errors were removed explicitly by varying the dwell time and rotation rate of the lap as a function of position on the mirror. Astigmatism was removed by varying the mirror rotation rate through each rotation so that dwell time increased in proportion to desired removal.

The final figure achieved with the mirror as a sphere meets the specification described in Section 2, as shown in figure 2. 


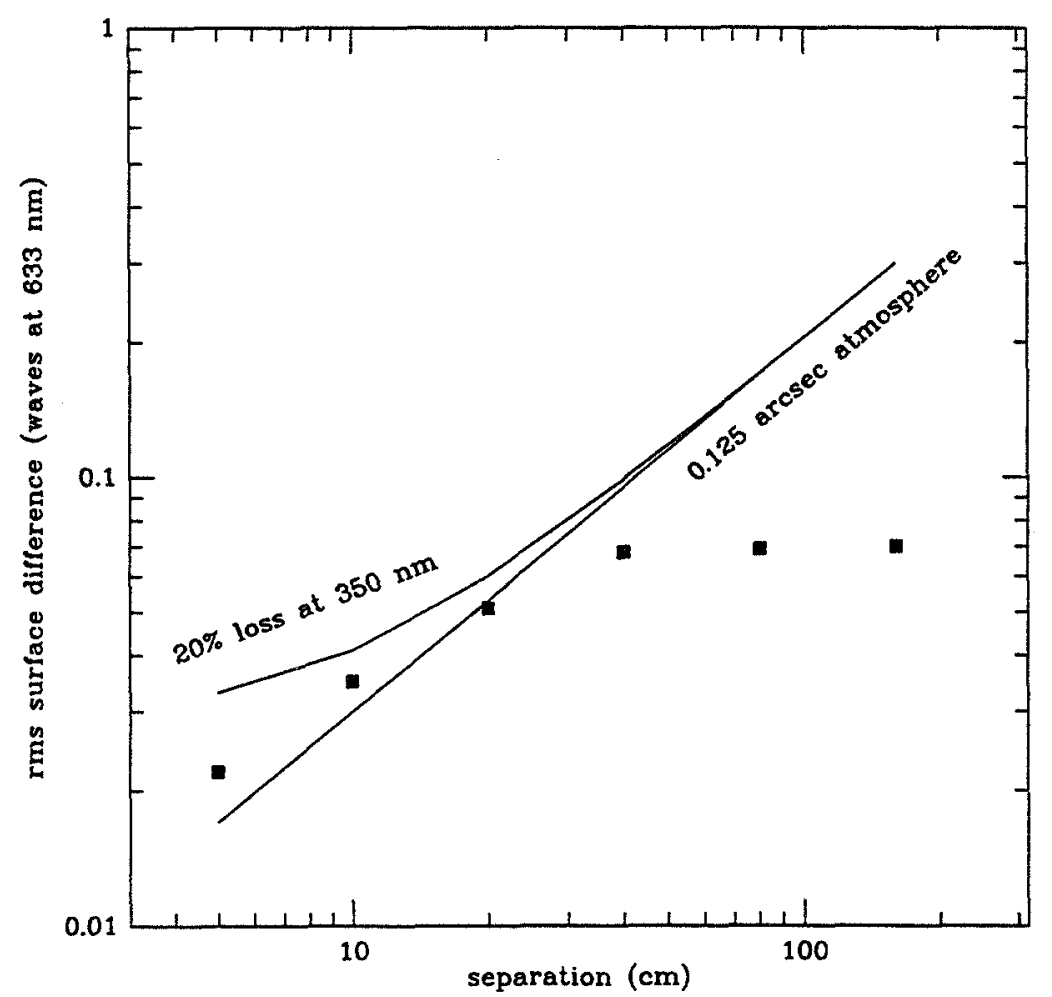

Figure 2. Surface error as a function of spatial scale on the 1.8-m mirror as a sphere. The straight line shows the surface error corresponding to the atmospheric wavefront distortion for $1 / 8$-arcsec seeing, and the curve includes an allowance for small-scale errors that scatter a small fraction of the light.

\section{Calibration and testing of the stressed lap}

The theory of plate bending establishes that we can produce very nearly the correct shape changes in the stressed lap with the distribution of edge moments that we apply. This was confirmed independently through finite-element analysis performed by Walter Siegmund. The theory can be used to obtain the forces required to bend the lap into the proper shape as a function of its position and orientation, but the accuracy of this prediction would be limited by our knowledge of the plate properties, tolerances in machining and assembly, and accuracy of the force measurements. Instead, we use plate bending theory only to design the lap, and determine force values empirically. Our only measure of actuator force is the displacement of the leaf spring shown in figure 1, which is the feedback signal for the actuator's servocontroller. We have made no attempt to calibrate this in terms of absolute force. We simply determine the feedback signals required to give the correct lap deformations.

We calibrate and test the lap by placing it on a metrology table consisting of three hard-point supports and an array of 32 spring-loaded displacement transducers located on a square grid that covers the $600-\mathrm{mm}$ diameter working surface. The lap deformations are 
computed as a function of the offset $r$ from mirror center to lap center, and orientation $\theta$ with respect to the direction to the mirror center. We choose a reference set of actuator feedback signals to correspond to the symmetric shape required for $r=0$. These values are fairly arbitrary as the pitch will take on the correct absolute shape for one position and orientation. The 32 displacements are read for these reference signals; all shape changes and all other signals are measured with respect to these reference values.

For a number of positions $(r, \theta)$, the actuator signals are varied to minimize the sum over the 32 sensors of the squared difference between measured and ideal displacement (Angel 1984). We achieve an rms error of $3 \mu \mathrm{m}$ or better over the entire range of $r$ out to $750 \mathrm{~mm}$, the maximum lap excursion. We need resolution of about $0.5 \mathrm{~mm}$ in $r$ and $4 \operatorname{arcmin}$ in $\theta$ to determine actuator signals to the required accuracy. The signals vary smoothly with $r$ and $\theta$, however, so we store them as smaller arrays of coarsely gridded values, and compute derivatives as finite differences between adjacent values. The control computer uses these arrays, with resolution of $25 \mathrm{~mm}$ in $r$ and $1.4^{\circ}$ in $\theta$, along with the encoder readings for $r$ and $\theta$, to interpolate actuator signals which are sent to the lap. Since this computation is done in real time, only a simple linear interpolation is possible. We can use a higher-order interpolation to go from the signals determined by calibration to the coarse tables used by the control computer, so a much smaller number of points, typically around 100 , need to be used for the calibration.

We believe that the accuracy of $3 \mu \mathrm{m}$ rms achieved during calibration is adequate to polish the mirror to its final figure. Currently the accuracy achieved during operation is limited to a value several times worse because of mechanical hysteresis in the system. Friction in the bearing located at the connection between the tension band and the leaf spring produces a bending moment that is not measured by the leaf spring's deflection and which exhibits hysteresis through the cycle of forces. This problem does not seem to have limited our ability to polish the mirror up to the present time, but we expect that it would eventually limit the accuracy of the mirror figure. It will be largely eliminated in a future implementation which replaces this bearing with a flexure pivot.

\section{Progress with the $f / 1$ asphere}

Following completion of the sphere, the mirror was generated as a paraboloid using the Large Optical Generator (Shannon and Parks 1984) at the University of Arizona Optical Sciences Center. The generated surface was accurate to $4 \mu \mathrm{m}$ rms and $20 \mu \mathrm{m}$ peak-tovalley, with subsurface damage extending only $30 \mu \mathrm{m}$ deep. It was therefore possible to start polishing with pitch directly, without going through the usual process of looseabrasive grinding with a sequence of grit sizes. The lap has removed material from the high zones left by the generator, as expected. As we write this paper the surface has not been lowered enough to meet the lowest zones left by the generator; about $80 \%$ of the surface now has a polished shine. The polished surface is extremely smooth, indicating that the lap is fitting the aspheric surface adequately. As long as the lap produces a surface that is 
smooth on small scales, we can control the overall mirror figure by varying the polishing strokes.

\section{Future polishing of larger primaries and other aspherics}

We are currently building a 1.2-m diameter stressed lap that will be used to polish a 3.5-m f/1.5 paraboloid starting in mid-1990. Laps of 2-2.5 $\mathrm{m}$ will be built for the larger primaries of the MMT upgrade project $(6.5 \mathrm{~m} \mathrm{f} / 1.25)$ and the Columbus and Magellan projects. These will use the same concept as the stressed lap for the 1.8-m mirror, with modifications to the actuators and force-measuring devices based on our experience with the first lap.

The stressed lap and polishing machine currently being used on the 1.8-m mirror will eventually be used to polish the large wide-field secondary mirrors for the 6.5- and 8-m primaries. These secondaries are convex hyperboloids roughly $2 \mathrm{~m}$ in diameter, with asphericity comparable to that of the $1.8-\mathrm{m}$ primary. The stressed lap does not care whether the surface is concave or convex, or precisely what shape changes are required, as long as they can be represented accurately as a sum of the three lowest-order bending modes. For the conic sections of revolution used for all classical telescope designs, a relatively large stressed lap can be used.

For off-axis conic sections and general aspherics, one can always represent any sufficiently small sub-aperture in terms of the low-order distortions that the stressed lap can produce. For a severe aspheric, as one increases the lap size, the higher-order distortions eventually become important and the lap will not fit the mirror adequately. This limiting lap size, however, will be many times greater than that of a passive lap which cannot accommodate any change of shape beyond the tolerance in fit.

\section{Acknowledgements}

The development of the stressed lap has been possible only through the combined effort of a number of scientists, engineers and programmers. We particularly acknowledge the contributions of Dave Anderson, Bob Nagel, Dick Young, and the Steward Observatory technical division.

\section{References}

Angel, J. R. P. 1984, Proc. IAU Colloquium 79: Very Large Telescopes, Their Instrumentation and Programs, ed. M.-H. Ulrich and K. Kjar, 11.

Angel, J. R. P. 1987 in Identification, Optimization, and Protection of Optical Telescope Sites, ed. R. L. Mills et al., 167.

Angel, J. R. P., Hill, J. M., Martin, H. M. and Strittmatter, P. A. 1989, Astrophysics and Space Science, 160, 55.

Brown, D. S. 1983, Proc. SPIE, 399, 12.

Lubliner, J. and Nelson, J. E. 1980, Applied Optics, 19, 2341. 
Martin, H. M., Angel, J. R. P. and Cheng, A. Y. S. 1988, Proc. ESO Conference on Very Large Telescopes and their Instrumentation (Garching: ESO), 353.

Shannon, R. R. and Parks, R. E., Proc. SPIE, 444, 271.

Tatarski, V. I. 1961, Wave Propogation in a Turbulent Medium (New York: McGraw-Hill).

\section{DISCUSSION}

Macchetto: Wouldn't you end up with different micro-roughness in different places with the stressed lap technique?

Angel: We expect that with large laps matched to the radius of curvature micro-roughness will be very small. This technique may be the best hope for generating optics with smooth surfaces. 
3, we b send

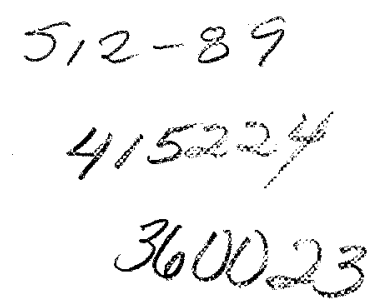

Large Telescope O-IR Astronomy from the ground

85

\section{N. Woolf}

Steward Observatory, University of Arizona

\section{Introduction}

What you would like me to say, is that large telescope diffraction limited observations can't be done from the ground. Failing that, you would like me to say it CAN be done from the ground. I bring you the worst message of all. We don't know whether it can be done from the ground. Or rather, that we suspect that some things can be done from the ground, and some can not be done from the ground, and it will take a great deal, of observational and experimental work to find where the boundary lies.

There are of course some things that will never be done from the ground, because the atmosphere absorbs too much. Thus the region from 3000 to $912 \mathrm{~A}$ is totally reserved for space observations. Similarly, telescopes on the ground will always have some surfaces with noticeably emissivity at ambient temperature. There will thus be an IR background that limits the sensitivity of equipment at wavelengths beyond 3 or 5 microns. And at some wavelengths airglow will make space the preferred location because of its reduced sky brightness. But in between lies the bulk of the region where astronomy has cut its teeth, and where we know most. That is the obvious region to advance with diffraction limited imaging by large telescopes.

\section{Adaptive optics}

The process for correcting images to diffraction limit in real time is called adaptive optics. There is the use of some kind of flexible surface to correct for wavefront errors put in by the atmosphere. There is the sensing and measuring of those errors to know how much to correct.

Because the refractive index of air is fairly constant across the visible and IR region, the wavefront error information sensed from one wavelength region can be used to correct at some other region. Because the accuracy of correction needed is reduced when the wavelength is longer, it is much easier to correct an infrared image in real time than an optical image in real time, and the image will stay corrected for a longer time, and be corrected over a wider field. The time and field diameter increase somewhat faster than the wavelength, so that there are somewhat more diffraction limited pixels in the correctable field at longer wavelengths. 
The length of time for which the image stays corrected, is the length of time for which the rms wavefront error stays within our diffraction limited tolerance of $1 / 10$ to $1 / 20$ wave. Because this time is very short in the visible, the correction must be carried out on a star with a substantial photon flux, somewhere between 8th. and 11th. magnitude. However there are experiments underway, using the telescope to project an artificial star by reflecting Sodium D lines off the upper atmosphere, and correcting on the image of that. If the process is successful, diffraction limited imaging will be available anywhere in the sky.

The next problem is that the wavefront errors arise at various layers throughout the atmosphere, while the correction is currently applied at a single surface which can at most be conjugate with a single layer of the atmosphere. As a result, the correction is only appropriate for a small area, the isoplanatic patch, around the light source and in consequence only a limited area of sky is corrected to diffraction limit. There are however possibilities of having more than one corrective surface, and so widening the region of sky. The expected final result is that visual images will be corrected across a patch of sky some 2 " to at most $20^{\prime \prime}$ in the visible, while at 10 microns, the corrected area will be some minutes of arc in size. The correction at longer wavelengths will be better and much easier, giving a longer coherence time, a larger isoplanatic patch and being possible on a fainter star if the initial seeing is superior. Adaptive optics is a way of improving the best sites, it is relatively poor at coping with the inferior ones.

Because a patch of Sodium light reflected from the upper atmosphere will show a parallax, the size of telescope is limited for having the source in the same isoplanatic patch for all parts of a large primary mirror. Conquering this problem will need a complex solution, but it is not in principal impossible.

What can adaptive optics achieve?

The first thing we have to realize is that the size of the isoplanatic patch is independent of the telescope size. In consequence, the number of diffraction limited patches that can be placed in a single isoplanatic patch increases as the primary mirror area increases. Even a 2 " patch can contain $2000 \times 2000$ pixels, if the pixel is $0.001 "$ across. However this corresponds to a telescope primary $100 \mathrm{~m}$ across. If we assume a standard ratio for the cost of ground and space facilities of 500:1, and cost being proportional to mirror area then such a ground telescope would cost about the same as a $4 \mathrm{~m}$ telescope in space. One difference is that, astonishingly, our technology is currently more geared to dealing with the problems of space.

If we had such a giant telescope, with such spectacular angular resolution, what would we do with it? There are three obvious areas of research. The first is studies of intrinsically bright objects, such as the surfaces of stars, the second is a study of the regions close to stars for objects related to those stars. The third is to say that the universe is similar in all directions, and so the regions close to the direction to stars is just like any other region, and one can study cosmology in them, and very well too, IF the telescope reaches sky background in the area we are observing. Thus the dark sky point source faintness limit of a $100 \mathrm{~m}$ diffraction limited telescope is under those circumstances 7 magnitudes fainter than the limit of a $4 \mathrm{~m}$ telescope, and $5 \mathrm{~m}$ fainter than the limit of a $10 \mathrm{~m}$ telescope. 
The background limit of telescopes with adaptive optics

If we observe near a star, using the star's light for adaptive optics, there is a concern that the scattered light from the star will exceed sky background. On the other hand if there is an artificial star made with sodium light, the sodium emission is easily filtered away. But we currently better understand how to use the real star as a source for adaptive optics. Thus it is appropriate to consider the scattered background around a star. For problems of planetary searches, from any platform, the problem of scattered light is a crucial one.

Ivan King (1971) published the profile of a star image, and it was later shown by two different groups that the profile consists of two parts, an inner region which is on the ground dominated by seeing, and an outer region which is called the aureole.

Following this talk, and at this meeting, a consensus developed that the aureole is the result of the residual polishing imperfections on the mirror. On the small scale, these are referred to as "orange peel" and "dog biscuit". The larger the scale of the structure, the closer in is the scattered light, thus orange peel and dog biscuit affect the outer parts of the aureole from about $10^{\prime \prime}$ out, ripple because of the use of small tools affects the area further in. There are possibilities of substantially reducing the ripple by (1) polishing aspheric surfaces with a stressed lap, (2) avoiding segmentation and controlling mirror shape at the support points, and (3) avoiding any obscuration in the beam by use of off-axis optics. The reduction of orange peel and dog biscuit seem likely to require some form of super polishing.

Some improvement in both large and small scale correction will be needed if we are to study cosmology using the areas of the sky close to stars. With current mirrors, the aureole at about $4500 \mathrm{~A}$ is fainter than the star by about 10 magnitudes per square arc second at a radial distance of $3^{\prime \prime}$, and at 9 " it is about 12.5 magnitudes fainter. The light at every part of the aureole is likely to be reduced by the square of the wavelength. You can see therefore, that at least in the visual there will be a very delicate question whether sufficiently faint stars can be used for adaptive optics that the sky background light will dominate at the outer part of the isoplanatic patch. The critical question is whether the large ground telescope concept is boxed in between wavelengths sufficiently short that the zodiacal light dominates airglow, and yet at the same time that the night sky brightness dominates inside the isoplanatic patch.

\section{Forms of Large Telescope}

Both on the ground, and in space there is considerable confusion about the relative role of large telescopes and interferometers. I do NOT regard them as interchangeable, at least at wavelengths below a few $\mathrm{mm}$. where one is in the photon-noise dominated regime. Equally, I regard them both as extremely important. The interferometer clearly has a better potential for highest angular resolution, and the single aperture is, for currently imaginable costs and associated aperture sizes, the leader in point source sensitivity.

The difference between a radio and an optical interferometer arises because the noise at radio wavelengths is dominated by wave-noise, or if you will by the statistics of the bunching of bosons, whereas in the O-IR region, the detection events are far fewer and are dominated by individual particle statistics. In consequence amplifiers are able to be 
relatively noise free at radio wavelengths and are very noisy at optical wavelengths, with even a perfect amplifier producing one noise event per unit time per unit bandwidth. (I am indebted to Dr. B. Burke for directing me to this conclusion). Thus the optical interferometer is forced to detect at the place where the light is brought together, while at radio wavelengths it is possible to amplify a signal, split this up, and get independent interference between beams. The problem shows up in the mapping mode, but need not show up in the spectroscopic observation of a single point source once its position has been determined.

Thus a single point source can be tracked and if the interferometer aperture is kept phased by variable delay lines (i.e. one sits on top of the bright fringe), and if perfect beamsplitters are assumed the device will reject the incoherent light of the background and so allow the same signal/noise ratio that would be obtained for the same area in the form of a single dish. ( This was pointed out to me by Dr. Angel). Unfortunately, for this configuration the light from many other areas of the sky is suppressed by interference because the interferometer impresses fringes onto the plane of the sky.

If however one instead attempts to map an area of sky, then one finds the light spread angularly into a pattern where a substantial fraction of the energy appears in low-surface brightness side-lobes (see for example the patterns shown by Meinel, Meinel and Woolf 1983). There is then a loss of signal/noise ratio if there is a sky background that is appreciable compared with the signal. Because of the background, the light in these low level frringes can be detected with at most an extremely low signal/noise ratio, and in consequence it hardly helps in the total observation. The problem appears to be reduced by, (1) using fewer, larger interferometer elements and moving them around to synthesize an aperture, with three elements being a probable optimum, or (2) having the interferometer spacings redundant, and in the extreme variant of this, in having all elements co-linear. Either of these solutions places considerable emphasis on aperture rotation synthesis.

It is interesting to consider three concepts intermediate between a telescope and an interferometer to try to balance the astronomical advantages of a single large instrument. In the NNTT 4-square concept (Angel and Woolf 1986), four close spaced large apertures were used to get somewhat higher angular resolution than for a single dish of the same area, and with a modest loss of point-source sensitivity in the sky-viewing mode. The versatile array concept (Woolf, Angel and McCarthy 1983) uses a similar 4 apertures in a linear non-redundant array, in which there was a greater loss of point sensitivity, though reduced by the co-linearity of the elements, and a much greater gain in angular resolution. The third (Angel, Woolf and Epps 1982) was the use of a non-circular aperture, a highly elongated one, with the use of rotation synthesis and tomographic image reconstruction.

This last seems to me to be worth far more consideration for large space and lunar telescopes as well as for giant telescopes on the ground. It has the full point source sensitivity of the aperture for spectroscopic observations, without any special efforts beyond those normal for adaptive optics on the ground, or alignment and phasing in space. Further, it is possible to map in various ways that allows the data to be analyzed either for optimum point source sensitivity or for maximum angular resolution 
Conclusions

There are no very simple conclusions. The balance of opportunities and costs of doing astronomy from the ground and space is a most difficult topic, even without the uncertainties that started this paper. I believe it is entirely appropriate to put substantial effort into concept development, and particularly into cost reduction for all schemes for giant astronomical facilities. Every dollar spent in this phase can pay off hundredfold in our eventual decision. It is in this phase that the normal committee type of compromise is least helpful. We need to discover the optimum, regardless of individual feelings.

Equally I feel that we cannot afford to overbalance our efforts either towards large telescope structures or towards interferometry. There will indeed be a time when we have to set a priority, and all pull together for a single device, and maybe even for a single platform, Earth, orbit or Moon. That time is not yet here. And equally, we are all responsible that EVERY concept is the very best one of its kind possible. We are here to get the best for astronomy, not to push anyone's pet scheme.

\section{References}

Angel J.R.P. and Woolf N.J. 1986 Ap.J. 301,478

Angel J.R.P. Woolf N.J. and Epps H.W. 1982 Proc. S.P.I.E. 332,134

King I. 1971 Pub. Astr. Soc. Pacific, 83,199

Meinel A., Meinel M. and Woolf N. 1983, App.Optics \& Opt.Eng. 9,150

Woolf N.J., Angel J.R.P. and McCarthy D.W.Jr. 1983, Proc. S.P.I.E. 444, 78 


$$
\text { omithes }
$$

\section{Session 4}

\section{Current Proposals and Studies}




\section{Page intentionally left blank}




\section{Considerations for a \\ Next Generation UV/Optical \\ Space Telescope}

$$
\begin{array}{rr}
43-39 & \begin{array}{r}
\text { M.E. Nein and S.H. Morgan } \\
\text { NASA - George C. Marshall } \\
360024
\end{array} \\
316 & \text { Space Flight Center }
\end{array}
$$

During the past 25 years, a remarkable scientific revolution has occurred in astrophysics as a result of convergence on two advancing fronts. First, instruments and telescopes have been developed to make sensitive measurements throughout the entire electromagnetic spectrum. Secondly, access to space has permitted observations above the obscuring and distorting "dirty window" of our atmosphere. Beginning around the middle of the next decade, a third major path - the availability of the permanently manned Space Station Freedom - will join with the earlier two capabilities, to not only continue this revolution, but to accelerate the quest for answers about the universe that have puzzled mankind for centuries. Beyond Earth-orbit, NASA is actively studying the possibility of a return to the Moon, which would provide a valuable platform for astrophysics observations during the next century.

The promise offered by space observatories will be dramatically illustrated when four observatories, the Hubble Space Telescope (HST), Gamma Ray Observatory (GRO), Advanced XRay Astrophysics Facility (AXAF), and Space

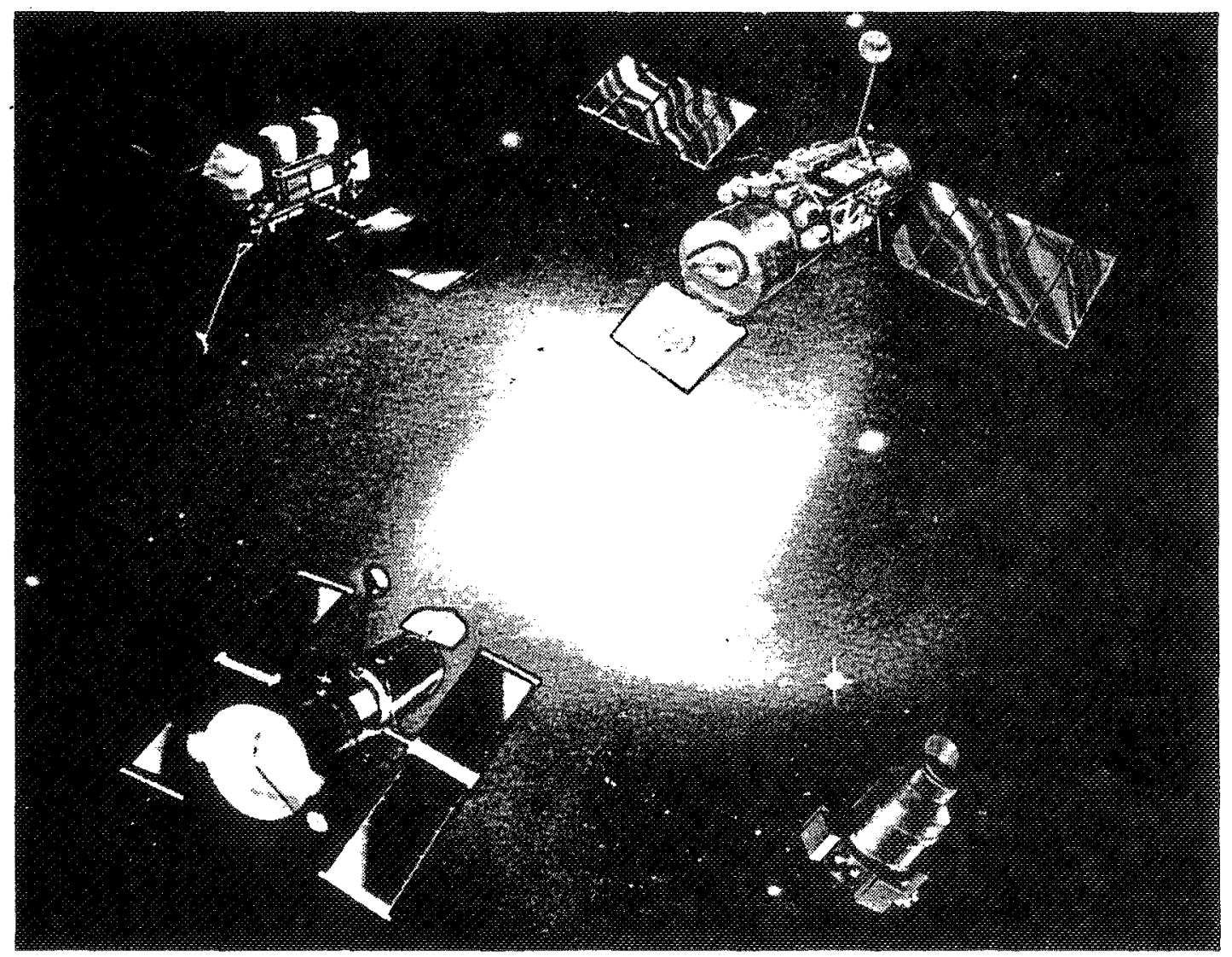

Figure 1. NASA's Great Observatories 
Infrared Telescope Facility (SIRTF), which form NASA's "Great Observatories" program, become operational (Fig. 1). These observatories will form the core of NASA's Astrophysics Program through the end of this century and early into the next.

By the mid-to-late 1990's, S.S. Freedom, associated co-orbiting platforms, space transportation systems, and supporting facilities will be able to support the Great Observatories. offer expanded capabilities for other astrophysics missions, and be available to enhance, and in some cases make possible, the successors to the Great Observatories. With the utilization of S.S. Freedom for repair and servicing and as a platform for observing facilities, such as the Advanced Solar Observatory (ASO), and to provide a base for assembly of large facilities, S.S. Freedom will be an integral part of astrophysics research. In fact, some of the proposed large observatories of the 21 st century may not be feasible without extensive assembly operations in orbit.

To maintain scientific continuity and momentum, to ensure that the Nation's leadership in astrophysics continues, and to establish the groundwork for future space observatories, the successors to the Great Observatories, the observatories of the 21 st century, are now being studied. In order for them to become a reality, it is vitally important to immediately begin assessing and planning the technology required for these future facilities.

Concepts for advanced large space telescopes which will follow the HST are being investigated by NASA and the scientific community. The very long development times and the great investment in resources to achieve one order of magnitude improvement over HST in both resolution and sensitivity are worthwhile objectives for detailed studies of the next generation of large space observatories.

Of the new design concepts being studied, the segmented mirror and phased-array telescopes have attracted the most attention for future UV/ optical space telescopes. Current technology, as demonstrated by the Multimirror Telescope, indicates that it is possible to break up a normally circular telescope aperture and separate the parts in order to effectively increase the aperture diameter without requiring increased collecting areas. Since the cost of telescopes has been estimated to vary approximately as the cube of the diameter, this is a very important consideration. Similar ideas have long been used in radio astronomy with remarkable success. Approaches such as these are now being examined in greater detail for advanced UV/optical telescopes in space, as well as future groundbased telescopes such as the New Technology Telescope (NTT) in order to reduce the weight and size of the subapertures. This would then allow transportation and assembly in space to produce a complete telescope. As studies have shown, there are many approaches to increasing the aperture and thus angular resolution and sensitivity. Figure 2 shows several representative concepts.
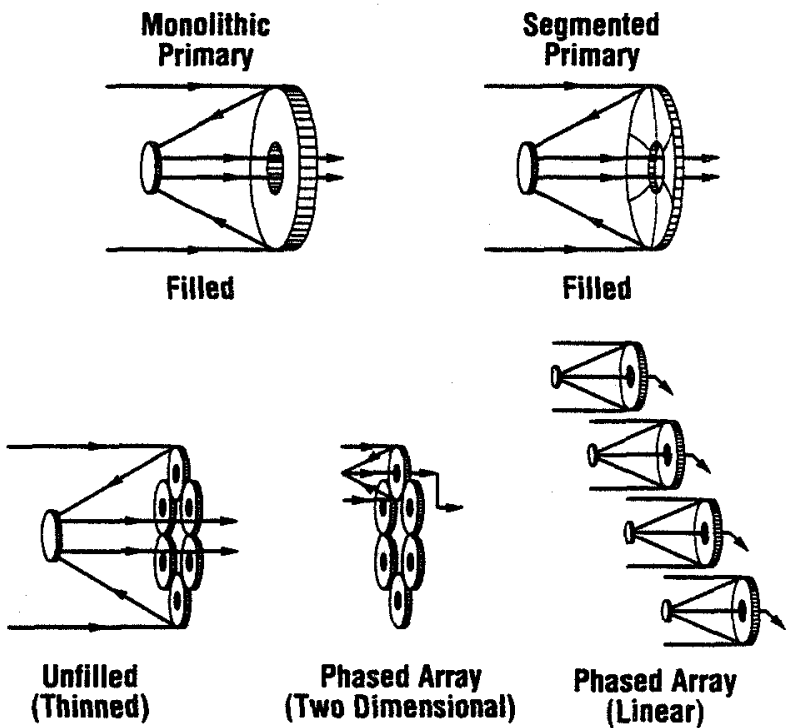

Figure 2. Aperture Options for Large SpaceBased Optical Telescopes 
In order to "thin" the aperture, several major considerations must be addressed to ensure that the optical performance remains acceptable. The modulation transfer function, as a measure of the optical performance, must not become zero anywhere except at the highest spatial frequency (corresponding to the diffraction limit of the circumscribed (full) aperture diameter). Otherwise, information at some spatial frequencies will be'lost. The practical consequences of this are reflected in careful selection requirements for subaperture spacing and location. Experimental results, reported elsewhere, seem to indicate that advantageous placement of the subaperture can be accomplished.

Although optical performance considerations play a major role in the selection of a telescope concept, other important aspects also become major trade criteria in the design of advanced space telescopes. Some of these criteria are listed in Figure 3, where various telescope concepts are compared. It is obvious that the traditional contiguous-filled circular aperture concept has excellent optical performance, allows testing of the complete optical train on the ground (although necessarily under degraded conditions because of the g-loading), avoids the complexities of orbital assembly, and does not require rotation of the telescope to construct an image. Unfortunately, the extreme weight and volume associated with this approach could exceed the transportation-to-orbit capability of projected future launch vehicles, unless extreme attention is paid to superlight-weighting of the mirror and telescope structure and the spacecraft components. The latter, however, normally has a high price tag associated with it. For example, a 10-m

\begin{tabular}{|c|c|c|c|}
\hline & Advantages & Disadvantages & $\begin{array}{c}\text { Advanced Technology } \\
\text { Development Auxilliary } \\
\text { Systems }\end{array}$ \\
\hline $\begin{array}{l}\text { Contiguous } \\
\text { Filled Circular } \\
\text { Aperture }\end{array}$ & $\begin{array}{l}\text { - Traditional Concept } \\
\text { - Excellent PSF } \\
\text { - Excellent Spatial } \\
\text { Frequency Coverage } \\
\text { - On-Ground Testing } \\
\text { - No Rotation } \\
\text { - No On-Orbit Assembly }\end{array}$ & $\begin{array}{l}\text { - Limited Resolution } \\
\text { - No Modularization } \\
\text { - Extreme Weight \& Volume } \\
\text { - Transportation (Ground \& } \\
\text { Space) } \\
\text { - Heavy Launch Vehicle }\end{array}$ & $\begin{array}{l}\text { - Structural Stability } \\
\text { - Launch Vehicle } \\
\text { - Transportation to Oper. } \\
\text { (GEO?) Orbit } \\
\text { - Mirror/Telescope Assembly }\end{array}$ \\
\hline $\begin{array}{l}\text { Segmented } \\
\text { Filled } \\
\text { Aperture }\end{array}$ & $\begin{array}{l}\text { - Optically Traditional } \\
\text { Concept } \\
\text { - Excellent PSF } \\
\text { - Excellent Spatial } \\
\text { Frequency Coverage } \\
\text { - No Rotation } \\
\text { - Light Weight } \\
\text { - Transportation (Ground } \\
\text { \& Space) }\end{array}$ & $\begin{array}{l}\text { - Off Axis Aspheres } \\
\text { - Complex Assembly } \\
\text { - Complex Alignment }\end{array}$ & $\begin{array}{l}\text { - Structural Stability } \\
\text { - Precision/Large Number } \\
\text { Segment Fabrication } \\
\text { - Precision Alignment \& } \\
\text { Control Actuators } \\
\text { - Optical Coatings }\end{array}$ \\
\hline $\begin{array}{l}\text { Mills } \\
\text { Cross }\end{array}$ & $\begin{array}{l}\text { - High Resolution } \\
\text { - Growth Capabilty } \\
\text { - On-Ground Testing } \\
\text { - Space Transportation }\end{array}$ & $\begin{array}{l}\text { - Limited Sensitivity } \\
\text { - Number of Reflections } \\
\text { - Field of View } \\
\text { - Image Reconstruction } \\
\text { - Beam Alignment } \\
\text { - Rotation }\end{array}$ & $\begin{array}{l}\text { - Optical/Mechanical Phasing } \\
\text { - Stable Structures } \\
\text { - Optical Coatings } \\
\text { - Fine Guidance Telescope } \\
\text { Coalignment }\end{array}$ \\
\hline $\begin{array}{l}\text { Segmented } \\
\text { Diluted } \\
\text { Aperture }\end{array}$ & $\begin{array}{l}\text { - Moderate Optical Path } \\
\text { Compensation } \\
\text { - Moderate Alignment } \\
\text { - Growth (Restricted to } \\
\text { Initial Aperture } \\
\text { - No Rotation }\end{array}$ & $\begin{array}{l}\text { - Limited Sensitivity } \\
\text { - Moderate Spatial Freq. } \\
\text { Coverage } \\
\text { - Ofi-Axis Aspheres } \\
\text { - No Ground Testing as a } \\
\text { Completed Telescope } \\
\text { - Size, Inertia } \\
\text { - No Modularization } \\
\text { - Difficult Assembly }\end{array}$ & $\begin{array}{l}\text { - Precision Alignment } \\
\text { Actuators } \\
\text { - Large Stable, Adj. Structures } \\
\text { - Large Off-Axis Mirrors } \\
\text { - Orbital Assembly } \\
\text { - Transportation to Oper. } \\
\text { Orbit } \\
\text { PSF = Point Spread Function }\end{array}$ \\
\hline
\end{tabular}

Figure 3. Typical Trade Considerations for Telescope Concepts 


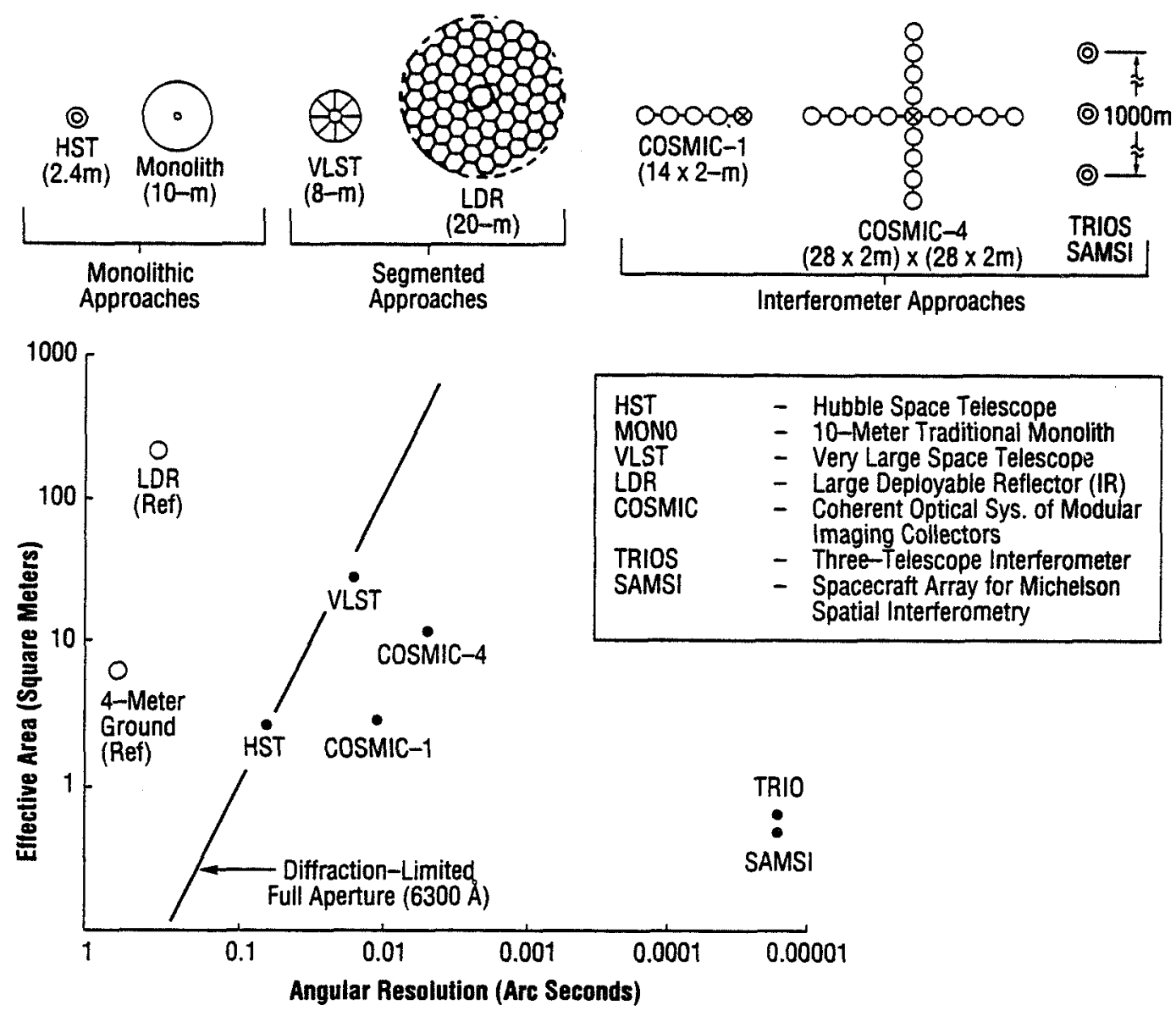

Figure 4. Approaches to Large Apertures

diameter monolithic mirror based on the HST design could weigh in excess of 70,000 lb and would be totally unacceptable. New lightweight concepts and materials or segmented, adaptive primary mirror concepts could be used to reduce the weight requirements, but will result in increasing the complexity of the mirror figure control. In a similar manner, advantages and disadvantages for other concepts must be weighed against scientific requirements, technological factors, and cost.

Many generically different approaches to a highresolution, high-sensitivity space telescope have been analyzed (Fig. 4). Of these, six representative concepts are shown in Table I, where they are compared on the basis of angular resolution and sensitivity to faint and extended sources. Figure 5 shows a series of representative artist concepts of UV/optical telescope concepts which have been studied at MSFC. Analyses of the various telescope designs have shown that there are two key enabling technology areas which must be firmly in hand before these advanced telescopes can even be considered for development: dimensional stability of the optical system and orbital assembly of the telescope.

Considerable technology development is in progress to address problems in the dimensional stability area. Specifically, the development of methods to improve mirror figure and surface accuracy of ultralightweight panels, sensors, and actuators for segment alignment, and element phasing, development of improved material stability and others are all under way. However, equally important areas such as transportation to orbit, on-orbit assembly using both astronauts 
Table I. Performance Comparison of UV/Optical Telescopes

\begin{tabular}{|lccccccc|}
\hline $\begin{array}{l}\text { Telescope } \\
\text { System }\end{array}$ & $\begin{array}{c}\text { Baseline } \\
(\mathrm{m})\end{array}$ & $\begin{array}{c}\text { Collecting } \\
\text { Area }(1) \\
\left(\mathrm{m}^{2}\right)\end{array}$ & $\begin{array}{c}\text { Aperture } \\
\text { Fill Factor } \\
(\%)\end{array}$ & $\begin{array}{c}\text { Mumber } \\
\text { of } \\
\text { Reflections }\end{array}$ & $\begin{array}{c}\text { Effective } \\
\text { Area (2) } \\
\left(\mathrm{m}^{2}\right)\end{array}$ & $\begin{array}{c}\text { Theoretical } \\
\text { Resolution } \\
\left(10^{-3} \text { arc sec) }\right.\end{array}$ & $\begin{array}{c}\text { Field of } \\
\text { View } \\
\text { (arc minutes) }\end{array}$ \\
\hline Filled Circ. Apert. & 10 & 66.8 & 85 & 2 & 44.9 & 14 & $\approx 1$ \\
MSFC-VLST & 8 & 42.7 & 85 & 2 & 28.6 & 17 & $\approx 1$ \\
COSMMC-1 & 14 & 11.0 & 7 & 8 & 2.6 & 10 & 0.05 \\
COSMIC-4 & 36 & 44.0 & 4 & 8 & 10.4 & 4 & 0.05 \\
GOLAY-9 & 13 & 19.5 & 15 & 6 & 5.9 & 10 & 0.5 \\
TRIO & 1000 & 1.3 & 0.0001 & 5 & 0.5 & 0.14 & $0.0017(0.1$ arc sec) \\
\hline HST & 2.4 & 3.9 & 86 & 2 & 2.6 & 58 & 2 \\
4-m Ground & 4 & 10.0 & 80 & 2 & 6.7 & 600 & 10 \\
\hline
\end{tabular}

(1) Includes $15 \%$ reduction for obscuration by Cassegrain mirror and fins

(2) Based on $82 \%$ reflectivity in visible for each optical surface

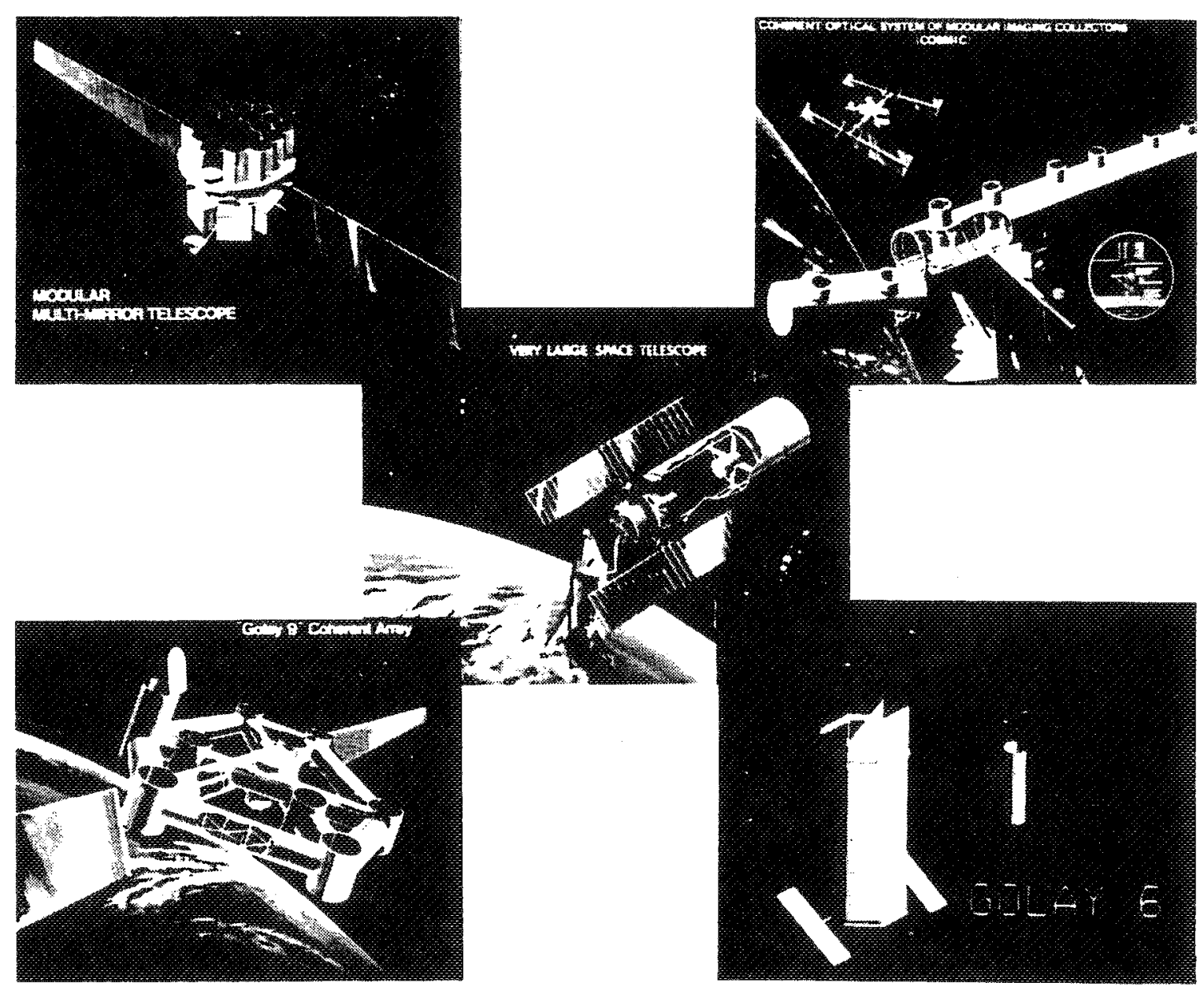

Figure 5. Advanced UV/Optical Space Telescopes Concepts 
and robotics, initial alignment, and long-term maintenance have only recently received attention. Early analysis shows that transportation to an orbital altitude of $300 \mathrm{nmi}$ with subsequent assembly requires launch capabilities of the order of 50 tons for a thinned primary mirror-type telescope with 10 -fold increase in resolution over the HST. This capability may be available early in the 21 st century. However, a high Earth orbit, especially a geosynchronous orbit (GEO), would be better for the observatory, since both gravity gradient and aerodynamic torques are minimized. For the large telescopes under consideration, these torques are the major environmental disturbances and are the primary drivers of momentum management and control system design in low Earth orbit.

Additional considerations for operating large observatories in high Earth orbit, or preferably GEO, are shown in Table II. Thus it becomes obvious that orbit-to-orbit transportation will be one of the major considerations in the development of future large observatories, affecting every aspect of the design. Since there are currently no indications in NASA's future mission models of an Orbital Transfer Vehicle (OTV) with the capability to transport 50 tons of payload to GEO, telescope designers must consider assembly of telescope modules at high altitude orbit locations either through robotics, by astronauts, or both. Alternatively, telescopes must be designed which can be accommodated by projected launch vehicles, even at the expense of some scientific capabilities. To operate these observatories at more accessible orbits will entail the problems highlighted in Figure 6. It is clear that assembly by robotics and/or man in GEO without the presence of a large operational base, such as a temporarily or permanently manned GEO station, will further complicate the design of these telescopes.

In order to better understand the key technology issues which will have to be resolved before serious design approaches can be advanced, several different generic telescope system concepts are now being analyzed by NASA. As

Table II. Orbit Considerations for Future Astronomical Observatories

\begin{tabular}{|c|c|}
\hline LEO & GEO \\
\hline $\begin{array}{l}\text { - Low observational efficiency } \\
\text { - No long exposure of sources without } \\
\text { interruption } \\
\text { - More complex radio transmission coverage } \\
\text { - High aerotorque } \\
\text { - High gravity gradient } \\
\text { - Frequent thermal cycling due to short period } \\
\text { day/night cycle }(5,600 / y r) \\
\text { - Higher power requirements (to cover night) } \\
\text { - Frequent reboost required }\end{array}$ & $\begin{array}{l}\text { - High observing efficiency } \\
\text { - Almost no sources eclipsed by Earth } \\
\text { - Less bright Earth induced stray light } \\
\text { - Long exposures without interruption } \\
\text { - Continuous real-time control } \\
\text { - Simplified scheduling } \\
\text { - Fast response to anomalies } \\
\text { - Efficient reaction to acquired science data } \\
\text { - High level of data transfer } \\
\text { - Direct link to ground } \\
\text { - Minimal attitude control } \\
\text { - Gravity gradient and aerotorque small } \\
\text { - Low level of mechanical excitations } \\
\text { - Elliminates short thermal cycling period present } \\
\text { in LEO ( 50/yr in GEO) } \\
\text { - Aids in obtaining accurate pointing and } \\
\text { tracking and stable optical figure }\end{array}$ \\
\hline
\end{tabular}




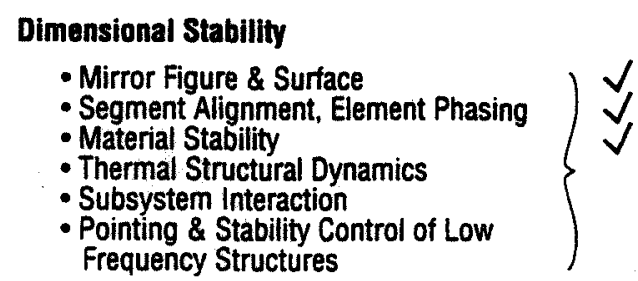

Orbital Requirements

- Transportation to Orbit

- Assembly

- Initial Alignment \& Maintenance of Alignment

- Orbit to Orbit Transfer

- Operations

- Maintenance \& Repair

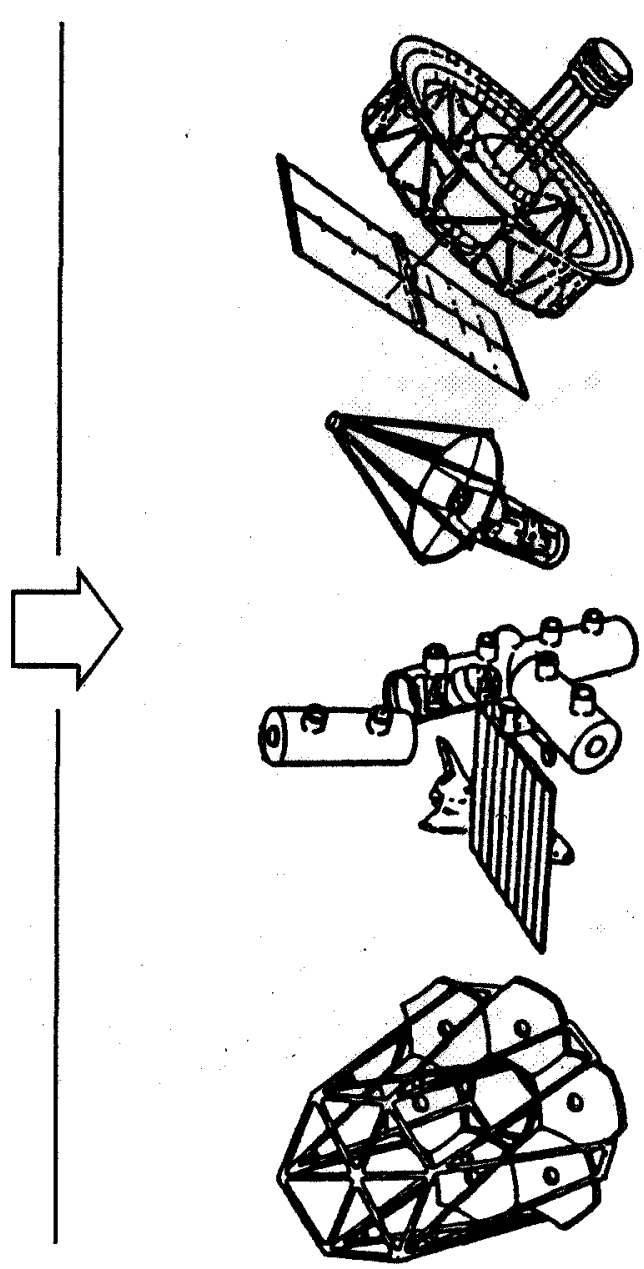

Figure 6. Key Enabling Technologies for Advanced Telescopes

pointed out by the Space Science Board's Task Group on Astronomy and Astrophysics in its study of major directions for space sciences during the 1995-2015 time period, astronomers will probably pursue two different approaches: (1) high resolution through interferometry, which means a large baseline between sensors, and (2) high "throughput" for imaging faint objects. This requires large 8- to $16-\mathrm{m}$ diameter mirrors.

In concert with these recommendations, MSFC has conducted studies of two representative advanced telescope configurations to further assess a range of key technical problems inherent in both interferometric-type telescopes and large contiguous primary mirror telescopes.
The interferometric telescope concept selected for technology investigations is a type representative of the one-dimensional Coherent Optical System of Modular Imaging Collectors (COSMIC) that was previously studied. The particular arrangement selected is known as a "Golay 9" array. This concept is optimum from the standpoint of providing an autocorrelation function of maximum compactness. The two-dimensional array of nine afocal telescopes arranged in a nonredundant pattern is shown in Figure 7. This concept has the advantages of being relatively compact, not requiring rotation to form an image, and allowing later expansion of the baseline by the addition of more telescopes that would feed the same beam combiner. However, there 


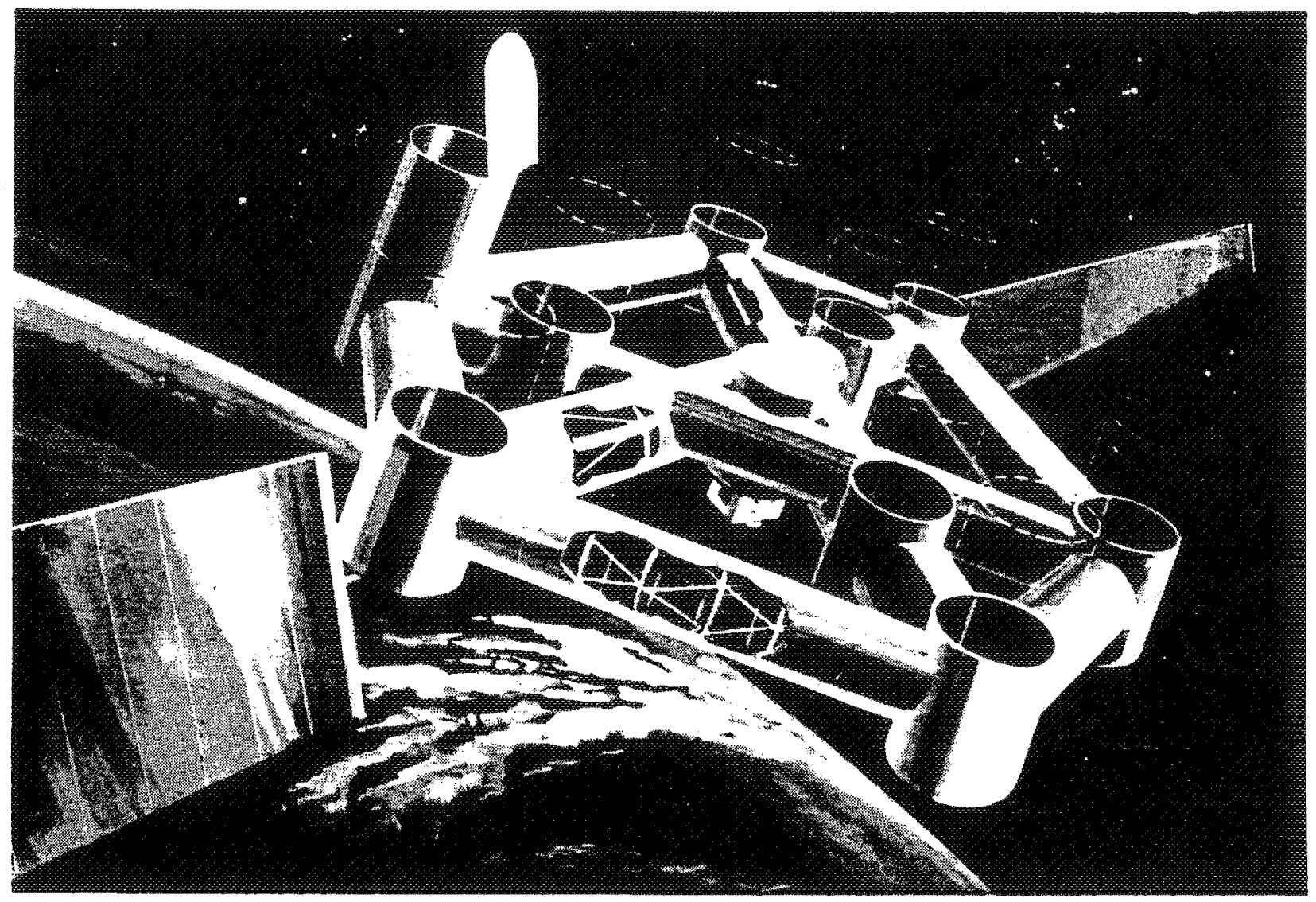

Figure 7. Two-Dimensional Coherent Array Telescope

are also disadvantages associated with these types of systems. They suffer from low UV throughput due to the eight or more reflections needed to bring the collected light to focus. In addition, the useful field of view (FOV) is very narrow, only a few tens of arcseconds (Fig. 8). This may not be too critical, however, since even a 10 -microrad FOV will require a detector array with 1 million resolution elements (for 10-nanorad telescope resolution). Other design benefits must also be considered, such as construction and checkout of the individual telescopes on the ground and transportation to and assembly in orbit. Furthermore, considerably less cross-sectional area is exposed to the micrometeoroid and space debris flux compared to large, contiguous aperture systems. The probability of micrometeoroid impact with the mirror surfaces is therefore reduced, allowing smaller light shields made from rigid material (a micrometeoroid bumper). This enhances the ability to control straylight increases during the telescope lifetime.
In order to investigate the technology requirements for a partially filled primary mirror telescope, the two-dimensional configuration shown in Figure 9 was selected. In this concept, the primary mirror consists of 18 off-axis segments of a single parent primary mirror feeding light to a common secondary mirror and thence to a focal plane behind the primary in a Cassegrain or Ritchey-Chretien arrangement. The nonredundant, two-dimensional partern provides the widest two-dimensional aperture separation that avoids zeros in the optical transfer function. The initial dilute aperture configuration requires some degree of image processing to obtain diffraction limited imagery. This concept, an unfilled aperture with a common secondary mirror, has a large FOV (recognizing that detector arrays may not be available to fill the focal plane). It allows a later increase of the sensitivity by the addition of segments to the primary and has a minimum number of reflections - thus a high UV throughput. The major disadvantage of this approach is 


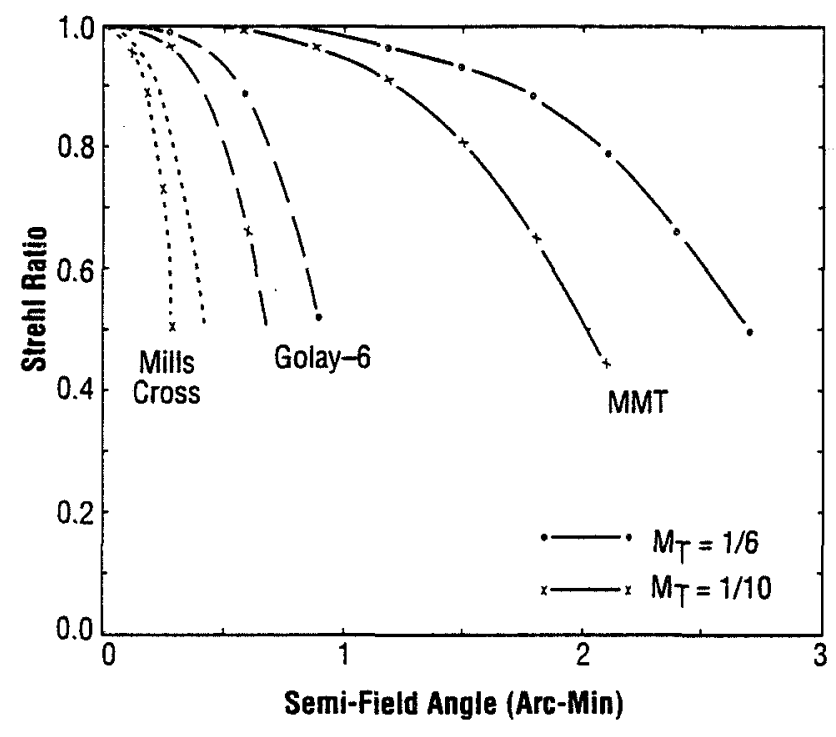

Figure 8. Field of View for Various Aperture Configurations that a very large outer structure is required to place the secondary mirror at the proper distance from the primary. The large dimensions pose problems, not only in control, but also in launch and assembly of the telescope modules. The system will only function as a complete telescope for the first time when the modules are assembled in space. Because of the large dimensions of the lightweight support structure, complete assembly and checkout on Earth is probably impossible or meaningless, at least from the standpoint of verifying optical performance.

Although studies of various telescope systems and the associated problems of orbital assembly of the telescopes are in progress, preliminary technology assessments have been completed and criticality and technology-readiness judgments have been made in order to scope the need and specific objectives of additional studies.
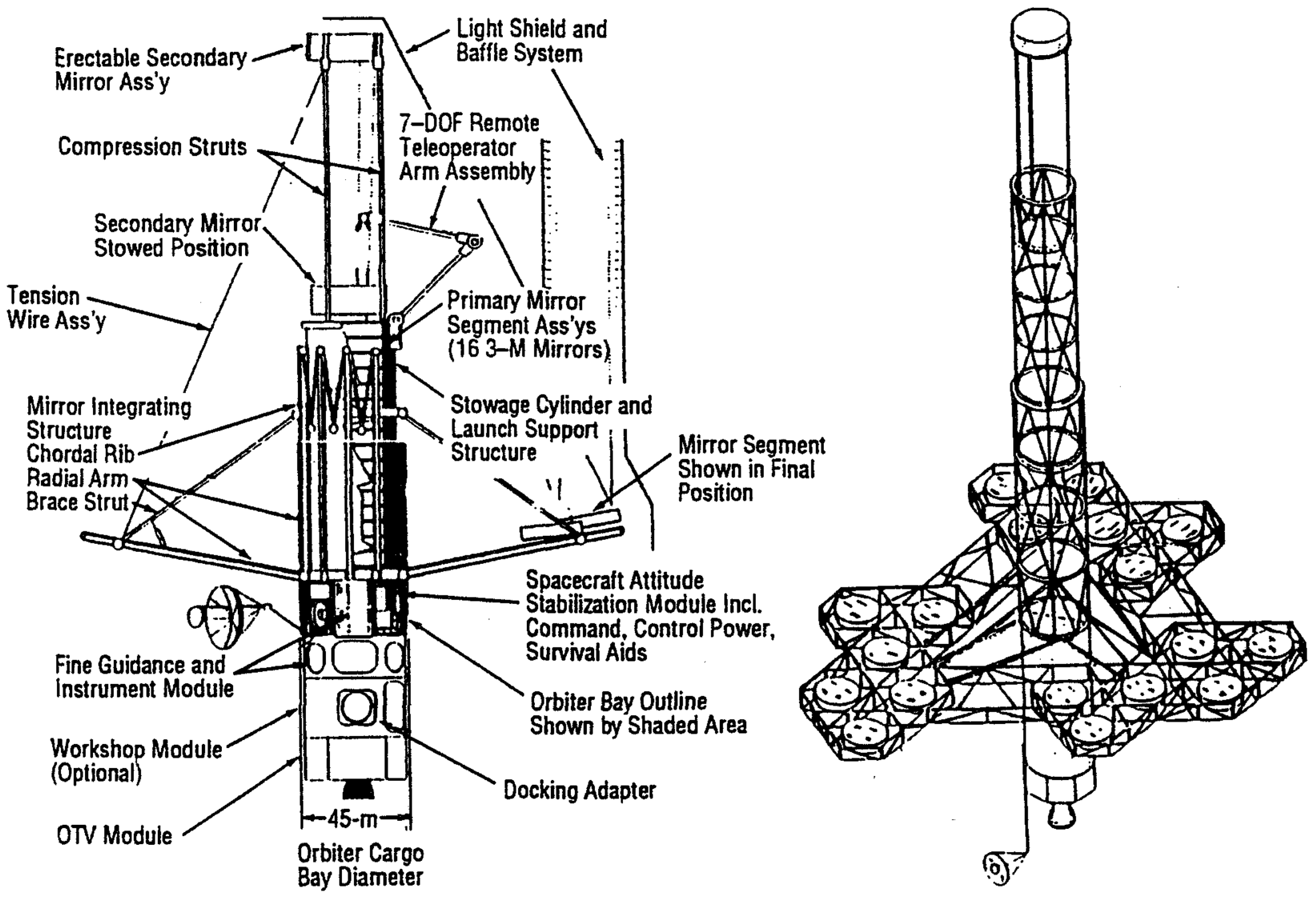

Figure 9. Thinned Primary Mirror with Common Secondary Mirror Telescope 


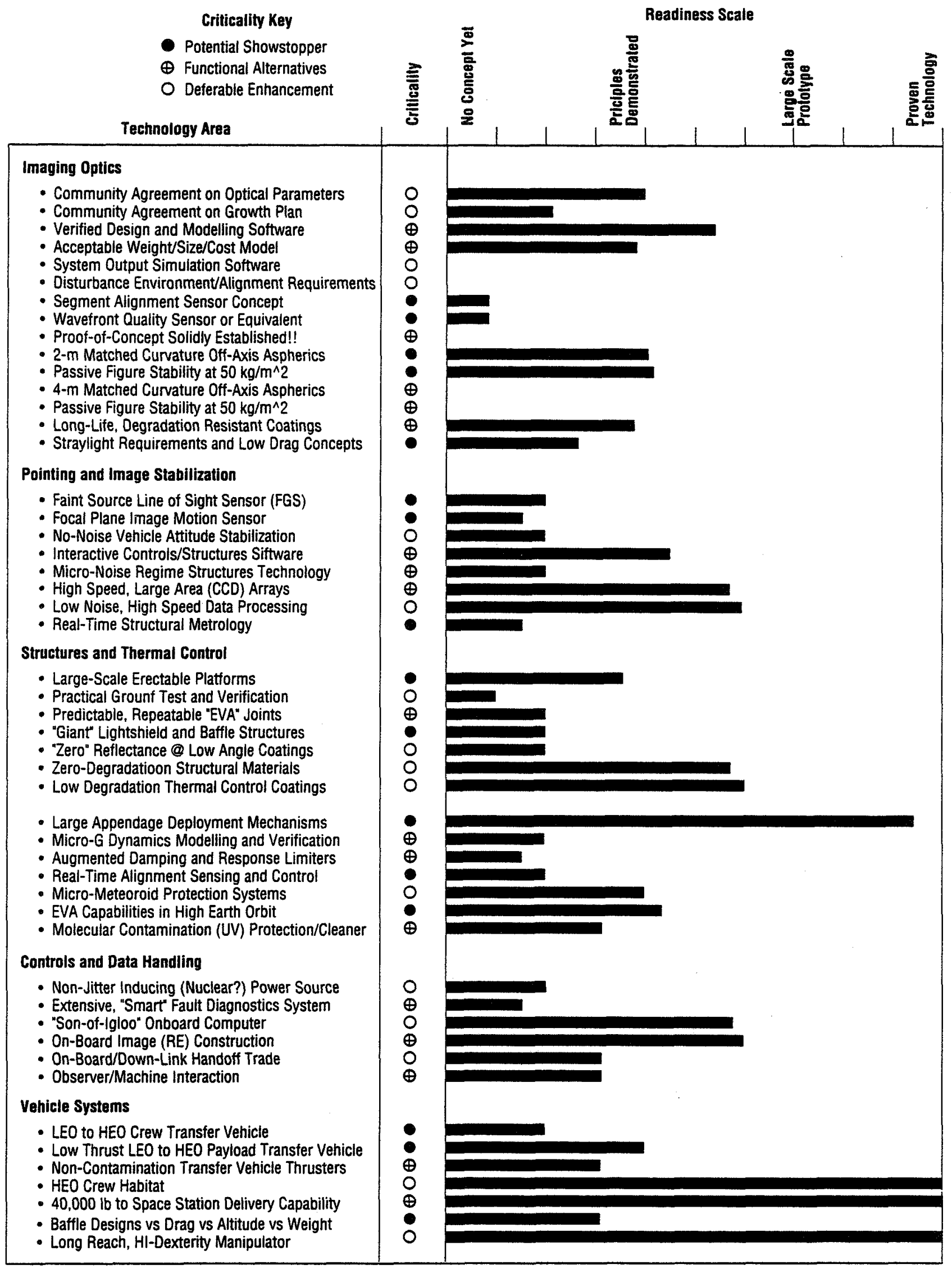

Figure 10. Technology Readiness Assessment for Advanced UV/Optical Telescopes 
These technology assessments are summarized in Figure 10.

Although the successors to NASA's Great Observatories will probably not be operational until early in the 21 st century, history has shown it takes at least 15 to 25 years from concept to flight of a large spaceborne astronomical telescope. Figure 11 indicates that the HST development cycle, spanning a period of 23 years, is not an anomaly. If one includes the precursor technology development projections for the AXAF, even at this early stage in the program, the forecast is a development time of about 25 years.

From the time observational astronomy began almost 400 years ago with the small telescope of
Galileo, to the sophisticated instruments of today, this discipline is driven by technology. The technology available for the next generation of space telescopes cannot be predicted, based solely on this discipline's demands. Rather, it depends to a large degree on the requirements of the other disciplines that use the capabilities of space. Therefore, it must be recognized that a technology development period of at least 5-10 years must be allocated in advance of any serious design efforts. Thus, it is appropriate and necessary that concept studies be conducted now to identify the key enabling technologies, not only for the eventual development of these advanced telescopes, but also for transportation and assembly requirements (Fig. 12). These requirements will have a major influence on telescope

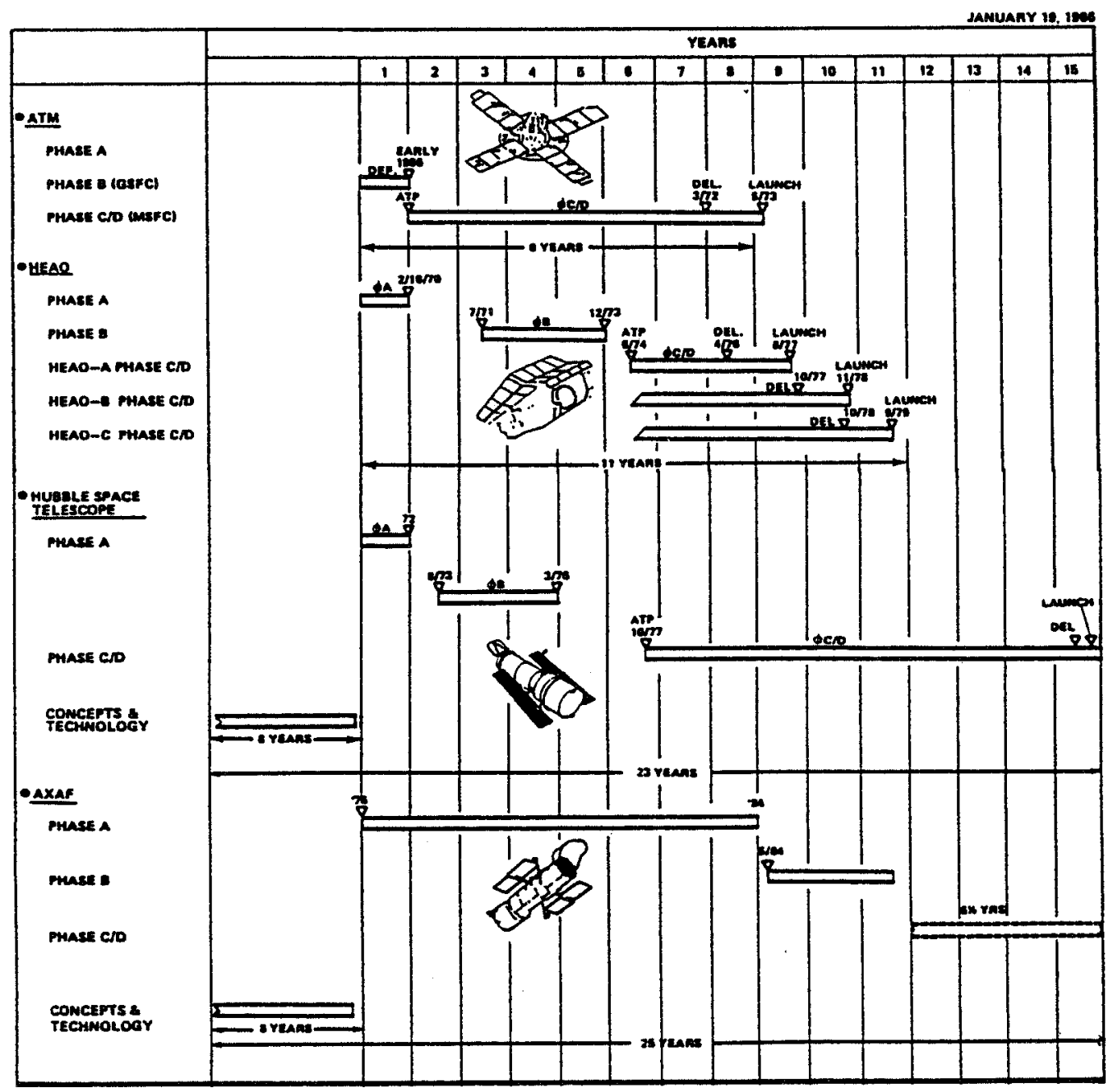

Figure 11. Historical Data on the Development Cycles of Space Telescopes 


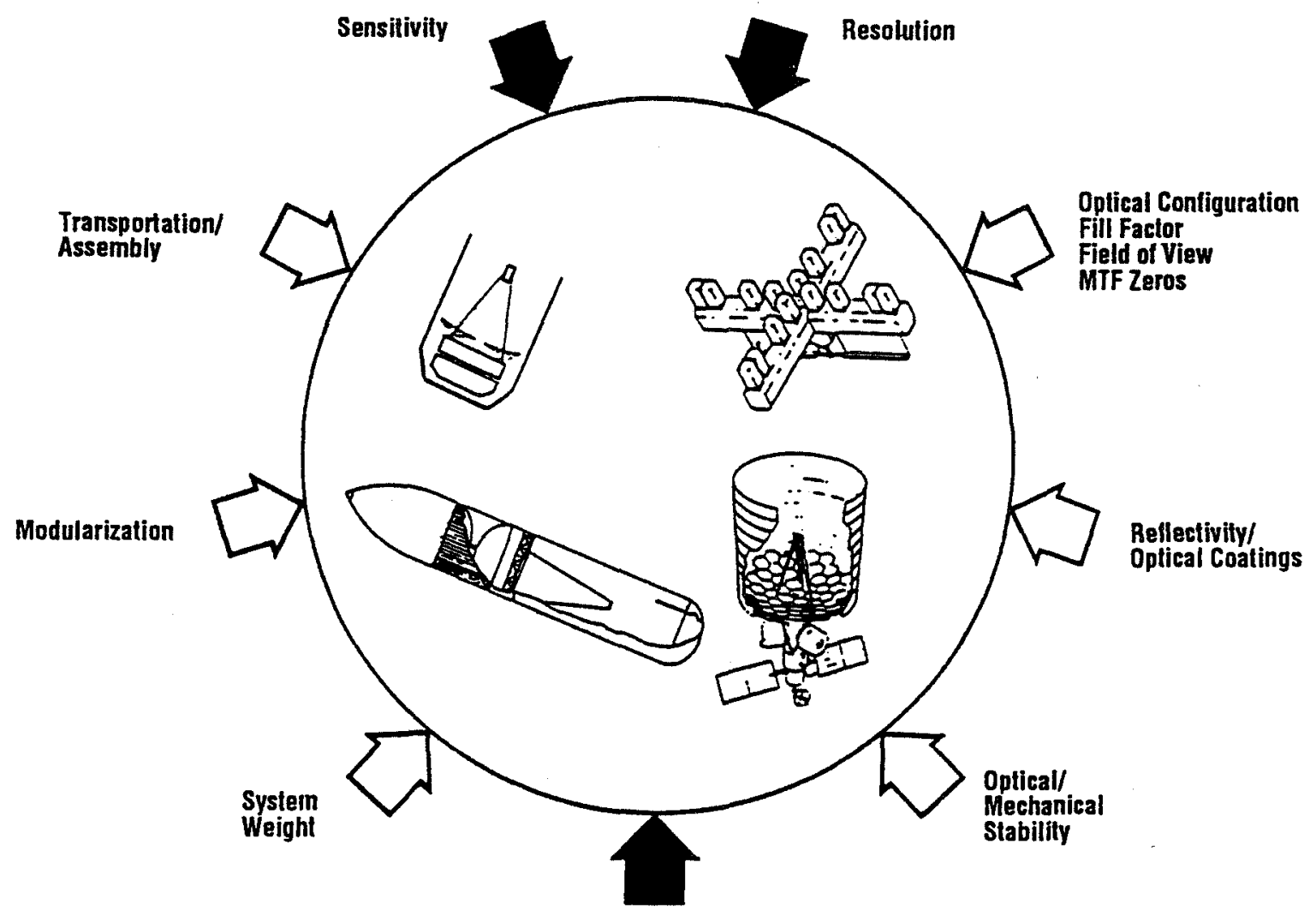

Program Cost

Figure 12. Generic Design and Technology Concerns for Advanced Telescopes

designs. As a matter of fact, the very configuration of the telescopes and apertures arrangement may be determined by the transportation systems available, orbital assembly, tools, facilities, and construction sequences which must be compatible with the man-machine interfaces.

NASA has recently expressed three major goals for the Agency:

- Advance scientific knowledge of the planet Earth, the solar system, and the universe beyond

- Expand human presence beyond the Earth into the solar system

- Strengthen aeronautics research and develop technology toward promoting U.S. leadership in civil and military aviation.

Consistent with the first and second goals and now specifically directed by the President's
"New Initiative for Space Exploration" is a return to the Moon (Fig. 13). This was, in fact, a recommendation of the National Commission on Space.

Since the Moon has no obscuring atmosphere, it would be an ideal platform for astronomical

- Continue observations with increasing sensitivity and angular resolution

- Use the unique science opportunities afforded by observations from lunar facilities for increases in resolution and sensitivity if cost effective

- Plan for evolutionary development of the lunar facilities

Figure 13. Next Century Astronomy Objectives 
observations (Fig. 14). A stable and low gravity environment would provide a platform for observations, such as interferometric measurements - both at radio and visible wavelengths. For example, the pattern of the Very Large Array (VLA) to make long baseline interferometry measurements in radio astronomy could be followed to construct a "Lunar Optical VLA" (Fig. 15) as proposed by Professor Bemard F. Burke of the Massachusetts Institute of Technology. A set of 1-m class telescopes would be deployed along a "Y"-shape, each arm $6 \mathrm{~km}$ long, with a maximum baseline of $10 \mathrm{~km}$. An angular resolution of 10 micro-arc seconds at $5000 \AA$, a four order of magnitude improvement over HST, could be possible with this system. The placement of other large telescopes, such as a 16- to $20-\mathrm{m}$ diameter UV/optical/IR system (Fig. 16) and other interferometric telescopes on the near or far side of the Moon offer interesting trade options; and certainly will affect the design of future space observatories, not only from the viewpoint of transportation requirements and assembly on the Moon by man and robots, but also from the aspects of modularity and commonality with orbiting space telescopes.

Exciting prospects await astrophysics research during the next 25 years. The Great Observatories, observations from S.S. Freedom, and specialized space missions will evolve to the development of space observatories with even greater capabilities. The demands for improved performance require aggressive long-term technology development. Moreover, these advanced observatories will no longer be guided by historical

\section{BENEFITS}

- Stable base for large telescopes (construction and operations)

- Stable baselines for elements of interferometers

- Hard vacuum for diffraction limited imagery

- 14 Earthday night time and slow rotation for long exposures

- Ideal for low temperature instruments

- Natural heat-sink

- Improved assembly and repair environment for manned and robotics activity

- Natural structures for construction of instrument supports or substrates

- Absence of magnetic field

- Absence of light/radio pollution

- Gravitational field
CONSTRAINTS

- Transportation to and landing on Moon

- Crew requirements for assembly

- Cost

- Priorities

- Dust contamination of optical systems, bearings, etc.

- Thermal environment

- Micrometeoroids and cosmic radiation

- Communication and remote control of observatories

- Gravitational field

Figure 14. Benefits and Constraints of Astronomical Observatories on the Moon 
approaches to development but must seek innovative responses to many diversified requirements. An accepted balance must be developed between the science requirements and program cost.

The studies discussed in this paper indicate that the technology requirements associated with the transportation to orbit and the assembly of these telescopes in orbit are major driving forces in the selection of generic design concepts. Ultimately, optical advances which are now becoming available through advanced manufacturing must be matched by technology advances in orbital operations, system modularization, and assembly by man and machine.

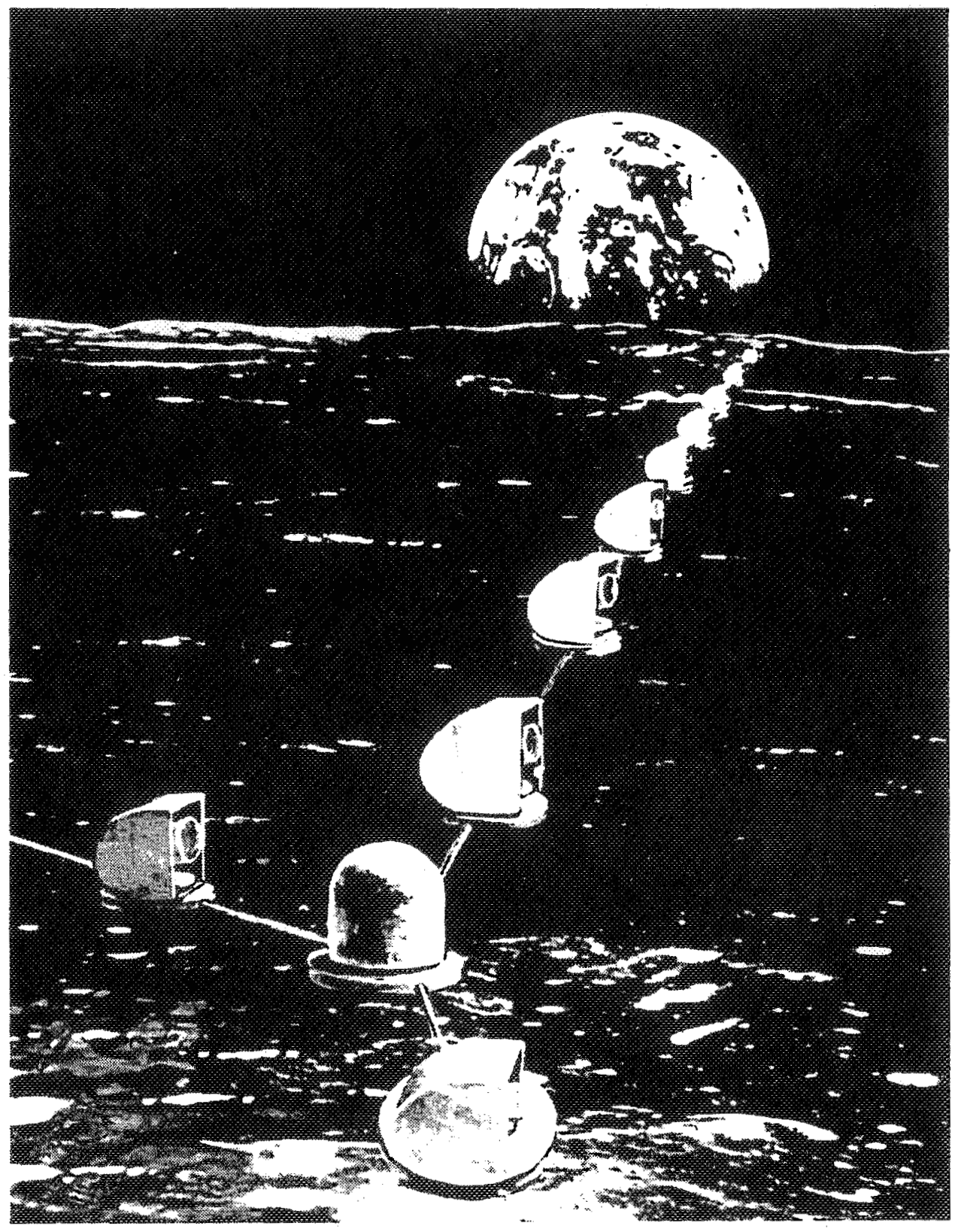

Figure 15. Lunar Optical VLA (LOVLA) 


\section{6 m UV/Visible/lR Modular Ring Telescope}

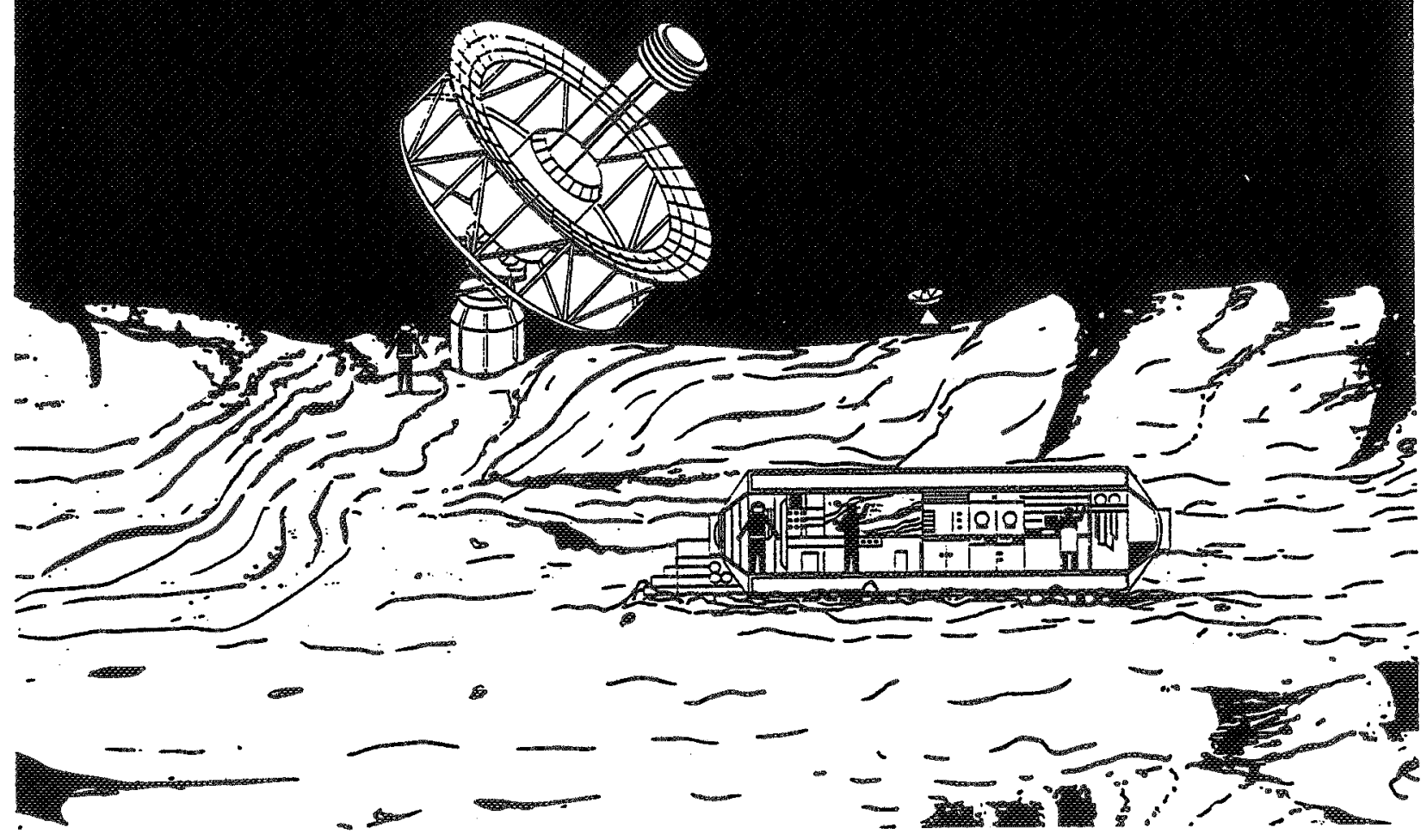

Marshall Space Flight Center August 30,1989

Figure 16. 16-m UV/Visible/IR Modular Ring Telescope 


\section{DISCUSSION}

Bender: How bad is the debris problem at $1500 \mathrm{~km}$ altitude, where you can get a fully illuminated sun synchronous orbit?

Nein: As shown in the graph below, the modelled surface area flux is a line straight above $700 \mathrm{~km}$, although there is a valley at $1300 \mathrm{~km}$ and a drop off past $1500 \mathrm{~km}$. However the second peak occurs at $1500 \mathrm{~km}$. This would not be a good location from a debris point of view and also because of the radiation environment).

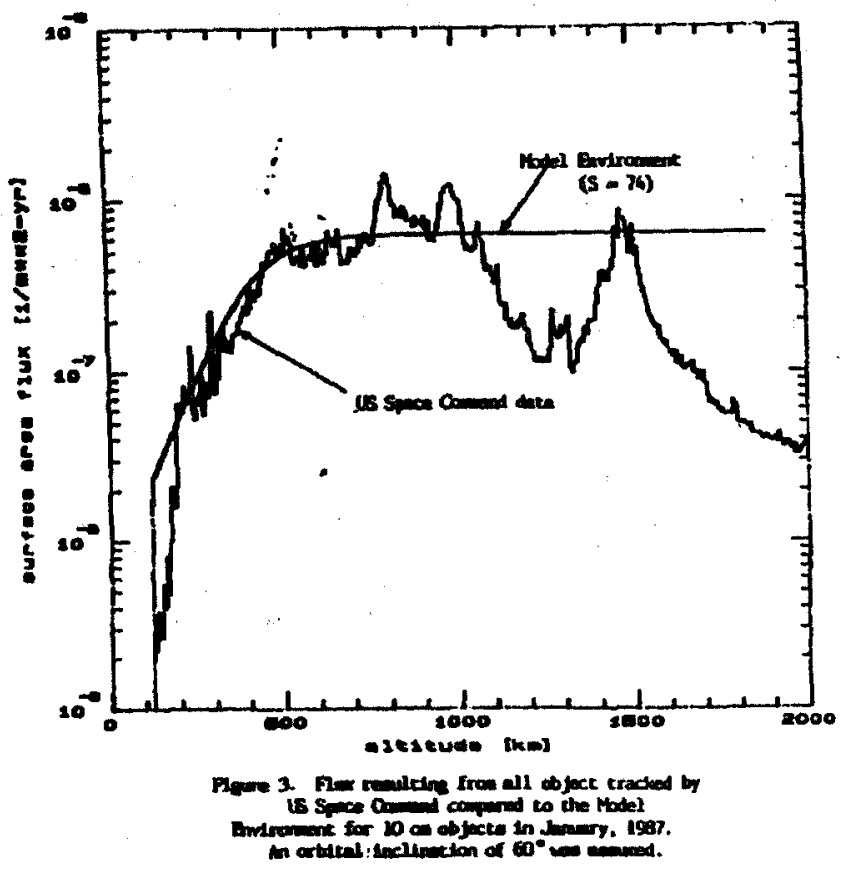

Disenhardt: Large impacts (greater than a few $\mathrm{mm}$ ) are due to micro-meteoroids and will be a problem in any orbit. $1500 \mathrm{~km}$ is a bad altitude.

Illingworth: What is the projected capability of OTV for weight/size from LEO to HEO?

Nein: The payload capability of an OTV can range from 13-30 klb depending on assumptions made for launch vehicle and engine. The volume available for the payload is about $15 \mathrm{ft}$ diameter by $20 \mathrm{ft}$ long.

Davis: What is the assembly time for large observatories?

Nein: Assembly time for the Galaxy 9 was estimated at about 800 EVA hours by MSFC. This is characteristic of all $10 \mathrm{~m}$ or larger observatories if restricted to launch by the shuttle (15' diameter payload bay). The assembly time via EVA is prohibitive and we conclude that for some time robots or automation are required. However, most $10 \mathrm{~m}$ size telescopes could be loaded without on orbit assembly by the Shuttle C Block II $10 \mathrm{~m} \times 30$ payload bay and $150 \mathrm{klb}$ to LEO, or possibly the ALS. 


\title{
IBIS: AN INTERFEROMETER-BASED IMAGING SYSTEM FOR DETECTING EXTRASOLAR PLANETS WITH A NEXT GENERATION SPACE TELESCOPE
}

\author{
David J. Diner \\ Jet Propulsion Laboratory, California Institute of Technology \\ Mail stop 169-237 \\ 4800 Oak Grove Drive \\ Pasadena, CA 91109

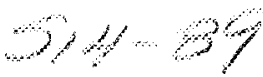 \\ (818) $354-6319$

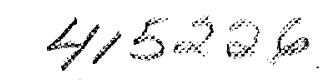

\section{INTRODUCTION}

The direct detection of extrasolar planetary systems is a challenging observational objective. As shown in Fig. 1, the observing system must be able to detect faint planetary signals against the background of diffracted and scattered starlight, zodiacal light, and in the IR, mirror thermal radiation. As part of a JPL study, we concluded that the best long-term approach is a 10$20 \mathrm{~m}$ filled-aperture telescope operating in the thermal IR (10-15 $\mu \mathrm{m}$ ) (Diner et al., 1988a,b). At these wavelengths, the star/planet flux ratio is on the order of $10^{6}-10^{8}$ (see Fig. 2). Our study supports the work of Angel et al. (1986), who proposed a cooled 16-m IR telescope and a special apodization mask to suppress the stellar light within a limited angular region around the star. Our scheme differs in that it is capable of stellar suppression over a much broader field-ofview, enabling more efficient planet searches. To do this, certain key optical signal-processing components are needed, including a coronagraph to apodize the stellar diffraction pattern, an infrared interferometer to provide further starlight suppression, a complementary visible-wavelength interferometer to sense figure errors in the telescope optics, and a deformable mirror to adaptively compensate for these errors. Because of the central role of interferometry we have designated this concept the Interferometer-Based Imaging System (IBIS). IBIS incorporates techniques originally suggested by KenKnight (1977) for extrasolar planet detection at visible wavelengths. The type of telescope discussed at this workshop is well suited to implementation of the IBIS concept.

\section{THE IBIS CONCEPT}

\subsection{Rationale}

IBIS is designed to detect terrestrial, as well as gas-giant type planets. The optimal spectral range for this purpose is $10-15 \mu \mathrm{m}$. Although the star/planet flux ratio improves at longer wavelengths (Fig. 2), for terrestrial-type planets the zodiacal light/planet ratio increases by more than an order of magnitude between 10 and $50 \mu \mathrm{m}$ (Fig. 3). Furthermore, the telescope cooling requirements become considerably more difficult as the wavelength increases (Fig. 4). Finally, the aperture size needed to achieve sufficient angular resolution will exceed $20 \mathrm{~m}$ if wavelengths longer than $15 \mu \mathrm{m}$ are considered.

IBIS uses several means to suppress the light from the parent star. The Lyot coronagraph is an effective means of suppressing diffracted light, with little penalty in the planetary signal. At the wavelengths envisioned for IBIS, the occulting disk will mask only a few Airy nulls, making it necessary to supplement the coronagraph with an additional starlight suppression method. As described below, this can be achieved with a rotational shearing interferometer (RSI). We require the RSI to reject $99 \%$ of the incident starlight. 


\subsection{Optical Schematic}

Figure 5 is a conceptual optical layout of the IBIS system. The primary and secondary image the star and planetary system onto an opening in the center of the quaternary. A tertiary mirror accepts the light diverging from the first image and forms an image of the primary on the active quaternary. The stellar image is then relayed to a point behind the primary. This optical layout is similar to a design for the Large Deployable Reflector.

The first stage of starlight suppression takes place at the second stellar image behind the primary mirror. Here an occulting mask blocks the light from the central star. Beyond this image a Lyot stop blocks diffracted light. The second stage of stellar suppression takes place in an RSI located behind the second focal plane. Because the RSI destructively interferes light reflected from diametrically opposite points on the primary mirror, effective cancellation requires that the wavefront phase be diametrically symmetric. This requires only the phase difference between each pair of points to be controlled to high accuracy, not the absolute wavefront. As a result, the figure tolerances on the primary mirror are relaxed by about an order of magnitude. Sensing of phase errors is envisioned to take place in a separate but complementary RSI operating at visible wavelengths. A dichroic beamsplitter would divide the incoming light into its visible and longer-wave components. Residual starlight in the visible RSI acts as a phase reference, generating a signal that is used to drive the deformable quaternary mirror.

\section{TECHNICAL BACKGROUND}

\subsection{Interferometer Modelling}

A rotational shearing interferometer produces superimposed images, one rotated by an angle $2 \theta$ about the center of the optical axis relative to the other. The quantity $2 \theta$ is the shearing angle, and results from a difference of $\theta$ in the angular orientations of the two fold mirrors in the interferometer arms. The general RSI concept is diagrammed schematically in Fig. 6a. Figure 6b shows the specific case discussed in this paper, in which the two roof mirrors are oriented $90^{\circ}$ with respect to each other, providing $180^{\circ}$ of shear.

For a point source illuminating the RSI, the pattern observed in the final focal plane is an image of the source and a ghost image at a position diametrically opposed to the source. With equal path lengths in each arm and a near-perfect conductor coating the roof mirrors, the interferometer will provide destructive interference at the center of the field. Thus, for a point source on the optical axis (the central star), the two images of the source null one another. On the other hand, for an off-axis source (the planet), the image and ghost image do not overlap and there isno interference in the focal plane.

Rejection of $99 \%$ of the incident starlight corresponds to a ratio of cancelled to uncancelled starlight, $\beta$, of $10^{-2}$ and a fringe visibility (contrast) of 0.99 . To determine the theoretical limits on fringe visibility, we have analyzed phase, amplitude, and polarization effects due to reflections within the interferometer. Following Roddier et al. (1978), it can be shown that the use of goldcoated roof mirrors in the interferometer results in our starlight rejection goal being met in both $\mathrm{P}$ - and S-polarizations.

\subsection{Fourier Optics Modelling}

A Fourier optics modelling program has been developed to simulate the effects of diffraction suppression techniques and wavefront errors on the feasibility of observing extrasolar planets. The stellar point-spread function for a $16-\mathrm{m}$ aperture operated at $12 \mu \mathrm{m}$ with a $25 \%$ bandwidth is shown in Fig. 7. Application of an occulting disk of radius 0.3 arcsec in the first focal plane, a pu- 
pil-plane Lyot stop that reduces the effective aperture diameter to $85 \%$ of the full diameter, and a RSI with a fringe visibility of 0.99 results in the point-spread function for an on-axis star shown in Fig. 8. The fraction of photons per pixel assumes that the detectors are sized so that 4 pixels cover the central Airy disk. Note the significant reduction in energy in the sidelobes of the stellar point-spread function over a several-arcsecond field-of-view. The peaks in sidelobe energy at 0.3 arcsec are due to diffraction at the edges of the occulting disk. This effect can be mitigated by using a partially transmitting, tapered occulting disk (Ftaclas et al., 1988).

The accuracy on the primary mirror required to achieve the performance shown in Fig. 8 is about $\lambda / 800\left(\lambda_{\text {vis }} / 40\right)$ if the deformable mirror is not used to make the wavefront errors diametrically symmetric. When the wavefront errors are made diametrically symmetric, the use of the deformable mirror and RSI simultaneously enables relaxation of the figure errors to about $\lambda / 80$ $\left(\lambda_{\text {vis }} / 4\right)$.

\section{PERFORMANCE CALCULATIONS}

Performance predictions are shown in Table 1 . We compare the performance of a $10-\mathrm{m}$ aperture operating at $10 \mu \mathrm{m}$ (mirror temperature $=100 \mathrm{~K}$ ) with a $16-\mathrm{m}$ system operating at $12 \mu \mathrm{m}$ (mirror temperature $=70 \mathrm{~K}$ ). In both cases, the mirror emissivity is 0.02 , the spectral bandwidth is $25 \%$, and the integration time is 40 hours. Stars are taken from the Woolley Catalog and the search distance is $25 \mathrm{pc}$. The zodiacal background used in the signal-to-noise calculations is an average of ecliptic equator and pole background fluxes. A planet is considered detectable if the ratio of planet flux in a pixel to shot noise from all background sources exceeds 4 and if the planet's angular distance from the star exceeds 2 Airy minima. Planet distances from their parent stars are determined by their equilibrium temperature and the stellar spectral class. Note the substantial improvement in performance resulting from increasing the aperture size, increasing the wavelength, and cooling the optics to a lower temperature.

\begin{tabular}{|lcccccc|}
\hline \multicolumn{7}{c|}{ Table 1. Predicted planet detection performance } \\
Planet type & "Mercury" & "Venus" & "Earth" & "Mars" & "Jupiter" & "Saturn" \\
Radius (km) & 2500 & 6100 & 6400 & 3400 & 70,000 & 60,000 \\
$\mathrm{~T}_{\text {eff }}(\mathrm{K})$ & 450 & 335 & 270 & 220 & 125 & 95 \\
$\begin{array}{l}\text { Number detectable } \\
\text { with 10-m telescope }\end{array}$ & 1 & 3 & 7 & 2 & 5 & 0 \\
$\begin{array}{l}\text { Number detectable } \\
\text { with 16-m telescope }\end{array}$ & 2 & 10 & 12 & 10 & 102 & 6 \\
\hline
\end{tabular}

\section{CONCLUSIONS}

The search for other solar systems is an exciting scientific objective. A filled-aperture 10-16 $\mathrm{m}$ class successor to the Hubble Space Telescope will be a promising platform for conducting such an investigation. The concept presented in this paper will provide sufficient suppression of the light from the central star to enable detection of both terrestrial and gas-giant type planets around many stars. Critical technology areas include IR detector arrays, thermal control, and adaptive optics. Developments in each of these areas will be needed to meet the long-range objectives of this program.

\section{ACKNOWLEDGMENTS}

The author wishes to thank the members of the JPL study group who assisted with this work: Yeou-Yen Cheng, Steven Gaiser, Robert P. Korechoff, Eldred Tubbs, and Valerie Duval. This research was carried out by the Jet Propulsion Laboratory, California Institute of Technolo- 
gy, under contract with NASA.

\section{REFERENCES}

Angel, J.R.P., A.Y.S. Cheng, and N.J. Woolf (1986). "A space telescope for infrared spectroscopy of Earth-like planets." Nature 322, 341.

Diner, D.J., J. van Zyl, D.L. Jones, E. Tubbs, V. Wright, J.F. Appleby, and E. Ribak (1988a). "Direct and interferometric imaging approaches for detecting Earth-like extrasolar planets." Paper AIAA-88-0553, 26th Aerospace Sciences Meeting, Reno, NV.

Diner, D.J., E.F. Tubbs, J.F. Appleby, V.G. Duval, S.L. Gaiser, D.L. Jones, R.P. Korechoff, E. Ribak, and J. van Zyl (1988b). "Comparison of imaging approaches for extrasolar planet detection." Optical Society of America Technical Digest Series 10, 54.

Ftaclas, C., Siebert, E.T., and Terrile, R.J. (1988). "A high efficiency coronagraph for astronomical applications." Optical Society of America Technical Digest Series 10, 62. 422.

KenKnight, C.E. (1977). "Methods of detecting extrasolar planets. I. Imaging." Icarus 30,

Roddier, F., C. Roddier, and J. DeMarcq (1978). "A rotation shearing interferometer with phase-compensated roof prisms." J. Optics (Paris) 9, 145. 


\section{TECHNICAL CHALLENGES FOR IMAGING}

SUN-LIKE STAR (5800 K)

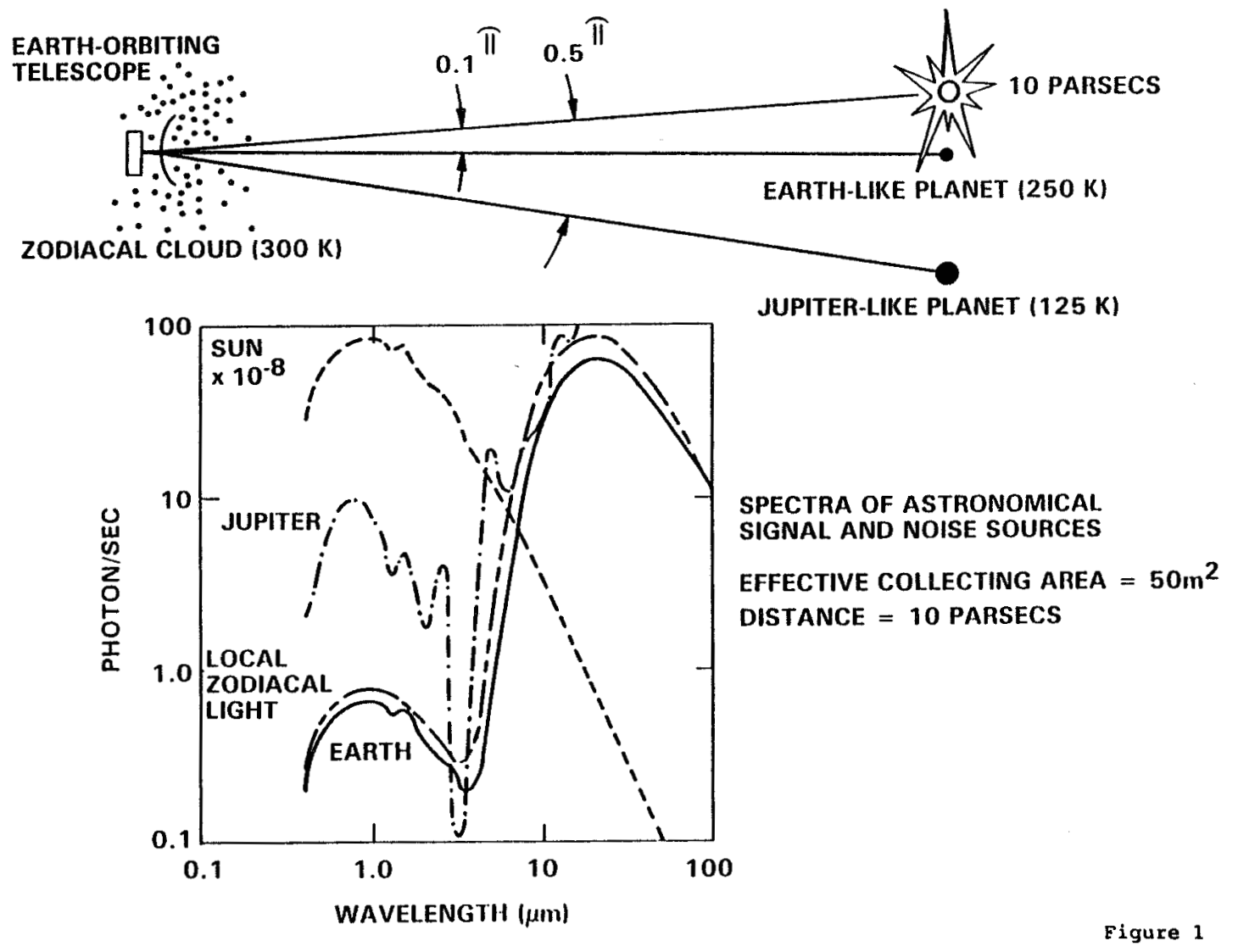

\section{SOLAR-TYPE STAR/PLANET FLUX RATIOS IN INFRARED}

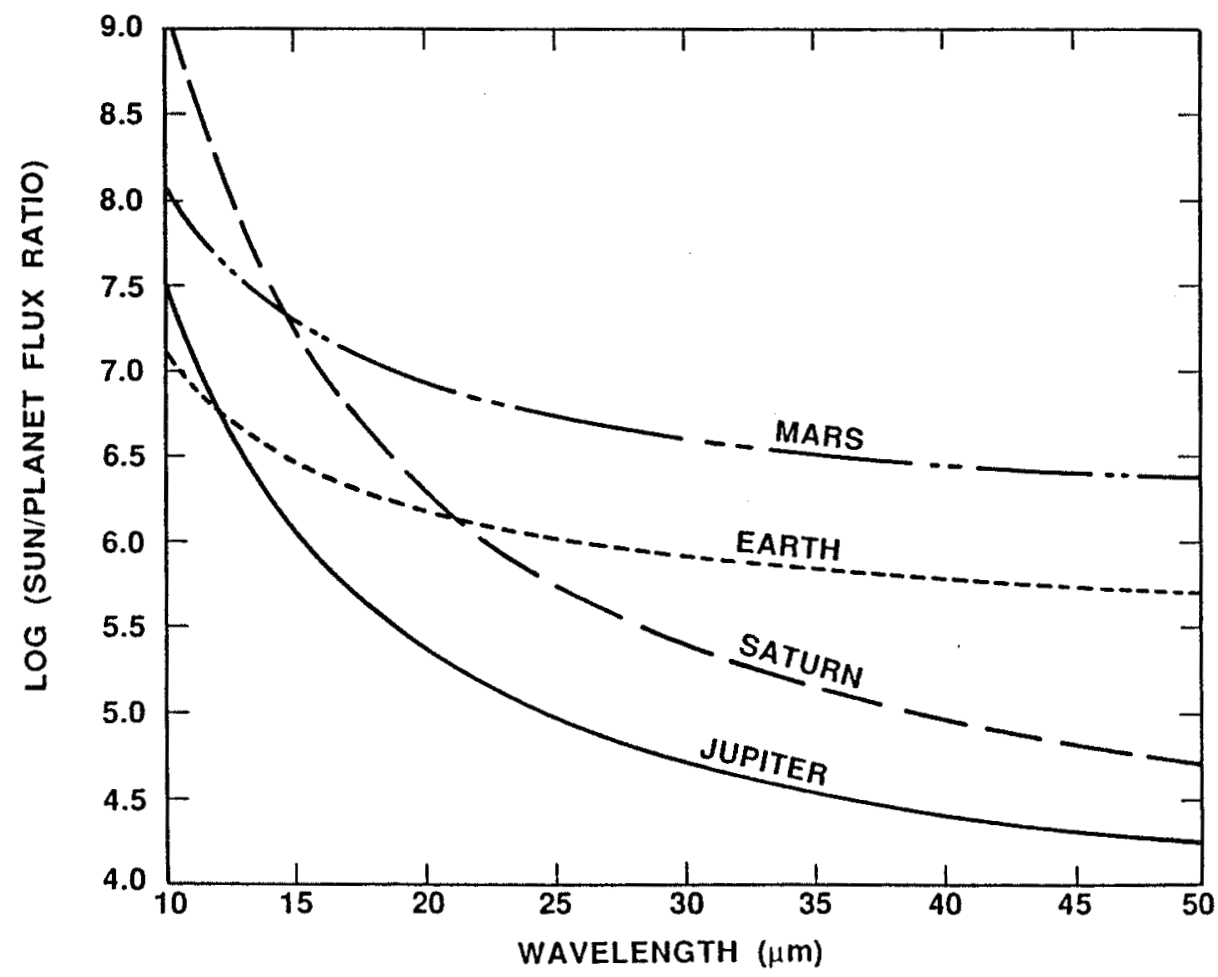




\section{ZODIACAL LIGHT/PLANET FLUX RATIOS IN INFRARED}

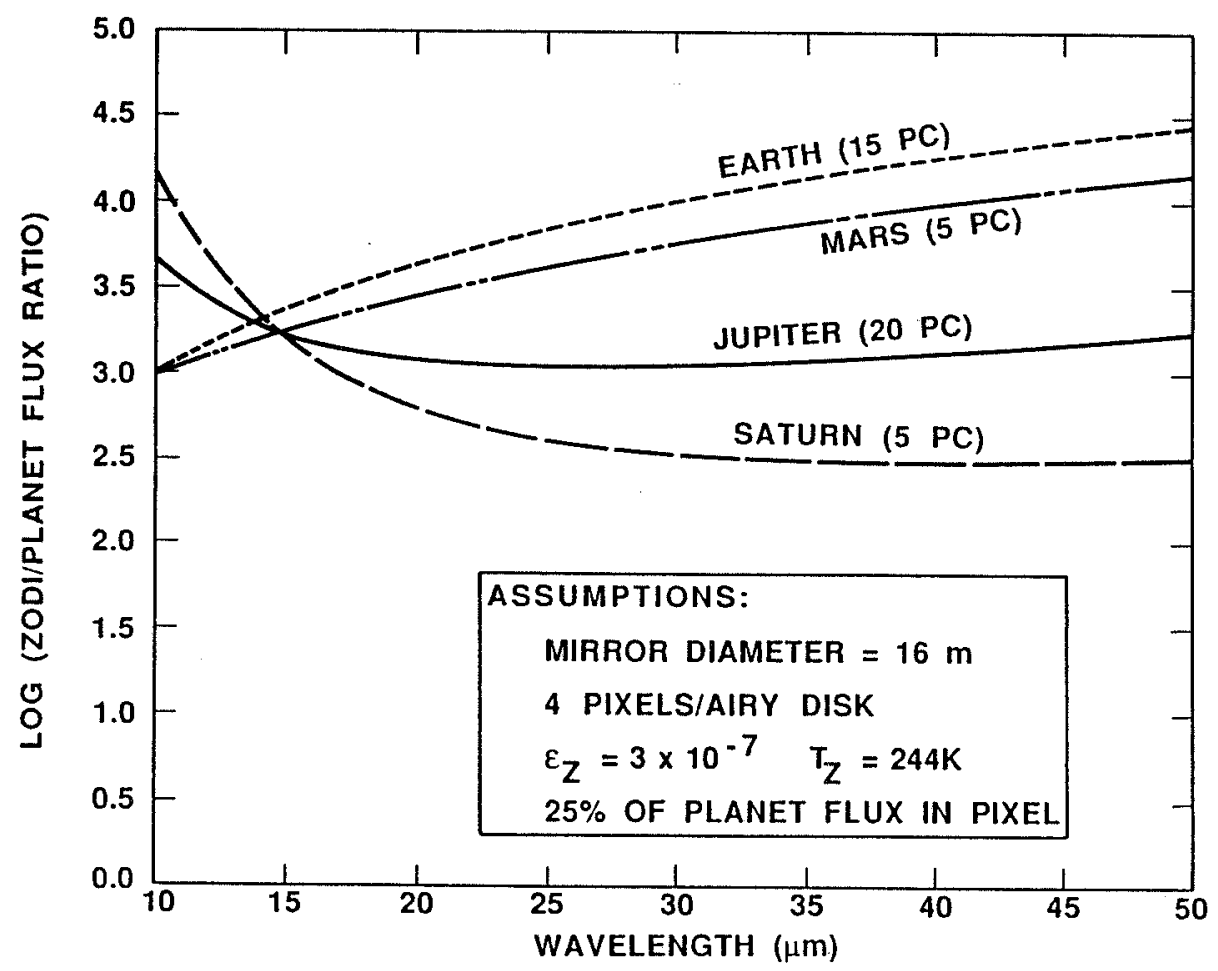

\section{MIRROR TEMPERATURE REQUIREMENTS FOR IR TELESCOPE}

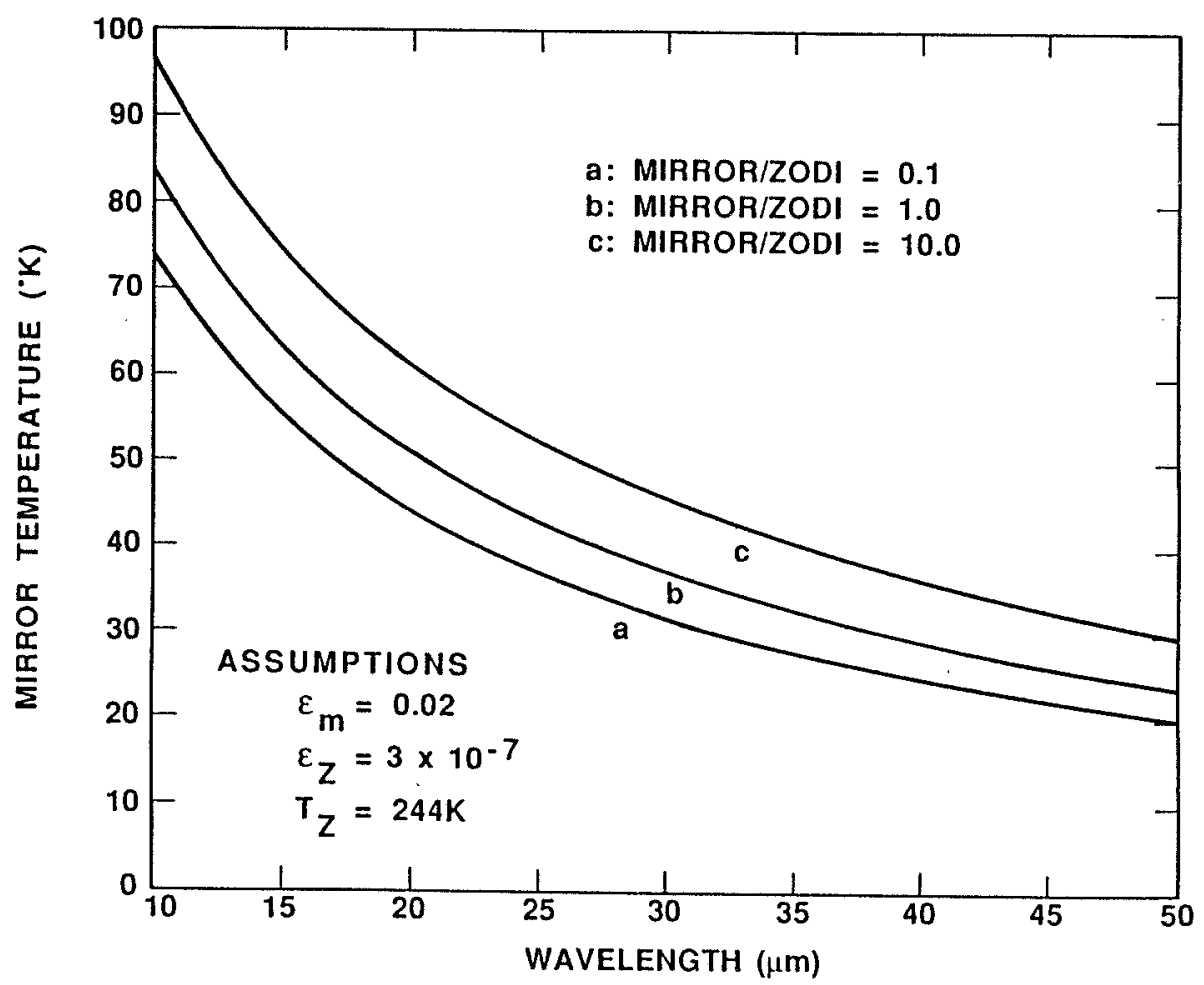

Figure 4 
INTERFEROMETER-BASED

IMAGING SYSTEM (IBIS)

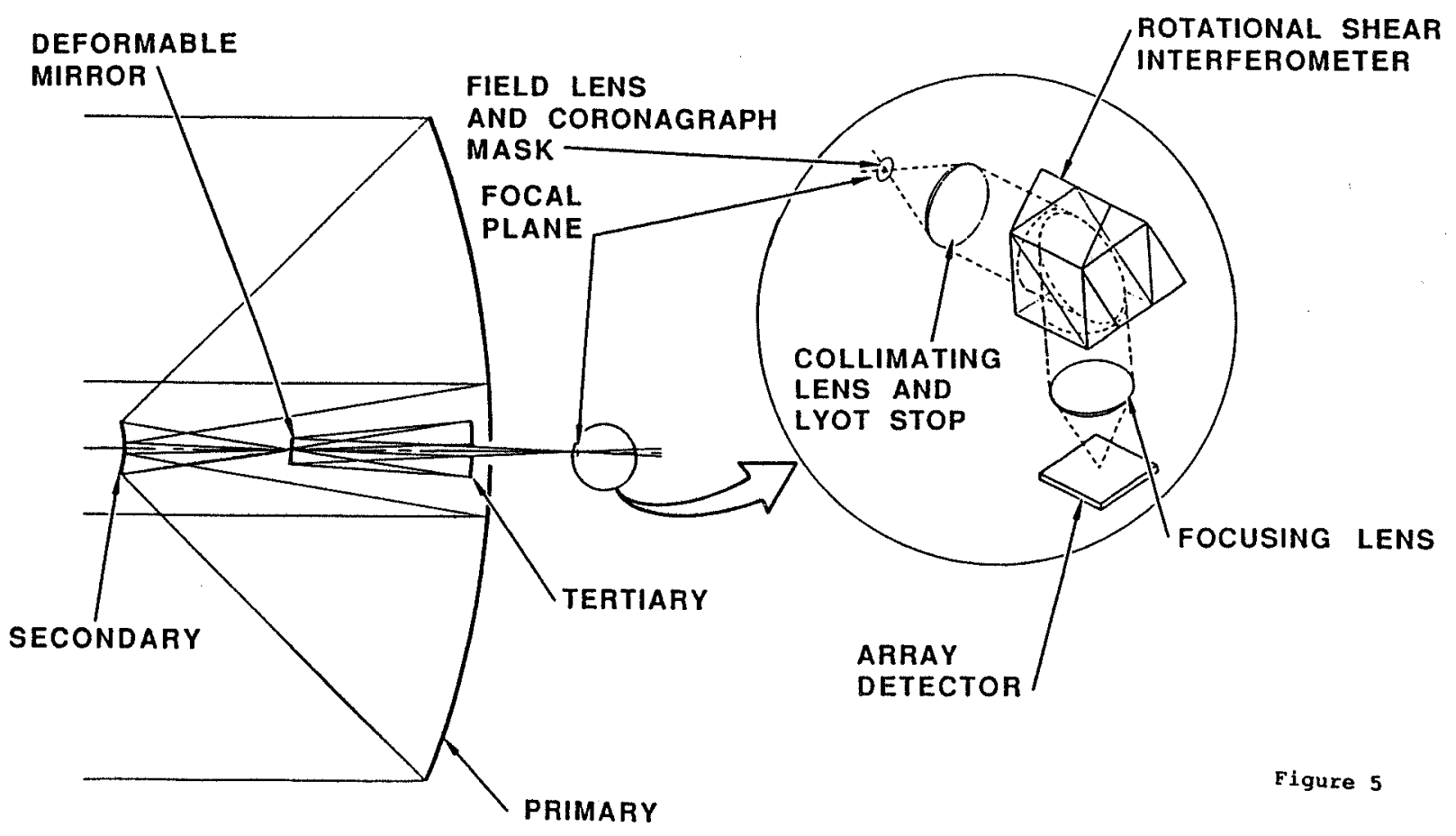

ROTATIONAL SHEAR INTERFEROMETRY

- OBJECTIVE IS TO SPLIT THE STAR WAVEFRONT AND RECOMBINE IT WITH A PHASE SHIFT TO PRODUCE DESTRUCTIVE INTERFERENCE

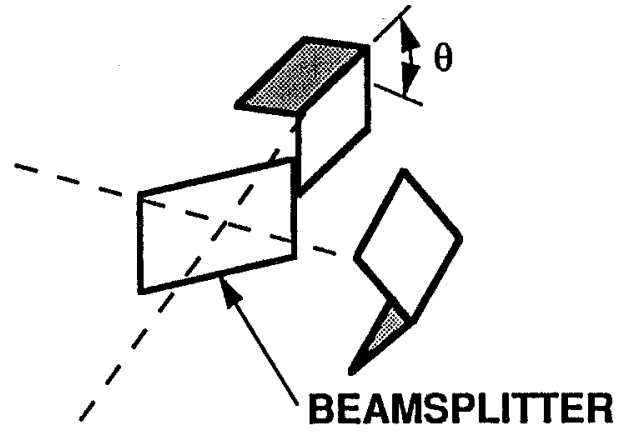

SHEAR ANGLE $=2 \theta$

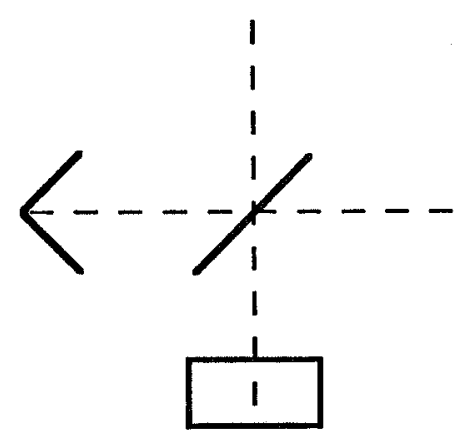

$\theta=\pi / 2$
A. ROOF MIRROR ORIENTATION FOR ARBITRARY SHEAR
B. ROOF MIRROR ORIENTATION FOR $\pi$ SHEAR 
STELLAR POINT-SPREAD FUNCTION

NO SUPPRESSION

I6-m diameter $\quad \lambda=12 \mu \mathrm{m} \quad \Delta \lambda / \lambda=0.25$

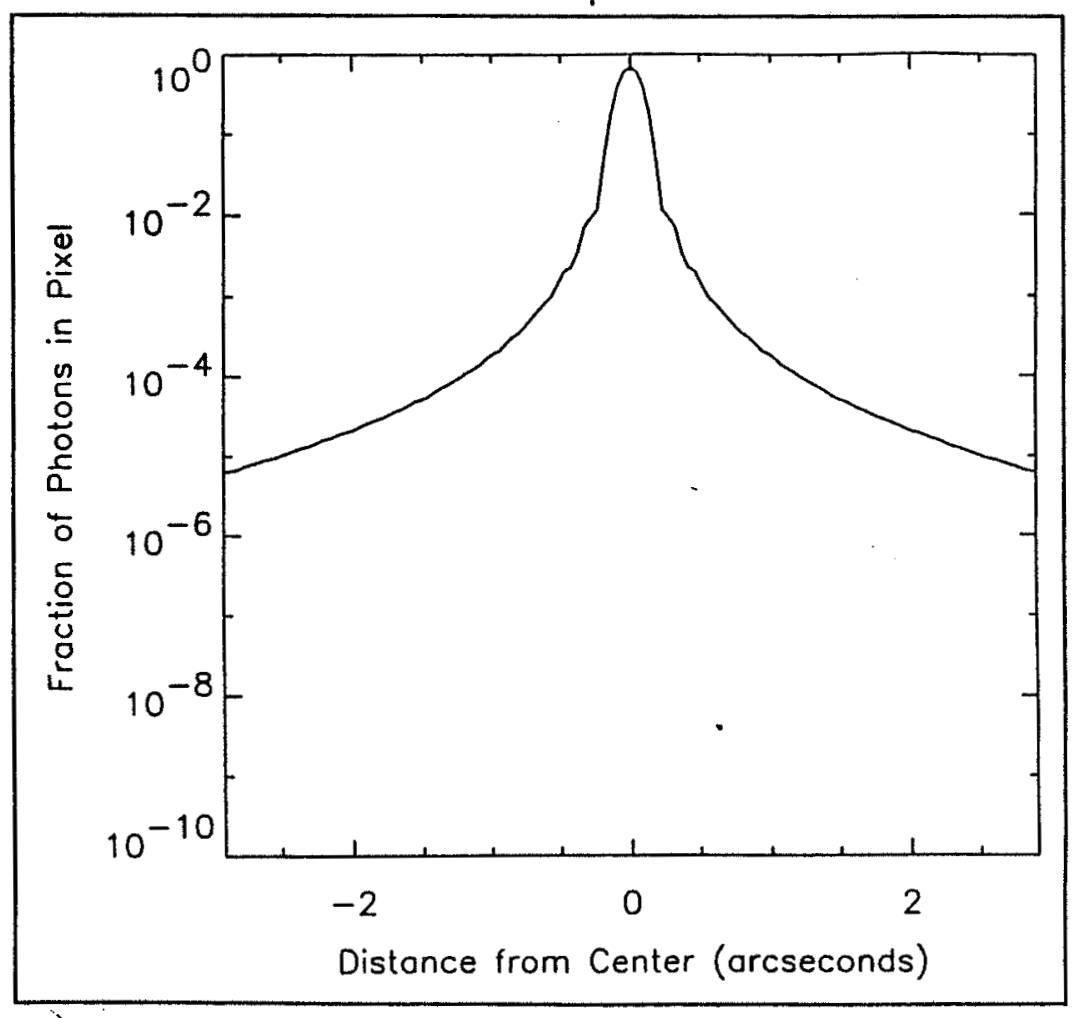

Figure 7

STELLAR POINT-SPREAD FUNCTION

WITH CORONAGRAPH AND INTERFEROMETER

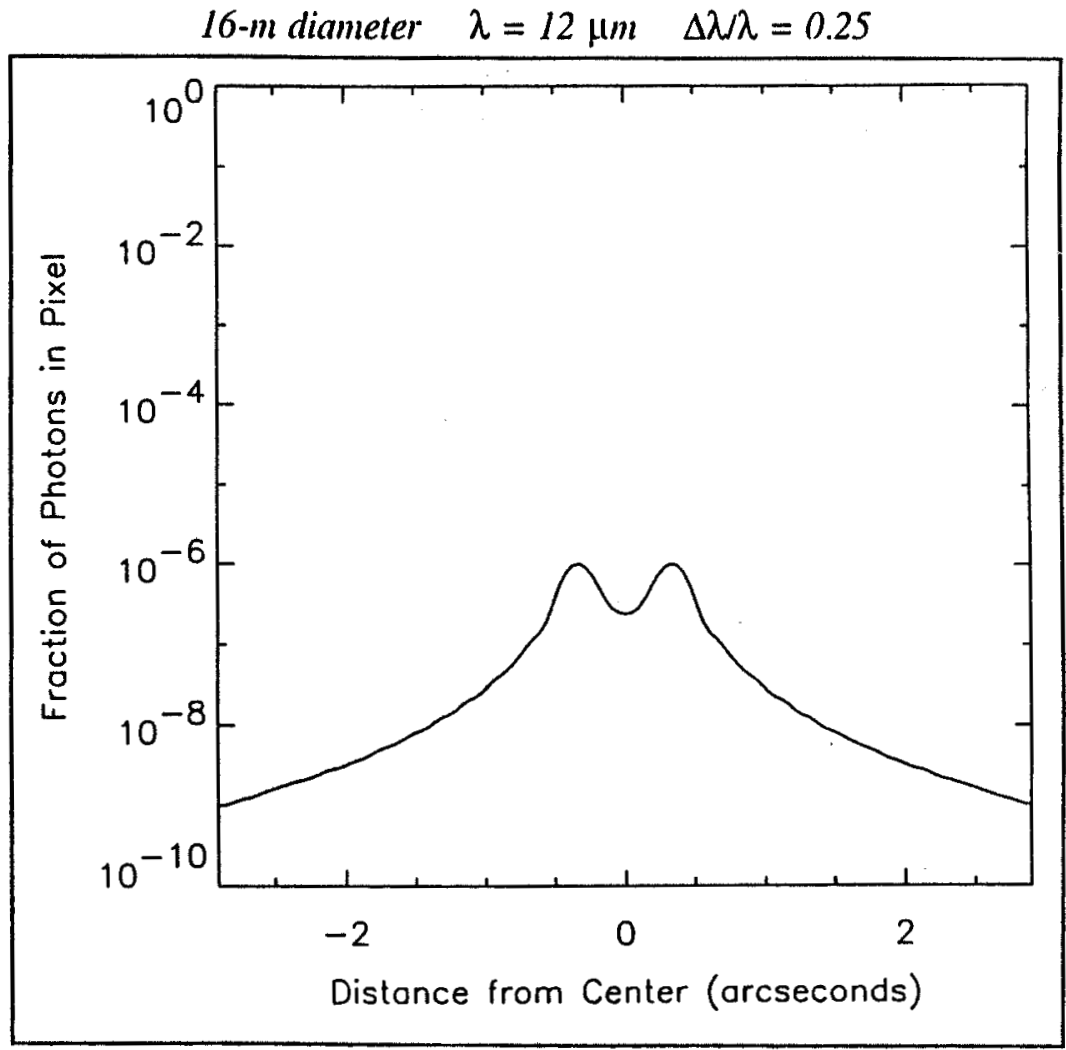

Figure 8 


\section{DISCUSSION}

Layman: Given a bulk mirror temperature of $100^{\circ} \mathrm{K}$, what detector temperature is required?

Diner: Probably about $20^{\circ} \mathrm{K}$.

R. Green: What are the requirements on the mirror scattering profile at the angular scales of interest here?

Diner: The figure requirement of $\lambda / 40$ at visible wavelengths is based on calculations of scattering due to mirror figure ripples on scales of $\sim 20-100 \mathrm{~cm}$. Using the rotational shearing interferometer and deformable mirror relaxes the requirements on the primary to about $\lambda / 4$ in the visible.

Layman: Do micrometeroroid induced surface pits $(1 \mathrm{~mm}$ to $100 \mathrm{~mm}$ pit size) give serious stray light problems for planet detection? (Assume about $10 \mathrm{pits} / \mathrm{ft}^{2}$ )

Diner: These small pits scatter light over a broad field of view. The most detrimental spatial scales for scattering are tens of $\mathrm{cm}$ to a meter, so the small pits, at least qualitatively, are not expected to degrade performance.

Woolf The benefits of the large filled aperture is that by minor modifications in the pupil plane you can configure an apodized aperture, an interferometer, or even something you have not yet dreamed up. The really worrisome problem is whether we will have enough control of phase and amplitude irregularities to benefit from these schemes.

\section{Diner: Right.}

Bely: How do you plan to guide the instrument? Using the parent star?

Diner: The one advantage of having the bright central star is that it provides a guiding and phase reference. There are a number of places a portion of the stellar signal could be picked off, such as the back side of the coronagraph mask. 
The Large Deployable Reflector

Paul N. Swanson

Jet Propulsion Laboratory

\author{
LDR SCIENCE HIGHLIGHTS \\ COSMOLOGY \\ SMALL SCALE BACKGROUND VARIATIONS \\ HIGH RED SHIFTED OBJECTS \\ GALACTIC EVOLUTION \\ CONFUSION LIMITED FOR $\mathrm{Z}>1$ \\ CI 157 MICRONS IS GOOD TRACER \\ INTERSTELLAR MEDIUM \\ FUNDAMENTAL CHEMICAL PROCESSES STUDIED BY SPECTROSCOPY \\ MOLECULAR, IONIC AND ATOMIC SPECTRAL LINES \\ STAR FORMATION / PROTOSTARS \\ MAP TEMPERATURE, DENSITY, VELOCITY FIELDS \\ COOLING AND COLILAPSE MECHANISMS \\ MASS LOSS, EJECTA, SUPER NOVAE (RECYCLED ISM) \\ PROTOPLANETS / EXTRA-SOLAR PLANETS \\ IRAS RESULTS \\ FAR IR MUCH MORE SENSITIVE THAN VISIBLE
}

\title{
LDR HISTORY
}

- 1978-1979 INITIAL STUDIES BY JPL AND AMES

- 1982 RECOMMENDATION BY NATIONAL ACADEMY OF SCIENCE

ASTRONOMY SURVEY COMMITTEE (FIELD REPORT)

- 1983 ASILOMAR I WORKSHOP - SCIENCE OBJECTIVES

- 1985 ASILOMAR II WORKSHOP - SYSTEM CONCEPT/TECHNOLOGY PLAN

- 1986 JPL ASSIGNED LEAD CENTER BY NASA

- 1987 ASILOMAR III WORKSHOP - TECHNOLOGY DEVELOPMENT STATUS

- 1988 RECOMMENDATION BY NATIONAL ACADEMY, SPACE SCIENCE BOARD 


\section{LDR: TWO - STAGE CONFIGURATION}

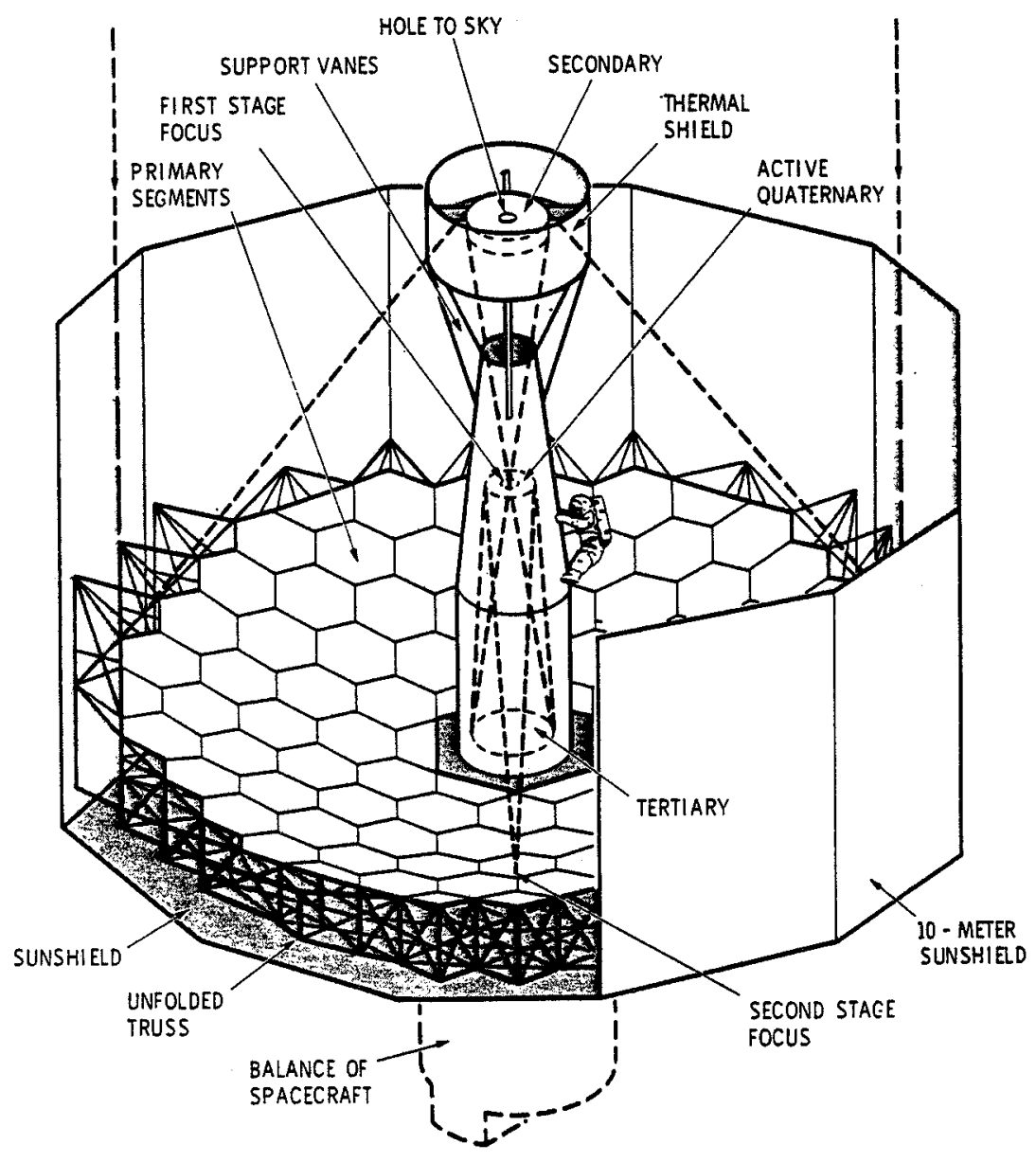

- DEDICATED ASTRONOMICAL OBSERVATORY FOR THE 1990'S

- 20M F/0.5, PASSIVE PRIMARY REFLECTOR, DIFFRACTION LIMITED AT 30-50 MICRONS

- FOUR MIRROR, TWO-STAGE OPTICS

- ACTIVE QUATERNARY MIRROR

- 84 LIGHTWEIGHT PRIMARY REFLECTOR SEGMENTS, 2M SIZE, <10 KG/M AREAL DENSITY

- OVERALL SURFACE ERROR <3 MICRONS RMS

- ACTIVE CONTROL SYSTEMS FOR FIGURE, POINTING, VIBRATICN

- SURFACE FIGURE MEASUREMENT SYSTEM

- SUNSHADE FOR THERMAL CONTROL

- FOCAL PLANE INSTRUMENTS COVERING 30-1000 MICRON SPECTRAL RANGE; CRYOGENIC, COHERENT NON-COHERENT

- SPACE STATION ASSEMBLY 


\section{SYSTEM REQUIREMENTS}

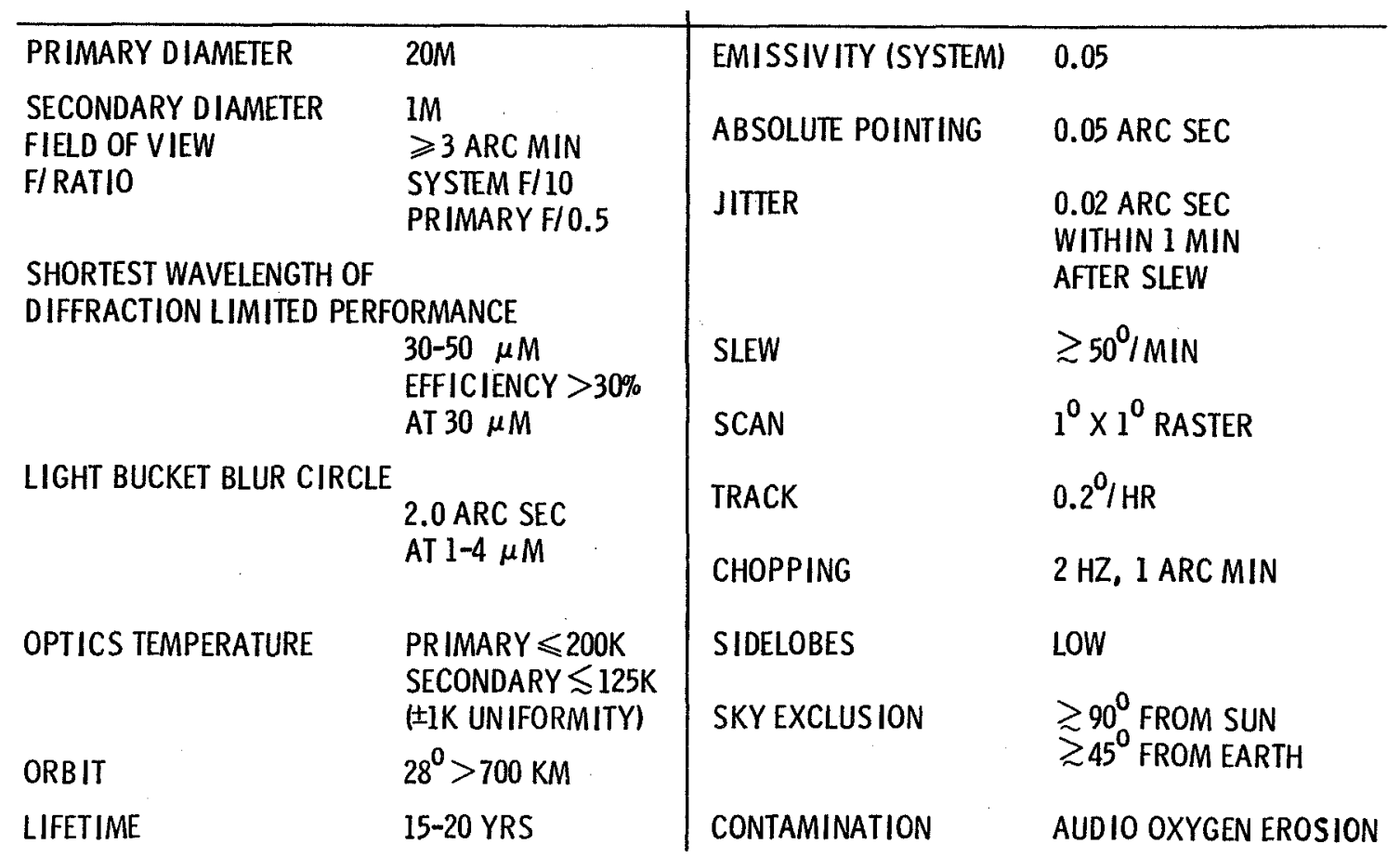

\section{LDR TECHNICAL CHALLENGES}

- LIGHTWEIGHT REFLECTOR PANELS

- SUBMILLIMETER COHERENT DETECTORS

- LONG LIFE SPACE CRYOGENICS

- CONTROL SYSTEMS FOR FIGURE, VIBRATION, POINTING

- LIGHTWEIGHT DEPLOYABLE BACKUP STRUCTURE

- SPACE ASSEMBLY, ALIGNMENT, TESTING 
DIAMETER VS. RMS SURFACE ERROR

FOR SOME WELL KNOWN TELESCOPES
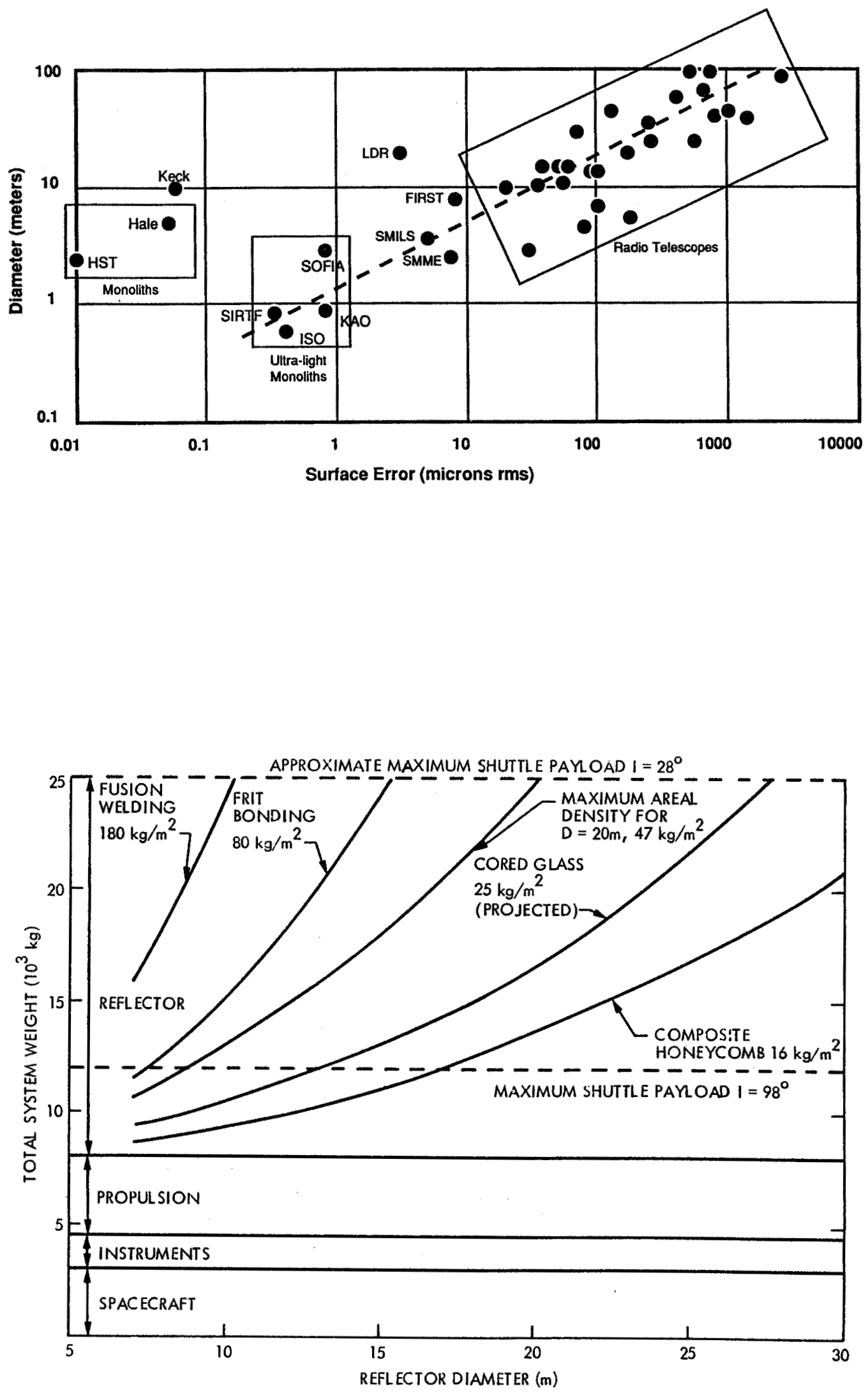


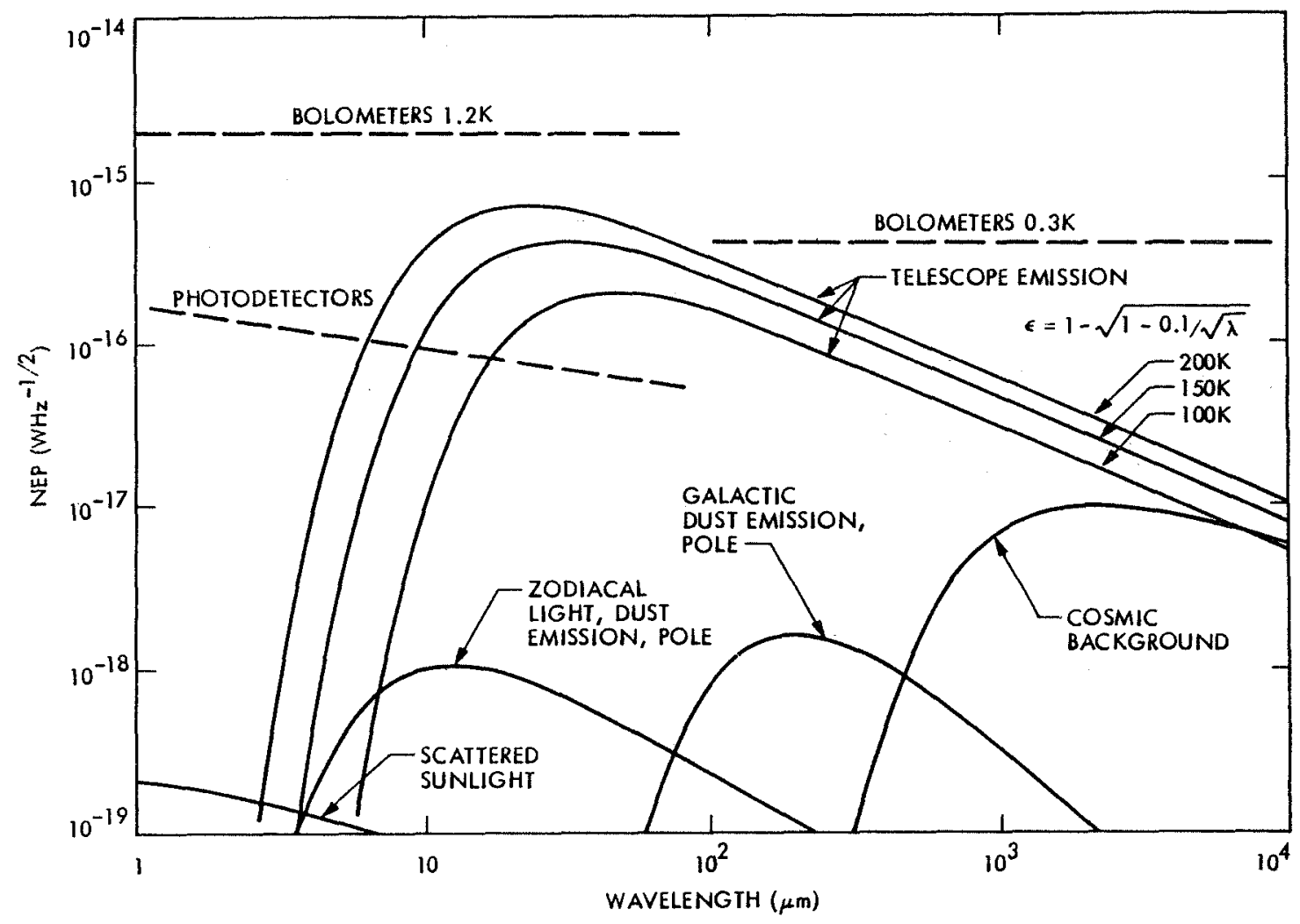

DISCUSSION

Illingworth: Leo Blitz's talk on star formation exemplified the need for wide waveband coverage for tackling fundamental astrophysical problems like star formation. The high resolving power of a 10$16 \mathrm{~m}$ at 1-10 $\mu \mathrm{m}$ nicely complements the ability of LDR to map cooler (high optical depth) structures in star forming regions.

Swanson: I agree. Furthermore, we shouldn't forget the other observational phase space directions, i.e., the synergistic high IR sensitivity of SIRTF and the 2nd spectroscopic capability of LDR.

Bely: You mentioned that you rejected a diluted aperture for LDR. Could you explain why?

Swanson: An interferometer requires a stable platform to precisely locate the elements. On earth (or the moon) this platform comes free. In space, one of the most difficult and expensive components is the large, ultra-stable platform required to hold the elements. Additionally, data reduction and mapping interpretation is more difficult and time consuming with an interferometer than with a filled aperture. 
* Reproduced from SPIE paper delivered in Orlando, Florida on April 2, 1989.

PAMEIA: High density segmentation for large, ultra-light, high performance mirrors.

J. D. G. Rather, B. L. Ulich, Kaman Aerospace Corporation G.Ames, A. Lazzarini, Kaman Instrumentation corporation E. K. Conklin, FORTH, Inc. and D. Korsch, Korsch Optics, Inc.

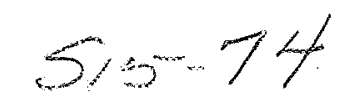

ABSTRACT $360026^{452 \% 2} p .13$

This paper provides the first public disclosure of an optical technology which makes possible important new opportunities for the construction of very large telescopes. This technology, developed by Kaman corporation in an Independent Research and Development program extending over the past several years, is generically known as PAMELA ${ }_{m}$, an acronym for Phased Array Mirror, Extendable Large Aperture. Kaman's patented PAMELA ${ }_{\mathbb{M}}$ approach leads directly to the ability to build rugged, diffraction-limited optical telescopes or beam expanders for ground-based or orbital deployment that have unprecedentedly low weight. In addition, the entire optical system will be fault-tolerant, leading to large expected savings in overall system cost and complexity. These attributes make PAMELA ${ }_{M}$ a prime candidate technology to benefit both civilian and military optical systems in such areas as laser applications, imaging, remote sensing, and communications. PAMELA $A_{m}$ will also have broad applicability to the needs of astronomy.

\subsection{INTRODUCTION}

The search for new, less expensive, and better ways to build and deploy large optical telescopes both on the ground and in space has received much attention over the past decade. Although numerous innovations have been attempted, optical systems built by existing methods are still very expensive, fragile, and demanding of time and talent. Breakthroughs in optical design, fabrication, and control are essential if a wider range of applications are to be addressed by large, lower-cost optical systems. Here we report such a breakthrough that should render large scale adaptive optics applications feasible.

The development of the technologies supporting Kaman's PAMELA ${ }_{\mathbb{M}}$ concept has been guided by carefully conceived studies of end to end strawman systems. The estimated primary mirror weights derived from these studies, if realized, would represent significant advancement of the state-of-the-art. Figure 1-1 provides a graphical comparison of the primary mirror weight (including all supporting structure in the mirror cell) for PAMELA $A_{n}$ mirror technology compared with existing achievements using more conventional optical technologies. The 
specific mass $\left(\mathrm{kg} / \mathrm{m}^{2}\right)$ of a PAMELA ${ }_{m}$ mirror will be at least four times lower than that of any previous mirror technology; and, more importantly, this specific mass will be preserved as the mirror aperture is scaled upward to 20 meters or more.

\subsection{DISCUSSION}

The idea of making large telescope primary mirrors from many small mirrors can be traced back well into the 19th century. Indeed numerous attempts have been made to build such telescopes; but, with only a few exceptions, they have functioned only as "light buckets" rather than true imaging devices because the individual small mirrors could not be kept phased to the requisite precision. If the irregularities in the optical surface exceed a few percent of the wavelength of light, diffraction limited performance cannot be achieved.

Kaman's PAMELA $A_{m}$ approach depends upon innovative improvements in the states-of-the-art of adaptive optics sensing, actuation, and control techniques. Basically, the PAMELA ${ }_{m}$ approach depends upon mass-produced small segments which can move at high frequencies with great precision to correct for optical errors both intrinsic and extrinsic to the telescope. This permits the use of very low-weight, easily deployable mounting surfaces for the segments. Though the mechanical structure and optical train may have many dynamical

\section{MIRROR AREAL MASS DENSITIES AS A FUNCTION OF SEGMENT SIZE}

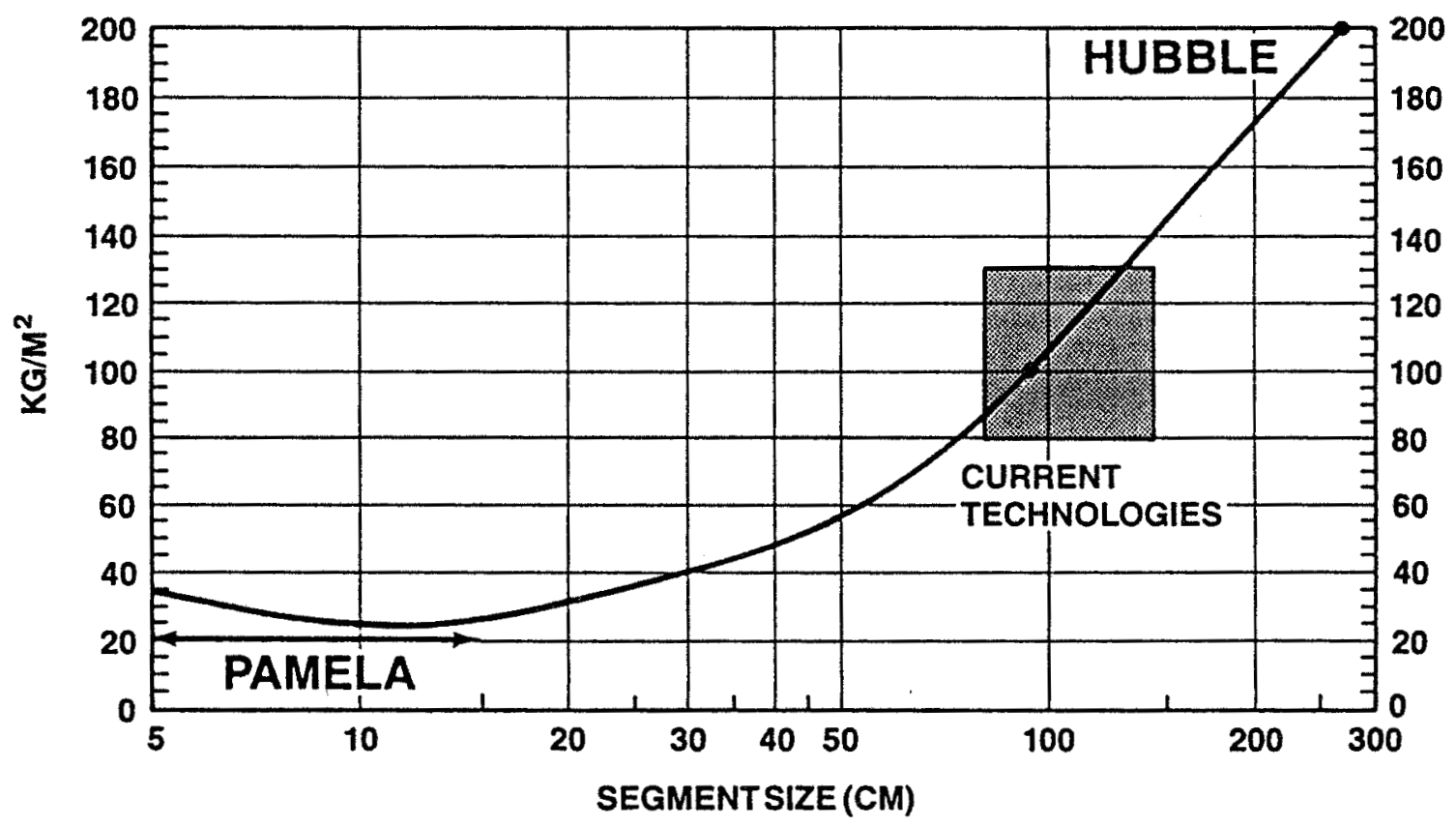

Figure 1-1. 
imperfections, the agile $\mathrm{PAMEIA}_{\mathrm{m}}$ segmented surface functions as a massive, parallel-processing automaton to generate the conjugate surface which cancels all of the errors, thus restoring nearly diffraction-limited performance.

All previously known adaptive optics wavefront correction schemes utilize methods for generating a so-called conjugate wavefront by reversing the spatial phase of a propagating wave at (or through) some sub-element of the optical system (such as a tertiary deformable mirror). Hence the optical ray bundles must be reduced to relatively small size in comparison with the primary optical element before the required conjugate wave corrections can be applied. These existing methods have several disadvantages compared with PAMEIA $\mathbb{m}_{\mathrm{M}}$ including limited spatial frequencies, limited phase adjustment ranges of only a few wavelengths, and limited ability to cope with the very large intensity associated with high power laser beams, to mention a few. The PAMELA $A_{m}$ approach circumvents all of these limitations.

While the PAMELA $A_{m}$ approach is described here as applied to the primary mirror of an optical system, the method is completely general and can be applied to any subsidiary element of the beam train as well. In most instances, however, great benefits of cost and system simplicity will result from putting the PAMELA $A_{m}$ segments on the surface of the large primary reflector.

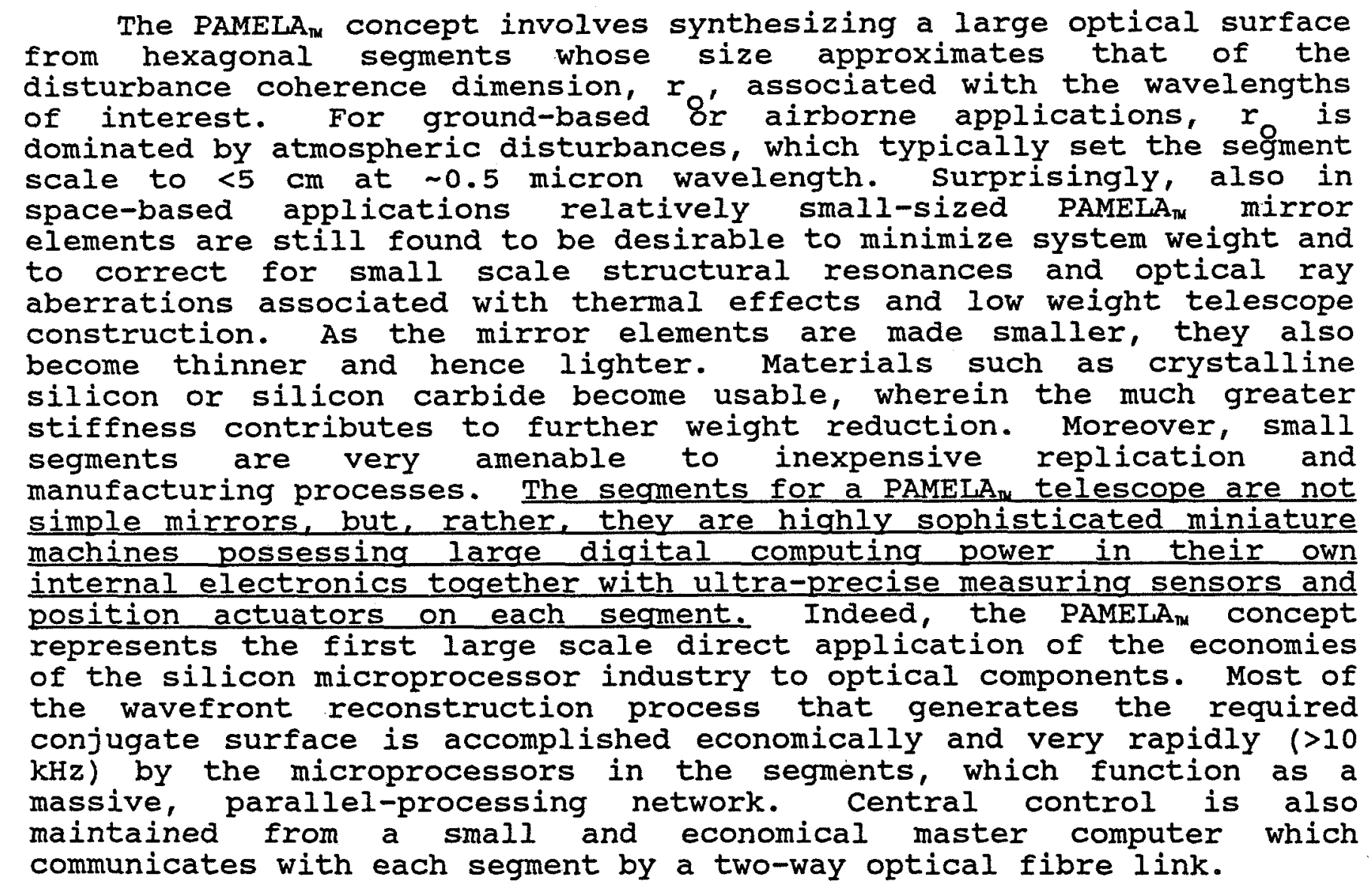


Figure 2-1 illustrates the PAMELA $A_{M}$ mirror concept, showing the individual segments grouped into clusters which in turn make up the mirror surface. Each of the individual segments has three electromagnetic piston actuators and three active inductive edge sensors (the other three alternate edges have passive elements for edge sensors associated with adjacent active segment edges). The piston actuators move in response to control signals from both a global wavefront error sensing device and the local edge sensors. The end result of precisely controlled segment motion is an optical surface that behaves like a phase conjugating continuous facesheet mirror, but with much larger dynamic range and much simpler and faster control algorithms and technology.

The long stroke afforded by the PAMELA $A_{m}$ actuators provides the uniquely important capability for modulo-zero corrections over $>200$ wavelengths (i.e. without $2 n \pi$ phase ambiguities). This property provides the stroke dynamic range to compensate easily for the largest wavefront aberrations that can be imposed by mechanical or thermal disturbances to the ray path. Hence, very lightweight support structures can be used to support the segments, which are, themselves, very light weight and thus highly mobile with only small power input to the actuators.

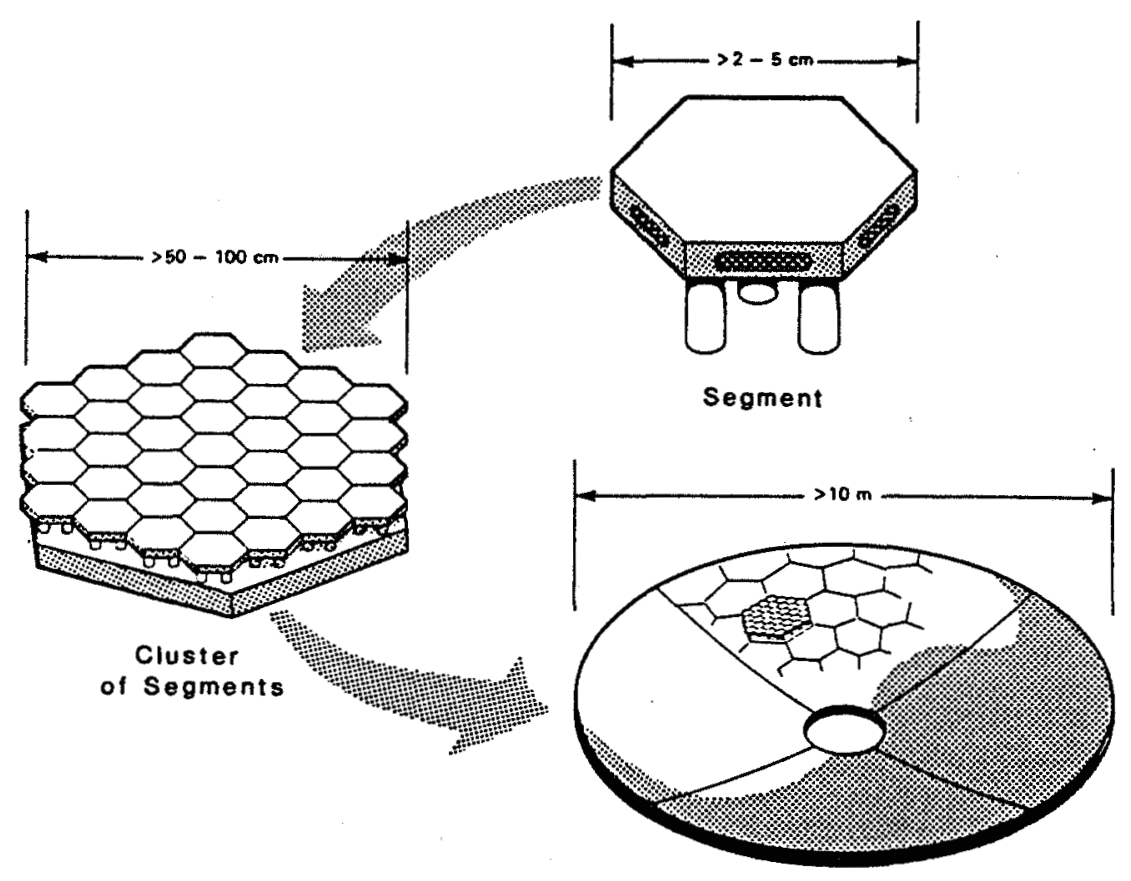

Fulf Aperture

Figure 2-1. PAMELA ${ }_{M}$ active segments will be mass produced in clusters which can be combined to form arbitrarily large apertures. Micro-electronics on each segment drives actuators to achieve a quasi-continuous surface which behaves as a phase conjugating membrane mirror having high frequency response and long-throw adaptive capability. 
This leads to large potential savings in weight and cost. Moreover, since the primary segments are demagnified back through the optical train, they can correct for small scale, large amplitude disturbances occurring anywhere in the telescope system, such as thermal distortions on secondary mirrors and vibration induced disturbances in the ray path.

The optical quality of images provided by highly segmented mirrors has been carefully compared to the quality of images from filled apertures. The amount of energy in the central lobe of the diffraction pattern is comparable to that for a filled aperture, and the image quality will be comparable. six-sided diffraction spikes will show only for very bright point sources, just as four sided spikes occur in conventional systems from the secondary mirror supports. Scattering of light from segment edges will lower image contrast very slightly, as predicted by the Ruze theory familiar to radio astronomers. As long as phase continuity is maintained, the images from a PAMELA ${ }_{n}$ array will be excellent. similarly, in the case of laser beam expanders, the strehl ratio will approach $87 \%$ at $0.5 \mu \mathrm{m}$ wavelength for the expected surface roughness of $0.025 \mu \mathrm{m}$, and the amount of power diffracted or lost between the inter-segment gaps will be less than 0.6 percent.

A set of baseline requirements were adopted during Kaman's PAMELA IR\&D effort. These requirements are listed in Table 2-1.

\section{TABLE 2-1 BASELINE CONCEPT FOR PAMELA}

* Large Aperture (scalable to area $>100 \mathrm{~m}^{2}$ )
* Many segments (scalable to $\mathrm{n}>20,000)$
* Active Wavefront Control on the Primary Mirror
* $\quad$ Large Dynamic Range ( $>100 \mu \mathrm{m}$ with 20 nm accuracy per
element)
High Temporal and Spatial Bandwidth (atmospheric and optics
train correction capability)
* $\quad$ Migh Power Throughput
* Nass-Produced Active Mirror segments (low cost)
* Modularity of Adaptive Surface and Control system

Since Kaman Corporation has developed and produced for many years a broad range of precision displacement measuring instrumentation, it was possible to develop hardware based on existing technology that has the potential for meeting the stringent requirements of PAMELA telescopes. Initial design and construction of these hardware components has been carried out, and the experimental data obtained from tests show the required performance characteristics.

Some of the key findings that evolved out of the IR\&D effort at Kaman are summarized below:

1) The segmented primary mirror using small, lightweight, essentially identical segments has very significant advantages in terms of ease of component fabrication, ruggedness of the system, 
overall system weight, and cost.

2) The formidable computing problem associated with reconstructing a visible wavefront over a large mirror surface from tilt measurements can be greatly reduced by use of the technologically attractive edge matching techniques to preserve phase and local parallel processing to simplify the control problem.

3) Edge matching of segments can be done iteratively with good convergence accuracy.

4) New edge matching sensors devised by Kaman are feasible on the edges of segments as small as $2 \mathrm{~cm}$.

5) New electromagnetic piston actuators, conceived and designed by Kaman, are needed since existing actuators (PZTs, PMNs, etc.) have inadequate stroke to achieve the large displacements required to correct errors in low-weight telescope structures. Moreover, the cost of Kaman's actuators is expected to be much less than that of conventional actuators.

The baseline concept adopted for PAMELA ${ }_{m}$ places additional constraints on the measurement and control process. Co-existence of a sensitive metrology system with an imaging or laser system leads to the need for multi-wavelength or broad-band operation, which implies a continuous or quasi-continuous surface; $2 n \pi$ ambiguities in the relative phase of individual elements are not acceptable. Near diffraction limited operation at visible wavelengths requires a closed-loop segment correction bandwidth of several $\mathrm{kHz}$ for typical composite system fluctuations. A general requirement for robustness and reliability in the system leads to a strong preference for distributed processing and as much local autonomy in the individual segments as possible.

In the extensive research performed by Kaman to date, numerous approaches for wavefront control were studied in detail. Three of them would work; each has advantages and disadvantages. However, when a full systems analysis approach was applied to the entire telescope system, one wavefront control approach was found to be the most practical, scalable, and inexpensive solution.

In this method, a global wavefront tilt sensor designed by Kaman provides two-axis tilt information to each segment via an optical fibre data link. The edge sensors on the segments are then used to derive additional piston information to drive the segments to a continuous surface condition. This method uses well-established technology which is very sensitive and inherently satisfies the requirement for non-dispersive performance at multiple wavelengths.

since segment tilt information is basically the local derivative of the phase (which is the required quantity), complex signal processing is required. A typical straightforward mathematical approach to the problem (frequently utilized in present adaptive optics systems) derives phase directly through the inversion of an 
extremely large matrix, on the order of the square of the number of segments, and requires a different solution for each permutation of missing or non-functioning tilt sensors. While this is feasible for small adaptive optics systems, it is very difficult for a system with potentialiy several tens of thousands of elements. However there exist a number of iterative methods which use tilt information directly to set the surface, and which appear practical for systems the size of PAMELA. These methods require a computation rate substantially higher than the phase disturbance bandwidth, but recent advances in microprocessor electronics make such computations not only practical but cost-effective. Thus, a systems analysis approach indicates Kaman's new measurement and control concept to be the preferred one. Its complexity lies not in the wavefront sensor, a difficult area, but in the computation algorithm, which is now well understood.

\subsection{VALIDATION}

The results of Kaman's studies provide the basis for our confidence that an edge-matched large array is feasible. Some interesting experiments can be reported.

1) Edge Sensors. An essential element of the proposed segmented mirror concept is the existence of a sensor which is capable of aligning the edges of adjacent segments in order to form what approximates a continuous "face-sheet" surface. This must be done to an accuracy of a small fraction of the wavelength of light. All existing sensing technologies were evaluated, with particular attention given to inductive, capacitive, and optical devices. The inductive sensor, which Kaman has investigated in depth, emerged as the most promising technique due to its inherently stable zero reference point, high resolution, wide dynamic range, low sensitivity to gap changes and environmental changes, and low complexity signal conditioning.

2) Circuit development, testing, and integration. First generation signal conditioning circuits have already been integrated on silicon using semi-custom cMos technology.

3) Computer modeling. Kaman has developed a computer model which facilitates simulation of the performance of large arrays of segments subjected to diverse perturbations.

4) Actuator design. To achieve the required Iong-throw displacement of the segments, a new type of actuator has been designed.

5) Segment Fabrication. Several preliminary segment fabrication techniques have been explored. The most promising thus far have utilized crystalline silicon and silicon-carbide for the substrate. Two types of silicon carbide fabrication methods are currently being evaluated under the ongoing IR\&D effort. Figure 3-1 shows one example of a test segment which is dynamically flat to $\lambda / 20$. 
6) Validator. A test device has been built which has successfully demonstrated edge match of a fully mobile center segment surrounded by displaceable segments. The edge sensors perform as expected, giving repeatable. White light fringes on a Michaelson interferometer. Figures $3-2$ and $3-3$ show the validator device and a typical fringe pattern.

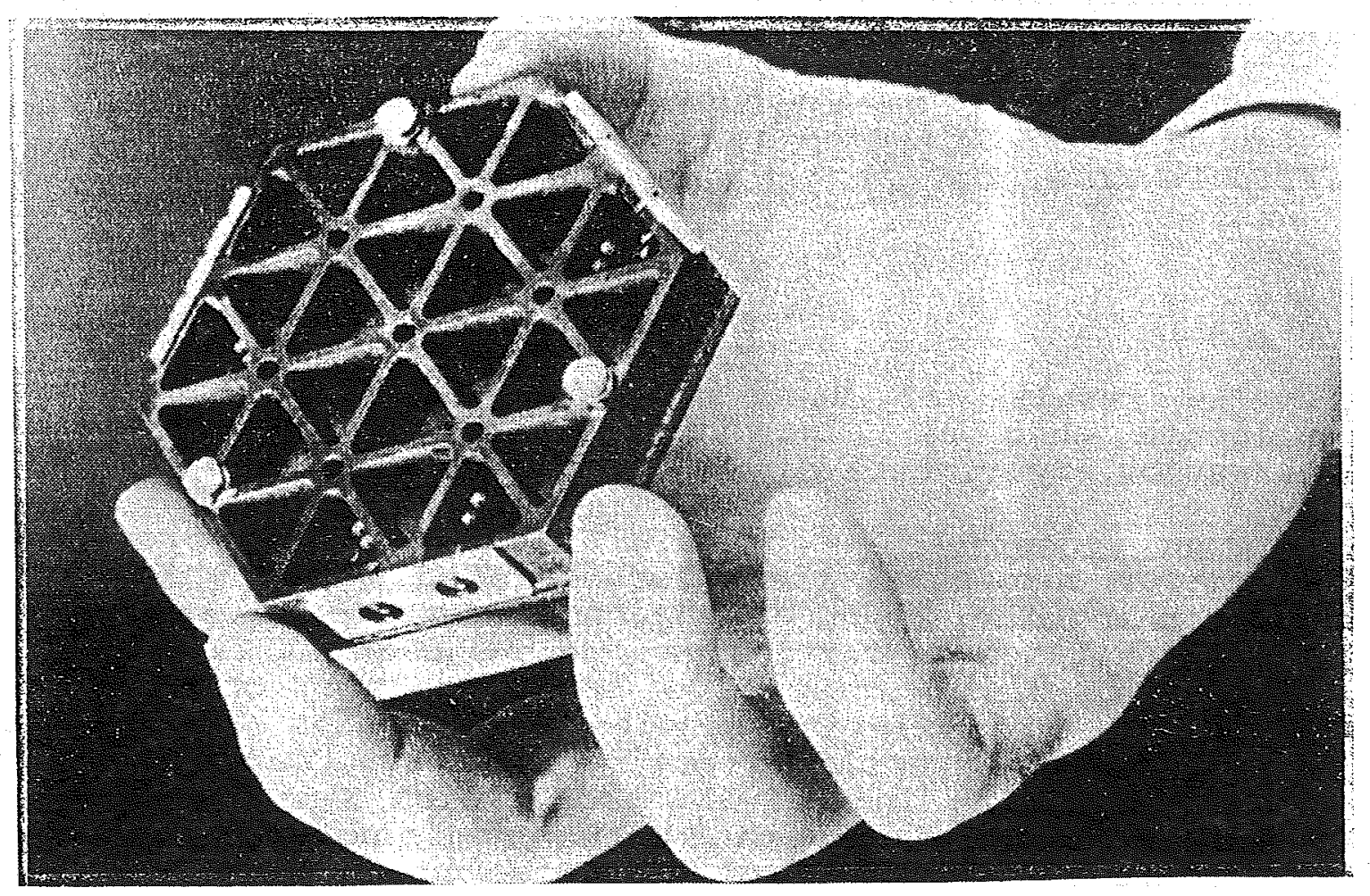

Figure 3-1. Silicon carbide segment (rear view). 


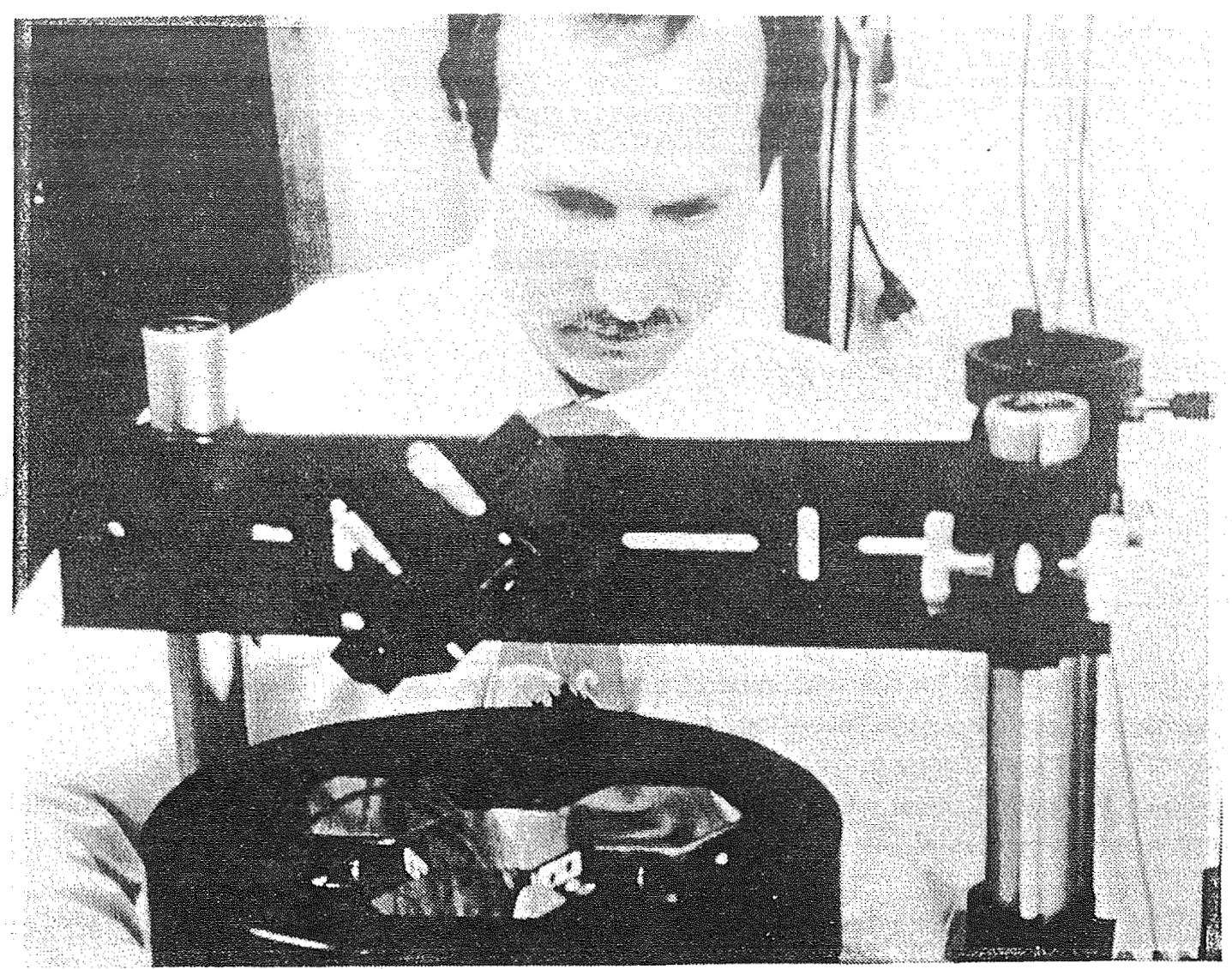

Figure 3-2. Dynamic segment validator.

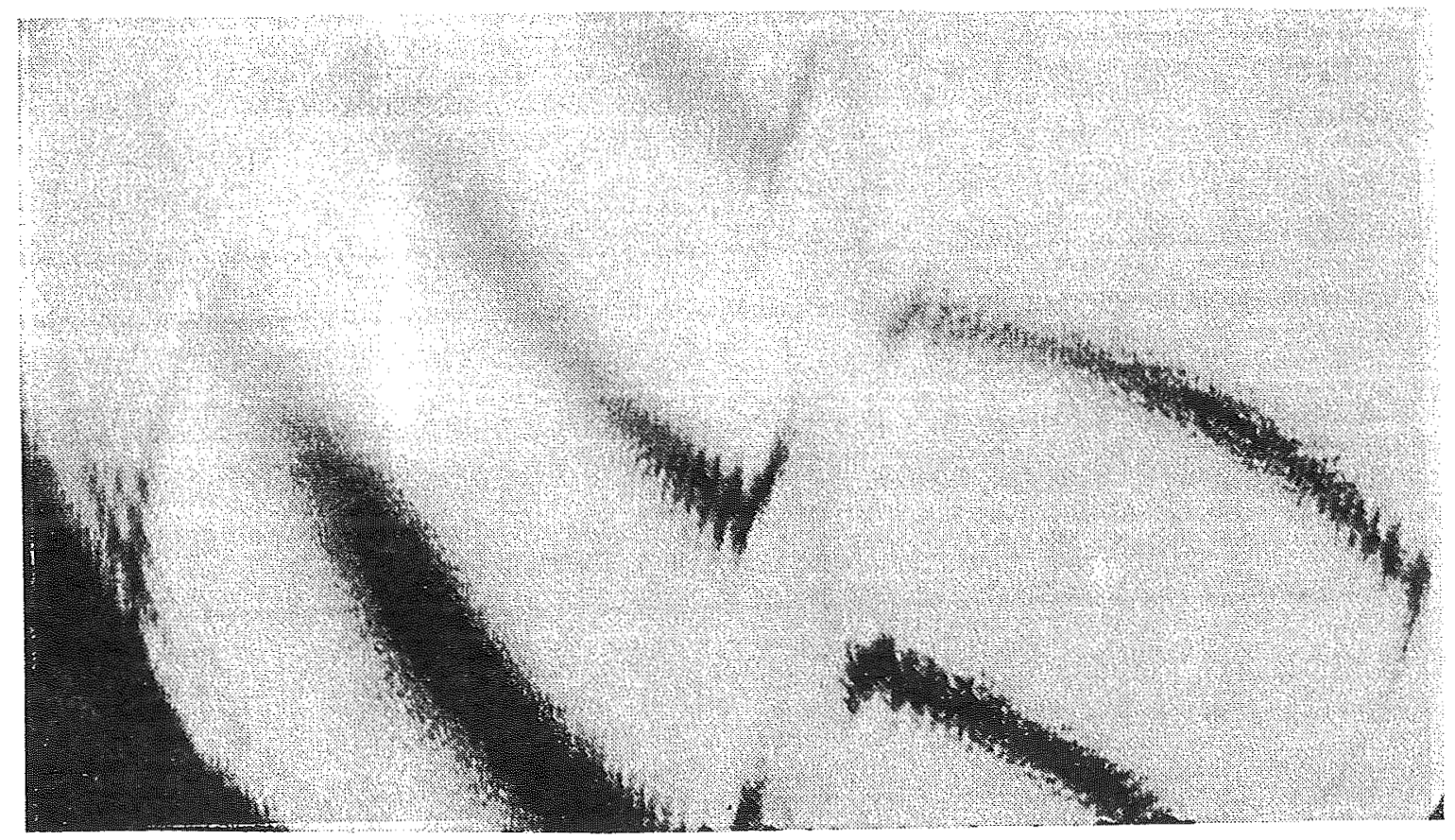

Figure 3-3. Continuity of fringes across gap between two edge-matched segments. 


\section{DISCUSSION}

Miller: What is the mirror surface? Is it silicon?

Rather: There are various ways of preparing the surface. The ones available now are a bonded silicon surface on the silicon carbide. There are a few manufacturers. One of the best is made by United Technologies Optical Systems in West Palm Beach.

Burbidge: You said that the cost is down by a factor 5-10. How do you demonstrate that?

Rather: It depends on the economies of mass-production. It is still uncertain but it comes from programs for evaluating the cost of mass-production.

Nelson: For a 10-16 meter telescope, how small do the segments have to be to approximate the surface with spherical segments?

Rather: There are many tradeoffs, but I would say no bigger than $15 \mathrm{~cm}$, and probably $7 \mathrm{~cm}$, but Korsch can answer that since he has been looking at this question.

Korsch: If the segments are sufficiently small, the shapes of Cassegrain-type and R/C-type primaries may be approximated by simpler segment shapes. The segment sizes allowing a spherical approximation will probably be too small (less than $1 \mathrm{~cm}$ ), but relatively simple toroidal shapes will most likely be adequate. 
Robert V. Stachnik

NASA Headquarters

\section{- RECENT HISTORY -}

- NUMEROUS EFFORTS CURRENTLY UNDER WAY FROM THE GROUND

- RAPID DEVELOPMENT OF ELECTRO-OPTICAL AND COMPUTING TECHNOLOGY

- INTERFEROMETERS SPECIFICALLY CITED AS SCIENTIFICALLY EXCELLENT BUT TECHNOLOGICALLY IMMATURE AT DELTA-CLASS EXPLORER REVIEW

- INTERFEROMETERS DID WELL IN SPACE STATION ATTACHED PAYLOAD REVIEW

- TWO WON SUPPORT AS CONCEPT STUDIES -

- ONE APPROACH - THAT PURSUED BY MIKE SHAO SPECIFICALLY CALLS FOR USE OF THE MOON AS A PLATFORM BUT THERE ARE MANY OTHER CONCEPTS PLANNED FOR FREE-FLIERS

- SCIENTIFIC FOCUS ON BOTH HIGH SPATIAL RESOLUTION IMAGING AND ASTROMETRY

- RELATION TO GRAVITATIONAL ASTRONOMY'S LASER INTERFEROMETERS

- ESA STUDY / HORIZON 2000

- JPL / ASTROTECH-21 STUDY

- baHcall paNel 


\title{
ADDITIONAL SPACE INTERFEROMETERS
}

\author{
- OASIS
}

RELATIVELY "FLOPPY" MONOLITHIC "Y" STRUCTURE HAVING UP TO 27 1-METER APERTURES

- RING TELESCOPE

ANNULAR APERTURE AND LONG FOCAL LENGTH

- VISTA

INDEPENDENT SPACECRAFT MICHELSON DESIGN WITH BEAMS SENT TO A CENTRAL COMBINING STATION

- TRIO

SIMILAR TO VISTA, WITH EMPHASIS ON PHASE CLOSURE FROM LARGE NUMBER OF SOLAR-SAIL SPACECRAFT AT LUNAR L5

- DEVICES TAKING SPECIAL ADVANTAGE OF LUNAR ENVIRONMENT

\section{INTERACTION: GROUND AND SPACE} INTERFEROMETRY

- GROUND: PROOF OF CONCEPT; TECHNOLOGY TESTBED; SCIENTIFIC CREDIBILITY

- GROUND: STELLAR DIAMETER AND SURFACE IMAGING, PRECISION ASTROMETRY

- SPACE: PHASED DEVELOPMENT

- SPACE: WIDENED SPECTRAL WINDOW, REMOVAL OF BOTH A MAJOR NOISE SOURCE AND ARTIFICIAL LIMITS ON BASELINE 


\title{
INTERACTION: MONOLITHIC AND
} SYNTHESIZED APERTURES

\author{
- A CONTINUUM ! \\ FILLED $=>$ VERY DILUTE \\ - RECALL THE EXPERIENCE OF THE RADIO \\ COMMUNITY \\ AND \\ LOOK AT THE VLT \\ - DESIGNING ANY NEW LARGE MONOLITH \\ TO BE COMPATIBLE WITH AN \\ ASSOCIATED ARRAY WILL BECOME \\ INCREASINGLY DESIRABLE \\ - SCIENCE, AT PRESENT, LOOKS \\ SOMEWHAT ORTHOGONAL. \\ THE DISTINCTION IS LIKELY TO \\ DISAPPEAR WITH TIME, AS HAS BEEN THE \\ CASE FOR THE RADIO COMMUNITY \\ - IN THE SHORT TERM, DESIGNS OF BOTH \\ SYNTHETIC AND MONOLITHIC APERTURE \\ INSTRUMENTS WILL ADDRESS \\ COMPLEMENTARY SCIENCE. THIS WILL BE \\ LESS TRUE IN THE FUTURE. DESIGNERS \\ OF BOTH TYPES OF INSTRUMENTS \\ SHOULD CONSIDER THE NEEDS OF THE \\ COMBINED COMMUNITY OF USERS
}


Michael Shao

Jet Propulsion Laboratory
$56-34$

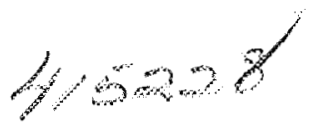

Abstract

This paper describes a concept for the direct detection of extra solar planets. The concept is based on a decade old idea from Bracewell but expanded. A long baseline interferometer is examined with two three meter telescopes, cooled to $70 \mathrm{~K}$ and a baseline of 30-50 meters. In space, this instrument would be able to detect an Earth sized planet around a solar like star at 10 parsec in approximately 1 hour of integration ( 5 sigma). The total number of candidate stars with detectable "Earths" number in the thousands.

Introduction

Several techniques have been proposed that in theory would be capable of detecting. Earth like planets around nearby stars. The IR long baseline interferometer technique described here, has a significant cost advantage of a factor of 10 to 30 , \$500M vs $\$ 5-15 \mathrm{~B}$ or $\$ 1 \mathrm{~B}$ vs $\$ 10-30 \mathrm{~B}$. The technique is based on using a long baseline interferometer with an achromatic nulling beam combiner to partially cancel the light from the star so that the IR light from the planet can be detected.

The major sources of noise in IR direct planetary detection are:

1)

2)

3)

4)

Light from the star, diffracted or scattered into the detector pixels that would otherwise detect planetary emission. Thermal emission from the optics

Zodiacal IR emission from dust within the solar system (near the earth's orbit, this dust has an emissivity of $1.0 \mathrm{e}-7$ and a temperature of $277 \mathrm{~K}$ ).

Zodiacal IR emission from the dust around the star where we are searching for planets.

In direction detection of extra solar planets at visible wavelengths, \#I in the above list is the most severe problem. At IR wavelengths, depending on the design of the instrument, any of the above four effects can dominate.

Bracewell in the mid 70's, first proposed the idea of using a space based IR interferometer to extra solar detect planets. A study of the concept by Lockheed for NASA Ames, was conducted with the detection of a Jupiter sized planet at 10 parsec as the goal of the "baseline" instrument. The detection of smaller but much warmer planets such as Earth, or Venus was not considered. It turns out that in many ways the detection these warmer planets with IR interferometry, is not significantly more difficult.

Fig. 1 shows the difference between detecting the emission of a extra solar planet at visible and IR wavelengths. By moving into 
Figure 1

\section{Relative Sun - Earth photon flux vs wavelength}

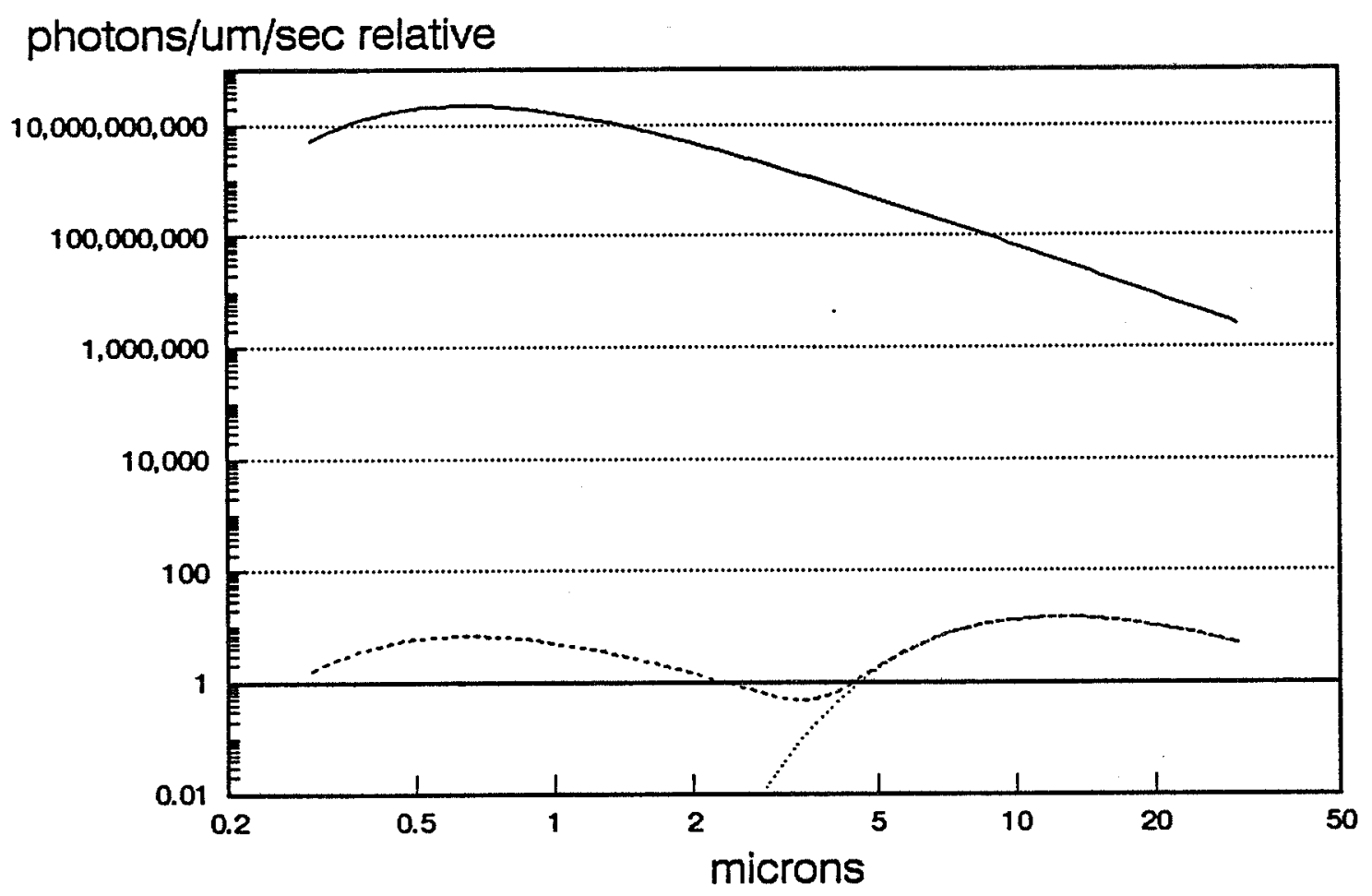

the IR two advantages are gained, one the contrast ratio between the star and planet improves by about 3 orders of magnitude, second, the number of IR photons per second from the planet is actualiy larger in the IR. For detection of Earth like planets, detection at 10 um is optimum.

While the detection of an Earth-Sun system at 10, 2.5 or 4 parsec is a useful objective, it is also important to determine the number of candidate stars around which a planet could be detected, if a planet were there. Here the advantage of a long baseline interferometer becomes apparent. An Earth-Sun system at 10pc would have an angular separation of 0.1 arcsec, requiring a baseline of 25 meters to resolve (or a 25 meter filled aperture telescope). However, the vast majority of stars in the solar neighborhood are much less luminous and the separation of an Earth like planet from the star would be much smaller, requiring corresponding longer baselines. While an interferometer with 2 meter apertures (15 $\mathrm{sq}$ meters) would be 10-30 times less expensive than a $16 \mathrm{~m}$ filled aperture telescope, it would be an additional order of magnitude less expensive than a 50-100 meter telescope that would be capable of detecting hundreds to a thousand planets that the $3 \mathrm{~m}$ interferometer would.

\section{IR Long Baseline Interferometer in Space}

For an example, we consider a long baseline interferometer with two 3 meter aperture telescopes, and a baseline of 30-50 meters. We assume that the baseline can be extended so that the star-planet is 


\section{Figure 2}

Planetary Achromatic Nulling Interferometer Concept

This is just 1 example (other achromatic nulling interf. exist)

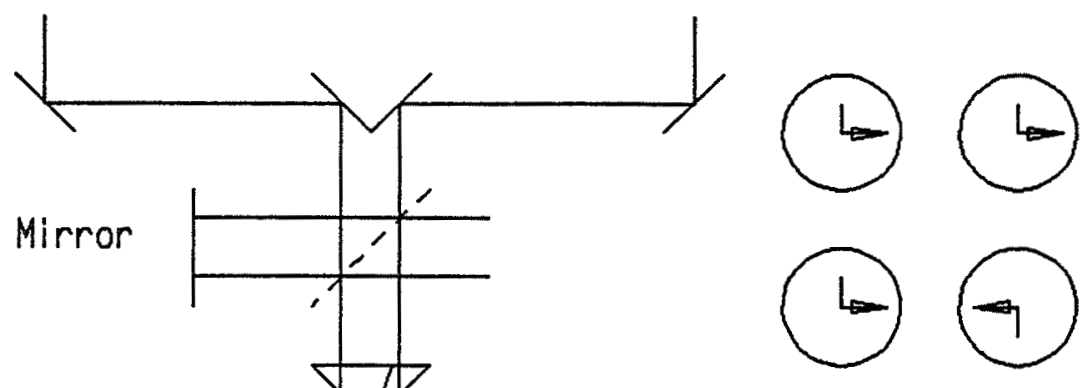

Corner cube

Eoch arn sees $1 T, 1 R$ off bean splitter

Normal bean splitter, not 50/50

Null due to polarization flip, not $\mathrm{QPD}$ shift, wideband

resolved by the interferometer. Fig. 2 shows the basic idea of the instrument. Iight from the two telescopes are brought together to an achromatic nulling beam combiner. In a conventional pupil plane interferometer such as the one studied by Lockheed, the starlight was nulled out by changing the path in one arm of the interferometer by $1 / 2$ wave. However, a physical change of $1 / 2$ wave at 10 um wavelength would not be exactly $1 / 2$ wave at $10.1 \mathrm{um}$. The interferometer in Fig. 2 gets around the problem by producing a null (dark fringe) at zero optical path difference by flipping the polarization of one of the interfering beams. This particular interferometer also uses the beam splitter in a symetrical way, light from both telescopes will use the beamsplitter once in transmission and one in reflection. This gets around the problem of fabricating a beamsplitter that is exactly 50/50. In fact, almost any dielectric beamsplitter with < $1 \%$ absorption will be capable of nulling out the starlight by a factor of 10,000 .

The pointing and phasing of the interferometer are important if a high degree of starlight cancellation is desired. Pointing errors will cause the interfering wavefronts to be tilted with respect to each other. The optical path difference must be almost equal for the null to be deep. Typically at 10,000 attenuation of stellar signal, the optical path must be equal to 20 nanometers rms. This implies the precision (optical figure) a factor of several less severe than the optical quality of the Hubble telescope optics. In addition the use of a single mode spatial filter before the detector could be used to relax the optics quality by an order of magnitude. 
Interferometric cancellation requires that the amplitude of the interfering wavefronts be equal to $1 \%$ for cancellation of 10,000 . This is met by using a dielectric beam splitter that has less than $1 \%$ absorption in a symmetrical way such as in fig 2 . In addition the pointing of the telescopes must not move the image more than 1/20 of the diffraction spot or about $1 / 30$ arcsec. Control of the optical paths in the two arms must also be controlled at the 20nm level. This would be accomplish by using a dichroic beam splitter to split the vis/near ir light to a separate interferometer. since the central star is quite bright centering the fringe to $20 \mathrm{~nm}$ at visible wavelengths is actually quite a bit simpler than the operation of a ground based interferometer that has to track the millisec fluctuations due to atmospheric turbulence.

\section{SNR calculation}

The SNR calculation is quite straightforward. The assumptions are:

1) The star is the size of the sun, a black body at $5700 \mathrm{~K}$.

2) The planet is the size of the Earth at 300K.

3) Both are a distance of 10 parsec (unit emissivity)

4) The local zodiacal dust is a gray body at $277 \mathrm{~K}$ and an emissivity of 7.0 e-8.

5) The dust around the distant star-planet is similar, $274 \mathrm{~K}$ and emissivity of $1.4 \mathrm{e}-7$. The emissivity is twice as high because in looking out of the solar system we only see $1 / 2$ of the dust.

6) The planetary signal is collected by 23 meter telescopes

7) The area-solid angle product for background is lambda squared 100 square microns

8) The optics is at a temperature of $70 \mathrm{~K}$ with $20 \%$ emissivity.

9) The detection bandwidth is $20 \%(9-11 \mathrm{um})$

10) The detector Q.E. is $50 \%$.

11) The interferometer cancels star light by a factor of 10,000

12) The center of the band is at loum

13) Assume that IR detector noise well below background of 10,000 photons/sec, eg read noise of $<<300$ electrons if detector is read out once per $10 \mathrm{sec}$.

$\begin{array}{lc}\text { source } & \text { detected photons/sec } \\ \text { zodi } & 6318 \\ \text { optics } & 1419 \\ \text { star } & 5183 \\ \text { planet } & 10.4\end{array}$

Time to achieve an SNR=5, 49.6 minutes

IV Interferometric Imaging

The above calculation was the SNR assuming the planet was on a bright (constructive interference) fringe and the star was in a nuli. Detection of the planet requires that the baseline length or orientation change. The changing baseline modulates the planet signal, which must then be demodulated to produce an image of the planet. If we consider a simplified scheme where four points in 
the UV plane is sampled by the interferometer, either by changing the orientation or length of the baseline, the signal would be the modulation of the planet signal. Since on the average the planet would be neither on the bright or dark fringe, but halfway in between, the total integration time needed would be a factor of four larger than the above 50 minutes because of the need to demodulate the signal.

An important consideration in the design of instruments that can detect extra-solar planets is the number of planets that the instrument can potentially detect. Current searches for planets with radial velocity techniques have yielded negative results for planets with masses between 10 and 80 Jupiter masses. A sufficient number of stars have been searched that the negative result is considered important. With the detection of Earth like planets an instrument must have the capability to search a sufficient number of nearby stars so that if no planets are found, the result is statistically significant.

Fig. 3 shows the distribution of the star-planet separation for the nearest 100 star assuming all of them have "Earths" around them. The most striking feature of this plot is that for the vast majority of stars, an Earth-like planet, eg one the size of Earth at $300 \mathrm{~K}$, would be less than 0.04 arcsec away from the star. At loum 0.04 arcsec is the diffraction limit of a 100 meter filled aperture telescope or an 80 meter baseline interferometer. From fig. $3, a$

rigure 3

\section{Distribution of Star-Earth Angle}

Nearest 100 stars (to 6pc)

\section{$\%$ of stars}

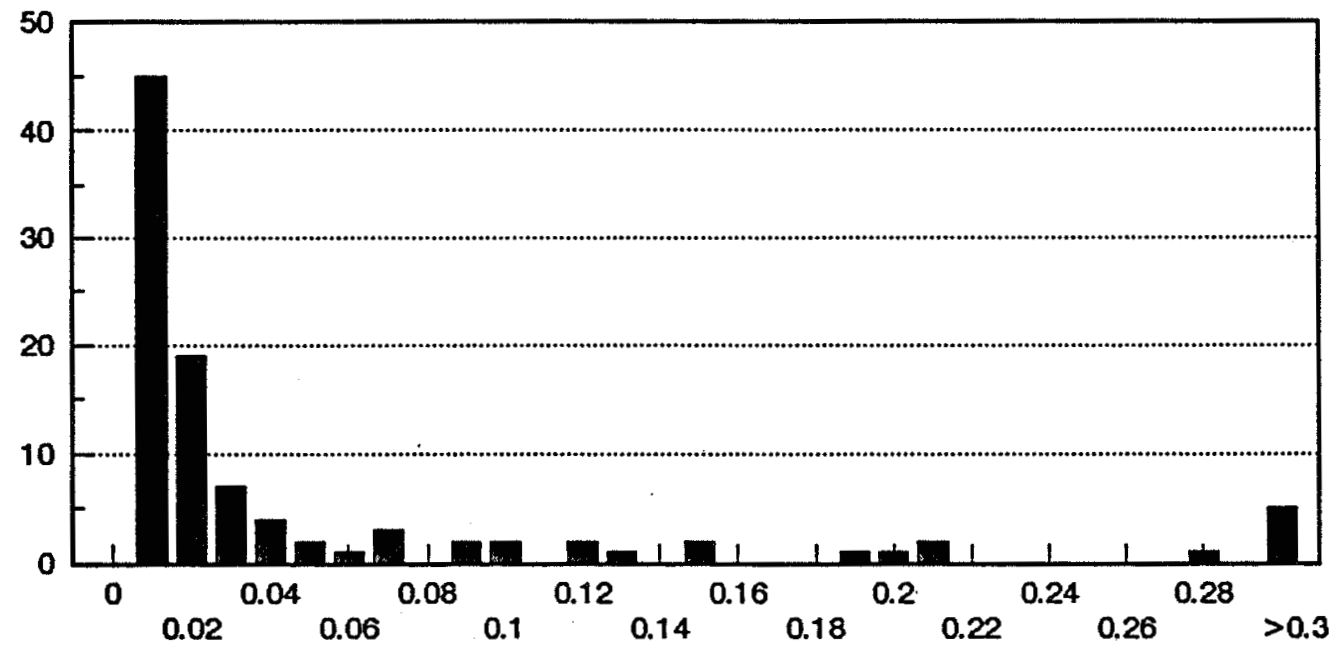

Angular separation between star-Earth (arcsec) 
$200 \mathrm{~m}$ baseline is needed to detect Earth like planets around 50\% of the nearest 100 stars (with 6 parsec). For stars further away than 6 parsec, on the average, longer baselines are needed. Radio astronomy went from filled apertures to interferometers because important scientific questions required very high angular resolution that was achievable in an affordable way only with multiple telescope interferometry. 100 or 200 meter telescopes in space or on the Moon are not practical or affordable in the foreseeable future. Three multi-hundred meter optical interferometers (ground based) are funded the Australian interferometer SUSI, the NRI imaging array and the ESO Very Large Telescope.

Interferometers have no intrinsic advantage over filled aperture telescopes. The advantage is economic, diffraction limited 100 to 200 meter filled aperture telescopes are extremely expensive, even at radio wavelengths, at optical wavelengths 100,000 times smaller, they are not affordable.

VI SNR summary

In the calculations above, an interferometer with 3 meter apertures was used to detect Earth like planets at 10 parsec. For stars closer than 10 parsec, the required apertures are smaller. In the above example, the nulling interferometer was assumed to cancel the starlight by a factor of 10,000. For stars with luminosity greater than the sun, the factor of 10,000 is not sufficient. Fortunately, the vast majority of stars in the solar neighborhood are less luminous and a factor of 10,000 is not needed. A large number of variables are involved and optimization of an instrument design is correspondingly complicated. As an example we consider the requirements for an interferometer to detect an Earth around Barnard's star.

Table 1. IR Interferometer for Direct Detection (Barnard's Star)

- Abs mag 13.2 Visible

- dist $1.9 \mathrm{pc}$

- Star/Earth ratio@10um 400,000 (600 times zodi)

- Interferometric cancellation factor of 600

- Star-Earth separation 0.012 arcsec

Baseline needed $250 \mathrm{~m}$

- Aperture needed $60 \mathrm{~cm}$

- Integration time $\sim 1 \mathrm{hr}$

- (30cm, *300, 16hrs) also works

Fig. 4 shows roughly, the degree of starlight cancellation required as a function of the star's absolute magnitude. Also plotted is the distribution of the density of stars as a function of absolute magnitude. Since the vast majority of stars are significantly fainter than the sun the degree of starlight cancellation (at loum) 
would be less than 10,000 in the 1 st example with $3 \mathrm{~m}$ apertures. With $3 \mathrm{~m}$ apertures, the interferometric cancellation needed is the flus ratio (star/planet) divided by 2500 to get star light equal the zodiacal light level.

Figure 4

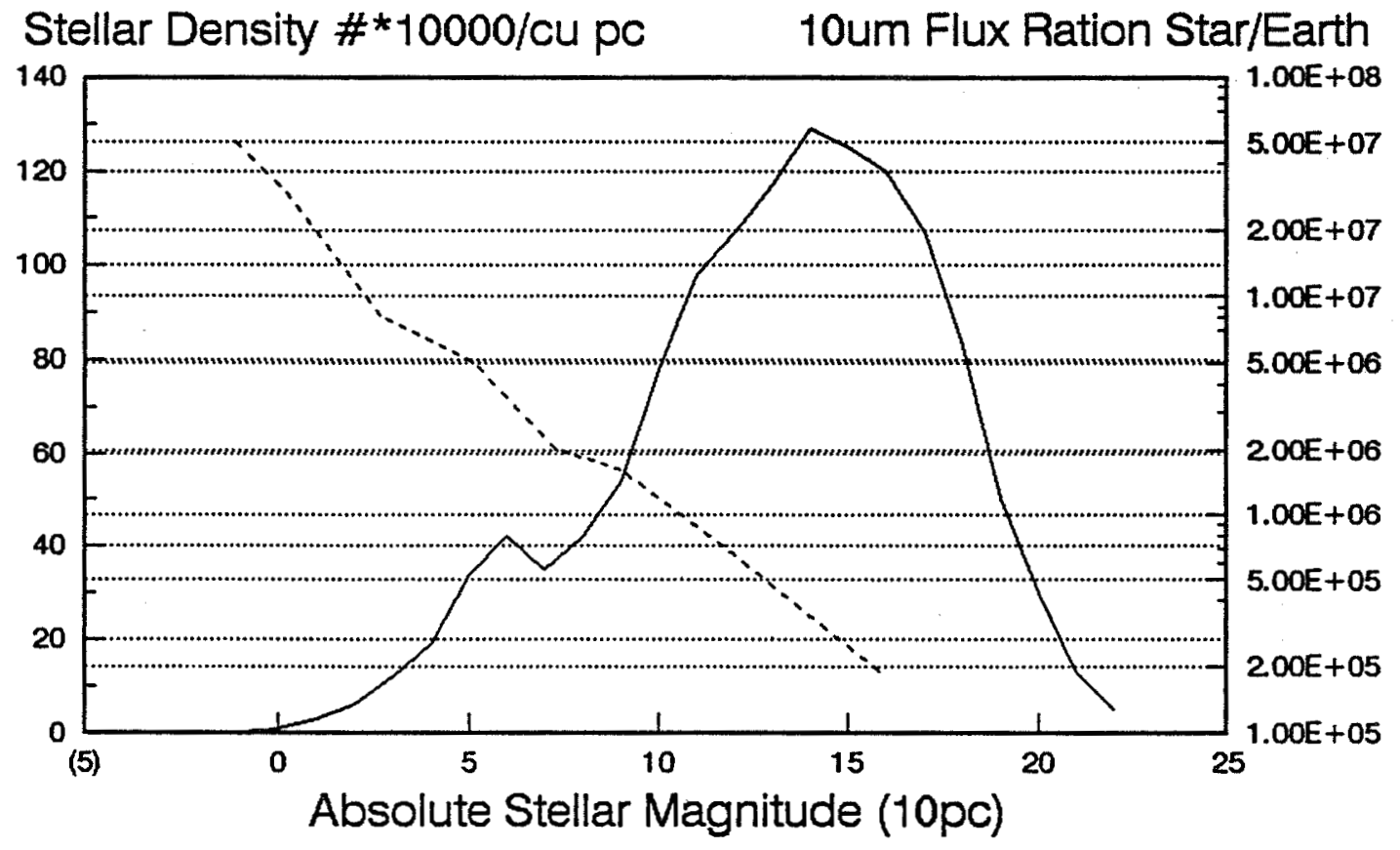

Density Flux Ratio

Fo $1 \mathrm{ch}$ Irtegration Zodiplanet=2500 (SNA =5)

Interferometric Cancelation $=$ F.R/2500

Fig 5 shows the number of candidate stars as a function of collecting aperture. With 3 meter apertures, the number of Earths that could be discovered, is over 1000 .

\section{\# Candidate Stars vs Aperture}

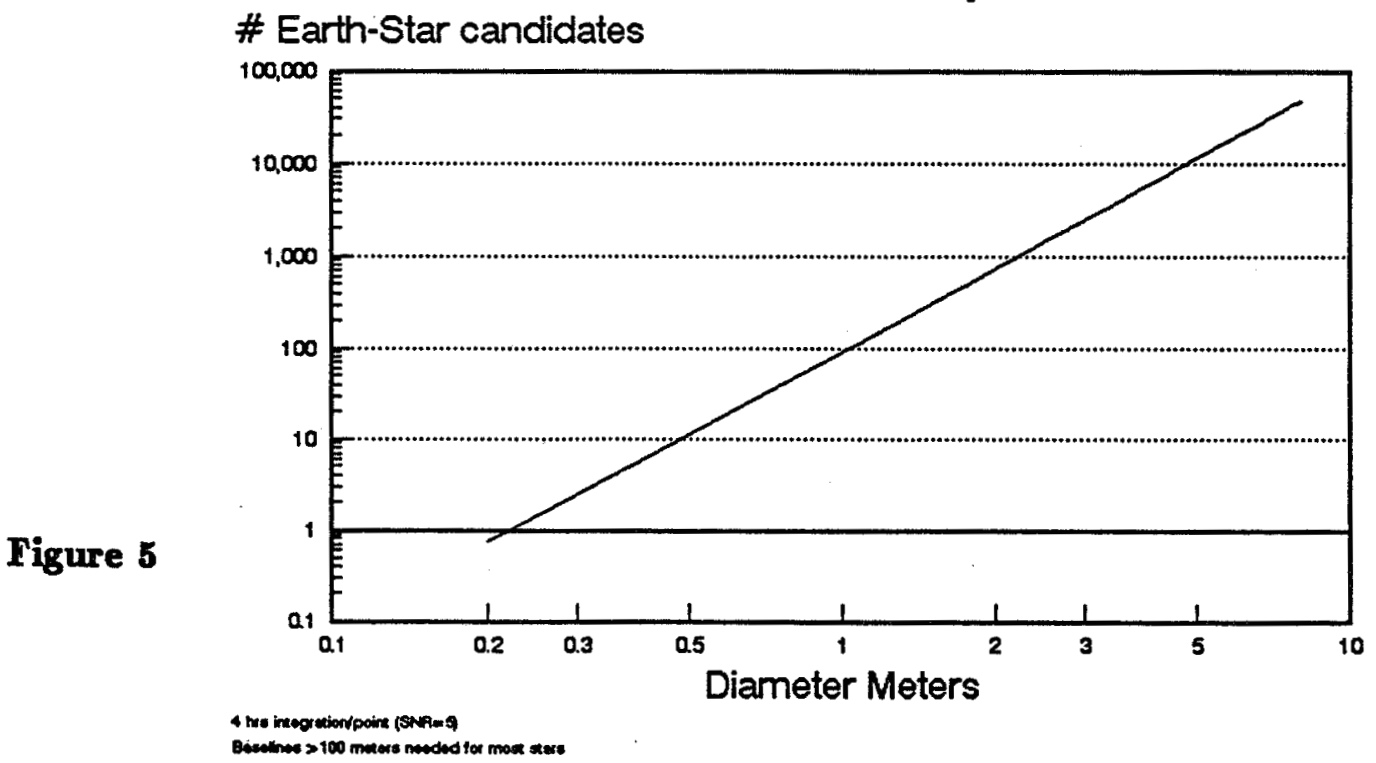




\section{Conclusion}

A number of techniques exist for the direct detection of extrasolar planets. Long baseline interferometry in the thermal IR has one compelling advantage. It is capable of detecting "Earths" around nearby stars with collecting apertures, and cost, an order of magnitude lower than other techniques. In addition, in the long run it is the only technique that can achieve the very high resolution needed ( $<0.01$ arcsec) to perform a complete survey of nearby stars for "Earth" like planets.

\section{DISCUSSION}

Angel: You have considered using a two-element interferometer with two 3-meter apertures. With a filled aperture and the apodizing system discussed yesterday, in an exposure of two minutes, an earth-like planet would be seen in a 0.25 " ring around a star at 4 parsecs. One would explore a region with 6 resolution elements in the image space unambiguously in 2 minutes at the 5 sigma level. For comparison, for the two 3-meter telescope interferometer, putting in the same conditions and the same zodical light "background", one would get a single measurement, not an image, at one fringe spacing at a single orientation, and that would take several hours. At the end we would know only that the fringe contrast would be slightly reduced by some object in the field. To make a map with any semblence of the detail from the filled aperture image, many orientations would have to be done. This would result in days of integration.

Shao: Let me respond to that. The standard algorithm is that the dynamic range of a map is the $\mathbf{S} / \mathrm{N}$ per $u-v$ point divided by the number of $u-v$ points. So if there are $25 u-v$ points each with a $S / N$ of 1 , that produces a map with a $S / N$ of 5 . That is the case for a large number of pixels. The radio maps are done that way. The $S / N$ per individual baseline can be quite low if you have a large number. The number of $u-v$ points needed are the number of pixels in the map that one is trying to reconstruct. In the case of the planet with the star nulled out there is only one pixel.

Angel: You have looked at using very large baselines with the interferometer. For the 16 meter telescope one can get to $0.1^{n}$ by masking out apertures, and so one can get out to $10 \mathrm{pc}$. Within $10 \mathrm{pc}$ there are enough stars that one could spend years with an interferometer, given the time that it takes to do a stellar system, and so it is not clear that there is an advantage over a filled aperture to having an interferometer with bigger baselines that goes below $0.1^{\prime \prime}$.

Sheo: Well, it really depends on the cost. Instead of two 3-meter telescopes one should really compare the cost of two 12-meter telescopes in an interferometer with 50 to 100 meter baselines to a filled aperture. You could look much, much further. You could go to $50 \mathrm{pc}$. The number then goes to thousends of objects. 
mlingworth: Planet detection is not the only science that will be done with either interferometers or filled apertures.

Shao: I agree that both interferometers and filled apertures have many science goals other than planet detection. In prior studies of interferometers for the direct detection of planets, a number of problems were identified that, upon more careful study have straightforward solutions, compared to the many problems of putting a large precise instrument in space.

mingworth: David Diner did a study comparing interferometers and filled apertures, for planet detection. He decided that a $10-20 \mathrm{~m}$ filled aperture was best. Why did Diner choose the filled aperture rather than the interferometer that you are proposing for planet detection?

Diner: I have several concerns with the interferometer. First, measurements must be made at many baselines and orientations and this multiplies the integration time. Second, the interferometer must be stable between collection of points in the $u-v$ plane, which puts very tight tolerances on the pointing of the telescopes and on thermal control of the optics. At the cold temperatures needed to detect planets, if the temperature of the mirror changes by a very tiny fraction of a degree that gives a change in the signal that might be interpreted as a planet. There were thus a number of problems with an interferometer that led us to choose a filled aperture.

Shao: I responded to the first of your concerns in my previous answer to Roger Angel. There are a number of technical problems such as the path length problem that we now have solutions to. Controlling to 5 nanometers we see as doable. For the thermal control problem we see using an array of detectors instead of a single detector as the means to solve this problem. In addition to the detector looking at the planet, we would look at the pixels 4-5 arcsecs away through the same optical system.

Kahan: A brief comment-your last viewgraph would suggest cost grows $>D^{2}$. In fact, in a production mode, and ignoring for the moment the issue of number of different segment shapes and the secondary mirror support dynamics, a number of studies suggest the cost of a fully populated segmented primary goes as $<D^{2}$. Considering the cost of the partially filled aperture support, it seems that the cost with a fully populated mirror is still very open.

Shao: Yes. For a spaceborne system that's likely to be the case at the moment. 


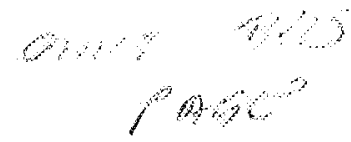

\section{Session 5}

Technologies for Large, High Throughput Wideband Optical Systems in Space 


\section{Page intentionally left blank}




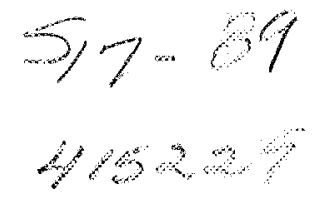

DESIGN CONCEPTS FOR VERY LARGE APERTURE, HIGH-PERFORMANCE TELESCOPES

Dietrich Korsch, Optical Science Consultant 10111 Bluff Drive, Huntsville, AL 35803

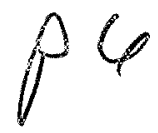

\section{SUMMARY}

The influence of the primary mirror on the performance and on the feasibibility of a very large telescope with extreme performance requirements is considered. A detailed study comparing aspheric- and spherical-primary systems, before selecting the mirror configuration, is recommended. Preliminary results are presented.

\section{INTRODUCTION}

When thinking about building a next generation space observatory which will undoubtedly take many years to complete and will, hopefully, operate for many more years, we must also think of the next generation of astronomers, who will eventually be the principal benificiaries of such a project. This means that we can not be satisfied with developing a system that only accommodates today's list of science objectives, as even those may change and more ambitious ones will most certainly be added in the years to come. It must, therefore, be our goal to develope a facility that serves not only as many science interests as possible, but that will also be able to grow to meet the challenges of the future.

Such a universality and flexibility can be achieved by applying the concept of modularization wherever possible. This not only facilitates servicing and repairing subsystems as it becomes necessary, but it also provides the opportunity to update and improve the overall system as the need arises and the state of the art progresses.

\section{DESIGN CONSIDERATIONS}

Based on today's state of the art the feasibility of a ten to sixteen meter telescope depends almost entirely on the feasibility of the primary mirror which is not only unprecedented in size, but also demands an unprecedented degree of precision.

The primary mirror, because of its size and because it is so tightly integrated into the overall system structure, cannot be easily replaced like many other subsystems of modular configuration. In addition to being the most crucial part of the telescope it will also be a permanent component and therefore requires extraordinary attention. A thorough study of possible mirror configurations, taking into account system performance, manufacturability and cost, should become a high-priority early effort. 
Regarding the primary mirror configuration there are basically two options to be considered.

Option 1: An aspheric primary.

The exact shape should be slightly hyperbolic to allow the formation of a Ritchey-Chrétien telescope, using a single secondary mirror. This would yield a telescope system with a moderate, high-resolution field of view, adequate to satisfy many of today's science objectives. In time the single secondary mirror could be replaced by a more sophisticated corrector optics which would create a telescope with a very wide, highly corrected field of view, allowing an increase of the variety and quality of observations.

The disadvantage of this option is the fact that the manufacturability of such a large and complex mirror to the required accuracy is at the present time extremely uncertain.

Option 2: A spherical primary.

A spherical primary could also initially be combined with an appropriate single secondary mirror which could then later be replaced by a more complex corrector optics. While the two-mirror system would provide only a very narrow, high quality field of view, it has been shown that a spherical primary with a threemirror corrector, for instance, can produce a telescope that out-performs the Ritchey-Chrétien,

The disadvantage of the spherical-primary concept is that, as a two-mirror system it might not perform adequately, even as a transitional solution. The advantages of a spherical primary, however, are manifold. It is the easiest and least expensive surface to fabricate; it is the surface that can be produced to the highest quality; it is very easy to test; the alignment of segments requires three degrees of freedom less than the segments of an aspheric mirror; and, perhaps most importantly, a spherical primary can be made now, that is, the probably highest risk factor can be eliminated immediately and time, effort and money can be used to develope the remaining, still demanding, but less risky parts of the system.

\section{PRELIMINARY DESIGN COMPARISONS}

A priliminary comparative study of the attainable performances of a sphericalprimary telescope with a three-mirror corrector and the classical (parabolic primary) and aplanatic (hyperbolic primary) versions of the Cassegrain-type telescope has been carried out. For comparative purposes the following common design parameters and performance goals were used:

$\begin{array}{ll}\text { aperture diameter } & : 10 \mathrm{~m} \\ \text { system focal length } & : 220 \mathrm{~m} \\ \text { maximum mirror separation: } & 15 \mathrm{~m}\end{array}$

field of view: 2 arcmin

resoluion : $50 \mathrm{nrad}$ (10 to $12 \mu \mathrm{m}$ pixel size) 
The telescope conigurations used for the comparison are diagrammetrically shown in fig. 1. A graph summarizing the performance analyses is shown in fig. 2. The residual aberrations are defined by the smallest circle surrounding the complete spot diagram. It was found that a spherical-primary telescope with a three-mirror corrector can be optimized to a higher degree than the aplanatic Cassegrain (Ritchey-Crétien). The arrangement of the three corrector mirrors as shown is neither the only possible one, nor necessarily the preferred one.

\section{CONCLUSIONS}

It is believed that the successful construction of telescopes with very large apertures depends almost exclusively upon the feasibility of the primary mirror. This is especially true, when stringent accuracy requirements are imposed in addition to the large size, as in the case of an HST successor. Trusting today in the availability of a large aspheric primary later could prove to be a cost$1 \mathrm{y}$, if not fatal mistake. A thorough investigation of the effects of the primary mirror configuration on the system performance and, even more importantly, on its feasibility is strongly recommended. 


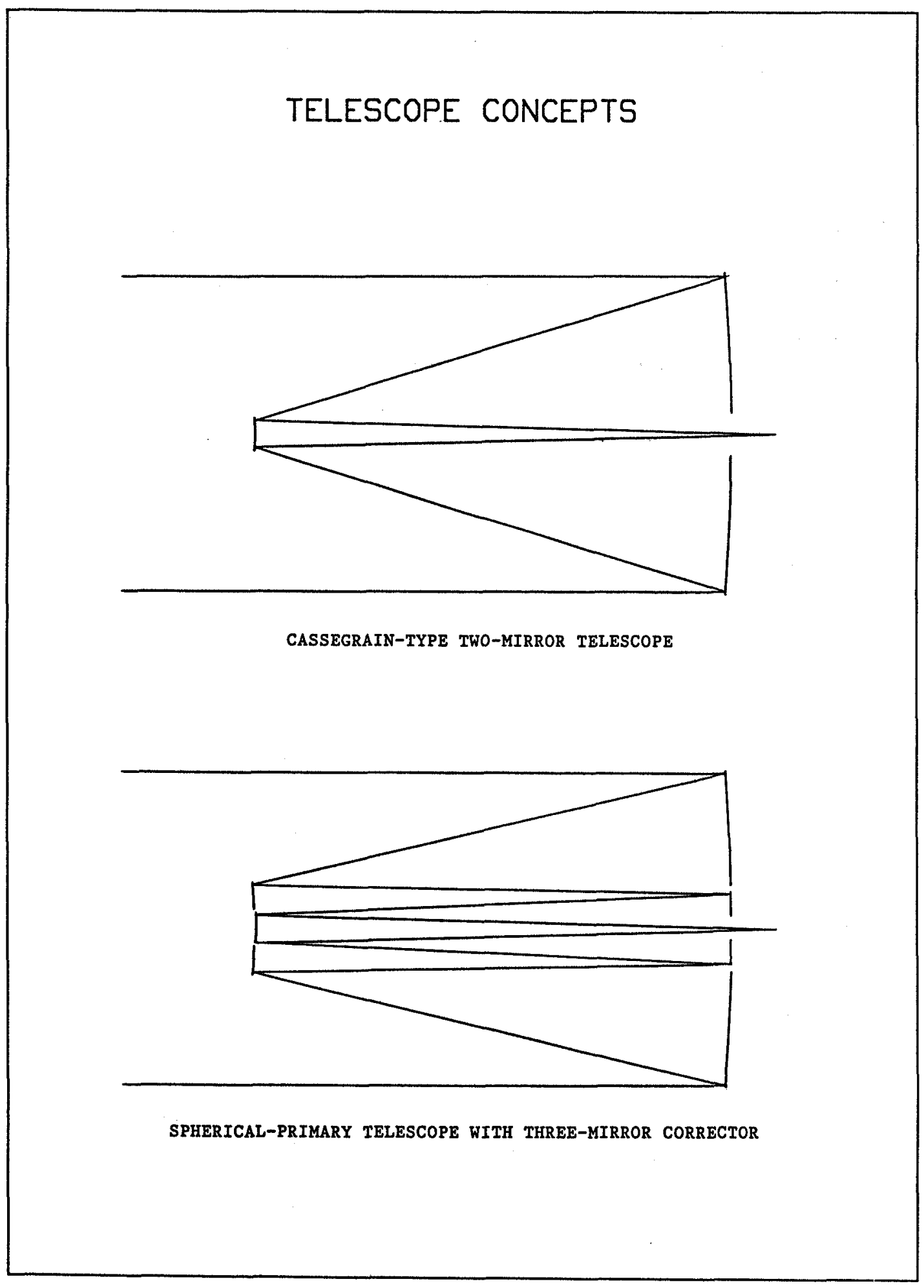

FIGURE 1 


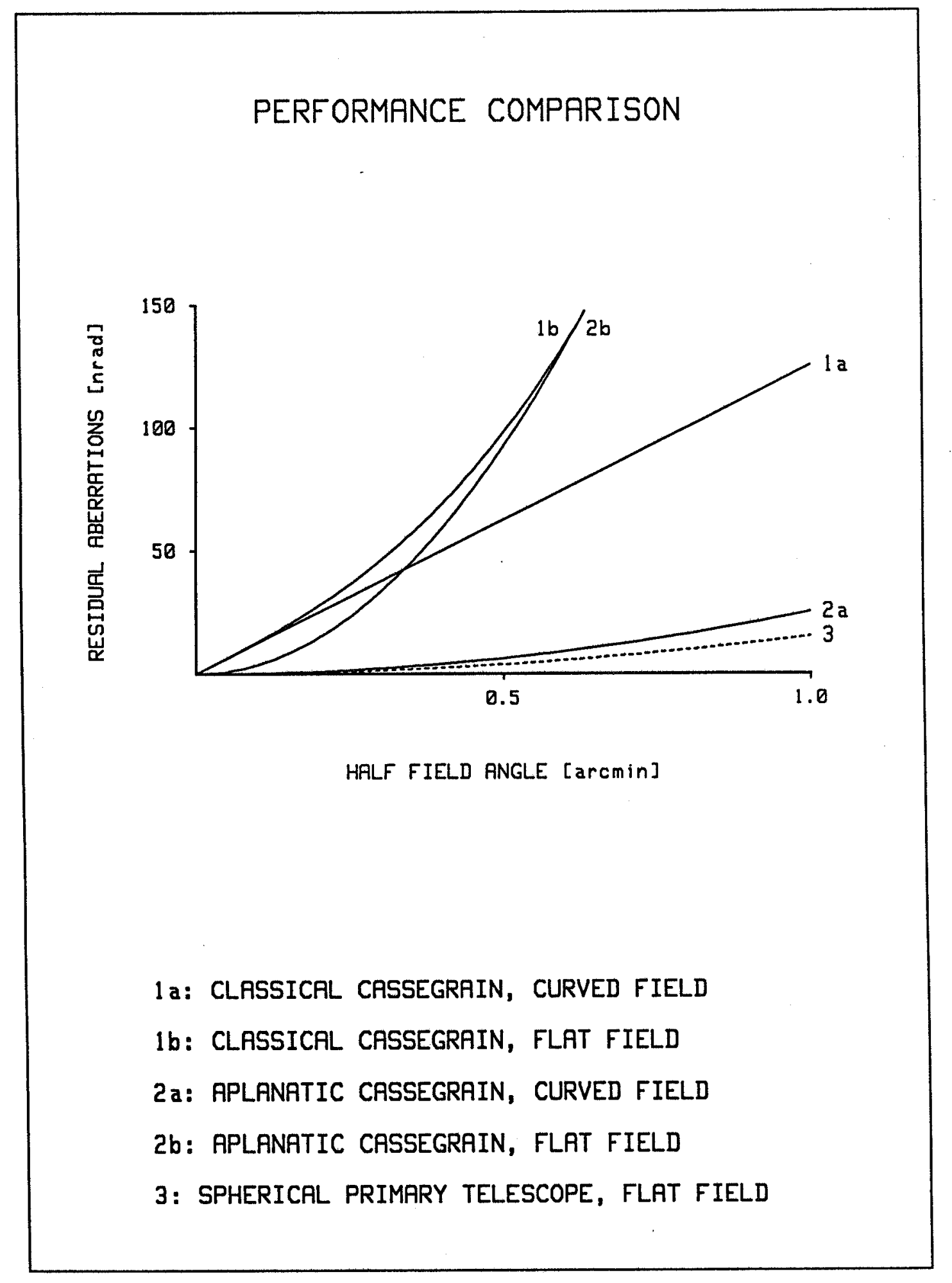

FIGURE 2 
Nelson: For a Ritchey Chretien system, how large a field of view can be approximated by a plane? The issue here is how large a single detector (in a mosaic) can be and still be planar. The individual detectors can be laid out to approximate the curved focal plane.

Korsch: It depends on the actual design parameters. For our example, the residual aberrations reach about pixel size $(10 \mu \mathrm{m})$ at the edges of a 1 arcmin diameter field.

Bely: Your proposed design includes 4 reflecting surfaces. Could you comment on the corresponding loss of throughput?

Korsch: This question is difficult to answer, because it depends on too many factors, such as coating materials, coating process, aging, etc. As an example, according to Action Research data, the efficiency, using a UV coating optimized for $250-360 \mathrm{~nm}$ ( $\mathrm{Ag}_{\mathrm{g}}$ and $\mathrm{Mg} \mathrm{F}_{2}$ ), drops to $64 \%$ after two reflections at $150 \mathrm{~nm}$.

Rehfield: Have you considered three mirror astigmat designs?

Korsch: A three-mirror telescope with an aspheric primary could meet the performance requirements. However, it seems that a spherical primary with a two mirror corrector cannot be corrected well enough.

Mlingworth: You have concentrated on a flat focal plane and noted that the field is quite limited with a curved focal plane. However, I do not feel that a curved focal plane is necessarily a major disadvantage. The instruments could accommodate such a curved focal plane and will generally have internal relay optics. I doubt also that we would try to have such a large magnification in one element. The structural requirements appear to be too large.

Korsch: There are certainly more important considerations than a flat focal plane as I have tried to point out in the paper. However, flatness of the field, at least over the area of an individual detector array (1/2 to 1 arcminute), may be required. The concern about a large secondary magnification is as familiar as it is unjustified. Interestingly enough, nobody is ever concerned about an afocal telescope which has an infinite secondary magnification. It is the vertex curvature that can cause fabrication and adjustment difficulties. 


\section{The Lunar Conflgurable Array Telescope (LCAT)}

\author{
Aden B. Meinel and Marjorie P. Meinel* \\ Jet Propulsion Laboratory
}

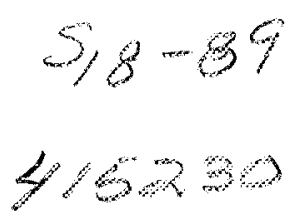

Introduction
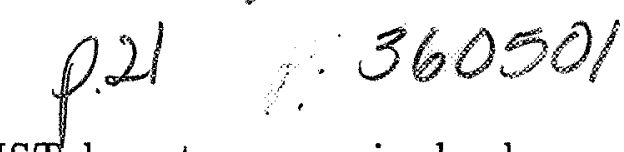

The desire for a much larger space telescope than HST by astronomers is clearly demonstrated by the attendance at this Workshop. The reality is that a much larger space telescope than the HST collides with cost scaling reality. Coupled with this reality is the fact that any multi-billion dollar science project must have broad-based support from the science community and solid political support at both Presidential and Congressional levels. The HST Successor is certainly in the same multi-billion dollar class as the Super Collider of the physics community, a project that has finally achieved the broad support base necessary for funding to follow. Advocacy of a bigger HST on the general grounds that 'bigger is better' will not be sufficient. A new concept needs to be developed that clearly diverges from scaling up of a traditional HST-type space telescope. With these realities in mind we have a few comments regarding the nature of a possible space telescope that may depart from what the organizers of this Workshop had in mind.

The national goal declared by the President is Space Station, the Moon and Mars, in that order. Space Station is a potential location where a large system could be assembled prior to being sent into a high orbit. It is not a desirable environment for a large space telescope. Mars is not relevant as an observatory site. The Moon is very relevant for reasons we will address. Our comments are based on the premise of a permanent Lunar Outpost.

One of the main arguments for a lunar telescope is a degree of permanency, that is, as long as a Lunar Outpost is maintained. In contrast, the relatively short lifetime of an orbiting telescope is a disadvantage, especially as a cost penalty. Access to a telescope in a $100,000 \mathrm{~km}$ orbit for refurbishment and resupply is a major problem with no solution in the present NASA planning.

A telescope in conjunction with a Lunar Outpost means the possibility for continual upgrading or modifying the telescope to meet changing science objectives. The two main technical disadvantages of the Moon are i) its gravity field and ii) direct Sun and Earth light. The gravity term is manageable. It also appears to be feasible to shield the telescope from direct sun and Earth light and from scattering from nearby lunar terrain. Thermal disturbances to the telescope also appear to be manageable by proper shielding, enabling the telescope to become as cold as if it were at a lunar pole crater. If these conditions are met, the telescope could be at a logistically convenient location near the Lunar Outpost.

We want to address a concept that is significantly different from those presented in the preliminary communications from Garth Illingworth in order to help fill in the matrix of possibilities. This option, moreover, is of special interest to JPL and could be an area where JPL can contribute in future studies.

\section{The Lunar Configurable Array Telescope (LCAT)}

The lunar telescope could well be the optical analogue of the VLA: the Lunar Configurable Array Telescope (LCAT). In tight clustering, similar to the MMT, it would

\footnotetext{
* The authors were unable to attend the meeting. This paper was read by J. Breckinridge who also answered questions on their behalf.
} 
produce a tight diffraction image suitable for feeding a high resolution image into the entrance aperture of analytical instrumentation. In dilute array it could become an interferometer of immense resolution capability through image reconstruction. JPL has prepared reports on the performance capabilities of lunar imaging interferometers that can be supplied upon request.

An LCAT is also a possibility for a very large space telescope operating in high Earth orbit. Here the problem is one of telescope lifetime compared to the usefulness of a given configuration and the number of weeks or months that a given configuration would be maintained. Configuration changes for VLA are scheduled months in advance to meet the science objectives of many astronomers.

This scheduling strategy is not appropriate for an orbital system, but it is for a lunar system. If the orbital system has a lifetime of only 5-10 years, configuration changes would be limited because of the intense demand for observation time. A full 10-year program could be developed for the array in its densest configuration. Similarly, a full program could be developed for the array in its interferometric configurations. The system lifetime would simply be too short to meet its potential for serving a broadest astronomical community. Not so for a lunar-based LCAT.

In our concept the most attractive aspect of LCAT is that the array consists of modest-sized fully-independent modules. This means that lunar-based astronomy could begin as soon as the first module is delivered to the Lunar Outpost. The cost scaling law is favorable, the political hurdle minimized, time to first light minimized, and industry assured of a production run sufficient to attract interest and effective support.

The optimum module size is clearly one that can be carried, fully assembled, in a single transport vehicle. This means a mirror that is the order of $2.4 \mathrm{~m}$ diameter, a size that is attractive because of existing industrial fabrication facilities and the baseline of experience to date with modules of this size. The alternative, if a large module is desired, is to at least have major subsystems fully assembled to minimize final assembly at the lunar base. One could foresee that there might be a manufacturing capability in the 4-meter size class, but the module cost then would be considerably higher. The answer as to whether a $2.4-\mathrm{m}$ module would be sufficiently large can readily be had by modeling procedures.

While the size we are proposing for each module is that of the HST, we do not propose that the module will either look or cost like the HST. The LCAT module would be much more compact and not have the expensive spacecraft modules necessary for orbital operation. The LCAT module would have the basic parts of any terrestrial telescope, with the beam combining module a separate subsystem delivered as a complete unit. The goal would be an LCAT module small enough and light weight enough that it can be delivered as space is available in the manifest.

Some of the main technical issues which need to be addressed are examined below.

Field of view. The field of view of the MMT is limited to a few arcmin by the size of the facets on the six-sided beam combiner. If a wider field is desired, a prime focus single mirror combiner can be used. It is still limited to a field of about 10 arcmin, but for a large telescope this size field is quite useful.

Added reflections. The beam combiner and path length compensation add at least three reflections. This can be serious for wavelengths below $0.2 \mu \mathrm{m}$. One is then caught between the desire on the one hand for the highest resolution, meaning an array of apertures rather than a single aperture, and on the other hand by a longer 
integration time. For the most extreme ultraviolet it would probably be preferable to operate the array as singles, and consequently at the resolution provided by a single.

Path length compensation. Path length compensation of a lunar array is small compared to that for an imaging interferometer on Earth. The rate at which the path length compensation system operates is small but over a much larger total pathlength change if a kilometer-sized array is used. An array like that proposed for project ALOHA by Labeyrie, where mobile modules move so as to keep path lengths constant, is a possible option. Further engineering analysis would be needed to determine if the ALOHA concept is a practical option for a lunar system. Demonstration of ALOHA on Earth is clearly a necessary precursor.

Polarization. The plane of polarization must be maintained throughout the reflections in the path length compensator / beam combiner optics; otherwise the diffraction pattern of the array will be compromised.

Diffraction / MTF effects. The diffraction pattern formed by an array of discrete apertures results in energy being distributed into an array of secondary maxima surrounding the central maximum. The cleanest diffraction pattern is clearly that of a single unobscured aperture. As the apertures are separated the number and intensity of these secondary maxima increase. For a non-redundant array the central maximum is at maximum sharpness and resolution but at minimum central spike intensity. What is not always appreciated is the seriousness of the effect of such an array pattern on the secondary maxima. Both the number and intensity total of the secondary maxima are maximized. This situation is very bad if one wants to feed an analytical instrument or record a direct image.

We feel that the LCAT concept should be given study attention along with the more conventional options for both orbital and lunar telescopes. This study should first be from a systems point of view. This would be followed by addressing technical issues such as the performance of LCAT in its densest clustering as well as for various configurations required for typical science objectives. It is easy to talk about beam combining. The real problems arise when one is faced with actually building a working system. These practical engineering aspects of beam combining mean that good concepts need to be developed and then analyzed. One objective of such a study should be to define precursor experiments that could be done on Earth to show that critical milestones can be met.

There is an interesting compromise between a single large aperture telescope and an interferometer that still makes use of the modular concept: A fixed, co-mounted cluster of seven modules, much like the MMT, plus outlying modules that would constitute the configurable interferometric array. The co- mounted cluster of seven would avoid path length compensation, give a reasonably tight diffraction pattern, and still have the cost and logistical advantages of modules that we discussed above.

Current related projects at JPL are addressing construction of a large imaging interferometer at a terrestrial site, location TBD (supported by the U.S. Navy), and design of an orbiting imaging interferometer of fixed baseline, supported by JPL funding.

\section{Acknowledgements}

The research described in this paper was carned out by the Jet Propulsion Laboratory, California Institute of Technology, under a contract with the National Aeronautics and Space Administration. 


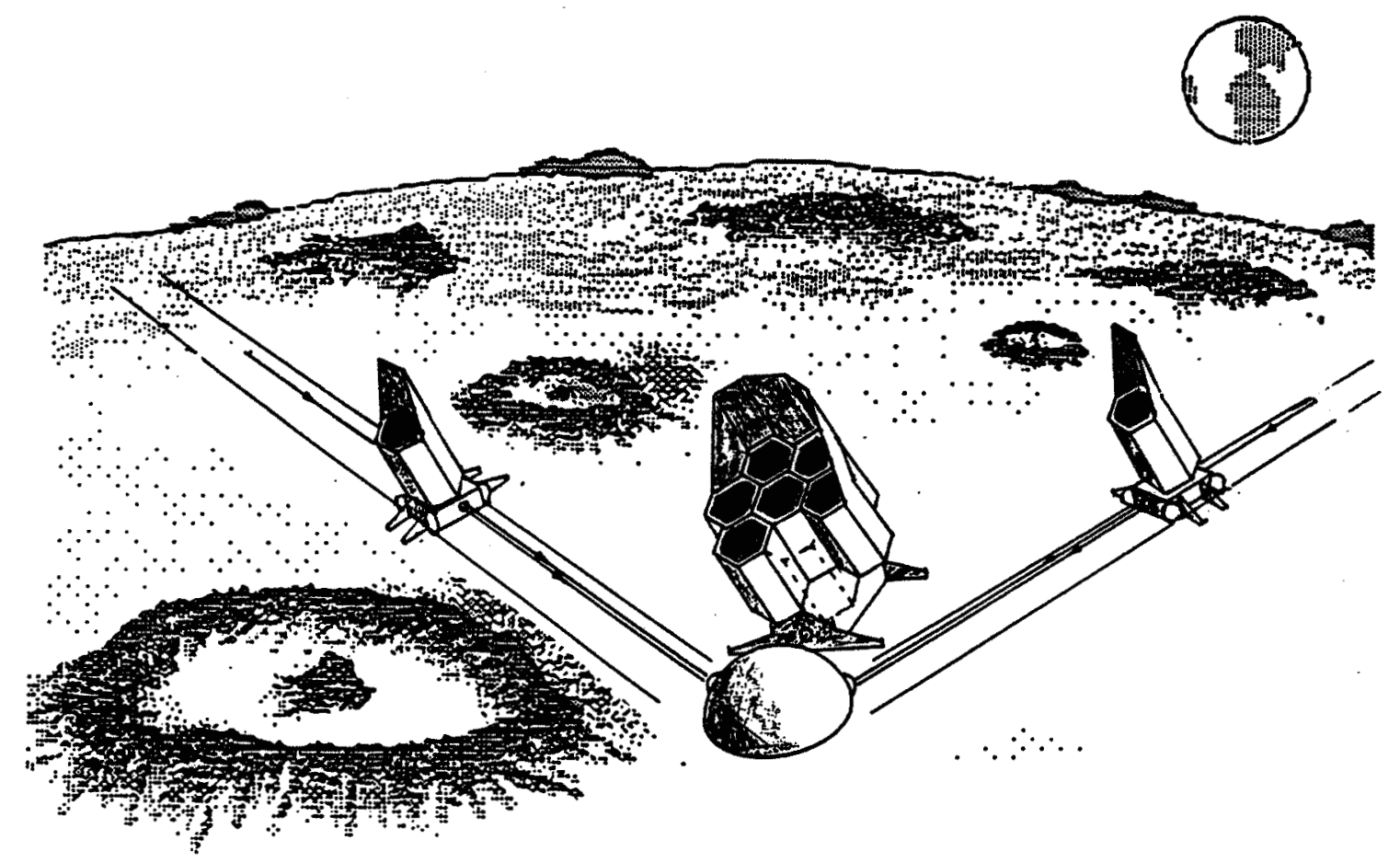

Artist concept of the Lunar Configurable Array Telescope (LCAT)

Standardized modules are delivered to Lunar Outpost as manifest permits. The modules are configured as they become available into a Large Aperture MMT with two or more configurable interferometric modules. Astronomy begins as soon as first module is de livered. The science objectives are similar to those of the NGST.

Advantages of lunar siting include: long operating lifetime, support from Lunar Outpost, vacuum environment, very low tracking rates, and ability to be reconfigured as science programs require. 


\section{DISCUSSION}

Woolf Recall that yesterday Roger Angel showed that while we are replacing the MMT with a single large aperture, with Columbus we are building a new MMT. The MMT concept is only appropriate if you have already started with the largest filled aperture that is possible. And if you want the same point source sensitivity as a 16 meter with thinly spread $4 \mathrm{~m}$ apertures, you need 256 of them.

Rolan: Extending the comment that Nick Woolf made, - as segment diameters rise, the full facesheet costs may drop, but one finds that reaction structure costs climb. (As diameters go up, bending rises by segment size to the 4th power; this has to be bought back with thickness, and thus the substrate cost can climb to offset pure faceplate based conclusions.) Thus, the optimum cost point for "5-tier" construction may be $<8 \mathrm{~m}$, especially when possible breakage is considered. Conversely, for "3-tier" designs (which are probably the preferred way to go), larger may be better.

Breckinridge: Yes, and it's certain that we need to do sufficient phase A studies to really let us get at some of these issues. I believe that NASA must have a much more aggressive Phase A system study than we had for HST. This is essential to: (i) identify trades between filled aperture and interferometry in terms of science payoffs; (ii) constrain cost; (iii) monitor technology development activities. The more money that is put up front in studies and analyses the cheaper the whole telescope is going to be (..APPLAUSE!!..).

mingworth: Let me make a point that is related to this discussion. There have been several workshops over the past few years where a rather rosy view has been taken about the potential of interferometry. There have been many scientists who have become concerned that we were starting down a path that was too narrowly defined in its technical focus without a clear understanding of the scientific goals of the astronomical community. The perspective has now become more sophisticated and an appreciation has developed that these approaches are complementary. If one looks at the observational phase space, the multiparameter phase space, there are regions which are optimally tackled by interferometry, and there are regions that are optimally tackled by large filled-aperture systems. The overlap is a grey area, which needs to be fleshed out scientifically and technically. I see this workshop as one of the first steps in this process. 


\title{
Large Optical Fabrication Considerations and Speculation about the overall NGST Configuration
}

\author{
Michael Krim \\ Perkin-Elmer Corp.
}

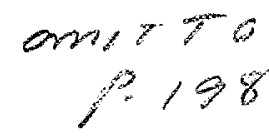

\section{Introduction}

It had been recognized at Perkin-Elmer, as elsewhere, that the the ability to efficiently produce optics in the eight to ten meter diameter class would be necessary for the next generation of optical telescopes. Depending on the application, specifically whether ground based or space based, these telescopes will have mirrors that, in general, are respectively monolithic or segmented. We believe that it is not necessary to distinguish between the two and that a manufacturing approach which is applicable to either type of mirror architecture is within our reach. This paper describes such an approach and the facility and equipment that would be required to implement it.

Since this conference is devoted to the science and engineering aspects of the Next Generation Space Telescope (NGST) and not mirror manufacturing in general, we have attempted to make our remarks about large mirror fabrication quite specific to that project. In this regard we will present a design concept for the NGST*, the intent of which is to provide the rationale for the selection of a particular mirror segmentation approach as well as to provide $\mathrm{s} 0 \mathrm{me}$ insight into other systems level design issues. Notable among these are the issues and considerations relating to system deployment and launch vehicle space constraints, i.e. real-estate allocation, and other mechanical aspects of the design. In any event it is exciting to speculate about what the large-scale design architecture of the NGST might be. It is happily reminiscent of the work that we did in the early 1970's when the configuration of the Hubble Space Telescope was first being "put to paper".

\section{Telescope Conceptual Design}

Why a Segmented Primary:

A concept for the overall architecture of the NGST is shown in Figure 1 where a sixpetal primary mirror, designed to facilitate automatic deployment in space without direct astronaut assistance, is utilized. The system architecture shown in the figure was evolved to meet the requirements and goals that were stated for the NGST. However, prior to discussing the logic behind the design, certain key assumptions were made at the outset. Firstly it was assumed that a launch vehicle sufficiently large to accomodate a monolithic, or fully assembled segmented, $10 \mathrm{~m}$ mirror would not exist in the USA's or anyone else's inventory in the 2010 to 2015 era. While the Advanced Launch System (ALS) is generally regarded as a $10 \mathrm{~m}$ vehicle, it would not be large enough to accomodate a fully assembled and erected $10 \mathrm{~m}$ system. As shown in the inset, a $10 \mathrm{~m}$ aperture telescope will have an

\footnotetext{
* This paper was written prior to receiving the preliminary systems description and data package prepared by Bely and Ilingworth. Any differences between what was assumed here and that data is completely unintentional. The conceptual design described in this paper was based only on the material contained in the announcement of the meeting and some (hopefully) educated guesses.
} 


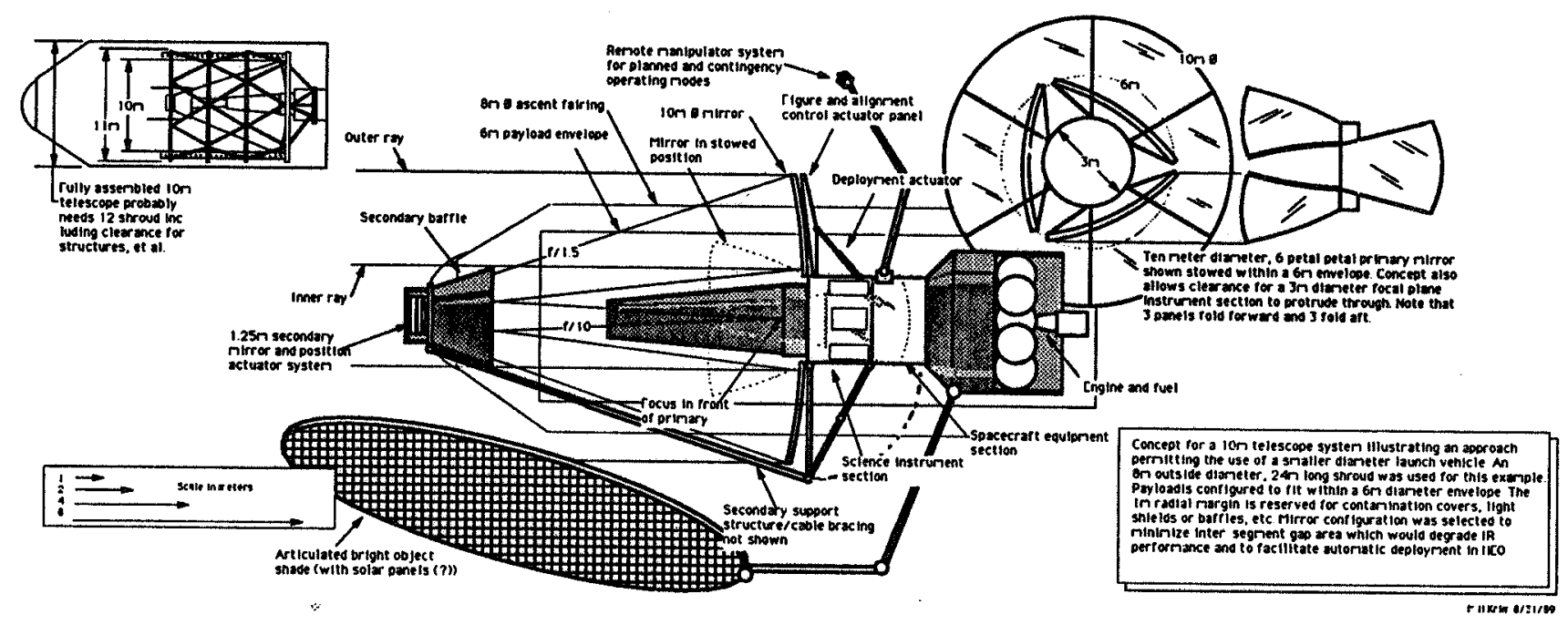

Figure 1 A Next Generation Space Telescope Concept

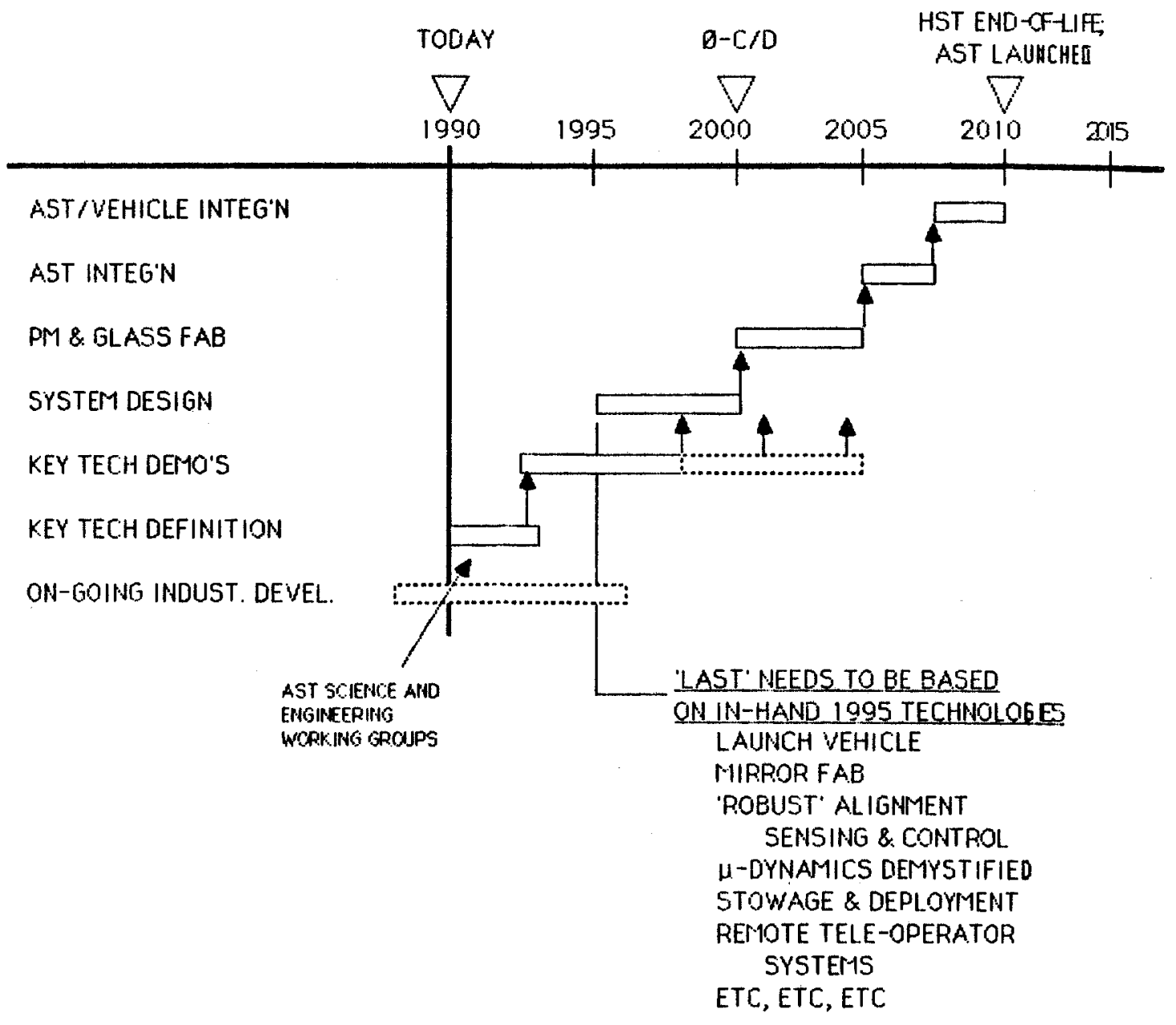

Figure 3 
overall diameter of 11 or so meters when baffling, structures and other system elements are accounted for. Adding shroud-to-structure clearances and making allowances for the fairing structure itself adds perhaps another meter. This suggests that a $12 \mathrm{~m}$ shroud would be realistic expectation.

Secondly, a segmented $10 \mathrm{~m}$ mirror system, including an integrating structure plus position control actuators, is probably a significantly lighter than a monolith of the same size. Presuming that a $10 \mathrm{~m}$ sandwich mirror is designed such that the maximum bending stress developed at a $7.5 \mathrm{~g}$ quasi- static launch acceleration does not exceed a safe stress limit of $2000 \mathrm{psi}$, it would weigh in the neighborhood of 50,000 lbs and be approximately 30 inches thick. This weight range is predicated on a family of mirror designs with 0.5 and 1 inch faceplates and a $10 \mathrm{x}$ areal density core. In Figure 2 we show the methodology used to determine the weight and proportions of these mirrors. The stress equation shown in that figure yields approximate answers only and is in no way intended as a substitute for rigorous analysis. While this mirror doesn't imply a final optimized design, it is felt to be representative of what might be achieved with a glass structure. This model further assumes that the mirror is supported at 3 points near its periphery. Some stress reduction can be achieved with supports at a $2 / 3$ 'rd radius location. The center deflection at 7.5 g's is on the order of 0.25 inches or about 9500 waves! It follows then that a (force) actuator system and s reaction plate would be needed to compensate for "some" non-recoverable launch strains. It follows also that an auxiliary multi-point launch support system would need to be extrémely stiff, and consequently heavy, if it were to be effective in limiting deflections for such a mirror.

On the other hand, $3 / 4$ of an inch glass distributed over a $10 \mathrm{~m}$ diameter would weigh only $7500 \mathrm{lbs}$. However a $3 / 4$ inch thick mirror segment in excess of 50 inches in diameter will require more than a simple 3 -point support system to limit stress levels to 2000 psi. Therefore thin shell (meniscus) mirrors of this type would need to employ displacement type actuators to couple it to a structurally efficient composite sandwich (or truss) support structure. These are "stiff" actuators which directly impart a displacement to the mirror as opposed to a "60ft" actuator such as employed on the HST which apply a force field to the mirror. This is an important but subtle difference. The idea here is to "....use structures for what structures do best and use mirrors as mirrors".

The sandwich mirror will require fewer but more powerful actuators than the thin meniscus mirrors. Both approaches will require reaction, i.e. support, structures. Qualitatively the weight of the actuator systems will be "a wash", at least to first order. In that case, the segmented meniscus approach could save about 25,000 lbs over a monolithic approach. The additional weight of the deployment mechanisms and possibly a robotic arm for teleoperator- assisted erection will still result in a positive weight advantage for the segmented approach.

Finally one might question the technical and economic feasibility of producing a $10 \mathrm{~m}$ diameter lightweighted (sandwich) mirror blank from a low expansion material such as Zerodur or Corning's ULE within the next several decades. For these reasons, the ability to efficiently produce a segmented primary mirror appears to be an important technology for any NGST concept. Implicit in this is also the technology needed to ensure the coalignment to fractional micron tolerances of the segments.

As tempting as it is to dismiss orbital assembly as something that could be accomplished by suited astronauts on a space station, the dependence of the NGST on the availability of such a resource was avoided. Even if it were assembled in low earth orbit 
FIGURE 2: MIRROR WEIGHT ESTIMATING METHOD

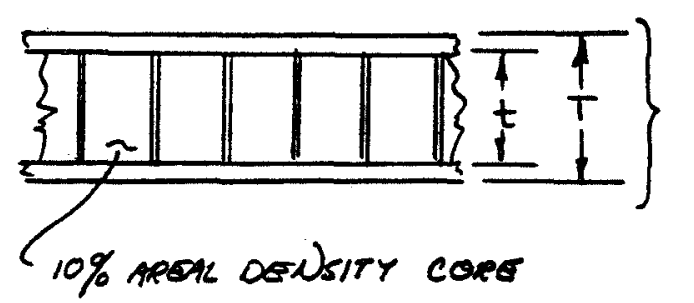

the "equivalent sou lo" thickness hang The SAME BenG STIFFNESS IS $t_{e}=\left(T^{3}-t^{3}\right)^{y_{3}}$

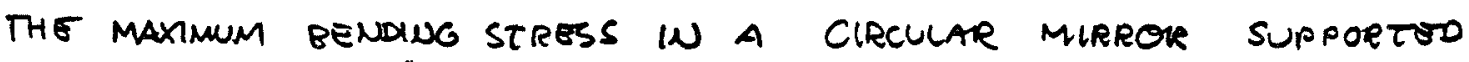
at 3 points $120^{\circ}$ apart. at the edo is

$$
\sigma_{\text {MAX }} \cong \frac{W_{g} T}{t_{e}^{3}} \quad \text { ' } g^{\prime} \text { is LOAD FACTOR }
$$

For $1^{\prime \prime}$ faceplates, $t=T-2$; for $1 / 2$ " Faceplates, $t=T-1$ FOR I" FACE, plates:

a) $t_{e}^{3}=T^{3}-(T-2)^{3}$

b) $W=\pi R^{2} \rho(T-.2 t)=\pi R^{2} \rho[T-.9(T-2)]=\pi R^{2} \rho(.1 T+1.8)$

c) $\sigma=\frac{\pi R^{2} \rho(.1 T+1.8) g T}{6 T^{2}+12 T-8}$

FOR. $5^{n}$ FACEPLATES

d) $r=\frac{\pi R^{2} \rho(.1 T+.9) T}{3 T^{2}-3 T+1}$

PRESUMING $T^{2} \gg T$

5') $\left.\sigma=\frac{\pi R^{2} P(.1 T+1.8) g}{6 T} ; c^{\prime}\right) r=\frac{\pi R^{2} p(-1 T+.9) g}{3 T}$

FOR $\sigma=2000$ pSI, THE OUSTALL MIRROR THICKNESS, FOR A $7.5 \mathrm{~g} l O A D$ can be calculated

$T_{1}=30^{\prime \prime}$ and $\omega=48,25+$ lbs

$T_{y_{2}}{ }^{n}=$ NO SOLUTION @ 2000 psi

185 
( LEO ) and that there was an available orbit transfer vehicle (OTV), the boosting of a fully assembled, relatively frail and flexible NGST represents a major controls, contamination avoidance, and structural dynamics challenge. Therefore we opted to configure the design to be automatically deployed in the specified high earth orbit (HEO) and to require only a single launch to achieve operational status. Finally, it is assumed that it would be desirable to achieve an operational date of about 2010 for the NGST. That is consistent with the HST's 15 year lifetime. Completing the NGST within 20 or 25 years from now implies that the technologies that will be used for it's design and construction are no more than extensions of what can be accomplished today, particularly with regard to materials. In fact it might be stated that the NGST cannot afford to use anything later than 1995 technology. We show this in Figure 3 where an attempt at a development schedule is presented. This schedule tacitly implies therefore that the primary mirror will be glass, such as Zerodur of Corning ULE, although the rapid emergence of new mirror materials such as silicon carbide may alter this conclusion in the near future.

A few comments about the concept drawing need to be made here. To a large extent, the drawing began with an outline drawing of the assumed launch vehicle which defined the envelope for the stowed NGST. An $8 \mathrm{~m}$ outside diameter ascent fairing with a $7 \mathrm{~m}$ diameter available for the payload was assumed. While such a vehicle does not exist today, it is within the range of capabilities that are being considered by the Air Force and NASA. For the purposes of this paper, volume constraints were considered to be more critical than weight. The ability to stow a $10 \mathrm{~m}$ system in a $7 \mathrm{~m}$ payload envelope and then automatically deploy it places far greater demands on on the resourcefullness of the designers than weight, at least in my opinion.

\section{Primary Mirror Segmentation:}

Segmentation concepts for primary mirrors encompass the (nearly) hexagonal shapes employed by the Keck and LDR designs, multi-row trapezoidal concepts often favored by metrologists since each row or circle contains identical facets but which are wasteful of material, and petals such as we are suggesting for the NGST. The principal motivation for the petal approach where each petal is in itself a monolithic segment is infra-red operation. To minimize self-emission or thermal noise arising from the telescope, the total area of non-reflecting surface between the mirror segments needs to be minimized. This is accomplished with the petal approach. A monolithic individual segments which might be pre-assemble and aligned on earth to take advantage of the deployment advantages of the petal configuration but the idea of reducing gap area between the segments would be defeated.

A six-petal approach was selected because it represents the minimum number of petals that can be stowed within the assumed $7 \mathrm{~m}$ diameter cargo bay or ascent fairing (shroud) payload envelope. This was illustrated in Figure 1 where three petals are folded forward and three back. The superscribed circle enclosing the segments is $6 \mathrm{~m}$ leaving margin between the assumed $7 \mathrm{~m}$ allowable payload envelope. At this stage of the concept, this margin needs to be reserved for mirror support structures, thermal control surfaces, and deployment mechanisms. The inscribed circle is $3 \mathrm{~m}$ which is sufficient for the telescope instrument science instrument.

Another petal approaches such as a "vegetable steamer", shown in Figure 4, in which all the petals are folded in one direction but overlap in sort of a spiral fashion was rejected because it forced the petal assemblies to be too thin for practical applications. Another 

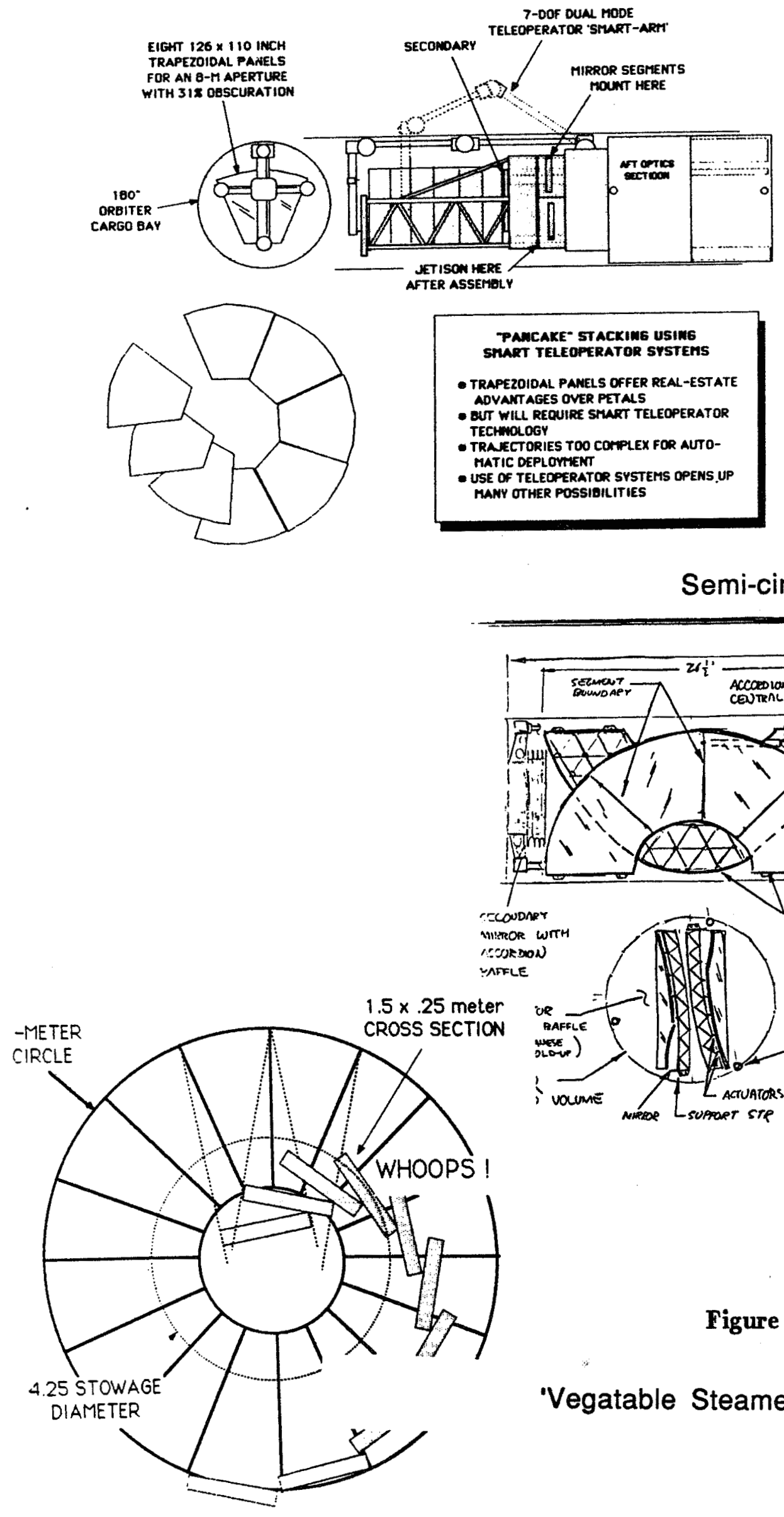

Semi-circular Segment Concept

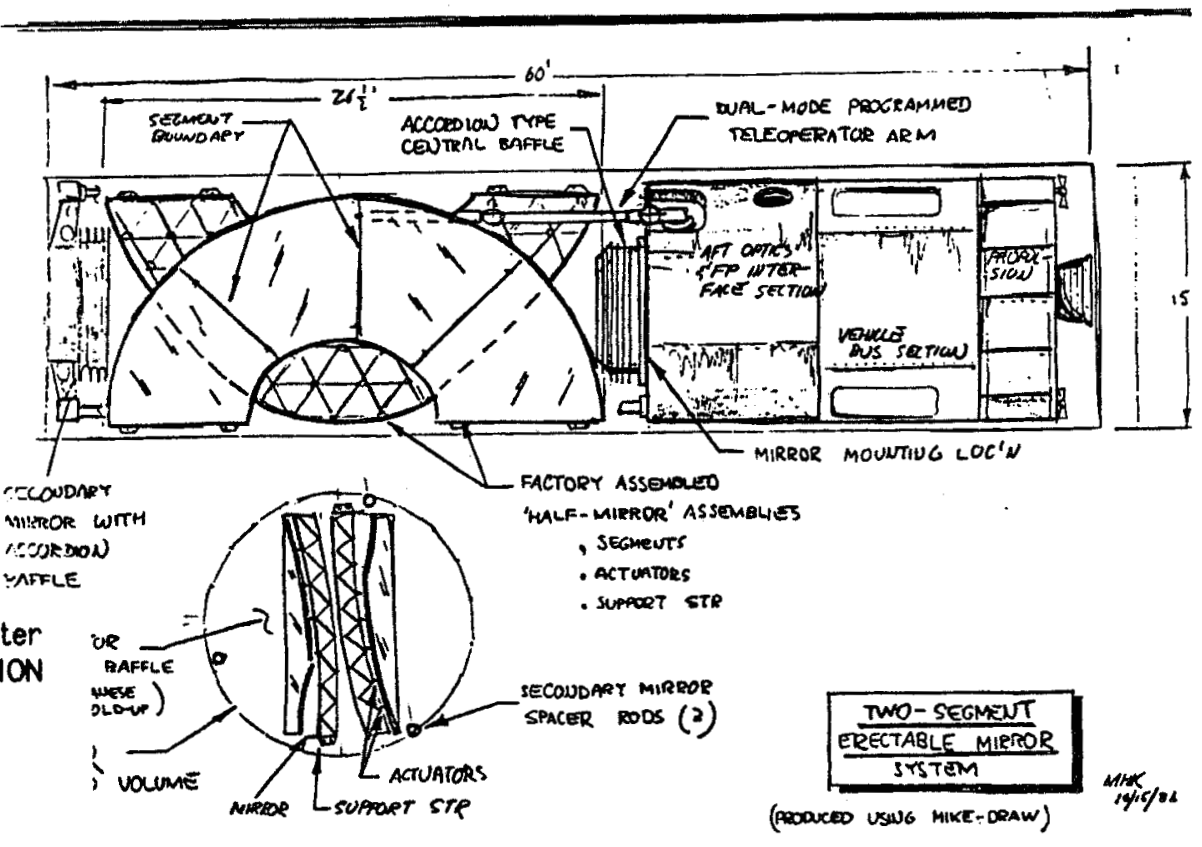

Figure 4

'Vegatable Steamer' Stowage Concept 
approach where the mirror was divided into only two segments, split along a diameter and stowed on either side of the central telescope structure, also shown in Figure 4, was considered as way of minimizing the inter-segment gap area. While it has many advantages from a mirror producibility and performance aspect, the lack of rotational symmetry was judged to be a disadvantage from an automatic deployment aspect. As shown in that figure, such an approach would include a robotic arm to 'maneuver' the segments and other system elements into position as an alternative to the more fully automatic deployment approach possible with the six-petal configuration.

\section{Figure Sensing and Control:}

The critical difference between segmented and monolithic mirrors is that the former requires that each segmented be generated and figured to an absolute radius of curvature while for a monolith, the radius of curvature only needs to fall within some nominal range defined (primarily) by the adjustment range of the secondary mirror. A rough estimate of overall wavefront error as a function of the difference in the radius of curvature between segments can be obtained from the equation

$$
\Delta W F_{r m s}=\frac{1}{3.46} \frac{d^{2}}{4 R} \frac{\Delta R}{R}
$$

where $d$ is the smallest segment dimension and $R$ is the nominal radius of curvature. The precision of $R$, expressed as $\Delta R / R$ is on the order of $310^{-6}$ for a wavefront error of a twentieth wave (rms) and for a nominal segment size of $2 \mathrm{~m}$ and a radius of curvature of $30 \mathrm{~m}$. This is equivalent to a gross saggita tolerance of 0.04 micrometers or about 1 fourteenth of a wave. Clearly this degree of precision is difficult, if not prohibitively costly (my opinion), to achieve if the segments were individually produced and/or if there were no means for adjusting curvature after the segments were actually completed. The four mirror telescope design advocated by Meinel and the LDR community is an elegant means of correcting radius of curvature errors arising from manufacture. For the NGST however, we felt that the additional two reflections imposed by the tertiary and quaternary mirrors might be too expensive from a throughput aspect in the short end of the operating spectrum. A more appropriate solution for this application we thought would be the use of shape (figure) control actuators which physically bend the segments to the correct curvature, if required, to correct for manufacturing errors. The figure control system, whether used directly on the primary mirror or on an image of it on a quaternary, also provides a correction capability for errors that might arise from the large temperature change between the shop and orbital operation at the desired 100 degrees Kelvin. Of particular concern is the effect of this spatially uniform temperature change on any CTE anisotropy that might (will) be present in the segment substrate. We will take advantage of the ability of the figure control system to correct low frequency aberrations and thereby relax the manufacturing tolerances in this range to about a half wave rms. The presumption here is that a properly designed actuator system can provide a wave of correction, at least in the long spatial frequency domain. In the mid- spatial frequency regime, beyond the (presumed) correction capability of the actuator system, the as-manufactured figure quality will be on the order of 0.01 waves. As with the Hubble Space Telescope (HST), our optics manufacturing approach is based on the use of small tools operating at a uniform and constant interface pressure with material removal being proportional to velocity of the tool path at any given point. This is accomplished at Perkin-Elmer with a machine referred to as the Computer Controled Polisher (CCP). 
The concept of a CCP capable of figuring a $10 \mathrm{~m}$ mirror will be described later in this paper, after the architecture discussions are completed.

The ability to compensate for long spatial frequency errors with actuators mitigates against the need for inherently rigid mirror segments. In fact, rigidity can be a detriment in the design of a figure control system (in certain instances). Provided that figure errors in those spatial frequencies equal to or shorter than the span between three (theoretically two) actuators are within acceptable limits ( 0.01 waves rms), the low spatial frequency errors can be relatively large, several waves is not unreasonable although we have not yet quantitatively explored just how inaccurate one could allow the optic to be before the sensing and control system becomes an impractical theoretical exercise.

It is conceivable therefore that the substrate could be a relatively thin sheet of material, a $25 \mathrm{~mm}$ ( $1 \mathrm{inch}$ ) for arguments sake, provided of course that it can be generated and figured. It is (well) beyond the scope of this paper to address the trades relating thickness, actuator density, sensing and control system complexity, structural integrity, weight, inherent reliabiity, and so on. Suffice for this discussion, the ability to generate, figure, and polish extraordinarily thin substrates is an important consideration in the manufacture of a NGST type mirror.

Other Interesting Speculations About the Concept: It was assumed that at least a dual focal length capability, for many of the same reasons this is the norm with terrestrial telescopes, would be desired. For illustrative purposes it was assumed that the visible and infrared final focal ratios were 10 and 20 respectively and that the primary mirror would be $f / 1.5$. Focal ratios ( $f / n o ' s$ ) greater than 20 were not considered for NGST because of the size related and exponentially increasing vibration and structural sensitivities. Final focal ratio-to-detector matching is as usual, an instrument responsibility. These choices were not based on any quantitative trades or analyses. An f/ 1.5 primary was judged to be as fast as one could go optically and still achieve a 20 or so arc minute total field of view. The selection of an $\mathrm{f} / 10$ visible and an $\mathrm{f} / 20 \mathrm{IR}$ focal ratios offers the ability to minimize the size of the secondary mirror to reduce self emission for IR operation. This could be at the expense of having two secondaries that could automatically be interchanged. This is probably a disadvantage from a weight, dynamics, and failure potential aspect. Several other more attractive approaches for achieving a dual focal length capability could be considered including the use of common $\mathrm{f} / 10$ fore-optics with the visible focal clearance and employ a tertiary to achieve a $2 \mathrm{x}$ final magnification as well as to provide an exit pupil for the location of a cold stop.

Unlike the HST, where a $3 \mathrm{~m}$ superscribed circle enveloped the instrument package, it may not be necessary to locate the NGST's final focus behind the primary. Presuming that a $3 \mathrm{~m}$ diameter instrument section is still reasonable, then the instrument section might be allowed to pass through a central hole in the mirror at the expense of a linear obscuration ration of 0.3 . A $1.25 \mathrm{~m}$ secondary with a magnification of 6.6 would produce an $\mathrm{f} / 10$ image which comes to focus approximately $0.6 \mathrm{~m}$ ahead of the primary. This is what is shown in the conceptual drawing.

Regardless of what form the final optical design might take, the secondary mirror needs to be supported by a structure which, from an idealistic IR performance standpoint, should not introduce any obscuration in object space and must not introduce any obstruction in (convergent) image space. An off-axis system would fulfil these desires. A structure such as the HST secondary mirror support truss would be a good practical compromise. While the the obscuration from the thin spiders legs are in object space, the rest of the structure 
is totally outside of the optical envelope. However, for all intents and purposes, a $10 \mathrm{~m}$ cylindrical truss such like the HST is a non-solution for the NGST, it is not amenable to automatic deployment. One attempt at a solution for a deployable secondary mirror support structure which doesn't intrude into image space is illustrated in the concept drawing. It consists (very conceptually) of an arrangement of telescoping tubes and rotary deployment actuators which can be stowed within the $7 \mathrm{~m}$ payload envelope. As shown in the drawing, the erected truss will not intrude into image space, meeting the self-imposed (inflicted?) goal for IR performance.

\section{Baffling:}

Bright object baffling, i.e. the earth, sun, and moon, is a persistent problem for giant, space deployable telescopes. The use of a positionable bright object blocking screen might prove to be a more easily implemented solution, albeit with (potentially severe) operational constraints. While on the subject of large distributed surfaces, the concept drawing does not show any solar arrays. Hopefully by the time NGST becomes operational compact power sources such as nuclear or radioisotope generators become politically as well as technically acceptable. The cumulative effect of submicron micrometeoroid impacts on the surface of the primary mirror is an issue that requires further evaluation. A baffle enclosure, even one composed of several layers of multi-layer insulation (MLI), can provide some protection against this source of degradation.

So much for thoughts and speculations about what the NGST might look like and why. Now I shall describe how the mirror described in the concept might actually be produced.

\section{Large Mirror Manufacturing options}

As we stated at the beginning of this paper, Perkin-Elmer along with others in the field, have been exploring alternative ways of producing optics in the 8 to 10 meter size range. Our experience lies with the successful application of small tools, the CCP described earlier, and we can extend that technology from the $90^{\prime}$ th wave, $2.4 \mathrm{~m}$ HST primary to the the $10 \mathrm{~m}$ NGST. All of the technology to accomplish this facility scale-up is currently in place and we believe that such a mirror could be completed in five years, including the production of the blank by Corning or Schott and the parallel construction of the building and the fabrication of the metrology and polishing equipment. Subsequent mirrors of that size, if there are any and we hope that there will be, could be figured at the rate of one every two years in that facility. The model of such an optical manufacturing facility is shown in Figure 5 and will be described more fully later.

\section{Segment Manufacture:}

In addition to the basic issue of scaling up our optical shop to accomodate 8 to $10 \mathrm{~m}$ HST type (monolithic) mirrors, the question of radius of curvature matching of individual segments to the kind of precision described earlier has been also been addressed at PerkinElmer. Making matched segments, one at a time, has successfully been accomplished under government contract. In this instance the segments were spheres, approximately $1 \mathrm{~m}$ in diameter with a focal length of $5 \mathrm{~m}$ (as I recall). While successful, this approach is 


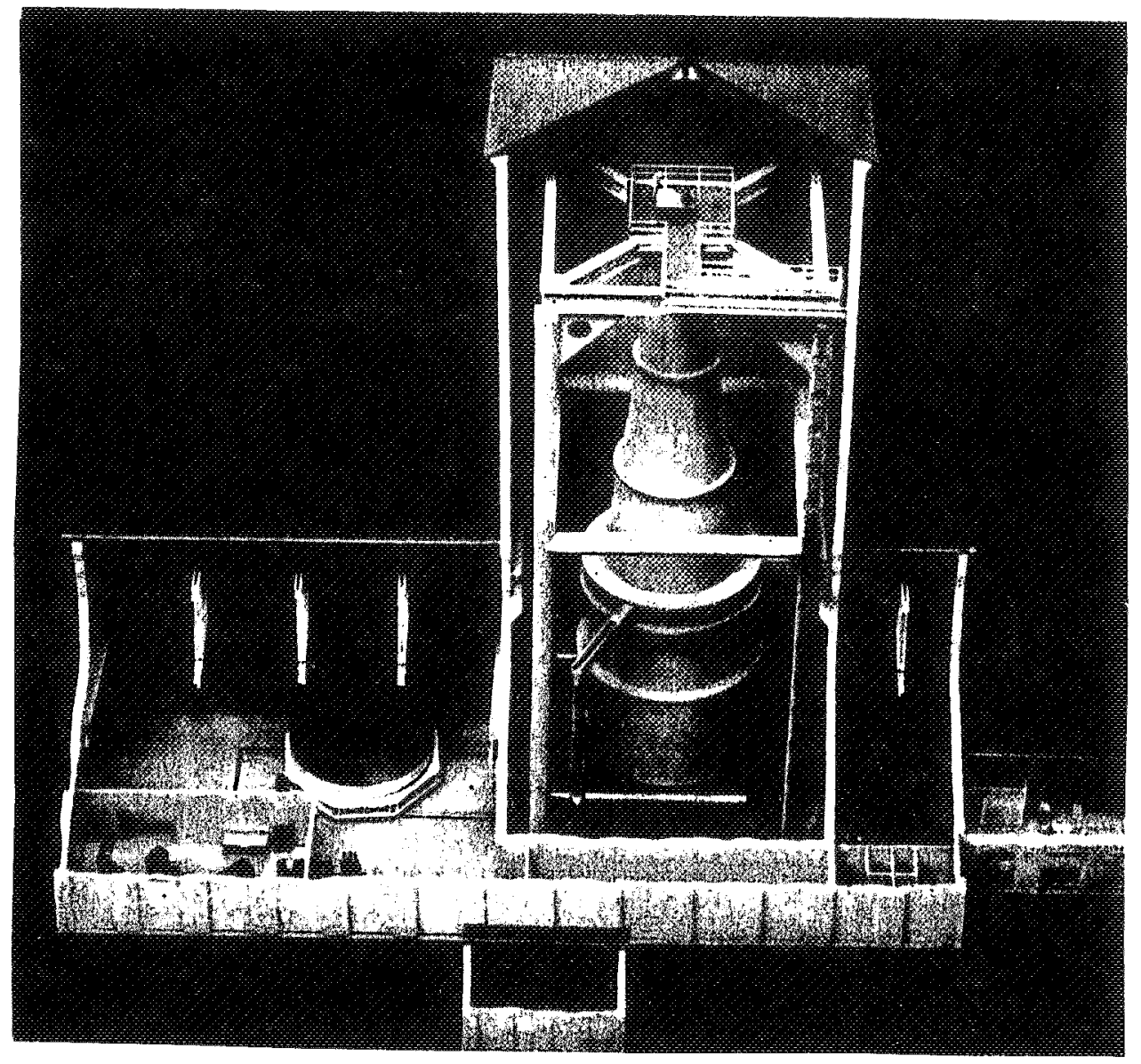

Figure 5: Model of the $10 \mathrm{~m}$ Optics Manufacturing Facility

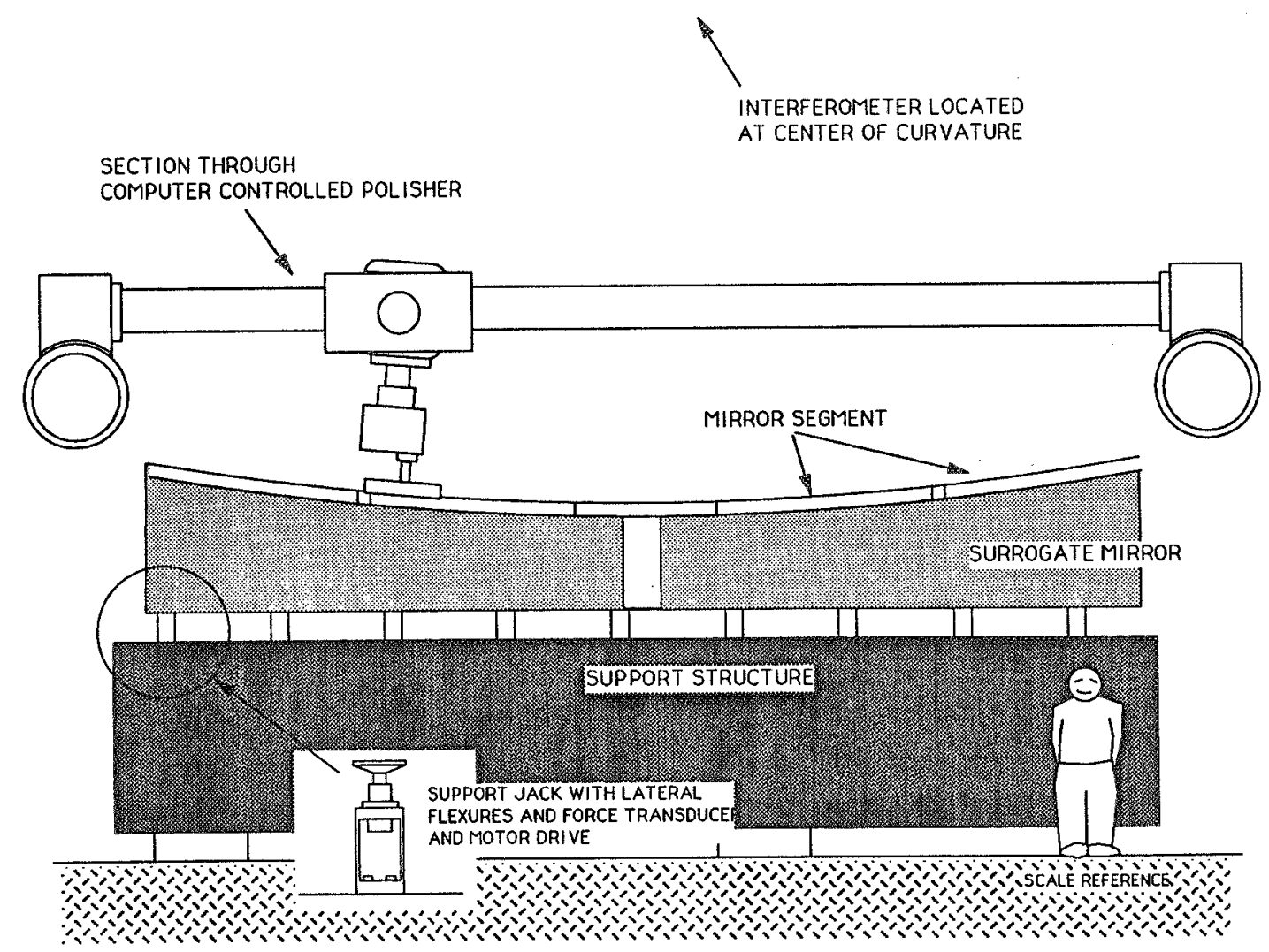

Figure 8 Method for simultaneously producing all segments with large CCP 
extremely costly in terms of schedule. As described earlier, curvatures can be adjusted to some extent with actuators, or as in the case of Keck, with preset "coercers", i.e. a "set and forget" actuator. Or at least that's what I think is being done. On another program that we were associated with, the Large Deployable Reflector (LDR), the concept of "semi-replication" was evolved where a graphite tool was used as a master for molding thin quartz substrates to a common radius of curvature. In this instance quartz was selected because of it's superior homogeneity. This was an important factor since the temperature change between fabrication and operation was several hundred degrees (F) and the $\Delta R / R$ precision had to be preserved. This process was subsequently demonstrated in a joint Perkin-Elmer/Hereaus program, under the DARPA umbrella program called Rapid Optical Fabrication Techniques (ROFT). In another program sponsored by NASALewis, we successfully laminated thin glass sheets to (nearly) matching CTE (coefficient of thermal expansion) composite substrates preformed to a spherical surface. These two programs are mentioned only for completeness since enormous material and process scaleup problems would need to be solved before they could even be considered as a candidate for the NGST mirror. From here on we shall confine our discussion to glass mirrors, albeit unusually thin by present standards.

The ability of the CCP to be scaled up to $10 \mathrm{~m}$ sizes begs the question, "Why not temporarily attach all the segments to a $10 \mathrm{~m}$ tooling plate and generate them all at once?". In principal, the concept is as illustrated in Figure 6. The idea here is to "fool" the CCP and/or the optical shop into thinking that they are working on a monolith. In this manner it is believed that all of the segments will have the correct curvatures, eliminating the $\Delta R / R$ concern described earlier. This is really an extension of the technique developed by Dr. Robert Leighton of the California Institute of Technology for the rapid and accurate manufacture of $10 \mathrm{~m}$ class sub-millimeter aluminum reflectors.

There are three issues that must be resolved for such a technique to work: what material should the tooling plate be made of; how are the segment substrates attached to the tooling plate; and what will be the extent of sleeking or other gap related 'damage' and how can it be controlled. Ideally the tooling plate would look very much like a relatively thick mirror and be constructed from the same material as the segments. The segments, presuming that they are constant thickness elements of a meniscus, would be attached to the concave front surface of the tooling plate. This front surface would be generated to a sphere whose radius of curvature matched the back surface of the segments.

In addition to the obvious choice of ULE or Zerodur for the tooling plate, a much lower cost and more readily available material, slip cast silica, has merit. Slip cast silica, as produced by the the Cerodyne Corporation, is used for high temperature tooling by the automotive and aircraft industries. It is cast, as implied by the name, from a fused silica slurry which may optionally contain a pebble sized fused silica aggregate which improves its strength and reduces shrinkage during the drying process. It is fired at a relatively low temperature (1100 degrees C) after casting to produce a stable, structurally sound shape which is about $95 \%$ as dense as the parent material. It can (probably) be cast over styrofoam or similar blocks to achieve for lightweighting if deemed necessary.

The key attributes for the attachment method to join the monolithic tooling plate and the segments include:

a) the ability to fill (the small but inevitable) voids between the the mating surfaces

b) the ability to set up without shrinkage or other mechanisms for inducing strain in the substrate 
c) the ability to allow the pieces to readily be separated after figuring

d) stability or the absence of flow characteristics such as exhibited by pitch

e) and sufficient stiffness so that the bondline doesn't act like an elastic foundation (and foul up the edges)

Candidate adhesives or bonding agents include quartz filled epoxies with a silver parting layer on the segment, quartz filled hard wax, certain of the Cerro near-room temperature melting metal alloys (used in the turbine blade industry for tooling purposes), as well as vacuum chucking. Development of a large scale segment attachment method is clearly a development area simply because it hasn't been done yet (we believe).

\section{Ten Meter CCP:}

Extending the CCP concept from its present $3 \mathrm{~m}$ capability, to $10 \mathrm{~m}$ is relatively straight forward. The CCP consists of an X-Y drive system which enables a (nominally) vertical spindle grinder to travel over the surface of the mirror. The spindle axis is gimbal mounted to provide a tilt degree of freedom allowing the spindle to be normal to the surface at any point. The spindle can be programmed to follow any desired path over the mirror surface, i.e. spiral, raster, or elliptical in the the case of an asymetric mirror used in an all reflecting Schmidt telescope. CCP is designed to work with unusually small tools (as small as 1 inch on the HST where midspatial frequency figure errors were less than a 150 'th of a wave) which operate at constant and uniform pressure. The amount of material removed at any specific point on the mirror is therefore proportional to the total integrated dwell time of the tool at that location. The tool velocity profile which defines the path the tool will follow and its velocity at any position along that path, is controlled by specially developed software.

This software is designed to convert interferometric data into actual machine command tapes. CCP simulation software which is based on specific tool material removal profiles and proven to be accurate on the HST, is used to verify the command tapes prior to an actual material removal 'run'. This software will be preserved as the machine size is scaled up. The surrogate mirror, or tooling plate approach, advocated for manufacturing the segmented NGST primary, greatly simplifies the metrology operations over what would be required with individual off-axis segments. Metrology for a $10 \mathrm{~m}$ optic and our envisioned facility for accomplishing it will be described next.

Unlike large 5-axis numerically controlled machines, the CCP is not a massive system. Because material removal is (simply) a function of dwell time and not vertical position of a tool, the CCP does not need to be extraordinarily rigid. Its precision is primarilly a function of metrology and the command tape algorithms and not the stiffness of the ways. Therefore expandability is readily accomplished without having to design and construct massive machine bases or turntables. In fact it should be noted that there are several commercially available precision X-Y machines that are large enough to adapt to a $10 \mathrm{~m}$ CCP. Notably among these are the Cincinnati Milicron modular and the Swedish Arboga line of precision (tape laying) machines.

\section{A Ten Meter Optics Facility:}

The $10 \mathrm{~m}$ optics manufacturing facility shown in Figure 8 evolved from a variety of considerations. These included:

a) vacuum metrology at 10e-4 torr to avoid optical noise caused by air turbulence over the 200 foot long optical path 
b) insensitivity of the 100 foot high metrology tower to ambient seismic and acoustic noise

c) in-situ metrology to minimize moving and handling the $60,000 \mathrm{lb}$ surrogate mirror (tooling plate and segment assembly) between figuring and metrology operations

d) the use of the CCP in a grinding mode to generate and aspherize the segments.

The ability of the CCP to operate with an abrasive wheel in addition to its more familar loose abrasive grinding and polishing mode reduces, or might possibly eliminate, the need for either a $10 \mathrm{~m}$ spherical generator or a precision grinder such as a Campbell. More importantly this would eliminate the need for a large precision turntable. Rough shaping of the surrogate mirror (tooling blank) can (probably) be as 'sloppy' as $\mathbf{0 . 0 2 5}$ inches or 1000 waves and still be within the capabability envisioned for the CCP in a wheel grinding mode. Since the CCP spindle path can follow almost any mathematically describable shape, and since the spindle has a two axis tilt capability, by commanding it to follow a series of concentric circular paths it can duplicate the kinematics of a classical generator without a massive turntable. Following this quasi-generating operation with a spiral path where the ' $\mathrm{Z}$ ' direction or spindle axis is also programmed, the (nominal) Imm or 0.040 inch of parabolization (or whatever) would also be accomplished without a turntable (and without a large Draper).

In the next section, we will describe a facility concept which is based on the use of this manufacturing approach with special emphasis on metrology.

\section{Facility Concept:}

As shown in Figure 8, the facility is essentially contained within a 150 foot high, 60 foot square tall building. This building is surrounded by a lower building which houses engineering offices, a small machine shop, and an area for integrating the mirror assembly with the shop support system. Because this operation requires high capacity overhead cranes and because the cranes cannot pass by the metrology tower support columns, this assembly operation is carried out in this support area. Once the integrated shop support system and mirror are wheeled into the tower and firmly jacked into position, we do not envision it to be moved again until after the final interferograms are completed.

\section{Metrology Tower:}

The metrology tower (Figure 9) is erected on a massive seismic mass, a 2000 ton concrete foundation which 'floats' on a thick bed of crushed rock. This foundation is separate from the foundation on which the rest of the building is constructed. The tower itself is constructed from four $\mathrm{lm}$ diameter $1 \mathrm{smm}$ steel columns braced to each other with a platform on top for the metrology equipment. To absolutely eliminate any resonance amplification of the basic tower modes or to prevent the existance of local, acoustically excited 'ringing' modes, the columns will be (partially) filled with gravel. We have designed the tower to have a 1'st mode of $1.3 \mathrm{cps}$, including the non-structural mass of the gravel. This is well above the noise spectra usualy associated with groundborne 'cultural' noise.

\section{Vacuum Tank:}

We had considered in-air (as was employed on the HST program), in helium, and in vacuum metrology. Ultimately we came to the belief that a vacuum approach is the least likely to provide 'regrets' during mirror fabrication. It is a conservative approach but 

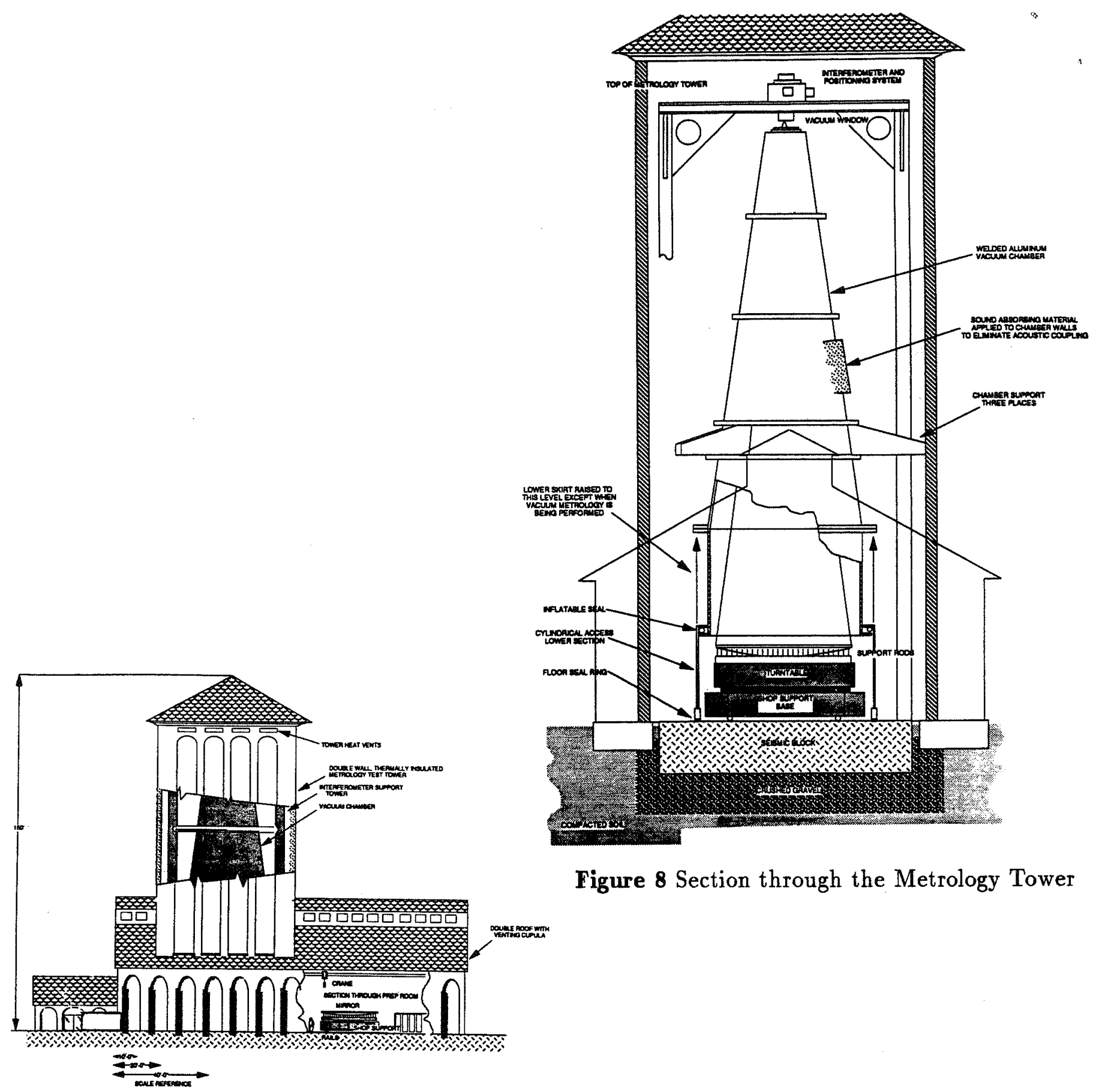

Figure 8 Section through the Metrology Tower

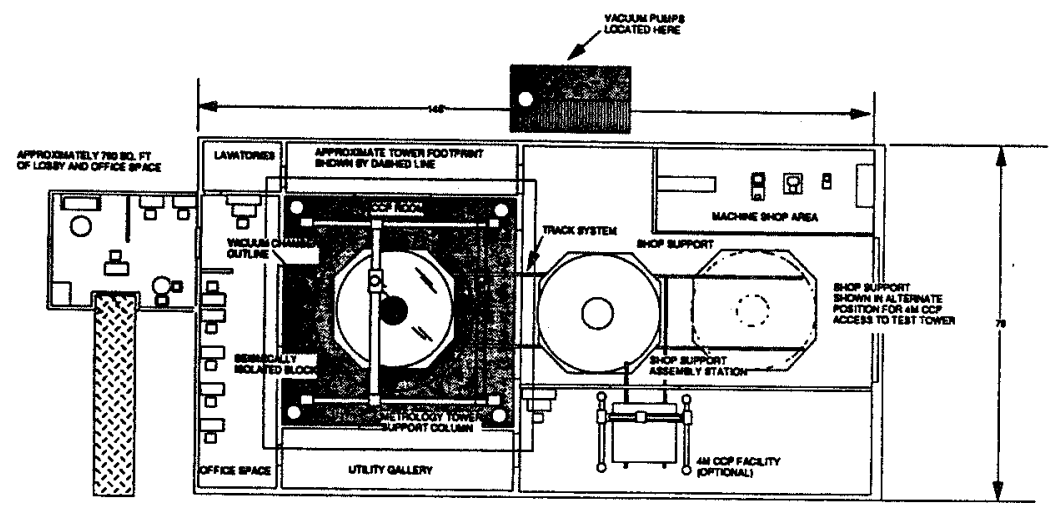

Figure 7 The Large Optics Facility as envisaged on the coast of Southern California 
one which we have absolute confidence in. In addition to the extremely long optical path length which itself might be a problem, stabilization times for HST metrology approached 6 to 8 hours in a closed chamber that had about tenth of the volume needed for the NGST. By eliminating the air, this stabilization time issue is eliminated. We believe that a mild $\left(10^{-4}\right.$ torr) vacuum can be achieved in the same or even less time. containment vessel would be required to contain the helium in any event. Rather than helium, it could be 'filled' with vacuum.

The vacuum chamber is suspended from the walls of the building and does not have any direct mechanical connection with the seismic mass or with the metrology tower. However the seismic block does serve as the lower closure for the chamber. We accomplish this by constructing the vacuum tank with a cylindrical skirt at its lower end. During figuring and polishing operations or when access to the mirror is needed, this skirt is raised approximately 18 feet above the floor. During those metrology operations when we will require vacuum, the skirt is lowered to the floor and an inflatable rubber-like seal will close the gap. As vacuum is increased, the skirt will be forced against the floor compressing a compliant gasket on its lower end. The inflatable seal is a barrier against the transmission of mechanical noise from the vacuum tank to the seismic mass and consequently the metrology tower.

\section{Metrology:}

Full aperture phase measuring interferometery (PMI) will be used for the NGST. It is important to note the inclusion of the words, 'full aperture'. We believe that working at the full circular aperture with all of the segments in place on the surrogate mirror, and consequently having symmetry about the optical axis, will lead to more accurate as well as more rapid metrology. The ability to simultaneously figure all of the segments in place is a distinct advantage of the CCP approach which is (conceptually) unlimited in size.

We investigated the effect of vibration on metrology accuracy, of particular interest with the interferometer located approximatly 100 feet above the mirror. Using a PMI modulation frequency of $500 \mathrm{cps}$ and assuming a (tentative) error allocation of 0.02 wave peak to peak, we determined that 6 microns of lateral motion occurring at the tower's natural frequency could be tolerated. This is equivalent to an acceleration response at the end of the tower of 40 micro-g's $(0.000040 \mathrm{~g}$ 's). At the base of the tower whose amplification at resonance will be extremely low as a result of the gravel fill, we estimated that disturbance levels on the order of 15 to 20 micro-g's could be permitted.

Measurements made on the shop floor of our Danbury facility, which is within a thousand yards of a major truck highway rarely exceeded 1.5 we do not believe that vibration will be a problem, despite the $30 \mathrm{~m}$ separation between the mirror and interferometer.

Unlike the HST where an all-reflective null corrector was employed, we believe that this would be impractical for the NGST primary. The reason is that the mirrors would have to be approximately $2 \mathrm{~m}$ in diameter (or so I'm told by the optical designers). The HST null corrector design cannot be simply scaled to the NGST because the $f /$ No of the latter is almost twice as 'fast' as the HST's 2.3. Suspending two $2 \mathrm{~m}$ mirrors on metrology mount systems whose precision needs to be equal. to the HST mount is judged to be an unacceptable and unnecessary risk. Therefore a refractive, or possibly a diffractive, approach will be employed. Obviously different nul correctors will be used for coarse IR and for precision visible interferometry. 


\section{Concluding Remarks:}

I apologize for a possibly too long and/or a too qualitative a paper. However I hope that some of the concepts and ideas contained in it were of interest and relevance to the NGST program. As for me, it is always exciting to start off with a blank sheet of paper and begin contemplating the architectural issues associated with a new and bold initiative such as the Next Generation Space Telescope.

\section{DISCUSSION}

Angel: A comment on the isolation of the tower. The tower that we are building in Arizona in the mirror lab (in the football stadium!) is very similar to yours. The resonant frequency is $10 \mathrm{~Hz}$. We have put it on isolators which give the concrete base a frequency of about $1.5-2 \mathrm{~Hz}$. We were concerned that if it was just put directly into rock, the isolation would not be adequate at the critical frequencies.

Krim: We based the design here on what has been done in Danbury. There we have an air system which can also be disabled if we wish. The reason that the air system is used is because of sources of vibration some several hundred yards away. The isolation system is quite adequate when these sources of vibrations occur. The resonant frequency of the tower was $6 \mathrm{~Hz}$ before the non-structural gravel mass was added. So the towers are probably quite similar.

Angel: Do you have any isolation at 1-2 Hz from the rock bed?

Krim: No. We don't have any significant disturbances at 1-2 $\mathrm{Hz}$ except at the 1-2 micro-g level. We have measurement data on this.

mingworth: The HST mirror is clearly one of the best ever produced. However, we have come to realize that the HST mirror will show some scattered light, particularly in the UV. This arises because of residual structure on the surface from the footprint of the small tools used in the computer controlled polishing. Have you thought about using technology such as the stressed lap approach that Roger Angel discussed? This will not leave structure on scales that are a particular problem for the UV-visible region.

Krim: This is an interesting question. We do need quite lightweight mirrors, with thin faceplates for future large telescopes. A problem arises when one uses relatively large tools which cover several cells and the resilience of the mirror differs from the rib to the center of the cell. A large tool will exert a non-uniform pressure profile and will rub away more material over the ribs. This introduces "quilting". One of the advantages of CCP is that we never span more than a cell, and so we avoid "quilting". We will be looking further at the question of the mid and high spatial frequency content.

mlingworth: It would be good to quantify whether the "quilting" is a worse problem for the image quality than the small tool effect.

Krim: We must remember that the HST mirror is a very conservative mirror compared to what we would be considering for the future. It has a faceplate that is an inch in thickness.

Nolan: So your conclusion is that bigger is better for the support structures. Recall Pamela, for example.

Krim: Yes, in our case, and for our design at least, it worked out this way. 


\title{
Large Segmented Optics Fabrication and Wavefront Control
}

\author{
J. Richard Vyce \\ Litton Itek Optical Systems
}

\subsection{NGST Technological Perspective}

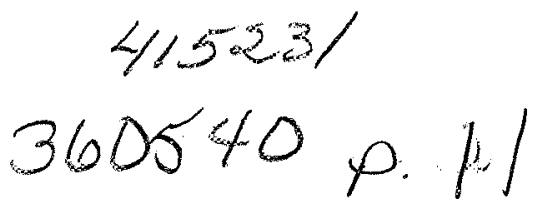

As a space based optical system to be implemented and operated for years or decades the NGST requires significant technological advances over the HST. Many, possibly all, of these advances are under development. Several have been and are being done at Itek; the scope of these efforts offers strong support for NGST feasibility.

Given the Workshop strawman NGST optical system of a segmented active primary mirror in a cassegrain-type configuration, its generic optics control system (Fig.1) is likely to follow those of other optical systems previously studied for various imaging and beam projection applications. The earliest of the latter at Itek, interestingly, is a 30 meter system (Fig.2) studied in 1975 for NASA Lewis as a laser projector for sending power from low earth orbit to a lunar station.

The elements in Figure 1 lead to the natural separation of NGST technology requirements into three distinct categories: the lightweight, deformable optical segments comprising the 10-16 meter pnmary facesheets; the active primary structure and activation mechanism; and adaptive optics sensors and control elements for maintaining good optical wavefront quality and stable pointing. These three technological areas will be considered in that order in what follows.

\subsection{Optical Tabrication}

\subsection{Keck Telescope Primary Optics}

The 10 meter f/1.75 Keck primary, comprising 36-1.9 meter hexagonal segments at 6 different radii, represents one realistic NGST primary option. Indeed, there was the reported 1988 announcement by the Soviet Academy of Sciences on their intention to pursue a space telescope with segmented optics of a "design similar to the Keck telescope".

Keck Telescope mirror fabrication, involving making the 36 segments plus a set of 6 spare segments, provides experience relevant to the NGST. Because the segments are a reasonably thick $10 \mathrm{~cm}$, it has been possible to make them utilizing the innovative stress polishing technique. However, this is unlikely to be possible on the necessarily thinner solid, or lightweighted, substrates of an NGST. Thus, Keck experience is relevant for the magnitude of surfacing and for the final test technique, but not for the surfacing technique. That is likely to be based on more general surfacing techniques such as Itek's Computer Controlled Optical Surfacing (CCOS) described below. CCOS was originally considered for final figuring on the Keck segments, but this was obviated by good stress polishing results, and use of a "warping harness" in the segment whiffletree supports for figure correction.

Steps in the Keck surfacing process are illustrated in Fig. 3. Stress polishing involves applying precise bending loads to an appropriately supported circular substrate, so as to deform the upper surface to the negative of the aspheric departure required in the finished 
surface. After polishing with a large lap to the required spherical radius as measured with a micro-inch bar profilometer each segment is unstressed and tested in the Autocollimating Test Facility (Fig. 4). It is then cut to hex shape, installed on its whiffletree mount, and remeasured in the ATF for warping harness adjustment. The ATF employs two movable flats and precise alignment metrology for autocollimation testing the segments in each of the 6 radial positions.

One infrequently encountered optical requirement, but a critical one in segmented mirrors, is the need for precise radius match among segments. This is achieved through measurement with the bar profilometer and radius control in polishing. The need for radius determination in ATF testing added significantly to the difficulty of its metrology.

After some debugging problems the Keck segment fabrication process is proceeding satisfactorily. Serial production is well underway with several segments having been completed and fabrication time continuing to drop as experience accumulates.

\subsection{Itek's CCOS (Computer Controlled Optical Surfacing) Process}

CCOS process is aimed at surfacing general aspheric elements both centered and off-axis with progressively greater efficiency. This process provides capability to manufacture 2 to $4-\mathrm{m}$ aspheric mirrors in less than half the time needed with the conventional technology. It is increasingly automated in its three major steps: figure generation, grinding/polishing, and testing. CCOS is essentially the robotic emulation of a skilled optician (Figs. 5,6) in which measured positive surface errors (bumps) determine rubbing commands for a nutating lap whose polishing "influence function" is well characterized. As with an optician, the process is iterative, but improving control of polishing parameters in CCOS is increasing the degree to which errors are corrected in a polishing cycle, thereby reducing the number of cycles and total surfacing time.

The mirror segment is first polished on an Arboga 5-axis machine which has circular symmetry about an external center and hence the polishing pad works in circular arcs. The pad is kept from rotating by a radius arm affixed at the center of symmetry.

To minimize the time between rubbing and testing, a CCOS machine is located adjacent to an optical test chamber. The work piece, blocking body and stand are on an air dolly which permits easy movement of that assembly between the machine and test chamber.

An important CCOS process adjunct is the laser profilometer, which serves two functions. It provides fractional micron measurement on ground surfaces, permitting use of fast fine grinding almost to final figure, thereby greatly reducing material to be removed at the slower polishing rate. It also makes the precise measurement of radius and off axis location required by segmented optics.

Interferograms of an earlier aspheric segment and a large aspheric demonstration mirror recently completed by CCOS, shown in Fig. 7, indicate the good performance of the process on difficult surfaces and the quality improvement in the recent mirror.

\subsection{Machine Lightweighting}

Cost and delivery of fused or fritted lightweighted mirror substrates can frequently be bettered by machine lightweighting of solid blanks. This is especially true for glassceramics whose material cost is low and which currently cannot be fused or fritted. Machine lightweighting has emerged as a useful option in lightweight mirror fabrication and has been applied at Itek to the largest lightweighted elements. 
The basic machining technique is relatively straightforward and has exhibited low risk comparable to other lightweighting techniques. After machining, acid etching is normally employed to relieve stresses in the grinding-damaged surface layer.

\subsection{Active/Adaptive Optics}

From the Generic Optics Control System block diagram (Fig. 1) a list of key NGST active/adaptive technologies has been compiled and addressed in terms of experience from specific programs or groups of programs at Itek (Fig. 8). For most technologies relevant experience has been accumulated from multiple programs over as long as two decades. A chronological listing of programs (Fig. 9) brings out some of their accomplishments, in many cases the first of their kind.

The following sections give examples of this work, first in active primary mirrors and then in adaptive optics.

\subsection{Active Primary Mirrors}

As early as the late '60s, there were concerns about providing ever larger diffraction limited space optical systems using passive technology alone. In the search for alternatives, development was started on active optics capable of being controlled by appropriate sensors to correct system wavefront errors ocurring during operation. An early 72-inch active optics breadboard served to test opto-mechanical design concepts, control algorithms, and closed loop operation.

The advent of real time adaptive optics in the early '70s, combined with the growing active optics experience led to investigation of large, lightweight, segmented active optics with characteristics consistent with space use. The first program of this kind was oriented to S-MWIR sensor application and thus was constructed for and tested at cryogenic temperature. In addition to breaking new ground in lightweighting, closed loop phasing and figure control through numerous actuators, and cryogenic operation, it also proved the viability of "negative figuring" the (repeatable) figure change from ambient to cryogenic test temperature.

Building on this experience, a more advanced active, segmented primary has been developed (Fig. 10) aimed at a specific space application and compatible with space flight after replacement of some electronic and mechanical components by space qualified versions. This mirror design was intended for scaling to larger size, and is believed to represent the most mature point of departure for an NGST primary of the Workshop strawman type.

\subsection{Adaptive Optics}

Adaptive optics of the kind currently used or planned stem from the 1973 21- channel breadboard predecessor of the Compensated Imaging System (CIS) (Fig. 11). The CIS, started in 1975, has operated on a 1.6 meter telescope throughout the ' 80 s to provide ground-based satellite imaging of unprecedented quality.

Adaptive optics technology has advanced about 11/2 generations beyond that in the CIS, in wavefront sensors, wavefront processing electronics, and deformable mirrors. From the beginning, wavefront sensors have had photon counting sensitivity and multi-kilohertz bandwidth. 
The first kilochannel wavefront sensor is used for SWIR laser wavefront diagnostics, and also incorporates a complete $100+$ channel closed loop wavefront correction system.

A pulsed, visible wavelength wavefront sensor of extremely high performance is designed for electronic scaleup to multi-kilo channels.

The first wavefront sensor for space use is part of a major Shuttle-based experiment scheduled to fly in 1991. Its design is based directly on the CIS wavefront sensor.

Deformable mirrors have improved substantially from that in the CIS, which required multi-kilovolts to produce one micron surface deformation. Current designs (Fig. 12) employ discrete multi-layer actuators to produce several microns of deformation from \pm 100 volt drive with wavefront quality suitable for the shortest visible wavelengths. Existing technology is of kiloactuator scale, and is capable of growth. The deformable mirror for the Shuttle wavefront control experiment is similar to those above but designed and tested to space and man-rated standards.

\subsection{Active Primary - Deformable Mirror Trade-Off}

An alternative to an NGST active segmented primary is a passive segmented primary with a deformable mirror (DM). Passive segments, if permitted to deform slightly under operational conditions, could be extremely lightweight. One of the small mirrors in the beam train could be made deformable to correct the primary deformations. The DM can also tilt and may be able to provide all necessary tilt correction. Phasing of the primary would still be necessary. The DM projection on the primary will be smeared several $\mathrm{mm}$ by the NGST field of view, but this is tolerable if small relative to the smallest deformed regions.

The basic trade off thus is the complexity and cost of assembled segments using large multi-part electro-magnetic actuators, versus monolithic segments and a relatively small DM using simple, monolithic ceramic actuators.

\subsection{Summary}

This limited review has covered many of the advanced optical technologies that may be required for the NGST.

All of those considered have been or are being pursued. At this level of examination there is no indication either of technology gaps or of "show stoppers" that would prevent attaining the desired NGST level of performance. 


\section{A 10 TO 16-METER NGST IS LIKELY TO REQUIRE ACTIVE CONTROL OF OPTICAL QUALITY AND POINTING}

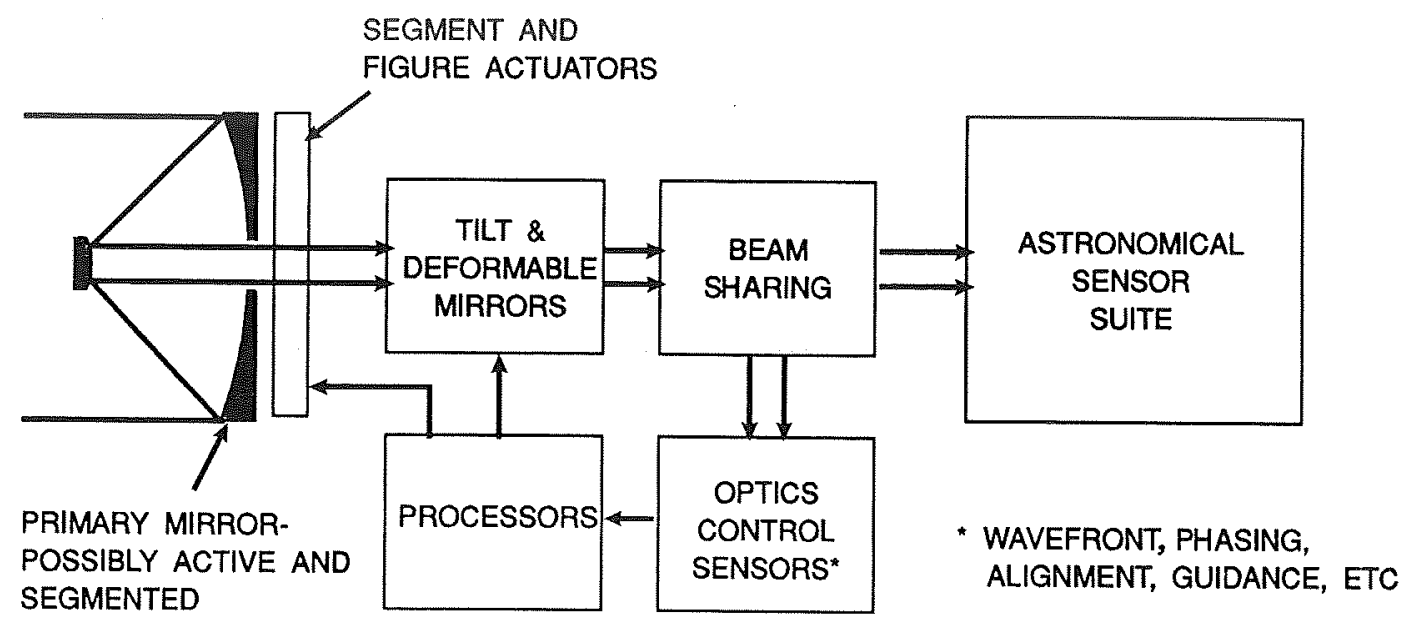

GENERIC OPTICS CONTROL SYSTEM

Figure 2

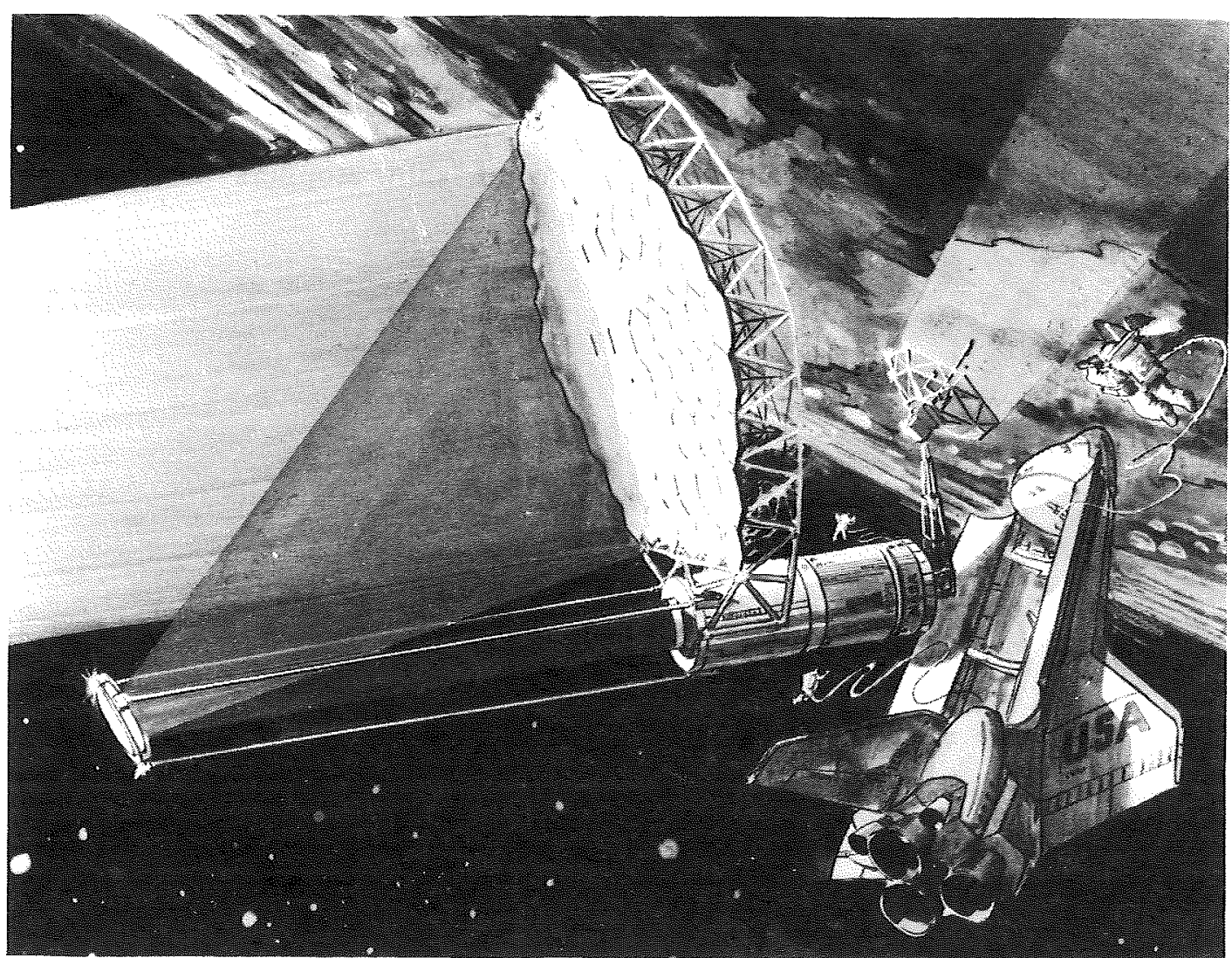


Figure 3

\section{KEY MANUFACTURING STEPS}

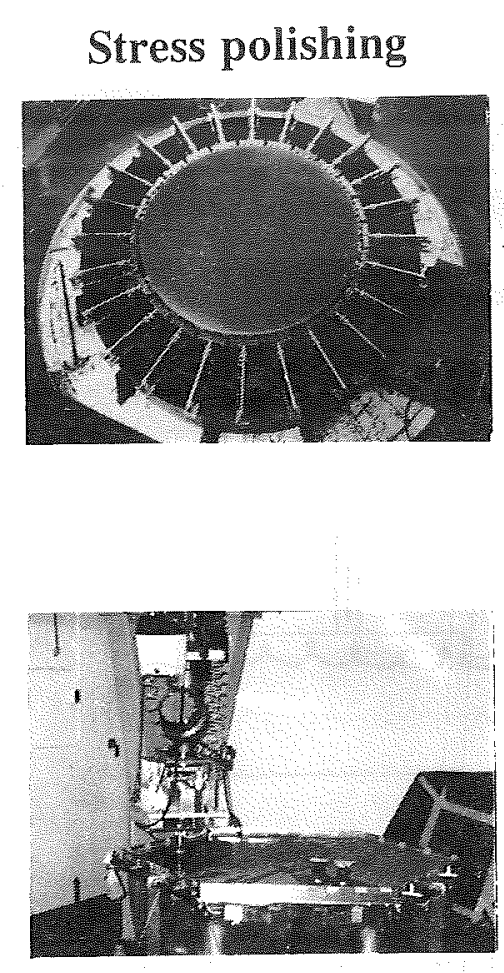

CCOS

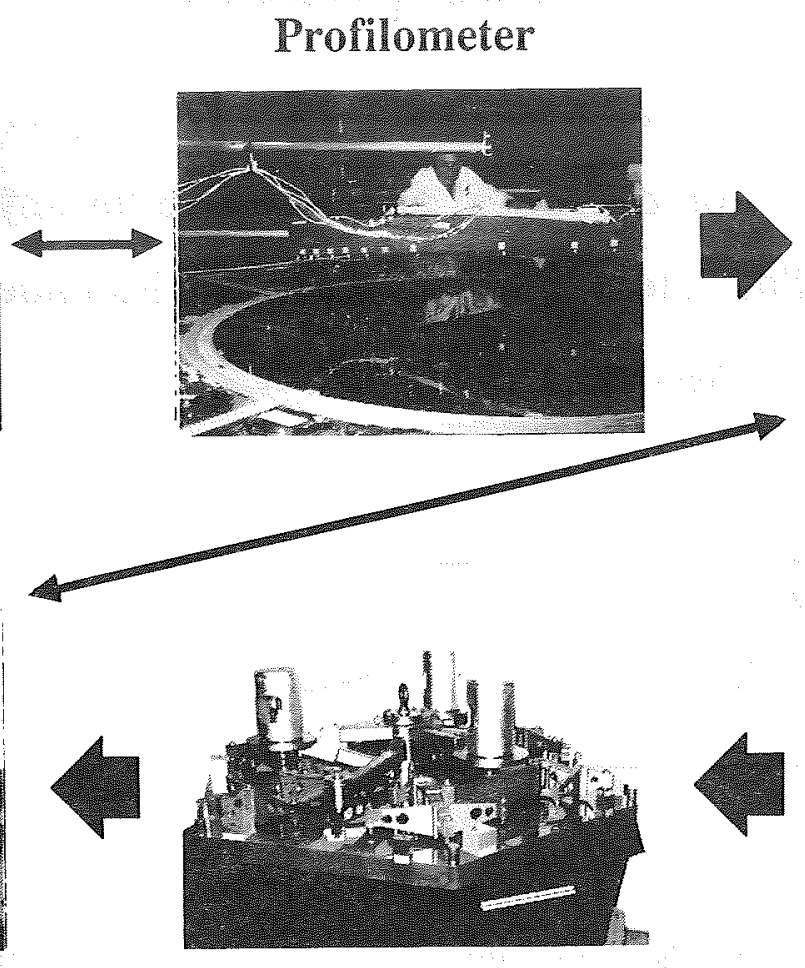

Whiffletree mount
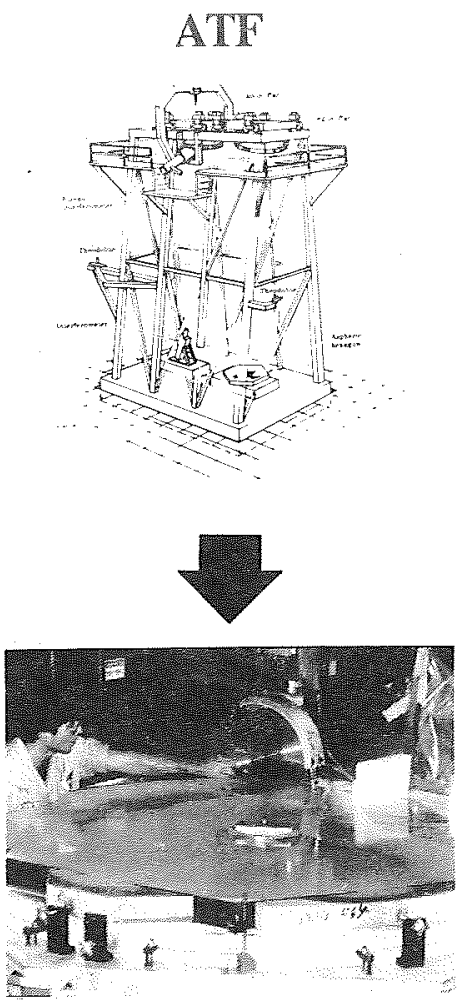

Hex cutting

Figure 4

\section{A PRACTICAL TEST FOR PRIMARY SEGMENTS}

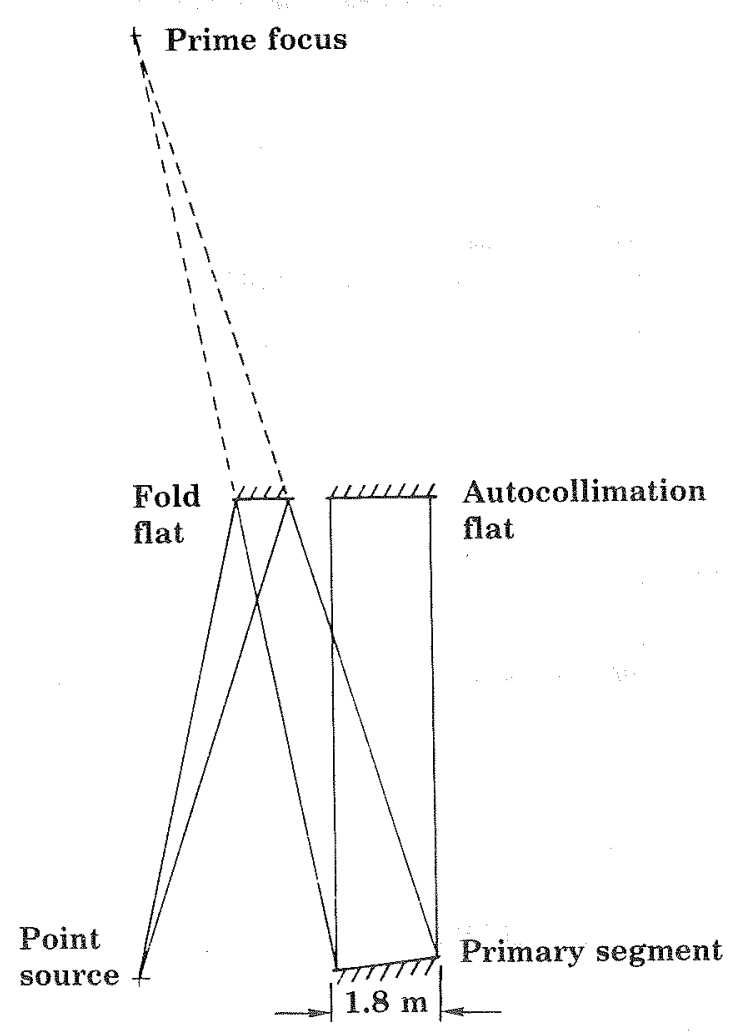




\section{CCOS PROCESS}

- Small orbital tool moves over optic under control of computer

- Figuring-Removal dependent on dwell time for any region

- Smoothing-Pad smooths surface irregularities under pad

Figuring

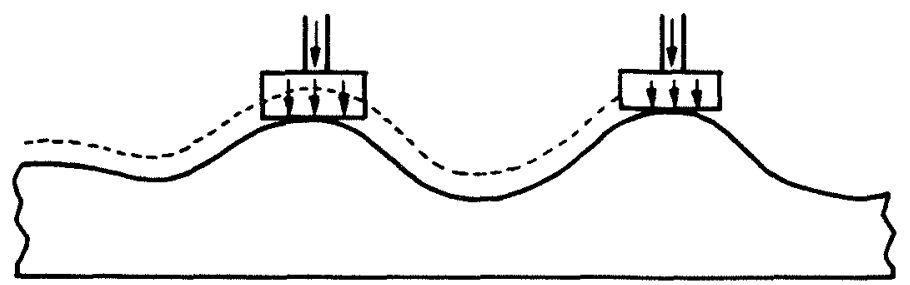

- Dwells on high regions

- Skips low regions
Smoothing

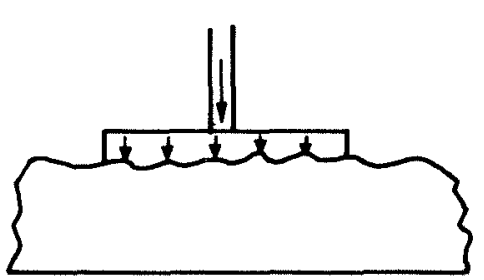

Figure 6

CCOS CYCLE

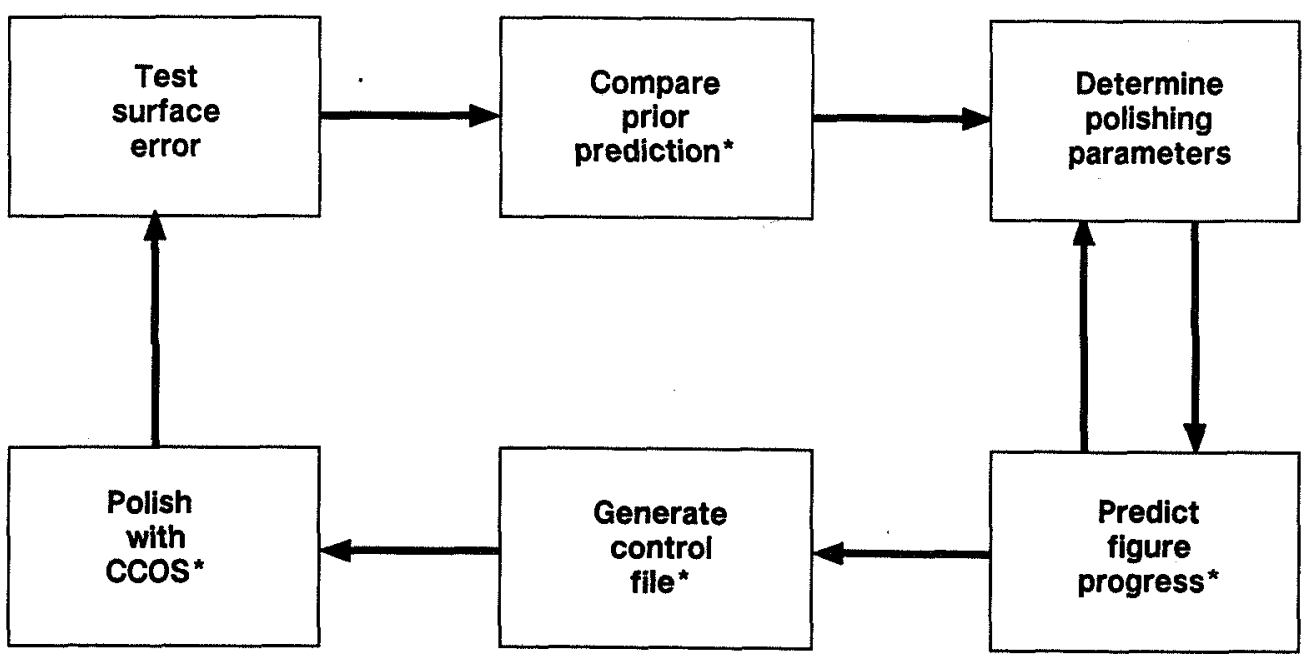

- CCOS used in iterative mannêr

- Tool applies more force on local high regions 


\section{CCOS FINAL INTERFEROGRAMS}

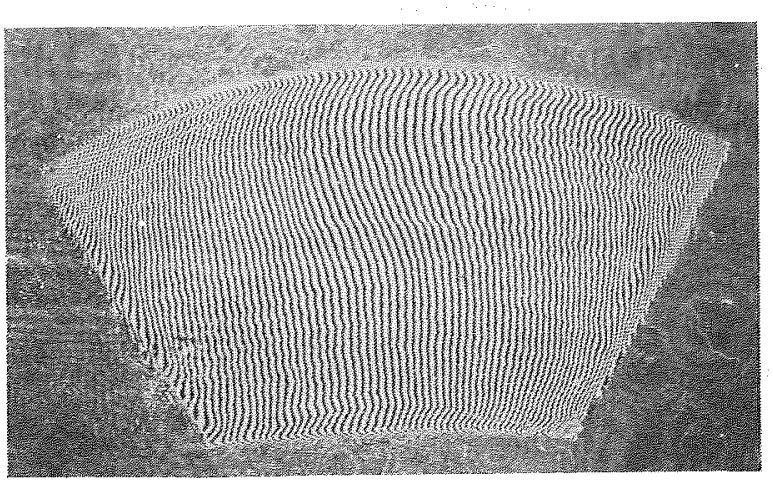

9754 Primary Mirror

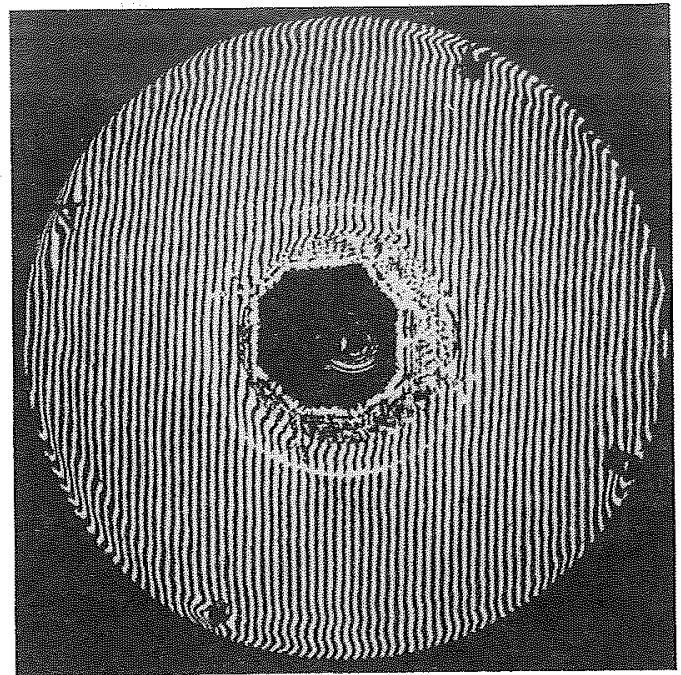

Rapidly fabricated mirrors worked to edges Rapid figure process

Figure 8

\section{PROGRAMS AT ITEK COVER MANY NGST TECHNOLOGIES}

Keck

$\begin{array}{ccc}\text { Starlab } & \text { Earlier } & \text { Adaptive } \\ \text { WCE } & \text { Programs } & \begin{array}{c}\text { Optics } \\ \text { Programs }\end{array}\end{array}$

Other

Programs

\begin{tabular}{|c|c|c|c|c|c|c|c|}
\hline $\begin{array}{l}\text { Large Segmented } \\
\text { Optics Fabrication }\end{array}$ & $x$ & $x$ & $x$ & & & $x$ & \\
\hline Active Mirrors & & $x$ & $x$ & & $x$ & & $x$ \\
\hline Space Applications & ; & $x$ & & $x$ & $x$ & & $x$ \\
\hline Cryogenic Primary & & & $x$ & & & & \\
\hline $\begin{array}{l}\text { Closed Loop } \\
\text { Wavefront Control }\end{array}$ & & $x$ & $x$ & & & $x$ & $x$ \\
\hline Closed Loop Phasing & & $x$ & $x$ & & & & $x$ \\
\hline Wavefront Sensors & & $x$ & $x$ & $x$ & $x$ & $x$ & $x$ \\
\hline Deformable Mirrors & & & & $x$ & $x$ & $x$ & $x$ \\
\hline Processors/Controls & & $x$ & $x$ & & $x$ & $x$ & $x$ \\
\hline
\end{tabular}




\section{PROGRAMS AND ACCOMPLISHMENTS}

\begin{tabular}{|c|c|c|}
\hline PROGRAM & ERA & ACCOMPLISHMENTS \\
\hline Active Mirror Experiments & 1970 & Active control understanding and algorithm development \\
\hline \multirow{3}{*}{$\begin{array}{l}\text { Compensated Imaging } \\
\text { System (CIS) }\end{array}$} & 1975 & First large scale operational adaptive optics system \\
\hline & 1975 & $\begin{array}{l}\text { First large, lightweighted, segmented, cryogenic active } \\
\text { optical system }\end{array}$ \\
\hline & 1984 & $\begin{array}{l}\text { First space-compatible, fast response, near-visible-quality, } \\
\text { segmented, active optical system; first serial production of } \\
\text { large off-axis segments }\end{array}$ \\
\hline Keck & 1986 & $\begin{array}{l}\text { First operational large, segmented optical system; } \\
\text { first serial production of large, off-axis, visible quality optics }\end{array}$ \\
\hline Advanced Adaptive Optics & $1980 \mathrm{~s}$ & $\begin{array}{l}\text { Large scale wavefront sensors, processors, and deformable } \\
\text { mirrors }\end{array}$ \\
\hline Starlab WCE & 1986 & First adaptive optics space experiment \\
\hline
\end{tabular}

Figure 10

\section{FULLY ASSEMBLED PRIMARY MIRROR}

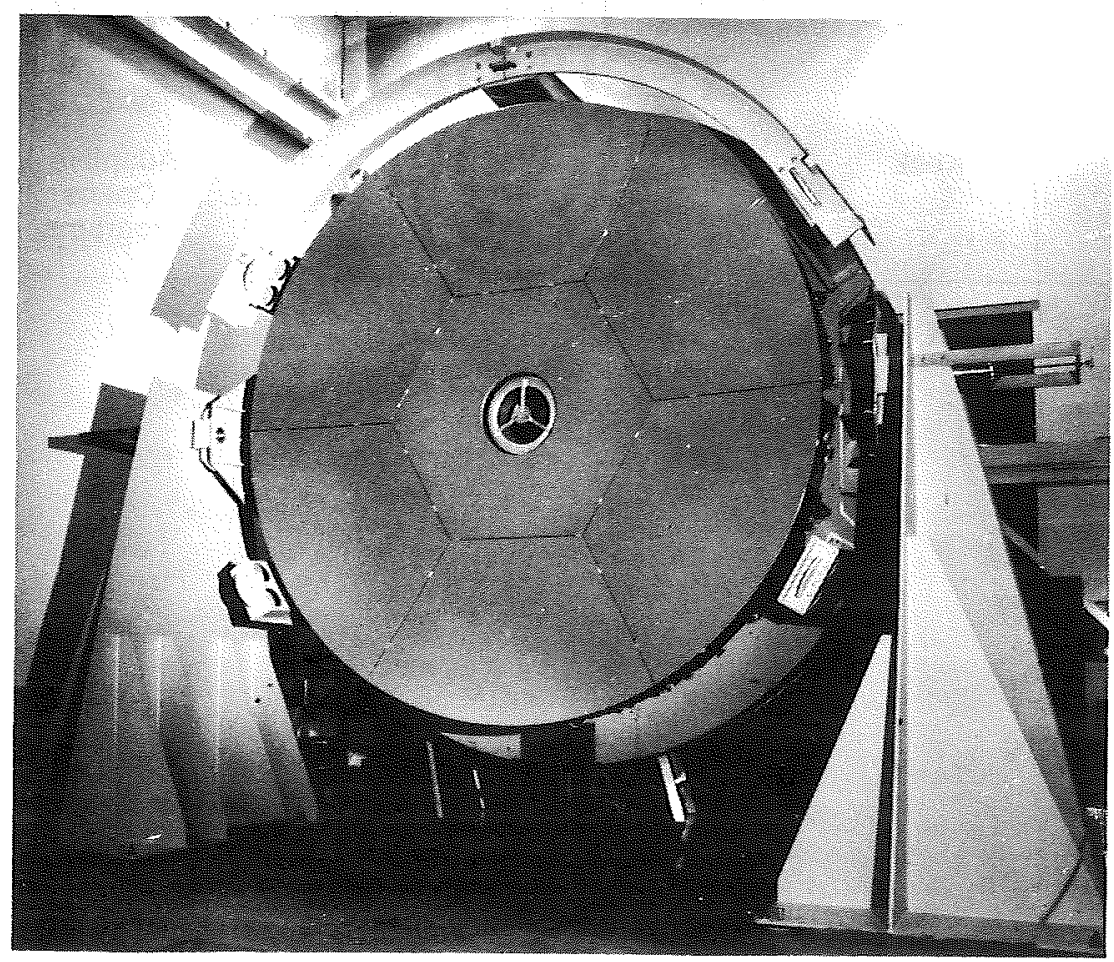


Figure 11

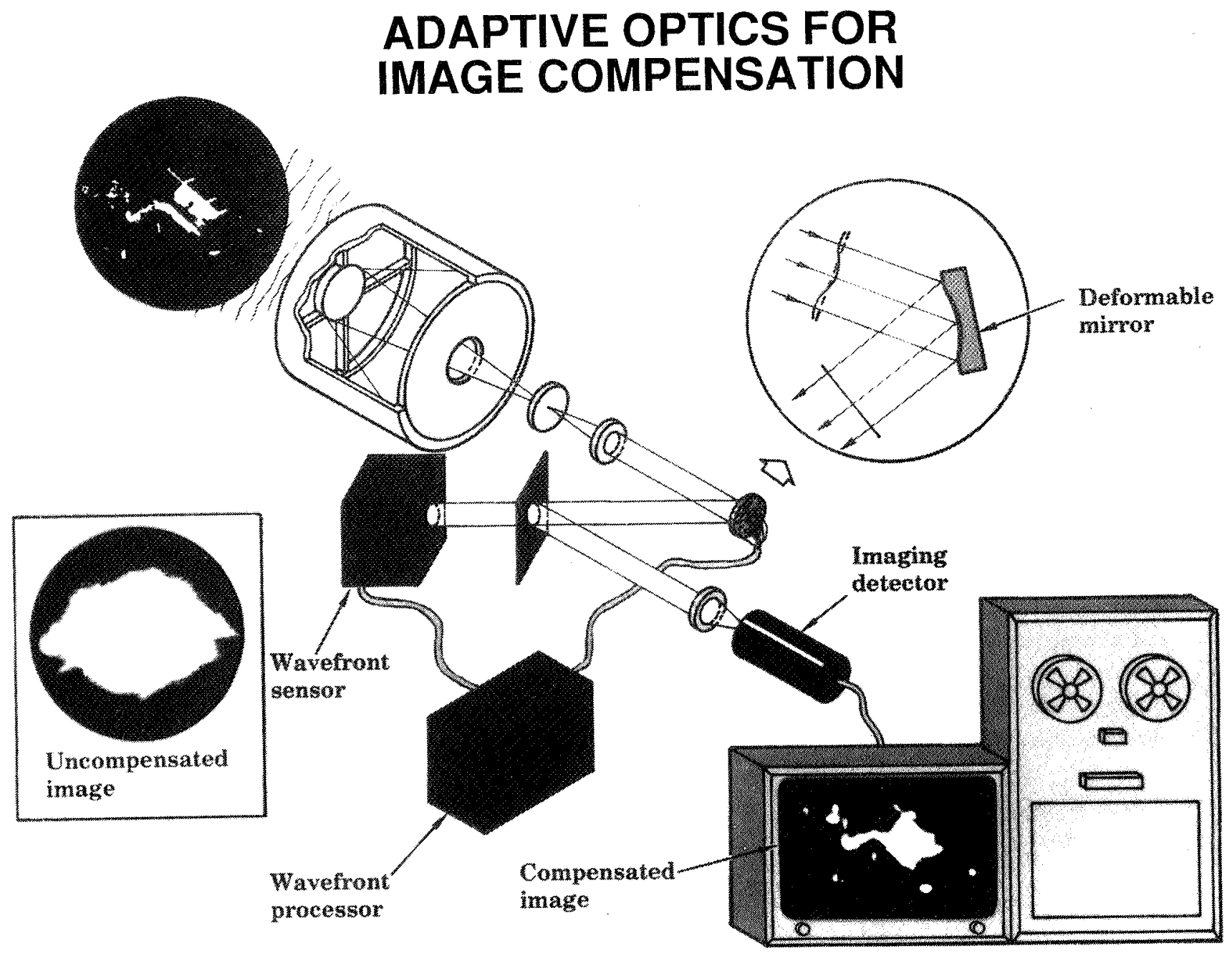

Figure 12

\section{LOW-VOLTAGE KILOACTUATOR MIRROR-PRINTED WIRING CONCEPT}

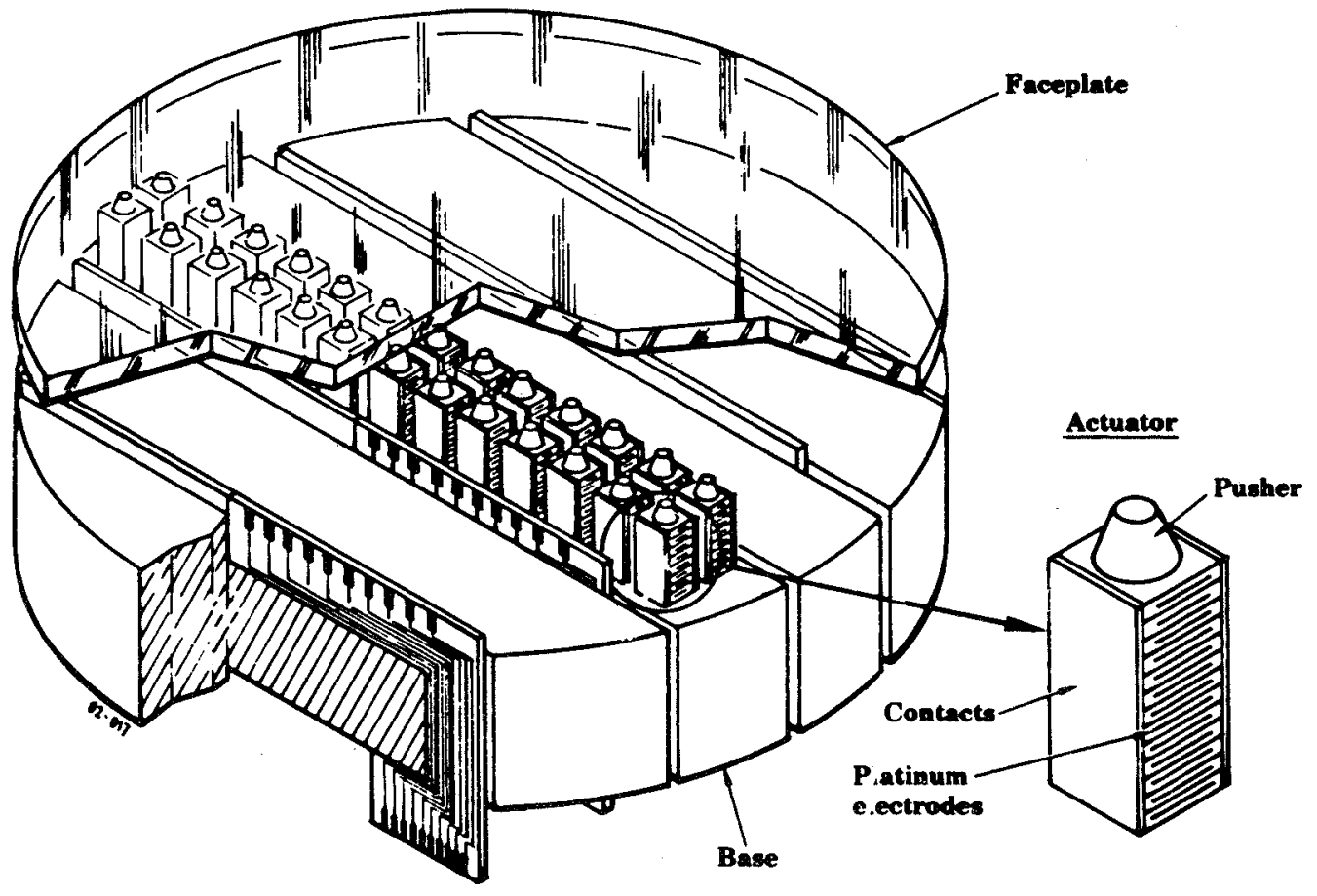




\section{DISCUSSION}

Angel: You got close to actually showing us what kind of quality you can get with computer controlled polishing. You showed an interferogram with rather closely-spaced fringes. You do phase-shifting interferometry and so real numbers are probably available. Can you tell us what quality can be obtained?

Vyce: I cannot say exactly what the quality is, but I am sure that it can be excellent for visible wavelength optics.

Angel: I can see wiggles in the interferogram so it is probably not adequate for ground-based systems, and almost certainly not for the space optics that we are talking about here.

Unidentified: Those are not corrected optics - that is an active mirror and is as manufactured without the corrections that will be applied.

Vyce: Angel is referring to the finer-grained structure which would not be corrected out. I am not certain that this is a finished unit, by the way.

Angel: There is a concern about computer-controlled polishing, and so I urge that when you come to talk to astronomers you give us numbers so that we can see how well the technique is doing.

Breckinridge: Are you doing any work on ion polishing?

Vyce: Not yet, but we may in the future.

Nelson: With regard to Angel's comments. Two of our mirrors for the Keck telescope had final polishing with CCOS and, ignoring the edge problems with CCOS, the mirrors were polished to surface errors below $20 \mathrm{~nm}$. There were edge problems and there were high frequency errors at the $10 \mathrm{~nm} \mathrm{rms}$ level that led us to go to a more cost-effective solution, namely warping harnesses. Since it is an iterative process, Itek may be able be able to do better. The edge effects were a particular concern to us because it is a segmented mirror.

Vyce: I think that we have a way of dealing with the edge effects and so we are checking that out now.

Kahan: Dick, would you agree that its fair to say that meter class optics can be made to visible quality levels in about 30 weeks, given today's CCOS techniques? This makes the primary mirror less of a cost driver, given the epic aerospace aspects of the total program.

Vyce: Yes. In fact, 30 weeks is prètty conservative. 
Fabrication of large and fast mirrors with extraordinarily smooth surfaces

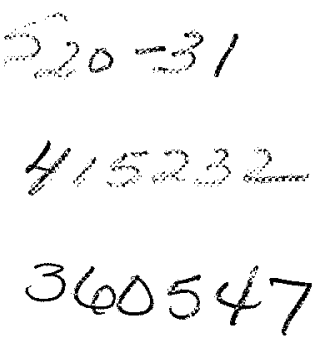

Ernst-Dieter Knohl

Carl Zeiss

\begin{abstract}
At the inception of the Next Generation Space Telescope (NGST) program we should ask what technologies are needed and are these technologies already available. It is clear that an important area is the fabrication of highly polished optical surfaces, in order to get high resolution images. For the NTT program Zeiss developed a fast polishing technique to generate super smooth surfaces, and patented it as membrane tool technology. The striking results of the ESO NTT demonstrate how powerful these new tools are: the concentration of energy of the $3.6 \mathrm{~m}$ primary turned out to be twice as good as the HST, and 50 times less expensive (Neglecting the atmospheric contribution to the image spread) Rapid metrology is essential for fast figuring process. Zeiss is running interferometry in "Real Time" averaging hundreds of frames in one evaluation. Therefore no vacuum chamber is needed. An adequate mirror support system for figuring and testing has been built for the NTT primary and has been qualified in interferometric tests. For an active supported mirror Zeiss prefers the thin, solid meniscus solution for best optical performance. With such designs, mirror diameters are limited to between 8 and $9 \mathrm{~m}$.
\end{abstract}

\title{
1. The conventional figuring technique
}

In the fabrication of a large aspherical mirror, the conventional technique is to begin with a spherical lapping step with area covering tools. Figuring of a spherical surface needs no special machine control just a turning workpiece and an oscillating tool. The next step is to polish the spherical surface. The third step is a interferometric test. Next aspherical deformation of the surface is done by lapping, and the asphere is polished. Finally, an interferometric test with a compensating system will give the input to the next polishing cycle. This process being repeated until the figure and polish are satisfactory.

This time consuming process was established to generate the optic of the $3.6 \mathrm{~m}$ telescope MPIA with $35 \mu$ deviation from the best fitting sphere and a F-number F/3.5. In four years a RMS wavefront aberration of $24 \mathrm{~nm}$ was reached. The first light of the MPIA telescope was in 1984 and Birkle has published "Operation and Test Results" in Ref.1.

With the same technology a second $3.6 \mathrm{~m}$ optic was fabricated for the Iraqi Observatory on Mt. Korek. Unfortunately the complete telescope is stored today in Baghdad.

\section{A new polishing technique to generate super smooth surfaces}

The ESO NTT optics was specified to have $80 \%$ energy within 0.15 arc sec diameter. This challenging requirement can only be met with new fabrication technologies. The NTT primary has a free aperture of $3.5 \mathrm{~m}$, a $F$-number $F / 2.2$ and about $200 \mu$ deviation from the best fitting sphere. To reach this goal Zeiss developed new tools for super smooth surfaces, a fast metrology with high resolution and an adequate support system. The results are striking and are published by ESO in Ref. 2 and 3, and show about a factor of three better energy concentration than standard techniques. 


\subsection{Membrane polishing tool technology}

The idea to use strip tools was initiated by experiments that Zeiss made during figuring of the ROSAT shells, illustrated in Figure 1. In the ROSAT program a super smooth surface quality was achieved:

- very small ripple amplitudes and

- surface roughness better than $2 \AA$ rms (Ref. 4, and Figure 2)

Later on in the ROFT program Zeiss developed the so called membrane tool for high quality optical surfaces in the range of $25 \mathrm{~nm}$ rms wavefront aberration with high wear rate (generating $100 \mathrm{~m}^{2}$ per year). This was the background used for the NTT primary fabrication with a flexible, stripe like tool and pressure control.

These tools turned out to be a great success in several respects

- they produce excellent wear rate, because of the great area of contact

- For the same reason, they produce extraordinarily smooth surfaces at frequencies associated with ripples.

- flexible stripe tools can follow aspheres as fast as F/1.0 where the conventional polishing technique fails.

If the tool wear rate is set by actuators under computer control, cycle time can be reduced drastically so that an $8 \mathrm{~m}$ mirror with a $50 \mathrm{~m}^{2}$ area can be finished within 2 years.

A special evaluation has been made of the surface ripple of the NTT primary (Ref. 5). A window varying between 25 and $550 \mathrm{~mm}$ was moved along the fringes and the amplitudes at the corresponding frequencies are shown in Figure 3.

The amplitudes of the MPIA and NTT primary are compared in Figure 4. At wavelengths between 25 and $75 \mathrm{~mm}$, and also between 225 and $550 \mathrm{~mm}$, the NTT mirror surface is smoother by a factor of 2 to 3 . The NTT intrinsic quality was shown to give an $80 \%$ encircled energy within 0.1 arc sec diameter.

\subsection{Fast metrology}

To reach an optimum rate in the figuring process all steps of the working cycle have to mesh with each other and have to be fast. In the past, interferometric tests could only be performed at nighttime because of vibrations of the test tower during the day. To overcome the influence of test tower seeing, vacuum chambers have been erected in the past, and they have been put on foundations damped by air to reduce vibration effects.

ZEISS developed an interferometer with 3-D stabilization of the optical beam with dynamic range up to $300 \mathrm{~Hz}$. This system was established first for the figuring process of the NTT primary and the process could be speeded up to one complete cycle per day. A stabilized interferogram can be digitized by CCD camera and the fast evaluation of many frames improves the measuring accuracy. The typical reproducibility of one frame is 0.5 $\mathrm{nm}$, with $N$ frames the reproducibility is improved to $0.5 \mathrm{~nm} / \sqrt{N}$.

Further development of the image processing hardware makes "real time" interferometry available at Zeiss. Now not ten but hundreds of frames will be taken and the air motion in the test tower can be directly made visible. By feeding the evaluated pressure function directly into the actuators, the figuring process can run in a closed control loop and the great wear rate of the large stripe tool is turned to full use. 


\subsection{Supporting System}

The advanced technology of active optics leads to more and more thin, solid mirrors with the most possible homogeneity. The aspect ratio of the NT'T primary was chosen $\mathrm{D} / \mathrm{h}=15$. New projects like SOFIA or VLT tend to $\mathrm{D} / \mathrm{h}=45$. Therefore the new mirror quality depends strongly upon the optimized supporting system. The mirror support during figuring has to guarantee the following functions:

- Supporting the mirror axial weight

- Carrying the axial and tangential force variations of the polishing loads

- Damping of the polishing loads

- Supporting for interferometry without imposing any constraints

- Active correction of long wavelength deformations to increase the dynamic range of the test system

During the NTT program Zeiss measured the quality of the support system by turning the mirror with respect to the supporting pads. Finally a perfect match was found between data from mirror support during fabrication and data from mirror support in the telescope cell . The beautifully sharp astronomical images are a direct consequence of the support quality.

Active correction makes sense only if the mirror body is solid and homogeneous. In case of a structured mirror the active bending corrects for long wave errors but introduces ripples with even steeper slope errors. This effect have been demonstrated with simple paper models.

\section{Limits of mirror dimensions}

In a study phase of the VLT program Zeiss developed all the technologies needed for $8 \mathrm{~m}$ mirror figuring (Ref. 6). The biggest useable monolithic glass mirror is between 8 and $9 \mathrm{~m}$ diameter for several reasons:

- fabrication of blank

- mirror handling

- transportation on road and by ship

Many ideas exist for the construction of larger primaries. For example the MMT solution or the Keck solution with all its attendant problems. To overcome the problems with figuring of off-axis aspherical elements, Zeiss is studying a $12 \mathrm{~m}$ primary made out of 4 segments. The idea is to assemble these segments at simulated zero-g into a monolithic blank, do the polishing and interferometric testing in the well known on-axis manner, to take the four segments apart for transportation and to align and assemble them again on site in the telescope cell.

Thorough calculations have still to validate this approach. But the great advantage of large coherent optics is obvious, and the approach is worth pursuing. 


\section{Conclusions}

- The largest monolithic mirrors that can be produced, handled and transported are limited to diameters between 8 and $9 \mathrm{~m}$.

- Two years is typically needed for figuring, preparation of supporting pads and testing to finish $50 \mathrm{~m}^{2}$ of super smooth optical surface corresponding to an encircled energy of $80 \%$ within 0.2 arcsec diameter.

- The great improvement of the NTT optics was achieved with stripe tools and actuators under pressure control, with fast IR and visible interferometry, and with unconstrained mirror support.

- The full advantages of active optics are only obtained if ripples at spatial frequencies above the controlled frequencies are accordingly reduced.

- Active correction of honeycomb mirrors makes no sense, while long wave deformations are corrected by active bending, short ripples caused by the structure occur with even greater slope errors.

- At Carl Zeiss rapid active tools to generate fast optics were developed and used in the NTT program. Real time metrology is available with correction of test tower seeing and vibration and adequate supporting systems have been used for mirrors with reduced stiffness.

Figures 5 illustrates the evolution of surface polish quality with time for the NTT. Figure 6 shows the time actually taken to figure mirrors of given aspheric deformation in three cases, and predictions for the VLT.

\section{References}

$1 \mathrm{~K}$. Birkle and U. Hopp, Das $3.5 \mathrm{~m}$ teleskop MPIA Operation and test Results, Mitteilungen der AG Nr. 68, 1987

2 R. N. Wilson et al., ESO Active Optics: the NTT and the Future, ESO Messenger No. 53, Sept. 1988

3 R. Wilson ESO, "First Light" in the NTT, ESO Messenger No. 56, June 1989

$4 \mathrm{~K}$. Beckstette, Aspects of Manufacturing Grazing Incidence X-Ray Mirrors, Carl Zeiss, D-7O82 Oberkochen, West Germany SPIE Vol . 645 (1986)

5 H. Geib, C. Kühne, E. Morgenbrod, How to Quantify Ripple, Proc. IAU Colloquium No. 79, April 1984

6 E.D. Knohl et al., $8 \mathrm{~m}$ Class Primary: Figuring, testing, handling, SPIE Vol . 1013 (1988) 
ESO-NTT M1 RMS - Werte

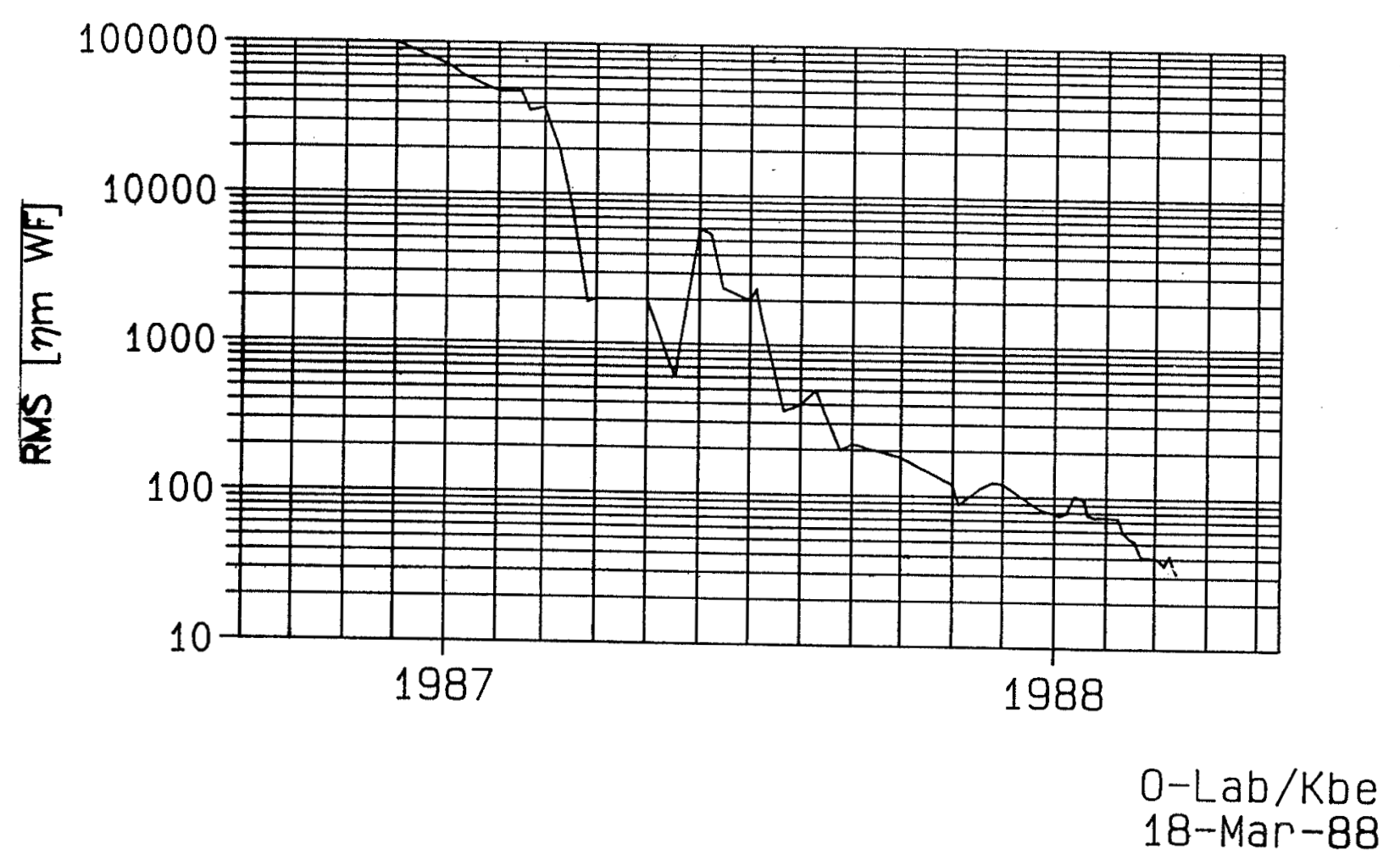

LARGE OPTICS FIGURING

STATE OF THE ART AT CARL ZEISS

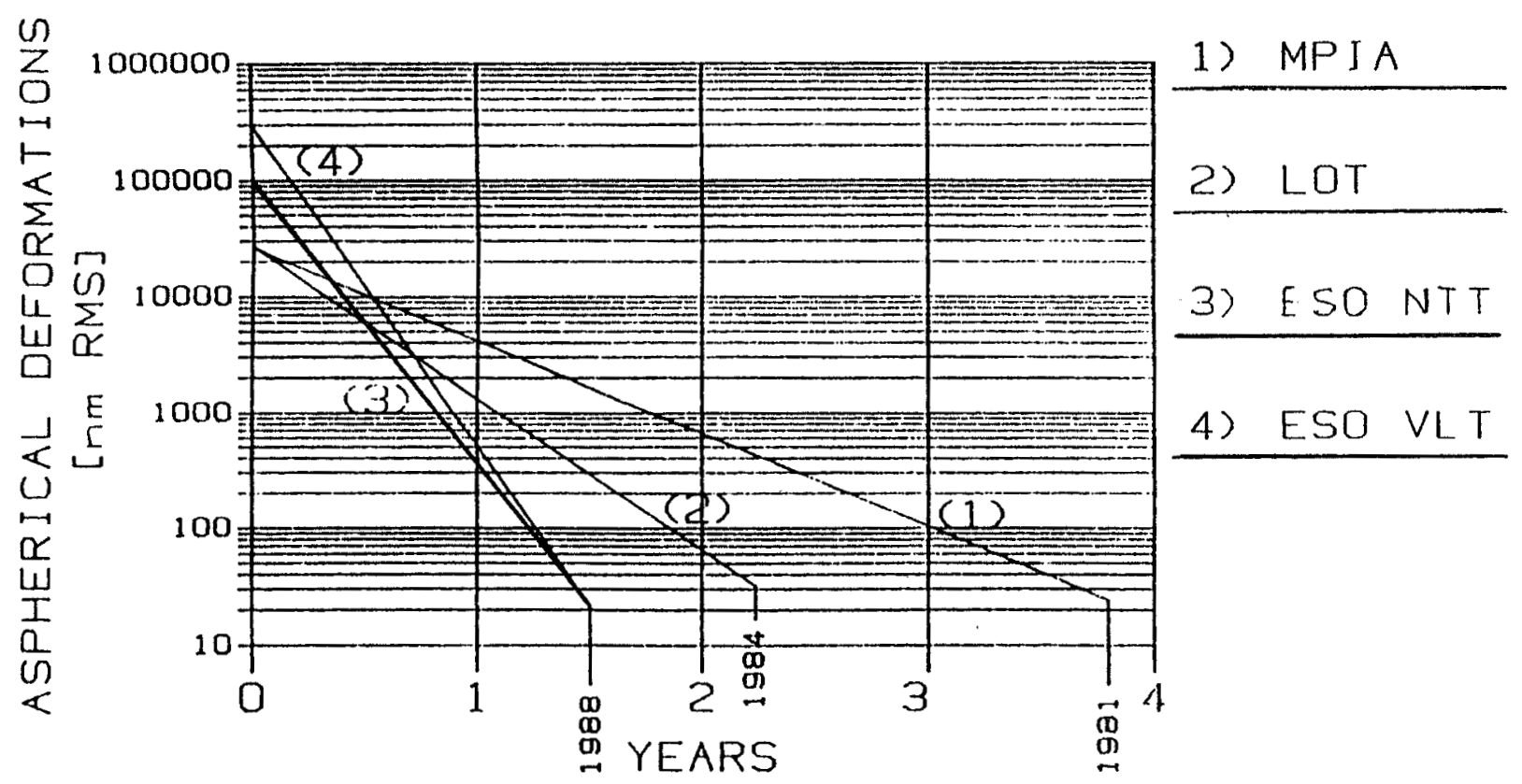




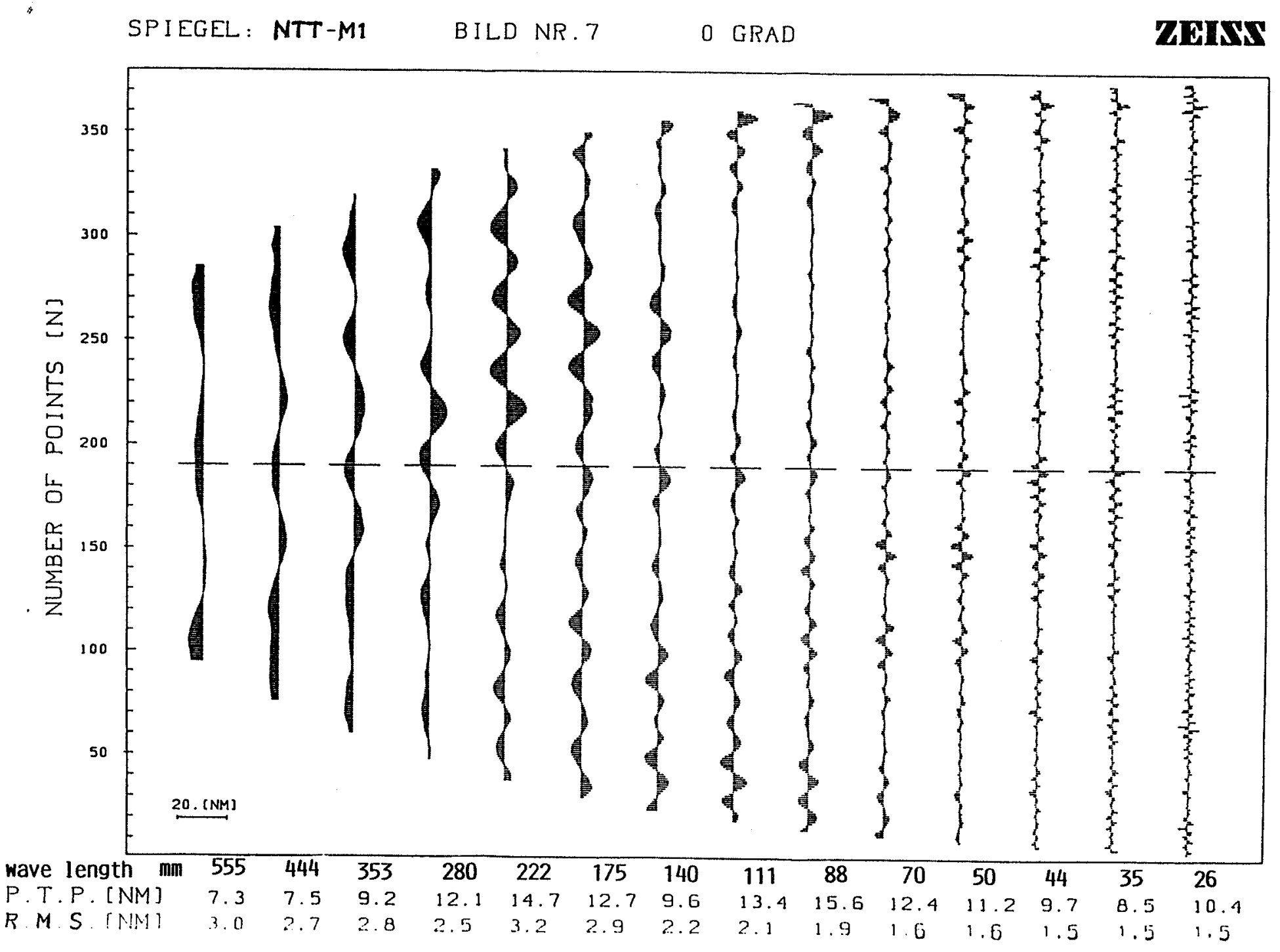

ESO NTT Primary

Improvement of surface smoothness

with respect to ripples

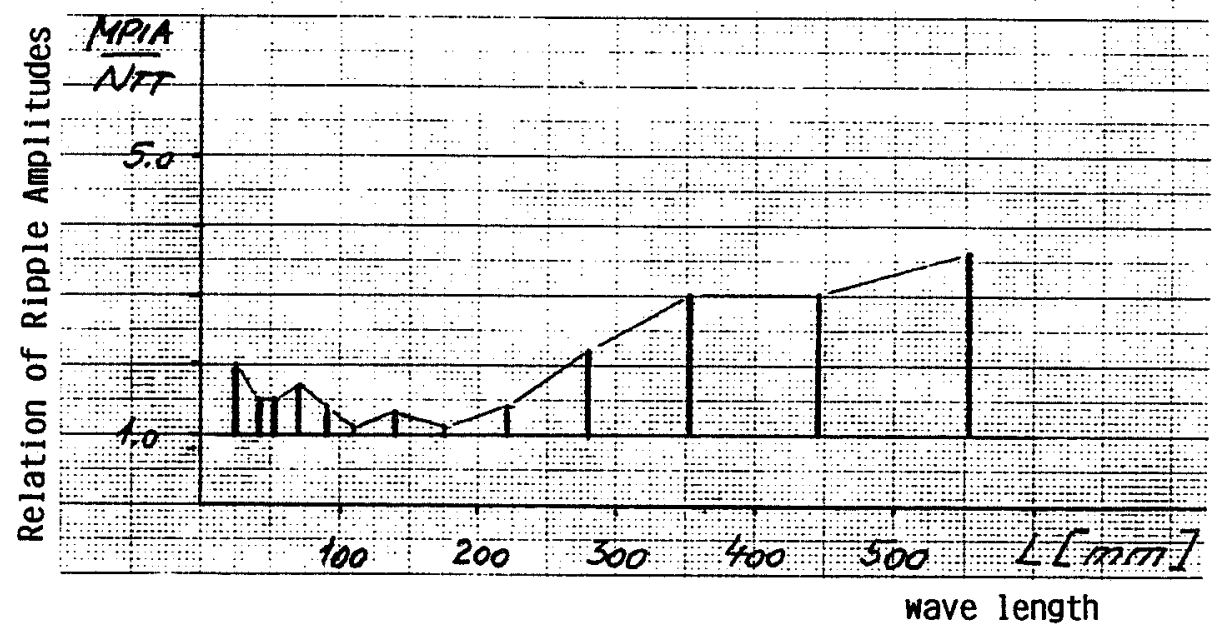




\title{
ZEIKX
}

\section{ROSAT}

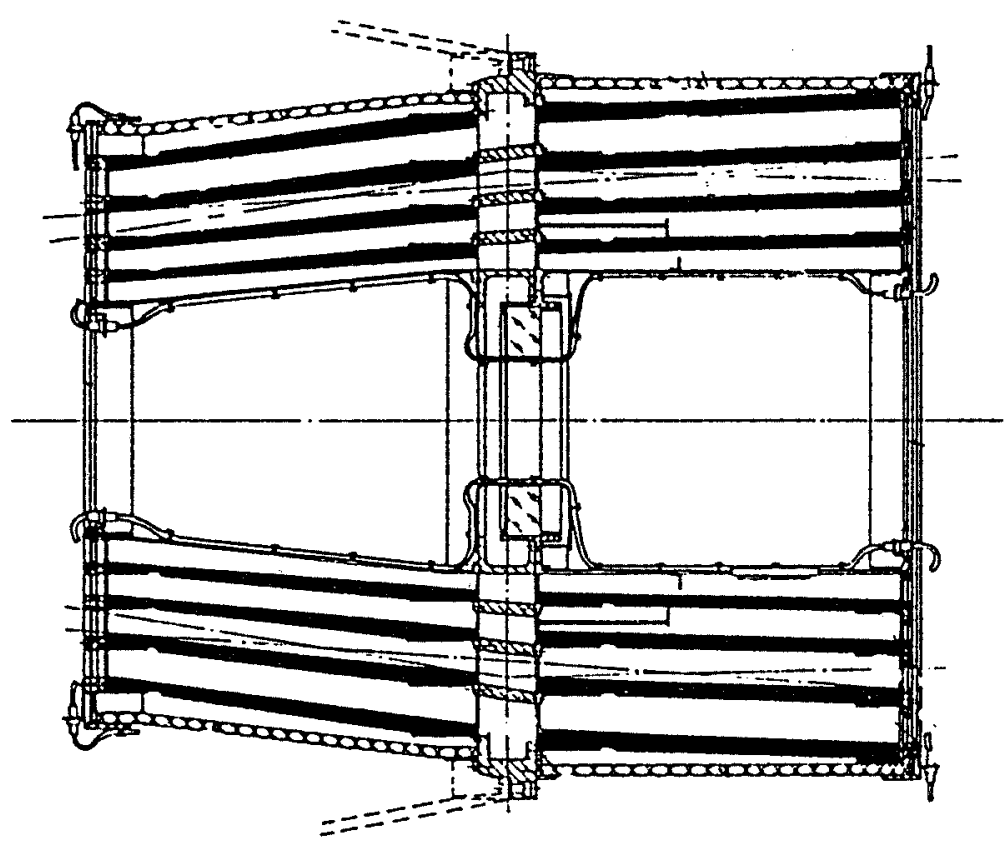

\author{
Wolter I Mirror System \\ 4 folded
}

max. Aperture $800 \mathrm{~mm}$ Focal Length $2400 \mathrm{~mm}$

Measured Resolution:

3.5 arc sec FWHM

Mirror material: ZERODUR

Surface Roughness better $2 \AA$ RMS

Contour of Hyperbola 2

after superfinish

within $0.05 \mu \mathrm{m}$ amplitude

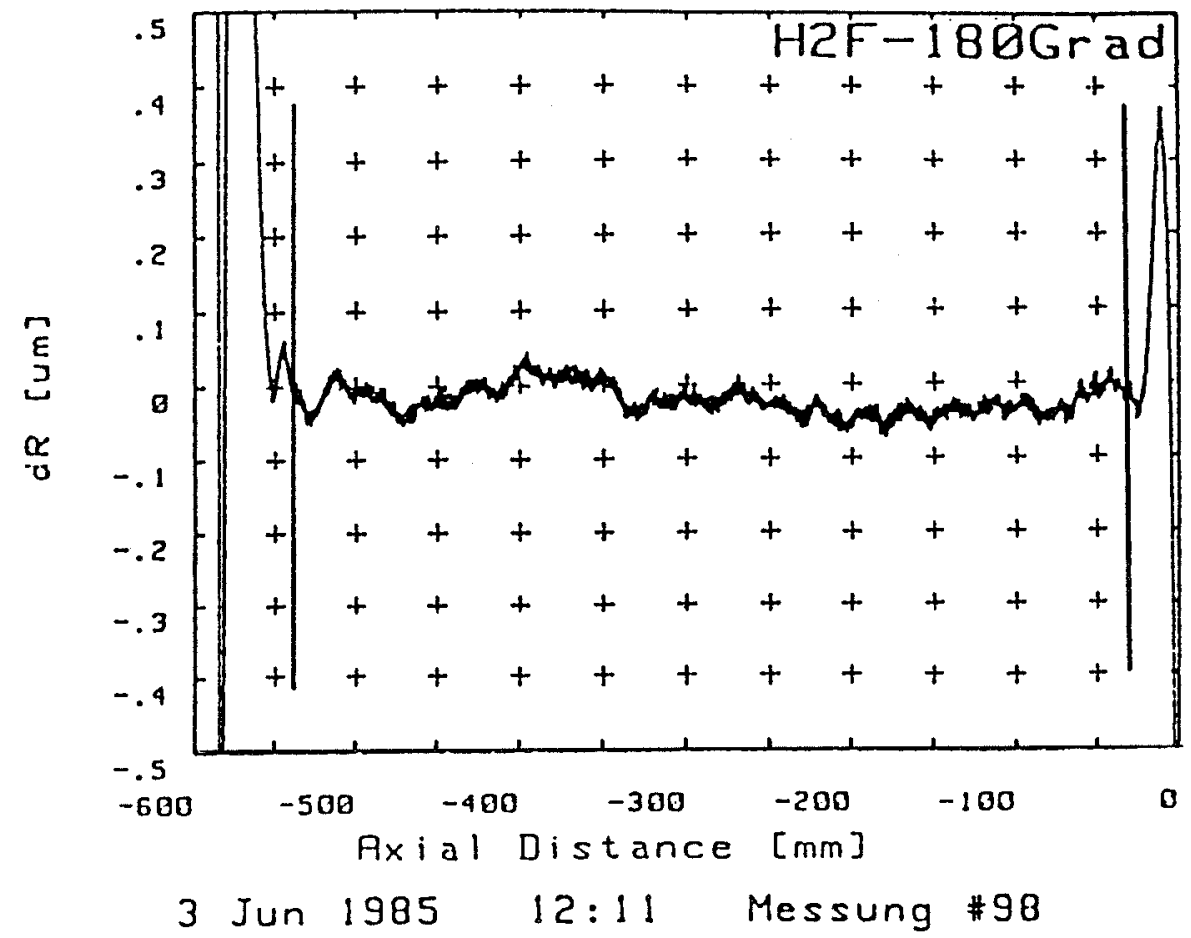




\section{DISCUSSION}

Miller: Can the membrane polishing be extended beyond 8 meters?

Knohl: Yes, we think that it could be extended beyond that point.

Mlingworth: Mike Krim noted a "print-through" problem with thin mirrors. For thin mirrors do you think that the technique will work without "print-through" of the support so that the resulting segments are smooth on small scales?

Knohl: The technique works on rotationally-symmetric mirrors. We are now working on the 12 meter DGT telescope and are discussing putting the segments together and polishing them as a monolith.

Krim: The "quilting" problem arises particularly in lightweight mirrors that are "pocketed" from the back. There are tradeoffs that can be made in the degree of lightweighting, the cell spacing, the faceplate thickness, and the tool pressure. It is a multiparameter (7?) tradeoff.

Knohl: Yes, you have to do an optimisation of the process.

Angel: On that topic, if you make a lightweight glass honeycomb structure for a large space telescope you are talking about really very thin faceplates - maybe a $\mathrm{cm}$ or less. Under any realistic polishing pressure, maybe a tenth of a psi, for example, the cells would have to be very small to avoid deflections. They would probably have to be an inch or two in size. The only advantage of a computer-controlled polisher in not "quilting" the surface is when the tool size is less than a cell size, say a cm or two. I find it difficult to see that this small tool advantage could be usefully realized for polishing a 16 meter mirror. Therefore I do not see that there is a domain where small tool polishers will be used for large, lightweight space optics.

Mlingworth: Have you thought about using your membrane lap technology on segments' surfaces by polishing all the segments at once in a support somewhat like that described by Mike Krim of Perkin Elmer?

Knohl: Yes, the membrane tool can be used for segments of any shape. In this case, the workpiece doesn't move and the membrane tool is oscillating in the surface plane. The point still being under discussion is the best set up for the metrology of off-axis segments. 


\section{Economical Production of Large Optics*}

David Pileri and William Prensky
$50,-3 /$

415233

Eastman Kodak Company, Federal Systems, Advanced Technology Products

Special Optics Fabrication, Rochester, New York 14650

\begin{abstract}
Optics for large systems are frequently ultra-lightweight and deformable by design. They often have unusual shapes and include non-rotationally symmetric surface figures. Surface roughness, subsurface damage, light scattering and laser damage threshold specifications are also frequently required. Manufacturing techniques being developed to address these stringent requirements will be discussed.
\end{abstract}

\title{
1. INTRODUCTION
}

As large, monolithic optical structures are no longer feasible, large optical systems typically incorporate multi-segmented arrays. ${ }^{1,2}$ Spaceborne systems can be assembled in orbit from segments that can be transported in smaller launch vehicles. Space-based and ground-based systems use active optics to compensate for distortions. Manufacturing these optical elements presents many challenges. Conventional processes, materials and methods are not capable of figuring these active optics in a cost effective manner. New, deterministic manufacturing techniques need to be developed. These techniques must not only be capable of accurately figuring large, complex, optical components; they must also support a performance level requiring minimum light scattering and minimum laser threshold damage (resulting from subsurface damage and surface microroughness). On-going process developments at Eastman Kodak Company have resulted in optical manufacturing technology that will provide the deterministic, cost effective fabrication of large state-of-the-art optical components.

\section{LARGE ASPHERIC OPTICS TECHNOLOGY}

Large primary mirror designs as shown in Fig. 1 are segmented representing a parent optic several meters in diameter. In order to reduce the areal density the mirror segments are either lightweighted, or consist of thin, solid facesheets (Fig. 2). Regardless of the configuration, the segments are deformable by design (under active actuator control in the system). Glass-ceramic or stable glass materials such as fused silica, ULE®, or Zerodur are likely candidates for substrates.

\subsection{Aspheric Curve Generation}

The generation of an aspheric profile (i.e., diamond grinding of the three-dimensional aspheric equation to within a few waves of nominal ) for axisymmetric optics is typically accomplished by spinning the optic about the optical/mechanical axis and moving a grinding head radially. This is not possible for large, off-axis elements. Therefore, reliable aspheric curve generation of unusually shaped, off-axis segments requires a generating machine with state-of-the-art size, stiffness, accuracy, and metrology. A machine of this caliber would also allow manufacturing large anamorphic designs and using ductile grinding techniques ${ }^{3}$ to produce a specular surface.

Process developments at Kodak have resulted in machinery capable of generating axisymmetric aspheric elements up to $0.8 \mathrm{~m}$ diameter, and to within $6 \mu \mathrm{m} \mathrm{p}-\mathrm{v}$ of nominal. Kodak has now expanded this process capability to large off-axis segments by commissioning Cranfield Precision Engineering, Ltd., to build a three-axis CNC generating machine with a working envelope of $2.5 \mathrm{~m} \times 2.5 \mathrm{~m} \times 0.6 \mathrm{~m} .4^{4}$. The OAGM 2500 (Off-Axis Generating Machine) pictured in Fig. 3 features a) a precision air bearing grinding spindle, b) an in-situ metrology system using a retractable air bearing measurement probe, c) a metrology frame based on distance measuring interferometers that reference to three precision mirrors, and d) a Granitan machine structure for stability yielding a total machine weight of 130 tons. The machine is expected to be on line in December 1989.

* This paper was presented by John Keller, Kodak 


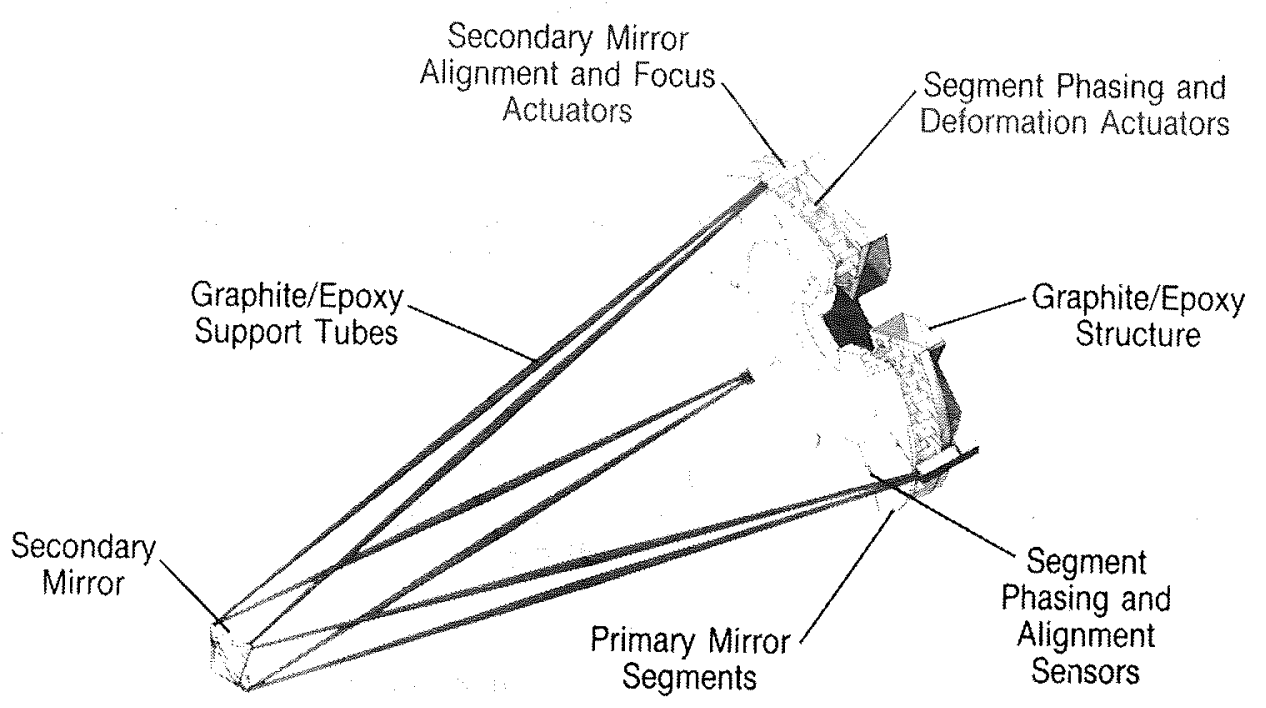

Fig. 1. Example of segmented primary mirror design.

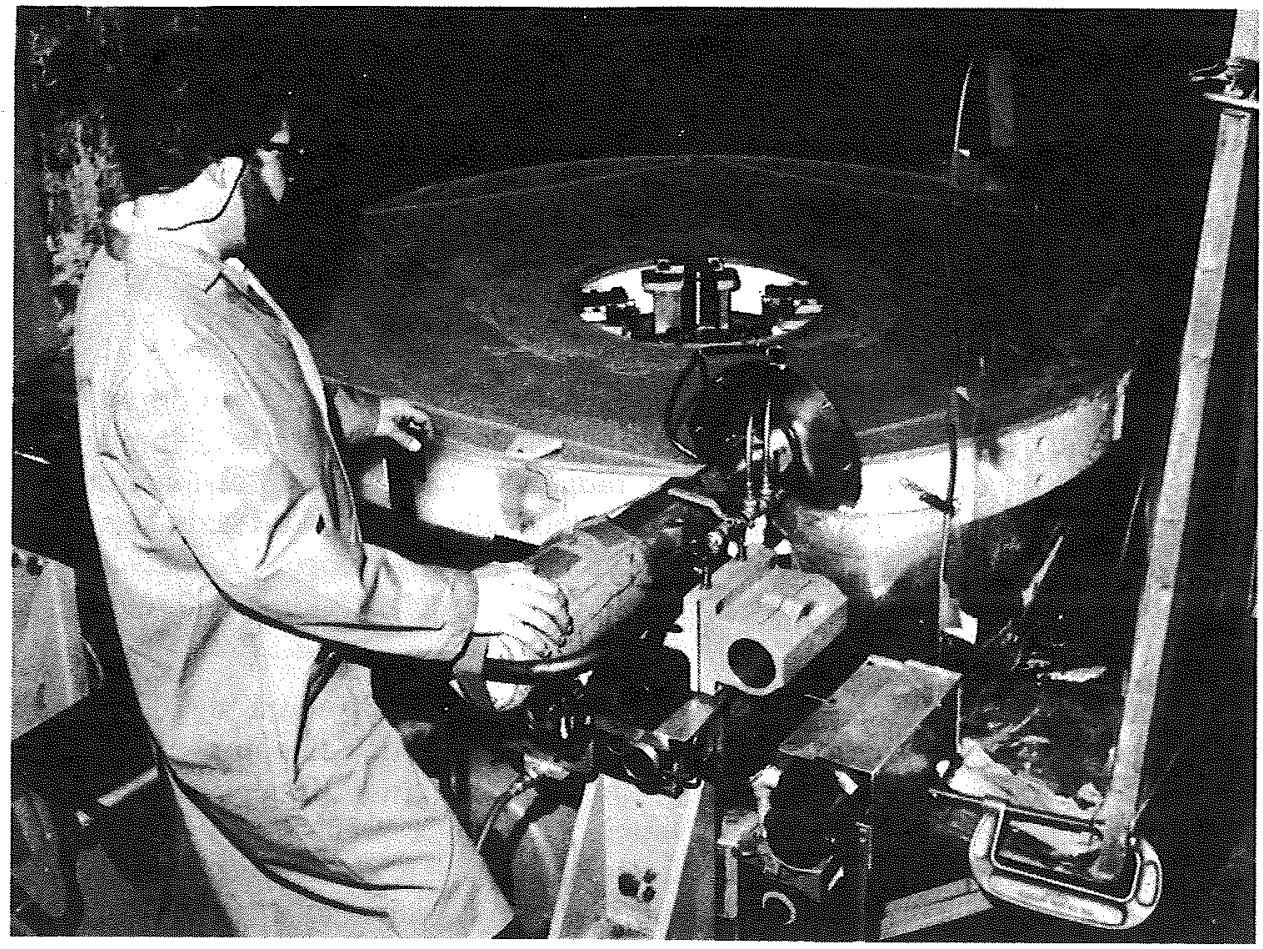

Fig. 2. Example of facesheet mirror blank. 


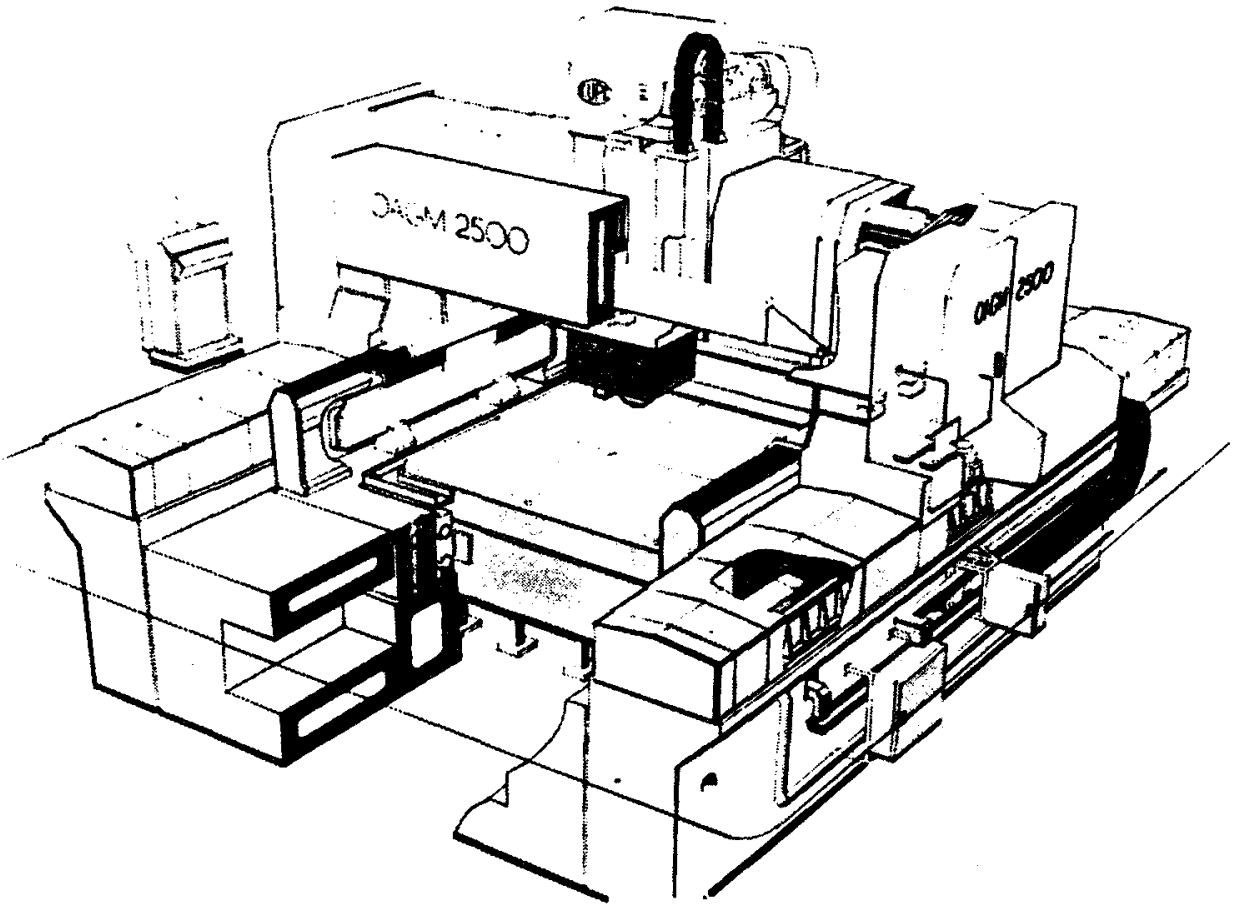

Fig. 3. Kodak's $2.5 m$ off-axis generating machine.

The OAGM 2500 will hold off-axis elements on the machine work table in an orientation that minimizes both sag and slope. The in-situ metrology system will reference the coordinate system of the machine and glass blank to the aspheric equation. The surface will then be diamond ground and profiled by the metrology system. Tool wear will be compensated and the map of the surface profile will be passed onto the next process step. The fine matte surface produced by this machine will not be sufficiently specular for interferometry. Further fine grinding will not be necessary, as the surface roughness and surface figure errors will be well within the envelope of correction for the subsequent small tool polishing operation. This machine may be capable of ductile grinding (the metrology system has $2 \mathrm{~nm}$ resolution). If this is possible, the specular surface produced will be ready for interferometric testing, thereby minimizing or eliminating the subsequent small tool polishing operation. Process development will evolve toward this end.

\subsection{Small Tool Polishing}

To remove subsurface damage from the previous aspheric generating operation and to achieve the surface specularity required for interferometric testing. Subaperture lap polishing is best suited to this operation because of the size and lack of symmetry in the shape of off-axis segments. Full aperture laps have difficulty conforming to non-symmetric shapes and are limited further by large aspheric departures.

The concept of small tool polishing consists of moving a small orbiting polishing lap over the optical surface under computer control. Because the removal profile of the lap is known, and because polishing parameters are held constant during the run, controlled amounts of material can be removed.

At Kodak, process development involving small tool polishing has been concentrated on understanding the process parameters that effect the tool removal function. To accommodate aspheric surfaces with various slopes and errors, computer modeling routines have been developed to choose the optimum tool removal function. The notorious edge effects caused by small tool polishing have also been modeled. Controlled experiments that compare predicted removal vs. actual removal yield results as shown in Fig. 4. Results indicate that this approach is deterministic. 


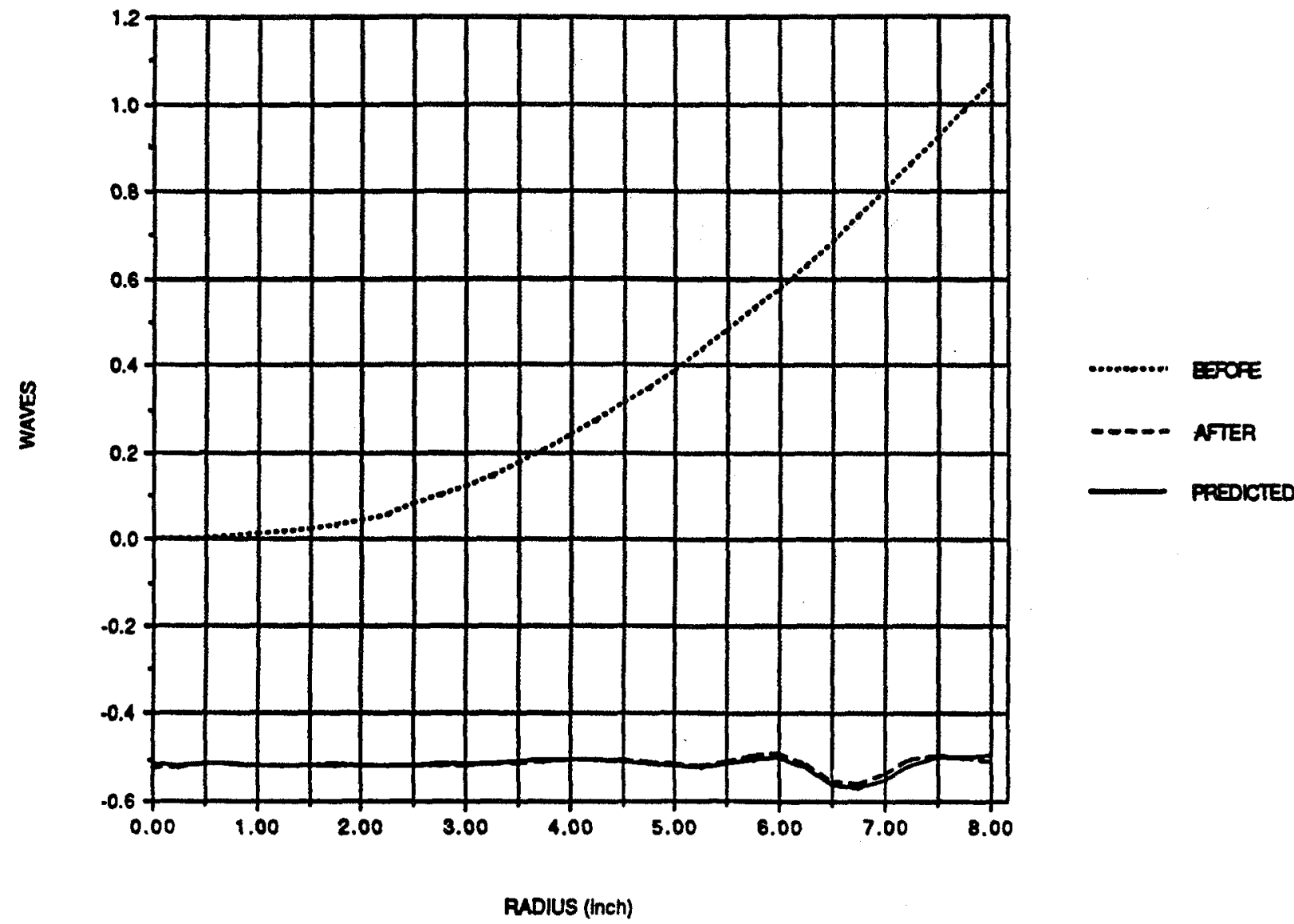

Fig. 4. Small tool polishing profile showing predicted edge ripple.

The roles of small tool polishing in large asphere production are a) removing subsurface damage left from the generating operation, b) making the surface specular for interferometric testing, c) correcting the figure to within a fraction of a wave of nominal, and d) producing a surface microroughness consistent with final performance specifications. Each small tool iteration will remove (gradually and efficiently) the subsurface damage of the previous step. The proper choice of polishing parameters will optimize convergence towards the nominal aspheric profile. Complications in final figuring caused by the edge ripple effect are not expected. Ion figuring technology will be used to complete the figuring. The cost advantage of this approach is that it requires fewer iterations. Tools with the optimum volumetric removal function can be chosen for efficiency first, and figuring second.

\subsection{Ion Figuring}

Ion figuring is the removal of material from the substrate, on the atomic level, by way of a momentum transfer from an ion beam. This technology has found applications in substrate surface cleaning, coating implantation, and surface modification. Application of this technology in optical processing has three major advantages. First, it is a non-mechanical process whose performance is determined by physical parameters that can be controlled and modeled. Second, only low level substrate heating takes place. Third, and most important, the use of a subaperture ion beam for figuring is analogous to small tool polishing practice (Table 1) with the following distinct advantage: the removal profile of the ion beam is insensitive to the edges of the optic and other geometry factors including unusual shapes and thin plate thickness. 5 
Table 1

SMALL TOOL POLISHING AND ION FIGURING ANALOGY

$\begin{array}{lcc} & \text { Removal Function Parameters } \mathrm{X} & \text { Path Profile = Figuring } \\ & \text { Dwell Time } & \\ \text { Small Tool } & \text { Obliquity } & \text { Rectangular } \\ \text { Polishing } & \text { Pad \& Orbit Diameter } & \mathrm{X}, \mathrm{Y}, \mathrm{Z} \\ & \text { Pad \& Orbit Speed } & \\ & & \\ & \text { Dwell Time } & \\ \text { Ion } & \text { Obliquity } & \text { Rectangular } \\ \text { Figuring } & \text { Beam Energy } & \mathrm{X}, \mathrm{Y}, \mathrm{Z} \\ & \text { Gas Type } & \\ & \text { Temperature } & \end{array}$

Kodak has supported process development in ion figuring and has significant experience with the substrates fused silica and silicon carbide using ion plasma etch on optics up to $100 \mathrm{~mm}$ in diameter. We have now increased this process capability by commissioning DynaVac Company, Division of Tenney Engineering, to build a vacuum chamber capable of ion figuring (Fig. 5). This chamber has a $2.5 \mathrm{~m}$ capacity. The ion source position is controlled by a five-axis CNC positioner $(x, y, z, \phi x, \phi y)$. The machine is expected to be on line in September 1989.

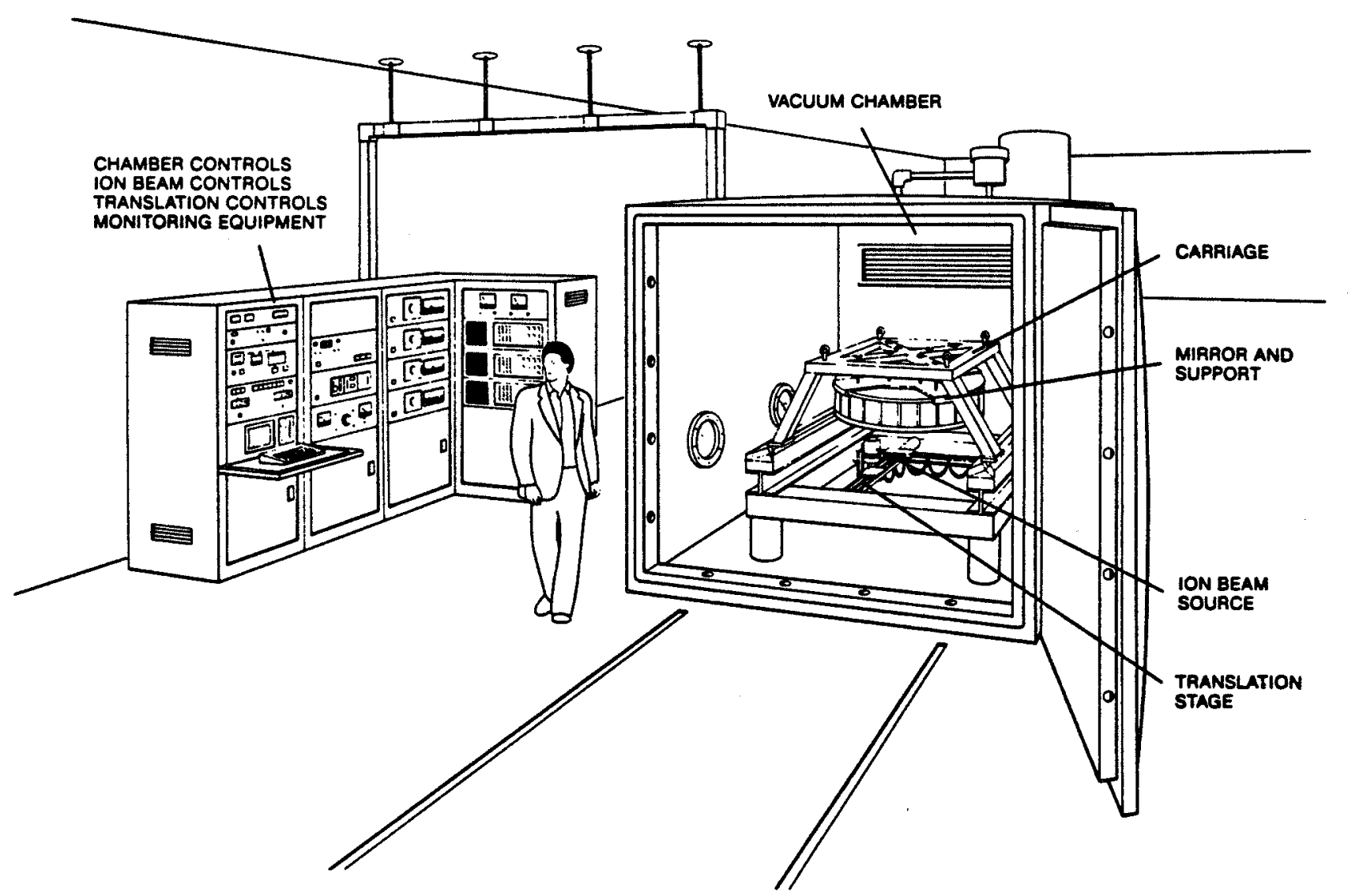

Fig. 5. Kodak's ion figuring system. 
With the IFS (Ion Figuring System), off-axis optical elements will be figured to their final specification. Interferometric data will be passed in closed loop fashion to the machine's controller for each iteration. Few iterations are expected, as this process is highly deterministic. The logical extension to this process development is a state-of-the-art machine that exhibits in-situ metrology and thin film coating capability. Process development will evolve toward this goal.

\section{LARGE PLANO OPTICS TECHNOLOGY}

Multi-segmented plano optics are also a design possibility. Again, these optics may be active by design, being either ultra-lightweighted (Fig. 6) or facesheets. Glass-ceramic or stable glass materials such as fused silica, ULE(W), or Zerodur(im) are likely candidates for substrates. We believe that surface figuring before ion figuring is best accomplished using planetary polishing techniques.

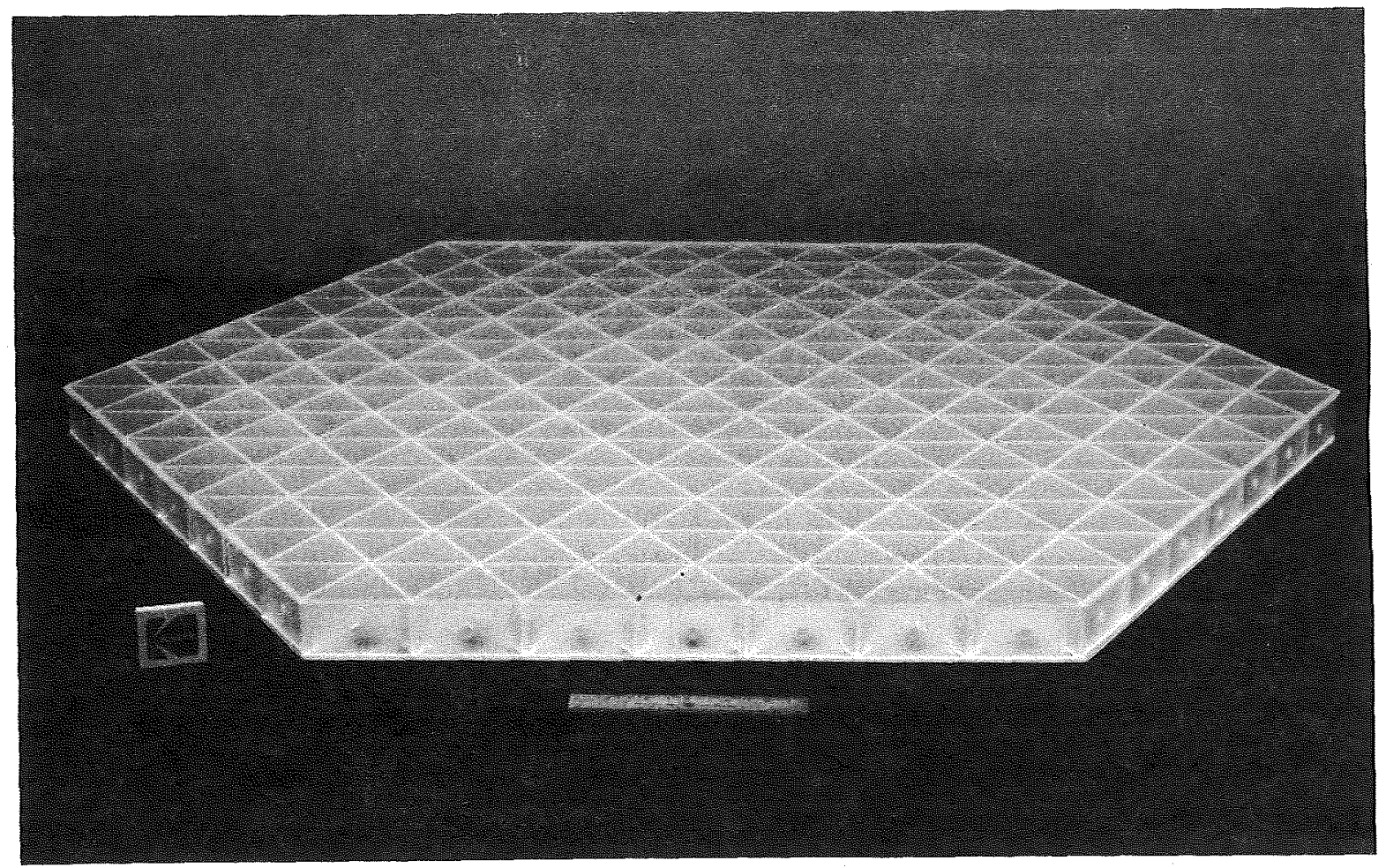

Fig. 6. Example of $1 \mathrm{~m}$ plano ultra-lightweight mirror.

\subsection{Planetary polishing}

Planetary polishing machines have a horizontal, annular-shaped lap, the flatness of which is maintained by a conditioner polishing on the surface along with two or more workstations. Within each workstation, optics are held in septums which are motor-driven to maintain rotational synchronization with the lapping table. Recirculating slurry systems are frequently used and environmental controls are necessary to maintain figure control of the lap. Planetary polishing is an efficient method of polishing large plano optics. Numerous optics can be processed simultaneously and stock removal can be carefully controlled, minimizing subsurface damage. Fig. 7 shows a $4 \mathrm{~m}$ planetary polishing machine designed and built at Kodak to support $1 \mathrm{~m}$ optics for the Lawrence Livermore National Laboratory's Nova Laser program. The table for this polisher is a single piece of granite weighing 33 tons. The conditioner is a 2.5 ton piece of fused quartz.

Final figuring for large plano optics no longer needs to be completed on the planetary polishing machine. Once subsurface damage is removed and the surface error is within a fraction of a wave of specification, ion figuring will be used to complete the manufacturing process. For large plano optics used in transmission ion figuring is an extremely attractive method to correct for inhomogeneities in the glass. 


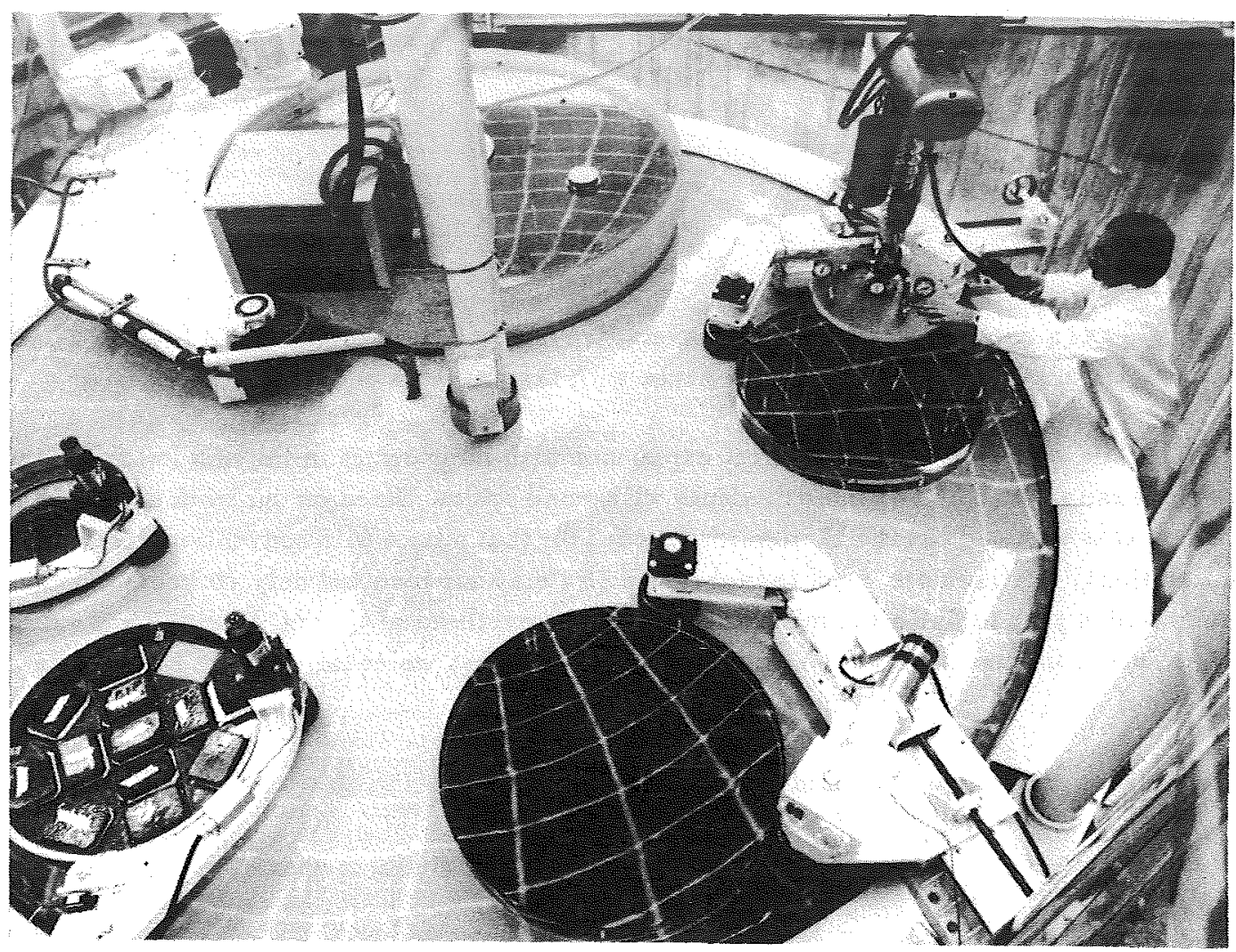

Fig. 7. Kodak's $4 \mathrm{~m}$ planetary polishing machine.

\section{CONCLUSIONS}

Kodak's new optical fabrication and finishing systems are undergoing final acceptance tests and should be operational by the end of 1989 . They will be usable with beryllium and other materials, as well as glasses. Their application will substantially reduce the time and cost traditionally associated with large optics. Kodak's developments have been driven by our growing concern that, despite growing needs for large optical systems, their deployment could be inhibited by expensive optical components.

\section{REFERENCES}

1 P.N. Swanson, J.B. Brekinridge, A. Diner, R.E. Freeland, W.R. Irace, P.M.McElroy, A.B. Meinel and A.F. Tolivar, "System Concept for a Moderate Cost Large Deployable Reflector (LDR)," Optical Engineering, Vol. 25, No. 9, pp. 1045-1054, September 1986.

2 D.C. Kline, "Space-based Chemical Lasers for Ballistic Missile Defense (BMD)." Proceedings of the International Conference on Lasers '87, F.J Duarte editor, pp. 205-217, STS Press, McLean, VA, 1988

${ }^{3}$ N.J. Brown and B.A. Fuchs, "Brittle to Shear Grinding Mode Transition for Loose Abrasive Grinding," OSA Technical Digest: Optical Fabrication and Testing, pp. 23-26, November 1988.

${ }^{4}$ P.B. Leadbeater, M. Clarke, W.J. Wills-Moren, T.J. Wilson, "A Unique Machine for Grinding Large, Off-axis Optical Components: The OAGM 2500," Precision Engineering, to be published.

${ }^{5}$ S.R. Wilson, D.W. Reicher and J.R. McNeil, "Surface Figuring Using Neutral Ion Beams," Advances in Fabrication and Metrology for Optics and Large Optics, J.B. Arnold and R.E. Parks editors, Vol. 966, pp. 74-81, SPIE, Washington 1989.

${ }^{6}$ L.N. Allen, "Precision Figuring of Optics by Ion Machining Processes," Conference on Lasers and ElectroOptics, Baltimore, MD, Vol. 11, April 1989. 


\section{DISCUSSION}

Nelson: I have a question regarding the convergence rate of ion polishing. I had heard that you could get accuracies of $90 \%$. What is your experience with this machine and your expectations?

Keller: While we haven't had any direct experience with large optics in the new ion facility, we have made some estimates based on our experience with small optics. Based on our work on the HST mirror we believe that we could cut the 18 iterations needed for that mirror with conventional techniques down to 3 iterations. Furthermore, even though those 18 iterations involved only 70 hours of polishing, it actually took a year to finish the mirror because of the overhead of testing, handling and setup. With a deterministic process such as ion polishing we believe that we could do a HST mirror in about a month.

Miller: Can you tell us what ductile grinding is?

Keller: Effectively it is like diamond turning where the material is sheared away instead of being "chipped" off.

Angel: Can you give us some feel for the size of the ripples that might be left on an aspheric surface from the polishing tool and the size of the ion beam needed to remove them?

Keller: We need some experience before we can give you some real numbers, but we do have some calculations.

Nelson: What is the maximum beam size that you could use?

Keller: The beam size could be 5 , or 10 or $15 \mathrm{~cm}$. It can be made quite large.

Tlingworth: Removal of the edge ripple from your example would suggest that you need ion beams that are a fraction (1/3-1/4) of the small tool size. Is that the size you use - several cm? If I understand correctly, this means that the "polished" surface from the "small tool" polishing would have to have the final desired smoothness on scales less than several centimeters.

Keller: Yes, 1/3 to $1 / 4$ will work, but the "up modes" of the ripple are Gaussian so the same size will work (faster removal). However, note that in one example, the low ripple mode was below the nominal surface. In practice one must keep the ripple zone "turned up." 
Super Smooth Optics for Extra-Solar Planet Detection

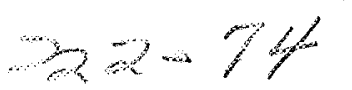

415234

\section{Richard J. Terrile} \\ Jet Propulsion Lab, Caltech, Mail Stop 183-501, Pasadena, CA \\ 360552 \\ 91109
}

and

Christ Ftaclas

Hughes Danbury Optical Systems Inc., 100 Wooster Heights Rd.,

Danbury, CT 06810

\begin{abstract}
The goal of imaging planets around the nearby stars has important scientific significance but requires the use of advanced methods of controlling diffracted and scattered light. Over the last three years we have undertaken a study of coronagraphic methods of controlling diffracted light and of figuring hyper-contrast optics. Progress in these two general areas have led to a proposed space-based, 1.9 meter diameter coronagraphic telescope designed specifically for very high performance in the imaging of faint objects near bright sources. This instrument, called the Circumstellar Imaging Telescope (CIT), relies on a new high efficiency coronagraph design and the careful control of scattered light by extremely smooth optics. The high efficiency coronagraph uses focal plane apodization in order to concentrate diffracted light more efficiently in the pupil. This allows convenient removal of the diffracted light by masking off parts of the telescope pupil while not sacrificing the center of the field. Reductions of diffracted light by factors exceeding 1000 are not only possible but are required in order to detect extrasolar planets. Laboratory experiments with this new design have confirmed the theoretical diffraction reductions to the limits of the optics used (factors of about 300). The extremely high efficiency of this coronagraph puts strong constraints on the narrow angle scattered light due to figure errors in the telescope mirror. Since planets orbiting nearby stars are expected at angular distances of about 1 arcsecond, it is in this small angular range in which scattering must be controlled. The figure errors responsible for scattering in this range come from mid-spatial frequencies corresponding to correlation lengths of about $10 \mathrm{~cm}$ on the primary mirror. A primary mirror about 15 times smoother than the Hubble Space Telescope mirror is required for the CIT. Laboratory experiments indicate that small test mirrors can be fabricated with existing technology which come within a factor of two of this requirement.
\end{abstract}

\title{
1. INTRODUCTION
}

In this paper we will discuss the application of super-smooth optics to the problem of the direct detection of extra-solar planetary systems. The two key technology developments which have enabled serious consideration of this scientific problem are in the control of diffracted light and in the fabrication of super-smooth optics. This paper will concentrate on the control of 
diffraction. Further discussion of mirror scattering and fabrication issues can be found in reference 1.

Over the last three years a joint program between the Jet Propulsion Laboratory (JPL) and the Perkin-Elmer Corporation (now Hughes Danbury Optical Systems Inc.) has been organized to define the requirements for optics capable of directly detecting extra-solar planetary systems from Earth orbit. The main conclusions from this study were that: 1) Direct detection of nearby extrasolar planets is possible from a meter-class orbital telescope designed to reduce diffracted and scattered light to levels about 1000 times below diffraction from the unapodized aperture. Furthermore, there are a wide range of scientific goals in planetary science and in astrophysics that are addressable with such an orbital telescope. 2) A high efficiency coronagraph was designed and tested in the laboratory which can reduce diffracted light to these required levels without sacrificing the center of the field of view and without dramatically reducing the effective aperture diameter of the telescope. 3) The requirements on the mirror figure demand a mirror about 15 times smoother than the HST primary at mid-spatial scale frequencies $(10 \mathrm{~cm})$. However, laboratory and fabrication experiments have shown that current metrology is capable of making such measurements and that small ( $30 \mathrm{~cm}$ diameter) test mirrors have been fabricated with nearly flight quality figures. As a result of these conclusions we have proposed a flight project called the Circumstellar Imaging Telescope $(\mathrm{CIT})^{2,3}$.

\section{THE CIRCUMSTELLAR IMAGING TELESCOPE (CIT)}

The CIT consists of a 1.9 meter diameter telescope in which small angle scatter has been greatly suppressed, mated to a camera that can implement a variety of diffraction control strategies. These strategies, including a new high efficiency design, are chosen to realize the performance inherent in the fore-optics and to maximize the scientific return of the mission. Thus both diffraction and scatter are comparably reduced unveiling the circumstellar environment. One arcsecond away from a bright point source the baseline CIT will have a background level more than 5 magnitudes per square arcsecond fainter than the Hubble Space Telescope (HST) leading to a much higher sensitivity near bright objects.

The key design features of the CIT are control of scattered light through use of super-polished mirrors, control of diffracted light through use of a coronagraph, and control of image motion through use of an internal fine pointing system. Computer modeling indicates that, with only moderate advances in mirror fabrication technology, the CIT will be able to detect Jupiter sized planets around solar type stars at distances out to ten parsecs.

\section{PLANETARY DETECTION AND CHARACTERIZATION}

At the very heart of Planetary Science lies the question of the uniqueness of life on Earth. Current theories entertain the idea that suitable conditions for life probably occur commonly throughout the galaxy on planets surrounding stars. Therefore, a corollary to this fundamental question is what is the nature and probability of planet formation. With our solar system as the only available model, clues to the nature of planet formation are derived from observations of current conditions. The planets orbiting the sun are co-planar, 
co-rotating and generally show a compositional ordering with distance from the sun. This regularity is thought to be a relic of the conditions during the formation of the sun and solar system. Models of the formation of the planets have them forming in a collapsing nebula or disk of material around the early sun. Indeed, recent observations of circumstellar disks indicate that these conditions do occur early in the lifetimes of stars. In order to gain a deep understanding of the entire process of planetary formation it is desirable to observe the various stages of evolution from the earliest stages of stellar formation to a survey of mature planetary systems. Recent progress has be made in detection and imaging of circumstellar disks, but direct planet detection remains the most challenging observational goal.

The most difficult aspect of direct detection of mature planetary systems is the large difference in brightness between the planets and parent star. In the example of Jupiter around the sun the brightness ratio in visible light is a factor of about 1 billion ${ }^{4}$. Furthermore, the maximum diameter a planet can have is about $10 \%$ greater than Jupiter before additional mass causes the core to become degenerate and the radius to decrease with added mass. The visual magnitude of Jupiter, viewed from 10 parsecs, is 25.9 at opposition, 27.4 at quadrature and 27.5 on average. Only the presence of a broad ring system could add to the equivalent cross section and therefore the brightness of a large planet. The challenge of direct detection of planets is not in the detection of a faint signal, but in separating this relatively faint planetary component from the dominant background glare of the parent star. Even for relatively nearby stars the angular separations of parent star and planet are an arcsecond or less. It is now recognized that the HST will not be able to directly detect extra-solar planetary systems because of the inherent levels of scattered light in the telescope $5,6,7$.

\section{CONTROL OF DIFFRACTED LIGHT}

The CIT baseline design includes diffraction control technologies which fully exploit the low scatter telescope optics. The CIT camera will use coronagraphic approaches to suppress diffraction near single bright in-field sources, as with the extra-solar planetary detection problem, and a pupil apodization approach to suppress the diffraction wings of all sources in crowded fields, as in observations of stellar populations in compact clusters.

Traditional approaches to diffraction reduction have involved either apodization (rolling-off or softening the edge of the entrance pupil to lower diffraction sidelobes), or coronagraphic re-imaging systems. The requirement for large diffraction reductions at sub-arcsecond scales imposed by the planetary detection problem argues against pupil apodization which extracts increasing penalties in effective collecting area for improved small angle performance ${ }^{4}$.

The coronagraph was first employed by Lyot $^{8}$ in 1934 to observe the solar corona. At the first focus the source is occulted and then the entrance pupil is re-imaged. The occultation results in the remaining diffracted light in the system concentrating in a ring approximately centered on the image of the edge of the entrance pupil. The pupil or Lyot stop masks off part of this ring so that when the light is re-imaged to a second focus the diffraction pattern is reduced in intensity. The same principle can be applied to secondary obscuration and secondary support structure so our subsequent discussion will 
concern itself with a simple circular unobscured pupil.

As a typical example of the classical coronagraph, when the occulting mask covers about 5 diffraction radii (i.e. 5 lambda/d) and the Lyot stop is $90 \%$ of the pupil image in diameter, the reduction in diffraction is about a factor of 100. Calculation of the pupil plane and first and second focal plane intensities for this case are shown in Figures $1 a$ and $b$. Closing down the Lyot stop in this case produces no further gains since the remaining light in the pupil plane is virtually uniformly distributed. The only way to improve the diffraction reduction efficiency of the coronagraph is to enlarge the occulting stop giving up valuable science field. For a 1.9 meter aperture the 5 lambda/d disk is already occulting the central arc second of the field which we considered marginally acceptable. Furthermore the hard edge of the occulting mask has rung through the system creating a diffraction peak centered on the stop edge further reducing the useful field. In short the classical coronagraph is inefficient, and performs worst where it's most needed, near the center of the field.

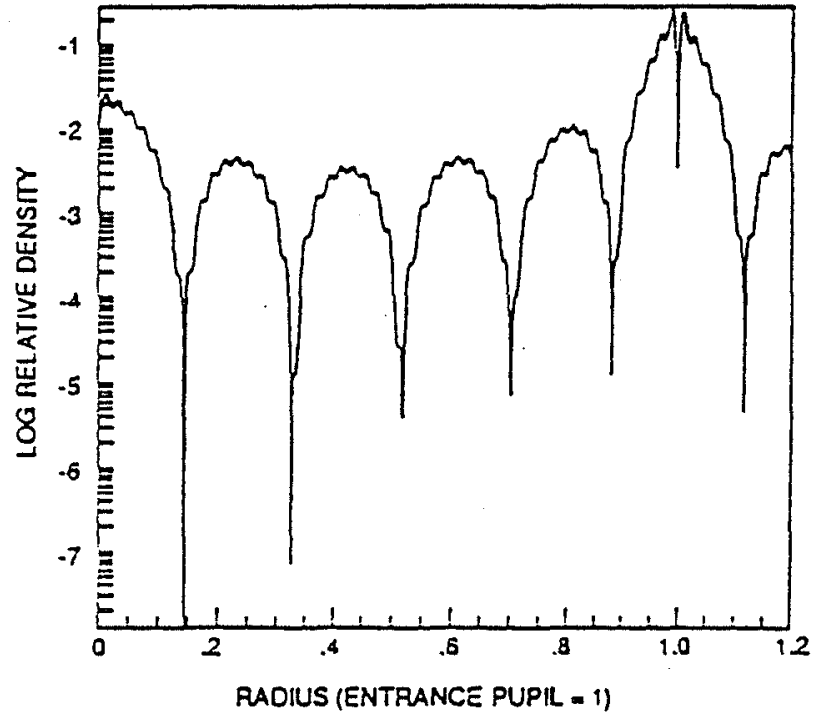

Figure 1a

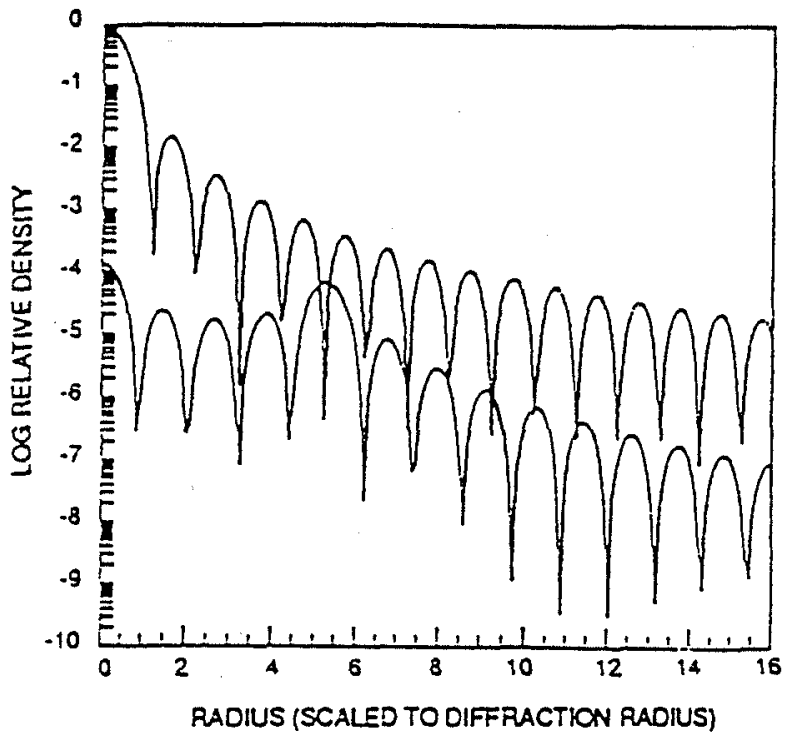

Figure 1b

Figures $1 \mathrm{a}$ and $\mathrm{b}$ show the distribution of light in the pupil of a Lyot type coronagraph and in the first and second focal planes, respectively. Note that in Figure la diffracted light is concentrated in a ring near the outer edge of the entrance pupil but is only reduced by about a factor of 10 in the center of the pupil. A $90 \%$ Lyot mask effectively removes the outer bright ring. Figure 10 shows the original star signal in the first focus (top curve) and in the second focus (bottom). Note that at the second focus light is reduced by about a factor of 100 but the edge of the occulting mask produces a bright ring of light at about 5 Airy rings.

The key to improving the performance of the coronagraph is to pack more diffracted light in the pupil plane ring. This suggests apodization in the conjugate or focal plane. In Figures $2 a$ and $b$ we show the same intensity plots as in Figures $1 a$ and $b$ but now the hard occulting mask has been replaced by $a$ graded transmission mask whose amplitude transmission varies as [1-exp $\left.\left(r / r_{0}\right) * * 2\right]$ with $r_{0}$ adjusted to give 508 intensity transmission at the 5 lambda/d point. The focal plane apodization has concentrated the diffraction pattern in the pupil plane into a single broad peak. The diffraction intensity reduction is comparable to the previous case for a 908 Lyot stop but now when the lyot stop 
is closed down from 90 to 808 diffraction intensity is reduced by more than an order of magnitude. For this hybrid coronagraph occulting mask transmission profile can be traded against pupil plane mask size to obtain optimal performance for a given target location and characteristic. For example, moving the half power points of the occulting mask out to 8 lambda/d allows the Lyot stop to be opened from $80 \%$ to just under 908 of the pupil diameter without changing final focus performance. Higher efficiencies $\left(>10^{4}\right)$ are achievable by appropriate adjustments of the pupil and occulting mask diameters.

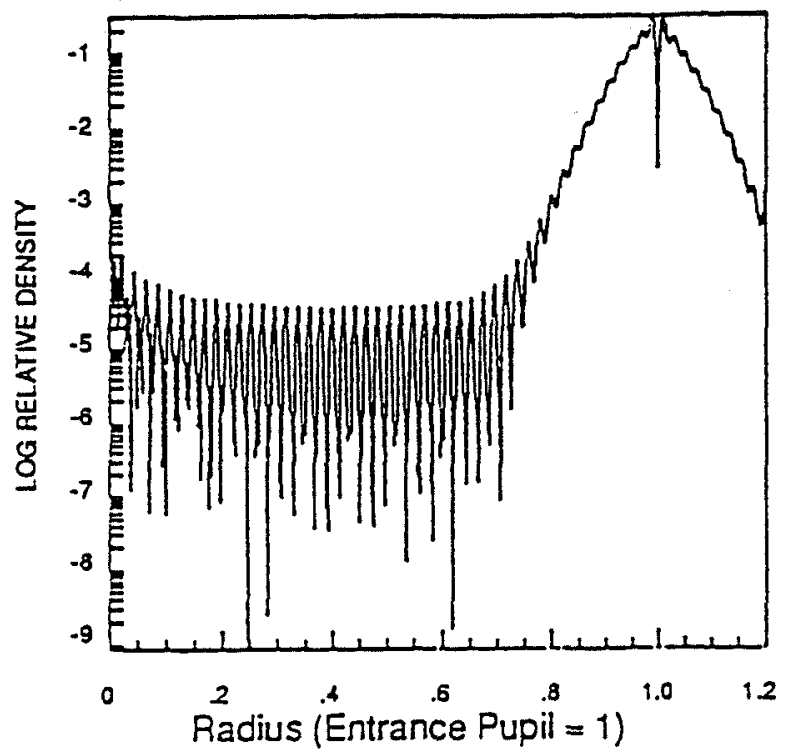

Figure 23

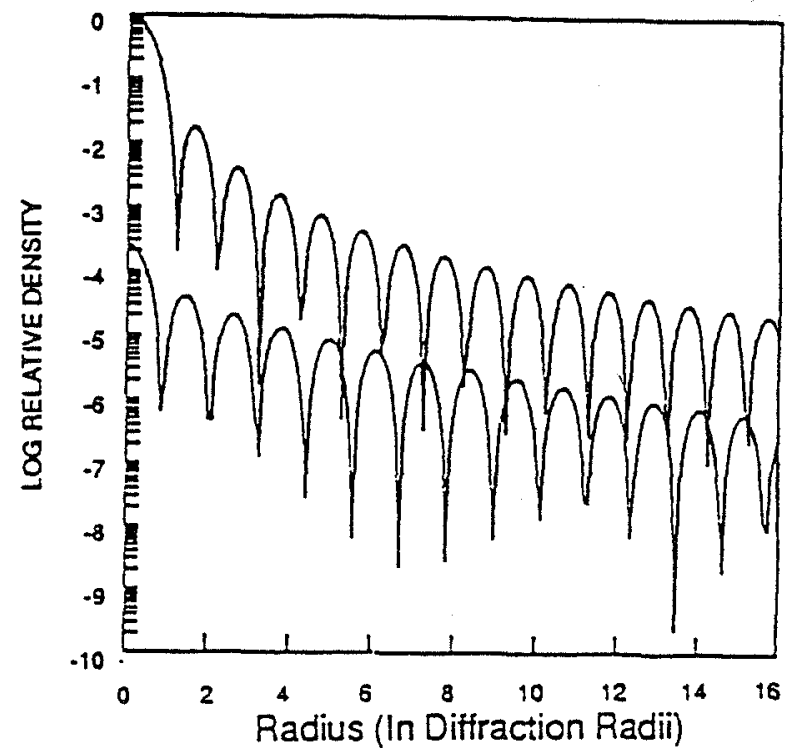

Figure $2 b$

Figures $2 a$ and $b$ are similar to Figures $1 a$ and $b$ but are for a high efficiency coronagraph using $a$ $90 \%$ Lyot mask. The diffracted light in the center of the pupil is now reduced by over three orders of magnitude and masking can be more effective (Figure 2a). An $80 \%$ Lyot mask would reduce diffracted light in the second focus by about a factor of 1700. Also because of the transparency of the occulting mask, the second focus (bottom of Figure 2b) shows a smooth distribution of light and high efficiency all the way to the center. Photographs of these cases from our coronagraphic breadboard are shown in Appendix B.

In addition to larger diffraction reductions and getting rid of edge ringing, the "soft" or "gaussian" mask used above offers two advantages critical to the performance of the CIT:

1. The mask is partially transmitting so imaging through the mask is possible all the way to the center of the field.

2. The system performs better at small field angles than large ones so that the "effective" diffraction efficiency, that is, the diffraction reduction scaled to the transmission of the mask is nearly constant up to a few diffraction radii.

Thus, over most of the field, the hybrid coronagraph enhances the brightness ratio of an off-axis source with respect to an on-axis source by a nearly uniform factor even when transmission losses through the mask are taken into account.

We have constructed a breadboard model of the coronagraph at Perkin-Elmer 
and results to date totally support the computer models. Our gaussian masks are made by direct playback through a microdensitometer onto Kodak Tech Pan film. They have a central photographic density in excess of 5 and conform quite closely to the required transmission profile. For the dimensions of our laboratory system the full width between $50 \%$ transmission points of these masks is $1 \mathrm{~mm}$.

We have compared the occulted point spread functions, and resulting pupil plane images for the hard and "soft" stop cases. We have verified to date that the run of intensity for the 908 Lyot stop follows model predictions for both the hard and soft edged stops. So far we have demonstrated diffraction reductions of 300 to 400 and are limited by the quality of the relay optics used in the laboratory set-up.

\section{CONCLUSIONS}

We have defined the observational constraints to directly detecting extrasolar planets around the nearby stars. The design of an instrument capable of meeting these goals requires the efficient reduction of diffracted and scattered light by about 3 orders of magnitude below the airy wings of the unapodized aperture. The diffraction reduction is achieved through the high efficiency coronagraph, which uses gaussian occulting masks in the first focus to better concentrate diffracted light in the pupil plane, and laboratory experiments confirm this high efficiency to the limits set by the experimental set-up. Scattering, due to mid-spatial scale figure error in the mirrors must also be reduce to a comparable level and fabrications of such quality mirrors has been demonstrated in the laboratory.

\section{REFERENCES}

1. Ftaclas, C. and Terrile, R. J. "Fabrication Experiments on Super-Smooth Optics for Direct Detection of Extra-Solar Planetary Systems." SPIE Proceedings, in press. (1989)

2. Terrile, R. J. "Direct Imaging of Extra-Solar Planetary Systems With A Low-Scattered Light Telescope." Bioastronomy - The Next Steps, G. Marx editor, Kluwer Academic Publishers, 125. (1987)

3. Terrile, R. J. "Direct Imaging of Extra-Solar Planetary Systems with the Circumstellar Imaging Telescope (CIT)." AIAA Paper 88-0555, 26th Aerospace Meeting, Reno, Nevada, Jan 11-14 (1988)

4. Lockheed Palo Alto Research Laboratory "Systems Level Feasibility Study for the Detection of Extrasolar Planets, Part 2, Apodized Telescope (APOTS)." LMSC-D676425, Lockheed. (1979)

5. Brown, R. A. "Efficiency of HST." STSCI News Letter, Vol. 3, No. 1.

6. Brown, R. A. "Direct Planet Detection: A Realistic Assessment of HST and Other Spaceborne Optics." Bioastronomy - The Next Steps, ed. G. Marx, Kluwer Academic Press. (1987)

7. Brown, R. A. and Burrows, C. J. "On the Feasibility of Direct Extrasolar Planet Detection Using Hubble Space Telescope." Icarus, in press. (1989).

8. Lyot, B. "A Study of the Solar Corona and Prominences Without Eclipses." Mon. Not. Royal Astron. Soc., 99, 580. (1939). 


\section{DISCUSSION}

Miller: Was the increased smoothness at the end due to the way in which the mirror was supported and the pitch was grooved, or was there some other process that was used?

Terrile: I am not aware that anything particularly unusual was done during the polishing. Some gains were made by stiffening the lap and controlling the mechanics of putting the mirror together. We are now interested in trying to see if the same gains can be obtained with small tools - with computer controlled polishing.

Burrows: If you scaled this to 16 meter, the diffraction curves would come down and the problem would be easier.

Terrile: That's a good point. With a 16 meter mirror you would be looking for the Jovian planets at many more rings out. So the Jupiter detection problem becomes easy because you would be at 50 - 100 rings out. But if you want to look at other problems like detecting earth-like planets, then you are back to the same difficulties.

Ilingworth: Have you thought about the practicality of doing these smooth surfaces with lightweight mirrors where you would have to worry about print-through from the ribs and supports to the surface?

Terrile: Yes, you have to worry about that. With planet detection we are talking about using spatial frequencies around $10 \mathrm{~cm}$, just the scale of the waffle pattern on a lightweight mirror. So we are very interested in the new lightweighting approaches, particularly those where the mirrors are lightweighted after polishing. Unless we can be shown otherwise, we will take a conservative approach and opt for a solid 2 meter mirror, with its weight disadvantage, for this problem of searching for Jovian planets. 


\section{Sensing and Control for Large Optical Systems}

\author{
Alan B. Wissinger
Perkin Elmer Corporation \\ Alan B. Wissinger
Perkin Elmer Corporation
}

Abstract
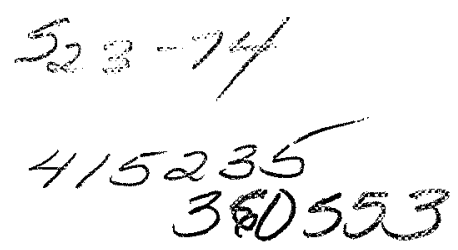

The paper provides an overview of the technology developed at Perkin Elmer for large optical system sensing and control. For future telescopes, sensing and control systems will be required to measure wavefront quality and alignment on-orbit without access to bright stars or to the primary mirror center-of-curvature. Examples of past technology developments (HST's OCS and the Sample Point Interferometer patent), current technology (laser radar and mirror sensing using holographic grating patches), and future technology (phase retrieval from the image alone) are reviewed.

\section{Introduction}

I originally organized this talk along the lines of the historic development of large optics alignment and wavefront sensing and control. However, in listening to other talks at this workshop, I realized that I could have gone back farther in technical history and made an interesting point.

A long time ago, Perkin-Elmer built a 36 inch balloon-borne telescope for Princeton University: Stratoscope II. The Stratoscope II system incorporated a means for alignment sensing and control. The sensing system consisted of a TV camera that produced a very detailed and enlarged image of a star, at about f/200. The control system consisted of Nick Woolf, sitting at the TV monitor in the Stratoscope control van. Nick observed the sharpness and symmetry of the image, and made decisions about how to move the secondary mirror of the telescope. He then commanded (via radio) the mechanism of the secondary mirror mount in one or more of its three degrees of freedom: longitudinal motion for focus and two lateral motions to reduce coma.

I mention this because we have come nearly full circle with our technology; the future technology we are developing at Perkin Elmer once again senses the image directly, but now computers and a special algorithm does what Nick did in his head, albeit with the ability to control more degrees of freedom. In between, we've developed a number of space-borne interferometric techniques for alignment sensing and control that I shall briefly describe.

\section{Requirements}

Table 1 lists typical summary requirements for alignment sensing and control for a large space-borne telescopic system such as the next generation Space Telescope. Since most ground-based optical metrology is done with the sensor at the center of curvature length of the mirror), it is not feasible to transfer these well-known techniques to space applications. Furthermore, sensing techniques which make use of bright stars (as in the Hubble Space Telescope) may not be applicable since the primary mirror of the next generation Space Telescope will most likely require continuous sensing and control of figure quality and segment alignment during long integration times on faint sources. In addition, it is desirable that sensing and control not interfere in any way with the astronomy mission, and, of course, it should be lightweight, efficient and reliable. 
Table 1. Alignment Requirements
Measure:
- Figure quality of optics on orbit without access to bright stars or center of curvature.
- Alignment of optical system
Desired features
- Non interference with mission; continuous measurement
- Applicable to space environment (lightweight, efficient, reliable)

\section{Historic Technology}

\subsection{Hubble Space Telescope Optical Control Subsystem}

The Optical Control Subsystem (OCS) of the Hubble Space Telescope (HST) will produce an interferogram of the optical wavefront sensed at three places in the telescope $\mathrm{f} / 24$ focal plane. With this information, the operators of the telescope will be able to correct focus, alignment, and figure of the primary mirror.

The sensing portions of the OCS is a white-light, radial shearing interferometer. The white light source is a bright star. Figure 1 shows a block diagram of the OCS (along with the Fine Guidance Sensor, which is housed in the same radial bay module). The OCS part of the diagram is enclosed by the outlining.

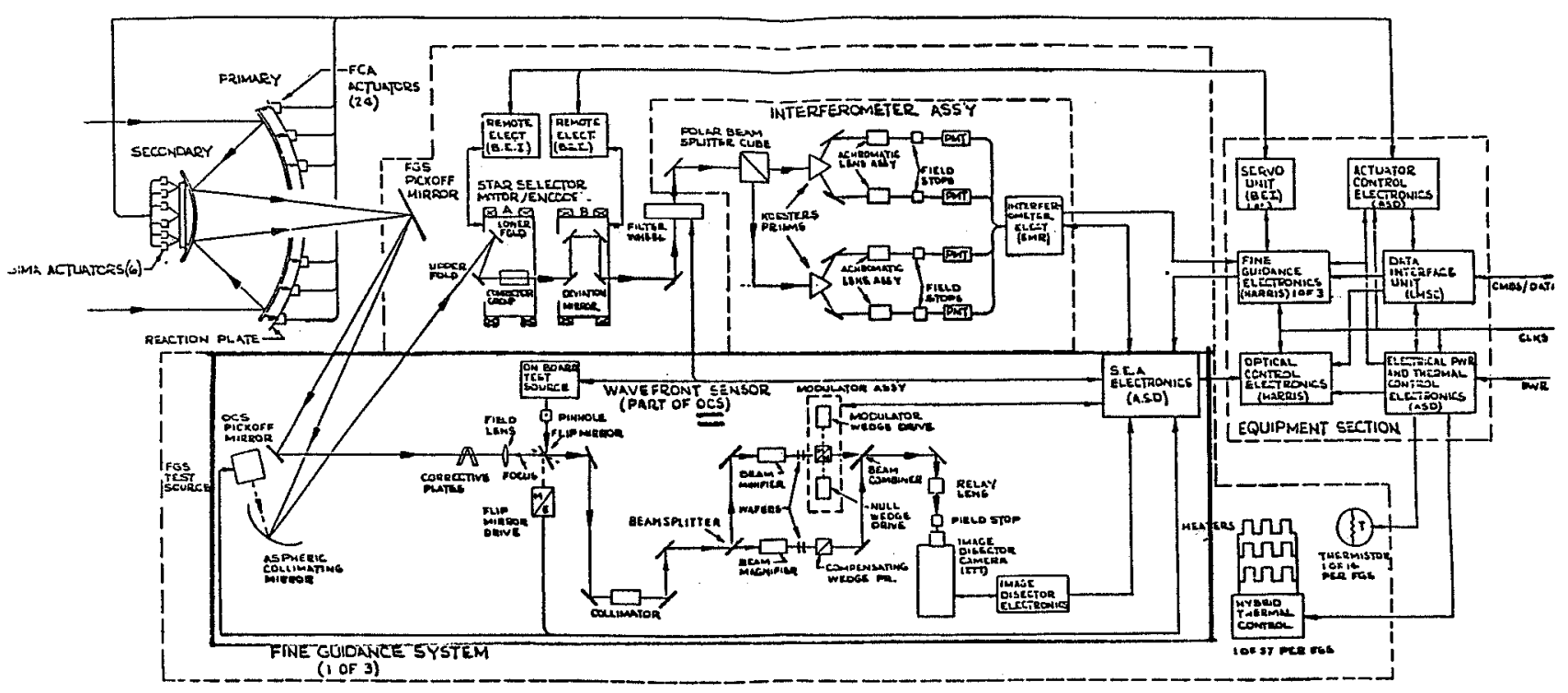

Figure 1. HST Optical Control System 
The main telescope is shown in the upper left part of the diagram, and shows the fivedegree-of-freedom secondary mirror mount and the primary mirror with its twenty-four figure control actuators. In operation, the image of a selected bright star is positioned on the OCS pickoff mirror (lower left). The light from the star is collimated, after passing through the astigmatism corrector plates and the field lens. The collimated beam is split by the beam splitter. One of the beams is magnified by several diameters while the other beam is demagnified by a similar amount. One of the two beams is then path-length modulated by a set of oscillating optical wedges. The two beams are then combined at the beam combiner, and relayed to an image dissector camera.

The interferometer reference beam is the larger of the two beams. In effect, a small portion of the primary mirror (the enlarged beam) becomes the reference for the entire mirror. The camera and signal processing electronics records the temporal phase (relative to the modulator) of the interference pattern and transmits that data to the ground. Further computer processing produces information about the state of focus, alignment and primary mirror figure. It is expected this procedure will be followed weekly in the early days of HST operation, and hopefully less frequently after favorable operating experience is gained.

\subsection{Sample Point Interferometers}

In the late seventies, a patent for the sample point interferometer was granted to Perkin Elmer. The purpose of the invention is to provide interferometric sensing of the position and figure of a mirror segment from a convenient location, i.e. behind either the secondary mirror or the primary mirror.

Figure 2, from the patent, illustrates the principle of operation. A mirror or a segment of a mirror (item 21, upper diagram - "Fig. 1") for which the position and figure quality is to be measured is fitted with a number of retroreflectors about the size of an eraser on a lead pencil. These retroreflectors might be arranged as shown in the inset figure at the right ("Fig.2"). A laser and lens arrangement (Items 10, 11, 12,20 and 22) illuminates the mirror and the embedded retroreflectors. The laser light reflected from the retroreflectors is combined with the light from the reference beam of the interferometer (item 15). The optical path length of the reference leg is modulated by moving the end mirror (17). The combined beams are detected by a photodetector array (item 18), where lens 22 produces an image of the mirror.

For each retroreflector (items $23 a, 23 b$, and $23 c$ ), there is a detector. The electrical signal output of these detectors is shown at the bottom ("Fig. 4"). If the optical path length to the retroreflectors is not exactly equal, a relative phase shift will occur as indicated by item 25. It is this phase shift that is the desired measurement. Measuring phase electronically is much more precise than measuring relative intensity, as in conventional interferograms.

Since the wavelength of the laser light is accurately known, the measured phase shift provides the mirror position actuator system with the signals needed to correct the position (or figure) of the mirror. Of course, the system must be initialized and calibrated since it can only measure changes from the start position, and must make continuous measurements and count waves to "remember" the initial position.

The center illustration ("Fig. 3") shows an embodiment where the laser and interferometer optics are conveniently located behind the primary mirror.

A number of alignment control systems have been built based on this patent. The system is extremely sensitive and is able to control to about a hundredth of a wave. 

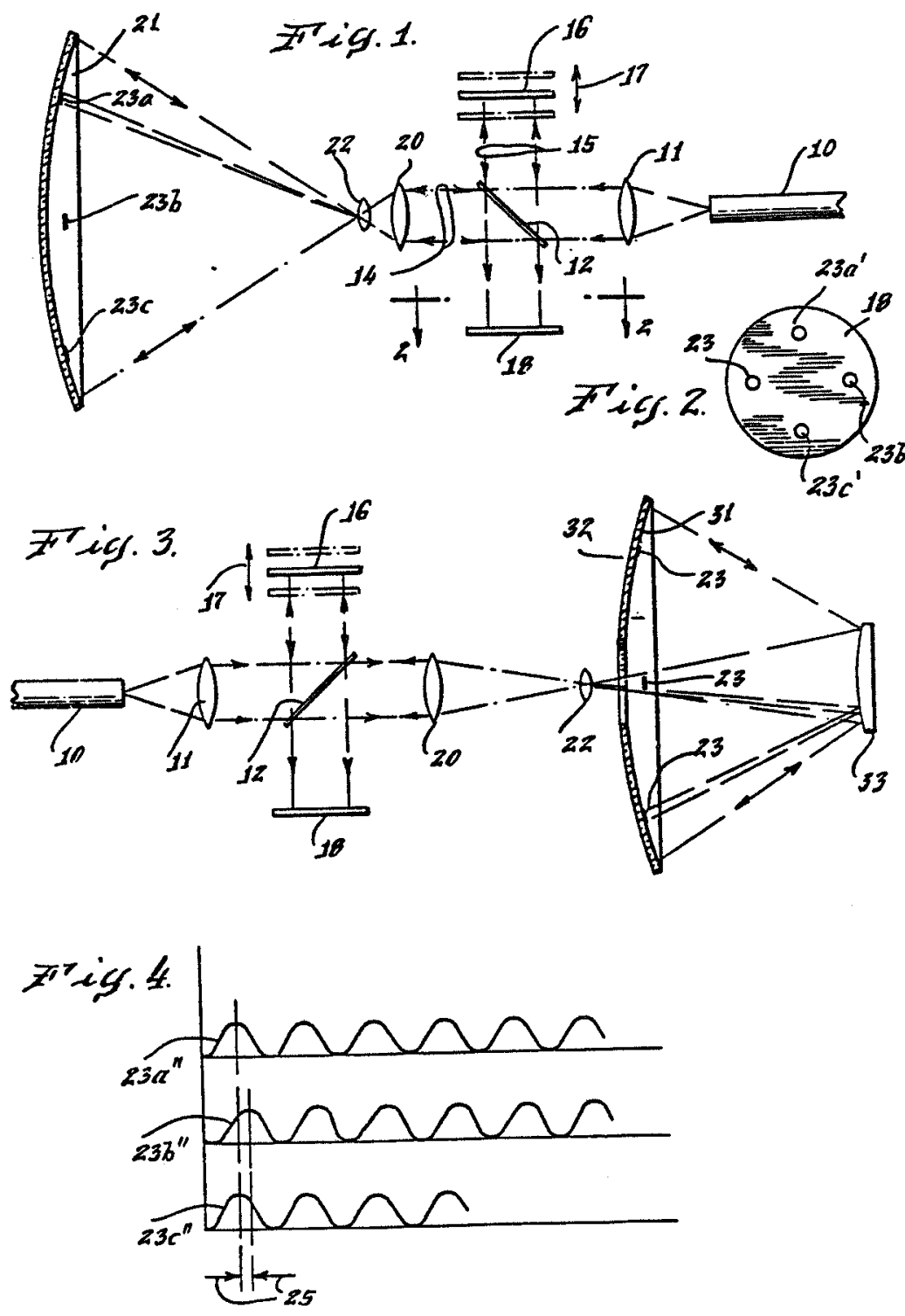

Figure 2. Sample Point Interferometer

\section{Current Technology}

\subsection{Holographic Grating Patches}

Holographic grating patches or holographic optical elements (HOEs) are currently employed in interferometry instead of the small sample point retroreflectors that I just described. The HOEs are the ultimate in representativeness, since they are actually etched directly into the optically-active front surface of the mirror. The diffractive efficiency of the HOE grating is controllable, meaning that the scattered light loss can be extremely small.

Table 2 lists some of the features of the holographic grating patches. Perkin Elmer is capable of manufacturing these HOEs as large as $25 \mathrm{~cm}$. Since they are computer 
generated, they can be designed for any arbitrary mirror surface and can form an image at any reasonably convenient place.

Table 2. Holographic Grating Patches

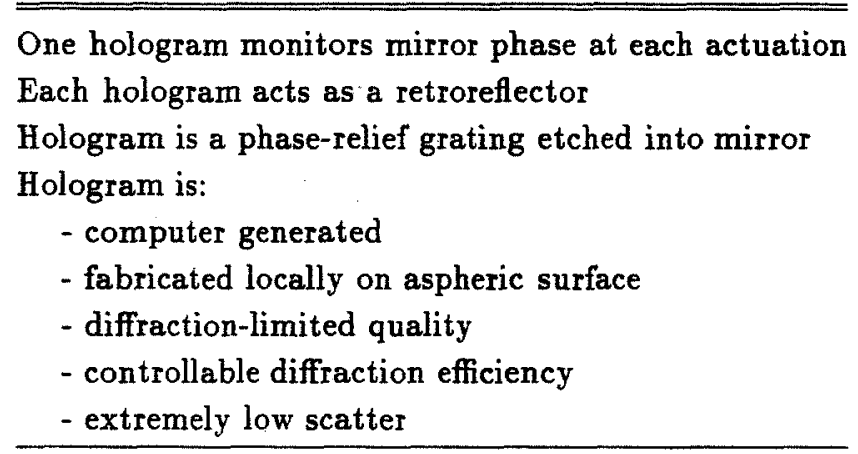

Figure 3 shows an application of the grating patch HOEs in the sample point interferometer application. They can also be used for alignment and focus monitors since the image produced by diffraction is of extremely high quality, and is bright (virtue of the laser illumination).

This technology is applicable to large optical systems having a smooth optical substrate, and permits exceedingly precise, unambiguous, and rapid measurements of the position and figure of the front surface of the mirror.

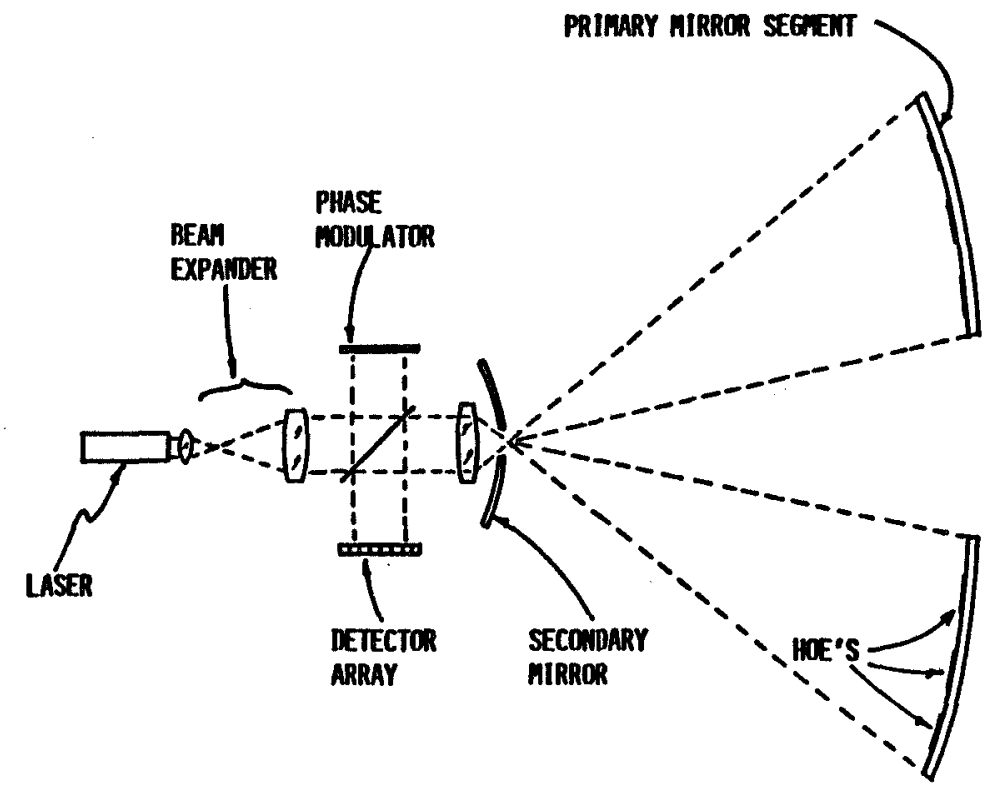

Figure 3 Sample Point Interferometer using HOEs

\subsection{Laser Radar}

The Applied Science and Technology Directorate at Perkin Elmer has recently produced a unique and very simple laser radar capable of unambiguous absolute distance measurements. The new laser radar incorporates a standard laser diode and an electronics box that modulates the diode current and decodes the laser return. The principle of operation is based on the fact that the backscatter from a target will form a front cavity 
for the laser. Generally, the front cavity effect is unwanted, but in this application, the front cavity is exploited to yield the desired distance information. Patents are pending at Perkin Elmer, and journal papers are available describing the application. (See DeGroot et al, Applied Optics, Vol. 27, No. 21, Nov. 1, '88; and Optics Letters, Vol. 14, No. 3, Feb. 1, '89)

Table 3 lists a number of applications for the laser radar. Of most interest to this workshop is the "Optical Feedback Metrology Tool" item. The sub-micron metrology application is applicable to rough as well as smooth surfaces.

We have presented to JPL the possible applications of laser radar for direct, frontsurface sensing for control of the Large Deployable Reflector (LDR) IR telescope that Paul Swanson told you about earlier at this workshop. Table 4 lists some of the main points that we made in our presentation.

The laser radar has been amply demonstrated in the laboratory and is ready for various systems applications.

Table 3 - Laser measuring tools under development

High-power Coherent Laser Radar:

- $100 \mathrm{~W}$ mode-lokded diode array source

- km operational range

- Absolute range and velocity of high-speed objects

Backscatter Modulation Laser Radar:

$-0.5 \mathrm{~mW}$ (eye safe) compact IR source

- $50 \mathrm{~m}$ operational range

- Absolute range and velocity with $1 \%$ accuracy

Short-range ranging and velocimetry instrument

- $5 \mathrm{~mW}$ UR source laser

- $3 \mathrm{~m}$ operational range

- Extremely compact, single and rugged design

- Both range and velocity to $1 \%$ accuracy

Optical Feedback Metrology Tool

- Sub-micron metrology of strongly aspheric optical surfaces

- Optical feedback geometry is compact and inexpensive

- Extrelmely aspheric surfaces can be accomadated

Position Tracking of Rotating Targets

- Compact, $1 \mathrm{~mW}$ IR source

- Tracks movement of rotating objects with 25 micron precision ( 0.001 inch)

- Can tolerate interupted beam and up to $1 \mathrm{~mm} / \mathrm{s}$ displacements without ambiguity

$-50 \mathrm{~cm}$ operational range (can be extended?) 
Table 4 - Laser Radar for PSR and LDR - Front Plane Sensing

1. Lock-up approach

- Concept: use phase sensitivity of backscatter modulation for monitoring position.

- Accuracy: $0.1 \mu$ to $1 \mu$ in space.

- No surface quality requirements.

- Could sense many points in parallel.

2. Absolute ranging

- Concept: multiple frequencies together with frequency chirp to remove phase ambiguities.

- No surface quality requirements.

- Principle demonstrated over $50 \mathrm{~cm}$ distance with $25 \mu$ accuracy.

- Potential for sub-micron accuracy with no ambiguity interval.

3. Mariage of interferometry with semiconductor laser radar

- Concept: Current laser diode based laser radar may provide technology support for established approaches, including sample-point interferometry.

- A compromise system involving holographic patches and laser diodes may be a viable near-term solution.

\section{Future Technology: Phase Retrieval}

As I mentioned in my opening remarks, our technology seems to be coming full circle as we are one again researching ways of controlling the phasing of mirrors directly from the image produced by the mirrors.

That the image contains the information needed for optical phasing can be appreciated through the following thought experiment. Imagine that the aperture of a diffractionlimited telescope were covered by a semicircular, 100plate over half of the area of the aperture. What would this do to the image?

Since the light forming the center of the image and passing through each semicircular zone will be a half wave out of phase with respect to the other half of each semicircular zone due to the action of the zone plate, the light will destructively interfere and the image will have a hole, rather than a peak, at the center. This is exactly analogous to having a segmented mirror system in which a semicircular half of the segments are one quarter of a wave ahead or behind the other half. Other phase errors will produce unique image aberrations.

Tables 5 and 6 summarize and outline our approach. Since we are in a competitive situation at the moment, I cannot tell you very much about the technique. It is being developed under Perkin Elmer IR\&D funding. We call it "Spatial Wavefront Estimation by Intensity Relationships" or SWEBIR. The inventor of the SWEBIR algorithm is Mr. 
Ed Siebert of P-E, and after all of his computer development is completed, I am sure you'll be able to find future journal articles by Ed.

\section{Table 5. Phase Retrieval}

- A family of mathematical approaches to determining optical
system figure errors from a star image
- Equivalent in performance to a white light interferometer,
but without hardware
- Can be used for figure capture and verification

Table 6. SWEBIR

Spatial wavefront estimation by intensity relationships

Computation of pupil function (figure and phase) from optical transfer function (OTF)

For point source (star):

- measure image (point spread function)

- compute Fourier transform to get OTF

- apply SWEBIR

For extended source (known):

- measure image

- compute Fourier transform

- divide by Fourier transform of known source to get OTF

- apply SWEBIR

SWEBIR is a very exciting development for large space telescopes since it promises a very minimum amount of sensing hardware in the telescope: a single CCD (and a computer). The computing requirements are not extraordinary.

\section{Conclusion}

A variety of technologies are available for the direct sensing and control of the large telescopes of the future. These range from direct observation of a star image to interferometric measurements of the wavefront or, alternatively, sampled optical path length, to laser radar. 


\section{DISCUSSION}

Rehfield: Is SWEBIR applicable to high spatial frequency wavefront errors?

Wissinger: Yes. The spatial frequency bandwidth is governed by the size and density of the detector array used to measure the PSF intensity.

Diner: I don't understand how you retrieve an OTF from the intensity image because you've lost phase information.

Wissinger: This is the purpose of phase retrieval. A comparison of algorithms is given by J. Fienup, Applied Optics 21, 2758 (1982).

Layman: Approximately what is time constant of a SWEBIR figure correction?

Wissinger: With a modest computer, the output should be expected in several seconds.

Vyce: Is the SWEBIR phase retrieval technique based on the 70s Gonzales' work more recently updated by Jim Fienugo of ERIM?

Wissinger: No. SWEBIR was developed at Perkin-Elmer. 


\title{
Requirements for Diffraction Limited Optics
}

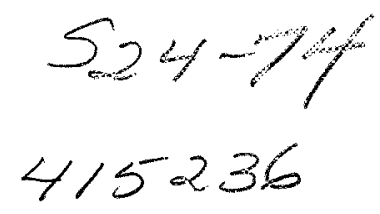

\author{
Christopher J. Burrows $\dagger$ \\ Space Telescope Science Institute
}

\begin{abstract}
We show that a ten meter 'diffraction limited' telescope can conduct a complete survey for a Jupiterlike planet in a similar orbit around any of 20 or so stars closer than 5 parsecs. It will see at least one planet in every close planetary system similar to ours. In addition to the scientific interest, such a survey has great popular appeal, and should figure prominently in the justification for funding a Next Generation Space Telescope (NGST) mission. If the telescope is capable of this very demanding mission, it will be suitable for a wide variety of other scientific investigations.

Certain design constraints must be met for the survey. In particular, we show that 'diffraction limited' means that both low and high spatial frequency errors on the wavefront are small in a definite sense. Low frequency errors lead to a loss in resolution. High frequency errors lead to a loss in contrast. A $10 \mathrm{~m}$ telescope that is not diffraction limited in both respects will not be able to conduct a complete survey. If the resolution is too low, the planet's image is smeared over too much stellar background signal, while if the contrast is poor, the stellar background is raised.

Two areas of critical technology emerge from these considerations. First, for high contrast imaging, fabrication and polishing techniques must be developed to yield smoother mirrors than those on HST. Then the wings of a stellar image will be dominated by the intrinsic diffraction profile, which can be largely removed by apodization. Second, for high resolution imaging, the conflicting requirements for a large, lightweight, and fast primary mirror with an accurate figure mean that active optics will be needed. We show that it will be hard to control the mirror surface with field stars. Either local sensing of mirror displacements will be needed, or the mirror must be intrinsically stable to a fraction of a wavelength over the full $10 \mathrm{~m}$ aperture for a typical time of many seconds. This implies critical developments are necessary in control and structure interactions.
\end{abstract}

360554

\section{Introduction}

Presently there are no direct observational constraints on the probability of planetary formation. The selection effects if we try to extrapolate from our own system are formidable! A strongly positive or negative result from a survey would be of great philosophical and scientific importance. Several schemes have been proposed for the detection of Jupiter or Earth-like planets around nearby stars. It is important to ensure that any search is complete over a substantial range of planet sizes, orbits (and temperatures), so that even a negative result is significant.

In this paper we derive technical constraints for a 10 meter space telescope given the specific scientific goal of conducting a meaningful survey for extrasolar planets. We show in Section 2 that such a telescope, if diffraction limited can achieve the goal. In Section 3 we indicate how performance changes as the telescope degrades from the ideal, and we indicate technological problems that will need to be addressed to ensure that the required image quality is achieved. The detailed discussion of the planet detection problem for the Hubble Space Telescope (HST) given in Brown and Burrows (1989, hereafter referred to as $\mathrm{BB}$ ) is extended here to the proposed $10 \mathrm{~m}$ aperture, and to include the effects of noise.

$\dagger$ Affliated with the Astrophysics Division, Space Science Department of ESA 
The result for HST was unfavorable because even with assumed perfect apodization, microroughness scatter degrades image contrast. We show that a $10 \mathrm{~m}$ aperture with a mirror that is about 3 times smoother than HST on the other hand would detect a Jupiter over a wide range of orbits and for a large number of stars.

If an infrared wavelength band is used where the planet is emitting radiation, the brightness ratio goes up relative to the visible, but unfortunately the contrast will go down by almost the same amount. Less obviously, as pointed out by Shao at this meeting, most nearby stars are much less luminous than the sun, so the equilibrium temperature of a planet at a given separation is lower, in the absence of internal heat sources. This seems to imply that a survey would require a larger aperture in the infrared, in addition to cooled optics. Coronagraphic techniques do not work well for a $10 \mathrm{~m}$ telescope in the infrared $(10 \mu \mathrm{m})$ at the separations $\left(<0^{\prime \prime} \cdot 2\right)$ where such a planet would be hot enough to be detectable around all but a handful of stars, although other interferometric techniques may be feasible.

If the telescope is designed to make the direct visible planet search possible, it will be ideal for a number of other scientific applications where high resolution and contrast are needed, such as the imaging of circumstellar or protostellar disks, or the environs of active galactic nuclei. For example, the possible protoplanetary disk around the $m_{V}=4$ star $\beta$ Pictoris has a surface brightness of $m_{V}=16 /$ arcsecond $^{2}$ at a separation of $6 "$, about one magnitude fainter than the seeing profile from the star. The $10 \mathrm{~m}$ NGST with a primary mirror that meets our specifications would have a scattered light level of $m_{V}=18 /$ arcsecond $^{2}$. Thus significant improvements over ground based observations would be expected. HST has a much higher predicted scattered light level, and hence may not offer any improvement over ground based measurements for a resolved extended source in a scattered light halo such as the $\beta$ Pictoris disk.

\section{Planet detection for a diffraction limited $10 \mathrm{~m}$ telescope}

Planet detection is limited by systematic errors which depend on the contrast achieved, and by photon noise. We examine each in turn.

On a diffraction limited telescope of aperture radius $R$, a stellar image has a peak intensity proportional to $\pi R^{2} / \lambda^{2}$, and an average asymptotic wing intensity proportional to $\lambda /\left(2 \pi^{3} R \theta^{3}\right)$, where $\theta$ is the angular distance from the star, $\lambda$ is the wavelength, and small corrections for the central obscuration ratio $\epsilon$ have been ignored for simplicity. The ratio of these surface brightnesses $\lambda^{3} /\left(2 \pi^{4} R^{3} \theta^{3}\right)$ is 17.8 magnitudes at one arcsecond for a $10 \mathrm{~m}$ telescope in the visible $(600 \mathrm{~nm})$. On the other hand, the brightness ratio at visible wavelengths of the sun and Jupiter at maximum elongation is about 22.3 magnitudes $\dagger$ $\left(10^{-9}\right)$. Given a star at 5 parsecs, a planet of Jupiter's size in a 5 AU orbit would be separated from it by one arcsecond. So the planet would be about $22.3-17.8=$ 4.5 magnitudes $(60 \mathrm{x})$ fainter than the background from the star, when at maximum elongation.

Observing a source with a relative brightness of $1 / 60$ on a spatially variable but measurable background is demanding but possible. Specifically, a reference image can be obtained by rotating the spacecraft around the line of sight to the star and taking a second otherwise equivalent control exposure. Both images need to be flat fielded, and have any

$\dagger$ From the observed geometric albedo of 0.44 , a phase function $\phi(90)=1 / \pi$ corresponding to a Lambertian sphere, and the observed Jovian radius and orbit. 
distortions removed and the control image would need to be derotated before subtracting. The stellar background should then almost exactly cancel. Sources of systematic error (such as pointing and flat field errors) would need to be carefully controlled. BB (1989) argue that for HST, such systematic effects limit the relative brightness to at best about $1 / 100$.

Photon noise, on the other hand is typically not a serious problem for the large aperture, provided a wide bandpass is used (justifying our choice of the average wing intensity above). In order to detect the planet, one can design an optimal linear filter, because the planet is unresolved so the expected signal is known, and the noise will have Poisson statistics. Within 5 parsec, Allen lists 50 stars. Of these, 23 are members of known multiple star systems and can probably be regarded as lower priority for a planetary search. A further 5 stars are fainter than 12th magnitude. This leaves 22 candidates, with magnitudes approximately evenly distributed between 6 th and 12th. In only one hour, Jupiter $5 \mathrm{AU}$ from an $m_{V}=6$ candidate, contributes about $2 \times 10^{4}$ counts, corresponding to a signal to noise ratio of $S / N=13$ (assuming an overall efficiency of 0.5 , a bandpass of $\Delta \lambda / \lambda=0.5$, and including noise from the reference exposure). If the candidate star has $m_{V}=9$ we would still have $S / N=3$ in one hour, and even the faintest candidates can be surveyed to this level in less than a day. $\dagger$

A $3 \sigma$ detection can be confirmed by examining the planet's rotated position - There should be a corresponding negative signal there. It seems desirable to do the detection in each channel separately to give a 'coincidence check', and hence eliminate problems caused by cosmic rays, or bad pixels for example. A more detailed analysis of the detection possibilities, including the effects of micro-roughness and apodization is given after their effects are discussed in the next section.

\section{Image quality}

A telescope can be regarded as diffraction limited when the images that it gives are not sensibly different from those obtained from a hypothetical perfect telescope of the same aperture size, and overall efficiency. In the previous section, the performance of such a hypothetical telescope was shown to be adequate for the planetary search problem, but any significant drop in performance relative to this standard would make detection impossible.

There are several different ways of failing to meet the 'diffraction limit': The aperture configuration can be suboptimum, the mirror can be contaminated with particulates (dust), the images can be distorted by aberrations, and the mirror surface can scatter light. All of these problems should be dealt with by careful attention in the design phase. Their detailed effects are examined and compared to HST in the following four subsections. HST is used as a reference because it is the best we have without NGST, we can learn from the experience in constructing it, and it was pushing the limits of our technology when constructed.

\subsection{Pupil obscurations}

The effect of small structures in the entrance pupil is to diffractively scatter light to large angles. In the case of HST, the secondary mirror spiders will produce diffraction

$\dagger$ For reference, under the above conditions, the telescope's limiting magnitude is 34.5 in 1 hour for $\mathrm{S} / \mathrm{N}=3$ with a detector read noise less than $3 e^{-} \mathrm{rms}$, and a background of $m_{V}=24 /$ arcsecond $^{2}$. 
peaks in two orthogonal directions, but otherwise do not disturb the image significantly. Clearly, a suitable spacecraft roll suffices to examine any region covered by the spikes. Although unobscured designs are possible, a $10 \mathrm{~m}$ telescope may also require secondary mirror supports in the beam. If so, they should be made as narrow as possible. Thinner spider fins scatter proportionally less power over a proportionally wider angle, while the width of the spikes remains of the order of the Airy disk, so the intensity decreases as the square of the width. In HST, further pupil obscurations are caused by the three primary mirror support pads. The effect of these is twofold: they raise the average intensity within the first arcsecond of the image, and more seriously, largely destroy the circular symmetry of the Airy rings. It is important to avoid any such pupil obscurations if at all possible. If a segmented approach is used in the primary mirror design, the effects of diffraction at segment edges would need to be assessed. Such effects can be minimized by making segment boundaries parallel to the unavoidable secondary mirror support structures (which need not be orthogonal), and by making the segments as large as possible.

Finally, a central secondary obscuration will cause the monochromatic Airy ring intensities to rise and fall relative to the inverse cube asymptote. The period of these fluctuations in Airy rings is the ratio of the outer to inner pupil radii $(1 / \epsilon)$. For HST, the central obscuration ratio, $\epsilon=0.33$, and this will give the images of bright sources a characteristic appearance with a repeating pattern of a relatively faint ring followed by two brighter rings. The NGST will probably have a much faster primary, hence a smaller central obscuration. The rings will therefore fall off more uniformly in intensity. These considerations are only relevant if the bandpass $\Delta \lambda / \lambda$ is less than $1 / N$ for Airy ring number $N$, and do not apply to a broadband planet survey. An alternative strategy is to look in a narrow bandpass near the minima of the Airy pattern. This improves the contrast, but cuts the signal to noise and search efficiency significantly. Such an approach should be used for follow-up observations only near the brightest stars, and we do not consider it further.

In conclusion, the aperture configuration will not prevent the ideal performance necessary for planet detection, provided that care is taken in the design of the primary mirror mounts so that they are not in the beam.

\subsection{Particulate contamination}

The second effect that can degrade the telescope performance is scattering by dust. Dust has two effects. First, it causes a loss in throughput of twice its covering factor. For example, a $5 \%$ covering of particulates will cause a $5 \%$ loss in the core of an image by absorbtion and large angle $\left(>>1^{\circ}\right)$ scattering, and a $5 \%$ loss by diffractive scattering. The second effect is that the diffractively scattered component leads to a loss in contrast. For example, a $d=20 \mu \mathrm{m}$ dust particle diffracts light over a region of about its Airy disk radius $1.22 \lambda / d=2^{\circ}$. This is the same irrespective of the aperture diameter. Hence if NGST ends up contaminated in the same way as HST (per unit area), the resulting halo will look the same. However, the image contrast will improve, because the central peak intensity will increase as the square of the diameter. HST has a limiting contrast caused by dust of about $10^{-11}$, while a similarly clean NGST would be 16 times better.

In conclusion, dust contamination affects the images less as the aperture size increases, and is not expected to be a major problem for the NGST, unless it is a moon based telescope where more dust is likely to gather on the mirror. 


\subsection{Mirror figure errors}

One effect of wavefront errors on the image is to lower the central peak intensity. The central intensity drops by the Strehl factor $\left(S=\exp \left(-4 \pi^{2} \sigma^{2} / \lambda^{2}\right)\right)$ for a small root mean square (rms) wavefront error $\sigma$, or if the wavefront error is large but occurs at high frequencies in the pupil, and has Gaussian statistics. If a $20 \%$ drop in peak intensity is regarded as acceptable, then the equation can be solved to show that $\sigma$ must be less than $\lambda / 13$. For larger wavefront errors, the peak intensity falls rapidly, and the desired survey would be impossible. The important point is that alignment and microroughness errors will also contribute to this total, so the primary mirror surface figure will need to be maintained to about $\lambda / 40 \mathrm{rms} \dagger$, over the entire aperture. This is a demanding specification and corresponds approximately to the performance of the HST primary. It is an order of magnitude more stringent than a typical groundbased telescope mirror specification. Furthermore it seems clear that the primary will need to be perhaps as fast as $F_{p}=1.2$ and lightweight for packaging reasons.

Nobody has proposed a method of building such a monolithic $10 \mathrm{~m}$ mirror that will passively retain its shape to this accuracy. Existing proposals generally involve either segmented, or meniscus construction, with an active control system. In each case, a characteristic length scale (either the segment size or the distance between actuators) is typically about $1 \mathrm{~m}$. Larger scales lead to tight positional tolerances on segments, or unacceptably thick membranes.

Specifically, if a segment is moved away from the mirror center, the rms wavefront residual after best correction for tilt and piston can be shown to be proportional to the radial displacement, the square of segment radius, and inversely proportional to the full aperture baseline squared, and $\mathrm{F} /$ ratio of the primary $\left(F_{p}\right)$ cubed. A detailed calculation shows that for $\lambda / 20$ wave rms at $500 \mathrm{~nm}$ and $F_{p}=1$, any radial displacement must be less than $7.5 \mu \mathrm{m}$ for one meter segments. If this degree of freedom is to be passively controlled, (as in the Keck telescope), it is clear that the segments cannot be much larger.

A wavefront tilt can be estimated to an accuracy of $\lambda / n$ if at least $n^{2}$ photons are detectedf. Furthermore, the correctable field size of the telescope is limited to about a radius of $2 \sqrt{\lambda F_{p} / D}$ where the astigmatism of a Ritchey-Chrétien Cassegrain becomes one wave. The field must be fully corrected or calibrated in order to be useable for wavefront measurements, so this seems a realistic bound on the achievable field.

For the $10 \mathrm{~m} F_{p}=1$ system we need a guide star density of 400 per square degree in order to get one in the field on average. Over much of the sky (away from the galactic plane) this means that guide stars will be 18th magnitude or fainter. It takes 1 second to collect 400 counts from an 18th magnitude star with a $1 \mathrm{~m}$ aperture (assuming an efficiency of 0.5 and a bandpass of $200 \mathrm{~nm}$ ). On the other hand, over the full aperture one collects enough light for overall pointing in about $10 \mathrm{~ms}$.

In conclusion, if the mirror surface is to be actively controlled in tilt, and field stars are to provide the position feedback, any disturbances must be less than $1 / 40$ wave over timescales of several seconds over the full $10 \mathrm{~m}$ aperture. The effects of any gravitational, ram pressure, thermal, and magnetic perturbations would all need to be shown to be below these levels over such timescales. Clearly, such a control system could not take out

$\dagger$ Corresponding to an rms wavefront error of $\lambda / 20$

$\ddagger$ This follows if one considers the Cramer Rao bound for estimating the phase of fringes $\sigma^{2}=$ $n^{-1} /\left(1-\sqrt{1-M^{2}}\right)$ for fringe contrast $\mathrm{M}$, or the statistical errors in a centroid. 
any structural normal modes (except perhaps loosely coupled components such as solar panels). Further studies should reveal if such a quiet design is possible, but it seems likely that such considerations will impose extremely tight constraints on the design, and we may well be forced to internal laser interferometry or direct metrology systems (such as capacitive sensors).

\subsection{Mirror surface roughness}

The final area of critical importance for planet searches is the mirror surface quality. The intensity $d P / d \Omega$ in the wings of the image of a point source can be written

$$
\frac{d P}{d \Omega}=\frac{S \lambda}{\pi^{3}(1-\epsilon) D \theta^{3}}+\frac{4 \pi^{2} G(2 \pi \theta / \lambda)}{\lambda^{4}}+\frac{c}{\lambda^{2}}
$$

Where, $G(K)$ is the two dimensional mirror surface power spectral density at wavenumber $K$. The first and third terms correspond to aperture diffraction and dust scattering. They have been shown to be acceptably small for the survey. BB (1989) consider all the available metrology for the HST primary. They deduced that a power law $G(K)=\alpha / K^{\beta}$ fits $\dagger$ the data with $\alpha_{H S T}=27100$ and $\beta_{H S T}=2.2$ for $G$ in $\mu \mathrm{m}^{4}$ and $K$ in $\mathrm{cm}^{-1}$. The first two terms are equal for any telescope with the same power spectrum slope when

$$
\left(\theta / 1^{\prime \prime}\right)^{0.8}(\lambda / 1 \mu m)^{-2.8}(D / 1 m)(1-\epsilon)=20\left(\alpha / \alpha_{H S T}\right)
$$

Hence, at $600 \mathrm{~nm}$, the microroughness scattering equals the diffraction scattering for HST $3^{\prime \prime} .6$ away from a point source. At greater angles, the wing intensity will fall approximately as the inverse square of the intensity, rather than the inverse cube expected from the diffraction limit.

Sayles and Thomas $(1978 \mathrm{a}, \mathrm{b})$ have shown that a power law description works for a large variety of surfaces, and that the index is often close to 2. King (1971) has shown that the observed profile from groundbased telescopes falls as the inverse square at angles greater than a few arcseconds. The classic Fried theory of atmospheric scattering can be shown to predict an inverse cube falloff. Hence it seems likely that the King observations are explained by microroughness scattering. If the $10 \mathrm{~m}$ telescope has the same surface polish as HST, then the crossover point is at 0 ".45. Therefore, the wing intensity would be significantly larger than we have assumed at angles in excess of 1 ", and the survey would be difficult. If the mirror is 2 times smoother than that of HST, the crossover moves out to 1 ".

If a suitable focal plane mask is included, covering of order $N$ Airy rings, then reimaging through an apodizing mask overlapping the exit pupil edges by of order $1 / N$ of the pupil diameter will exclude most of the diffractively scattered light. If the masks have suitably blurred edges, doubling the size of either improves the suppression factor by an order of magnitude. One arcsecond corresponds to $N=100$, so it is easy to design a coronagraph that gives essentially perfect suppression of the Airy profile, with very small loss in throughput, at least beyond about $0^{\prime \prime} ! 1$. This technique does not work for light scattered by microroughness, so that one will always ultimately be limited by the quality of the mirror surface.

$\dagger$ The fit is uncertain particularly because the bandpasses of the metrology considered in deriving it are not well defined. 
If the mirror polish power can be improved by about a factor of 2 (a factor of 1.4 in amplitude) on the relevant scales $(1-10 \mathrm{~cm})$, over that predicted for HST then the planet search is feasible. An order of magnitude improvement would be desirable because both contrast and signal to noise would improve correspondingly, leaving more margin for error and a smaller planet would be detectable. Such a specification is reasonable. There are indications that the HST primary is not as good as some ground based telescopes in this respect. The specifications on the secondary polish can be about $1 / \epsilon$ less stringent, because the beam diameter is smaller on the secondary.

Microroughness imposes a practical limit on contrast. A goal should be to fabricate a mirror that has a surface that is 3 times smoother in amplitude than the HST primary. The telescope would then be 'diffraction limited' out to 8 arcseconds from a bright source.

\section{Planet detection with apodization and microroughness present}

If the specifications developed in Section 3 are met, a Jupiter-like planet would be detectable at $5 \mathrm{AU}$, from any of about 20 stars at 5 parsec. Some of the 20 candidates are in fact closer, or the planet may be at a different distance from the star. Therefore, we consider the effects of the angular separation explicitly here, and combine the effects of signal to noise and contrast limitations to derive the available search space.

First, if the star is closer, but the planet is assumed to be in the same radius orbit, the apparent angular separation increases linearly, so the contrast will go up as the the inverse square or cube of the distance (depending on whether one is microroughness or Airy diffraction limited). So we are considering a worst case by assuming all the candidates are at their maximum distance.

Second, if the planet orbit is changed but for a fixed distance to the star, the planet brightness decreases quadratically with angular separation. Thus, in the diffraction limit one gets a linear growth in the ratio of the planet to stellar surface brightness with increasing angular separation, until the image becomes dominated by sky background (or other effects such as microroughness scattering). For a star with $m_{V}=6$ the profile remains brighter than a sky brightness of $m_{V}=24$ /arcsecond ${ }^{2}$ out to a separation of $15 "$. For $m_{V}=12$ the sky is reached at $2 "$. The planet at $15 "$ is 5.9 magnitudes fainter than at 1 ", so a Jupiter-like planet at 15 " from a 6 th magnitude star at 5 pc would have a brightness of $6+5.9+22.3=34.2$. A detection should be possible throughout the range 1 " to 15 " and is limited at the low end by low contrast and systematic errors, and at the high end by poor signal to noise. On the other hand, in the region where the image is microroughness limited, the ratio of the planet to stellar surface brightness will be constant, until the sky brightness becomes the limiting factor. In this case, one is limited by signal to noise for short exposures (one hour), but by contrast for longer exposures (10 hours). The microroughness limit will be reached if a suitable coronagraph is used.

All of these considerations are illustrated in Figure 1, which shows the available search space both with and without microroughness scattering. The area indicated as feasible applies to the detection of a Jupiter-like planet in orbit around a star at 5 parsec. Therefore, angular separations in the Figure correspond numerically to the size of the orbit relative to Jupiter's. The grey area defining the limits of detectability corresponds to a signal to noise in the range 2 to 4 and a contrast in the range $1 / 64$ to $1 / 128$. For the bottom panels, all the Airy profile contribution is assumed removed by apodization, and the microroughness scatter is taken to be a factor of 2 better than that predicted for HST (although we recommended a factor of 9 in Section 3 ). 

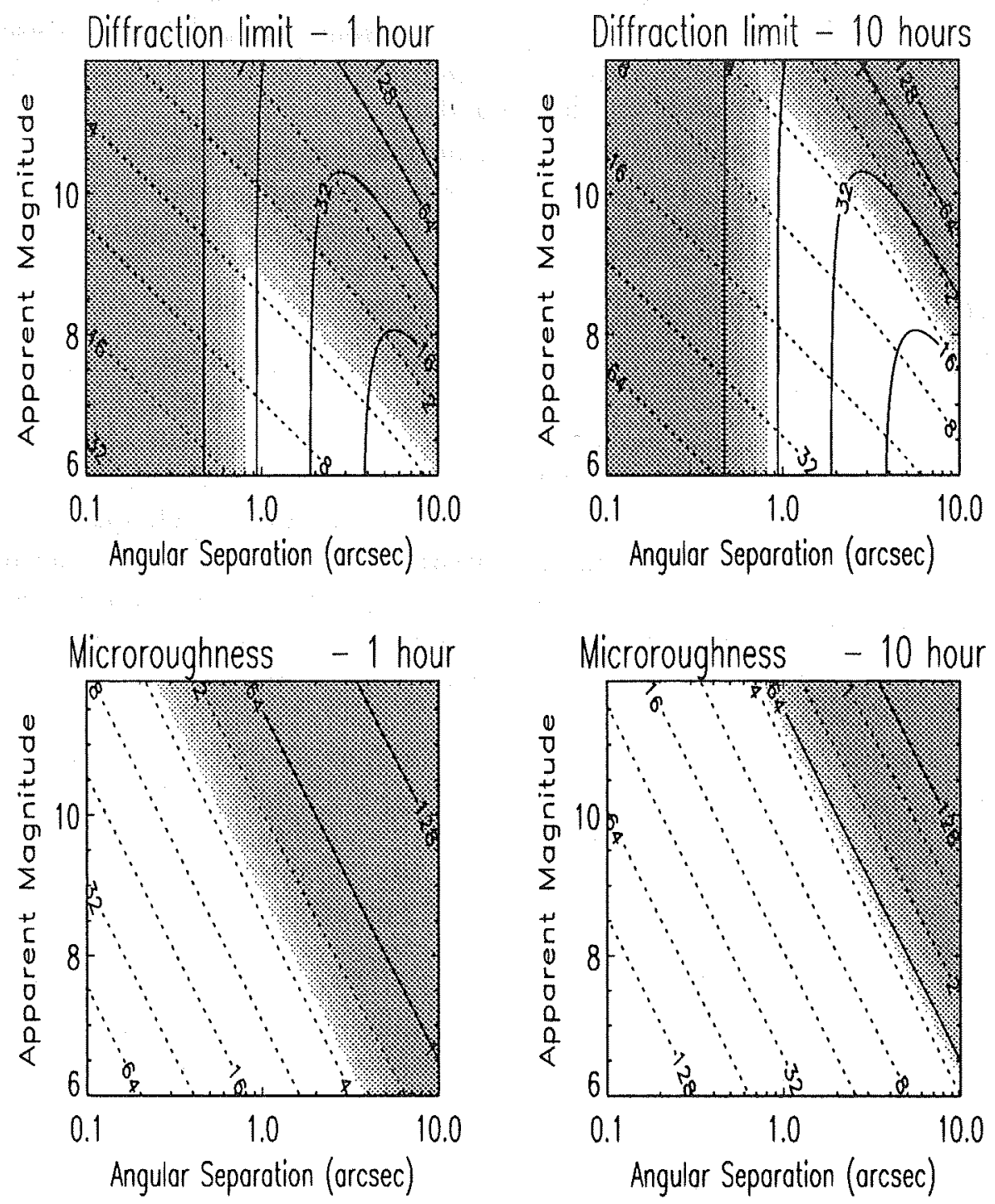

Figure 1: Feasible search space in white, as a function of the star's apparent magnitude, and the angular separation from it of a Jupiter-like planet. The panels correspond to 1 and 10 hours of integration, when limited by diffraction or microroughness. The dotted contours map the signal to noise ratio, while the solid contours correspond to contrast. 


\section{Conclusions}

A $10 \mathrm{~m}$ diffraction-limited telescope can directly detect a Jupiter sized planet at 5 parsecs, where over 20 single candidate stars are available for a survey. The planet would be detected when it passes through maximum elongation if it is least $5 \mathrm{AU}$ and less than about $50 \mathrm{AU}$ from the brighter stars. Therefore, even a non-detection would be a scientifically valuable result, provided synoptic observations cover half the orbital period (6 years for Jupiter around one solar mass). If a coronagraphic camera is used the planet would be detectable throughout most of the orbit.

Conversely, a significant decrease in the peak intensity, or increase in the wing intensity would make this experiment impossible with a $10 \mathrm{~m}$ aperture. In the microroughness limit, increasing the aperture size improves the contrast ratio as the square. Although the experiment is feasible with a $10 \mathrm{~m}$ mirror that is a factor of 2 smoother than the HST primary, an order of magnitude improvement would vastly improve the results, and expand the search space. A significantly smaller aperture, even if made proportionally smoother would suffer from severe signal to noise problems for the survey considered here.

Planet searches are not the only scientific driver for the development of the NGST, but the goal has great popular appeal, and should figure prominently in the justification for funding such a mission. Furthermore, as we have shown, mission requirements flow naturally from such a goal, in areas as diverse as aperture configuration, mirror quality, pointing stability, detector performance, and mission lifetime.

If these ambitious goals can be met, other scientific projects requiring high contrast and resolution, and accurate reproducable pointing, would of course also benefit. HST has a much higher predicted scattered light level, and hence may not offer any improvement over ground based measurements for a resolved extended source in a scattered light halo such as the $\beta$ Pictoris disk. The NGST will provide us with higher resolution and sensitivity. With careful design it will also provide higher contrast. Such image quality is vital for the application that may be a primary driver for the development, and will also serve well in other investigations.

\section{Acknowledgements}

It is a pleasure to thank Robert Brown and Pierre Bely for many useful discussions, and Jan Koornneef for a careful reading of the manuscript.

\section{References}

Brown, R. A. and Burrows, C. J., 1990. Icarus, Submitted

Sayles, R. S. and Thomas, T. R. 1978a. Nature, 271, 433

Sayles, R. S. and Thomas, T. R. 1978b. Nature, 273, 573

King, I. 1971. Publ. Astron. Soc. Pacific, 83, 199 


\section{DISCUSSION}

Angel: I have a comment relating to on-board metrology using guide stars. The situation is easy compared to doing adaptive optics on the ground. You can take stars at much larger angles if you use something like a Hartmann test, for example. You know what the aberrations are going to be at large fields. So once you have made a calibration, you can either do it by calculation or explicitly with the telescope. It seems to me that fields up to several arcminutes are quite feasible, because the aberrations will still be such that a Hartmann test will work. You can use brighter stars from a wider field because wavefront distortion of the guide star can be quite large.

Burrows: My main object in identifying the guide star problem is to bring it to people's attention. The field can no doubt be enlarged somewhat with corrective optics. However, the uncorrected aberrations increase as the square of the field angle, and I have already assumed that they can be calibrated or corrected to something like $\lambda / 20$ over a field where they are as large as one wave. It is an order-ofmagnitude calculation. The problem that I have is that even if you make it a 5 arcminutes or 10 arcminutes field you still need an extremely quiet structure over long periods. Furthermore, for many active optics concepts, people talk about correcting over diameters that are much smaller than a meter. I agree that one can push any of the individual assumptions but we will need to push several of them at once. It seems to me that it will be extremely challenging to do that. So perhaps we will need instead to put effort into developing on-board metrology that will make the structure rigid internally without external references.

Breckinridge: Is there an observing program to take place during the flight of the Space Telescope which will measure the performance and the performance degradation of large mirrors in space for the purpose of planet detection and characterization?

Burrows: Yes. One of the Observatory level tests to be conducted during the Science Verification period is specifically designed to measure the wings of the point spread function out to about 12 arcsec. These wings provide the background against which any planet must be detected. The mirrors are not expected to experience degradation on orbit at length scales of centimeters, and so the performance in this region is not expected to change. It will be monitored with the science instruments as the mission progresses. It will be interesting to compare the results of this test with predictions made on the basis of the pre-launch metrology. I know of no case of a mirror where there is both well-established metrology and good measurements of the profile. So maybe we have to be careful when saying that we understand the wing profile. HST may be the first case where both of these measurements are done.

mingworth: The timescales and size scales ( $1 \mathrm{~s}$ and $1 \mathrm{~m}$ ) that can be constrained with the signal from an 18th magnitude star do not seem to me to necessarily be a severe problem - But I would appreciate some feedback from the speaker and the audience on this!

Burrows: The $1 \mathrm{~m}$ constraint seems reasonable from stiffness and weight considerations. The 1 second constraint is much harder to justify, and I have identified it as an area for further work. Clearly, thermal, 
gravitational, magnetic and possibly atmospheric drag stresses become relevant on these timescales in addition to mechanical normal modes that may be excited in the structure by the pointing system in response to those perturbations.

Layman: The 1 second sample time calculated for $\lambda / 20$ position measurement accuracy (18 mag star,

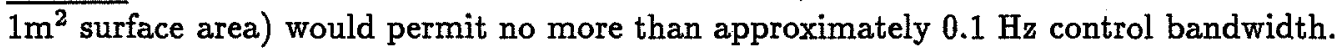

Burrows: One would need to be concerned if the area to be controlled was less than a meter, such as might be required for a meniscus of for small segments.

Unidentified : A comment of on the dust coverage. For AXAF the end- of-life budget for coverage is $510^{-5}$. As you noted, dust only affects the Airy profile at large radii, but it still matters.

Burrows: Dust has been a serious concern for the HST project, and the measured $2 \%$ coverage is expected to increase by $1 \%$ or so during launch. For a given dust distribution, larger apertures have less contrast degradation.

Angel: For a telescope on the moon one can dump the vibrational energy into the ground. The moon also has the great advantage of lacking the exciting mechanisms (e.g., wind) that cause us so much trouble on earth. For a spacecraft you need to avoid vibrational frequencies which are higher than some critical frequency. So you have to think about what is going to excite it, and how quickly you can dump it.

Stockman: I believe I can comment on Roger Angel's question about how vibrations are damped in space. On HST, the structure is made extremely stiff so that all natural frequencies are above 10 $\mathrm{Hz}$. However, the solar arrays have very low frequencies (about $0.2 \mathrm{~Hz}$ ) and their effects and slow thermal effects are removed by the pointing control system. The Pointing Conrol System and all other disturbances are controlled or damped to minimize the excitation of high frequency modes. 
Precision Segmented Reflectors (PSR)

Richard Y. Lin

Jet Propulsion Laboratory

$$
\begin{array}{r}
555-74 \\
415237 \\
360555 p 4
\end{array}
$$

\section{PSR PROGRAM}

- AN ELEMENT OF CIVIL SPACE TECHNOLOGY INITIATIVE (CSTI)

- OBJECTIVES

- DEVELOP ENABLING TECHNOLOGies for LARGE LIGHTWEIGHT SEGMENTED REFLECTOR SYSTEMS FOR SPACE APPLICATIONS

- VALIDATE TECHNOLOGIES BY MEANS OF A SYSTEM DEMONSTRATION

- APPROACH

- FOCUSED DEVELOPMENT

- SOME ADVANCED RESEARCH

- Will serve as a fUtURE TECHNOLOGY TEST BED

- JOINT OAST \& OSSA PROGRAM: MANAGED BY OAST

- participating centers

- JPL \& LaRC, WITH JPL LEAD

- Resources

$\sim$ \$20M

- FoUR yeARS PROGRAM

- FY'88 - FY'91

- Program completion CAN be extended

- 4 MAJOR ELEMENTS 
TECHNICAL OBJECTIVES

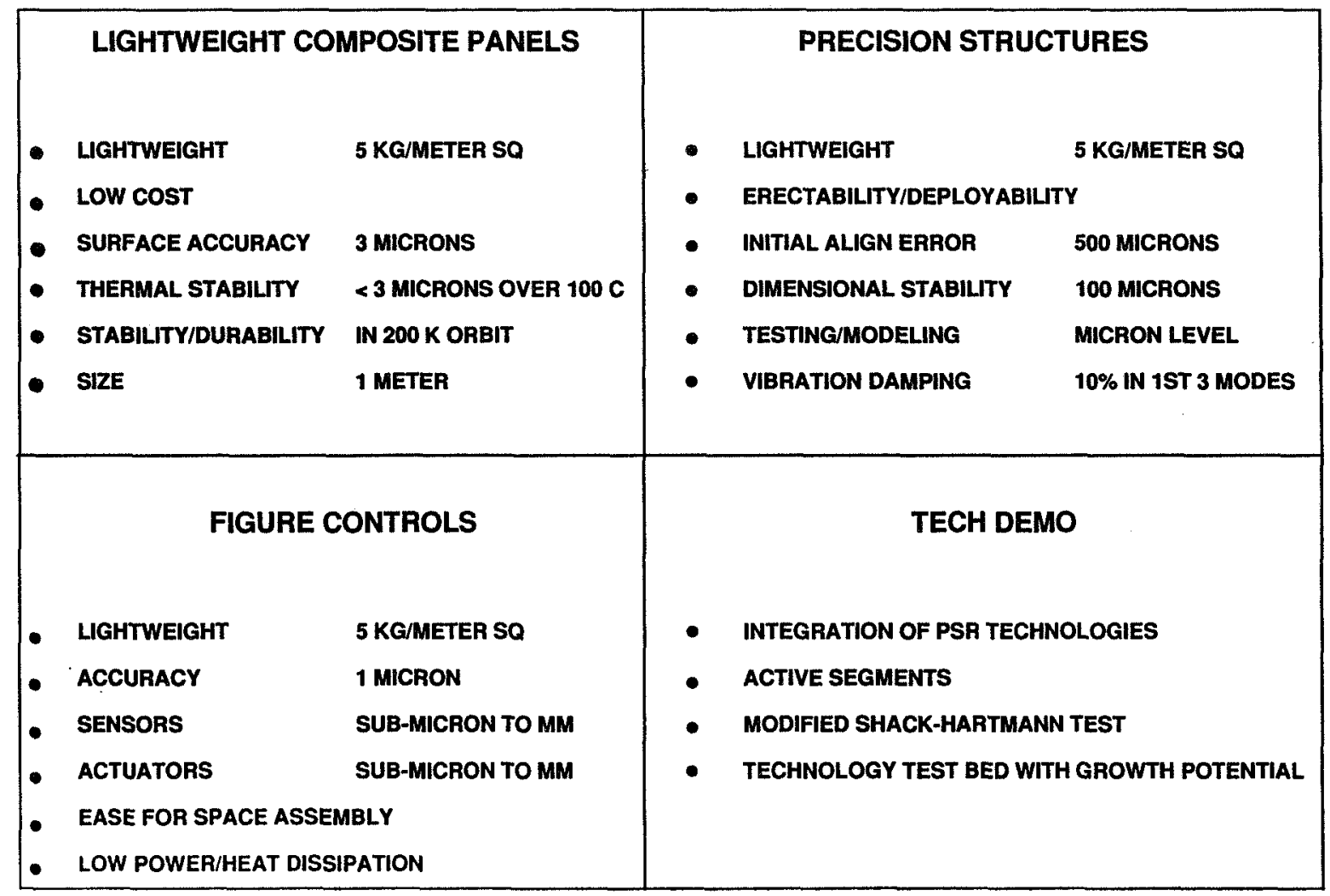

\section{FOUR MAJOR ELEMENTS}

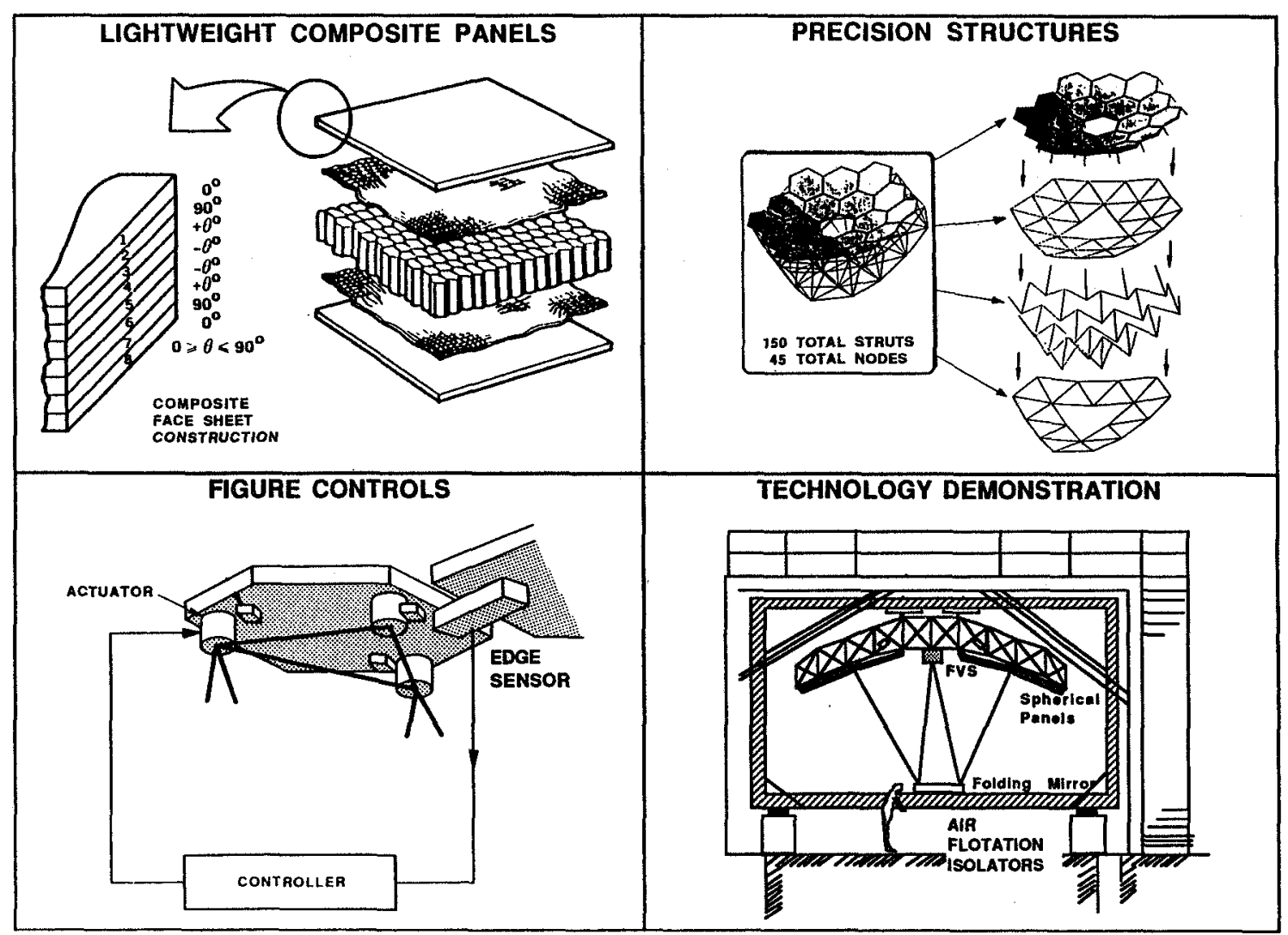




\section{TWO-RING ERECTABLE PRECISION SEGMENTED REFLECTOR}

(Tetrahedral Truss Design)

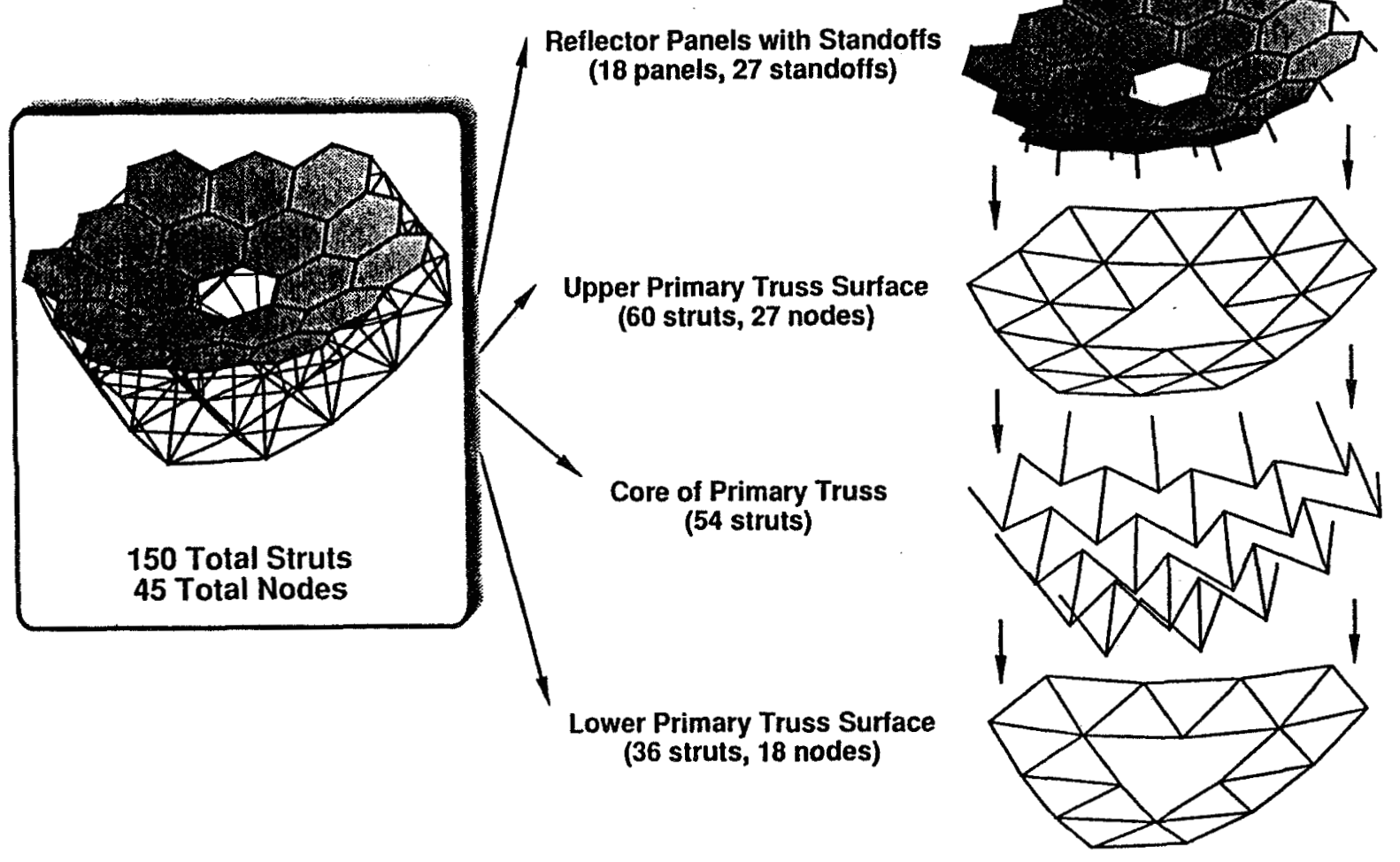

\section{SUMMARY}

- Current PSR technology development is intended for LDR-like applications:

- Large segmented reflector systems

- Lightweight composite panels at $3 \mu \mathrm{m}$ RMS

- Precision lightweight structure at $500 \mu \mathrm{m}$

- Active figure control at $1 \mu \mathrm{m}$ RMS accuracy

- $5 \mu \mathrm{m}$ RMS overall surface accuracy

- Development of PSR technologies to date has demonstrated the potential of:

- Lightweight composite panels at $0.1 \mu \mathrm{m}$ RMS via polishing \& re-finishing

- Shape control of composite panels by imbeded actuators

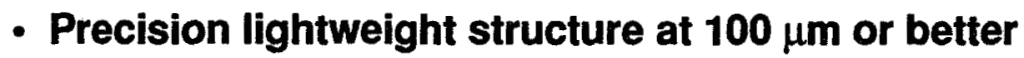

- Smart structure strut that can suppress vibrations and change its length 


\section{DISCUSSION}

Nelson: What limits the stability of the structure? You have 100 microns as an estimate. Is it water outgassing or thermal variability? What might you expect for a 10-16 meter space telescope structure like this?

Lin: In the development of space structures, one of the key components are the joints. Currently these are aluminum which has a large coefficient of thermal expansion compared to graphite epoxy. So we are interested in pushing that joint design towards titanium, for example, which has a much smaller coefficient of expansion, or even graphite epoxy. But the challenge is how does one design a structure that is lightweight, but also at the same time does not have hysteresis, non-linearity or free play. That is our challenge.

Unidentified: Have you done any testing of these structures at cryogenic temperatures?

Lin: First, we have done about a dozen tests on the panels, one being a low temperature test in a thermal vacuum chamber. It was done with a goal of calibrating the overall system, and so it wasn't really intended to get the low temperature performance of the panels. But we believe that at the low temperatures, about $200^{\circ} \mathrm{K}$, that the surface accuracy changes would be about 5-6 microns rms. This is from analysis. In addition, we are in the process of carrying out actual tests. We have, however, done a lot of low temperature testing in the materials area using coupons. This materials development is being done in parallel and one can do a lot very useful testing using coupons. 
ASCIE: An Integrated Experiment to Study Control Structure Interaction in Large Segmented Optical Systems

by Jean-Noel Aubrun and Kenneth R. Lorell
Lockheed Palo Alto Research Laboratory

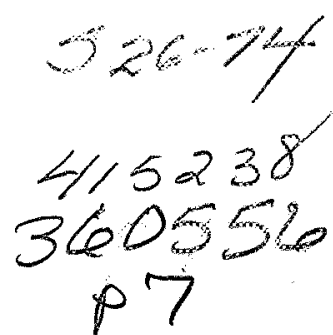

Large segmented reflector systems

The use of a segmented primary mirror is one of the major design concepts for the new generation of large ground and space-based telescopes. The W. M. Keck Ten-Meter Telescope (TMT), or NASA's planned Large Deployable Reflector (LDR) are typical examples of this approach.

In a segmented reflector the mechanical rigidity and geometric aecuracy are supplied solely by the support structure. Imperfections in the manufacturing process, deformations due to gravity loads, thermal gradients, slewing and tracking dynamics, and structural vibrations make it imperative that the positions of the segments be actively controlled. For example, the TMT segment alignment system requires 162 sensors, 108 actuators, and a special control system to align its 36 segments.

An important characteristic of such systems is that the supporting truss is very light (even for ground-based telescopes like the TMT), thus very flexible, with usually low natural damping. As a result, interactions between the segment alignment control system and the structural dynamics are expected to occur.

\section{Motivation for the development of an integrated experiment}

The interaction between the control system actuators and sensors with the dynamics of the supporting structure seriously limits the performance of the system. A recent analytical study done by Lockheed [refs 1, 2 and 3] of the TMT that modelled the full structure, actuator and sensor set, and control system operation showed that control system stability was seriously affected by dynamic coupling between the segments through the support structure. Tests performed on a single segment and support cell conducted at the Lawrence Berkeley Laboratory failed to predict this phenomenon because they did not account for the effects of collective motion and coupling in the full system. While analysis can be very effective in predicting major behavior, there are numerous practical problems that must be solved and tested with real hardware. Moreover, the design and implementation of a multi-actuator, multi-sensor control system for large flexible segmented reflectors (LFSR) has never been experimentally validated. There was thus a need to develop a test bed that could support strong interdisciplinary studies to develop and validate the emerging LFSR technology.

\section{ASCIE Objectives}

A unique ground experiment called the Advanced Structures/Controls Integrated Experiment (ASCIE) has been conceived and developed as a means of performing meaningful laboratory experiments for the design, implementation, and validation of control strategies for large flexible systems with distributed optical elements, and in particular for large 
segmented telescopes. The ASCIE test bed has been designed to support a number of interdisciplinary studies that address major technical challenges of LFSRs. One of the immediate objectives of this project concerns the study of structures/controls interaction in LFSRs. However the scope of ASCIE is of a more general nature. Topics such as structural control (e.g. active damping, vibration suppression, disturbance alleviation) or pointing and slewing techniques for LFSRs will also be addressed using the ASCIE System.

The near-term goal for the ASCIE is to demonstrate in the laboratory a fully operating TMT-like segment alignment control system with a level of performance comparable to that required for a real telescope. This study will provide a means to investigate the CSI phenomenon in a real structure and compare it to analytical predictions. Longer term goals include substantial improvements in bandwidth and disturbance rejection through the use of advanced control techniques.

\section{ASCIE Features}

The ASCIE structure shown in Fig. 1 consists of a 2-meter, 7-segment actively controlled primary mirror supported by a light, flexible truss structure. The optical system emulates that of an $\mathrm{f} / 1.25$ Cassegrain telescope and utilizes an actively controlled secondary mirror. The six peripheral segments are controlled in three degrees of freedom using specially developed precision actuators. Segment alignment is obtained through the use of edge sensors whose signals are processed by the control system which then generates the commands for the actuators. One of the unique features of the ASCIE is its optical scoring and calibration system which eliminates the requirement that the segments have real optical surfaces. Small optical flats combined with a special faceted secondary mirror reflect laser beams onto an array of linear position-sensing photodetectors.

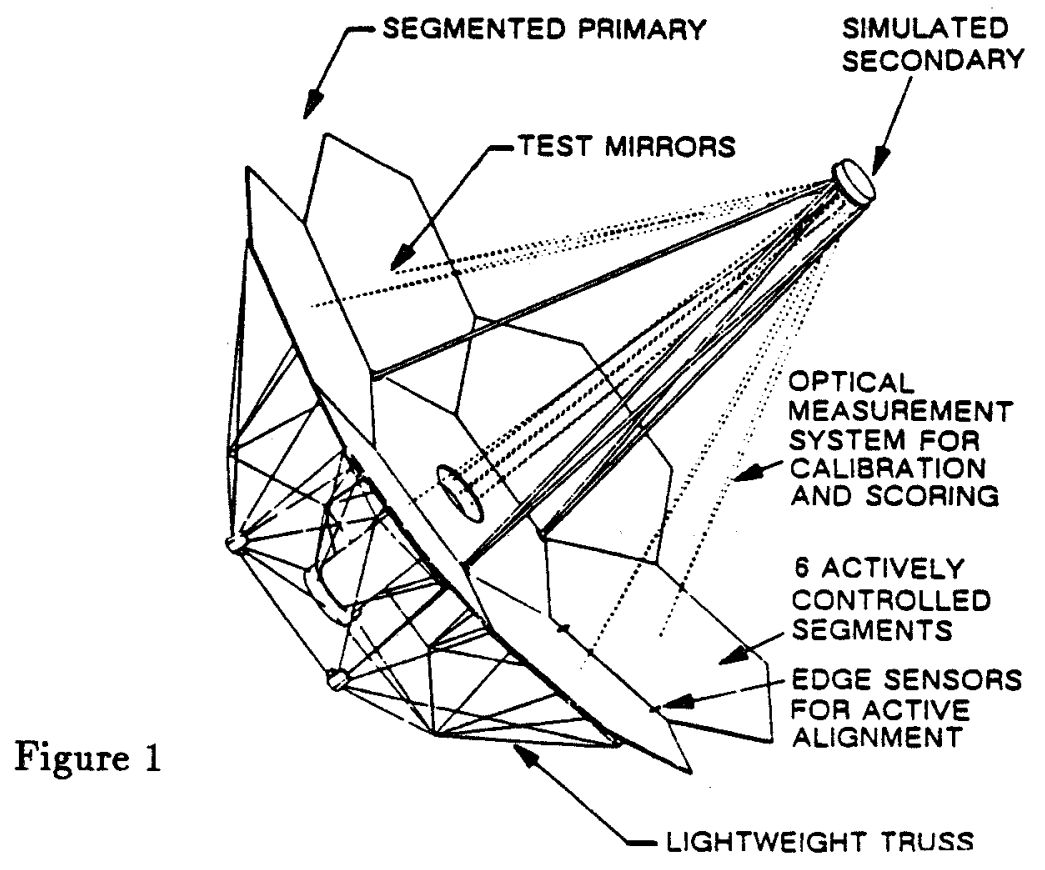

The active control of the secondary mirror is necessary to provide correct initial alignment of the primary segments. It will also be used to improve image stability and to simulate a chopping secondary, a feature found in all infrared astronomical telescopes [Refs 4 and 5]. Controlling the secondary mirror to stabilize the image at the focal plane is another example of a non-collocated system where CSI pays an important role [Ref 6]. 


\section{ASCIE structure dynamics properties}

The ASCIE structure was designed to replicate the complex dynamic behavior that characterize large segmented systems. Typical of such systems is the modal grouping due to the high degree of symmetry of the structure. For a perfectly rigid support structure, the segments and their supporting mechanisms (e.g. subcell and actuators) have almost identical dynamic properties and thus can be viewed as $\mathrm{N}$ identical oscillators at the same frequency. For ASCIE 18 modes of vibration related to the segments will occur at more or less the same frequency. However, because the support structure is in reality quite flexible, coupling between the grouped oscillators produces two results. First, the resonant frequencies tend to spread slightly by moving away from each other [Ref 7]. The second, and more significant effect in terms of CSI, is the creation of global or collective modes in which the segments as a whole behave as a continuous sheet rather than as individual pieces. These modes effectively couple one part of the mirror to another, creating adverse interactions that did not exist when considering individual segment dynamics. Fig. 2 shows a comparison between the modal frequency histograms of ASCIE and of the Keck telescope. A great similarity can be observed. This behavior is quite different from that of a beam-like structure as also shown on the figure. In addition the ASCIE structure was tuned to have its significant modes around $12-15 \mathrm{HZ}$ to be relevant.

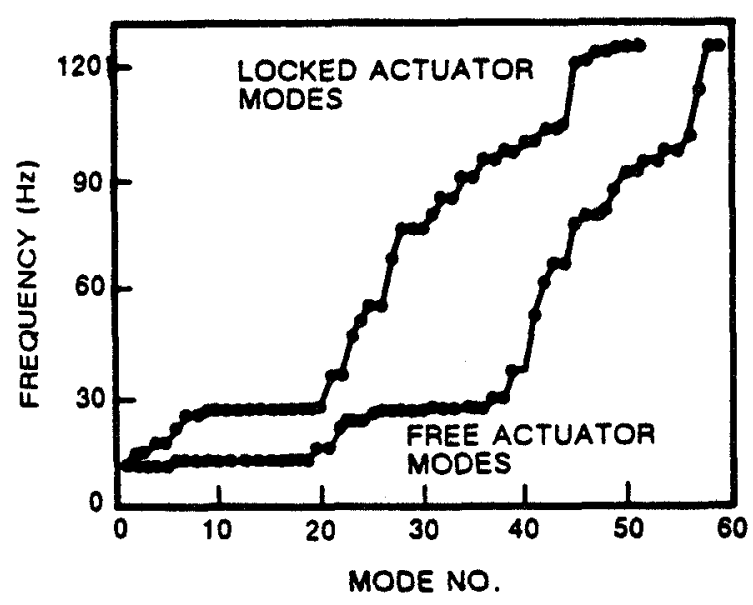

a. ASCIE

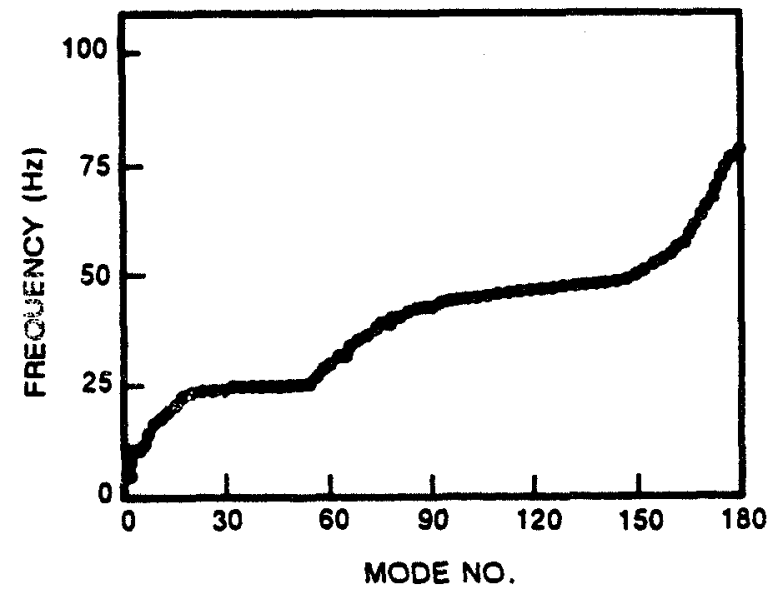

b. Histogram of TMT Modal Frequencies

Figure 2

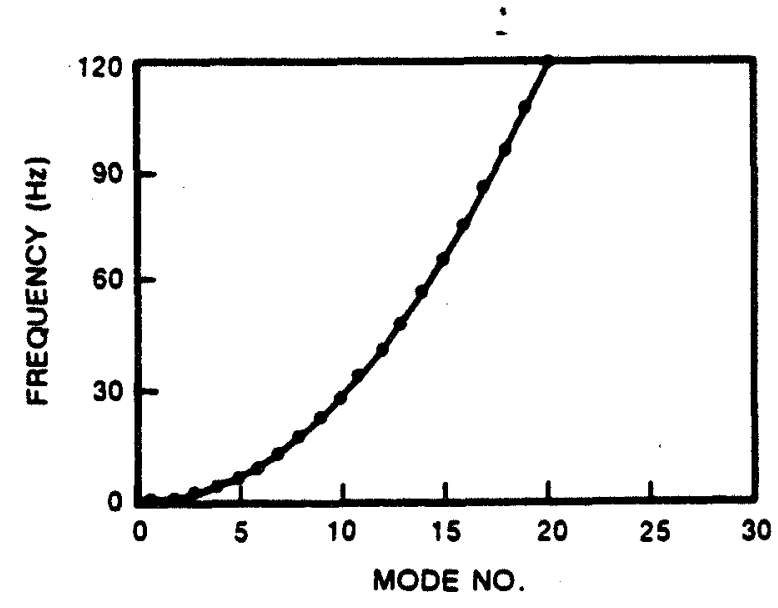

c. Cantilever Beam 


\section{ASCIE Control system principle}

The segment alignment control system is similar to that of the Keck telescope. It utilizes a self-referenced system of edge sensors providing a set of error signals that are processed through a special algorithm to obtain the piston and tilt errors for each individual segment. Corrections based upon these errors are applied, through proper electronic compensation, to the actuators controlling position and tilt of each segment (Fig 3 ). In such a centralized control system where the actuators are driven by signals from all the sensors, structural dynamics can couple the actuators to all the sensors through global modes of vibration, thus resulting in potential instability.

Figure 3

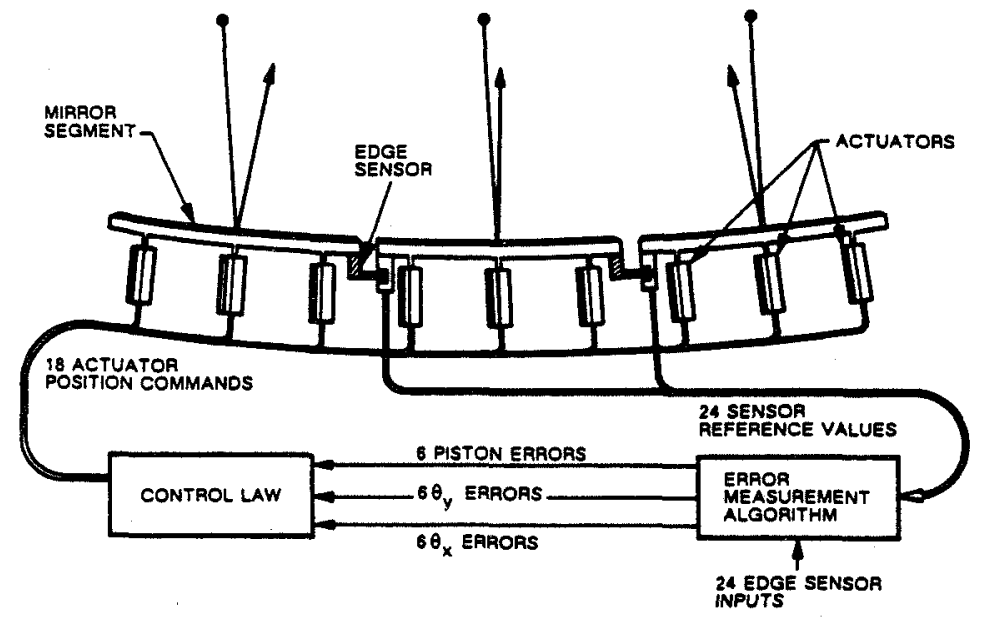

\section{ASCIL edge sensor system}

The edge sensors used for ASCIE are small inductive position sensors as shown in Fig 4a. This type was preferred to the capacitive sensors used for the Keck telescope because of their commercial availability, price and performance characteristics. Also they are rugged and easy to use. The sensors are mounted on tabs attached to the segments, thus directly measuring the relative displacement shown in Fig $4 \mathrm{~b}$. The offset from the true edge is a very important factor for the full observabiliity of the system and it must be optimized in order to obtain the best sensitivity and noise performance. The redundancy (24 sensors to measure 18 degrees of freedom) also helps to reduce noise, is important for reliability, and will permit fault detection and accommodation studies. Since these measurements are relative, a reference must be chosen to relate them to the absolute axis of reference. In ASCIE, the central segment is taken as the reference.

Figure 4a

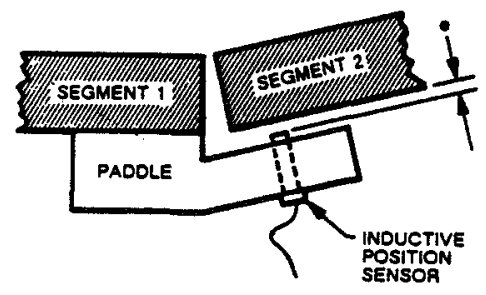

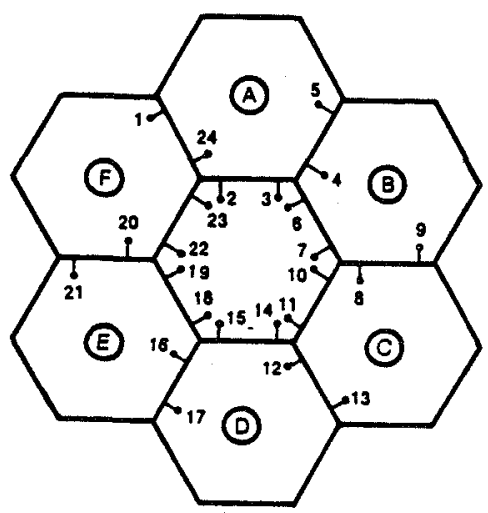

Figure 4b 


\section{ASCIE optical calibration and scoring system principle}

Fig. 5 shows the principle of an optical system that directly measures the tilt errors associated with each segment. It emulates a more complex wavefront sensor based on holographic patches, for example. The laser beam coming from the central tube reference is split into six equal beams by a special faceted secondary mirror. Each of the beams reflects on a small flat mounted on the corresponding segment reflects back on the same facet of the secondary mirror and finally focuses on a two-axis photodetector. The photodetector provides two electrical signals directly proportional to the position of the laser spot in two orthogonal directions. This optical system has a sensitivity of 0.1 arcsecond and is used to initially align the segments. After this operation the control system remembers the set points of all the edge sensors and maintains them in the presence of disturbances. The optical system is not a part of the control loop but can be used as an independent scoring system to evaluate the performance of the segment alignment control system.

Figure 5

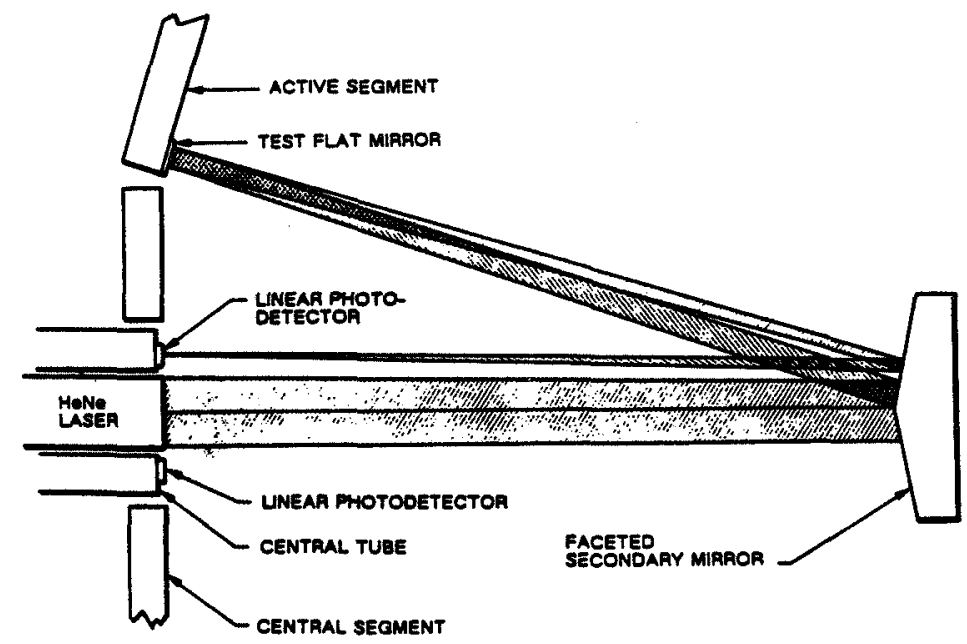

\section{ASCE single element alignment test (piston and tilt errors)}

The segment aligment errors during closed-loop operations are shown in Fig. 6a for piston, and in Fig. 6b for the two tilt axes ("Petal" denotes a folding motion of the segments toward the optical axis). The residual error is due essentially to seismic and sensor noise. By contrast with the previous plot, the traces are here perfectly centered, i.e., the thermal drift has been completely eliminated. The level of performance that has been achieved (about $30 \mathrm{~nm} \mathrm{rms}$ in piston and less than 0.05 arcsecond in tilt) is

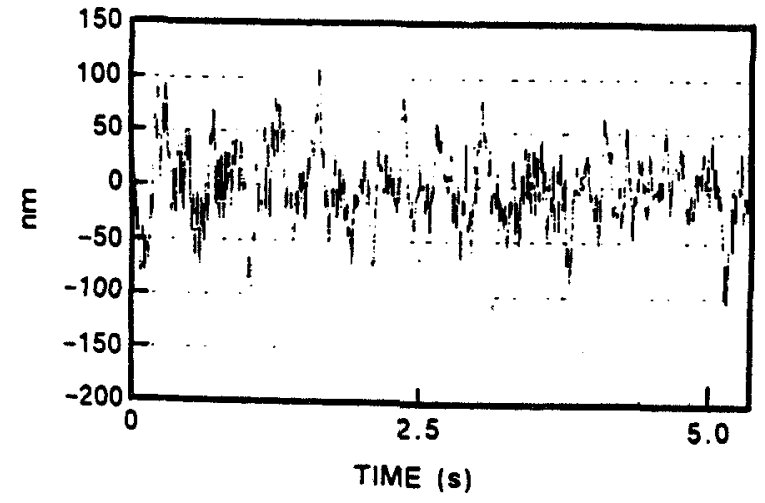

Piston error

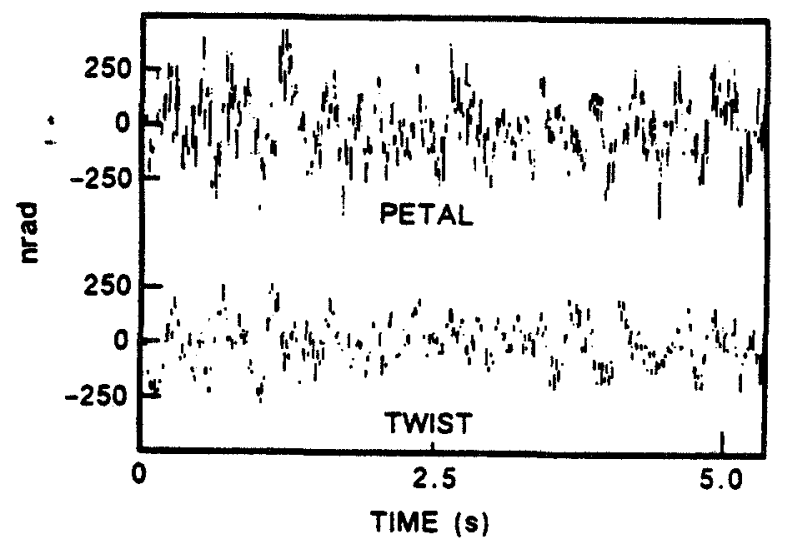

Tilt error 
comparable to that of the Keck telescope requirements and thus represents a major step in validating the technology for large flexible segmented optical systems.

\section{Conclusions}

This paper has presented a description of the ASCIE experimental setup, a generic test bed for several essential technologies. In particular its multi-input, multi-output, non-collocated control system and its complex structural dynamics, characteristic of large segmented systems make it an ideal test bed for CSI experiments. The high accuracy of its measurement system will make it possible to investigate the dynamics of microvibrations and its implication for thie CSI phenomenon.

\section{References}

1. Aubrun, J-N, Lorell, K.R.,Havas, T.W., and Henniger, W.C.: An Analysis of the Segment Alignment Control System for the W- M. Keck Observatory Ten Heter Telescope. Final Report. Keck Observatory Report Number 143, Dec 1985

2. Aubrun,J-N., Lorell, K.R.,Mast, T.S., and Nelson, J.E.: Dynamic Analysis of the Actively Controlled Segmented Hirror of tne W. M. Keck Ten Meter Telescope. IEEE Control Systems Magazine, Vol. 7, No. 6, Dec 1987

3. Aubrun, J-N, Lorell, K.R., Havas, T.W., and Henniger, W.C.: Performance Analysis of the Segment Alignment Control System for the Ten-Heter Telescope. AUTOMATICA, Vol. 24, No. 4, Jul 1988

4. Sridhar, B.,Lorell, K.R., and Aubrun, J-N.: Design of a Precision Pointing Control System for the Space Infrared Telescope Facility. IEEE Control Systems Magazine, Vol. 6, No. 1, Feb. 1986

5. Lorell, K.R. and Aubrun, J-N.: Active Image Stabilization for Shuttle-Based Payloads. Proceedings of the 1986 American Control Conference, June 18-20, Seattle, Washington

6. Parsons, E.K.: The Jitter Beam: an Experiment Demonstrating Pointing Control on a Flexible Structure. 1988 American Control Conference (Atlanta, Georgia), June 1988

7. Sridhar.,B., Aubrun, J-N., and Lorell, K.R.: Analytical Approximation of the Dynamics of a Segmented Optical System. Proceedings of the International Federation of Automatic Control gtn Symposium on Distributed Parameter Systems, June 30 July 2, 1986, UCLA, Los Angeles, California 


\section{DISCUSSION}

Nelson: What is the sensor noise with the actuators off, that is, the high frequency noise? Also, what is the sensor noise when the actuators are turned on and running - that is, the sensor residuals?

Lorell: The baseline sensor performance has a floor around $17-20 \mathrm{~nm}$, and that is inherent in the sensors. When the loop is closed and the system is running with 6 panels, we get approximately $200 \mathrm{~nm}$ of piston error and 800 nano-radians of tilt error.

Nelson: And that is measured by the optical system, and not the sensor system?

Lorell: That is the sensor system, since we have not yet calibrated the optical system.

Kahan: What was the forcing function?

Lorell: It was the lab floor. Next year we hope to apply specific loads.

Bely: Have you an explanation for the plateau on the frequency response curve?

Lorell: Yes, it is the collective motion. We saw this on the Keck models. These big segmented reflectors are like coupled spring-mass systems. Jerry Nelson has a computer animated movie of the vibration modes of the Keck telescope from $6 \mathrm{~Hz}$ up to about $50 \mathrm{~Hz}$. There are modes where the motion of one panel ripples through the structure and causes the other panels to vibrate. For example, the model for the Keck telescope that we did some time ago for the whiffletrees had a natural frequency of $25 \mathrm{~Hz}$. What we saw were peaks at $23.9 \mathrm{~Hz}, 24.2 \mathrm{~Hz}, 25.0 \mathrm{~Hz}, 25.2 \mathrm{~Hz}$ and so on. The $25 \mathrm{~Hz}$ whiffletree frequency spreads out - the energy spectrum spreads out. The mirror acts, for example, like a membrane with very densely packed modes and so classical structural control becomes much more difficult. 


\title{
Control Structures Interaction (CSI) Technology
}

\author{
W. E Layman \\ CSI Task Manager \\ Jet Propulsion Laboratory
}

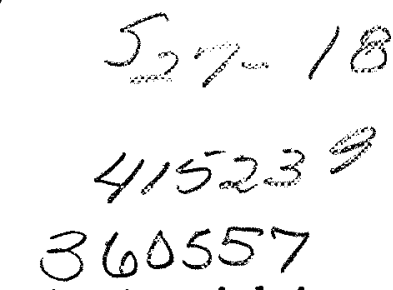

Control Structures Interaction (CSI) technology for control of space structures is being developed cooperatively by JPL, LaRC and MSFC for NASA OAST/RM. The mid-'90s goal of JPL's CSI program is to demonstrate with analysis, ground and flight tests, the super quiet structures needed for large diffraction-limited instruments such as optical stellar interferometers and large advanced successors to the Hubble Space Telescope. Microprecision CSI technology is intended as a new "building block" for use by the designers of large optical systems. The thrust of the microprecision CSI technology effort is to achieve nanometer-levels of space structure stability/accuracy with designs which employ otherwise conventional spacecraft technologies.

Without CSI, an ambitious (advanced in size or dynamic range) large optical system would demand major state-of-the-art advances in areas of conventional spacecraft technology, such as:

1) Ultra-quiet reaction control wheels, tape recorders, antenna and solar panel drives.

2) Ultra quiet cryogenic coolers, IR chopping mirrors, oscillating gratings, etc.

3) Ultra-stable/ultra-stiff/ultra-quiet and highly-damped structural materials and joints.

4) Ultra-tight temperature control, etc.

Even on a first, less ambitious, large optical system, incorporation of CSI technology is desirable in order to assure healthy performance margins. By incorporating CSI into the design, project scientists can be more confident of achieving needed performance, and project managers can be more confident of meeting project schedules and budgets.

Since optical interferometer missions require extreme structural and optical stability and accuracy, JPL's CSI team is developing a Focus Mission Interferometer (FMI) as a technology-driving challenge-problem generally representative of large optical system technology needs. The FMI finite element models, control system designs, and performance analyses will be available to the large optics design community for reference as they develop future large optics design concepts and mission proposals. The CSI design methodologies demonstrated on the FMI will be applicable to all large microprecision optical systems.

JPL design experiences have indicated the following CSI technology development areas are especially applicable to large optical system projects:

\section{Active structural members}

Microprecision control of vibration and static deformation requires the development of precision active members. Strut members are presently being tested which incorporate internal sensors and piezoelectric elements capable of nanometer-level positioning. A single strut, incorporated into a conventional truss structure, has initially demonstrated vibration response reduction by factors on the order of 30. Advanced CSI structure/control hierarchical architectures, control designs, and active member usages are being developed with a goal of reducing vibration response at critical optical elements by factors of 1,000 to 10,000 . 


\section{Control/structures design methods}

Quick early estimates of system performance are needed for pre-project studies of large optical systems which incorporate CSI. The JPL and LaRC CSI teams are cooperatively developing expedient CSI analysis/design tools for quick global (structure/control/optical) system stability/accuracy prediction. Also, CSI design strategies, and architectures (disturbance isolation, structural response suppression, optical element position compensation, local control, global control, proof mass actuators, active members, etc.) are being evaluated and documented in a CSI Handbook. Transition from conceptual studies to flight-projects will demand CSI design/analysis tools with expanded capacity and accuracy, for exact detailed design and exact performance prediction. JPL and LaRC will be cooperatively developing these tools as well, as part of the CSI technology development effort.

\section{Microdynamic effects characterization}

To assure meeting the stability/accuracy performance requirements demanded by large optical systems, structural modeling for nanometer-level control must be based on accurate microdynamic stiffness and damping characteristics. Nanometer-level stiffness and damping characteristics of materials and joints can differ significantly from those observed at the macro level, but little data exists in this "microdynamic" range. The JPL CSI team is building a special microdynamic test machine to measure force and displacement characteristics for representative structural components (materials, joints, dampers, actuators, struts, etc.) at the nanometer level. Additional tests on the microdynamic machine and from other sources will be compiled to characterize typical flight vibration sources and possible bizarre microdynamic noise sourccs such as thermal "creak," creep strain relief "popping" (structural annealing), loaded joint "micro-ratcheting" (micro-stick slip), etc.

\section{Ground and flight test validation of CSI methods}

The new CSI technology will be valuable to flight projects, only if its performance and reliabilily are convincingly demonstrated in ground and flight tests. To this end, JPL, LaRC, and MSFC are operating CSI ground test beds to validate various aspects of CSI technology. (Academia and industry are also involved in the test programs through individual contracts and through a guest investigator program.) The JPL micro-precision test bed will exercise active actuators and passive dampers to quiet vibration responses induced by flight-like on board disturbances. At structural resonance frequencies of 5 $\mathrm{Hz}$ and above, one of the test bed goals will be to demonstrate differential light path interference fringe stability in the regime of 10 nanometers rms. (Below about $5 \mathrm{~Hz}$, laser beam air-path stability will preclude 10 nanometer-class experiments.) Long range plans include CSI flight experiments to assure that findings in the ground test programs remain valid in a space environment. 


\section{JPL Control Structures Interaction Technology Program}

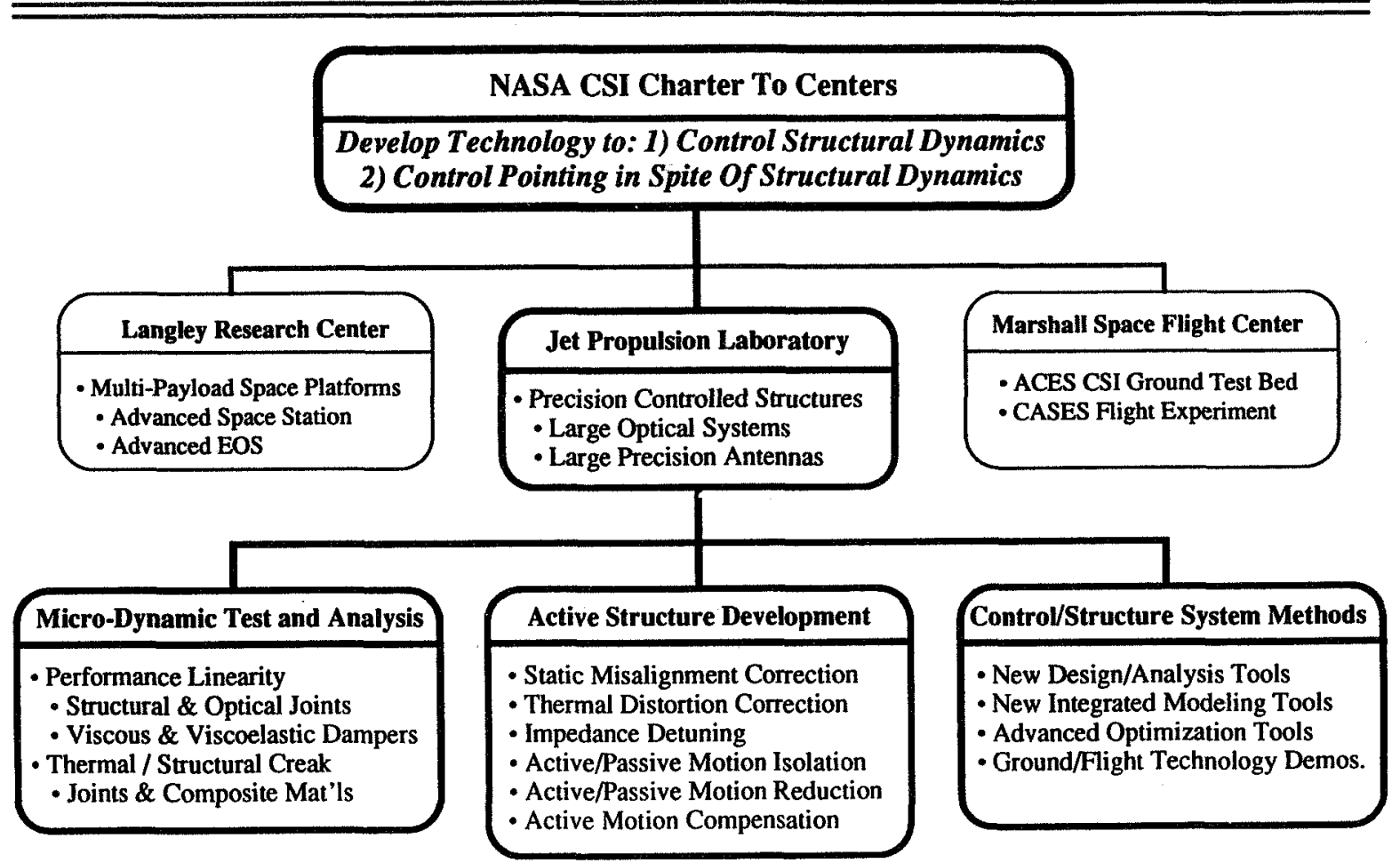

JPL CSI Technology Goal Setting

\begin{tabular}{|c|c|c|}
\hline Space-Interferometer CSI Needs & Large-Space-Optics CSI Needs & Micro-Precise-Antenna CSI Needs \\
\hline $\begin{array}{l}\text { - "Quiet" Space Interferometers } \\
\text { - Synnott Study Team, Others } \\
\text { - “Active” Space Interferometers } \\
\text { - Focus Mission Interferometer }\end{array}$ & $\begin{array}{l}\text { - Large Space-Station Telescopes } \\
\text { - Large EOS Telescopes } \\
\text { - Large Free Flier Telescopes }\end{array}$ & $\begin{array}{l}\text { - Large Sub-Millimeter Antennas } \\
\text { - Low Earth } \\
\text { - Geosynchronous } \\
\text { - Heliocentric }\end{array}$ \\
\hline \multicolumn{3}{|c|}{ Micro-Precision Control Structures Interactions: Technology Goals } \\
\hline $\begin{array}{l}\text { - Sub-Micron Motion Isolation } \\
\text { - Low Cost/Pwr/Wt } \\
\text { - } 20 \text { to } 40 \mathrm{~dB} \text { at Struct Freqs } \\
\text { - Reliable/Ground Testable }\end{array}$ & $\begin{array}{l}\text { - Sub-Micron Motion Reduction } \\
\text { - Low Cost/Pwr/Wt } \\
\text { - } 20 \text { to } 40 \mathrm{~dB} \text { Reduction } \\
\text { - Reliable/Ground Testable }\end{array}$ & $\begin{array}{l}\text { - Sub-Micron Motion Compensation } \\
\text { - Low Cost/Pwr/Wt } \\
\text { - Static and Dynamic Correction } \\
\text { - } 20 \text { to } 40 \mathrm{~dB} \text { Compensation } \\
\text { - Reliable/Ground Testable }\end{array}$ \\
\hline $\begin{array}{l}\text { - Microdynamic Creak Assessment } \\
\text { (Energy, Frequency, Displacement) } \\
\text { - Characterize Joint Classes } \\
\text { - Characterize Material Classes } \\
\text { - Make Practical Design Solutions }\end{array}$ & $\begin{array}{l}\text { - Make CSI Preliminary Design Practical } \\
\text { - Faster, Easier Modeling } \\
\text { - Faster, Easier Analysis/Design } \\
\text { - Integrate The Structure and The } \\
\text { Control Design Processes }\end{array}$ & $\begin{array}{l}\text { - Validate CSI Technology } \\
\text { - Develop Ground Test Methods } \\
\text { - Correlate Analysis With Tests } \\
\text { - Prove Results With Flight Tests }\end{array}$ \\
\hline
\end{tabular}




\section{JPL CSI Technology: Active Structure}

- Active Structures Include:

- Actuators: Electromechanically Extensible Truss-Struts (and Shells)

- Integral In The Primary Structural Paths

- Small Motion Control: High BW $(\approx 1000 \mathrm{~Hz})$ (nanometers-to-100 micron)

- Large Motion Control: Quasi-Static (millimeters to centimeters)

- Local Sensors: Displacement, Velocity, Acceleration, Force, Temperature, etc.

- Collocated With Each Actuator

- Local Control Loops: High Bandwidth, (Typically Analog for Speed)

- Active Control Of: Damping, Stiffness, Thermal Contraction, etc.

- Active Structures Can Provide:

- Structural Property Control (Factor-of-Ten to Factor-of-One-Hundred) Such As:

- Damping Changes to Reduce Vibration Response

- Stiffness Changes to Detune Resonant Modes

- Force Isolation From Disturbances

- Active Motion Compensation

- Thermal Expansion Reduction (Factor-of-Ten to Factor-of-One-Hundred)

\section{JPL CSI Technology: Micro-Dynamic Test \& Analysis}

- Micro-Dynamic (Motion Under 10 micron) Issues

- Structural Stiffness and Damping

- "Micro-Behavior" Deviation From "Macro-Behavior"

- Non-Linear Continuous...Load-Stiffening, Load-Softening

- Non-Linear Discontinuous...Backlash, Stick-Friction

- Static Position Maintenance

- Material and Joint Micro-Creep and Hysteresis Behavior

- Bizarre Disturbance Sources

- Thermal Creak

- Micro-Creep Strain Relief "Popping"

- High Frequency Ratcheting of Load Changes

- Micro-Dynamic Assessment Approach

- Conduct Micro-Dynamic, Static,and Thermal Load/Displacement Tests On:

- Structural \& Optical Materials, Joints, Dampers, and Actuators

- Identify/Develop Components Having Acceptable Micro-Dynamic Behavior

- If Necessary, Develop Analysis/Design Methods Which Work Despite Significant Micro-Dynamic Effects 


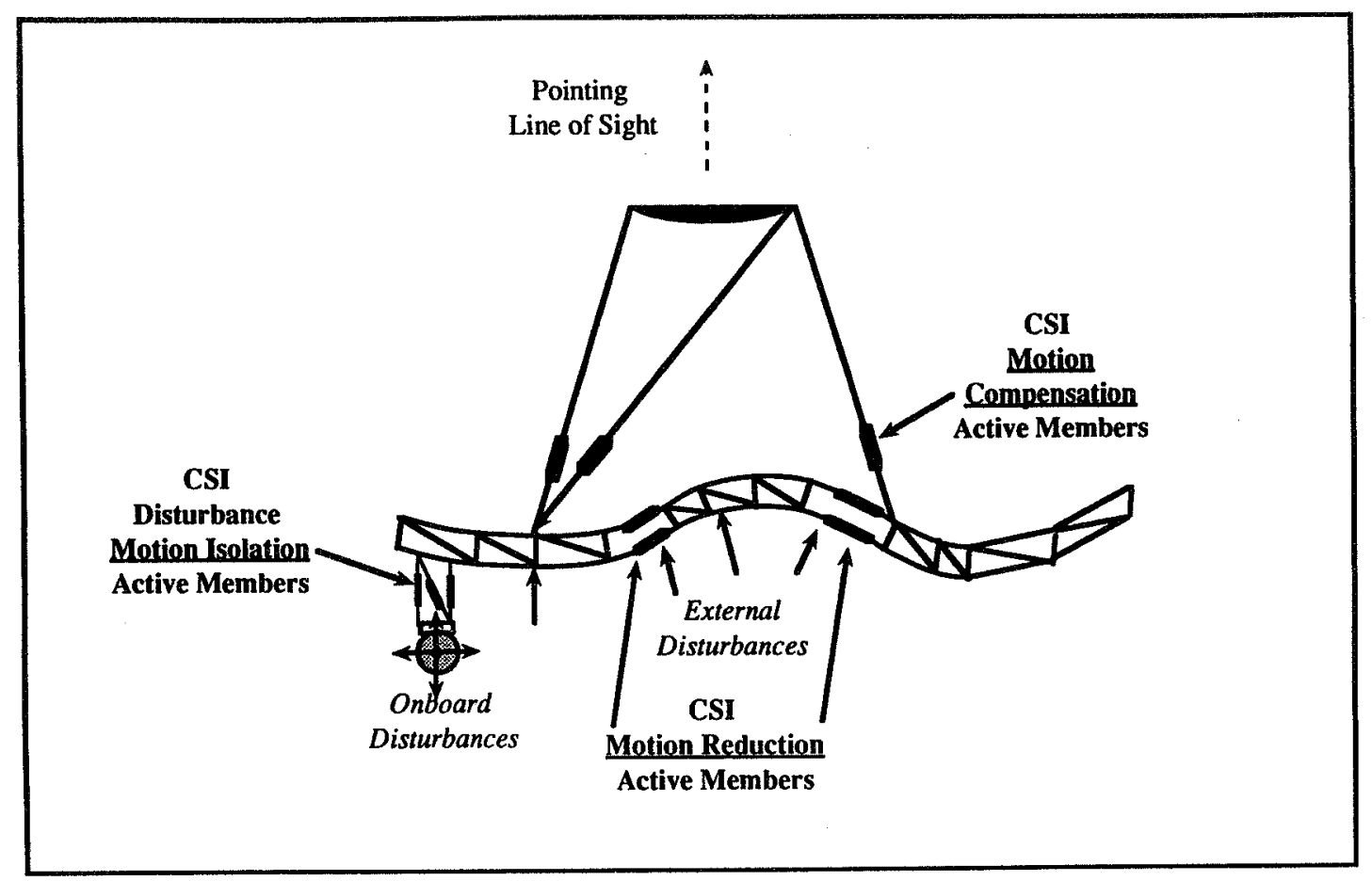

\section{JPL CSI Preliminary Specifications: Active Structure Driver}

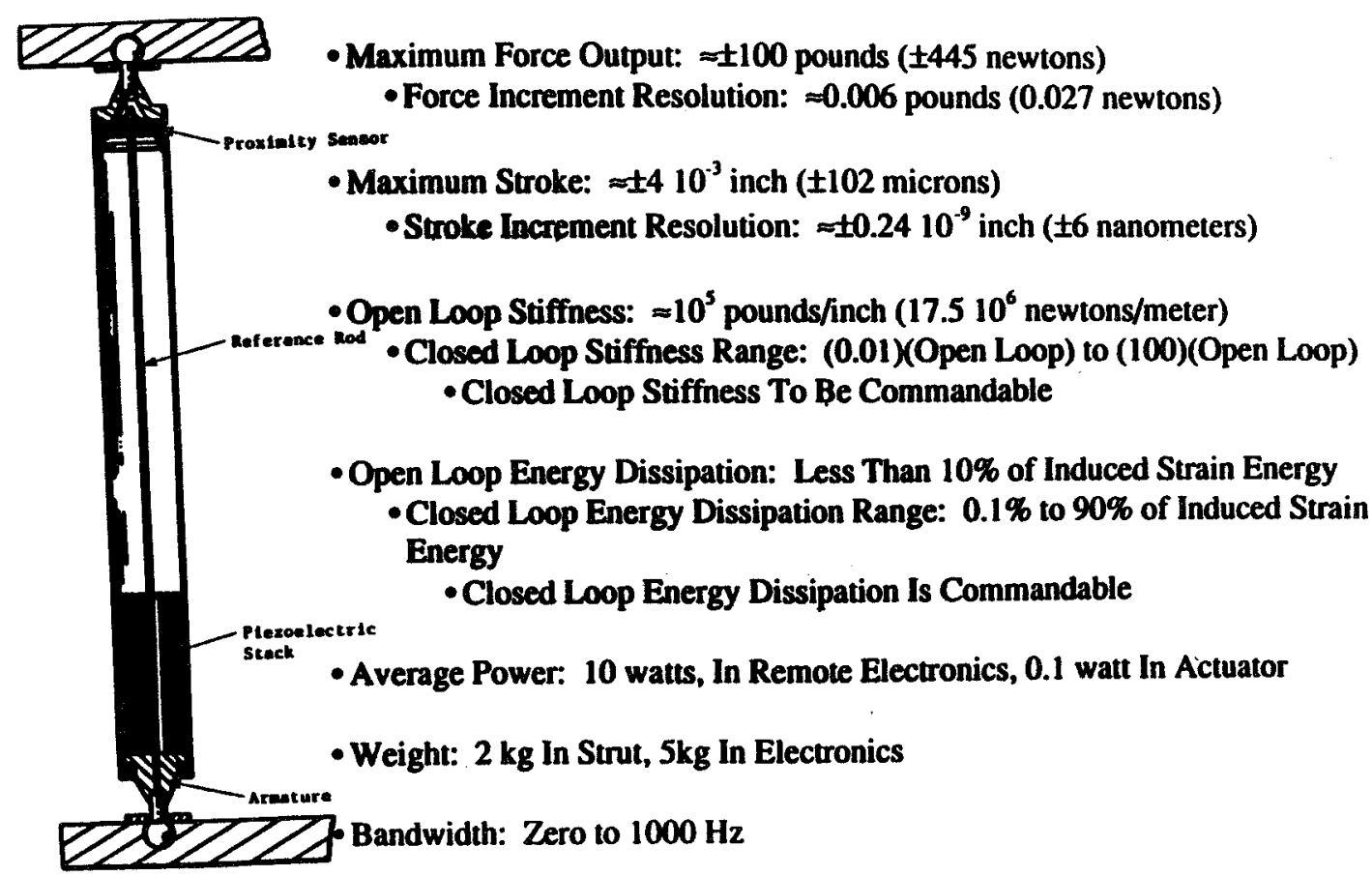




\section{VIBRATION ENVIRONMENT AND CONTROL APPROACH}

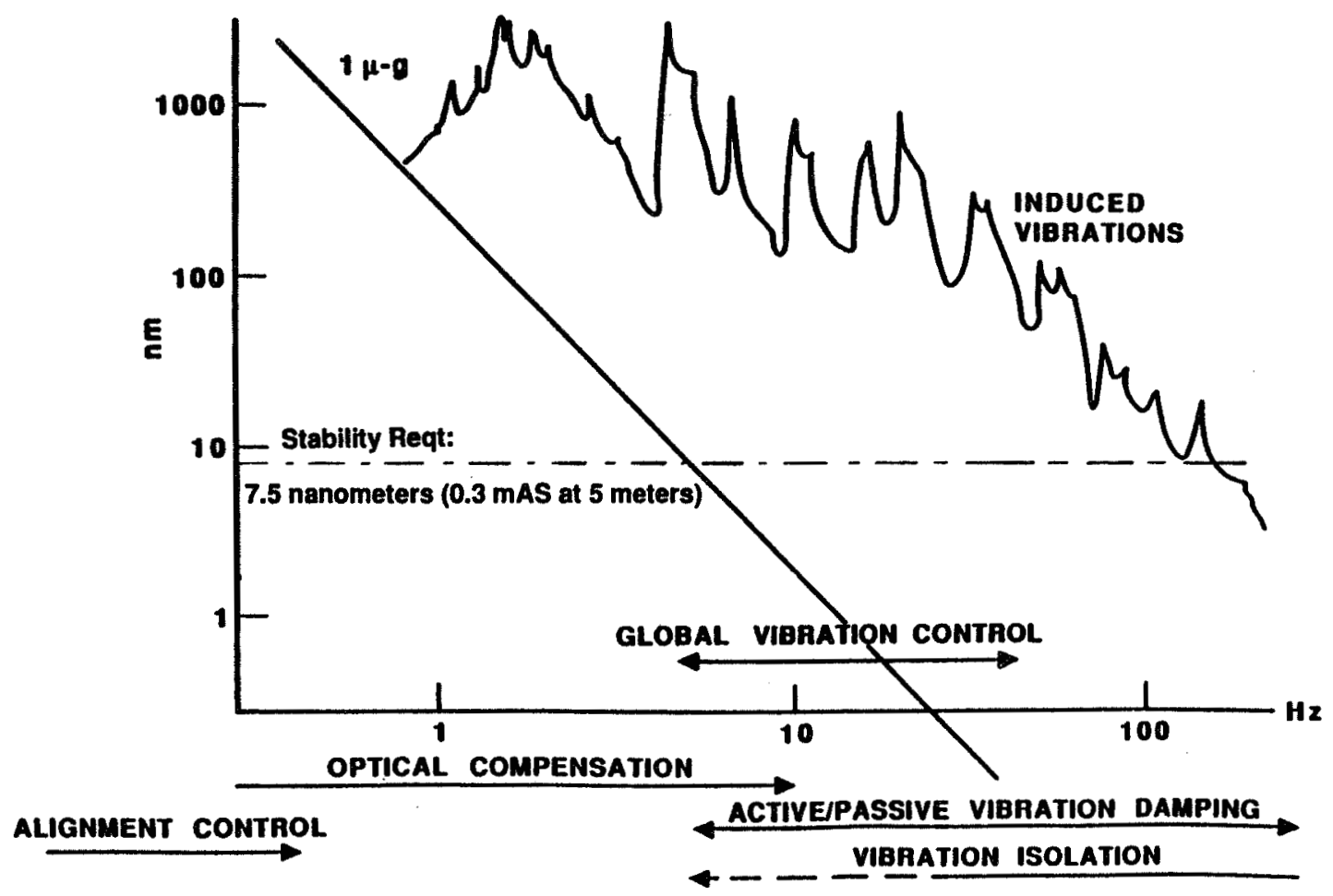

\section{JPL CSI Technology: Active Structure Test Results}
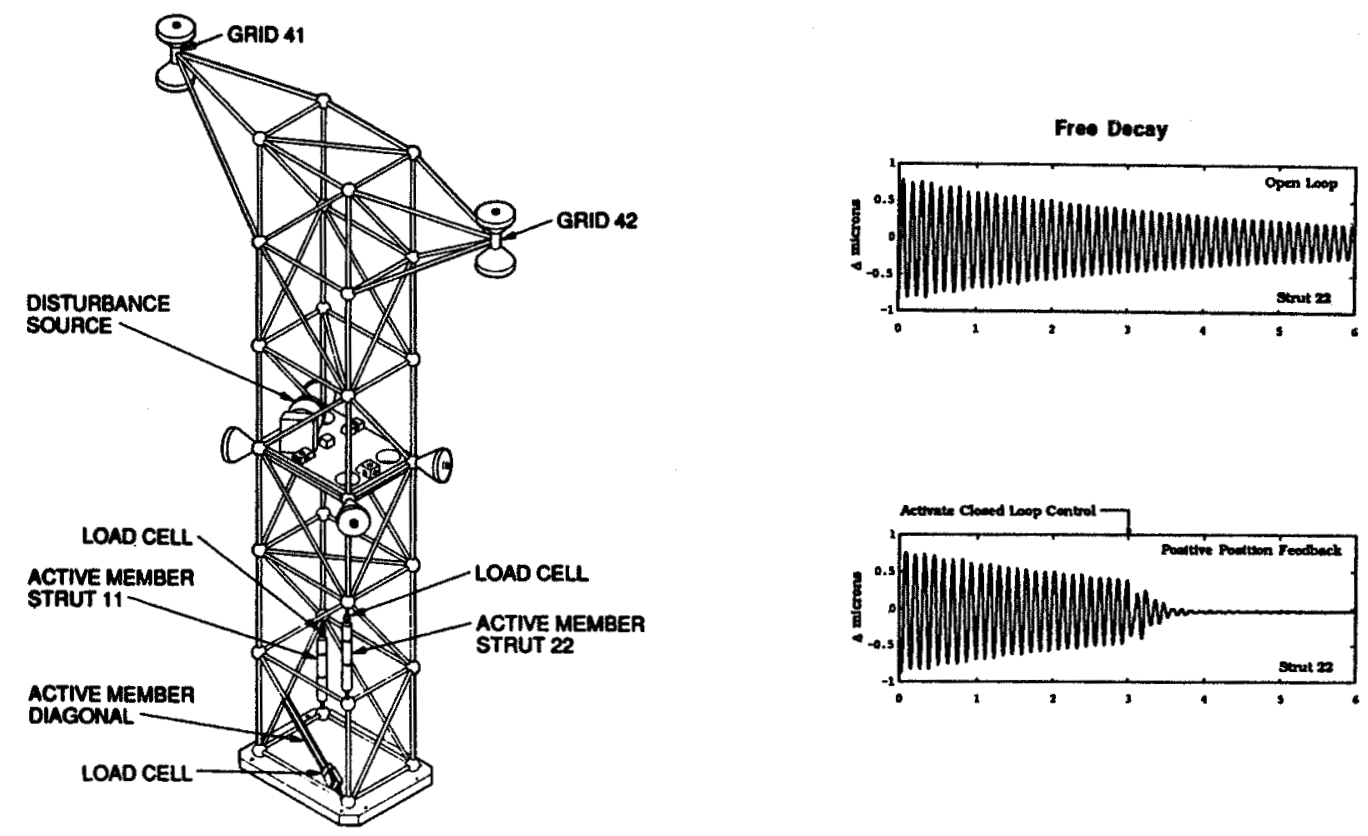


\section{DISCUSSION}

Bely: What gain in mass over conventional systems can we expect from an active structure?

Layman: In a representative calculation, about 100 times the stiffness of support structure (5 to 10 times the mass) was required to obtain as low a structural vibration response with a conventional structure as was achievable with a controlled structure. There is no good rule of thumb for conventional structural equivalents to controlled structure. It may be impractical to solve many future problems with a conventional structure. 
Pointing Control System for a 10 meter Space Telescope

Dominick Tenerelli

Lockheed Missiles and Space Co

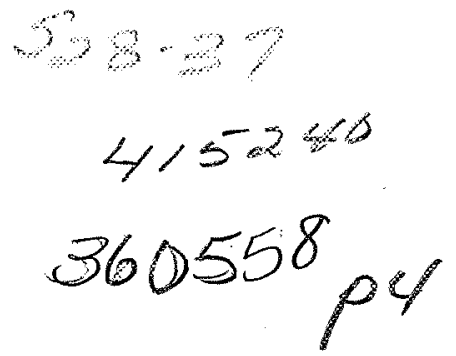

10 METER

UV-VISIBLE-IR

OVERYIEW

TELESCOPE

OQVEATYE-

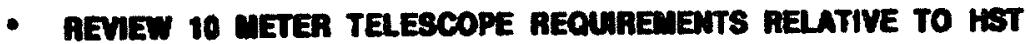

- OSCUSS MUPROVEd JITER RECUIRED RELATME TO hST

- descuss tonove actuaton due to labgen inemina of the 10 meter telescope

10 METER

UV-VISIBLE-IR

TELESCOPE

POINTING CONTROL SYSTEM REQUAREMENTS

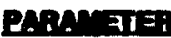

10M:Tris

MST

co:

Pondme acciancy 10 mes

$10 \mathrm{~mm}$

Condentant with

ist requilowents

Ponirive

STacumr

1.4 mos in villowe

0.5 mine in UV

$7 \mathrm{~mm}$

AITUEA

Thuckens

O.5 map up to 0.21 ereacedience

20 ming, 0 to 0 at

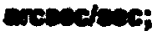

30 mon, ace 6

D.21 erowatese

CUTE STARS

LARE AMCIE

MNESUMP

25 aremb currounche the colence field (17) mag etare)

35 erconin enrounding the

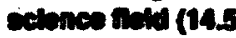
meg simes)

Requines roduction in friser rolutive to IST

For HST the errer heludere polaling, miner end uncintanduced curere.

Dimaner etare (theter of 17 and hure collinellog cret (moler of 10$)$ bringenes

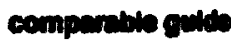
cour enougy bor

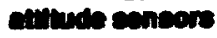

$90^{\circ}$ in 18 minutas

Larger inertite of 10 mener triencepe will nopule lover mection whed cepability 


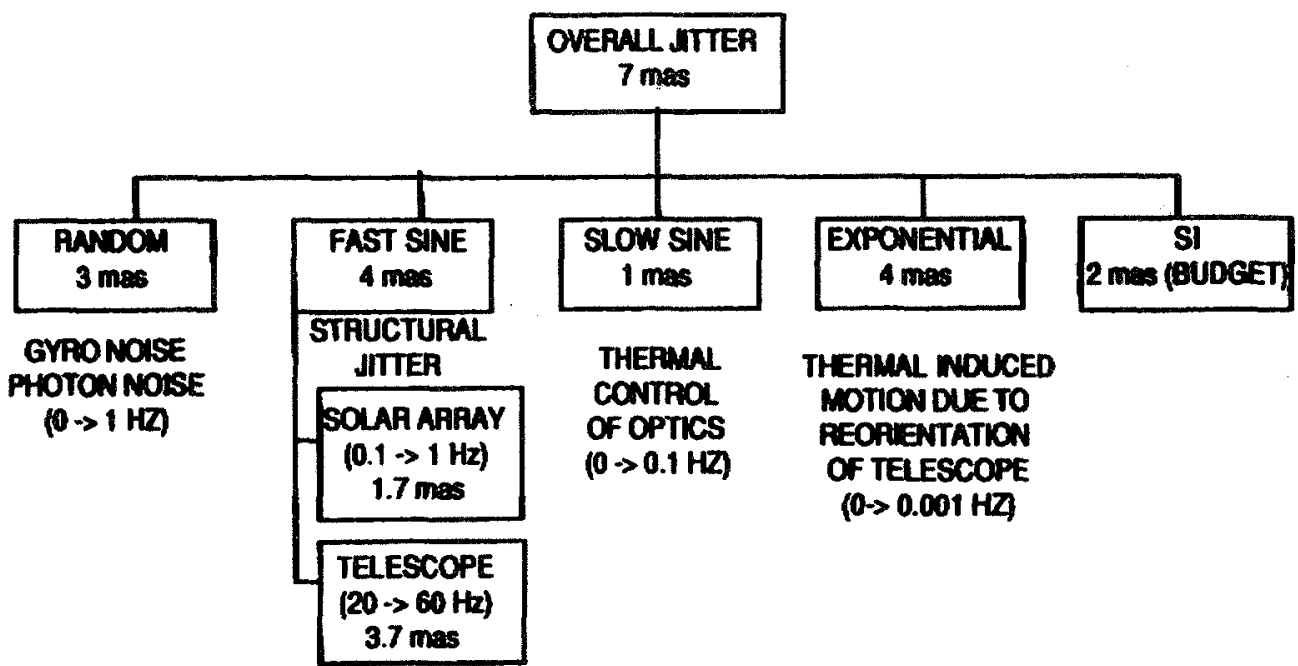

- CONSIDERATION OF A TWO LAYER CONIROL SYSTEM, I.E, BODY PONTIMG AUEMENTED BY MMGE MOTION COMPENSATION (IMC). TO ACHEVE 1.4 MAS NN THE VISBBLE ( 0.3 MAS IN THE UV) WDKCATES A HIGH BWNDWDTH FINE GUIDANCE SENSOA WLL BE REOU WED TO REDUCE ERPORS STNCE SOME ERAOA SOURCES HAVE FAECUENCIES UP TO GO HZ, OR MORE.

- TO ACHEVE LOM PHASE ERROR W TRACKRG THE POINTING DEMATIONS, THE IMC SENSOR MUST HAVE A BANDWDTH 5 - 10 TMES HIGHER THAN THE DISTUABANCE, A CONDITION HARD TO SATISFY FOA THE 17 MAG GUIDE STAR.

SUMAARY: REDUCTION OF ERPOR SOUACES AND PAECISE BOOY PONNTMG WIL BE KEY TO ACHIEVMG THE JITTER REQUFEMENTS.

10 METER UV-VISIBLE-IR TELESCOPE

TYPICAL RWASTRUCTURAL JITTER

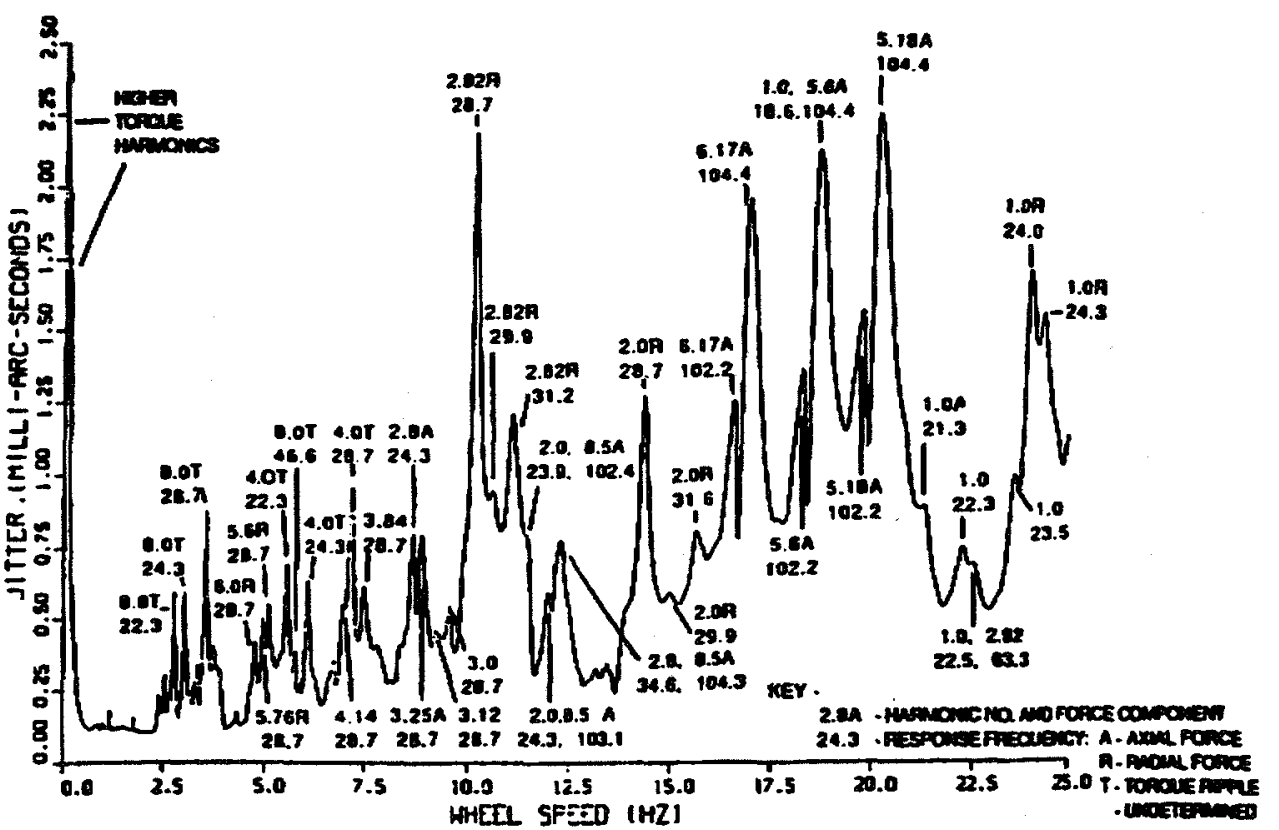

- BgON 10 HE WhEE SPEED WHE DOMUMNT SOUACES OF DSTURBBNCE ARE THE REACTION WHEFA TOROUER RIPPLE THERE ARE A FEW COMPONENTS OF AXILL MND RADUL FORCE.

- ABOVE 1OHZ THE RWA FOACES DOAMMTE

- THE FRECUENCIES OF THE DISTURBAANCES VARY FAOM 22 TO $104 \mathrm{HZ}$ 
10 METER

UV-VISIBLE-IR RWA MANEUVERING FOR THE 10 METER TELESCOPE
TELESCOPE

- HST AS PRIMAPLY TOROUE LMITED FOR MAMEUYERS, NOT MOMENTUM LMTED.

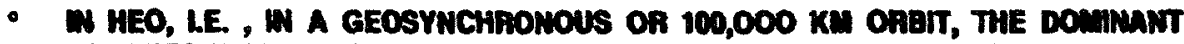

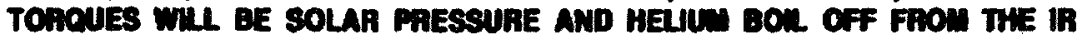
IMSTRUHENTS, DOTH OF WHICH ARE SMAL COMPARED TO THE TORQUES ON HST W THE LOW EATTH OABT.

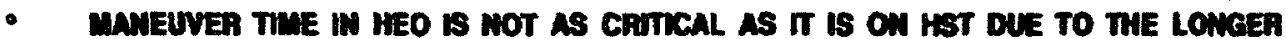
ORBT PEROD, AND HEUCE LONGER TME PERIOD FOR WHCH THE TARGET IS AYAILABLE.

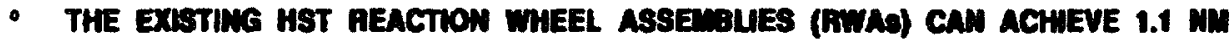

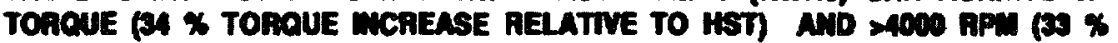
monENTU INCREASE RELATTE TO HST) wm MaOR ELECTRONIC MODIFICATIONS.

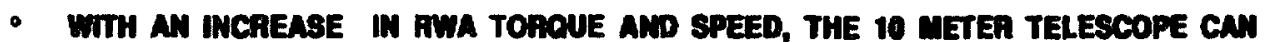
ACHEVE ESSERTMLY HST MANEUUER PERFOAHANCE MTH VEHCLE UNERMAS

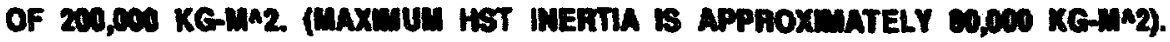

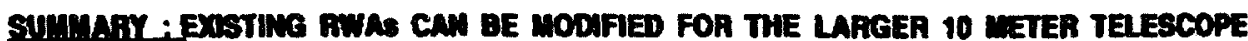 \\ MERTIA.
}

RWAs vs CMGs

Bach actuator has its assets. The CMG can provide high torque, high momentum starage capablity and, the force disturbances tend to be at fixed frequencies. The high torque capability of the CMC is not needed for the 10 meter telescope. The fixed frequencies of the CMG rotor forces and torques make it attractive when viewed relative to the fact that the changing $R W A$ wheel speed causes the RWA to "sweep" through the teleacope structural modes. For example, if the HST bearing were used in a CMG which had a rotor frequency of $100 \mathrm{~Hz} 16000 \mathrm{RPM}$, the bearing forces would hove a small component due to the retainer at $34 \mathrm{~Hz}$. The other dominant forces and moments would be at 100 Hz, $282 \mathrm{~Hz}, 516 \mathrm{~Hz}, 560 \mathrm{~Hz}$, et al, frequencies which could be efiectively reduced by an isolator, If required. Also. the CMG could incorporate a capability to reset its spin opeed over a small range. e.g. I $5 \%$ about the $100 \mathrm{~Hz}$ nominal speed to avold troublesome structural modes. Most CMG development to date has been for large devices. Relatively few small devices have been bullt fone example ts the astronaut manned maneuvering unith, and none have not been concemed with ulturalow force, moment and torque ripple noise in the development of these devices.

The HST RWA can meet the maneuvering requirements and has been extenatvely characterbed relative to its noise producing error sources.

Using "today's persective" on the 10 meter telescope, which is many years from actual hardware implementation. it is better to spend any available technology funds to pursue a low noloe RWA at this time. 
10 METER

UV-VISIBLE-IR

TELESCOPE

SUMMARY

- JITTER PRESENTS THE TECH WOLOGY CHALLENGE IT IS EXPECTED THAT THE 1.4 MAS (20\% OF HST) JITTER REQUIAEMENT CAN BE MET WITH DESIGNS USAMG AVALABLE ATTTULE CONTROL ACTUATORS EUIPLOYED M PAOVEN HST CONTROL LAWS AND USER INTERFACES. THE O3 MAS ( $4 \%$ OF HST MTTER REQUREEMENT FOR UV WLL REQUAE ADDMONAL DEVELOPMIUT OF LOW NOSE GYROS, RWAS AND "QUIET" STRUCTURES.

- PAECISTON BOOY PONTING COUPLED WTH REDUCTONS IN DLSTUABANCES WUL BE THE PANAE CAMDIDATE TO WEET THE 10 METEA JTTER REQUIREMENTS DUE TO THE DIFFICULTY W OBTANEUG A SUTTABLE IMC SENSOR.

- USE OF IMC WLL REQURE DEVELOPUENT OF A HKG BANDWDTH SENSOR. THE STAR ENERGY AVALABLE AT 17 MAG DOES NOT APPEAR TO SUPPOAT A HGH BANDWDTH SEISOR. BRIGHTER GUDE STARS ARE REQURED, IIPL. YUUG A LARGER FGS ACOUESTION AREA.

- RWAS ARE AVALLAbLe TO MEET THE 10 METER MANEUVER REQUIREMENTS. TORGUER RIPPLE AND FOACE DESTURBANCE REDUCTIONS ARE REQUARED TO MEET THE JTTER RECUAREUENT.

- candidate technology developients

- FGS WITH LARGE FIELD OF VIEW AND POTENTIL MC CAPABHLTY

- RWA FORCE, WOLENT AND TORQUER RIPPLE REDUCTION

- GYRO NOSE REDUCTION, BOTH SENSOR AND ELECTRONICS

- "QUIET" STRUCTUAES WITH LOW MODAL COEFFICIENTS AMDIOR MGH DAMPMG

- RIGOROUS CONTROL OF DISTURBANCES FROM SIS AS WELL AS VEHICLE EQUPUENT, EG. , TAPE RECORDERS

- USER COMMAND INTERFACE BETWEEN GROUND SEGMENTS AND WTH THE VEHCLE

\section{DISCUSSION}

Layman: Are the reaction wheels larger than for HST?

Tenerelli: Yes, about $30 \%$ larger.

Unidentified: You noted that some people at LMSC feel that you should use CMGs, Controlled Moment Gyros, instead of reaction wheels. Why use reaction wheels?

Tenerelli: Because of problems with CMGs on Skylab there is a bias against CMGs and towards reaction wheels amongst some folks. In our organisation there is a strong element who feel that CMGs should be the momentum compensation device that is used. But we can make either work.

Stockman: What was the structure you assumed for your analysis for a 10 meter telescope?

Tenerelli: We assumed that we would have a metering structure and a focal plane structure that would have at least the stiffness of HST. 


\title{
Moving Target Tracking for a Next Generation Space Telescope
}

\author{
David R. Skillman \\ HST Project Office, Goddard Space Flight Center
}
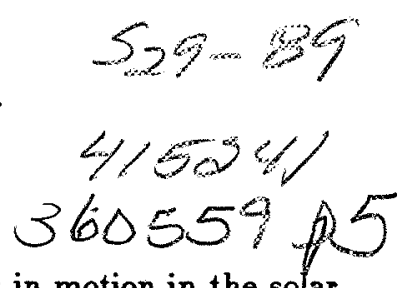

The Hubble Space Telescope required a complex method for tracking targets in motion in the solar system, due mostly to atmospheric drag effects. A NGST in high earth orbit, or on the Moon, would not suffer these drag effects, thus mitigating the tracking problem. Many of the HST problems arise from the 'open-loop' nature of the tracking method. An NGST would be better off if it could use 'closed-loop' tracking of all targets, both fixed and moving. A method to accomplish this is proposed. Methods to reduce the jitter and lower the ambient temperature of the optics via a tethered optical system are proposed.

\section{Introduction}

The Hubble Space Telescope required a complex method for tracking targets in motion in the solar system. Most of that complexity is driven by the low orbit of HST and the attendant atmospheric drag effects. Target tracking must be 'open-loop' on HST since vehicle tracking is done on the guide stars, not on the target. This puts a burden on the predictive capabilities of the ground system.

The track of a moving target is usually known in terms of celestial coordinates. The ability of a spacecraft to follow this path in its own coordinate system is limited by the quality of the registration between the two.

Jitter is a concern for both fixed and moving targets. The jitter in HST comes from those parts of the spacecraft that move during an observation. If the jitter-inducing components could be isolated from the optical system, a much better performance could be obtained.

\section{The effects of atmospheric drag on moving targets}

In order to understand why atmospheric drag is a problem, the following chain of reasoning is presented:

a) In order to plan the observing schedule efficiently, one wants to have the schedules planned well in advance.

b) Targets in the solar system have a substantial parallax motion due to the orbiting motion of the spacecraft. This spacecraft- induced parallax motion of the target can only be tracked if the position and velocity of the spacecraft are well known in advance.

c) The position of the spacecraft rapidly becomes unpredictable if atmospheric drag is significant. Variations in solar activity can dramatically affect the drag. For HST the position uncertainty can grow to the order of a $1000 \mathrm{~km}$ in a month. At Jupiter this would correspond to pointing errors of hundreds of milliarcseconds.

These three considerations show that it is impossible to produce a tracking profile (spacecraft attitude as a function in time in the control system coordinates of the spacecraft) that will remain accurate for execution a month or more in the future.

This problem was solved on HST by generating all tracking paths in geocentric coordinates and then having the HST flight computer perform the coordinate transformation from geocentric to spacecraft centered coordinates. The orbit ephemeris of the HST in 
the flight computer is corrected every few days (and can be updated within 12 hours if needed) to remove the drag error.

A NGST in high earth orbit, or on the Moon, would not suffer these drag effects, thus mitigating the tracking problem. Thus, for the low drag locations the generation of tracking paths, even in spacecraft coordinates and for times well in advance of observation, is simplified considerably and are not a significant design driver either in the spacecraft or ground system area. A low earth orbit for a NGST would be susceptible to the same problems faced by HST and would need similar solutions.

\section{The roll error problem}

The track of a moving target is most naturally known in terms of celestial coordinates. Right ascension and declination coordinates are not the optimal representation since the distance to the target is also needed to allow for parallax variations. Typically it is best to use an inertial X,Y,Z system since most ground system and flight system elements use the GEI (geocentric earth inertial) coordinate system. The path is then described as the time evolution of the three components of the position vector. The ability of a spacecraft to follow this path in its own coordinate system is limited by the quality of the registration between the two.

The roll error between celestial and spacecraft systems is the hardest to control. For HST this error will be of the order of 30 arcseconds and is set by the resolution of the fixed head star trackers and the knowledge of their alignment to the spacecraft. This roll error manifests itself as a pointing error which increases proportionally to the track length. This error is of the order of 30 milliarcseconds at the end of a three arcminute length track.

For a NGST with a pointing stability requirement below a milliarcsecond, roll reference star positions would have to be known to an arcsecond or better and the star tracker would have to have a resolution of better than an arcsecond. Astrometric stars would probably be required for these roll control stars (i.e. Hipparcos stars). The nominal three arc minute track length used for HST is predicated on the width of the FGS pickoff mirrors. A telescope that is responding to true science requirements may want track lengths much longer. (Halley at closest approach was moving 3 arc minutes in 15 minutes of time.) Such longer track lengths make the roll knowledge even more crucial for moving targets.

Again, this roll problem is due in part to the inability to 'closed-loop' track on the target. If the moving target itself is used to generate an error signal, this roll issue disappears.

Traditionally a dichroic beam splitter or pellicle is inserted near the focus to divert light into a guiding device. An alternate way to be able to view a target while also performing a science observation would be to intercept some of the light directly at the prime focus of the primary while allowing the bulk of the light to trace a nominal path through the rest of the telescope optics. A camera at the prime focus could then generate the necessary error signals for the pointing control system (Figure 1).

The advantages of this approach would be that the amount of intercepted light could be controlled by geometry rather than by index of refraction changes or by partial reflections. No pickoff mirrors need to be devoted to the guidance system. The roll axis would be controlled by the fixed head star trackers, thus only one guidance camera is needed. 


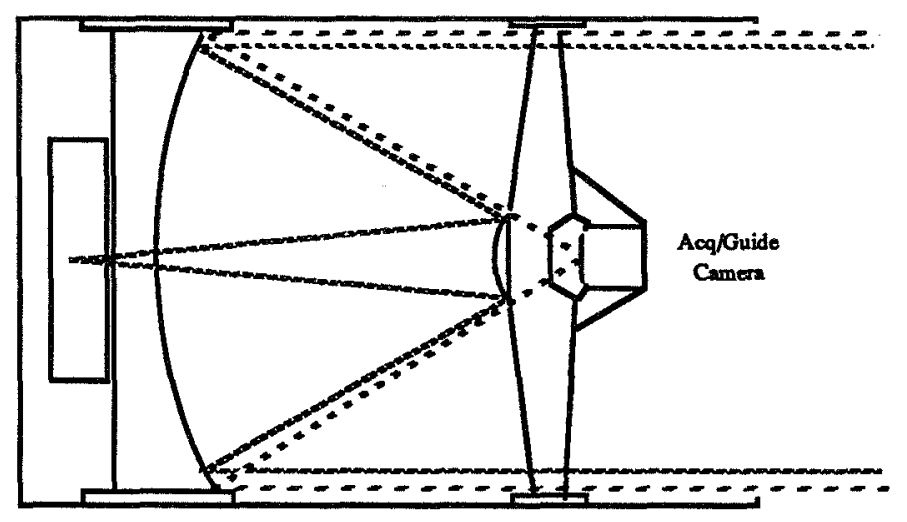

Figure 1

\section{Jitter control}

Moving mechanisms and excitable structural modes provide most of the high speed jitter. These sources include solar array movement, solar array excitation modes, reaction wheels, antenna pointing/tracking motions, and tape recorders. The isolation and control of these sources demands much attention and consumes resources. A simpler approach would be to divide the spacecraft up into a noisy platform and a quiet platform. This is only possible in a high earth orbit where gravity gradient and aerodynamic torques are ignorable.

Figure 2 schematically outlines a division of the spacecraft into two platforms. All mechanically noisy subsystems are moved into a support module. This support module houses the reaction wheels, the telecommunications antennas, the solar array, and the tape recorders. In addition, it contains quiet subsystems such as transponders, magnetic torquing coils, batteries, local gyros, and the flight computer. This platform is sun pointed with low precision (a few degrees). Segregation of these components also reduces the harness size for the spacecraft.

The optical system, with its science package, is housed in a separate platform. The two are connected by a flexible cable which contains two wires and an optical fiber. The wires carry power to the optical section and the optical fiber carries data bi-directionally. The quiet platform must have its own gyros and magnetic coils. These gyros would be fiber-optic gyros and would have fiber coils that span large segments of the body. The magnetic torquer coils would also be body-spanning.

The two bodies are coupled via magnetic fields. Each platform can generate at least a dipole field in any direction. The solar pointing platform generates a base field and the optical platform generates a torquing field to interact with the base field. The dipole created by the optical platform will be perpendicular to the base field (Figure 3). As the optical platform slews, the fields will remain fixed relative to each other, generating maximum torque.

Since a dipole in a gradient will experience a net force, the interacting fields can also be used to position the optical platform relative to the support platform. As the optical platform torques against the base field, the reaction wheels in the support platform will act to maintain the solar pointing of the support platform. Thus the slewing motion of 


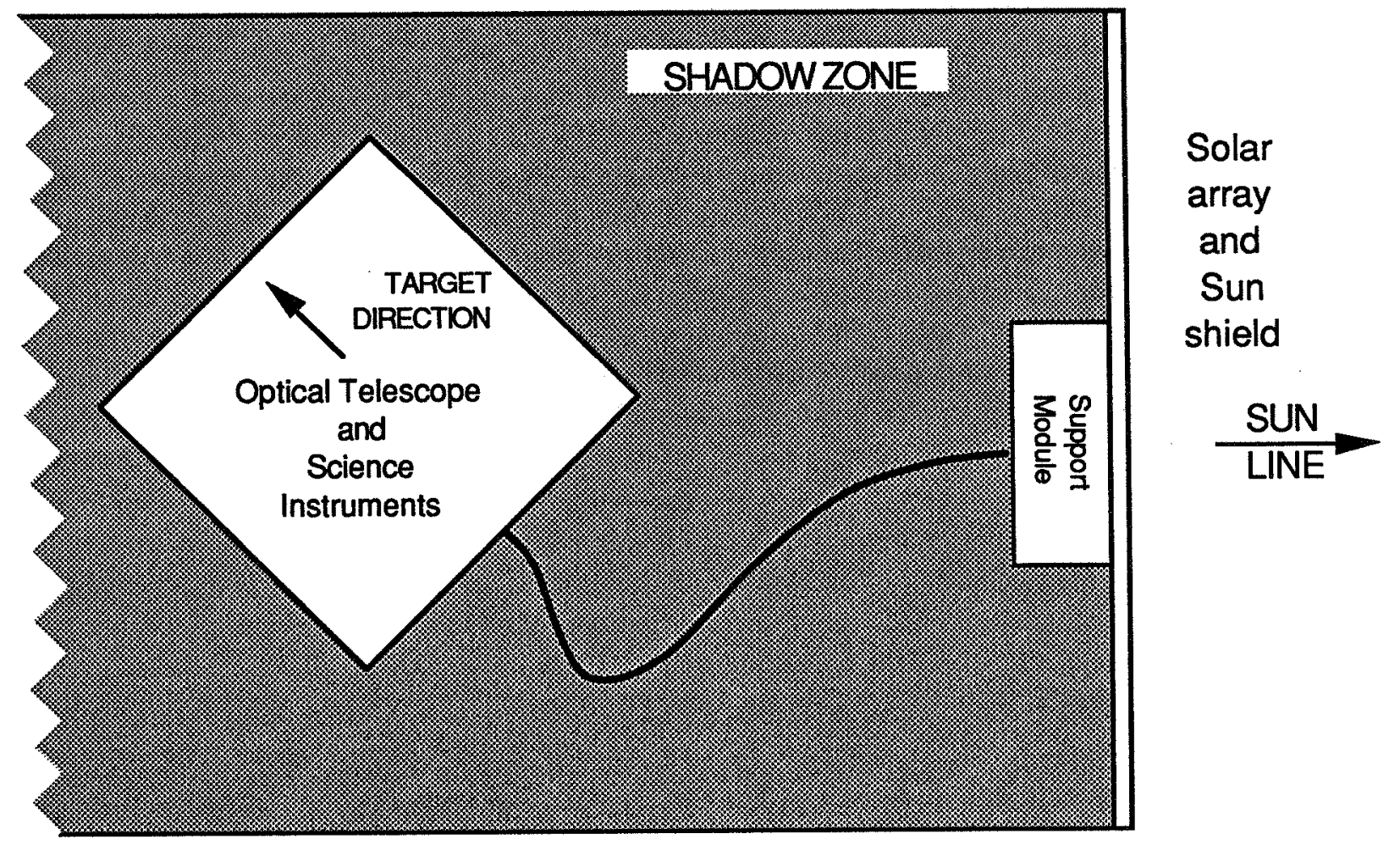

Tigure 2

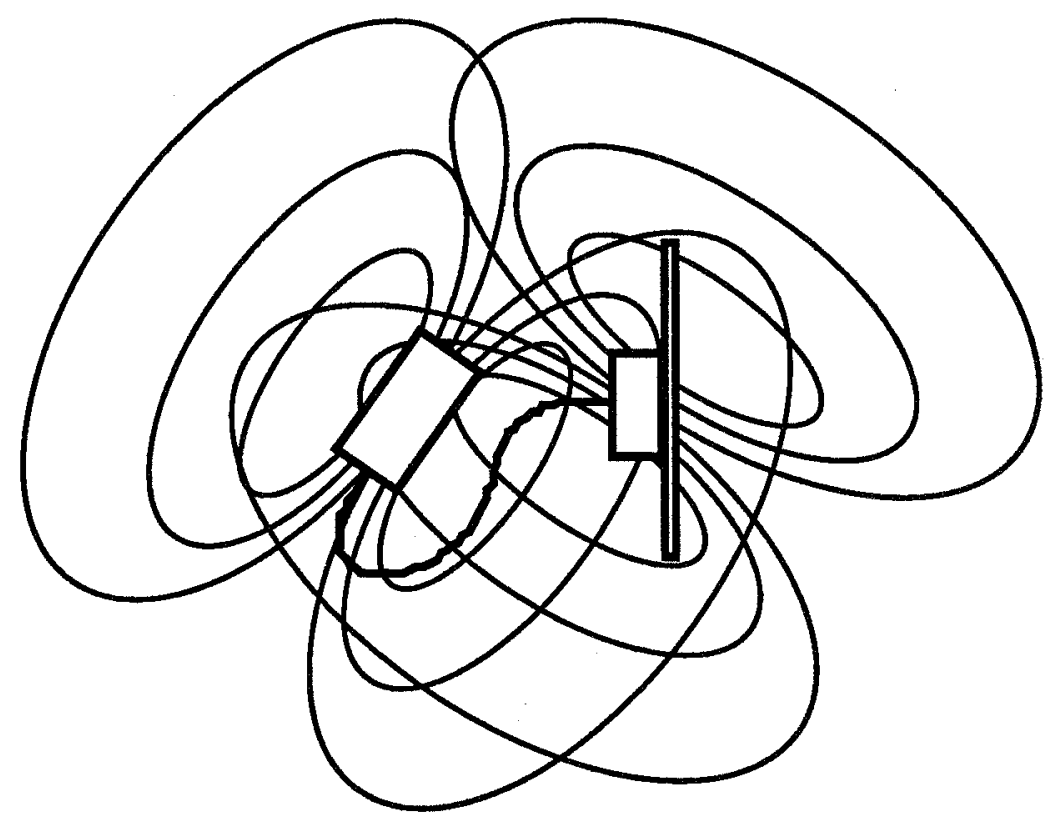

Figure 3 
the optical platform will be reflected in the increased reaction wheel speed of the support platform.

The large circular solar array/solar shield structure is designed to cast a shadow region in which the optical platform can operate. This provides a number of benefits. The first benefit is that the passive temperature of the telescope can be lowered considerably. This will lend itself to achieving lower optical surface temperatures for infrared performance and for lower ambient detector temperatures. Power from the tether will supply heat to those instrument components that prefer higher temperatures. The second benefit is that the telescope line of sight can come closer to the sun than 90 degrees. This allows access to more of the sky and allows observations over longer time windows. Inner solar system objects such as Venus and comets can also be followed closer to the Sun.

\section{Conclusion}

The primary conclusions of this paper are that closed-loop tracking of both fixed and moving targets is highly advantageous, that fixed head star trackers of high resolution are needed for roll control, that an alternate method exists for imaging the target on both the science instrument and the guidance camera, and that a dual platform offer substantial vibration isolation.

\section{DISCUSSION}

Mlingworth: David, you made an interesting point about the difficulty of doing planetary observations from the lunar poles. However, I do not think that we are likely to face that problem. As good as these sites would be for astronomical telescopes, I cannot see us getting access to these sites in our lifetimes, especially for the two telescopes needed for full sky coverage. 


\title{
Passive Cooling of a 10 meter Space Telescope
}

\author{
A Preliminary Investigation \\ Philip J. Tulkoff \\ Swales and Associates, Inc.
}

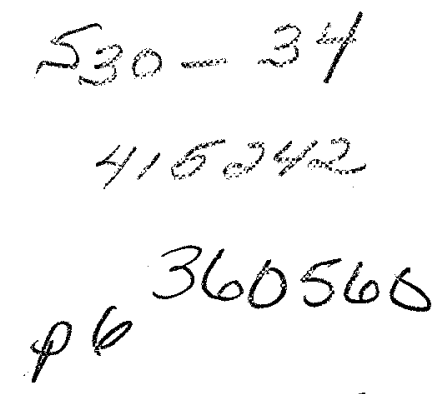

\section{Objectives}

The objective of this study was to determine to what steady-state temperature the primary mirror of a 10 meter telescope in space could be driven down to by purely passive means. Factors such as orbit, spacecraft orientation, geometric and thermal design, thermal coatings, and power requirements were all considered within this study.

\section{Assumptions}

The spacecraft structure was assumed to be approximately 12 meters in diameter and 12 meters high. The upper cylindrical section of the structure houses the primary and secondary mirrors and acts as the telescope's baffle and sun shield. The lower portion of the spacecraft called the instrument module (I/M) houses the scientific instruments and the spacecraft subsystems such as power, attitude control, and communication. Figures 1 and 2 show views of the external geometry as described above.
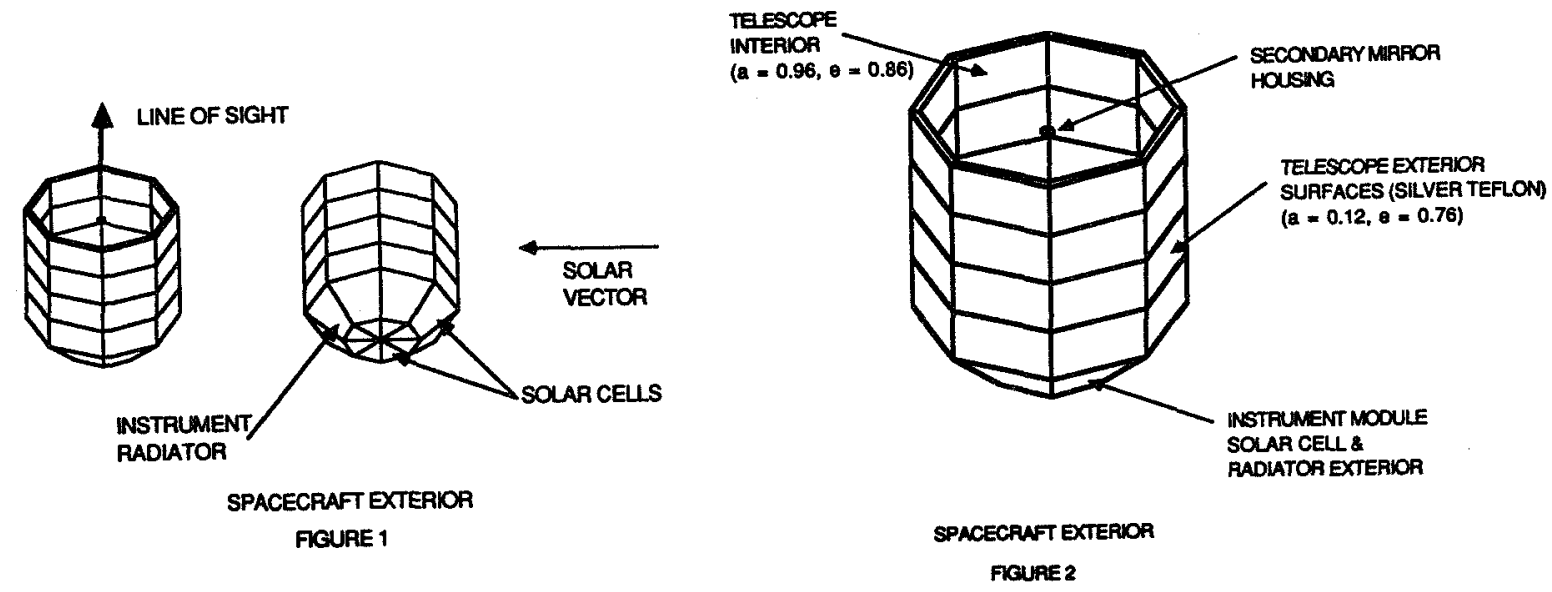

The primary mirror housed in the telescope section was assumed to be made entirely of aluminum. The face sheets of the mirror were 1 centimeter thick and the core was comprised of an aluminum foam. The active mirror surface was modeled as having a thermal emittance of 0.05 . The secondary mirror was contained in a shroud at the top of the telescope section to protect it from stray light. The mirror was assumed to be 1 foot in diameter and also had an active mirror surface with an emittance of 0.05 . The hole in the middle of the primary mirror was not modeled in order to simplify the geometric model.

The external surfaces of the telescope section were assumed to be covered with silverized teflon to provide for a low alpha/e thus reducing the absorbed solar flux on the sides of the spacecraft. All internal surfaces of the telescope section were assumed to be optically black. 
The power requirement was assumed to be 5 kilowatts and was supplied through body mounted solar cells attached to two areas of the $I / M$ to take advantage of the range of possible solar angles that the spacecraft might encounter. The other areas of the $I / M$ were used as thermal radiators to control the temperature of the instruments and spacecraft subsystems. The thermal radiators were coated with silverized teflon to provide for an efficient radiator surface as well as reduce solar inputs. Figure 3 shows a view of the bottom of the spacecraft and depicts the location of the solar cells and the radiator panels.

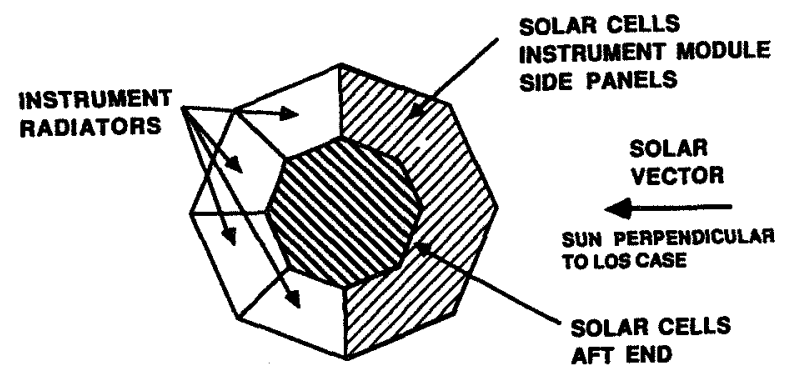

SPACECRAFT AFT VIEW

FIGURE 3

The telescope and $1 / \mathrm{M}$ sections were modeled as two separate elements. The only interaction between the elements was through a radiation coupling between the bottom of the primary mirror and the top closeout of the I/M. No conduction from these two sections was considered in order to simplify the modeling and due to the lack of structural detail. This method also allowed for comparisons between the solar flux effects on the primary mirror and the radiation heat leaks from the $\mathrm{I} / \mathrm{M}$ to the telescope.

The side panels of the telescope section were not conductively coupled to one another due to the lack of structural definition. This assumption does create the worst possible gradient between structural panels around the spacecraft's circumference allowing the temperature results to be as conservative as possible.

\section{Environmental Parameters}

All temperatures were based upon upon orbital average flux results calculated by using the Simplified Shuttle Payload Thermal Analyzer (SSPTA) software program. This allowed for steady state orbital average temperature predictions to be made. A transient temperature response would have required an estimate of the mass of various spacecraft components and structures which was not available. Three spacecraft orbits were considered in the study using spring equinox for the earth orientation. For each of the three orbits two spacecraft orientations were considered, the sun direction parallel to the line-ofsight (LOS) and the sun direction perpendicular to the LOS (Figure 4). Nominal values for solar UV, earth IR, and albedo were used for flux calculations $(429.0 \mathrm{BTU} / \mathrm{hr} \mathrm{ft} 2,75.0$ $\mathrm{BTU} / \mathrm{hr} \mathrm{ft} 2$, and 0.30 , respectively).

The three orbits considered were as follows:

- Sunsynchronous $960 \mathrm{~km}$ altitude - fully illuminated (6am - 6pm)

- Geosynchronous - $95 \%$ sun time

- High Earth Orbit (HEO) - $28^{\circ}$ inclination - $100000 \mathrm{~km}$ altitude 


\section{Thermal Model}

The spacecraft was modeled with 156 active surfaces which were combined to form 102 nodes for temperature calculations. Multi- layered insulation (MLI) was used in several areas on the spacecraft to minimize heat gain from solar inputs and warmer surrounding structures. The bottom of the primary mirror was assumed to be covered with MLI to prevent interactions with the warmer I/M located below it. The sides of the telescope were covered with MLI to prevent excessive heat inputs to the mirrors and to reduce the circumferential gradient around the structure.

Four different modeling assumptions were made for each orbit/ spacecraft orientation combination. The assumptions were as follows:

Case A: No I/M coupling to the telescope allowing for only environmental heat flux inputs to effect the mirror temperature. No MLI on the anti-sun side of the telescope to allow for maximum heat dissipation to space.

Case B: No I/M coupling to the telescope. MLI around the entire telescope circumference.

Case C: No conduction coupling from the $\mathrm{I} / \mathrm{M}$ to the telescope. Assumed that the $\mathrm{I} / \mathrm{M}$ portion of the spacecraft operated at $20 \mathrm{~F}$ and that a radiation coupling from the top of the I/M to the bottom of the telescope section existed. MLI around the entire telescope circumference.

Case D: No conduction coupling from the $I / M$ to the telescope. Assumed that the $\mathrm{I} / \mathrm{M}$ portion of the spacecraft operated at $20 \mathrm{~F}$ and that a radiation coupling from the top of the $I / M$ to the bottom of the telescope section existed.No MLI on the anti- sun side of the telescope.

\section{Results}

A series of twenty-four cases were run as a result of the three orbits, two spacecraft orientations, and the four modeling assumptions. The results are summarized in Table 1.

All cases that assumed no I/M coupling result in primary mirror temperatures that were determined strictly by environmental flux leakage through the MLI and in some cases by the combined effect of earth IR or albedo which enters the telescope aperture. The sunsynchronous orbit was warmer than all other cases considered due to its low altitude which caused higher fluxes incident on the telescope external surfaces. On the sun parallel to the LOS orientation and sunsynchronous orbit enough flux gets into the telescope to cause the primary mirror temperature to rise almost $45 \mathrm{~K}$ above the sun perpendicular case.

The geosynchronous orbit showed similar temperatures for the parallel and the perpendicular to LOS cases. A careful examination of the absorbed fluxes showed that the primary mirror temperature in the sun perpendicular to the LOS case was determined by the solar and earth IR fluxes incident on the exterior of the telescope. In the sun parallel to the LOS case, there is no solar flux and less earth IR on the telescope exterior than in the previously mentioned case. The temperature of these two cases are extremely similar because a total of 2.8 watts of earth IR flux are absorbed directly by the primary mirror in the sun parallel case and no flux is absorbed directly by the mirror in the other case. This is because in the sun parallel to the LOS case the telescope is pointing directly at the earth as it passes the 12:00 position in the orbit. 
Table 1.

\begin{tabular}{lc}
\hline Case & $\begin{array}{c}\text { Primary mirror } \\
\text { temp. }\left({ }^{\circ} \mathrm{K}\right)\end{array}$ \\
\hline
\end{tabular}

Sunsynchronous Orbit - Sun perpendicular to LOS
A. No I/M coupling, no MLI anti-sun side
143.
B. No I/M coupling, full MLI
109.
C. $20 \mathrm{~F} \mathrm{I} / \mathrm{M}$ coupling, full MLI
120.
D. 20F I/M coupling, no MLI anti-sun side
146.

\section{Sunsynchronous Orbit - Sun parallel to LOS}
A. No I/M coupling, no $\mathrm{MLI}$ anti-sun side
188.
B. No I/M coupling, full MLI
159.
C. $20 \mathrm{~F} \mathrm{I/M}$ coupling, full MLI
163.
D. $20 \mathrm{~F} \mathrm{I} / \mathrm{M}$ coupling, no MLI anti-sun side
190.

\section{Geosynchronous Orbit - Sun perpendicular to LOS}
A. No I/M coupling, no MLI anti-sun side
91.
B. No I/M coupling, full MLI
97.
C. $20 \mathrm{~F} \mathrm{I/M}$ coupling, full MLI
105.
D. 20F I/M coupling, no MLI anti-sun side
96.

\section{Geosynchronous Orbit - Sun parallel to LOS}
A. No I/M coupling, no MLI anti-sun side
93.
B. No I/M coupling, full MLI
96.
C. 20F 1/M coupling, full MLI
105.
D. 20F I/M coupling, no MLI anti-sun side
98.

\section{High-earth Orbit - Sun perpendicular to LOS}
A. No I/M coupling, no MLI anti-sun side
85.
B. No I/M coupling, full MLI
94.
C. $20 \mathrm{~F} \mathrm{I/M}$ coupling, full MLI
104.
D. 20F I/M coupling, no MLI anti-sun side
90.

\section{High-earth Orbit - Sun parallel to LOS}
A. No $I / M$ coupling, no MLI anti-sun side
N/A
B. No I/M coupling, full MLI
N/A
C. 20F I/M coupling, full MLI
78.
D. 20F I/M coupling, no MLI anti-sun side
66.
R. 20C I/M coupling, no MLI anti-sun side
73. 
Another observation that comes from the examination of the temperature results shows that the addition of the $20^{\circ} \mathrm{F} \mathrm{I} / \mathrm{M}$ sink adds $5^{\circ} \mathrm{C}$ to the primary mirror temperature in the case with no anti-sun side MLI and $8^{\circ} \mathrm{C}$ to the fully insulated case. This result is caused by the additional effective radiator area caused by having no insulation on the anti-sun side of the telescope. The fully insulated case does not allow the extra heat to dissipate as effectively.

The High Earth Orbit yields the lowest temperatures of all cases examined. The absorbed flux tables showed that no earth IR is absorbed by the spacecraft due to its extremely high altitude. The two sun parallel to the LOS cases with no $\mathrm{I} / \mathrm{M}$ coupling were not considered realistic for the following reasons. Due the the spacecraft orientation, sun parallel to LOS, zero solar flux strikes the side of the telescope and due to the high orbit no earth IR is absorbed. In addition, without any $\mathrm{I} / \mathrm{M}$ coupling there would be absolutely no heat input to the telescope section which would yield a primary mirror temperature equal to absolute zero which is unrealistic.

An examination of the sun perpendicular to the LOS case for HEO shows that case $A$ and $\mathrm{D}$ have both dropped $6 \mathrm{~K}$ as compared to the Geosynchronous orbit case due to the lack of any earth IR inputs. Case $\mathrm{B}$ has dropped only $3^{\circ} \mathrm{K}$ due to the fully insulated telescope section. Case $\mathrm{C}$ decreased only $1^{\circ} \mathrm{K}$ due to the insulated telescope section and the added heat input from the I/M.

The sun parallel to the LOS shows an even greater improvement over the comparable geosynchronous cases. This is because at HEO there is zero absorbed IR and with this orientation no solar flux strikes the telescope. The primary mirror temperature is determined strictly by the heat absorbed from the $\mathrm{I} / \mathrm{M}$ and the ability of the mirror to reradiate the heat out to the telescope walls or directly to space. The case with no MLI on the anti-sun side of the telescope is the coldest case as would be expected. For comparison purposes an additional case was run with a $20^{\circ} \mathrm{C}\left(67.4^{\circ} \mathrm{F}\right) \mathrm{I} / \mathrm{M}$ sink with no anti-sun side MLI and the sun parallel to the LOS for an HEO orbit. This last case showed a primary mirror temperature of $73^{\circ} \mathrm{K}$ as compared to $66 \mathrm{~K}$ for the same case with a $20^{\circ} \mathrm{F} \mathrm{I} / \mathrm{M}$ sink.

A summary of the results is presented in Table 1 below which outlines the highest and lowest temperatures achieved for each case. In addition, the primary concerns regarding the thermal design are pointed out. In the sun synchronous orbit, the temperature of the $\mathrm{I} / \mathrm{M}$ radiators are high due to the earth IR and albedo inputs. Of even more concern is the flux that enters the telescope baffle and becomes trapped thus raising the primary mirror temperature. In the geosynchronous orbit, some IR flux enters the telescope raising the mirror temperature. The $\mathrm{HEO}$ orbit avoids the problems of incident flux entering the telescope but is much more sensitive to heat leaks from the $I / M$ to the telescope section.

Table 2. Results Summary - Primary Mirror Temperatures $\left({ }^{\circ} \mathbf{K}\right)$

\begin{tabular}{lccc}
\hline \hline Orbit & Highest temp. & Lowest temp. & Concerns \\
\hline Sun-synchronous & 190 & 109 & Radiator temperature \\
Geosynchronous & 105 & 91 & Earth IR in telescope \\
High-earth orbit & 104 & 66 & Heat leaks to mirror \\
\hline
\end{tabular}




\section{Conclusions}

This preliminary investigation indicates that a mirror temperature in the 70 to $100^{\circ} \mathrm{K}$ range appears attainable through purely passive cooling. Orbit selection is a major factor. If a temperature approaching $70^{\circ} \mathrm{K}$ is desirable for scientific reasons, low earth orbits which are strongly affected by earth radiation must be avoided and a geosynchronous or high earth orbit is to be preferred. An additional advantage of the high earth orbit is that the earth flux input on radiators is so negligible that the constraint on spacecraft orientation disappears.

This study also indicates, however, that the mirror temperature is fairly sensitive to heat leaks from the spacecraft power dissipating components. The Instrument Module should be designed to run as cold as possible, and extreme care should be exercised in the mechanical/thermal design in order to minimize heat leaks.

An attempt should be made to balance the heat input to the mirror resulting from external fluxes and internal heat dissipating components regardless of spacecraft orientation. This would eliminate primary mirror temperature drifts as the spacecraft moves from one target to the next.

\section{DISCUSSION}

Bender: Can you keep the absorbtivity as low as 0.12 over 15 years?

Tulkof: This study did not address the questions concerning coatings in any great depth. It is doubtful that the coatings absorbtivity would remain as low as 0.12 over 15 years which must be taken into account when a more comprehensive thermal design is done. The question of thermal coating stability is currently being addressed for the Polar Orbiting Platform which is being designed at the Goddard Space Flight Center. Another possible source of information will be the examination of the coating on the LDEF experiment which is due to be retrieved by the Shuttle early in 1990.

Schember: Can your discuss the specifics of your solar panel modelling, especially optical properties, $\mathrm{W} / \mathrm{m}^{2}$ at the beginning and end of life, and efficiencies? Also, what is the average solar panel temperature and the heat leak from the solar panel to the instruments?

Tulkoff A detailed look at the solar panel was not conducted in this study since they would have only a secondary effect on the primary mirror temperature. The solar panels would primarly affect the $1 / M$ temperature. Optical properties used in the study were $\alpha=0.82$ and $e=0.78$ which are nominal properties. A more comprehensive study would include BOL and EOL properties in any analyses. The solar cell efficiency was assumed to be approximately $10 \%$ and the total area available for mounting solar cells was $775 \mathrm{ft}^{2}$. The incident solar flux was assumed to be $420 \mathrm{BTU} / \mathrm{hr} \mathrm{ft}^{2}$. The structural design of the I/M can be used to optimize the external configuration of the solar cells. The exact angle of the I/M side (the truncated cone shape) can be used to best place and orient the solar cells. In the sun perpendicular to the LOS case the output of the solar panels at $10 \%$ efficiency was approximately 2600 Watts at temperatures from 17 to $88 \mathrm{C}$. However, this was below the desired $5 \mathrm{~kW}$. In the case where the sun vector is parallel to the LOS, the cells produced $5950 \mathrm{~W}$ with cell temperatures ranging from 88 to $123 \mathrm{C}$. 


\section{CRYOGENICS FOR SPACE OBSERVATORIES: TECHNOLOGY, REQUIRENENTS, ISSUES}

Helene Schember, Ph.D. Jet Propulsion Laboratory California Institute of Technology Pasadena, CA 91109

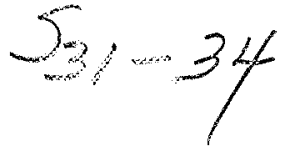

415243

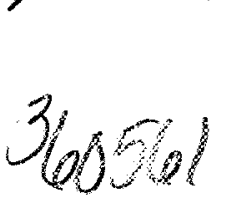

\section{Summary}

The ability to provide cooling at cryogenic temperatures is a critical enabling technology for many of the next generation of space-based observatories and instruments. This report discusses the state of cryogenics technology and identifies and categorizes the various methods of cooling.

\section{Nomenclature and Acronyms}

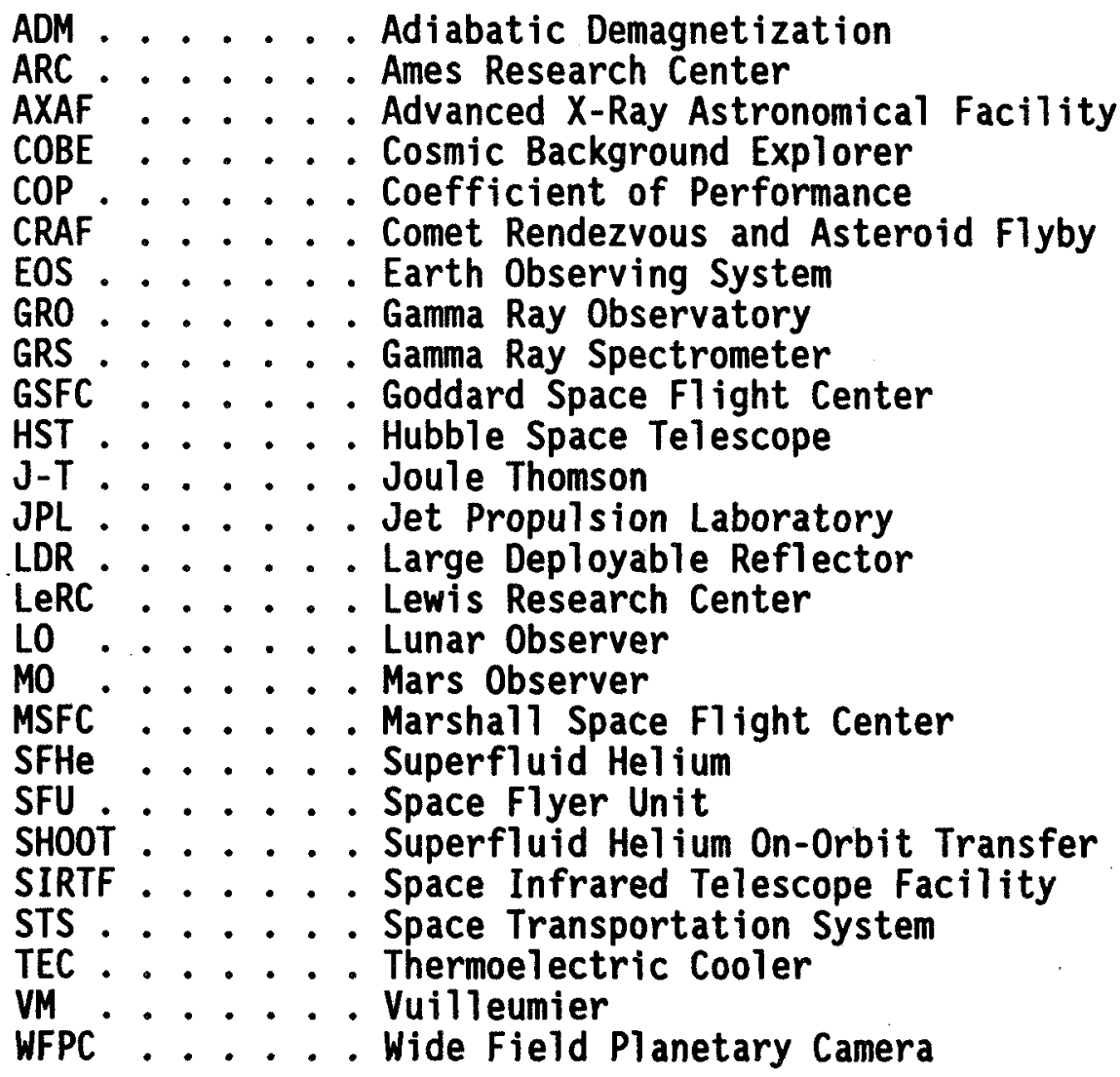




\section{Background: Spacecraft Cryogenics Experience}

The planning, analysis, design and testing of spacecraft cryogenic systems fall within the charter of spacecraft thermal control engineers. The range of temperatures included in the cryogenics regime is somewhat flexible, sometimes considered to begin as high as $200 \mathrm{Kelvin}$, but usually taken to be $150 \mathrm{~K}$ and below. A number of spacecraft and instruments have operational temperatures in the cryogenic range. Table 1 lists a sampling of spacecraft and instruments requiring cooling to cryogenic temperatures. A number of projects are presently under active study, design, test and/or analys is in the cryogenics area, including: Mars Observer, Lunar Observer, SIRTF, EOS, LDR, AXAF, EOS and others. In addition, a great variety of research and development of enabling technology in cryogenics is ongoing at NASA, including work on transfer of superfluid Helium (GSFC), dilution refrigeration (GSFC, ARC, JPL), cryogenic propellant transfer (LeRC), pulse tube refrigerators (ARC), adaptation of mechanical refrigeration to instruments (JPL), sorption refrigeration (JPL), magnetic refrigeration (GSFC, JPL, ARC), cryogenic heat pipes (GSFC) and long lifetime cryogenic dewars (ARC, JPL, MSFC).

\section{Cryogenics Technologies}

\section{Overview}

The technology used to provide cooling at cryogenic temperatures may be divided into four fairly distinct categories. These cooler categories are: thermoelectric, radiative, open cycle, and closed cycle refrigeration. As a generality, the categories are 1 isted in order of decreasing state of maturity (or flight readiness) and, perhaps not coincidentally, in order of decreasing minimum achievable temperature. There are, naturally, some exceptions to this rule of thumb.

It is important to recognize that thermal control at cold temperatures requires attention to both the temperature and the heat load (or capacity) at that temperature. These two critical pieces of information along with the orbit and spacecraft attitude lay the groundwork for the selection of the best method for cooling a particular device. One Watt of cooling at $20 \mathrm{~K}$ is much harder to achieve than one Watt at $200 \mathrm{~K}$. Coolers also require greater input power to produce the same cooling capacity at lower temperature. Other technical or systems considerations, such as allowable temperature cycling, instrument duty cycles, sensitivity to vibration, cooldown time, contamination environment and lifetime (operating and shelf), play a role in cooler selection. The heat load on a cooler is considered to be made up of three parts shown schematically in Figure 1. The first part, usually referred to simply as the "heat load from the detector" (or sometimes the instrument heat load) is the heat which enters through the device and which must be removed to maintain temperature. This heat load comes from the actual signal to the device, the device dissipation and any parasitic heat load carried through the detector. The second component of total heat load is called the parasitic heat load or the parasitics, and is the radiative and conductive heat leak from the warm environment (i.e., spacecraft) around the device. The final contribution to the total heat load is the environmental heat load, which consists of the heat load from the Sun, and, if applicable, from planetary thermal emission and solar reflection. Radiative heat loads from distant parts of the 
spacecraft may be included in this category.

\section{Thermoelectric Coolers}

Thermoelectric coolers or TECs are small, lightweight and completely vibrationless solid-state devices which provide refrigeration by using the Peltier effect (the reverse effect is called Seebeck). These devices require a source of electrical power to provide cooling. They are sometimes described as reverse or powered thermocouples, because an electric current produces a heat flow from the cold face to the hot face of the TEC. The current status of these devices is mature, in that they have a long history of successful application. At the present time, there are no significant developments underway in TECs, other than periodic improvements in materials.

TECs are sometimes not considered to be part of the cryogenics toolbox, because they are limited to the very high end of the temperature region. They operate most efficiently at temperatures above $200 \mathrm{~K}$, and possibly as low as $170 \mathrm{~K}$. Below this temperature the efficiency, even for an optimized, staged device drops so low that no heat load can be supported at the cold face. TECs are generally cascaded: Marlow Industries makes a popular 6-stage device for cooling to temperatures below $200 \mathrm{~K}$. In staging, the hot face of the lower stage is in close thermal contact with the cold face of the upper stage, and so on. This makes it possible for the TEC to provide a greater temperature differential from the hot side to the cold side. It must be noted that the hot face of the TEC will be fairly close to the cold face and provision must be made to conduct or radiate the heat away from this face. The heat at the final hot face may be a large quantity and is equal to the heat load at the cold face divided by the overall TEC efficiency.

Past experience with TECs dictates close attention to thermal expansion and interfacing of the device. The TECs on the HST Wide Field Planetary Camera, carrying $60 \mathrm{~mW}$ at $178 \mathrm{~K}$, had specially designed flexible silver straps to

accommodate movement during thermal cycling. Petrick (1987) has described the selection and interfacing of TECs to detectors.

\section{Radiative Coolers}

Radiators or radiative coolers are completely passive and vibrationless. Heat from a source is passed to the radiator which in turn radiates to space. High emissivity surfaces $(\epsilon \geq 0.94)$ are generally required on cryogenic radiators, in order to bring them to very low temperatures. Depending on the heat load and environment, passive radiators can reach temperatures as low as $75 \mathrm{~K}$ in practice. Missions to outer planets or small cold bodies where the environmental heat loads are very low can support even lower temperatures.

Although radiator technology is quite mature, there are developmental tasks currently underway. Because a radiator is so sensitive to its environment, varying mission requirements and scenarios require individual design and analysis. Sun-synchronous orbits are preferred, in general, although they are not always possible, as in the case of lunar orbit. The heat loading from the environment must be minimized along with that due to parasitics. A number of very clever and imaginative radiator designs have emerged in the last 10 years 
which successfully reduce the parasitic and environmental loading, allowing lower temperatures and higher capacities. These designs make use of multistaging, special low conductivity supports and highly specular surfaces to reduce the conductive and radiative loads on the coldest stage due to parasitic heat leaks and environment. Specular surfaces are extremely reflective and are usually made from highly polished metal for which reflects incoming thermal radiation at same angle as the angle of incidence. (Nonspecular surfaces exhibit diffuse reflection in which incoming rays of thermal radiation are reflected hemispherically following a cosine law.) Specular surfaces permit the designer to strictly limit the environmental heat loads reaching the highly emissive (and thus highly absorptive) cold radiator surface. Furthermore, specular surfaces allow radiative parasitic heat leaks to be directed away from the radiator, as best exemplified by the V-groove radiator concept. The drawback is that these surfaces must be kept very clean -- contamination which diminishes the reflectivity or specularity could be disastrous.

Since the capacity of the radiator is directly proportional to its area, and proportional to the difference between the fourth powers of the radiator and the surrounding cold sink temperatures, cryogenic radiators can be quite bulky and heavy. The placement of the radiator can severely limit the allowable spacecraft attitude. These issues may mean that although a radiator may be a possible solution, it may not be a practical one.

\section{Open Cycle Coolers}

Open cycle coolers are moderately complex systems which expend a fluid to provide cooling. The fluid is not recaptured for reuse, although it may be channelled during its rejection in order to reduce parasitic heat loads. There is a great deal of developmental work currently being performed in the area of open cycle coolers. There are three subclasses of open cycle coolers: First, the liquid storage or dewar type, in which either the heat capacity of the fluid or the latent heat of vaporization is used to absorb the heat load. The second type is fluid storage with Joule-Thomson $(\mathrm{J}-\mathrm{T})$ expansion. In this method the fluid is stored at high pressure and then throttled through a J-T expansion valve to a lower pressure; cooling is produced through the expansion process. The fluid must be below its inversion temperature or the throttling will not produce cooling. Most fluids have reasonably high inversion temperatures, however there are some exceptions such as Helium $(40 \mathrm{~K})$ and Hydrogen $(204 \mathrm{~K})$. The third method, solid cryogen storage, relies on the latent heat of sublimation to maintain temperature. Due to the difficulty of managing cryogenic liquids, only systems employing solid cryogens, superfluid He and compressed gasses have been used on spacecraft.

Open cycle systems are inherently lifetime limited; once the fluid has been expended, cooling ceases and the assembly assumes its equilibrium temperature. Once the heat loads and temperatures have been established, open cycle coolers may be considered from two vantage points: Either a lifetime requirement is imposed, in which case the quantity of cryogen required may be calculated, or the quantity of cryogen is specified (by specifying an allowable volume or mass), and theil a lifetime may be calculated. Refill systems have been proposed to extend the lifetime of cryogenic dewars, however, significant development and testing are required in this area. The implementation of a 
refill or replacement system is the only method available to extend the lifetime of an open cycle. In the absence of a resupply mechanism, most designers attempt to achieve a minimum heat load to provide the longest lifetime possible. IRAS operated for 10 months; SIRTF 1 ifetime is estimated at 6 years.

The temperature range for open cycle systems is extremely large ranging down to below $2 \mathrm{~K}$. (Helium is used for the very lowest temperatures.) Figure 2 shows operating temperatures for a sampling of typical expendable fluids. Only single phase cooling can occur above the critical point. A useful limit on the low temperature is given based on sublimation of the solid cryogen. Saturation conditions dictate this lower limit because the cryogen is in equilibrium with its vapor. To maintain a constant temperature, the pressure must remain constant, thus the vapor produced by sublimation must be vented, with 0.1 Torr being the practical lower pressure limit which is possible given current vent valve capabilities. The lower temperatures shown in Figure 2 correspond to the saturation temperature at this low pressure.

Systems relying on J-T expansion may experience serious reliability problems due to clogging of the valve. Contaminants tend to freeze out on the small orifice used as the J-T. Other methods have been developed to avoid the clogging problems with only moderate success, among them capillary tubes and porous plugs. Vibration is only a minor issue in open cycle systems, as valves are the only source of acoustic noise. This vibration can essentially be eliminated through proper design.

There have been a number of open cycle systems flown and many are currently in the planning stages. An important consideration to support these systems is the expended fluid, which may itself be a source of contamination to sensitive surfaces on the spacecraft. In addition, for reasonable lifetime, these systems can be very large and bulky. A major component of the spacecraft total mass will likely be the cooling system. Finally, these systems are sensitive to the heat loading -- a small change in heat load (say milliwatts) may drastically change the sizing/lifetime. As an example of this, Figure 3 displays the lifetime sensitivity of the LDR Helium dewar to heat load variations.

\section{v. Closed Cycle Coolers}

There is an extremely large variety of closed cycle coolers for use at cryogenic temperatures. The status of most of these coolers for use on spacecraft must be considered developmental, but there is vigorous activity in this area. The "coefficient of performance or COP" is often used as a reference when considering the performance of a closed cycle cooler. The cop of a refrigerator is defined as the ratio:

$$
\text { COP = Refrigerator capacity/Input power }
$$

The Carnot COP is the upper limit (ideal case) for any refrigerator at the given cold $\left(T_{c}\right.$ ) and hot $\left(T_{h}\right)$ temperatures:

$$
C O P=T_{c} /\left(T_{h}-T_{c}\right)
$$

Refrigerator efficiencies are given as the ratio of the actual refrigerator COP to the Carnot COP, either as a fraction or a percent. The efficiencies of cryogenic refrigerators range from less than one percent to about 60 percent. 
The Walker classification scheme separates all refrigerators into two classes: Recuperative and Regenerative. A Joule-Thomson closed cycle cooler is a prime example of a recuperative type refrigerator. In the $\mathrm{J}-\mathrm{T}$ type refrigerator, cooling is provided as in the open cycle J-T, but now the low pressure fluid is collected on the down side of the throttling valve, repressurized and returned placed back in the high pressure line. The term recuperative comes about because the cold fluid on the low pressure side is allowed to exchange heat with the incoming high pressure fluid through the adjoining walls of the tubing thus recuperating the heat. These systems have the same weaknesses as the open cycle J-T systems. A critical element in a closed J-T system is the compressor which provides the high pressure gas for expansion. There have been a number of problems resulting from contamination by, or leakage through, the seals of mechanical compressors. Certain types of coolers do not use mechanical means to provide compression for the refrigeration cycle. The systems, the foremost of which is the sorption refrigerator, use other, nonmechanical compression techniques, such as heating, or physical or chemical adsorption. These refrigerators are still developmental, but show strong promise for long lifetime, quiet cooling.

A number of mechanical coolers employing the Stirling cycle have recently emerged and are rapidly being assessed by the spacecraft thermal community. Stirling cycle coolers are examples of the regenerative class of refrigerators. A regenerator is a heat exchanger made of a porous matrix which acts as a type of thermodynamic sponge. Hot and cold fluids flow through the matrix in a cyclic succession, either passing their heat to the matrix or absorbing heat from it. The Stirling, Vuilleumier (VM) and GiffordMcMahon refrigerators all employ some type of regenerative heat exchange scheme. The Stirling refrigerator employs one or more pistons to achieve the pressure variation which drives the cooling. In a VM refrigerator, there is no piston but simply a displacer moving the fluid from a hot space to a cold space cyclicly. Gifford-McMahon refrigerators differ from Stirling and VM machines in that they use valves to control the flow of the working fluid. All rely on mechanical compression with heat exchange and expansion to produce cooling. Systems which use mechanical compression are subject to vibration. The piston of several popular Stirling type coolers operates at near $40 \mathrm{~Hz}$, a range which may affect the optics or sensor. Since these coolers have moving parts, lifetime is also an issue. Several new coolers which have the potential to provide substantial cooling near $77 \mathrm{~K}$ claim long 1 ifetime due to innovative design and special care in manufacture. Currently available, commercial coolers, however, have predicted lifetimes of only a few months. A number of programs are currently underway to raise the maturity level of a family of Stirling-cycle-based cryocoolers. JPL and GSFC, among other NASA centers have proposed tasks specifically aimed at testing, characterizing and integrating the latest generation of coolers from such sources as British Aerospace, Phillips/Magnavox, Lockheed, Lucas, Hughes and others.

Pulse tube, or thermoacoustic refrigerators, are in an active stage of development. These refrigerators are closely related to Stirling machines and require only one moving part, viz., the (room temperature) oscillating piston or diaphragm. Refrigeration occurs within a tube connected to the pressure wave generator when the thermal relaxation time between the gas and the tube is comparable to a half period. Low temperatures of approximately $60 \mathrm{~K}$ have been reported. Although still early in their development, they have the potential for long lifetime operation. 
The efficiency of regenerators drops off with decreasing temperature, and regenerative coolers are unable to operate much below $15 \mathrm{~K}$. Extremely low temperatures (below $10 \mathrm{~K}$ ) can be reached using other techniques currently being researched. All refrigerators rely on a cycle that makes use of entropy variations as a function of temperature and some other variable, such as magnetic field; electric field, chemical potential, concentration or surface tension. (In a conventional refrigerator this second variable is pressure). Extremely low temperature refrigerators use some novel means to provide this other variable. Among these are the dilution refrigerator, various kind of magnetic refrigerators relying on the magnetocaloric effect, and the He-III evaporation refrigerator. All of these refrigeration schemes rely critically on the ability to provide a very cold operational environment, which means that some other more conventional cooler is used as an upper stage to provide cooling to, say, 10 or $20 \mathrm{~K}$. Adiabatic demagnetization refrigerators are currently basedi ined for SIRTF and AXAF to provide the extremely low temperatures $(<1 \mathrm{~K})$ needed by the detectors.

\section{Application Issues - Sensors and Detectors}

Detectors and sensors are cooled to reduce the flux of stray radiation and thus improve signal quality. In general the cooling requirements of various detectors may be summarized as:

\section{Detector Type}

IR Detectors

Cooled Optics

Sub mm SIS mixers

Far IR and sub mm detectors

Bolometers
Temperature range (K)

10 to $100 \mathrm{~K}$

20 to $200 \mathrm{~K}$

1 to $20 \mathrm{~K}$

1 to $8 \mathrm{~K}$

0.1 to $2 \mathrm{~K}$
Heat Load (W)

$10^{-1}$ to $10^{-3}$

varies, $\sim 10^{-3}$

$10^{-2}$

$10^{-3}$ and below

$10^{-6}$

A representative plot showing cooling temperature versus power for various detectors is shown in Figure 4. Also shown on the figure are diagonal lines representing the input power required for a Carnot (ideal) refrigerator operating at the given temperature and heat load. 


\section{References}

Gasser, M. (ed.), "Refrigeration for Cryogenic Sensors," Proceeding of the Second Biennial Conference on Refrigeration for Cryogenic Sensors and Electronic Systems, NASA Conference Publication 2287, December, 1982.

Hudson, R.D., Infrared System Engineering, Wiley, New York, NY, 1969.

Petrick, S.W., "General Approach to Cooling Charge Couple Devices Using Thermoelectric Coolers," Optical Engineering, 1987.

Rapp, D., "Sensor Cooling Handbook," JPL D-6483, June, 1989.

Walker, G., Cryocoolers, Plenum Press, New York, NY, 1983.

Wolfe, W.L and Zissis, G.J. (eds.), The Infrared Handbook revised edition, Environmental Research Institute of Michigan, Ann Arbor, MI, 1989. 


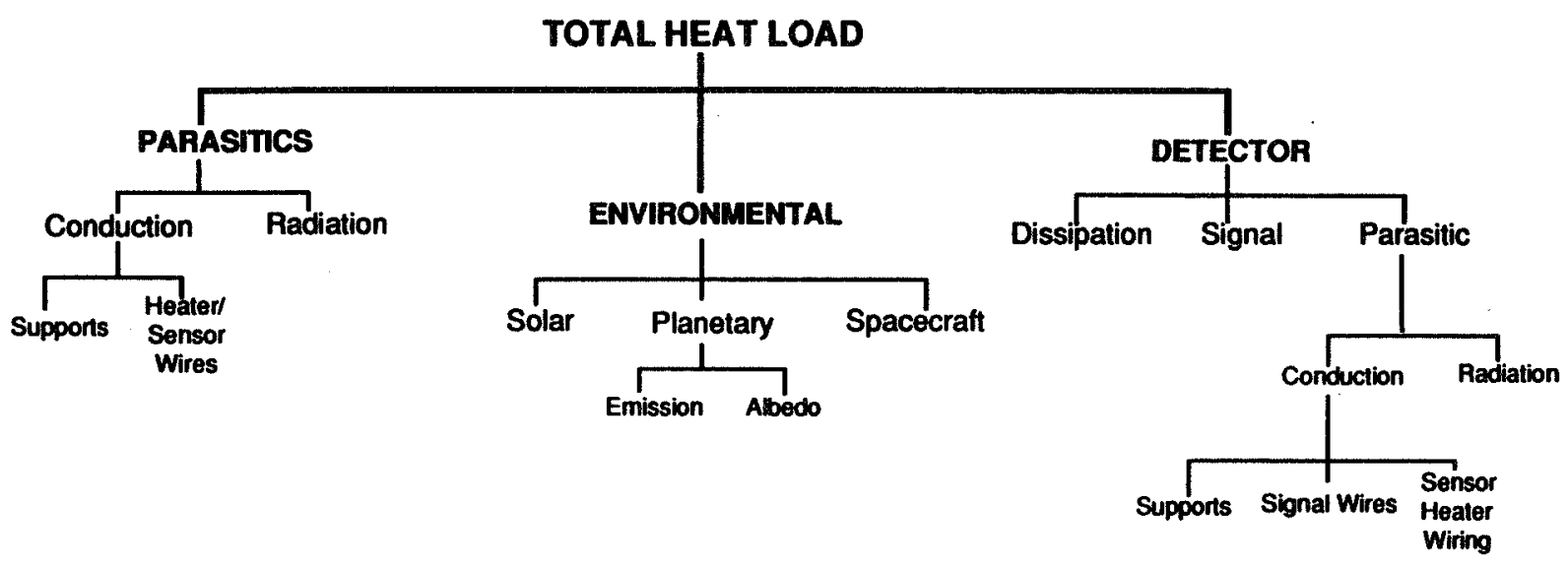

Figure 1. Breakdown of heat loads for spacecraft cryogenic cooling.

$\begin{array}{ccccccc}\text { Spacecraft } & \text { Instrument } & \begin{array}{c}\text { Temperature } \\ (K)\end{array} & \begin{array}{c}\text { Required } \\ \text { Lifetime }\end{array} & \begin{array}{c}\text { Heat Load } \\ \text { (miwatts) }\end{array} & \text { Type } & \text { Remarks } \\ \text { Voyager } & \text { IRIS } & 200 & 5 \text { years } & 500 & \text { Radiator } & \text { Flown } \\ \text { HEAO-C } & \text { GRS } & 92 & 8 \text { months } & 50 & \text { Solid Cryogen } & \text { Flown } \\ \text { Galileo } & \text { NIMS } & 75 & >3 \text { years } & 50 & \text { Radiator } & \text { In Flight } \\ \text { STS } & \text { ATMOS } & 75 & 2 \text { weeks } & 500 & \text { Stirling Refrig } & \text { Flown } \\ \text { STS/Spacelab } 2 & \text { SFHE } & 1.5 & 1 \text { week } & - & \text { Liquid Cryogen } & \text { Flown, SFHe } \\ \text { IRAS } & - & 2 & 1 \text { year } & 80 & \text { Liquid Cryogen } & \text { Flown, SFHe } \\ \text { COBE } & - & -2 & 18 \text { months } & 80 & \text { Liquid Cryogen } & \text { In Flight } \\ \text { HST } & \text { WFPC } & 178 & 3 \text { years } & 60 & \text { TEC } & \text { Built } \\ \text { STS } & \text { SHOOT } & <2 & 1 \text { week } & - & \text { SFHe transfer Demo } & \text { Designed } \\ \text { AXAF } & - & 0.1 & \text { N years } & & \text { ADN Refrigerator } & \text { Designed } \\ \text { GRO } & - & >200 & & & \text { Standard thermal } & \text { Designed } \\ \text { STS } & \text { SISEX } & 120 & 2 \text { weeks } & 1000 & \text { Stirling Refrig } & \text { Designed } \\ \text { MO } & \text { GRS } & 100 & 3 \text { years } & 200 & \text { V-Groove } & \text { Designed }\end{array}$

Table 1. Examples of spacecraft and instruments employing cryogenics technology 


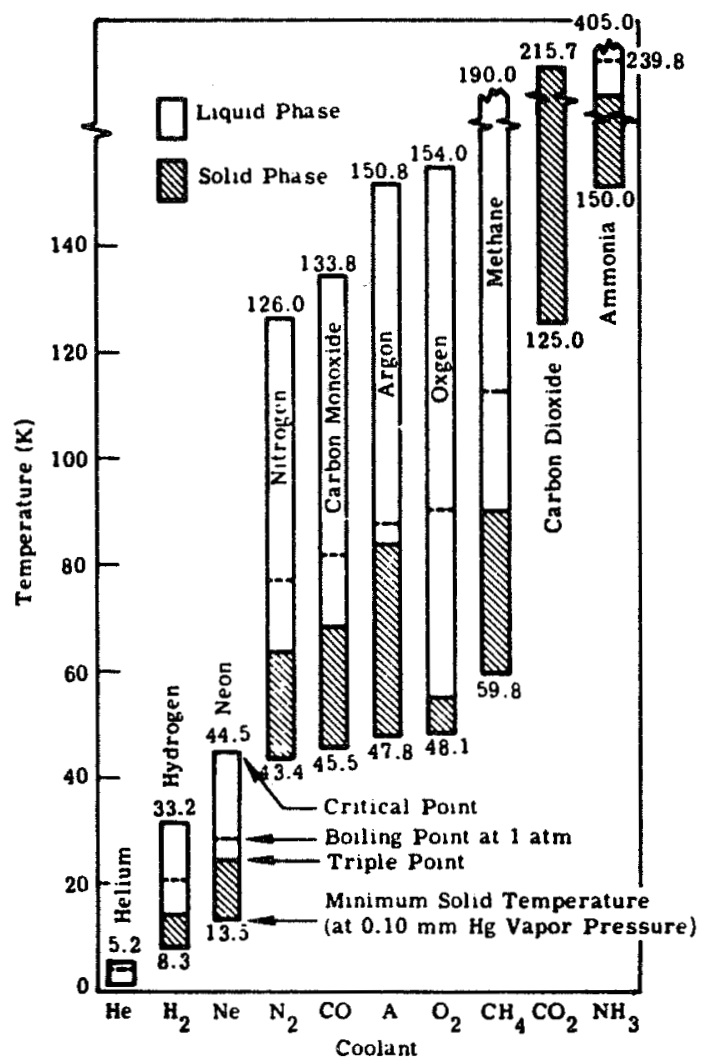

Figure 2. Operating temperature range of selected expendable coolants. (from Donabedian, 1989)

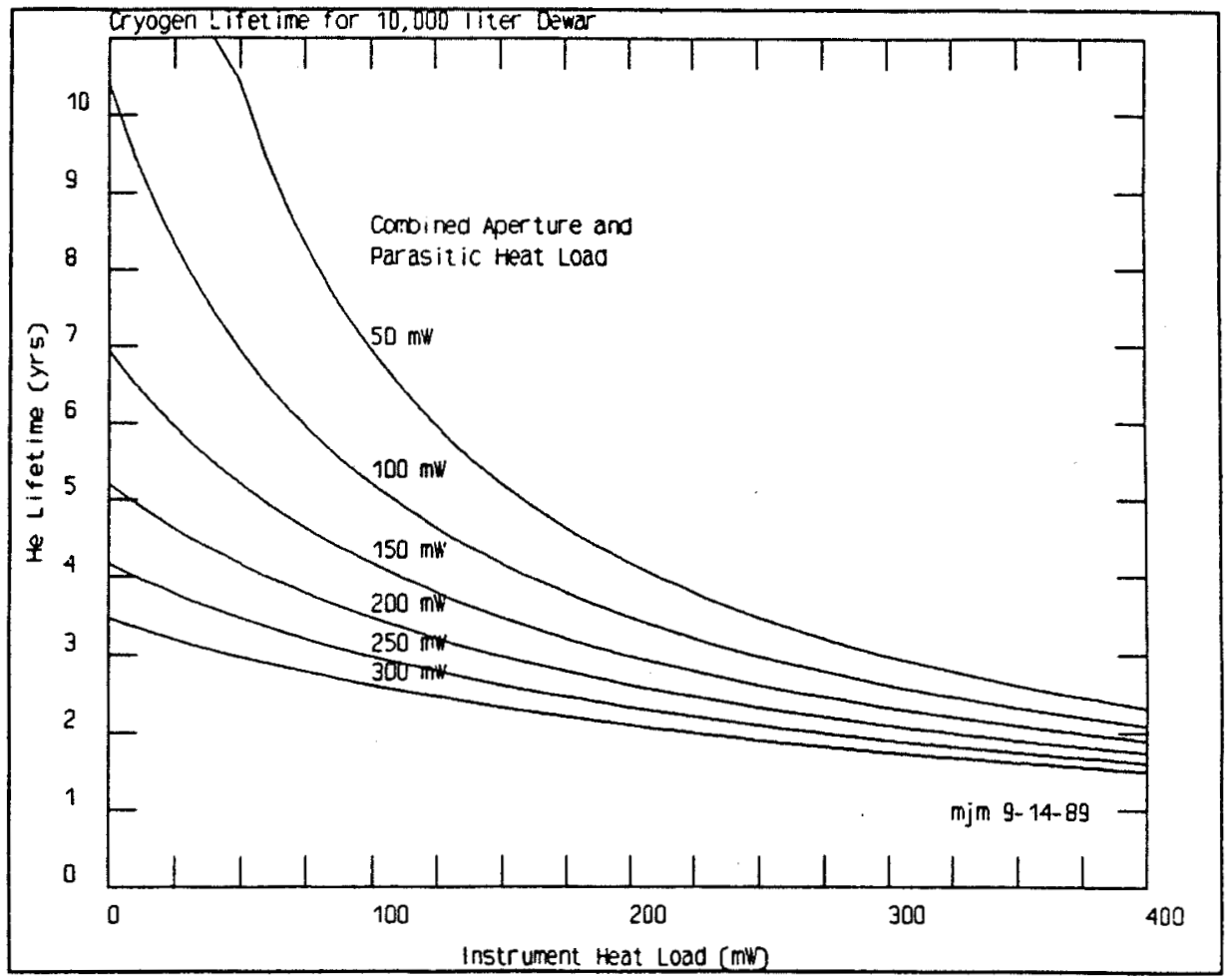

Figure 3. Variation of cryogen lifetime for a 10,000 dewar of SFHe as a function of heat load for LOR. (courtesy M.J. Mahoney, JPL) 


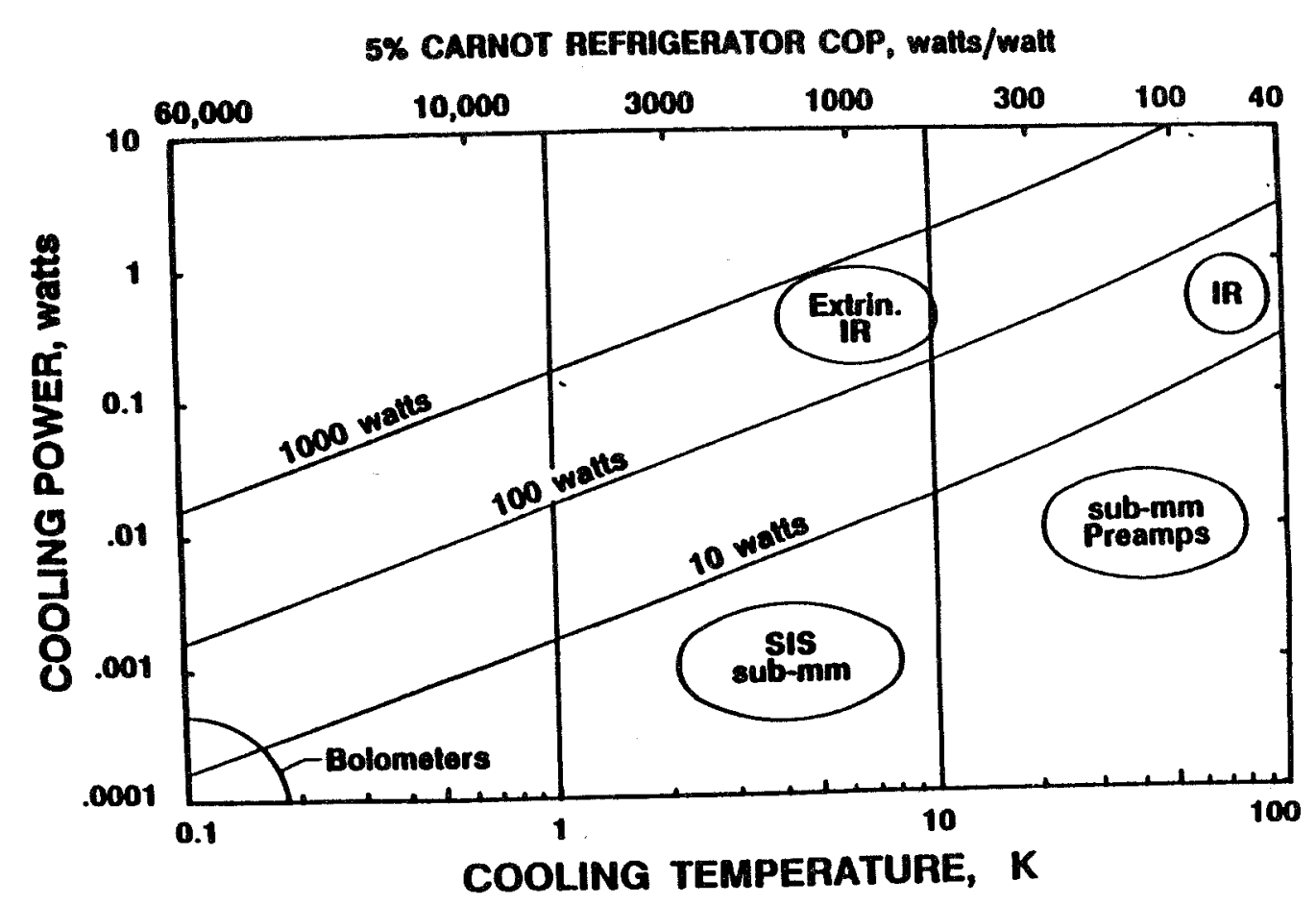

Figure 4. Detector Cooling Requirements versus Refrigerator Temperature and Performance.

(Figure courtesy of R. Ross, JPL)

DISCUSSION

Stockman: Since most spacecraft and instrument designers are conservative, we do not have much experience with closed-cycle coolers. Do you know of upcoming missions which will provide useful lifetime and performance data?

Schember: EOS will have Stirling coolers. Many other spacecraft uses are proposed, as is a full complement of tests. (Funding for a series of tests on various coolers is limited and sporadic). The lifetime will probably not be as long as one might be interested in for these telescopes (5 years?).

Disenhardt: The Oxford Stirling Cooler has been run on the ground for long periods, but apparently with some problems.

Schember: The Oxford Stirling Cooler (single unit) has been on life test for approximately 3 years. It has run continuously, except for power outages, electrical problems, etc. It is not the production model British Aerospace cooler, however.

Thompson: It will be useful for this afternoon's panel to discuss cryogenics and cooling as a discriminator between lunar telescopes and free flyers.

Schember: I agree with this remark. 


\begin{abstract}
S)
$332-89$

Visible and Ultraviolet Detectors for High Earth Orbit and Lunar Observatories

Bruce E. Woodgate

NASA/Goddard Space Flight Center

Abstract

The current status of detectors for the visible and UV for future large observatories in earth orbit and the moon is briefly reviewed. For the visible, CCDs have the highest quantum efficiency, but are subject to contamination of the data by cosmic ray hits. For the moon, the level of hits can be brought down to that at the earth's surface by shielding below about 20 meters of rock. For high earth orbits above the geomagnetic shield, CCDs might be able to be used by combining many short exposures and vetoing the cosmic ray hits, otherwise photoemissive detectors will be necessary. For the UV, photoemissive detectors will be necessary to reject the visible; to use CCDs would require the development of UVefficient filters which reject the visible by many orders of magnitude. Development of higher count rate capability would be desirable for photoemissive detectors.
\end{abstract}

\title{
Charge Coupled Devices (CCDs)
}

\section{Current status of CCDs}

The very high quantum efficiency (QE) of CCDs in the visible and near IR makes them the detector of choice in most ground-based astronomical observations. For example, they were used in over $95 \%$ of observations at Kitt Peak last year.

The largest format CCD built to date is the Ford $4096 \times 4096$ with 7.5 micron pixels (Janesick, 1989b,d). Tektronix have built the largest physical size CCD, the 2048x2048 with 27 micron pixels (Blouke et. al., 1989). At least three manufacturers have built 2048x2048 CCDB, and at least five build 1024x1024 CCDs. Some of these have been made 3-side buttable, allowing 2-row mosaics to be built to increase further the effective format.

Pixel sizes range from 6.5 to 52 microns.

Read noise levels have been achieved in the laboratory of 1.5 to 4 electrons rms, using single sampling speeds of 6-20 microsec. Improvements have been made by carefully reducing capacitances affecting the readout amplifier region. Multiple non-destructive readouts using the "skipper" technology have achieved less than one electron rms readout noise with 32 reads. This could be used with realistic total read times only for small regions of interest.

Charge transfer efficiencies (CTE) of .999999 have been achieved for point source charges greater than 10000 electrons at -70C. However, CTEs are generally different in the parallel (imaging) region, and in the serial registers, and are charge level, readout method (inverted or not), temperature, and readout time dependent. They are also altered by radiation damage, as will be discussed below.

Dark current is important in the use of CCDs in space, since it is generally not easy to cool to -100 to $-150 \mathrm{C}$ as on the ground. Good CCDs achieve at room temperature typically 1 nanoamp $/ \mathrm{cm}^{2}$ non-inverted, 0.1-0.2 nanoamp $/ \mathrm{cm}^{2}$ partially inverted, and 0.01-0.02 nanoamp $/ \mathrm{cm}^{2}$ fully inverted. The latter corresponds to 1 electron per pixel per hour at 
-70C. Operating fully inverted requires the use of an extra implant during manufacture (called Multi Phase Pinned (MPP)) to restore a useful full well.

The full well depends strongly on pixel size, ranging from about 10,000 to over a million electrons. Typically for a 21 micron square pixel, the full well is 300,000 electrons non-inverted, or 130,000 electrons inverted (MPP).

Sensitivity uniformity between neighboring pixels is typically $2-3 \%$ non-inverted, and $1 \%$ inverted or partially inverted.

Nonlinearity is typically less than $1 \%$, and often less than $0.1 \%$ away from the full well limit.

Quantum efficiencies vary widely with wavelength, illumination direction and surface treatment. Results are shown in Table I. Recently the Tektronix backside treatment with anti-reflection coating has achieved $85 \%$ at $700 \mathrm{~nm}$ and $65 \%$ at $400 \mathrm{~nm}$ (Reed and Green, 1989). Separately, $80 \%$ at $900 \mathrm{~nm}$ was obtained by Ford and Photometrics using deep high resistivity silicon and an anti-reflective coat (Schempp, et. al., 1989).

Quantum efficiency hysteresis (QEH), where the sensitivity is dependent on previous illumination, can occur in backside illuminated CCDs at wavelengths where the photon is absorbed close to the surface, usually in the blue and UV. It can be eliminated by careful shaping of the internal backside electric field by UV flooding, biased thin metal gates, or implants followed by annealing, or by using fluorescing coatings.

Modern CCDs generally have no residual image (except after radiation damage), do not require a fat zero, and recover from a fully saturated condition after one frame erasure.

\section{Future Possibilities}

Larger format CCDs would be desirable to obtain larger fields of view. Current CCDs are very far from the formats of photographic plates. Small pixel $8192 \times 8192$ CCDs are probably achievable now, although larger silicon wafers such as are being used in Japan would be desirable to obtain good yields. For CCDs of $2048 \times 2048$ pixels and above, it would be desirable to provide more than the current 4 amplifiers, with several along the serial registers, in order to keep the readout time within bounds. The Space Telescope Imaging Spectrograph (STIS), (Woodgate et.al.,1987), will take $80 \mathrm{sec}$ to readout the whole array through one amplifier, and $20 \mathrm{sec}$ through all 4 amplifiers. Larger arrays will be achieved using buttable CCDs, so multiple readouts will inevitably occur.

Wavelength extension to 1.6 microns would be possible by using Germanium instead of Silicon. This development is beginning at JPL with fabrication at Ford.

CCDs are potentially useable through the UV into the X-ray region. Good progress is being made in maintaining sensitivity through the $U V$, by careful treatment of the thinned backside (Peckerar et al 1987; Stern et al, 1987), but the process is not yet fully established through the UV. Also, avoiding thin UV-absorbing contaminants on surfaces requires extreme care and materials selection and control of the environment, even with warm surfaces. It is yet more difficult with a cold CCD, as has been found with the WF/PC program (Westphal, 1988). Rigorous cleaning, bakeout and sealing as for vacuum tubes is probably necessary.

Even then, the main problem of using CCDs in the UV is not solved. Many astronomical sources, such as cool stars, planets, or galaxies containing cool stars, have 8-9 orders of magnitude more visible light emission than in the UV. It is therefore necessary, 
for spectrographs and even more for cameras, to remove the visible light to avoid contamination of the UV to be observed. Possible methods include alkali metal filters (not so far successful), crossed polarizers, or tandem gratings. Even if future developments were to make these successful, it is unlikely that the CCD/filter combination would rival UV photocathodes for quantum efficiency.

The use of non-destructive readout in the "skipper" amplifier can allow less than one electron readout noise, over limited regions of interest, at the expense of very long readout times (Janesick, 1988a and 1989a). Possibly this method could be used in a datadependent mode, with the number of non-destructive reads being dependent on the signal level of the first read, or by inserting a preliminary non-destructive amplifier ahead of the "skipper". Alternately, the development of low-capacitance lower noise JFET technology on the chip would allow sub-electron readout noise without multiple reads. This could be useful in measuring photon energy directly in the EUV, as is done now with X-ray CCDs, or for combining large numbers of images in the high cosmic ray environments above the earths geomagnetic shield, as will be discussed below.

\section{Radiation Damage}

CCDs can be damaged by particle radiation from the earth's trapped (Van Allen) radiation belts. In low earth orbits (LEO) a few hundred kilometers high, spacecraft pass through the lower edge of the inner belt at the South Atlantic Anomaly (SAA) at low orbit inclinations, and also through the polar caps at high orbit inclinations. The electrons in the SAA are relatively easily shielded against with about $1 \mathrm{~cm}$ of aluminum, but the protons with energies up to a few hundred Mev can only be partially shielded out. The HST Wide Field and Planetary Camera (WF/PC) by using considerable weight for shielding, with $1 \mathrm{~cm}$ of tantalum, has limited the predicted 5 year proton dose to 600 rads (Gunn and Westphal, 1981). This problem could be greatly reduced by launching with a very low orbit inclination, as was done for the Small Astronomical Satellites, including UHURU, but this would reduce the available weight for a given launch vehicle from Cape Canaveral, or would require the use of a foreign equatorial launch site such as San Marco or Kourou, with lower launch weight capabilities.

Recent tests by JPL at U.C. Davis, and by GSFC and Ball Aerospace at Harvard, have shown that protons can be much more damaging to CCDs than an 'equivalent' dose (measured in rads) of gammas, for example from cobalt-60 (Janesick,1989c; Delamere et. al.,1989; Brown et. al.,1989). This is because gammas produce ionization damage, where holes migrate to insulating layers, act as traps and alter potentials in a manner highly dependent on the dopant and applied electric fields, while the protons also produce lattice displacement damage. It can be highly misleading to demonstrate 'radiation hardening' of electronics by gamma testing, when the use environment is mainly protons.

Figure 1 shows results of average dark current tests for three different CCDs. A new lot of Tektronix 1024x1024 MPP 21 micron pixel CCDs was built for the STIS program. One of these chips was subjected first to $5 \mathrm{kRads}$ of cobalt-60, which had a relatively small effect, and then to 2 kRads of protons, which had a much larger effect. Even so, the average dark current was only $10-17$ electrons/pix/hour at $-80 \mathrm{C}$ after irradiation, when operated fully inverted. However, an earlier commercial Tektronix 512x512 CCD showed much higher dark currents both before and after irradiation. A WF/PC II Texas Instruments CCD showed no effect with 600rads of protons at 30 electrons/pix/hour at -95C (Trauger,1989 private communication). 
These results are quite encouraging. However, a large number of pixels become 'hot', or have dark noise much higher than the average, when irradiated. Preliminary results suggest that these pixels were already abnormal prior to irradiation, which brought out latent defects.

The lack of smearing of the lines in test images of a grid pattern of 6 electrons recorded signal, taken with an unirradiated STIS Tektronix 1024x1024 test CCD, suggests excellent charge transfer efficiency (CTE), even at very low signal levels. Figure 2 shows a composite graph for several CCDs, of CTE/pixel as a function of charge. Note that the measured CTE for the same unirradiated Tektronix 1024x1024 CCD above is well below that required for STIS. It also appears inconsistent with the 6 electron image. The measurement was made using the Edge Pixel Extended Response (EPER) method (Janesick et al, 1988). We need to understand the effects of CTE as measured by the different test methods on the photometric and resolution properties of CCDs. The effects of proton damage on CTE are also shown. At each charge level the CTE degrades monotonically with proton dose, and the degradation increases to lower charge levels. It is easier to express the damage by the Charge Transfer Inefficiency (CTI =1-CTE). A rough empirical fit to the data at $-60 \mathrm{C}$ shows $\mathrm{CTI} / \mathrm{krad}=0.14 \times \mathrm{Q}^{-0.9}$, where $\mathrm{Q}$ is the charge in electrons. This ignores the different pixel sizes, and the effects of temperature and readout time. The parallel CTE improves rapidly as the temperature is lowered down to -140C. The serial CTE is much less affected by the protons. These two effects may be due to the proton-induced trap lifetimes being between the parallel and serial readout times at $-60 \mathrm{C}$, but longer than either at $-140 \mathrm{C}$ (Janesick, 1989c,d). This suggests a strategy for future missions of running the CCDs very cold, and doing a light flood and erase before exposing. Meanwhile, other techniques avoiding the need for consumable cryogenics, such as a duct within the readout channels, and the effect of nitride insulators are being investigated.

The damage problem can be reduced by using higher orbits which remain above the radiation belts. Highly elliptical orbits which go through the radiation belts will generally have a worse problem than the low orbits which just skim through the SAA. Geosynchronous orbits remain in the upper radiation belts which contain mainly high energy electrons which cannot be completely shielded out. While their damaging effects are limited, they can produce an increased background for data taking, as seen with IUE. Circular orbits with more than 2-4 day periods, or Lagrangian orbits, or siting on the moon, will avoid this trapped radiation damage problem. However, similar levels of damage can occur from the occasional very large solar flare. The levels are very unpredictable, since one large flare can produce more damage than a decade of smaller

flares. The damaging particles are a mixture of gammas, $\mathrm{X}$-rays, protons, neutrons and heavier ions.

\section{Cosmic Ray Background}

While the effects of particle damage may be less in high orbit than in low, apart from the risk of a large solar flare, the effect of the primary cosmic rays on the detector background is much worse. Figure 3 shows the energy spectrum of cosmic ray protons (the dominant particle - see question discussion below), for various orbits. The 'free space proton' curve, above the earth's magnetic shield, shows a total flux 10-20 times higher than for a 32 degree low orbit, or 200 times higher than the flux at the earth's surface. It is instructive to calculate the amount of shielding required to reduce the flux to the level found at mountain observatories on the earth. From the 'free space proton' curve, 
we must stop protons less than $10 \mathrm{Gev}$ energy. At an energy loss rate of $0.5 \mathrm{Mev} / \mathrm{mm}$, we need 20 meters of silicon. The detailed calculation for moon rock requires knowing the density and composition of the moon down to these depths. It is suggested that a lunar observatory using CCDs should direct the beam from the telescope down to a focal plane of order 20 meters below the moon's surface. Measurements of the radioactivity of lunar soil show that is is less than that of granite. Clearly, shielding detectors on a spacecraft against primary cosmic rays is impractical.

The charge deposited in a typical thin CCD from a cosmic ray is about 1000 electrons, with a broad charge distribution. A 15 minute exposure in high orbit would leave 5-10\% of the pixels affected, so that it would be impossible to detect faint images from individual exposures. Ground-based astronomers typically limit their exposures to about an hour to limit the probability of a cosmic ray hit in a pixel to about $0.1 \%$. However, the advantages of retaining the high quantum efficiency of CCDs in the visible and near IR are considerable. What is necessary to remove cosmic ray hits from the images?

Table 2 shows the allowed integration times to clean a combined image of cosmic ray hits by comparing individual registered images, and rejecting any pixel with counts significantly greater than the minimum value. A $2048 \times 204821$ micron pixel CCD is assumed (STIS case), with three cosmic ray environments; $0.01 \mathrm{~cm}^{-2} \mathrm{~s}^{-1}$ for the earth's surface and 20 meters under the moon, $0.1 \mathrm{~cm}^{-2} \mathrm{~s}^{-1}$ for low earth orbit (HST), and 2 $\mathrm{cm}^{-1} \mathrm{~s}^{-1}$ for high orbit (proposed 10 meter site). Perfect sub-pixel registration between images is assumed, to minimize the effects of mismatch of edges in the structure of the target image, and each cosmic ray count is spread amongst 4 pixels of the re-registered images. For example, to obtain a total 25 minute exposure in the high orbit case, it must be split into four 6.3 minute exposures. At low signal/noise, for spectroscopy where the diffuse background is small, combining the images also increases the effective readout noise, and increases the exposure time needed for a detection. The Table shows the additional exposure factor required for a signal/noise of 5 . This analysis assumes a low density of hits in each image, and ignores any difficulties in distinguishing the cosmic ray hits from real image structure, for all intensity values and slopes.

A feature of the above analysis is that some of the pixels (e.g. $2.2 \%$, or 9000 out of 4 million, in the case above for a $25 \mathrm{~min}$ exposure in high orbit) have only the count value from one individual image of the set to contribute. It would be necessary to subdivide further the total image to assure a reasonable minimum signal to noise in each pixel. For example, to ensure all pixels have at least half the images with real data, $(\mathrm{N}-1)$ images should be added to the $\mathrm{N}$ in the analysis above.

Even these exposure factor increases are small compared to the differences between CCD quantum efficiencies and photocathodes in the near IR, so that CCDs should not be ruled out for high orbits, especially for broad band imaging, where diffuse background typically dominates over detector background. More sophisticated computer analyses and real CCD simulations of these effects are required before basing a high orbit mission on using CCDs.

\section{Photoemissive Detectors}

Detectors based on photoemissive photocathodes are much less sensitive than CCDs to radiation damage and to radiation background while taking data. Their primary advantage is that photocathodes can be selected which are sensitive to the UV and insensitive 
to the visible/IR. In addition they can be used for fast timing applications without adding readout noise.

Their primary disadvantage in the visible and near IR is that there are no photocathodes that can compete with CCDs for QE. For example, in the blue at 400nm the best photocathodes are bialkalis, peaking at $20-25 \%$ QE compared to a good CCD with $65 \%$, and in the red at H-alpha the best trialkali photocathodes have about 7\% QE compared to a good CCD with $85 \%$. Another problem, particularly for broad band imaging, is their limited upper counting rate, particularly when used in photon counting modes.

\section{UV Photocathodes}

The difficulties of using CCDs in the UV are discussed above, including the sensitivity to scattered or leaked light from the visible, and contamination of the cooled surface by UV-absorbing layers.

Photocathodes are available such as CsTe $(<300 \mathrm{~nm})$, CsI $(<170 \mathrm{~nm}), \mathrm{KBr}(<$ $130 \mathrm{~nm}), \mathrm{MgF}$ (<110 nm), which are sensitive below and insensitive above these cutoff wavelengths. UV sensitivities can vary from QEs of $10-25 \%$ for semi-transparent cathodes to $30-80 \%$ for opaque cathodes. High purity cathodes can cutoff sharply with QEs of $10^{-4}$ to $10^{-6}$ to the long wavelength side of the cutoff wavelength. Primarily for this reason photoemissive detectors with CsI and CsTe cathodes, MultiAnode Microchannelplate Arrays (MAMAs), were selected for the STIS instrument.

\section{Timing}

Many readout schemes using photocathodes readout the event promptly, or store for times short compared to unintensified CCDs. This makes them competitive even in the visible for tasks such as speckle imaging and spectroscopy, astrometry, flare stars and pulsars, and many applications in medical, laboratory, and military situations. Prompt readout detectors can identify pulse arrival times to less than one microsec, if needed.

\section{Radiation effects}

Cumulative radiation damage in photoemissive detectors, except for intensified CCDs, are not expected to be a problem in orbital situations. Cathodes, intensifiers, and metal grid readout structures are all much more robust to radiation damage than are CCDs. However, care should be taken to avoid some operations through the radiation belts which could result in bombardment of the photocathode by feedback ions, or heating of the anode.

Background counts produced by cosmic rays are also greatly reduced in photoemissive detectors compared to CCDs. Woodgate and Fowler (1988) studied these effects for low and high earth orbit, including the effects of Cerenkov, fluorescence and phosphorescence in detector windows. A cosmic ray passing through the detector usually produces a single count, equivalent to one detected photon, instead of over 1000 for a CCD. Further, some readout methods (such as the MAMA) can provide some internal anti-coincidence when the cosmic ray interacts with the surface of the intensifier structure producing a coincident signal, although the magnitude of this reduction was probably overestimated by Woodgate and Fowler. Even without anti-coincidence, the high orbit background corresponds to one count per pixel in $\mathbf{2 2}$ hours for $\mathbf{2 5}$ micron pixels, allowing extremely long exposures without effective cosmic ray background. 


\section{Intensifiers}

The signal from a photocathode is extracted, and intensified onto an anode by an electrostatic or electromagnetic or microchannelplate (MCP) imaging intensifier. The first two accelerate each photoelectron to several thousand volts as input to the next stage, which must convert the signal into sufficient charge to be detected electrically, either as direct input into a diode array or CCD, or indirectly in a phosphor. The MCP multiplies the number of electrons, producing $10^{5}-10^{7}$ electrons from each primary photoelectron as input to the next stage, enabling the direct collection of this charge and position measurement of the event by some 'crossed wire' encoding scheme.

Electrostatic and electromagnetic intensifiers with adequate gain are relatively large and heavy and require shielding from stray fields, but do not of themselves significantly limit the upper counting rate and therefore the dynamic range of the detector. MCPs can be compact (about $1 \mathrm{~mm}$ long and up to $100 \mathrm{~mm}$ diameter), low mass, and work in strong ambient fields, but current designs with sufficient gain to detect individual photoevents limit the local upper counting rate to a few hundred events per pixel per second, limiting the dynamic range of the detector. While MCP intensified detectors may be quite suitable as astronomical spectroscopic detectors, their use as broad band detectors in cameras will be restricted to faint objects.

\section{Readout Methods}

A class of readout methods which encode each recorded photon event promptly such that they may be time-tagged, that is each event is recorded with a two-dimensional position and a time, is possible using the increased electron charge output by a MCP. These detectors may also be used in accumulate mode by adding counts into an image memory. This class includes the Multi-Anode Microchannel Array (MAMA) (Timothy, 1983; Timothy and Bybee,1986), selected as UV detectors for the STIS and SOHO programs, the Wedge and Strip as selected for EUVE (Siegmund et. al. 1986), the Delay Line Anode (Lampton, Siegmund and Raffanti, 1987), and the Mepsicron (Lampton and Paresce, 1974). As photon-counters using MCPs at high gain, they are all limited in local counting rate by the MCP. In addition their total counting rate over the whole array is limited by the readout electronics speed to $10^{5}-4 \times 10^{6}$ counts/s in current designs.

Alternatively, intrinsically framing readout schemes can be used, where the intensified electron signal is temporarily stored and then readout sequentially into memory or a tape recorder or downlinked directly via telemetry. The storage medium can include a CCD (Broadfoot, 1987; Stapinski, Rodgers and Ellis, 1981; Williams and Weistrop, 1984; Green, 1989; Jenkins et al 1988; Swartz et al, 1988; Poland and Golub, 1988; Chen, 1987), or a 'vidicon' (Boksenberg, 1975; Machetto et. al., 1982; Boggess et al, 1978). If the frames are readout sufficiently rapidly that only one event is recorded in each pixel in each frame, then the detector may be used as a photon counter. Then the upper count rate is usually even more severely constrained than for the MCP, since it can only reach a fraction of the frame rate. For example the Faint Object Camera of the HST becomes non-linear at less than the frame rate of $30 / \mathrm{s}$. Such a photon counter can centroid individual events to improve internal resolution and effectively increase the format size. The on-the-fly processing required often further decreases the upper count rate limit. The charge storage medium, CCD or 'vidicon', can alternatively be read out in analog mode, recording the charge representing many photons, as in the IUE detector. The SEC Vidicon used in 
IUE has a limited dynamic range, with only a factor of about 10 between noise level and saturation, which has required a choice between sensitivity and signal-to-noise. Using a CCD in analog intensified mode could in principal help reduce the effect of recording cosmic rays by adjusting the gain to adjust the relative charge representing a photon. The cumulative radiation damage problem would still occur, but the effects could be reduced by operating at higher charge levels. The concept used in the one dimensional Digicons, as used in the HST Faint Object Spectrograph and High Resolution Spectrograph (Beaver and McIlwain, 1971), with prompt readout of a diode array, is difficult to extend to two dimensions because of the large number of amplifiers needed.

Assessing the possibilities for development in the future, the major unmet need is for a UV camera capability with high dynamic range (ability to cope with targets brighter than a 23rd magnitude $B$ star when used with a 10 meter telescope), and large format. One development path is to devise higher count rate $\mathrm{MCPs}$, where the potential distribution can be restored much more quickly to the depleted channel. Using separate materials for secondary emission and for establishing the potential distribution could help. Also a possible method is a discrete dynode MCP constructed of several layers of alternate resistive and conducting sheets with holes. Such improvements would extend the utility of the majority of modern intensified detectors, which use MCPs. A temporary expedient of scanning the image over the detector has been used successfully at Ball Aerospace to move bright points to a fresh location until the MCP has recovered. This works with a dilute image consisting of bright features on a relatively dark background, such as a star field, but does require a mechanism such as a continuously nutating mirror. Another development path would be to convert current framing detectors using photon counting to also distinguish many events per pixel per frame in analog mode. This is being done at GSFC with the detector for the Coronal Diagnostics Spectrograph instrument on SOHO (Poland and Golub, 1988). It would be particularly attractive if it could be used with the opaque photocathode method with its higher quantum efficiency in the UV (Jenkins et al, 1988).

\section{Energy Resolving Detectors}

In the X-ray region, CCDs are used to simultaneously determine the two dimensional position and energy of each recorded photon, by measuring the charge generated by ionization as the X-ray is absorbed. Similarly, if the thermal noise of detection is made sufficiently low, and the photon energy can be distributed in a proportional way, as charge or heat, in principle the energy of a UV, visible, or IR photon could be measured.

One approach, which has been developed for particle detection, is to hold a colloid of equal sized superconucting grains just below their superconducting transition temperature in a magnetic field. A photon absorbed by a grain will slightly heat it, temporarily allowing the volume of magnetic field excluded by the Meissner effect to collapse slightly. The field change and its position could be sensed by four SQUID detectors. Currently only X-ray energies and above have been measured, but with high signal to noise. Temperatures in the 0.1-4 $\mathrm{K}$ range are required (Drukier, private communication).

An avalanche photodiode counting individual photoelectrons out to 28 microns has been developed by Rockwell (Petroff, Stapelbroek and Kleinhans, 1987). If the amplification is stable, and a mechanism could be found for an optical photon to deposit its energy into many photoelectrons using a low bandgap material, this could form the basis for an energy resolving optical imager. 
These and other methods using cryogenic noise reduction would require considerable sustained funding to develop into useful practical detector systems, but the advantage of having simultaneous two dimensional imaging and spectroscopy is also very large.

\section{Summary and Recommendations \\ Visible/Near-IR Detectors}

Because of their high quantum efficiency, CCDs are the preferred detector for the visible and near IR for most purposes. Larger formats are desireable, up from the current $10^{6}$ pixels to $10^{8}-10^{9}$ pixels. A combination of monolithic pixel number increases and mosaic arrays are needed, with multiple simultaneous outputs to maintain reasonable readout times. Further quantum efficiency optimization is needed to extend the highest efficiencies over the full wavelength range.

Rejection of cosmic ray hits from the images is a significant problem in orbit, and very severe in high orbit above the earth's magnetic shield. Methods of combining many frames should be simulated and tested for effective rejection in the presence of real images before committing to a high orbit mission using CCDs. Further reductions in readout noise will be necessary to maintain signal to noise when many images are combined. The problem can be avoided on the moon by burying the CCDs under 20 meters of rock.

Radiation hardening of CCDs against proton damage as well as against ionizing radiation is required, especially for maintaining the charge transfer efficiency. Both manufacturing and operational considerations are important, including temperatures, time scales and voltage distributions. UV Detectors

For extension of CCD use into the UV, visible rejecting filters would be desireable, but contamination of cold CCDs by UV absorbing films is also severe.

Currently, photoemissive detectors are preferred in the UV because of their ability to reject the visible and being able to operate without cooling. Larger formats are desireable, which in some designs will require mosaicking of both intensifiers and readout arrays. Some further quantum efficiency improvements may also be possible. Matching detector front surfaces to curved focal planes is desireable for large format cameras and for spectroscopy.

The primary unmet need is for higher dynamic range for UV cameras. Both higher rate MCPs and analog use of framing cameras should be pursued. Energy Resolving Detectors

Long term consistent and sustained development efforts would be required to obtain detectors which could provide simultaneous energy and two dimensional position measurement, but they would be highly desireable.

\section{Orbit Selection}

It is important to consider the radiation damage and cosmic ray rejection aspects of available and developable detectors when selecting the best orbit for a mission. For example, a highly elliptical orbit of a few days period, ideal from the point of view of observing duty cycle and launch mass, is the most difficult for CCDs for both radiation damage and cosmic ray rejection. A low orbit with very low inclination to the equator, such as those used for the Small Astronomy Satellites, would be most favorable for CCD performance, although more difficult for launch mass and operations. 
I thank Jim Janesick for providing his viewgraphs for this talk, from which much of the CCD discussion was derived, and for the continuing flow of broadly distributed memos which keeps many of us up to date on the latest results and ideas.

\section{References}

Note: The several references to the Proceedings of the Conference on CCDs in Astronomy, Tucson, Sept 1989, ed. G.Jacoby, are shown as PCCCDA below.

Beaver,E.A., and McIlwain,C.E. 1971, Rev. Sci. Instrum. 42,1321.

Blouke,M.M., Delamere,A. and Orbock,J., 1989, PCCCDA.

Boggess,A. and 32 others, 1978, Nature 275,372.

Boksen berg.A., 1975, in Image Processing Techniques in Astronomy, eds. C. deJager and $H$. Nieuwenhuijsen (Boston, D. Reidel) p59.

Broadfoot,L., 1987, Geophys. Res. Lett. 14,4.

Brown,L.W., Rebar,F., Fowler,W., Yagelowich,J., Ziegler,M., Becker,J. and Reed,R., 1989, PCCCDA.

Chen,P., 1987, Bull.AAS, $19,746$.

Delamere,A., Orbock,J. and Murata-Seawalt,D., 1989, PCCCDA.

Delamere,A., Atkinson,M., Rice,J., Blouke,M. and Reed,R., 1989, PCCCDA.

Green,R.F., 1989, NOAO Newsletter 17, March 1.

Gunn,J.E. and Westphal,J.A., 1981, SPIE Solid State Imagers for Astronomy, 290,16.

Janesick,J., Elliott,T., Bredthauer,R., Chandler,C. and Burke,B., 1988, SPIE X-ray Instrumentation in Astronomy, San Diego, Aug88.

Janesick,J., JPL Distributed Memos, 1988 Jan 19, and 1989 Aug 1.

Janesick,J., JPL Distributed Memos, 1989 Aug 2.

Janesick,J., JPL Distributed Memos, 1989 Aug 28.

Janesick,J. 1989, PCCCDA.

Jenkins,E.B., Joseph,C.L., Long,D., Zucchino,P.M., Carruthers,G.R., Bottema,M. and Delamere,A., 1988, SPIE Ultraviolet II 932,213.

Lampton,M.L. and Paresce,F. 1974, Rev. Sci. Inst. 45,1098.

Lampton,M.L., Siegmund,O.H.W., and Raffanti,R. 1987, Rev. Sci. Inst., 58,2299.

Machetto,F. and the FOC Team, 1982, The Space Telescope Observatory NASA CP2244, ed. D.N.B.Hall.

Peckerar,M.C., Bosiers,J.T., McCarthy,D., Saks,N.S. and Michels,D.J., 1987 Appl. Phys. Lett. 50, 1275.

Petroff,M.D., Stapelbroeck,M.G. and Kleinhans,W.A., 1987, Appl. Ph. Let. 51,406. Poland,A.I. and Golub,L., 1988, An Intensified CCD for SOHO, NASA Report.

Reed,R.and Green,R. 1989, PCCCDA.

Schempp,W., Griffin,F., Sims,G. and Lesser,M., 1989, PCCCDA.

Siegmund,O.H.W., Lampton,M.L., Bixler,J., Chakrabarti,S., Vallerga,J., Bowyer,S., and Malina,R.F., 1986, JOSA-A, 3,2139.

Stapinski,T.E., Rodgers,A.W. and Ellis,M.J., 1981, PASP 93,242. 
Stern,R.A., Catura,R.C., Kimble,R., Davidsen,R.F., Winzenread,M., Blouke,M.M., Hayes,R., Walton,D.M. and Culhane,J.L., 1987, Optical Engineering 26,875.

Swartz,M., Epstein,G.L. and Thomas,R.J., 1988, NASA/GSFC X-682-88-2.

Timothy,J.G., 1983, PASP 95,810.

Timothy,J.G. and Bybee,R.L., 1986, SPIE Ultraviolet Astronomy 687,109.

Williams,J.T. and Weistrop,D., 1984, SPIE 445,204.

Westphal,J., 1988, Report to the HST Science Working Group, May 4.

Woodgate,B.E. and Fowler,W., 1988, Proceedings of the Conference on High Energy Radiation in Space, Nov87, Sanibel Island,FL, eds. A.C.Rester and J.I.Trombka. (AIP Conference Proceedings No.186.)

Woodgate,B.E. and the STIS Team, 1986, SPIE Instrumentation in Astronomy VI 627,350 .

\section{DISCUSSION}

Johnson: The speaker mentioned a 20 meter thickness of lunar soil and rock for shielding. Silberberg and coworkers at NRL have calculated the depth of lunar soil required as 400 to $700 \mathrm{grams} / \mathrm{cm}^{2}$ to bring the annual dose equivalent to less than $5 \mathrm{rem} /$ year. They were looking at the protection required for long term human habitation on the moon. It appears that 2 to $31 / 2$ meters of compacted lunar soil would be adequate for cosmic rays, their secondaries, and giant flares (e.g., the flare of February 1956). See Silberberg in Lunar Bases and Space Activities in the 21st Century, W. W. Mendell, editor, Lunar and Planetary Institute, Houston, Texas, 1985.

MacKay: In your estimate of cosmic ray event rates, did you take into account the much higher rates that could come from secondary events absorbed by any surrounding structure?

Woodgate: No. According to J. Trombka, the primary protons can produce a somewhat larger number of secondary neutrons in surrounding structure, depending on its thickness and composition. Typically in interplanetary space, about 2 protons $\mathrm{cm}^{-2} \mathrm{~g}^{-1}$ produce about 5 fast neutrons $\mathrm{cm}^{-2} \mathrm{~s}^{-1}$. These fast neutrons can partially thermalize in large spacecraft (e.g. about 1 meter of aluminum), especially if low density materials such as hydrogen in fuel or water are present. The thermal neutrons can easily be removed by small quantities of boron or rare earths, as in a paint, for example at the interior of a shield. The fast neutrons will not produce ionization, and will mostly pass through the detector undetected. A very few will recoil, and may produce an event which must be vetoed. The number of these events will be much less than the number of primary protons, but should be quantitatively assessed for each situation, as should the damage produced by the neutrons. 
TABLE 1. TYPICAL CCD QUANTUM EFFICIENCIES

\begin{tabular}{|l|cccc|}
\hline \multicolumn{1}{|c|}{ WAVELENGTH (nM) } & 200 & 400 & 600 & 900 \\
\hline $\begin{array}{l}\text { FRONTSIDE ILLUMINATION } \\
\text { (1500 A THREE-PHASE } \\
\text { POLY GATES) }\end{array}$ & 0.0 & 0.08 & 0.45 & 0.2 \\
$\begin{array}{l}\text { FRONTSIDE: OPEN-PINNED } \\
\text { PHASE }\end{array}$ & 0.18 & 0.3 & 0.55 & 0.3 \\
$\begin{array}{l}\text { BACKSIDE ILLUMINATION } \\
\text { (OE-PINNED, UV FLOODED) }\end{array}$ & 0.3 & 0.5 & 0.6 & 0.3 \\
$\begin{array}{l}\text { BACKSIDE; (ION-IMPLANTED, } \\
\text { ANNEALED, AR COATED) }\end{array}$ & -- & 0.65 & 0.85 & 0.8 \\
\hline
\end{tabular}

\section{TABLE 2. REMOVAL OF COSMIC RAY HITS FROM CCD IMAGES}

\begin{tabular}{|c|c|c|c|c|c|}
\hline NUMBER OF IMAGES & 1 & 2 & 3 & 4 & 5 \\
\hline $\begin{array}{l}\text { PROBABILITY OF A HIT } \\
\text { IN EACH PIXEL. IN EACH } \\
\text { FRAME, } p\end{array}$ & $2.5 * *-7$ & .0005 & .0063 & .022 & .048 \\
\hline $\begin{array}{l}\text { MAX INTEGRATION TIME } \\
\text { TO ELIMINATE HITS BY } \\
\text { VETO AT: }\end{array}$ & & & & & \\
\hline $\begin{array}{l}\text { EARTH SURFACE } \\
(0.01 \text { CM**-2 S**-1) }\end{array}$ & $2.4 \mathrm{~s}$ & $\begin{array}{l}27 \mathrm{~m} * \\
(54 \mathrm{~m})\end{array}$ & $\begin{array}{c}5.8 \mathrm{~h} \\
(17.4 \mathrm{~h})\end{array}$ & -- & -- \\
\hline $\begin{array}{l}\text { LEO (HST) } \\
(0.1 \text { CM**-2 S**-1) }\end{array}$ & $0.24 \mathrm{~s}$ & $\begin{array}{r}2.7 \mathrm{~m} \\
(5.4 \mathrm{~m})\end{array}$ & $\begin{array}{r}35 \mathrm{~m} \\
(1.8 \mathrm{~h})\end{array}$ & $\begin{array}{l}2.1 \mathrm{~h} \\
(8.4 \mathrm{~h})\end{array}$ & -- \\
\hline $\begin{array}{l}\text { HEO (10 METER) } \\
(2 \text { CM**-2 S**-1) }\end{array}$ & $0.012 \mathrm{~s}$ & $\begin{array}{c}8 \mathrm{~s} \\
(16 \mathrm{~s})\end{array}$ & $\begin{array}{c}1.8 \mathrm{~m} \\
(5.4 \mathrm{~m})\end{array}$ & $\begin{array}{l}6.3 \mathrm{~m} \\
(25 \mathrm{~m})\end{array}$ & $\begin{array}{l}13.5 \mathrm{~m} \\
(1.1 \mathrm{~h})\end{array}$ \\
\hline $\begin{array}{l}\text { EFFECTIVE READOUT } \\
\text { NOISE (STIS } \\
\text { TECHNOLOGY) (ELS) }\end{array}$ & 2.7 & 3.8 & 4.7 & 5.4 & 6.0 \\
\hline $\begin{array}{l}\text { COUNTS REQUIRED FOR } \\
S / N=5 \text { DETECTION }\end{array}$ & 31 & 35 & 39 & 42 & 45 \\
\hline $\begin{array}{l}\text { EXPOSURE FACTOR } \\
\text { INCREASE FOR SAME S/N }\end{array}$ & 1.0 & 1.13 & 1.26 & 1.35 & 1.45 \\
\hline $\begin{array}{l}\text { \# FOR A } 2048 \times 2048 \text { DETECTOR WITH } \\
4 \text { PIXELS/HIT (TO ALLOW FOR SUB- } \\
\text { * FRAME TIMES } \\
\text { (TOTAL TMES) }\end{array}$ & $\begin{array}{l}\text { Ix21 MICRC } \\
\text { EL REGISTF }\end{array}$ & $\begin{array}{l}\text { PIXELS, } \\
\text { THONS }\end{array}$ & & & \\
\hline \multicolumn{6}{|c|}{$\begin{array}{l}\text { DAVEATS: APPLIES TO LOW DEVISITY OF HITS, BLAINK FIELDS. } \\
\text { PROBLEMS WITH HIGH DENSITY OF HITS, MAGE STRUCTURE } \\
\text { SLOPES, SUB-PIXEL REGISTRATION, NOT MUCH USED IN IRAF } \\
\text { EVEN WITH } \$<.0005 \text {. }\end{array}$} \\
\hline
\end{tabular}




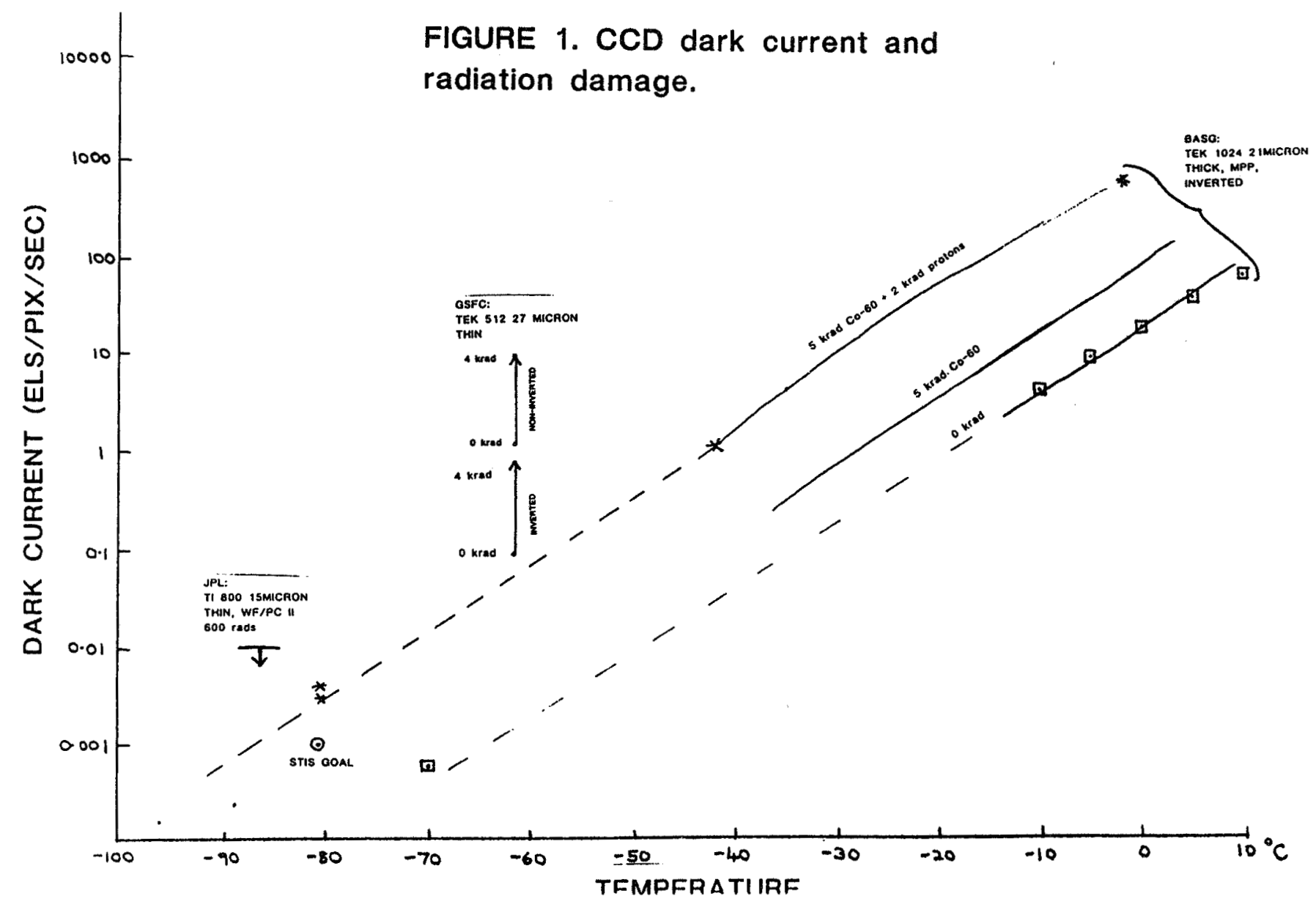

FIGURE 2. CCD charge transfer

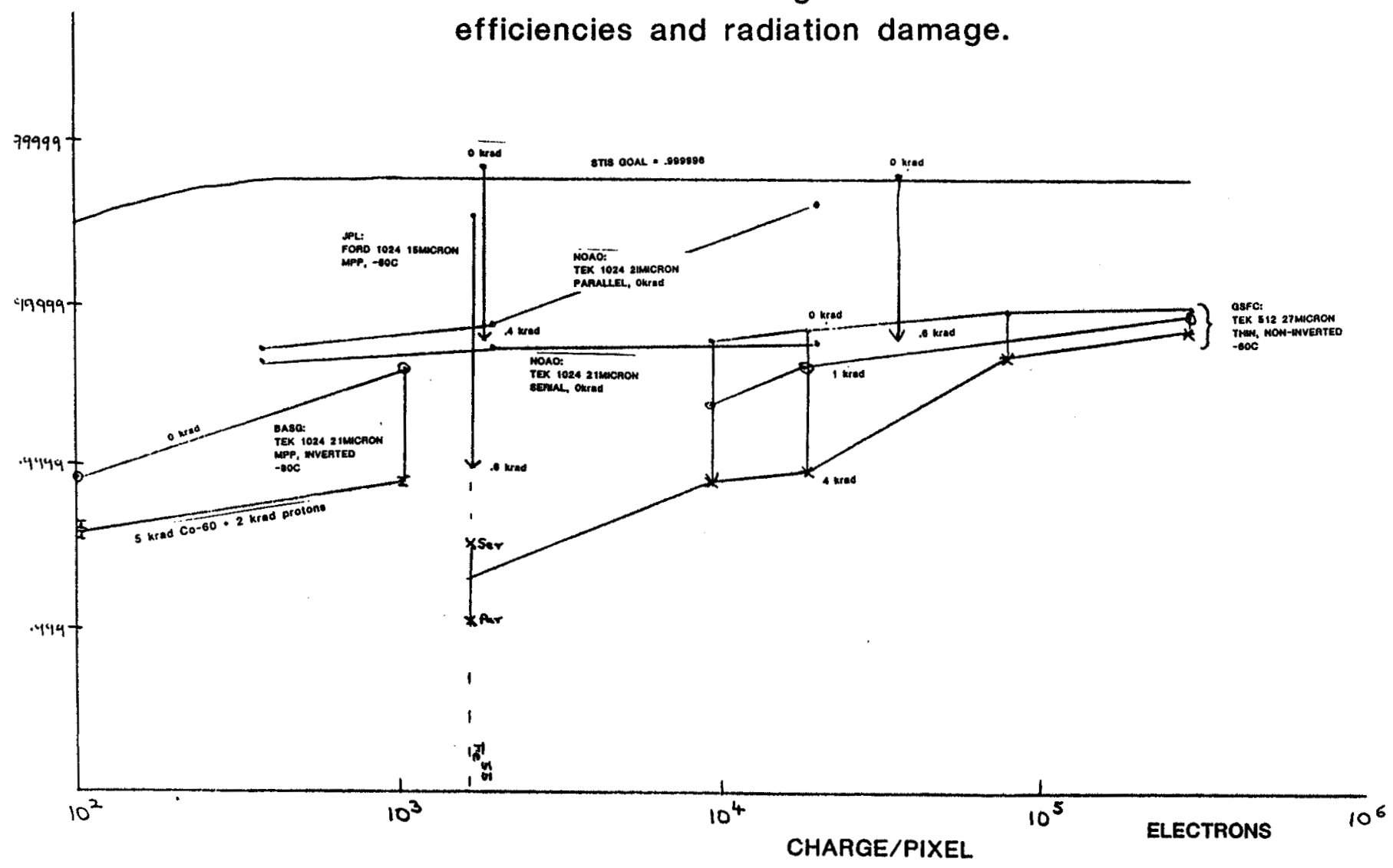


FIGURE 3. Cosmic ray proton

energy spectrum.

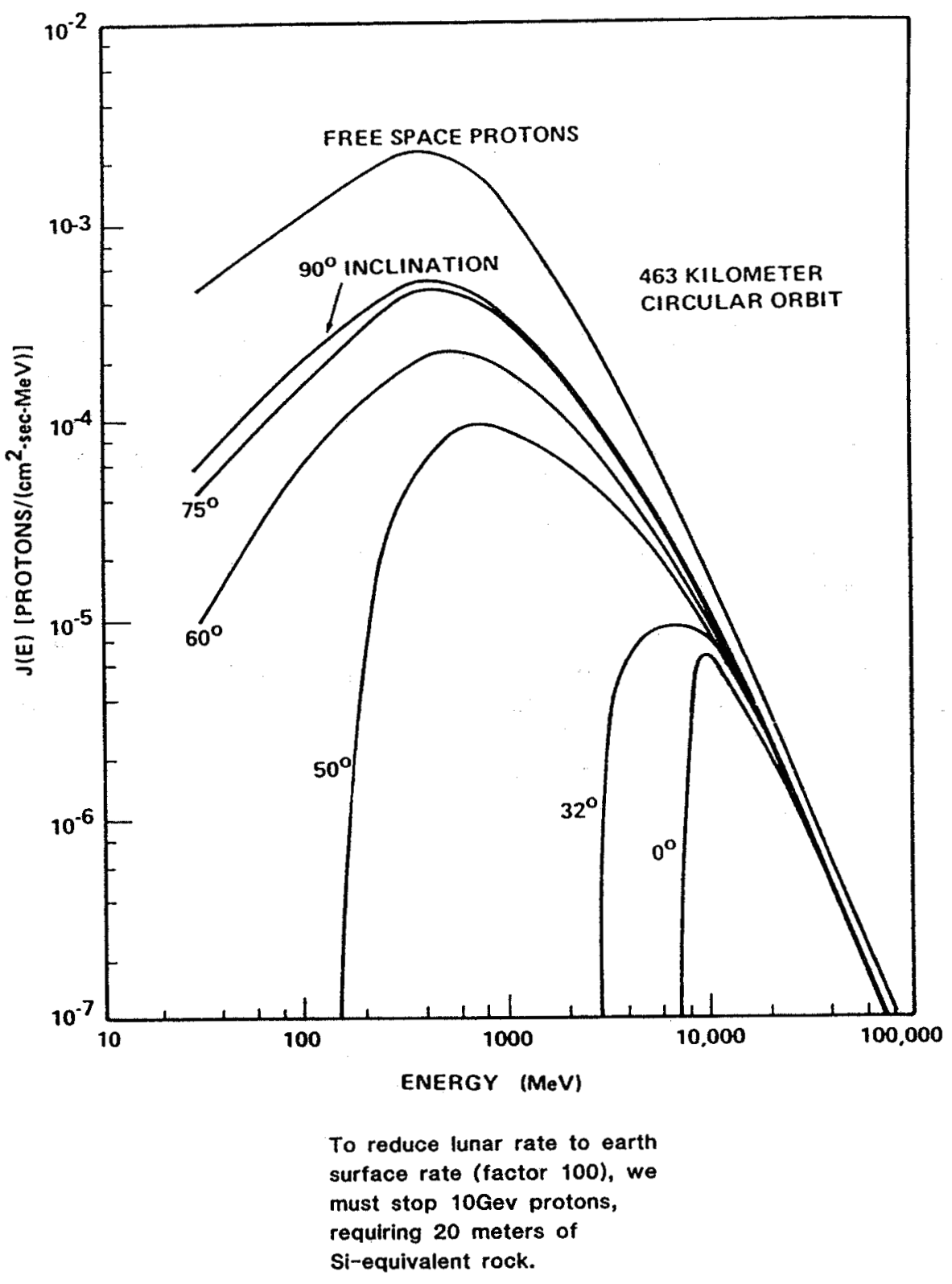




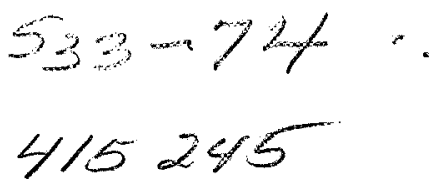

Infrared Detectors for a $10 \mathrm{M}$ Space or Lunar Telescope

Rodger I. Thompson

360569

Steward Observatory - University of Arizona

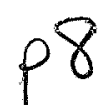

\section{Introduction}

The rapid advance of infrared detector technology makes it useless to attemps a prediction of the state of the art at the time of instrument definition for the next large space telescope. On the other hand, prediction of the opportunities presented by such a telescope is relatively straight forward. As a baseline this presentation assumes a 10 meter diameter telescope operating at $100^{\circ} \mathrm{K}$ with a total emissivity of $10 \%$. Whether the telescope is space or lunar based is not a factor in this analysis. The characteristics of the telescope and natural background set the environment for the detectors. The goal of future detector development is a total noise performance limited only by the background presented to the detector. In addition the detector should have the highest possible sensitivity or quantum efficiency possible, a large enough format to easily cover the useful field of view, readout modes which are easily accomodated, and enough immunity to radiation to operate with impunity in the expected radiation environment.

In the presentation on goals for detector development the low background spectral region between 1 and $4 \mu \mathrm{m}$ is treated differently from the high background $4-10 \mu \mathrm{m}$ region. This conference is limited to the region shorter than $10 \mu \mathrm{m}$; therefore, the longer wavelength regions will not be discussed here.

\section{Background environment}

The background environment of the detector is set by the thermal emission from the telescope optics at long wavelengths and by the zodiacal background at shorter wavelengths. Here the zodiacal background consists of both a scattered component and a thermal emission component. The thermal emission is characteristic of a diluted $265 \mathrm{~K}$ blackbody. The zodiacal background is modeled by the following function:

$$
Z(\lambda)=\frac{1.5110^{9}}{\lambda^{3}}+6.010^{-8} B B(\lambda, 265 K)
$$

where $\mathrm{Z}$ is in photons per $\mathrm{cm}^{2}$ per second per micron per steradian and BB is the blackbody flux in the same units. This equation approximates the zodiacal flux at approximately 45 degrees above the ecliptic.

Figure 1 shows the expected background based on the zodiacal flux and the radiation from the telescope. The pixel size is the diffraction limit which scales as $\lambda^{2}$ in area. The bandwidth is $50 \%$ of the wavelength which scales as $\lambda$. The product of pixel size and bandwidth cancels the $\lambda^{-3}$ dependence of the scattering term of the zodiacal light. For this reason the background is flat in the spectral region out to $3 \mu \mathrm{m}$ where the thermal emission begins to dominate over the scattered light. It is also evident that the thermal emission from the telescope begins to dominate over the zodiacal light at longer wavelengths. For true natural background limited operation, the telescope should be at a temperature of $60 \mathrm{~K}$ or colder. The minimum photon flux at short wavelengths is about 1 photon per second. 
Figure 1

\section{BACKOROUND PHOTONS PER SECOND DIFFMCTION LUMTED PDXes 60\% ENDWIOTH}

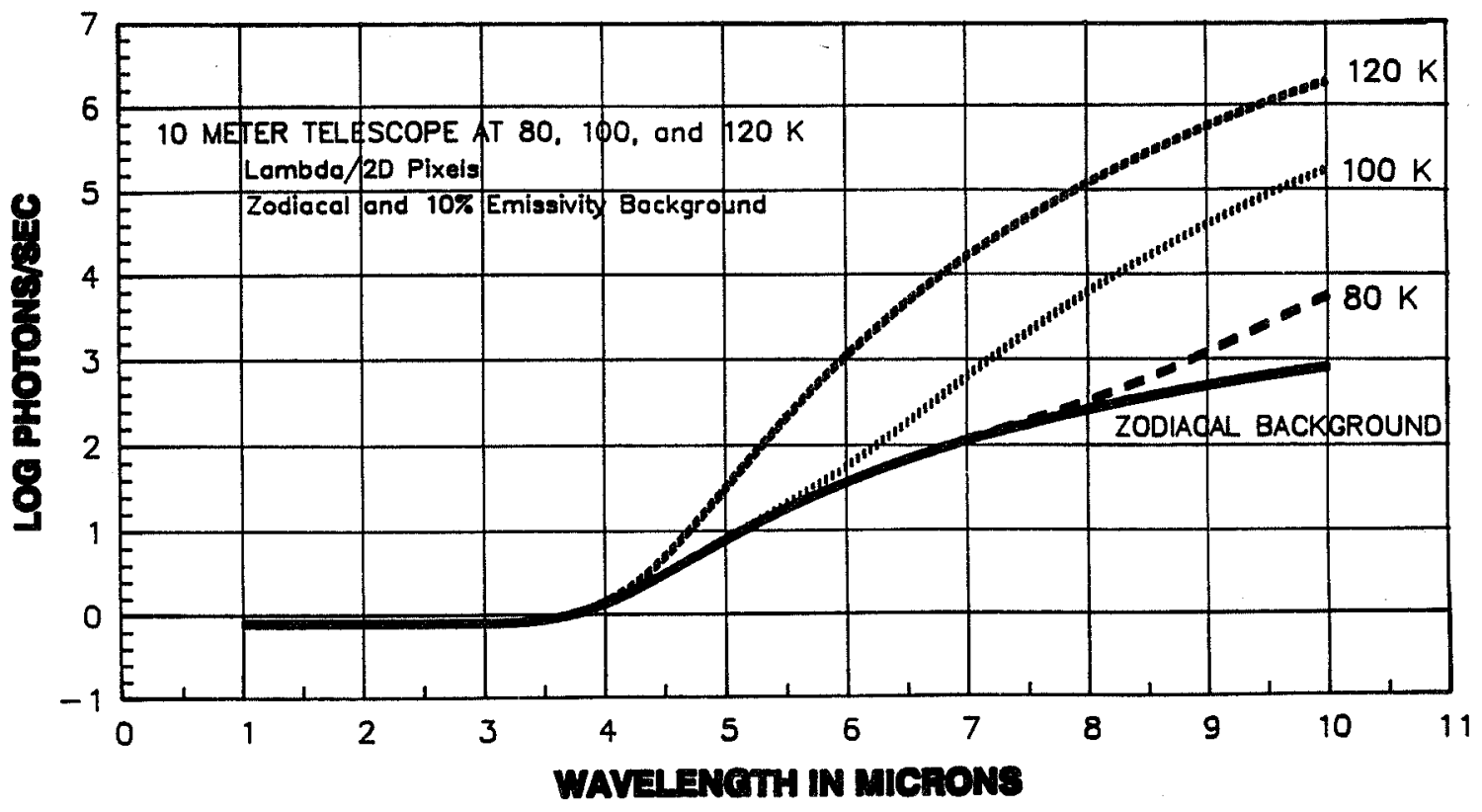

Figure 2

\section{BACKGROUND PHOTONS PER SECOND DIFFRACTION LIMITED PIXELS $R=10,000$ SPECTROSCOPY}

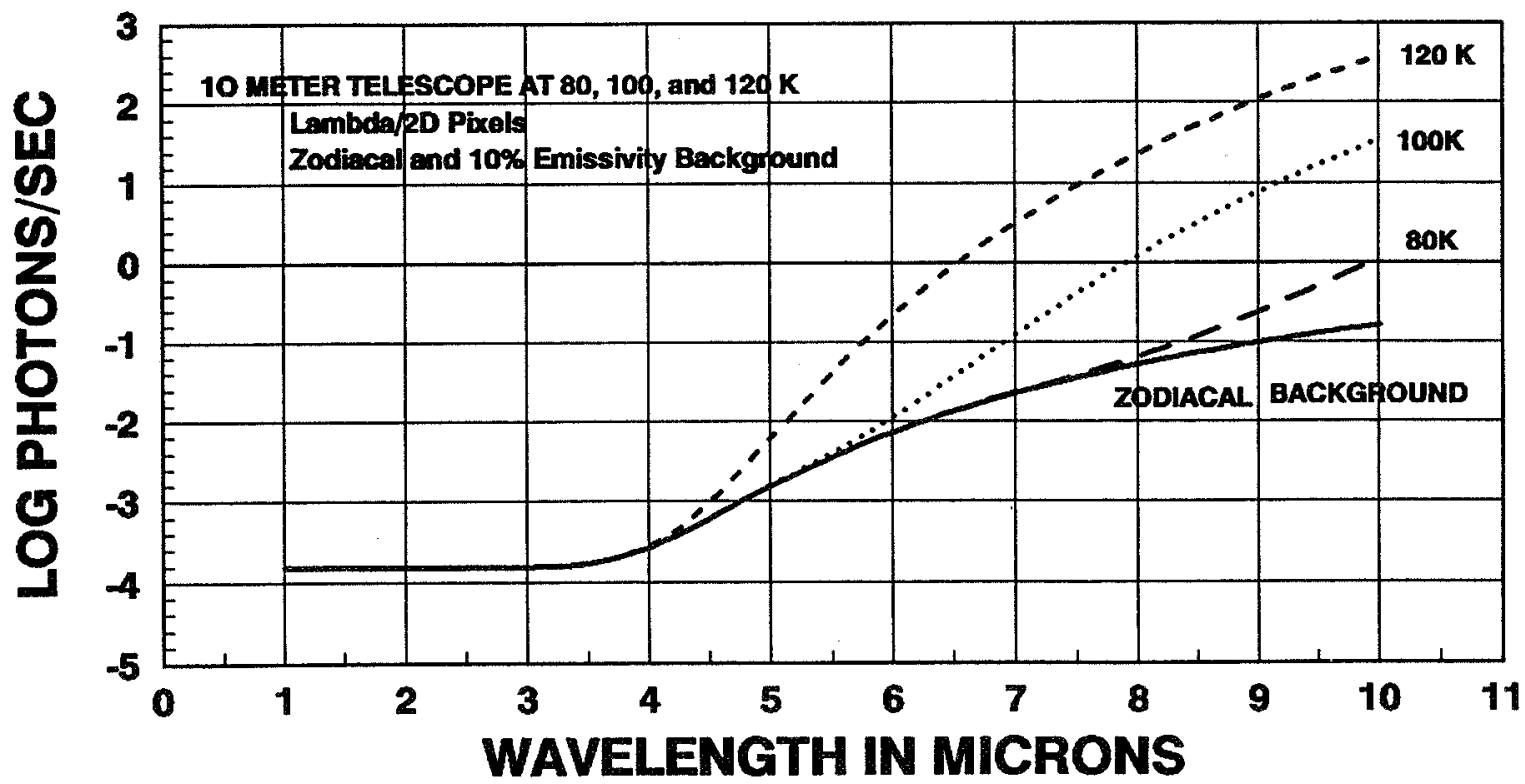


The spectroscopic background is the same as the imaging background except reduced by the much higher resolution. In Figure 2, the background is plotted for a bandwidth of $10^{-4}$ instead of 0.5 as in the imaging plot.

The region between 1 and $4 \mu \mathrm{m}$ has background fluxes substantially below any of the current detector dark currents. This will have to be a primary area of development to exploit the spectroscopic opportunities. The detectors will also require a large amount of radiation shielding to achieve the low photon background performance levels.

The low background in the 1 to $3 \mu \mathrm{m}$ region, coupled with the cosmological redshift offers a unique opportunity for a very deep survey of the Universe. The most advantageous region is 3.0-3.5 $\mu \mathrm{m}$ where galaxies at high redshift have their peak but where the thermal emission is not yet dominant. The warm temperature of the HST mirror starts to dominate at wavelengths longer than $1.7 \mu \mathrm{m}$ and has a much smaller mirror than the proposed telescope. The cold telescope has a distinct advantage in this area.

\section{Imaging goals: 1-4 $\mu \mathrm{m}$}

The HgCdTe $256 \times 256$ arrays developed by the Rockwell Corporation for the second generation HST instrument NICMOS represent present status of detectors in the short wavelength region. These are hybrid detectors which bond a HgCdTe photodiode array to a silicon switched MOSFET readout. The performance levels for $2.5 \mu \mathrm{m}$ cutoff arrays are given in Table 1. Comparison of the performance levels with the detector environment for imaging shows that the dark current is on the order of the background flux. Some reduction of the dark current will be advantageous but the detectors are near the requirements for imaging. Since the dark current rises steeply with cutoff wavelength some development will be needed to extend the low dark current performance to $4 \mu \mathrm{m}$ cutoffs.

For short integration times the read noise dominates over dark current and background as the detector noise source. Reduction of the read noise from 30 electrons to 10 electrons results in background limited performance after integrations on the order of 100 seconds. Further reduction is of course desirable but again the present detectors are close to the requirements. Extension of the pixel format to $1024 \times 1024$ will cover the expected field of view. This can be done with mosaicing via focal plane splitting. Unless the pixel sizes are reduced to approximately $10 \mu \mathrm{m}$ it will be difficult to extend a single hybrid to $1024 \times 1024$ due to the thermal mismatch between the sapphire substrate which carries the $\mathrm{HgCdTe}$ and the silicon multiplexer chip. Direct deposlt of $\mathrm{HgCdTe}$ on $\mathrm{Si}$ may be the answer for larger arrays.

Further refinement of the $\mathrm{HgCdTe}$ growth process should yield quantum efficiencies in the $80 \%$ range throughout the wavelength band. Current detectors can reach this Q.E. at various wavelengths but no detector to date has this efficiency over the whole wavelength region. 
Table 1. Imaging Performance of HgCdTe Array Detectors

Present status:

Dark current $<1 \mathrm{e} / \mathrm{sec}$ at $60 \mathrm{~K} ; 2.5 \mu \mathrm{m}$ cutoff wavelength

Read noise of $30 \mathrm{e}$

Average quantum efficiency is $65 \%$

$256 \times 256$ pixel format with $40 \mu \mathrm{m}$ pixels

Time to background limit for telescope is 1150 seconds

Development goals:

Dark current nearly adequate, extend performance to $4 \mu \mathrm{m}$

Reduce read noise to below 10 e; gives a time to background

limit for the telescope of less than 100 seconds

Increase quantum efficiency to $80 \%$

Extend the format to $1024 x 1024$ to cover the expected

field of view; larger format applicable at $1 \mu \mathrm{m}$

Develop monolithic $\mathrm{HgCdTe}$ or $\mathrm{HgCdTe} / \mathrm{Si}$ manufacturing processes

\section{Imaging goals: $4-10 \mu \mathrm{m}$}

Blocked Impurity Band (BIB) detectors represent the best current performance levels in the $4-10 \mu \mathrm{m}$ spectral region. The combination of high background and the excellent performance levels shown on Table 2 indicates that these detectors already meet most of the performance requirements for the proposed telescope. Since these detectors have significant gain in the readout the equivalent electron noise and dark current must be scaled by the gain. All of the performance figures presented have been adjusted for the gain effect. Some controversy still remains over the appropriateness of the scaling.

Production of large pixel format arrays still needs development from the current $10 \times 50$ to the required $512 \times 512$ format. There is potential for almost a factor of two gain in quantum efficiency. Finally the sensitivity of the detector to changes in the background flux needs to be corrected.

Table 2. Imaging Performance of BIB arrays

Present status:

Dark current is $4 \mathrm{e} / \mathrm{sec}$ (scaled by gain)

Read noise is 12 electrons (scaled by gain)

Average quantum efficiency is $45 \%$

$10 x 50$ pixel array format

At natural background limit after 1.2 seconds at $7 \mu \mathrm{m}$

At $100 \mathrm{~K}$ telescope limit in less than 1 second

Sensitive to background changes

Performance goals

Increase quantum efficiency to $80 \%$

Increase pixel format to $512 \times 512$ to match telescope field of view Decrease sensitivity to background changes 


\section{Camera performance}

Figure 3 indicates the time needed to achieve a signal-to-noise of 10 in a single pixel versus the flux in Janskys falling on that pixel. For diffraction limited sampling the flux from a point object is spread over several pixels, therefore, the limiting flux for a single object is modified by the area in pixels of the point spread function. Since this varies for different cameras we have simply given the results in terms of limiting flux per pixel. The wavelengths of 3 and $7 \mu \mathrm{m}$ are chosen as being typical of the band. Again the pixels are chosen to be diffraction limited and bandwidth as $50 \%$ to calculate the background flux on the pixel. The change in slope of the curves from 1 to $1 / 2$ occurs when the background or dark current noise dominates the read noise. When the observation is read noise limited the signal to noise improves directly with integration time. For dark current or background noise the signal to noise improves as the square root of the time. Shot noise or source noise has some effect on the $7 \mu \mathrm{m}$ curve since it has a read noise of only 4 electrons whereas the shot noise for a signal to noise of 10 is 10 electrons in the absence of other noise. The $3 \mu \mathrm{m}$ curve has a read noise of 30 and is not significantly affected by shot noise for a signal to noise of 10 .

\section{Tigure 3}

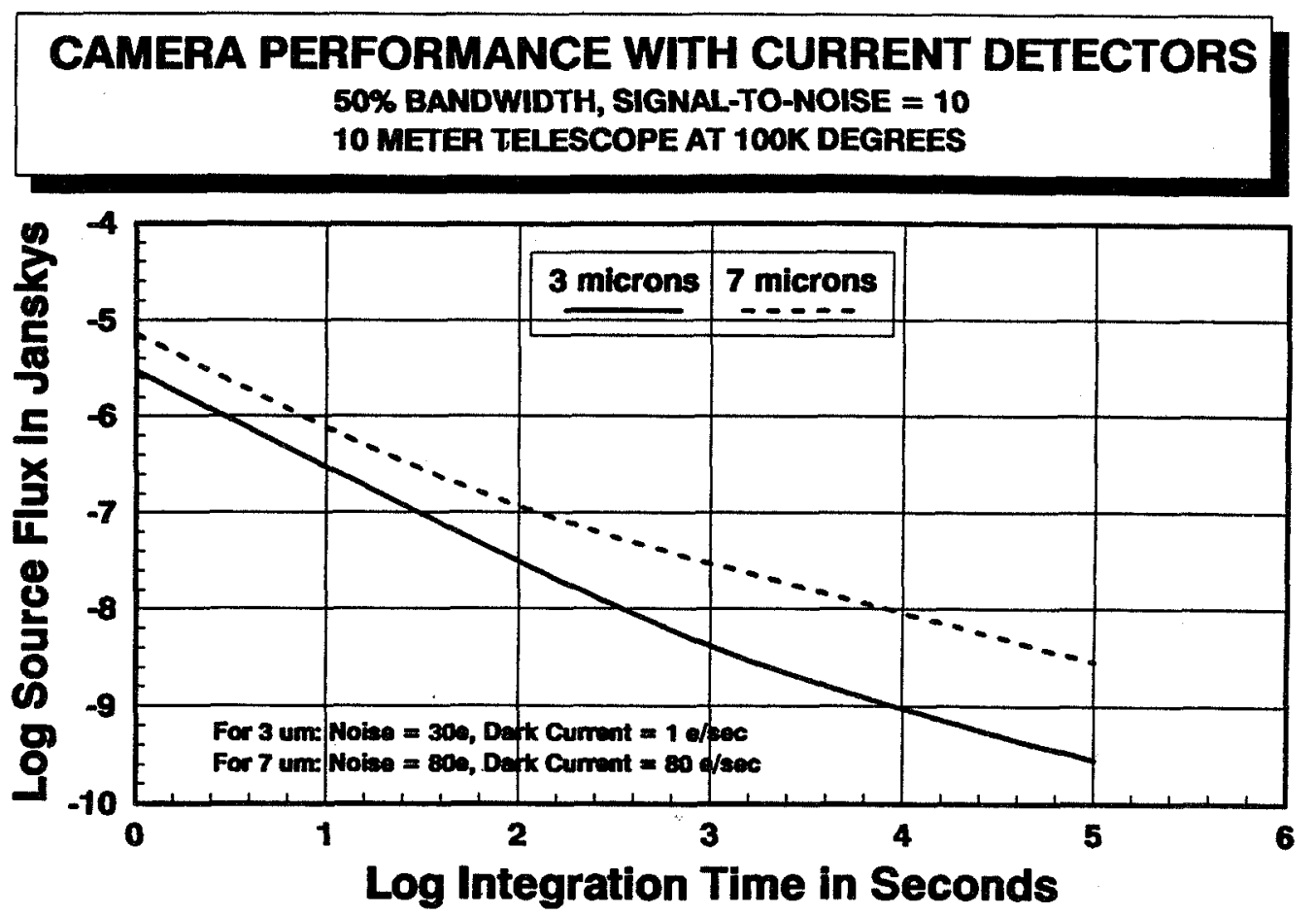

\section{Spectroscopic goals $1-4 \mu \mathrm{m}$}

The performance levels for current 1-4 $\mu \mathrm{m}$ detectors are shown in the Table 3 , which is of course similar to that of the imaging performance levels. However, the goals are signiflcantly different due to the low background flux on the detectors. The primary goal is the reduction of dark current by as large a factor as possible. The radiation environment must be considered in setting these goals since without a very large amount of shielding, the radiation flux will greatly exceed the photon flux in the spectroscopic bandwidth. 
Current $\mathrm{HgCdTe}$ detectors are not at the theoretical limit of the dark current. Gains of several hundred are theoretically possible, although the source of the excess dark current is not well understood. It will have to be the astronomical community which drives the materials research to achieve the low dark currents as most other applications are well served by the currently achievable dark current levels.

The read noise goal of 3 electrons comes from the qualitative requirement that a useful spectrum should have a signal to noise of at least 10 which requires 100 photons with a shot noise of 10. At 3 electrons the read noise is signiflcantly less than the shot noise. Read noises of 3 electrons and less have been achieved with optical CCDs which gives some confldence that similar noise levels can be achieved with the infrared arrays.

For high resolution spectroscopy larger formats are required to accommodate cross dispersed spectra and multi-object spectroscopy with flbers. Formats of 2048x2048 are useful in this context. Image plane splitting and reimaging may be more difficult in the spectroscopic mode and therefore a strong driver for the alternatives to the hybrid tech niques described earlier.

Table 3. Spectroscopic Performance of HgCdTe array detectors

Present status:

Dark current $<1 \mathrm{e} / \mathrm{sec}$ at $60 \mathrm{~K} ; 2.5 \mu \mathrm{m}$ cutoff wavelength

Read noise of $30 \mathrm{e}$

Average quantum efficiency of $65 \%$

256x256 pixel format with $40 \mu \mathrm{m}$ pixels

For $R=10,000$ Spectroscopy, never at the telescope background limit

Development goals:

Reduce the dark current by 10,000 or to radiation limit

Reduce the read noise to below 3 e; at this level the read noise does not significantly affect a $\mathrm{S} / \mathrm{N}=10$ Spectrum

Increase average quantum efficiency to $80 \%$

Extend the format to at least $2048 \times 2048$ to accommodate multi-object cross dispersed spectroscopy

\section{Spectroscopic goals: 4-10 $\mu \mathrm{m}$}

The goals for the detector development in the $4-10 \mu \mathrm{m}$ range are similar to those of the $1-4 \mu \mathrm{m}$ region. At the short wavelength end the same reduction in dark current is required. At $10 \mu \mathrm{m}$ a reduction of about 40 is required to reach the zodiacal limit. If the telescope is not cold enough to reach the zodiacal limit less stringent requirements may be accommodated. 
Table 4. Spectroscopic Performance of BIB Arrays

Present status:

Dark current is $4 \mathrm{e} / \mathrm{sec}$ (scaled by gain)

Read noise is 12 electrons (scaled by gain)

Average quantum efficiency is $45 \%$

$256 \times 256$ pixel array format

At the natural background limit after $5700 \mathrm{~s}$ at $7 \mu \mathrm{m}, \mathrm{R}=10,000$

At $100 \mathrm{~K}$ telescope limit after 1,500 seconds at $7 \mu \mathrm{m}, \mathrm{R}=10,000$

Performance goals:

Dark current reduced by 10,000 or at the radiation limit

Read noise $<3$ electrons for $\mathrm{S} / \mathrm{N}=10$ spectra

Increase quantum efficiency to $80 \%$

Increase pixel format to $2048 \times 2048$ for multiple

object cross dispersed spectroscopy

\section{Spectrometer performance}

The predicted spectrometer performance (Figure 4) has two distinct regions, similar to the imaging performance chart. At short integration times the read noise dominates and the limiting flux vs time relation is linear. At longer integration times the dark current limits the $3 \mu \mathrm{m}$ spectrum and the natural background limits the $7 \mu \mathrm{m}$ spectrum. In both of these cases the limiting flux decreases with the square root of the time. The higher long wavelength background causes the cross over of the two curves around 200 seconds.

Figure 4
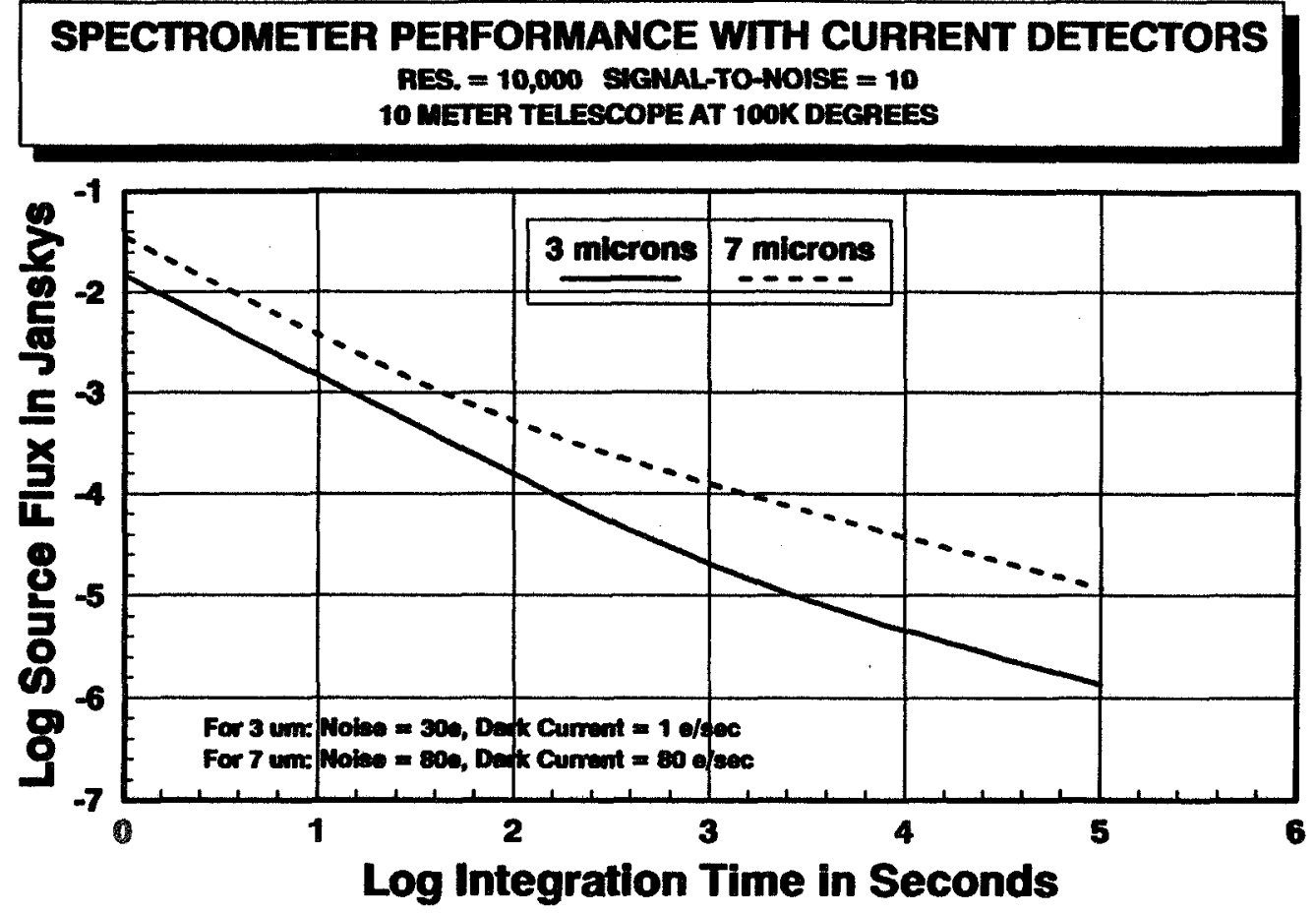


\section{Conclusions}

The basic conclusions of this presentation are listed below.

1. The performance levels of current near and mid-IR detectors are near the broad band imaging requirements of a $10 \mathrm{~m}, 100 \mathrm{~K}$, Space Telescope.

2. Current detector technology does not meet the spectroscopic opportunities of the telescope.

3. Even a present detector performance levels, the telescope offers a dramatic increase in detectivity.

4. Telescope temperatures near $60 \mathrm{~K}$ are required for true background limited performance.

The predominant consideration in the conclusions is the background flux on the detector since the remaining considerations of field of view etc. are more qualitative than quantitative. The radiation sensitivity of the new detectors is still under study.

It is clear that even the current infrared array performance levels make a $10 \mathrm{~m}, 100$ $\mathrm{K}$ telescope a very exciting prospect. Future lowering of read noise and dark current levels will greatly increase the spectroscopic performance levels and will to a lesser degree enhance the imaging capabilities. Emphasis should be applied to finding the best detector array to exploit the region around 4 microns where the background is near a minimum and the flux from highly redshifted objects will be at a maximum.

In order to exploit the low background levels of such a telescope, significant effort needs to be applied to reducing the radiation levels on the detectors and to hardening them against radiation. Particularly important is the hardening against energetic protons which is the main component of the background high energy radiation. There is a tradeoff on telescope location which will be important in this context. Although it imposes operational constraints, the low earth orbit of HST has a significantly lower radiation density than the high earth orbit proposed for the next telescope. Location on the lunar surface may allow more shielding by either attached material or shielding by lunar material. From a scientific production viewpoint there is not yet a clear discriminator between orbital and lunar locations.

\section{DISCUSSION}

Diner: Can you say anything about arrays at 10 microns or larger?

Thompson: I am not an expert in this area, perhaps other people could comment.

Disenhardt: Si BIB detectors will operate to $28 \mu \mathrm{m}$. We should consider making $\lambda_{\text {mam }}=30 \mu \mathrm{m}$ for this telescope, even if its not background limited, its still far better than observing from the ground, and complements LDR. Also SIRTF PIs are doing tests on IR detectors with protons and $\gamma \mathbf{s .}$

Moos: What do we know about radiation damage?

Thompson: We have just begun radiation testing of short wavelength cut off $\mathrm{HgCdTe}$. It recovers immediately from $\gamma$-ray and e-radiation doses equivalent to the SAA. We still have to do proton and long duration tests.

Rehfield: What temperature was the Rockwell Array operating at for demonstration?

Thompson: The array was operating at liquid $\mathrm{N}_{2}$ temperature, $77^{\circ} \mathrm{K}$. The NICMOS array will be operated at $60^{\circ} \mathrm{K}$ where the dark current will be about a factor 10 less. 


\section{Page intentionally left blank}




$$
\begin{aligned}
& \text { mo pros } \\
& \text { powe }
\end{aligned}
$$

\section{Session 6}

Space Logistics 


\section{Page intentionally left blank}




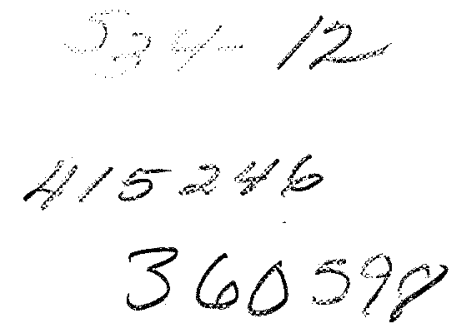

\title{
Advantages of High vs. Low Earth Orbit for SIRTF
}

\author{
Peter Eisenhardt* and Michael W. Werner, NASA-Ames Research Center
}

\section{Introduction}

While the subject of this workshop, which we'll refer to as ET (for Enlightenment Telescope), is a dazzling successor to the Hubble Space Telescope, its location is unlikely to be the Low Earth Orbit (LEO) used by HST. Locations suggested for ET include High Earth Orbit (HEO) and the moon. The first space telescope to occupy HEO will be the liquid helium cooled Space Infrared Telescope Facility (SIRTF - Figure 1). (For reasons given in Section 3.3.2, we do not consider geosynchronous orbits, such as that occupied by the International Ultraviolet Explorer telescope, to be in the HEO class.) The selection of HEO for SIRTF was the outcome of a recent study led by the Ames Research Center which showed significant advantages for SIRTF in HEO vs. LEO. This article summarizes the main results of that study. We begin with a review of SIRTF's rationale and requirements, in part because the IR capabilities and low temperature proposed for ET make it something of a successor to SIRTF as well as to HST. We conclude with some comments about another possible location for both SIRTF and ET, the Earth-Sun L2 Lagrangian point.

\section{SIRTF's Rationale and Requirements}

The fundamental rationale for a cryogenic telescope in space is shown in Figure 2, which compares the infrared background brightness of the earth's natural astrophysical environment with that encountered by an ambient temperature telscope operating within the atmosphere. The natural astrophysical background radiation includes contributions from the zodiacal dust cloud, diffuse galactic dust, and the $3 \mathrm{~K}$ cosmic background. These natural backgrounds are more than a million times lower than those characteristic of Earthbound ambient temperature telescopes, a reduction comparable to the difference between night and day in visible sky brightness. The minima in the natural backgrounds around 3 and $300 \mu \mathrm{m}$ are particularly noteworthy, as sensitive observations in these windows may provide unique views of the distant, early universe. Because the limiting sensitivity improves with the square root of the background brightness, even a modest sized cryogenic space telescope, such as SIRTF, can have a thousand or more times the sensitivity of a large ground based telescope. SIRTF's goal is therefore to reduce both the thermal emission of the telescope and scattered and off-axis radiation sufficiently so that its performance can be "natural-background limited" at wavelengths between 2 and $\gtrsim 200 \mu \mathrm{m}$.

SIRTF will be NASA's Great Observatory for infrared astronomy. Most of the observing time over the SIRTF mission lifetime (currently estimated as 5 years with a substantial safety margin) will be used by general observers drawn from the broad astronomical community. The SIRTF instrument complement, selected in 1984, will provide a broad range of capabilities (Table 1). Scientific objectives of the Multiband Imaging Photometer and the Infrared Array Camera include imaging of objects ranging from comets to galaxies, mapping of extended regions of star formation, and deep surveys at SIRTF's limiting sensitivity. Scientific objectives of the Infrared Spectrograph include

\footnotetext{
"Department of Astronomy, University of California, Berkeley, CA 94720
} 


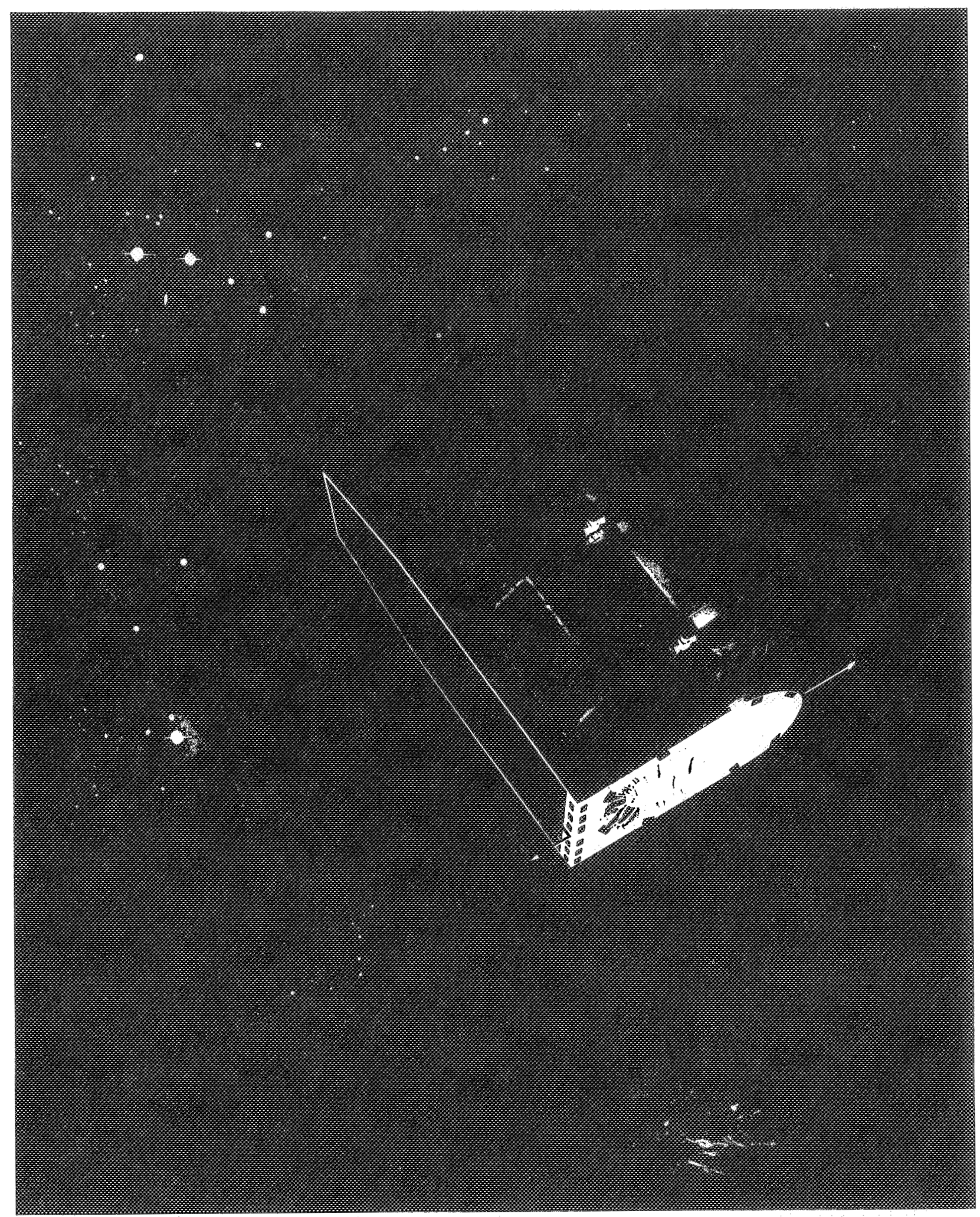

Figure 1: The Space Infrared Telescope Facility (SIRTF), shown in its new high altitude Warth orbit (HEO) configuration. 
ATMOSPHERIC TRANSMISSION

MOUNTAIN TOP
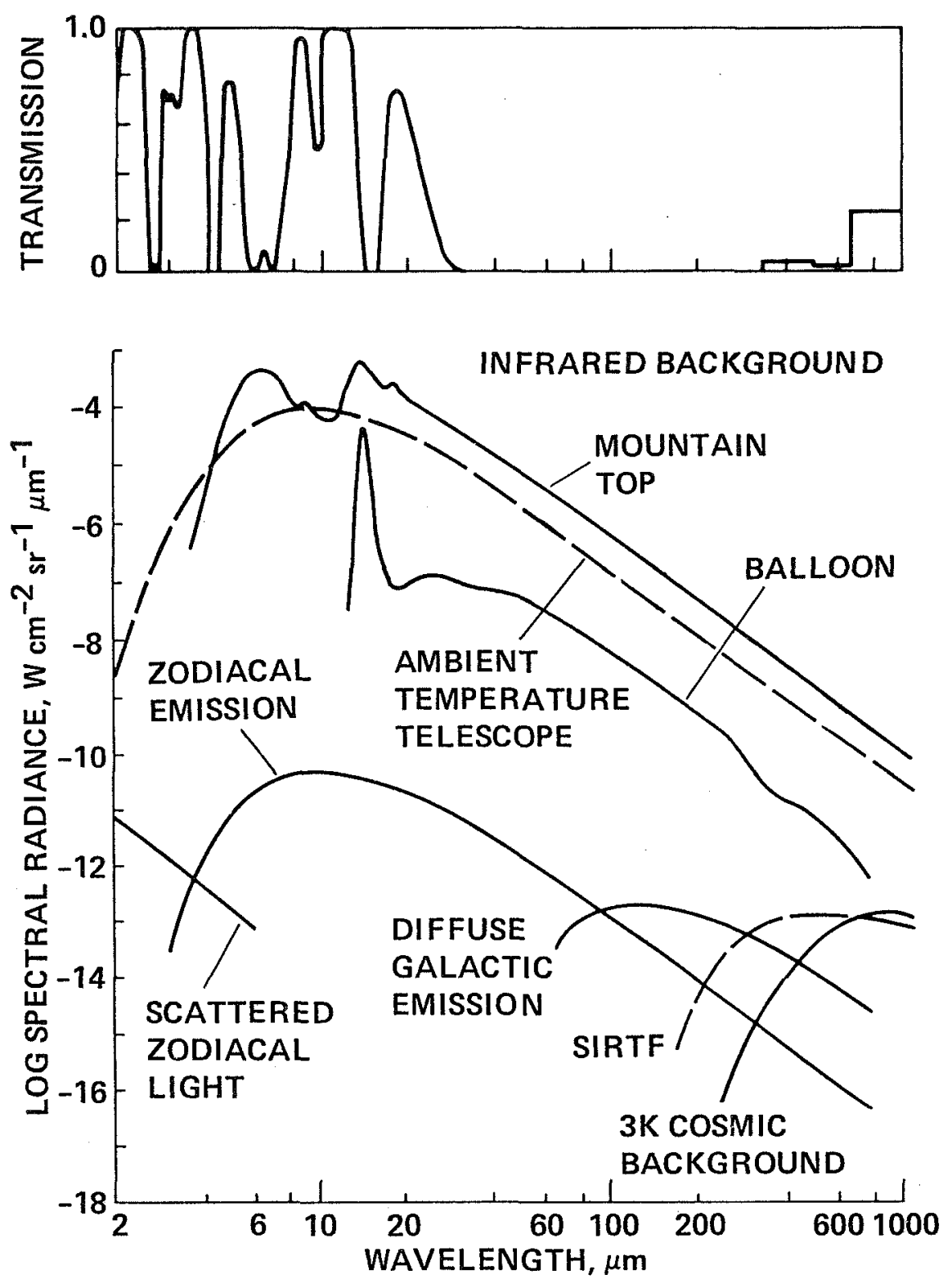

Figure 2: Atmospheric transmission and background fluxes for infrared astronomy. The upper panel shows the atmospheric transmission seen from a good mountain top observatory, illustrating that much of the infrared spectrum is completely inaccessible from the ground. Solid curves in the lower panel show the infrared background flux in three observing environments: a good mountain top observatory, high balloon altitudes, and space. Atmospheric background flux at airplane altitudes is intermediate between the curves for the mountain observatory and the balloon. The natural astrophysical background radiation in space is the sum of scattering and emission by zodiacal dust grains, emission by diffuse galactic dust, and the $3 \mathrm{~K}$ cosmic background radiation. Dashed curves show the background contributions from optimized infrared telescopes: an ambient temperature instrument (such as would be used from the ground, airplanes, or balloons) and SIRTF. The total background at the infrared detector is the sum of the natural and telescopic contributions. 
Table 1: SIRTF Instrumentation Summary

\begin{tabular}{|c|c|c|}
\hline Instrument & Principal Investigator & Characteristics \\
\hline $\begin{array}{l}\text { Infrared Array Camera } \\
\text { (IRAC) }\end{array}$ & $\begin{array}{l}\text { G. Fazio, Smithsonian } \\
\text { Astrophysical Observa- } \\
\text { tory }\end{array}$ & $\begin{array}{l}\text { Wide field and diffraction limited imag- } \\
\text { ing, } 1.8-30 \mu \mathrm{m} \text {, using arrays with up to } \\
256 \times 256 \text { pixels. Simultaneous viewing in } \\
\text { three wavelength bands, selectable filters. } \\
\text { Polarimetric capability. }\end{array}$ \\
\hline $\begin{array}{l}\text { Infrared } \\
\text { (IRS) }\end{array}$ & $\begin{array}{l}\text { J. Houck, Cornell Uni- } \\
\text { versity }\end{array}$ & $\begin{array}{l}\text { Grating spectrometers, } 2.5-200 \mu \mathrm{m} \text {, using } \\
\text { two dimensional detector arrays. Resolv- } \\
\text { ing power from } 100 \text { to } 2500 \text {. Low and high } \\
\text { resolution options at most wavelengths. }\end{array}$ \\
\hline $\begin{array}{l}\text { Multiband Imaging Pho- } \\
\text { tometer for SIRTF } \\
\text { (MIPS) }\end{array}$ & $\begin{array}{l}\text { G. Rieke, University of } \\
\text { Arizona }\end{array}$ & $\begin{array}{l}\text { Background limited imaging and photom- } \\
\text { etry, } 3-200 \mu \mathrm{m} \text {, using small arrays with } \\
\text { pixels sized for complete sampling of Airy } \\
\text { disk. Wide field, high resolution imaging, } \\
50-120 \mu \mathrm{m} \text {. Broadband photometry and } \\
\text { mapping, } 200-700 \mu \mathrm{m} \text {. Polarimetric ca- } \\
\text { pability. }\end{array}$ \\
\hline
\end{tabular}

studies of composition and physical conditions in planetary atmospheres, the interstellar medium, and external galaxies. The spectrograph will also be used to determine the nature of objects discovered in SIRTF's surveys, many of which will be too faint to be studied in detail from other platforms. SIRTF's instruments are more fully described in Ramos et al. (1988). More complete discussions of SIRTF's scientific objectives and potential can be found in Rieke et al. (1986) and in a series of articles in Astrophysical Letters and Communications (Vol. 27 No. 2, pp. 97 ff., 1987).

SIRTF's scientific objectives require not only high sensitivity but also excellent performance in many other areas. The resulting system parameters and requirements are listed in Table 2. Also shown for comparison are the parameters for the Infrared Astronomical Satellite (IRAS). SIRTF's gains over the IRAS survey in wavelength coverage, spatial and spectral resolution, sensitivity, and lifetime call to mind the gains of HST over the Palomar sky survey, as befits a Great Observatory. SIRTF's biggest gains over IRAS result from the fact that its instruments will be equipped with large arrays having up to 65,000 detector elements. The current status of IR detector development is the subject of the 1989 Proceedings of the 3rd Infrared Detector Technology Workshop, C.R. McCreight, editor.

We have also listed in Table 2 the corresponding parameters for ET, assuming a temperature of $100 \mathrm{~K}$ and $10 \%$ emissivity. Using diffraction limited pixels, SIRTF and ET will see the same background levels from 2 to about $6 \mu \mathrm{m}$, beyond which the thermal emission of ET starts to dominate. This region includes the $3 \mu \mathrm{m}$ cosmic "window", and extends to wavelengths where extinction coefficients are only a few percent of that in visible light. ET's wavelength range should probably extend to $30 \mu \mathrm{m}$, for overlap with the Large Deployable Reflector, and because much of this regime is unobservable from the ground. ET's larger collecting area will provide over one hundred times more signal per pixel than SIRTF for unresolved sources, which translates directly into improved sensitivity from 2-6 $\mathrm{mm}$. Even with its higher temperature, ET's sensitivity will be about nine times better than the $6 \mu \mathrm{Jy}$ achieved by SIRTF at $10 \mu \mathrm{m} \mathrm{(5} \mathrm{mJy} \mathrm{is} \mathrm{a} \mathrm{comparable} \mathrm{sensitivity} \mathrm{from}$ current ground based telescopes), equal to SIRTF's at $20 \mu \mathrm{m}$, and only two times less than SIRTF's at $30 \mu \mathrm{m}$. ET integration times to achieve the same point source sensitivity at $10 \mu \mathrm{m}$ will be ten thousand times shorter than for a groundbased $10 \mathrm{~m}$ telescope such as the Keck, even if adaptive 
Table 2: Comparison of SIRTF, IRAS and ET System Parameters

\begin{tabular}{llll}
\hline \hline Parameter & SIRTF & IRAS & ET \\
\hline Mirror Diameter & $95 \mathrm{~cm}$ & $60 \mathrm{~cm}$ & $10 \mathrm{~m}$ \\
Wavelength Coverage & $1.8-700 \mu \mathrm{m}$ & $8-120 \mu \mathrm{m}$ & $0.1-10 \mu \mathrm{m}$ \\
Diffraction-Limited Wavelength & $2.5 \mu \mathrm{m}$ & $\sim 15 \mu \mathrm{m}$ & $0.1 \mu \mathrm{m}$ \\
Angular Resolution at Wavelength $\lambda$ & $\lambda / 4 \mu \mathrm{m}$ arcsec & $\gtrsim 15$ arcsec & $\lambda / 40 \mu \mathrm{m}$ arcsec \\
Pointing Stability/Accuracy & $0.15 / 0.15 \operatorname{arcsec}$ & $2 \operatorname{arcsec}$ & $0.0005 / 0.01 \mathrm{arcsec}$ \\
Sensitivity: ${ }^{3}$ & & & \\
$\quad 3 \mu \mathrm{m}$ & $0.5 \mu \mathrm{Jy}$ & & $5 \mathrm{nJy}$ \\
$10 \mu \mathrm{m}$ & $6 \mu \mathrm{Jy}$ & $70 \mathrm{mJy}$ & $0.7 \mu \mathrm{Jy}$ \\
$\quad 60 \mu \mathrm{m}$ & $150 \mu \mathrm{Jy}$ & $70 \mathrm{mJy}$ & \\
Number of Detectors & $\gtrsim 100,000$ & 62 & $210^{8}$ \\
Spectral Resolving Power & $\gtrsim 2000$ & 20 & $10^{5}$ \\
Mode & Observatory & Survey & Observatory \\
Lifetime & $>5$ years & 10 months & Many years \\
\hline
\end{tabular}

${ }^{1}$ One sigma in 500 seconds of integration for SIRTF and ET, and in one survey pass for IRAS.

optics are used to eliminate atmospheric seeing. For extended sources, ET provides images in a given integration time with the same signal to noise ratio per pixel as SIRTF out to $6 \mu \mathrm{m}$, but with over ten times the angular resolution. Of course these gains are made only for a relatively small part of SIRTF's spectral range, but they illustrate how potent the combination of large aperture and low temperature is in the infrared.

\section{SIRTF Mission Options Study}

NASA's decision following the Challenger accident to employ a mixed fleet of launch vehicles encouraged the SIRTF team to explore alternate mission approaches to achieving SIRTF's science objectives. In an initial study phase concluded in mid-1988, a circular orbit at an altitude of approximately $100,000 \mathrm{~km}$ above the Earth was selected for detailed study and comparison with the $900 \mathrm{~km}$ altitude baseline mission (Figure 3). These two altitudes are, respectively, just above and below the Earth's Van Allen belts, whose trapped energetic particles would degrade detector performance (see Section 3.3.2). The rules of the comparison stipulated that both missions satisfy the requirements in Table 2 and that both be capable of supporting the complement of three instruments selected for SIRTF.

The HEO mission concept outlined below is the result of six months of intensive study headed by the Ames Research Center with support from the SIRTF Science Working Group, the Jet Propulsion Laboratory, the Marshall Space Flight Center, and the Lewis Research Center. An alternative HEO option, in which SIRTF is flown at the Earth-Sun L2 Lagrangian point has been proposed by the Goddard Space Flight Center, and is briefly discussed in Section 3.4. The LEO SIRTF mission is discussed in more detail in Werner et al. (1986). Further optimization of the HEO concept will take place during the SIRTF Phase B studies beginning in 1990. The current schedule for SIRTF calls for initiation of the final design and fabrication activities (Phase C/D) in 1993, leading to launch in 1998. 
$900 \mathrm{~km}$ altitude

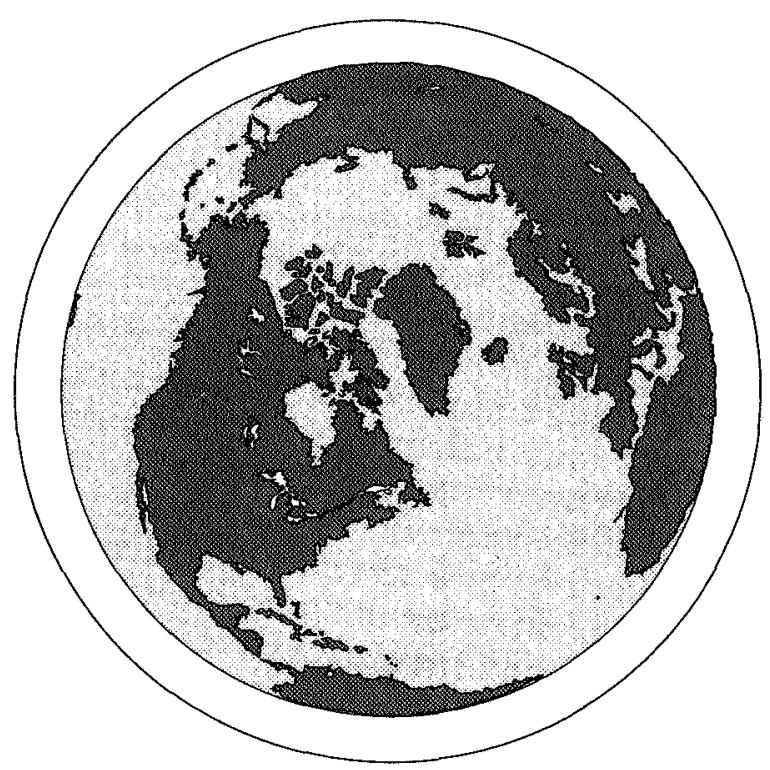

$100,000 \mathrm{~km}$ altitude

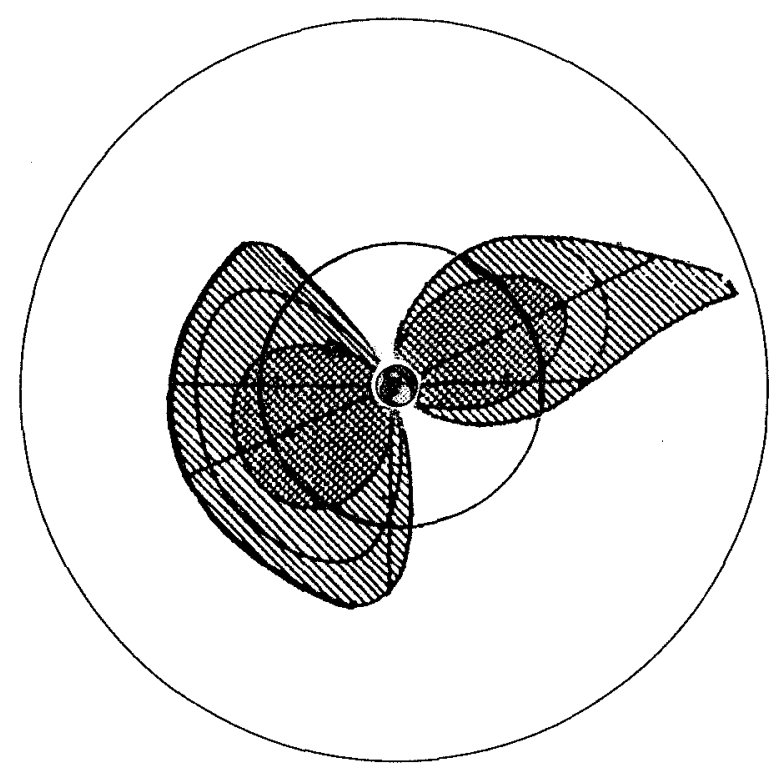

Figure 3: Relative size of the Earth in comparison to low Earth orbit (LEO - left), and high Earth orbit (HEO - right). Also shown on the right are the relative size of a geosynchronous orbit $(36,000$ $\mathrm{km}$ altitude) and of the Van Allen belts (adapted from Ness 1969). The inclination of LEO and HEO for SIRTF is $28.5^{\circ}$, while the Van Allen belts are shown in the noon-midnight plane (inclination $90^{\circ}$ ) with the sun to the left. The tilted axis of symmetry of the belts is the geomagnetic equator.

\subsection{The HEO Mission}

The new mission will use a Titan IV/Centaur to launch a $5 \mathrm{~m}$ long, approximately 4,500 kg SIRTF into a $100,000 \mathrm{~km}$ altitude, $28.5^{\circ}$ inclination orbit with a period of $\approx 100$ hours. Seen from this altitude, the Earth subtends an angle of less than $7^{\circ}$ (v8. $122^{\circ}$ in LEO), 80 it is possible to maintain a flexible viewing strategy while constraining the telescope to point no closer than $80^{\circ}$ to the Earth or Sun limb. The thermal load due to the Earth is much smaller in HEO than in LEO, and the high orbit allows the use of a fixed solar panel which shades the telescope. Together, these effects lower the predicted temperature of the telescope outer shell to $\lesssim 110 \mathrm{~K}$. Eclipses are infrequent in this orbit; the time and date of launch can be selected to orient the orbit so that the spacecraft passes through the Earth's shadow only a few days each year. Reaction wheels provide slewing and fine pointing capability. Environmental disturbance torques are small, but momentum control requires the use of cold gas because the Earth's magnetic field is too weak for momentum dumping at 100,000 $\mathrm{km}$. The helium boil off from the cryogen system and a small tank of compressed helium gas will be used for this purpose. Science and engineering data will be stored on-board the spacecraft and downlinked 8 hours per day via fixed omnidirectional antennae to the $26 \mathrm{~m}$ dishes of the Deep Space Network.

Figure 4 shows a cutaway view of the telescope concept for SIRTF. The IRAS heritage is shown in the annular cryogen tank, containing 4000 liters of superfluid helium which cools the optics and baffles, and in the truncated "sugar scoop" aperture shade, which allows the telescope to point as close as $80^{\circ}$ to the Earth and Sun limb. The optical system is an f/24 Ritchey-Chrêtien design with a $95 \mathrm{~cm} \mathrm{f} / 2.3$ primary mirror. The secondary mirror will be used for conventional chopping at the longer wavelengths and for flat-fielding and scanning procedures at the shorter wavelengths. Within the multiple instrument chamber, a rotatable dichroic tertiary mirror will direct the entire seven arcminute field of view to whichever instrument is in use, while the optical image is passed to a fine guidance sensor which will be used with the spacecraft gyros to provide pointing and stabilization. 


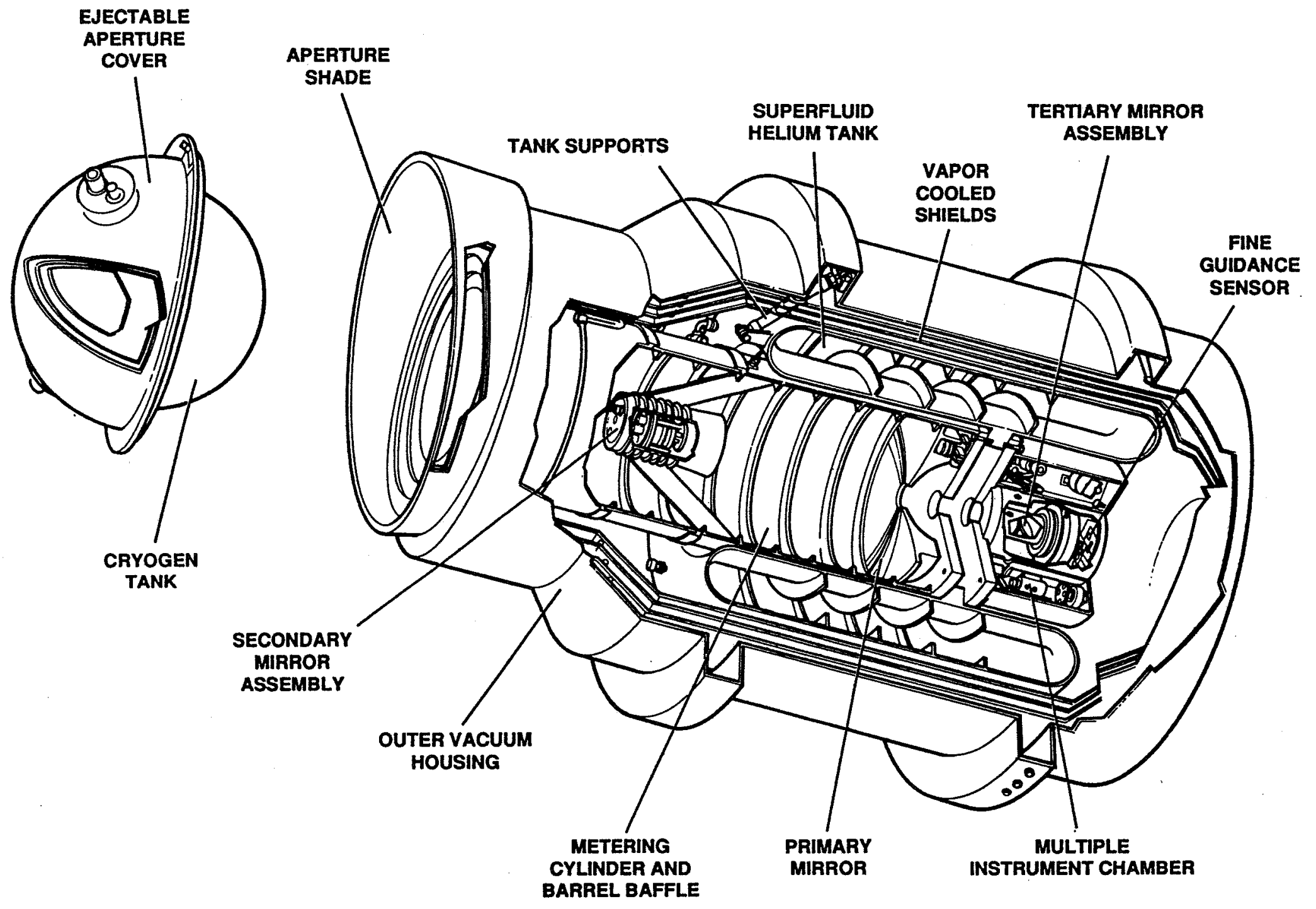

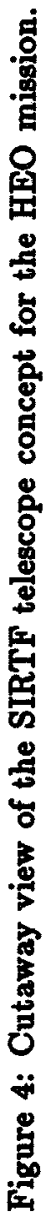




\subsection{Technical Concerns}

The two principal technical concerns which arose during the study of the HEO SIRTF option were in the areas of launch vehicle capability and lifetime. These issues are fundamental for ET as well. The present HEO baseline configuration for SIRTF has a mass of $\mathbf{4 3 7 0} \mathbf{~ k g}$. By comparison, the expected Titan IV/Centaur launch capability to $100,000 \mathrm{~km}$ for a SIRTF sised payload is at least $5770 \mathrm{~kg}$. It is felt that the $1400 \mathrm{~kg}$ difference ( $36 \%$ of the current mass excluding the helium) is adequate margin in this critical area. However, launching a $\gtrsim 15,000 \mathrm{~kg}, 12 \mathrm{~m}$ wide ET into HEO is clearly a formidable challenge. (Note that the Titan's launch capability to geosynchronous orbit is only slightly larger than to HEO).

The cryogen lifetime is an issue for SIRTF in HEO because on-orbit cryogen refill, which was to be used in LEO to achieve the five year lifetime, will not be available in this option. Preliminary optimisation of the $\mathbf{4 0 0 0}$ liter HEO system yields an estimated useful on-orbit cryogen lifetime (exclusive of losses due to launch holds, on-orbit cooldown, etc.) of six years, giving a 20\% margin over the requirement. The dramatic increase over the 2.5 year lifetime predicted for the same size dewar and the same instrument complement in LEO reflects:

1. the lower outer shell temperature (110K vs. 220K) achievable in HEO due to the greatly reduced heat load from the Earth and to a lesser extent to the shading provided by the fixed solar panel; and

2. reduction by more than an order of magnitude in the "aperture load" - the power radiated into the dewar by the aperture shade - which results from the fact that the aperture shade in HEO is both smaller and colder than in LEO.

Cryogen refill may be an issue for ET also: ET's IR detectors will almost certainly require temperatures lower than can be achieved using passive cooling. The difficulty of access to HEO after the initial launch may be a broader concern for ET, because of the possible need for on-orbit servicing in general to achieve long life, and for on-orbit assembly as a way to reduce the launch diameter and/or mass.

\subsection{Comparison of HEO and LEO Missions}

\subsubsection{Overall Conflguration}

Figure 3 shows the relative sizes of the Earth and SIRTF'S orbit for the HEO and LEO missions, while Figure 5 compares the general configuration of the orbiting observatories in the two missions. Both missions have an orbital inclination of $28.5^{\circ}$ to maximize the launch capability and to permit servicing in the LEO mission. The 280 -fold reduction in solid angle subtended by the Earth in HEO leads to the thermal advantages cited above and also permits more freedom in telescope pointing, even though the solar and terrestrial avoidance angles are larger in HEO. These larger avoidance angles cannot be satisfied when SIRTF passes directly between the Earth and Sun in the LEO mission. The $122^{\circ}$ angle subtended by the Earth permits Sun and Earth avoidance angles no greater than $59^{\circ}$ at these times, which occur for several orbits approximately every 28 days. The use of the larger avoidance angles $\left(80^{\circ}\right)$ in HEO leads to a smaller aperture shade, which in turn permits the forward portion of the telescope baffle tube to be shortened while maintaing the required rejection of stray radiation. The reduction in aperture shade size and forebaffle length lead to the overall shortening of the system shown in Figure 5. Note also in Figure 5 that the solar arrays and antennae are fixed in the HEO system but must be deployable and steerable in LEO. The dewar, optical system, and instruments are essentially identical in the two concepts. Thus the analysis and technology work done on these system elements for LEO carries over directly into the HEO system. 

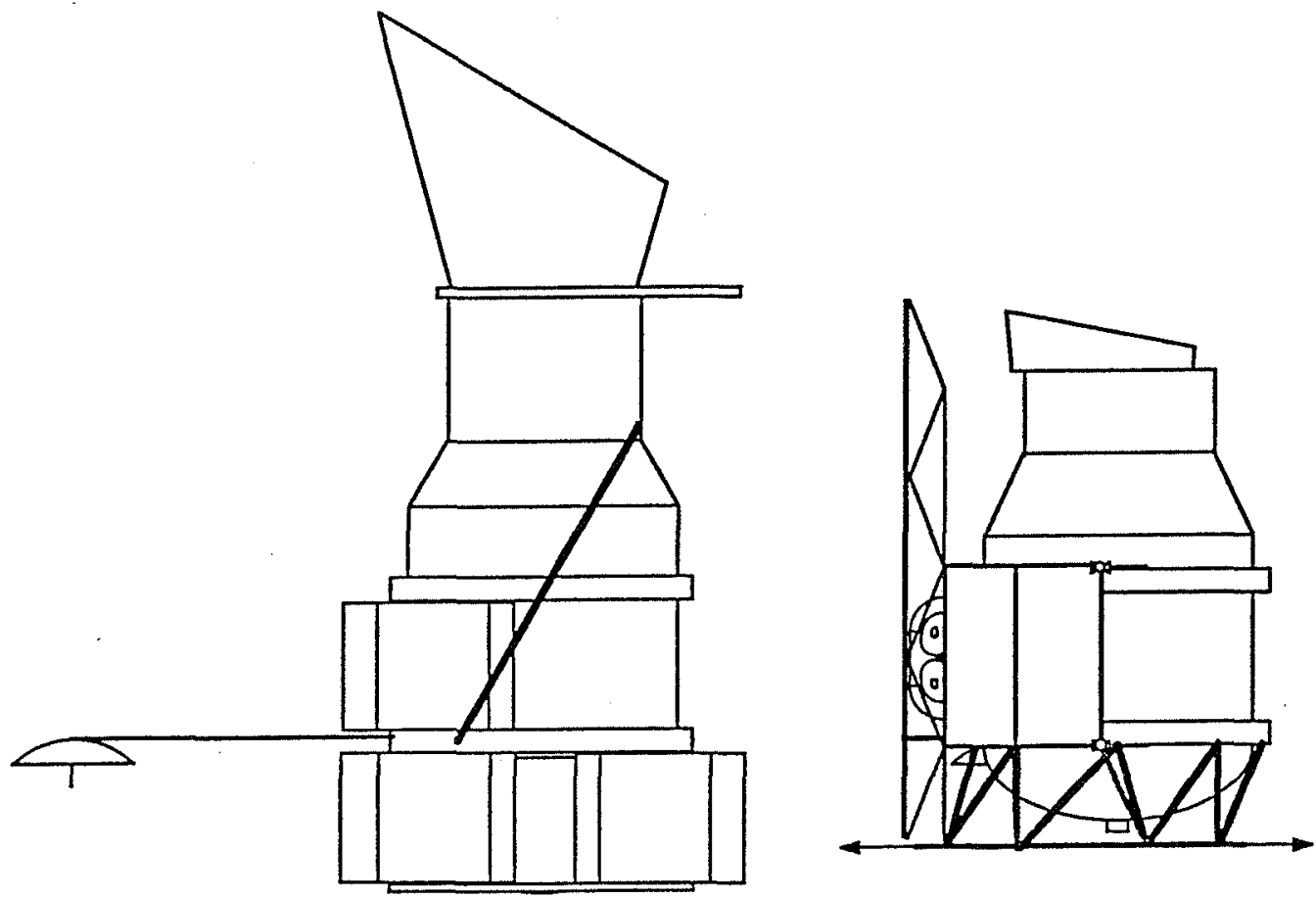

Figure 5: General configuration of the LEO (left) and HEO (right) SIRTF concepts. The masses and lengths of the satellites are $6620 \mathrm{~kg}$ and $4370 \mathrm{~kg}$; and $8 \mathrm{~m}$ and $5 \mathrm{~m}$ respectively.

\subsubsection{Ionizing Radiation Environment}

The locations of the orbits considered in the study were largely driven by the ionizing radiation environment, which has three main components: particles trapped in the Earth's magnetic field, galactic cosmic rays, and energetic particles produced in solar flares (see Stassiniopoulos and Raymond 1988 for a comprehensive discussion).

Protons with energies up to $500 \mathrm{MeV}$ are trapped in the Earth's magnetic field above an altitude of $\approx 1000 \mathrm{~km}$, while trapped electrons with energies up to $7 \mathrm{MeV}$ are found to $70,000 \mathrm{~km}$ altitude in the solar direction, and substantially farther in the anti-solar direction. These regions define the Earth's Van Allen belts, and the LEO and HEO altitudes are, respectively, just below and somewhat above them (Figure 3). In LEO, the main radiation effects are due to the intense fluxes of trapped protons encountered during passages through the South Atlantic Anomaly (SAA), a low lying region of the Van Allen belts. These passages occur almost every orbit and render about $25 \%$ of the time unsuitable for observations. Because these high fluxes alter detector response, additional time would be required for post-SAA annealing and recovery. Analysis by J. Mihalov at Ames of satellite data shows that in Heo, fairly high electron fluxes are still present at 70,000 km altitude in the anti-solar direction, and that moving to $100,000 \mathrm{~km}$ substantially reduces these. While the electrons are easily shielded, the $\gamma$ ray bremsstrahlung they produce is not, but at HEO the "hit" rate from bremsstrahlung is below that of cosmic rays (see below) for all but a few percent of the time.

Note that geostationary orbits (GEO) at $36,000 \mathrm{~km}$ altitude are in the heart of the electron belt and are therefore a very noisy place for sensitive astronomical detectors: it is for this reason that IUE, when not near apogee in its elliptical 24 hour orbit, has "bright" time. Another problem with GEO is that the Earth's thermal load is no longer negligible (see the paper by Phil Tulkoff). Because this misconception was voiced by several speakers at the workshop, it bears repeating: HEO is not GEO! 
The second source of ionizing radiation is galactic cosmic rays, mainly protons and alpha particles whose differential energy spectra peak near $1 \mathrm{GeV} /$ nucleon. These high energies are impossible to shield against in a spacecraft, although the Earth's magnetic field and atmosphere are effective shields, and they are modulated by solar activity. Integrated fluxes in HEO range from 0.4 (at solar maximum) to 1 (solar $\mathrm{min}$ ) per $\mathrm{cm}^{2}$ of surface area per second, while in LEO the range is from 0.15 at the geomagnetic equator to 0.6 at high geomagnetic latitudes (Mason and Culhane 1983). In cases where performance is read noise limited, and the cosmic ray hit rate limits the maximum integration time, this will lead to a reduction in sensitivity in HEO vs. LEO.

The third component is solar flare particles. Again, because of geomagnetic shielding, these are much less of a problem in LEO. However, they are less energetic than galactic cosmic rays and therefore onboard detector shielding is effective. With SIRTF's expected shielding, we estimate the solar flare particle hit rate will exceed the cosmic ray rate in HEO only a few percent of the time even during solar maximum. The rare (one or two per solar cycle) anomalously large solar flares provide most of the cumulative radiation dosage in HEO; in LEO the cumulative dose is higher and due to SAA passages.

Thus ionizing radiation effects are a concern in either orbit, and radiation testing continues to be an important part of the SIRTF detector technology program.

\subsubsection{Operations}

On-orbit science operations in HEO will benefit from the reduced Earth solid angle, the long orbital period (100 hours vs. 100 minutes in LEO), and the absence of the South Atlantic Anomaly (SAA - see section 3.3.2 above). In addition, the contamination constraint introduced in LEO that the telescope not point into the "wind" created by the orbital motion of the spacecraft does not apply in HEO, where the atmospheric density is negligible. Depending on the relative location of the Sun, Earth and spacecraft, SIRTF can view instantaneously 14 to $33 \%$ of the sky in HEO but only $<1$ to $12 \%$ in LEO. In HEO, there are zones over the orbit poles ( $\approx 200$ square degrees in total) which can be viewed continuously, and accessible targets elsewhere in the sky can typically be observed for 50 or more consecutive hours. By contrast, there is no direction which can be viewed continuously in LEO, because the combination of Earth limb and "wind" avoidance constraints limit the maximum viewing time per target to 15 minutes. These and similar considerations suggest that the observation planning and scheduling process will be much more straightforward in HEO. Maintaining the Earth avoidance constraint in LEO means that tens of minutes in each 100 minute orbit must be spent in large angle slews. Observing efficiency simulations for LEO including sky visibility, slewing, and time lost to the high proton fluxes in the SAA, indicate that the average efficiency (fraction of time on-target) would be about $45 \%$. The corresponding efficiency in HEO is estimated at $90 \%$. We would expect similar gains for ET as compared to HST.

\subsubsection{Risk and Complexity}

The HEO mission appears to have lower operational risk for SIRTF than the LEO option. In LEO the performance and system lifetime depend critically on maintaining the cleanliness of the telescope and the aperture shade, which are subject to contamination both by the residual atmosphere and by problems which could occur during a servicing mission. In addition, an emergency safe-hold mode is simply achieved in HEO by pointing at the constant viewing zone, while in LEO a complex series of pointing constraints must be continually satisfied. Finally, the use of fixed solar arrays and antennae in HEO eliminates the risk of failure during deployment or operation of these mechanisms.

\subsubsection{Scientific Performance}

The features of the HEO mission summarized above improve the scientific performance of SIRTF in many ways, including the following areas of particular importance: 
- The doubled on-target efficiency means that the HEO mission will support twice the number of investigations, and produce twice the quantity of data, as the LEO mission.

- The longer on-target times and greater sky accessibility in HEO will allow SIRTF to operate in a true observatory mode, in which a single scientific investigation can be scheduled in an unbroken block of time. Data from the completed observation will be available to the observer more quickly, and will be more uniform and easier to calibrate and reduce, than if it were obtained on many successive orbits in the LEO mission.

- The long wavelength ( $>100 \mu \mathrm{m}$ ) performance will be improved in HEO. SIRTF's sensitivity at these long wavelengths will be influenced by radiation from the telescope itself. To reach natural-background limited performance at $300 \mu \mathrm{m}$ requires a forebaffle temperature of $7 \mathrm{~K}$ or below; the predicted temperature is $4 \mathrm{~K}$ in HEO and 8 to $14 \mathrm{~K}$ in LEO.

The longer on-target times and much less frequent eclipses in HEO imply that the temperature will be more stable as well as lower. These effects together should allow the HEO mission to achieve far better performance in the cosmic window at $300 \mu \mathrm{m}$, where recent results (Matsumoto et al. 1988) indicate that many important cosmological questions can be investigated. For ET, this thermal stability may be important to meet its pointing and image quality requirements.

- Performance in the $3 \mu \mathrm{m}$ window may also be improved in HEO. The extremely low background photon rate means that observations (especially spectroscopy) near this wavelength are likely to be limited by detector read noise in 15 minutes, the maximum integration time in for SIRTF in LEO. Under these circumstances, $n^{2} 15$ minute integrations must be combined in LEO to equal the sensitivity of a single $(n \times 15)$ minute integration in HEO. However, the higher cosmic ray rates in HEO will probably limit continuous integration times to about an hour, depending on pixel size and the fraction of "hit" pixels acceptable.

- The high galactic latitude sky (latitude greater than $60^{\circ}$ ) can be observed at any time during the HEO mission, but is accessible only about $25 \%$ of the time in LEO. This is important because many of SIRTF's (and ET's) most important scientific objectives, including deep cosmological surveys, will require extended access to high latitudes.

The importance of these advantages for ET is obvious.

\subsection{The L2 HEO option}

An alternative HEO option, in which SIRTF is flown at the Earth-Sun L2 Lagrangian point (1.5 million $\mathrm{km}$ in the anti-solar direction), has been examined by Ronald Muller of the Goddard Space Flight Center. L2 is still being considered for SIRTF, and should certainly be considered for ET. This mission would use a Titan IV and modified Centaur to place SIRTF into a halo orbit about the metastable L2 point. Because no fuel is needed to circularize this orbit, the Titan IV launch capability is substantially greater to L2 than to a $100,000 \mathrm{~km}$ circular orbit (or to GEO). Midcourse corrections and stationkeeping are needed to achieve and maintain this orbit. As seen from L2, the Earth, Sun, and Moon all lie in the same direction, and therefore viewing constraints are minimal, and no aperture shade is required. Even lower outer shell temperatures than for the $100,000 \mathrm{~km}$ orbit may be achievable. The chief concerns which kept the L2 mission from being adopted as SIRTF's baseline were: the Centaur upper stage development required; and the long transit time (over 30 days) with course corrections, which may require the aperture be covered to avoid contamination, and therefore reduce cryogen lifetime. 


\section{Summary}

The consensus of the SIRTF Study Office at Ames, the other NASA centers which participated in the Mission Options Study, and the SIRTF Science Working Group is that the high orbit offers significant scientific and engineering advantages for SIRTF. These advantages have been summarized above. As a result, the HEO mission has been adopted by NASA as the new baseline. This selection of an orbit optimized to maximize the scientific productivity of the mission, together with the dramatic recent advances in IR detector performance, has brought the promise of SIRTF close to realization.

Perhaps the fact that LEO has not been mentioned as a candidate for ET is an indication of how compelling the advantages of HEO are. The L2 point may be an even better choice for ET than a $100,000 \mathrm{~km}$ circular orbit, while many other factors need to be considered in evaluating a lunar based site. (We remind the reader again that geosynchronous orbit is not a desirable location for sensitive astronomical detectors.) Of these locations, launch capabilities are greatest to L2 and smallest to the lunar surface, while post-launch access for servicing or assembly is poorest for L2, and best for a lunar site (once a lunar base is established).

No matter where it is located, ET will enlighten us with the answers to myriad astronomical questions, including many raised by discoveries made by SIRTF in its high Earth orbit.

\section{References}

Matsumoto, T. et al. 1988, Ap.J. 329, p. 567.

Mason, I.M., and Culhane, J.L. 1983, IEEE Transactions on Nuclear Science, NS-80, p. 485.

Ness, N.F. 1969, Reviews of Geophysics, 7, p. 97.

Ramos, R., Hing, S.M., Leidich, C.A., Fazio, G., Houck, J.R., and Rieke, G. 1988, Proc. SPIE 973, p. 2.

Rieke, G.H., Werner, M.W., Thompson, R.I., Becklin, E.E., Hoffmann, W.F., Houck, J.R., Low, F.J., Stein, W.A., and Witteborn, F.C. 1986, Science 231, 807.

Stassinopoulos, E.G., and Raymond, J.P. 1988, Proc. IEEE 76, 1423.

Werner, M.W., Murphy, J.P., Witteborn, F.C., and Wiltsee, C.B. 1986, Proc. SPIE 589, p. 210.

\section{DISCUSSION}

Darnton: In view of the fact that liquid He is light and SIRTF is heavy, small spacecraft weight reductions afford large potential liquid He dewar volume increases. Is the 4000 liter volume fixed, or is NASA considering larger possible cryogen loads?

Fisenhardt: The 4000 liter volume was used for comparison with the LEO baseline. Larger volumes are being considered for HEO as part of SIRTF's Phase B studies.

Angel: A comment on the optics. The Kodak ion polishing technique would be ideal for getting the desired optical performance when cold. The nearly-final optics could be cooled, tested, and then in one pass all the surface aberrations resulting from the cooling could be fixed up. 


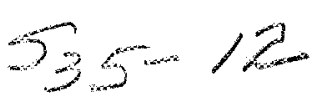

Orbital Sites Tradeoff Study

\author{
J. D. Neill*, P. Y. Belyt, G. Miller*, and A. Spigler
}

\begin{abstract}
A comparative study of typical Earth orbits for their impact on certain spacecraft and science instrument parameters was conducted using models and formulae from the literature and models specifically derived for this study. We conclude that low Earth orbits are undesirable because of high disturbance level and space debris and favor high Earth orbit sites.
\end{abstract}

\title{
1 Introduction
}

The purpose of this study is to determine what would be the best site in space for a large astronomical observatory operating in the optical and near-IR. To that end we selected a number of parameters affecting the performance and operation of a telescope in space and compared them as a function of the orbits. Some parameters were chosen because of their impact on attitude control and the lifespan of the satellite. Others were chosen because of their impact on the efficiency of science observations. The orbits chosen and their elements are listed in Table 1.

For the purpose of this study the baseline telescope is a 10 meter full aperture mirror with a closed tube extending just beyond the secondary. We adopted the nominal parameters for this telescope as presented in Table 2. The baffling prohibits the telescope from pointing within $90^{\circ}$ of the Sun. The Hubble Space Telescope (HST) was also included in the study and its parameters are also presented in Table 2 . We chose to include HST in the study because the properties of its mission are well studied and this allowed us to check our results.

Table 1. Orbital Elements

\begin{tabular}{lll|rrrrrr} 
Parameter & & Unit & LEO $^{a}$ & LEO $^{b}$ & SS $^{c}$ & GEO $^{d}$ & HEO $^{e}$ & L5 $^{f}$ \\
\hline Semi-major axis & $\mathrm{a}$ & $\mathrm{km}$ & 6978 & 6978 & 7278 & 41378 & 106378 & 384000 \\
Eccentricity & $\mathrm{e}$ & & 0 & 0 & 0 & 0 & 0 & 0.055 \\
Inclination & $\mathrm{i}$ & $\circ$ & 28 & 5 & 90 & 0 & 28 & 5.1 \\
Long. of Asc. Node & $\Omega$ & $\circ$ & 0 & 0 & 126 & 0 & 0 & 335 \\
Arg. of Perigee & $\omega$ & $\circ$ & 0 & 0 & 0 & 0 & 0 & 0 \\
Period & $\mathrm{P}$ & $\mathrm{min}$ & 97 & 97 & 103 & 1396 & 5243 & 39456 \\
Altitude & $\mathrm{H}$ & $\mathrm{km}$ & 600 & 600 & 900 & 35000 & 100000 & 356502 \\
\hline
\end{tabular}

${ }^{a}$ Low Earth Orbit, NASA Launch.

${ }^{b}$ Low Earth Orbit, ESA Launch.

cSun Synchronous Orbit.

${ }^{d}$ Geosynchronous Orbit.

${ }^{e}$ High Earth Orbit.

${ }^{f}$ Earth-Moon Lagrange Point (4 or 5).

${ }^{*}$ Space Telescope Science Institute, operated by the Association of Universities for Research in Astronomy for the National Aeronautics and Space Administration

'Affiliated to the Astrophysics Division, Space Science Department, European Space Agency

tAstronomy Programs, Computer Sciences Corporation 
Table 2. Satellite Characteristics

\begin{tabular}{ll|rr} 
Parameter & Unit & 10-meter & HST \\
\hline Mass of Spacecraft & $\mathrm{kg}$ & 22700 & 11600 \\
Average Cross-Section & $\mathrm{m}^{2}$ & 180 & 30 \\
Gravity-Pressure Centers Offset & $\mathrm{m}$ & 3 & 1.5 \\
Solar Radiation Torque & $\mathrm{N}-\mathrm{m} \times 10^{-3}$ & 4.5 & 0.38 \\
Reflectance Parameter & & 1.9 & 1.9 \\
Sun Avoidance Angle & $\circ$ & 90 & 50 \\
Moment of Inertia Vector & $\mathrm{kg}-\mathrm{m}^{2}$ & & \\
$\mathrm{X}$ & & 80000 & 31000 \\
$\mathrm{Y}$ & & 80000 & 76600 \\
$\mathrm{Z}$ & & 210000 & 78000 \\
\hline
\end{tabular}

\section{Model}

An orbit simulator program was developed in FORTRAN on a VAX computer ${ }^{1}$ to determine the parameters under consideration using the spacecraft and orbit characteristics as inputs.

Most of these parameters depend, to the first order, on the semi-major axis of the orbit alone. The sky coverage and magnetic field strength depend on the unique position of the spacecraft. An average value for these parameters was derived by calculating the spacecraft position (accounting for precession) at 1/10th orbit intervals over the span of a year. Smaller sampling intervals were tested and we found that no change larger than 1 percent was encountered down to and including a sampling interval of $1 / 100$ th of an orbit.

The calculation of the sky coverage fraction for the Sun synchronous orbit involved simplifications (made to permit obtaining results from the simulator in a reasonable amount of time) that we later found to introduce larger uncertainties than for the other orbits. A more accurate calculation would take into account the seasonal variation of the terminator with respect to the orbit plane, and would carry higher order terms in the calculation of the precession. The uncertainty due to these simplifications may be very large for the sky coverage parameter.

\section{Orbit Parameters}

The parameters calculated in our orbit models are described below. We limited ourselves to parameters which are of major importance in the design and operation of an astronomical observatory in space operating at optical and near-IR wavelengths. However, we did not consider the thermal aspects which are covered by a different study (Tulkoff) reported in these proceedings. Also the logistical problem of how to place or maintain a spacecraft at any of the orbits in this study has not been considered.

\subsection{Orbit Lifetime}

The lifetime of a satellite due to aerodynamic drag in a given orbit was calculated from Wertz with extrapolation to the Sun synchronous orbit. For orbits with radii greater than $900 \mathrm{~km}$, aerodynamic drag is negligible. The drag coefficient for both satellites under consideration was assumed to be 1.75. All models of spacecraft lifetime are approximations since the atmospheric density fluctuates considerably. Our calculations used a constant atmospheric density given for a low solar activity

\footnotetext{
${ }^{1}$ VAX is a trademark of the Digital Equipment Corporation.
} 
year. The estimates presented here may be in error by as much as $50 \%$ depending on the actual solar activity. The orbital lifetime due to aerodynamic drag was found to be 7.3 years for the 10 meter telescope in a low Earth orbit (22.5 years for HST). The lifetime at higher Earth orbits is effectively infinite.

\subsection{Torque}

The gravity gradient torque was calculated using the moments of inertia and the angular velocity of the satellite. The solar radiation and aerodynamic torques were calculated from the solar radiation and atmospheric pressures and the offset between the center of gravity of the satellite and the center of pressure for the worst case satellite orientation. The solar radiation pressure was taken to have a single value $\left(4.4 \times 10^{-6} \mathrm{~N} / \mathrm{m}^{2}\right)$ for all of the orbits under consideration. The atmospheric pressure was calculated from Wertz and the same qualifications mentioned above regarding the fluctuation of the atmosphere also apply here. The torque was calculated assuming a circular orbit and a constant cross section perpendicular to the motion of the satellite.

\subsection{Damage}

The probability that the satellite would suffer severe damage over a 15 year period due to both meteoroids and space debris was calculated by extrapolating a study investigating these factors for HST (Lockheed). The level of space debris was updated to reflect projections for the year 2000 (Kessler, et. al.). We were unable to locate any data on space debris at orbits higher than 1500 kilometers and therefore the probability of damage at the geosynchronous orbit is likely to be higher than what has been calculated for the meteoroid level alone.

\subsection{Magnetic Field}

The magnitude of the geomagnetic field vector was calculated using the International Geomagnetic Reference Field (Leaton).

\subsection{Sky Coverage}

Sky coverage is defined as the fraction of the sky that is observable once occultations and avoidance zones due to off axis bright objects are taken into account. Bright object avoidance zones were calculated as the areas on the sky in which scattered light at the focal plane was greater than that of the zodiacal light. The attenuation factor of the telescope baffling system was calculated using the simplified method developed by Greynolds. The angle of avoidance from the limb of the body was calculated as a function of distance from the Earth and the Moon. The avoidance angle for the Sun was assumed constant over the range of orbits considered in this study.

The lunar phase was taken to be constant at full illumination to simplify the calculation. For the Earth a plane was defined at the satellite whose normal points toward the Sun. Whenever the Earth was on the Sun side of this plane the Earth was considered to be seen as fully dark and whenever the Earth was on the anti-Sun side it was considered to be fully illuminated. This simplification was made with the assumption that the errors produced would average to zero over a year. When fully dark, 200 kilometers was added to the radius of the Earth to avoid sky seen through the Earth's atmosphere.

The total angular area for the resulting avoidance zones was calculated taking overlaps and eclipses into account and compared to the total sky area to derive the fraction of sky available to the satellite at a given time. The average sky coverage fraction as well as the upper and lower limits were calculated over a period of a year. 


\section{Results and Discussion}

The results of the model are summarized in Figures 1-3.

From Figure 1 and the discussion in section 3.1, it is obvious that the effect of the atmosphere on a voluminous spacecraft such as the one considered is very large for low Earth orbits and may still be significant at the Sun synchronous orbit altitude. This effect becomes negligible very quickly at altitudes higher than Sun synchronous.

The gravity gradient torque dominates the torques to be overcome by the attitude control system of a satellite at low Earth orbits and is still significant at geosynchronous altitudes. At altitudes higher than Sun synchronous, the torque due to solar radiation dominates.

It is interesting to note how little the meteoroid damage probability changes with different orbits (Figure 2). Most of this change is due to the Earth's gravitational focusing of the ambient meteoroid field. The effect of meteoroids trapped at the Lagrange points was not considered although we note that these particles would have very low velocities relative to a satellite at one of the Lagrange points.

The space debris damage projected to the year 2000 presents a serious concern for the Sun synchronous orbit. Lower Earth orbits are depleted of space debris by orbital decay, but particles above these altitudes are fairly stable. The smaller number of spacecraft at higher altitudes combined with the larger volume of space results in a much lower probability for damage. This may not not be true at the geosynchronous orbit due to its popularity. We were unable to acquire data on the geosynchronous orbit, but due to the large number of spacecraft and launch equipment at this orbit there may be enough space debris to make the probability for damage non-negligible.

The average fraction of sky available increases with distance from the Earth and is essentially limited by the Sun avoidance angle (Figure 3). The high maximum values shown result from eclipses of the Sun, during which nearly the entire sky is available for a short period of time. The minimum values correspond to periods when two bright solar system bodies are at opposition.

As noted above the sky coverage fraction presented for the Sun synchronous orbit is highly uncertain. With the $90^{\circ}$ avoidance angle assumed for the Sun we can estimate the average sky coverage fraction would be somewhat less than 0.5 due to the Earth and the Moon.

Our model does not include observing efficiency as a parameter, and a detailed study would require a simulation of the observations and schedules. However an estimate of the maximum efficiency can be obtained by a analytic model in which the important factors are the overhead time per observation and the average duration of an observation. Figure 4 shows the maximum efficiency of primary observations (i.e. excluding any parallel science) as a function of the exposure time for the high Earth orbit, given two possible values for the overhead time per observation. Also shown is the estimated efficiency of HST observations from a simulation by Johnston. The greater efficiency of the high Earth orbit compared to the HST is caused by the same factors which determine the fraction of sky available at a given orbit, namely, the avoidance angles of the Earth, Sun and Moon. The further these bodies are from the satellite, the more efficient the observatory and the more sky available for observations. Note also that for the high Earth orbit, the efficiency increases much more rapidly with an increase in exposure duration (or a decrease in overhead time) compared to a lower orbit.

\section{The Lunar Bases}

Our original model was limited to Earth orbits. We had not anticipated the enthusiasm for a Lunar base at the conference but have since made some calculations which are discussed below.

Many of the parameters we considered for free orbiting satellites would not be a factor for a telescope mounted on the Moon. Torques would no longer present a serious design problem and damage due to space debris would also not be a factor. Severe damage due to the ambient meteor 

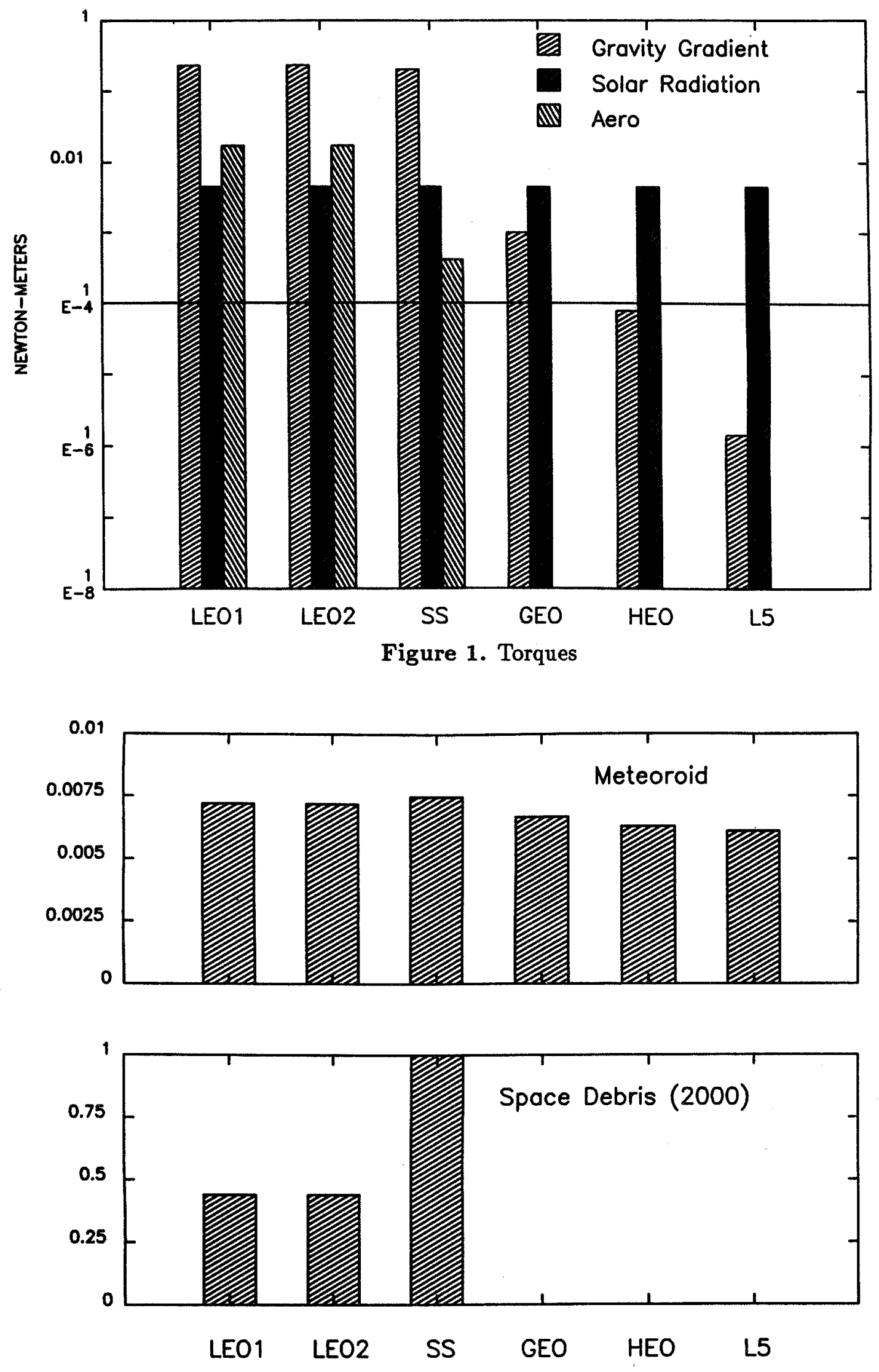

Figure 2. Probability of Damage (15yr) 


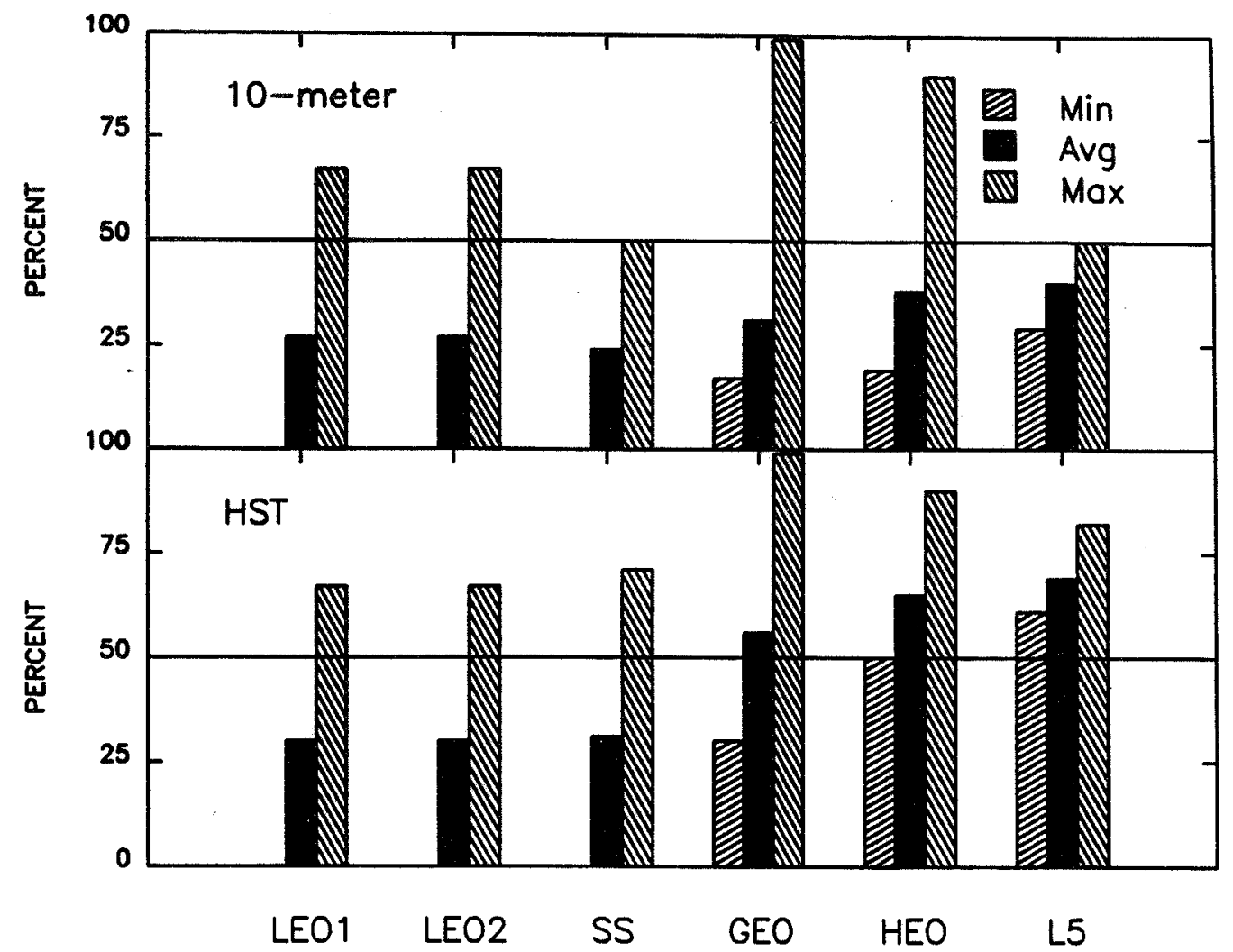

Figure 3. Sky Coverage

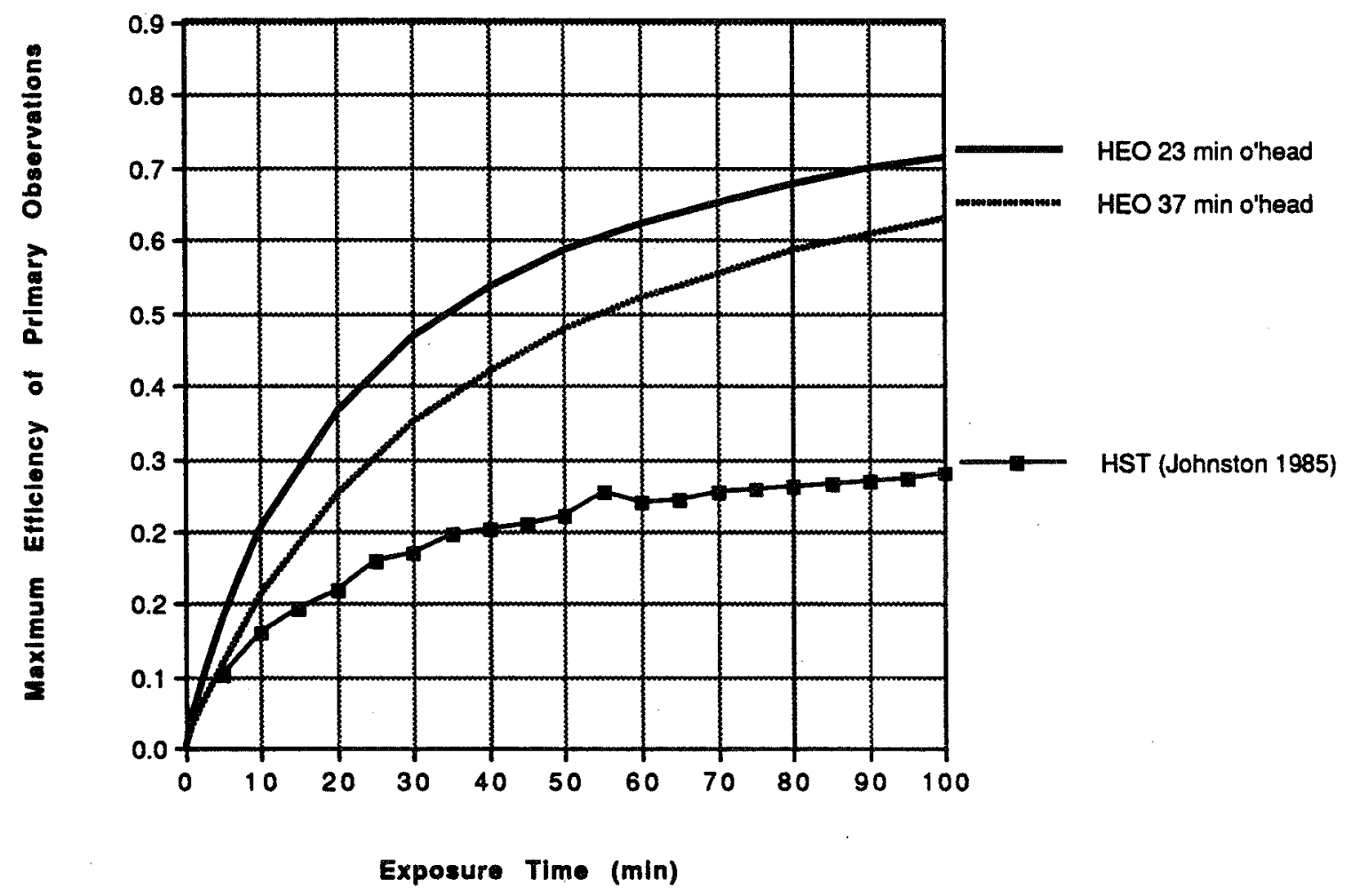

Figure 4. Observatory Efficiency 
field would still be a consideration, but we estimate that over a 15 year period the probability for severe damage would be less than one tenth of one percent.

We calculated the fraction of sky available from two Lunar sites; an equatorial site located at the sub-Earth point, and a polar site. We assumed that the Lunar observatory would be mechanically constrained to point within $70^{\circ}$ of the zenith, and that the bright Earth could be shielded to within two degrees of its subtence. We have also assumed that no observations would take place when the Sun was above the horizon due to thermal and scattered light problems.

For the equatorial site we calculated that the fraction of sky available on the average would be only 0.15 . This is due primarily to the constraint imposed by the Sun. This can be improved to a value of 0.33 if the observatory were placed at one of the poles of the Moon in a crater whose walls would shield the observatory from the light of the Sun and the Earth. Contact with the Earth could be maintained through a relay station placed on the rim of the crater. The Earth would be shielded from an observatory placed in a crater anywhere along the limb of the Moon. Shielding the Earth in this manner adds about one hundredth of the sky to the average fraction available.

It should be noted that large areas of the sky would be permanently unavailable to a Lunar observatory. An equatorially placed observatory would be able to minimize this area at the sacrifice of overall efficiency. A better way to alleviate this would be to place two observatories on the Moon, one in each hemisphere.

\section{Conclusions}

We conclude that if it were equally feasible to place a space telescope at any of the orbits under study, the best choice would be either the high Earth orbit or the Lagrange orbit. Observing efficiency would be greatest there and attitude control problems would be minimized. In addition the probability of damage by space debris is minimized.

A Lunar based observatory offers essentially the same advantages as that of the high Earth orbits except that it would retain the traditional problems of limited sky coverage encountered by ground based telescopes.

\section{$7 \quad$ References}

Greynolds, Alan W. 1980, "Radiation Scattering in Optical Systems", proceedings, SPIE, Vol 257, p.39.

Johnston, M. 1985, internal communication.

Leaton, B. R. 1976, "International Geomagnetic Reference Field 1975", Trans., Amer. Geophysical Union $(E \oplus S)$, Vol 57, p.120.

Lockheed 1989, "Hubble Space Telescope Meteoroid Protection Analysis", Lockheed Report, LMSC/F158270C.

Kessler, D. J., Grun, E. and Sehnal, L. 1985, "Space Debris, Asteroids and Satellite Orbits", JASR, Vol 5, No. 2.

Tulkoff, P. 1989 "Passive Cooling for Low Temperature Operation", these proceedings.

Wertz. James R., ed. 1978, Spacecraft Attitude Determination and Control, D. Reidel Publishing Co., Dordrecht, Holland. 


\section{DISCUSSION}

Swanson: How can you put 23,000 kg in anything except a low Earth Orbit? This is the zeroth order issue. If this cannot be answered, most of the other orbital issues tradeoffs are irrelevant.

Neil: Our study demonstrates the penalty paid by having a limited lift capability and provides some fuel for the fire warming the efforts to increase our launch capability. It is also true that the Soviet Union does have this capability.

Mallama: Your analysis of the sun synchronous orbit was interesting, but it only considered one possible altitude. Precession rate is a function of altitude and inclination. Thus, a wide range of altitudes could be chosen by adjusting the inclination. One way to avoid thermal cycling is to put the nodes 90 degrees from the solar longitude.

Neil: We have selected the traditional $900 \mathrm{~km}$ altitude because it is just below the Van Allen belt. And yes, you are right, the 6 a.m.-6 p.m. orbit is the best (minimizes thermal cycling and supplies continuous solar power). 
THE MOON AS A SITE FOR ASTRONOMICAL OBSERVATORIES

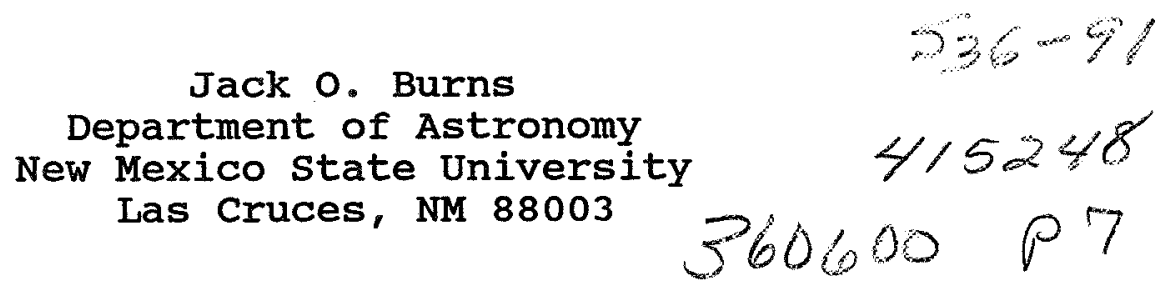

ABSTRACT: The Moon has a number of important advantages as a site for future astronomical observatories. These include a very low density atmosphere and ionosphere, extraordinary surface seismic stability, slow thermal cycling times, natural cryogenic environments within polar craters, very low light levels especially on the lunar far-side, a small gravitational field, lunar regolith which can be used to shield CCD detectors from cosmic rays, and an abundance of natural resources. These characteristics suggest that the lunar surface is best suited for high resolution imaging at uv/optical/IR wavelengths using either large filled aperture telescopes or interferometric arrays.

\section{INTRODUCTION}

For the past five years, a small but active group within the astronomical community has been investigating possible observatories for the Moon in the 21 st century (see e.g., Burns and Mendell, 1988). These studies were in large part motivated by a recognition of the limitations imposed by observations from the surface of the Earth or from low Earth orbit (LEO). For example, the plasma frequency of the Earth's ionosphere (10 MHz) is such that low frequency radio observations are not possible from the Earth's surface. Similarly, leakage of manmade interference through the ionosphere, atmospherics, and auroral kilometric radiation make low frequency observations very difficult in LEO. on the other hand, the far-side of the Moon is shielded from these low frequency emissions. At optical and infrared wavelengths, atmospheric emissions or high optical depths make observations challenging from the ground. In LEO, the still significant atmosphere will produce higher than ideal backgrounds due to molecular gas excitations from spacecraft motion and scattering of light by dust. The Moon, and especially the lunar far-side, will offer astronomers one of the darkest skies in the inner solar system.

Following the first conference on Lunar Bases and Space Activities of the 21st Century (Mendell, 1985) at the National Academy in 1984, three workshops dedicated to the topic of lunar observatories were held. The first such workshop took place in Houston in 1986 following the AAS meeting (Burns and Mendell, 1988). This conference was devoted to discussions of general ideas on lunar telescopes at all wavelengths and included cosmic 
rays. Over 100 astronomers, space scientists, and engineers attended. This was the first time that a broad cross-section of the astronomical community was gathered together to focus on possible telescopes for the Moon. Several novel ideas emerged from this workshop that would make full utilization of the Moon's advantages for astronomy. The second workshop was held in Albuquerque in February, 1988. This meeting was devoted to a lunar far-side very low frequency array (Burns et al., 1989). The third workshop was held in February, 1989, also in Albuquerque. The topic focused on a lunar optical-uv-IR interferometer (Burns et al., 1990). Together, these three workshops demonstrated that the Moon may very well be the best location in the inner solar system for a variety of telescopes that will follow on the Great observatories.

All three workshops and related engineering studies (e.g., Johnson and Wetzel, 1988) had a single simple but important underlying assumption. The basic assumption was that astronomy will not be the primary driver in the establishment of a lunar outpost. Rather, political considerations will motivate such a national or international enterprise. Importantly for astronomy, though, will be the transportation and lunar surface infrastructure that will put into place. Astronomers may be able to "piggy-back" upon this infrarstructure in building initially simple, and later more complex, astronomical observatories on the Moon at relatively lower costs. The recent address by President Bush at the Air and Space Museum coupled with NASA's renewed interest in the Moon may bring this political scenario to realization. Such a program may begin in the first decade of the 21st century (see Figure 1). We must be aware, however, that a strong coupling between the manned space program and lunar observatories limits our independence and links the fate of the lunar telescopes (and possibly the future of space-based astronomy) with that of the lunar base program.

\section{ADVANTAGES OF THE MOON FOR ASTRONOMY}

Early on in our studies of lunar observatories, we recognized that the Moon has some very significant and attractive features conducive to astronomy (Smith, 1988; Burns, 1988). This is particularly true if one is interested in designing high resolution filled apertures or interferometric arrays that operate from the ultraviolet through the infrared.

Relative to the Earth, the Moon is a nearly airless world. Its atmospheric density is less than that of LEO, $2 \times 10^{5}$ molecules $/ \mathrm{cm}^{3}$, and its total atmospheric mass is only $10^{4} \mathrm{~kg}$ (Hoffman et al., 1973). Thus, the atmosphere of the Moon will permit full diffraction-limited imagery providing mirror surfaces are sufficiently accurate. It will also allow us to build phase coherent interferometers at uv to IR wavelengths.

Equally important, the Moon offers a large and stable platform upon which large aperture telescopes or imaging interferometers can be constructed. This seismic stability is a crucial characteristic. Typical ground motions, as measured by Apollo, 
are about $1 \mathrm{~nm}$ (Goins et al., 1981). Moonquakes are relatively rare and shallow, registering $1-2$ on the Richter scale. Because the subsurface layers of the Moon consist of meteoritic rubble, seismic waves are damped at short distances from their origin relative to that for quakes on the Earth. Although there are surface distortions produced by solar and terrestrial tides, the simplicity of the Moon as a body allows us to accurately predict and correct for these ground motions. Such stability is a fundamental environmental attribute for an optical interferometer where one must know baselines between optical elements to within a fraction of a wavelength. In principle, an interferometer can be built using free-flying spacecraft in Earth-orbit. However, when the baselines are large $(>1 \mathrm{~km})$, station-keeping of the individual elements becomes costly and complex (Burns et al., 1990). The lunar surface provides the necessary stability without complications inherent in free-flyers.

The Moon also offers an advantage over LEO in terms of slow thermal cycling times. In LEO, spacecraft move in and out of sunlight every 50 minutes. This places severe constraints on the design and materials used for observatories. Therefore, either an active cooling system or a passive sunscreen is needed depending upon the operational wavelength. The Moon, however, has a much longer thermal cycle with two weeks of daylight and two weeks of darkness. In addition, the temperature change from day to night (about $280 \mathrm{~K}$ ) occurs rapidly at $5 \mathrm{~K} / \mathrm{hr}$ (Keihm and Langseth, 1973). Thus, one might envision building a telescope that can operate in the two temperature extremes with re-calibration occurring for operation in the day or night conditions. The long cycling time on the Moon would make such a design viable.

Along these same lines, there appear to be craters near the lunar poles that are permanently shadowed with equilibrium temperatures as low as $60 \mathrm{~K}$ (Burke, 1988). Such an environment is particularly attractive for infrared telescopes where both the optics and the superstructure must be cooled to reduce the thermal noise background. The disadvantage of these polar sites is the limited sky area that is available.

Because of tidal locking between the Earth and Moon, one side of the Moon always faces toward the Earth (near-side) and the other side faces away (far-side). Such an orientation, however, is a distinct advantage for astronomy. The lunar far-side offers observers a natural radio-quiet zone (especially below $30 \mathrm{MHz}$ ) and the lowest background light levels during night-time in the Earth-Moon system. For an optical/IR telescope, the far-side sky background is dominated by zodiacal light near the ecliptic plane and the galactic (stellar) plus extragalactic diffuse light above and below ecliptic.

The fact that the Moon has some gravity $(1 / 6 \mathrm{~g})$ is considered an advantage for building large structures. Gravitational torques can be used for construction with techniques akin to that on the Earth. LEO is at a disadvantage here. Also, the gravity of the Moon produces an insignificant loading on a 16-m optical/IR mirror in comparison to that on the Earth or that due to thermal loading. Finite element analyses of lunar telescopes have demonstrated that very light weight backup structures can be used to support large 
monolithic apertures (Akgul et al., 1990).

Any space environment above LEO is directly exposed to the

the full energy spectrum of cosmic ray protons and nuclei. This is a serious limitation for solid state electronics and detectors such as CCDs. However, the lunar soil (regolith) could be used to shield both astronauts and electronics from this cosmic radiation. Generally, several meters of regolith will absorb enough cosmic radiation to reduce hazards for human habitats to tolerable levels (e.g., Mendell, 1985). One can envision placing CCDs several meters below the surface in a fashion similar to current coude focus spectrographs on Earth. Finally, the Moon is the only other space environment relatively nearby that has raw materials available. Ceramics, aluminum, and a superstrength glass with tensile strength comparable to steel and a low coefficient of thermal expansion are present. By the middle of the next century, such materials may be mined and processed thereby reducing the costs, incurred by transport, of building telescopes on the Moon.

\section{CONCLUSIONS}

The above characteristics suggest that the Moon is best suited for high resolution imaging at ultraviolet through infrared wavelengths. The lunar surface is an excellent site for filled aperture, dilute aperture, and interferometric arrays. If there is a single theme to be advocated for lunar telescopes, it is the concept of high angular resolution. The resulting high spatial resolution will open new classes of astronomical problems for study, from extrasolar planets to cosmology.

\section{ACKNOWLEDGEMENTS}

I would like to thank NASA, particularly C. Pilcher and $M$. Duke, for providing funding for these studies and the workshops. I am grateful to our many colleagues who participated in the workshops, for illuminating discussions on the characteristics of the Moon and lunar telescope designs. I would particularly like to thank J. Taylor, S. Johnson, and N. Duric for their efforts.

\section{REFERENCES}

Akgul, F., Gerstle, W.H., and Johnson, S.W. 1990, preprint. Burke, J. D. 1988 in Future Astronomical Observatories on the Moon, ed. J. O. Burns and W. W. Mendell, NASA Conference Publication \#2489, p. 31.

Burns, J.O. 1988 in Engineering, Construction, \& Operations in Space, ed. S.W. Johnson \& J.P. Wetzel, (American Society of Civil Engineers, NY), p. 1083.

Burns, J. O. and Mendell, W. W., editors, 1988, Future Astronomical observatories on the Moon, NASA Conference Publication \#2489. 
Burns, J.O., Duric, N., Johnson, S., and Taylor, G.J. 1989, A Lunar Far-Side Very Low Frequency Array, NASA Conference Publication, in press.

Burns, J. O., Johnson, S. W., and Duric, N. 1990, A Lunar OpticalUV-IR Synthesis Array, NASA Conference Publication, in press.

Goins, N. R., Dainty, A. M., and Toksoz, M. N. 1981, J. Geophys. Res., 86, 378 .

Hoffman, J. H., Hodges, R. R., and Johnson, F. S. 1973, Proc. Lunar Sci. Conf. 4th, 2865.

Johnson, S.W. and Wetzel, J.P., editors, 1988, Engineering. Construction, \& Operations in Space, (American Society of Civil Engineers, NY).

Keihm, S. J. and Langseth, M. G. 1973, Proc. Lunar Sci. Conf. 4th, 2503.

Kulkarni, S. 1990 in A Lunar Optical-UV-IR Synthesis Array, NASA Conference Publication, in press.

Mendell, W.W., editor, 1985, Lunar Bases and Space Activities of the 21st Century, (LPI: Houston).

Smith, H.J. 1988 in Future Astronomical Observatories on the Moon, ed. J.O. Burns and W.W. Mendell, NASA Conference Publication \#2489, p. 37.

Thompson, A. R., Clarke, B. G., Wade, C. M., and Napier, P. J. 1980, Ap.J. Suppl., 44, 151.

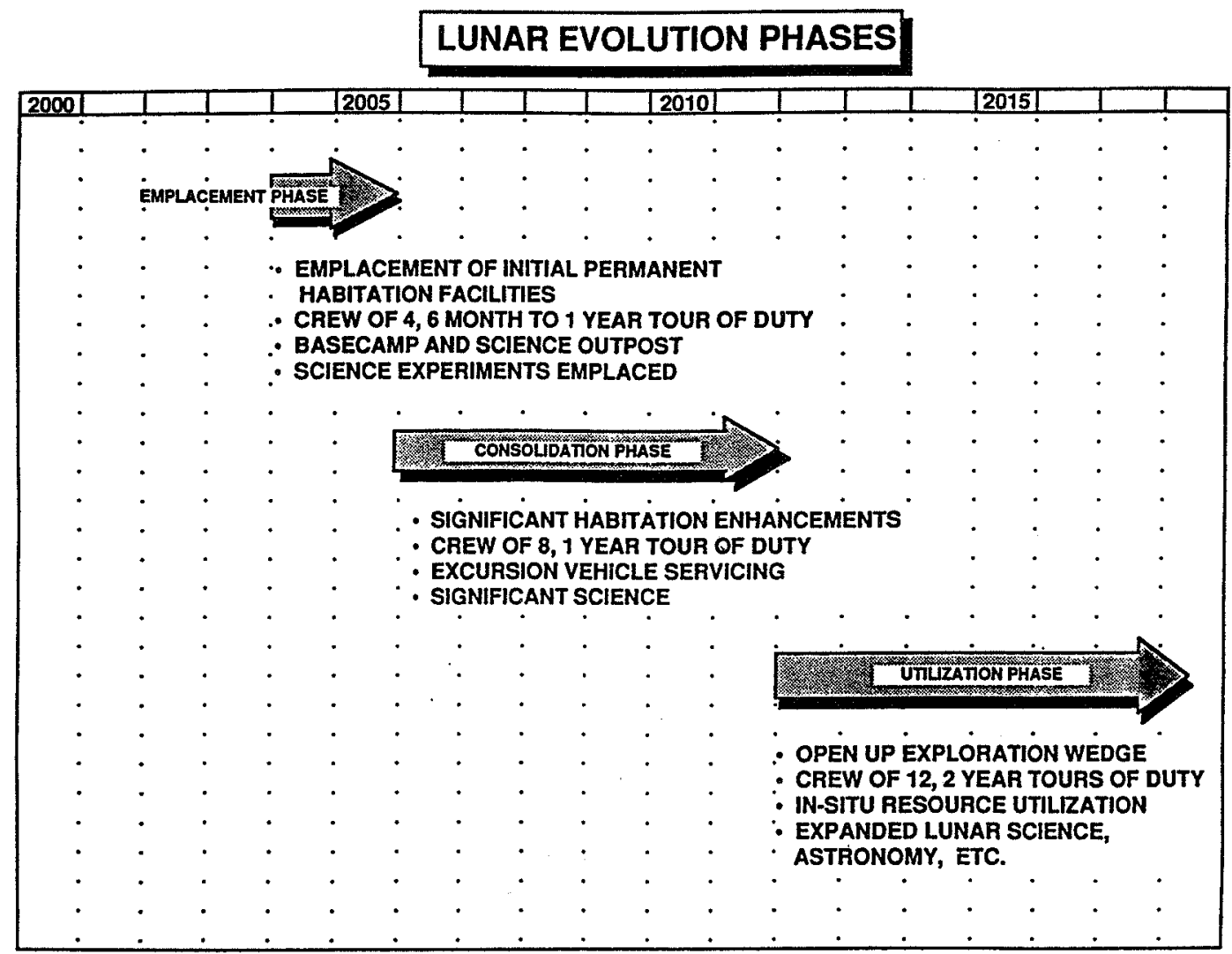

Figure 1. Current (i.e., September, 1989) phases for the lunar outpost program. 


\section{DISCUSSION}

Stockman: You mentioned that the lunar surface is very quiet. Yet I recall that one of the discoveries of the Apollo days was that moonquakes were quite persistent. Can you explain this?

Burns: There are two main categories of lunar seismic signals, based on the depth at which they originate. Almost all occur deep within the Moon at depths 700-1100 km; on average, about 500 deep events were recorded each year during the 8 years that the Apollo network operated. These deep moonquakes are related to tidal forces. The less frequent $(\simeq 5 /$ yr $)$ shallow quakes occur at depths of $<200 \mathbf{~ k m}$. Overall, seismic activity on the moon is drastically less than on Earth, with much smaller amplitudes. Seismic waves are intensely scattered near the lunar surface. This causes the energy of the waves arriving at a given point to be spread out, so the damaging effects of a moonquake are much less than those of an earthquake of the same magnitude.

Bely: I was intrigued by your proposal to design a lunar telescope to operate at two distinct temperatures. Could you expand on that?

Burns: One possibility is to operate an optical/IR telescope in two modes. Optical during the day and IR at night. Thermal cycles are long enough to make this practical. One needs to see if such a hybrid, thermally deformable telescope is possible in detail.

Johnson: We should try to use the telescopes both in the daytime and at night. It may require heating of some elements at night, and baffling and shielding during the day. We don't want to throw away the observing time and so we should try to adjust the system to operate in both environments.

Angel: One note of caution about interferometry. You have a very stable baseline on the moon. However, it will not be pointing in the direction that you want to point. So you have to chase around to find the null of the interference fringes, just like you have to do on earth. So there is a lower scale of separation for interferometers where free flyers have a very big advantage because you can point them. That makes beam combining very much better, with much wider fields. One needs to look at the transition point, that is, the size, where the problem of the stability of the baseline overcomes the disadvantage of not pointing in the right direction. The experience on a fixed surface, on the earth, shows that is it easy to do interferometry when the elements point as a single unit. Whereas, when you spread them out over a surface and have to make path length corrections you add a considerable complication.

Burns: The tradeoffs between optical interferometers and single aperture systems have really not been addressed yet, particularly for the moon, and those tradeoffs really need serious study.

Mlingworth: I agree, and would emphasize the need for a broad technical and scientific evaluation. The statement is often made that experience has shown that the future for radio astronomy is with interferometers, and so the jump is made to the statement that the future in optical-IR is also with 
interferometers. I think that there are real technical differences in these two areas that need to be explored and understood to define the realms of applicability of interferometers and filled apertures. To say that interferometers are the future overstates the potential of interferometric systems.

Burns: The technology is fundamentally different in the optical and the radio. Radio interferometry is made so simple and so elegant because of the availability of heterodyne receivers. The technology is well developed in the radio, it is well understood, and it is very flexible. We do not have heterodyne receivers in the optical and so it is different and harder to make predictions. Interferometry may be the best way to go, but it is not proven yet.

Mlingworth: We should define the $\mathrm{S} / \mathrm{N}$ that is needed for observations of astronomical objects that play an important role in a variety of fundamental problems, and compare the interferometry and filled aperture approaches.

Burns: Even with the technological leaps that we have made on the ground to make interferometers function through our turbulent atmosphere, we are still dealing with relatively primitive interferometers. Progress in space may allow us to leap some of the technological hurdles and to see if the basic technology will give us interferometers that will be productive in a very general sense. 
Required Technologies for A 10-16m UV-Visible-IR

Telescope on the Moon

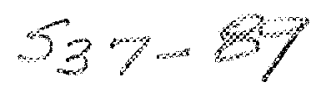

Stewart W. Johnson 1

John P. Wetze $1^{2}$

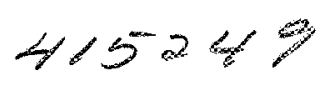

ABSTRACT

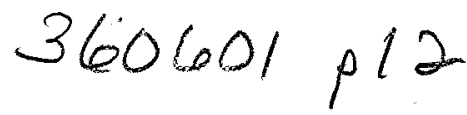

A successor to the Hubble space Telescope, incorporating a 10 to 16 meter mirror, and operating in the UV-Visible-IR is being considered for emplacement on the Moon in the 21st Century. To take advantage of the characteristics of the lunar environment, such a telescope requires appropriate advances in technology. These technologies are in the areas of contamination/interference control, test and evaluation, manufacturing, construction, autonomous operations and maintenance, power and heating/cooling, stable precision structures, optics, parabolic antennas, and communications/control. This telescope for the lunar surface needs to be engineered to operate for long periods with minimal intervention by humans or robots. What is essential for lunar observatory operation is enforcement of a systems engineering approach that makes compatible all lunar operations associated with habitation, resource development, and science.

\section{INTRODUCTION}

A 10-16m UV-Visible-IR Telescope has been proposed to take advantage of the unique nature of the lunar environment. Other observatories proposed include the Very Low Frequency Array (VLFA) for radio astronomy (Douglas and Smith, 1985), the Moon-Earth Radio Interferometer (MERI) (Burns, 1985; Burns, 1988), and the Optical Interferometer (Burke, 1985). With each proposed telescope, there are a myriad of engineering issues to be resolved. Figure 1 shows examples of technology development considerations for a 10-16m UV-Visible-IR telescope.

\section{THE CHALLENGE}

The next great initiative in astronomy may be a Grand observatory on the Moon. As a consequence of the interest in returning to the Moon early in the 21st Century, there are possibilities for astronomy to take advantage of the long lunar nights and the vacuum and stable body of the moon for a first class optical astronomy facility. A 16 meter active segmented mirror provides possibilities for detection of extrasolar planets and investigation of galactic origins and evolution. Galaxies may be resolvable in early forms at red shifts of 1.4. Quasistellar objects may be used as probes to sound the

1 Principal Engineer, Advanced Basing Systems. BDM International, Inc., 1801 Randolph Road., S.E., Albuquerque, NM 87106.

Associate Staff Member, Space Systems. BDM Internationa1, Inc. 
intergalatic medium. Roger Angel of the Arizona Steward Observatory offers the prospect, with a 16 meter instrument, of using the ozone as a tracer of life on distant planets. These are exciting prospects. The possible schematic of this 10-16 meter telescope is shown in Figure 2.

Large Telescope (10 to $16 \mathrm{~m}$ UV-Visible-IR)

- Requirements

- System definition and specifications

- Site selection and characterization

- Control capability (stringent requirements limiting differential settlements, tide compensation)

- Lunar surface layout requires locating and modifying a suitable site

- Dynamic response of lunar soil to movement of telescopes

- Preservation, cleaning, and renewal of optical surfaces and coatings

General Technology needs

- Automation, telepresence, and robotics for construction, operations, and maintenance

- Human factors considerations (man-in-the-loop) and realistic artificial intelligence interaction

- Stiff, stable, light-weight structures from modern composite metal matrix or other selected materials either transported from Earth or made on the moon

- Data gathering, storage, processing, and transmission

- Thermal control, cryocoolers, heat dissipation and heaters as appropriate

- Power sources to serve lunar outpost requirements

- Potential applications of superconductivity

- Mobility on the surface (robots/human)

- Earth to moon and return transportation

- Test and evaluation of system

- Self-organizing failure characteristic prediction/detection and remote correction

Figure 1. Examples of Technology Development Considerations for a 10-16m UV-Visible-IR Telescope

\section{ADVANCED TECHNOLOGIES AND CRITICAL ENGINEERING ISSUES}

A major difficulty in determining what the critical engineering issues are for the large optical telescope. on the Moon is that the instrument is in the early planning stages. The identification of critical engineering issues is somewhat arbitrary predicated on judgement as to observatory design and types of materials and technologies to be used. There will be many significant components such as foundations and supporting structures, thermal control systems, power, communications and control, and data processing and transmission (Johnson, 1988).

Each of these significant components suggests a set of critical engineering issues which can be addressed from the point of view of required technologies to make the telescope perform in an acceptable way. Figure 3 lists the significant new technologies discussed in this paper which will be required for the telescope.

\section{CONTAMINATION/INTERFERENCE ISSUES}

One of the challenges facing telescope designers and operators
is coping with natural and operations-induced sources of
contamination/interference (Figure 4) on the Moon. Sources of
contaminants and interference will have implications for all aspects
of the lunar astronomical observatory performance (Figures 5 and 6 ).




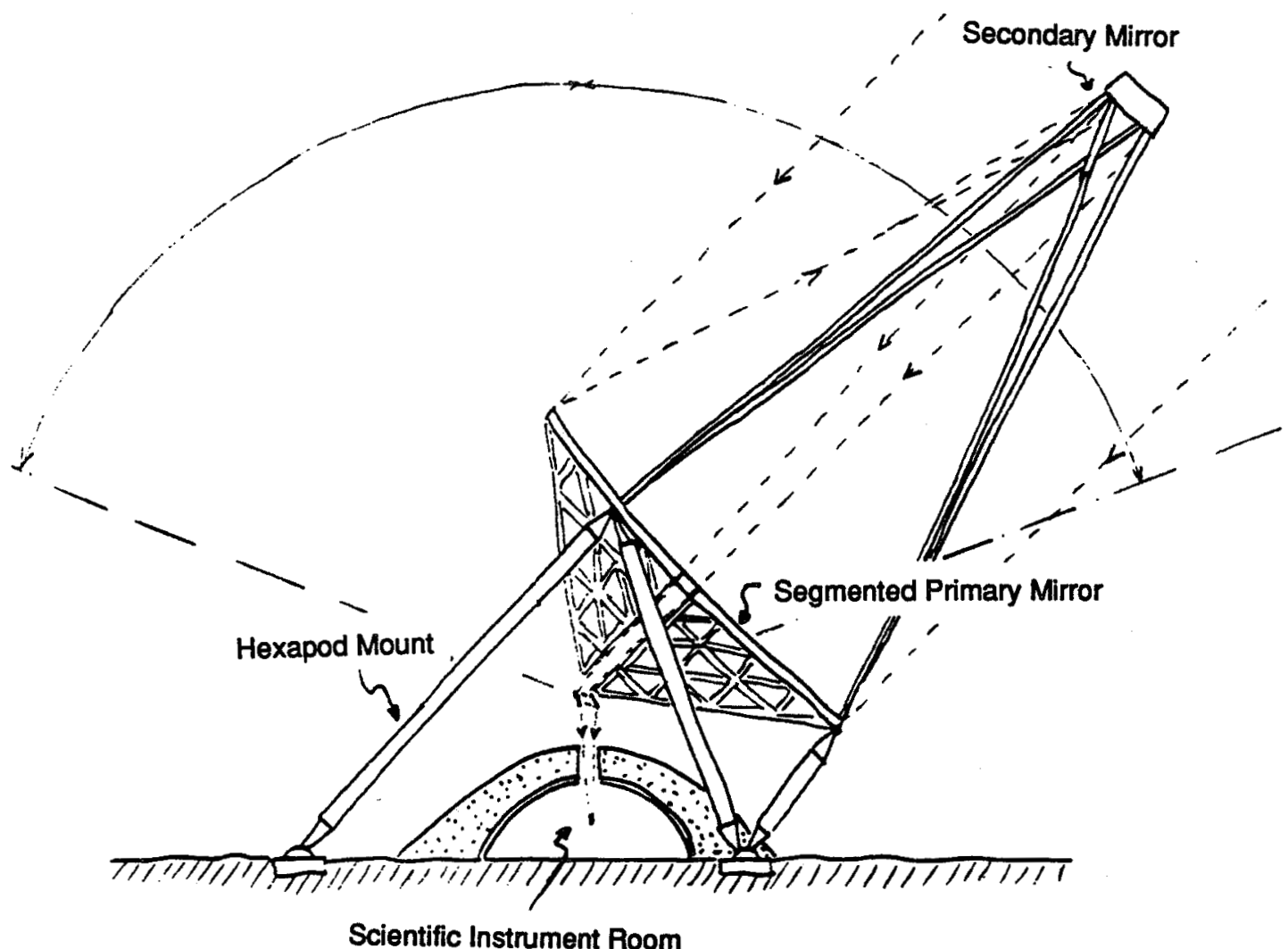

Figure 2. Simplified Concept for a 16 Meter Lunar-Based Telescope (after P. Bely, August 1989)

Contamination/Interference Control Test and Evaluation Construction

Power and Cooling/Heating

Stable Precision Structures

Optical Systems

Parabolic Antennas

Shielding

Communications and Control
Manufacturing:

Terrestrial

In-space

Lunar

Autonomous/Semi-autonomous:

Deployment

Operations

Maintenance

Figure 3. Technologies for Lunar Telescope 
Fine-grained particulates from the lunar surface - stick to surfaces

Meteoroid impacts - loft debris; cause surface pitting

Gases - stick to surfaces

- Natural

- Induced by operations

- rocket plumes

- outgassing from excavations/fill in soil and mining/manufacturing

- outgassing from suited workers

Radio frequency - interference problem for radio astronomy/communication

Ground shock/vibrations both natural and operations induced - problem for optical interferometers/other instruments

Other:

- Reactor radiation

- Waste heat from power sources

Figure 4. Some Contamination/Interference Sources and Implications

\begin{tabular}{|l|l|}
\hline Possible Contamination/Interference & Possible Countermeasures \\
\hline $\begin{array}{l}\text { Gases "sticking" to optical surfaces and } \\
\text { changing optical properties }\end{array}$ & $\begin{array}{l}\text { Reduction of effluents at } \\
\text { source. Technology to } \\
\text { purge and renew } \\
\text { surfaces }\end{array}$ \\
$\begin{array}{l}\text { Fine-grained particulates from lunar } \\
\text { regolith adhering to optical surfaces } \\
\text { and other surfaces }\end{array}$ & $\begin{array}{l}\text { Dust mitigation tech- } \\
\text { nologies (reduce } \\
\text { operations generating } \\
\text { gases); clean-up tech- } \\
\text { nologies }\end{array}$ \\
$\begin{array}{l}\text { Radio trequency interference with } \\
\text { broad-band data transmission' } \\
\text { reception }\end{array}$ & $\begin{array}{l}\text { Frequency allocation and } \\
\text { transmitter standards }\end{array}$ \\
$\begin{array}{l}\text { Ground shock/vibrations interfering } \\
\text { with nanometer precision alignments }\end{array}$ & $\begin{array}{l}\text { Alignment sensing/adjust- } \\
\text { ment in real time; shock/ } \\
\text { vibration isolation at tele- } \\
\text { scope; shock suppression } \\
\text { at origin; ke日p-out zones }\end{array}$ \\
\hline
\end{tabular}

Figure 5. Instrument Contamination/Interference and Possible Countermeasures for a 10 to $16 \mathrm{~m}$ UV-Visible-IR Telescope 
Contamination effects research

- Determination of effects

- Development of acceptable standards

Modeling of the mechanisms of contamination

Critical diagnostics/measurements program for lunar surface contamination

- Material/structural samples deployed to lunar surface and data collected

- Verification/comparison of model to results of data collection

Development of contamination prevention and cleaning techniques

Figure 6. Some Recommended Contamination Technology Programs for Lunar Surface Astronomy

Particulates and gases deposited on surfaces can significantly alter optical and thermal properties of surfaces and degrade performance. They can defeat the important attributes of delicate coatings and scatter light, create assembly and erection problems (particulates), and lead to problems in electronics. This paper first looks at some contamination/interference control technologies needed and then deals with selected other technologies for lunar observatories.

Telescopes on the Moon may tend to be surrounded by transient atmospheres resulting from manned and unmanned operations in the vicinity (Burns, 1989). That there will be a transient gas cloud is evident from the work of investigators ( $T$. H. Morgan, personal communication) interpreting measurements from atmospheric detection instruments on the Apollo Lunar Surface Experiments Package (ALSEP). Under a worst-case scenario, the "cloud" of transient atmosphere could degrade astronomical observations. The "cloud" density will be dependent on relative rates of contaminant generation and removal. Removal is by collisions with solar wind protons, diverging orbits of particles, expansion into space, decomposition and evaporation, and entrapment or sticking in the lunar soil or regolith.

Particulate and gaseous deposits on critical surfaces of astronomical instruments on the lunar surface may occur as a result of both natural and man-made environments. Deployment and emplacement will involve vehicles and perhaps suited construction workers outgassing water and other by-products of metabolism and suit functions.

Required power and communication units may be sources of unwanted heat, radiation, and radio frequency interference. Surface operations for emplacement of observatories may involve excavation, compaction, trenching and fill operations which will accelerate and disperse particulates and liberate gases. 
Contamination control is a prime area of concern for virtually any telescope installation. Contamination control technologies required for telescopes to be based on the Moon include protection of precision surfaces and parts through the life cycle including manufacture, assembly, test and checkout, transportation, landing, erection/deployment, and lunar surface operations/maintenance. Safe techniques to remove contaminants at any stage in this life cycle are needed. Obviously, means to detect and establish the nature of contaminants are required so that the severity of the contamination problems can be monitored and appropriate countermeasures can be taken.

Particular attention is needed to ascertain the implications of long-term lunar surface operations for accumulation on surfaces of contaminants such as fine-grained particulates, products of outgassing of materials, and propulsion products.

There are needs for investigations to improve our understanding of optical and thermal control coatings, their behavior, and interactions with contaminants and radiation environments on the lunar surface. The processes of contamination and contamination removal can be modeled to assist in predictions of the severity of problems developing as a result of various operational scenarios. To develop useful models will require an improved understanding of the physics of surface deposition and better characterization of the lunar environments, both natural and operations-induced. The longer-term goal will be to develop techniques for surface cleaning and coating restoration in situ on the lunar surface.

Johnson, Taylor, and Wetzel (1989), discuss environmental effects on astronomical observatories. They relate to experience with recovered Surveyor 3, Solar Max and other parts exposed to the lunar and orbital environments. The results they present are instructive in formulating future contamination control technologies.

\section{TEST AND EVALUATION TEGHNOLOGIES}

A methodology and facilities and resources are needed to assure that systems concepts for a lunar astronomical observatory can and will be modeled and tested adequately at various stages of conceptualization, research, development, fabrication, and preparation for launch. The goal is to avoid unpleasant surprises after arrival on the lunar surface. Questions to be resolved by a test and evaluation process relate to the operational effectiveness and suitability of the observatory systems. Effectiveness questions for test and evaluation are those tied in with performance such as pointing and tracking accuracy and precision, resolution, and image quality. Suitability questions relate to reliability, maintainability, and supportability of the telescope operational systems on the lunar surface. All of the suitability questions are of enormous importance when the logistics line of support is from the Moon to the Earth.

Early involvement of test and evaluation methodologies will start at the telescope system concept level to make adaptation possible to assure testability. Ground-based simulators will be needed to verify interoperability and autonomy of telescopes. Systems for 
calibration of telescope systems are an important aspect for the prelaunch modeling, test, and evaluation process.

\section{MANUFACTURING TECHNOLOGIES}

Two types of manufacturing capabilities should be pursued to support lunar-based astronomical observatories. One set of capabilities will be on Earth and the other eventually on the Moon. Terrestrial manufacturing of telescopes will be aimed at producing very lightweight, reliable and packageable components of observatories for shipment to and deployment on the Moon. One example will be composites manufacturing which requires technology development for coatings, joints, fabrication techniques, and complex fixtures for support of steerable dishes and mirrors for radio astronomy and optical astronomy. Parts should be produced so that they are interchangeable where possible (e.g., the struts supporting mirrors and dishes). Optics and electronics suitable for long-term use at a lunar observatory require special care in manufacturing to avoid faults and impurities that lead to subsequent degradation and failure.

In the area of manufacturing, the prime technology issue is producibility. Required for the 10 to $16 \mathrm{~m}$ UV-Visible-IR telescope are capabilities to manufacture, assemble, inspect, test, and maintain high quality at reasonable cost. U1timately, some components may be manufactured from lunar materials on the Moon--requiring a whole new set of manufacturing technologies.

\section{CONSTRUCTION TECHNOLOGIES}

Mobility and transportation with minimal environmental impact are key elements in the deployment of the observatory and its components on the lunar surface. Transportation of components to the lunar surface will, for example, require safe and secure packaging to preserve the integrity and cleanliness of delicate optical and other elements. Deployment and erection sequences must be carefully preplanned so that components match up in spite of temperature variations from component to component and with time. Technologies for deployment should minimize the needs for intervention by construction workers in space suits. Teleoperated cranes may serve as backup for automated off-loading of components from arriving payload packages. Ways will be needed to prevent the accumulation of fine-grained particulates from the lunar regolith on mating surfaces of contiguous elements of the observatory. Confidence in deployment and erection technologies will be critical in determining the future success of the observatory.

The emplacement of the proposed telescope on the Moon will require the capability to maneuver vehicles in remotely controlled (teleoperated) or preprogrammed operational modes. A variety of terrains will be encountered including small and large craters, boulder fields, hills and valleys.

\section{AUTONOMOUS OPERATIONS AND MAINTENANCE TECHNOLOGIES}

Autonomous operation and maintenance of telescope systems on the Moon is a goal that will be difficult to achieve because of the unpredictability of the problems that will be encountered. Allowance 
stould be made for teleoperation and maintenance workers in space suits if unanticipated difficulties arise. Prelaunch test and evaluation efforts on Earth will focus on various aspects of teleoperated operation and maintenance to predict and resolve difficulties before arrival at the Moon.

The vehicle associated with the telescope should be able to operate in several different modes as needs dictate change from manual operation to local teleoperation or to remote teleoperation, or perhaps to autonomous operation and hybrid modes. Technical issues with the vehicle design relate to vehicle size and mass, load carrying capacity and range, communications and control, number of wheels (or tracks), manipulator capabilities, power, and how the vehicle copes with the environment (e.g., the soil, rock, and terrain; vacuum; meteoroid impact; radiation; extremes of temperature; and diurnal cycles of solar radiation). The robotic vehicle system that supports the construction on the lunar surface will be required to support all phases of the effort including transport according to the predetermined plan, emplacing a communications/computer station, and performing maintenance and repair tasks. The vehicle must have flexibility to meet unanticipated needs such as coping with the unexpected, unusual terrain, soil variability, and layout adjustments.

The prime power source for the lunar astronomical observatory and associated facilities will be either solar or nuclear or a combination. Power requirements will probably be much less than $100 \mathrm{KW}$. Solar arrays appear to be suitable if backed by sufficient energy storage capacity (batteries or regenerative fuel cells) to continue operations during the lunar night. There is a strong need for development of regenerative/rechargeable power storage devices both large and small for use with solar energy devices to furnish power during the 14 Earth-day lunar night. One option for the next generation battery is a $\mathrm{Na} / \mathrm{S}$ battery being developed at the Aero Propulsion Laboratory at Wright-Patterson Air Force Base, Ohio (Sovie, 1988). Radioisotope thermoelectric generators also are possible power sources although they are inefficient and generate relatively large amounts of heat. Focal plane arrays for optical telescopes on the Moon will need to be cooled. Much technology development is required for cryocoolers to fill this need. One option is the development of an integrated radioisotope-fueled dynamic power generator and cryocooler to cool the focal plane arrays.

\section{STABLE PRECISION STRUCTURES TECHNOLOGIES}

Technology is required for large, stable, precision structures to support observatory components on the moon. Geometrically precise structures using advanced materials such as metal matrix composites are needed. These structures can be designed to have the required very low coefficients of thermal expansion.

The supporting structures for large optical telescopes on the moon need attention to isolation from disturbance, structures and controls interaction, and testing issues as portrayed in Figure 7. Operation of the telescope on the Moon will involve large structures which must be precisely aligned and which must track with high precision. Technologies will be required to measure surface accuracies and to make adjustments if needed (Figure 8 ). 


\section{Disturbance Issues}

- What are the critical disturbances?

- Natural - seismic shock, thermal

- Operations induced - ground shock, vibrations

- What mitigation technologies are applicable?

- How can disturbances be characterized and mitigation approaches formulated?

\section{Structures Issues}

- What approaches can be taken to build light-weight, high-stiffness structures optimized for the lunar $1 / 6 \mathrm{~g}$ and extreme thermal environments?

- Structural parameters - how ascertained?

- Improved models (computational)

- Test and instrumentation challenges

- Optimization

- Assembly/erectioninspection

Control Issues (for orienting mirrors)

- Control - structure interactions

- Transients and damping in structures optimized for $1 / 6 \mathrm{~g}$

- Experiments and tests of control mechanics

\section{Testing Issues}

- Ground testing on Earth vs. on Moon

- Scaling of terrestrial structures tests to larger structures at $1 / 6 \mathrm{~g}$

- Measurements/instrumentation for terrestrial//unar use

\section{Figure 7. Issues Relating to Large Structures to Support Opitcs}

- Surface accuracies

- Precise demountable segments

- Stable frameworks

- Easily transportable pieces

- Disassemble/reassemble without loss of accuracy

- Means for adjustments

- Mounts with pointing accuracies

- Foundations in lunar regolith

Figure 8. Technology Development for 10 to $16 \mathrm{~m}$ UV-Visible-IR Telescope

\section{OPTICAL SYSTEMS TECHNOLOGY DRIVERS}

There are many technology drivers for these optics. They include optical coatings that resist delamination, optics that are stress free after manufacture, and refractive materials which do not darken or develop color centers. Refractive materials should have low scatter. Adaptive optics will be important for lunar optical telescope applications. Actuator and controls development and power and thermal control for adaptive optics should be pursued. 
For mirrors on the lunar surface, active cleaning and contamination control techniques will be needed. Polishing techniques need to be improved; renewable coatings may be required. Materials used for telescopes need to be thermally stable. The appropriate degree of coating hardness against the ultraviolet and $X$-ray environments of the lunar surface will be needed. As always, the telescope optics will require the necessary vibration isolation.

Material systems should be developed to function as protective shields for antenna structures and mirrors against the worst extremes of the lunar thermal environment and the micrometeoroid environment.

\section{COMMUNICATION AND CONTROL TECHNOLOGIES}

There are many requirements on the communication system for the lunar astronomical observatory. Communication satellites in lunar orbit may be needed. At an observatory site on the far side of the Moon, communication antennas will be needed for uplink and downlink which are high-gain, lightweight, and have low power consumption. Frequency and bandwidth selection for communications must be compatible with radio astronomy operations.

\section{CONCLUSION}

The need for all the observatories under consideration and for all extraterrestrial facilities is to engineer them with technologies that make it possible to perform well for long periods of time with minimal intervention by humans or robots. Better astronomy can be done if contamination and interference (gases, particulates, ground shock, and extraneous $\mathrm{RF}$ radiation) resulting from nearby operations can be kept to very low levels by limiting the need for nearby operations. An obvious need is to strive for facilities compatibility in lunar surface operations at various sites by controlling and reducing functions (e.g., proximity of mining operations or rocket launch pads to optical astronomy facilities) that lead to undesirable consequences: This need for compatibility implies the enforcement of a broad-based systems engineering discipline to all lunar engineering, construction, and operations.

\section{ACKNOWLEDGMENTS}

The support of The BDM Corporation, The University of New Mexico, and The National Aeronautics and Space Administration (Grant NAG 9-245) Johnson Space Center in the preparation of this paper is acknowledged. We thank T. H. Morgan for perceptive comments on the paper. The authors are grateful for the advice and support by $J$. Burns, N. Duric, J. Taylor, W. Mendell, M. Duke, B. Roberts, and Pierre Bely. Appreciation is expressed to Jean $\mathrm{K}$. Helmick and Clara P. Peele for preparation of the manuscript.

\section{APPENDIX 1. REFERENCES}

Akgul, F., W. Gerstle, and S. Johnson (1988). Presentation on a Lunar Steerable Parabolic Dish design for Radio Astronomy, at Space 88 Conference, Albuquerque, NM, Aug. 29-31, 1988. 
Burke, B. F. (1985). "Astronomical interferometry on the moon." Lunar bases and space activities in the 2lst century, W. W. Mende11, ed., Lunar and Planetary Institute, Houston, Tex., 281-291.

Burns, J. O. (1985). "A moon-Earth radio interferometer," Lunar bases and space activities in the 21 st century, W. W. Mende11, ed., Lunar and Planetary Institute, Houston, Tex., 293-300

Burns, J. O., et al, (1989). "Artificially generated atmosphere near a lunar base," LBS-88-024, Second Symposium on Lunar Bases and Space Activities in the 21 st Century, NASA-JSC, Houston, TX

Burns, J. 0 . (1988). "2lst century astronomical observatories on the Moon," Engineering, Construction, and Operations in Space, S. W. Johnson and J. P. Wetzel, editors, American Society of Civil Engineers, N.Y., N.Y., 1083-1094.

Douglas, J. N., and Smith, H. J. (1985). "A very low frequency radio astronomy observatory on the moon." Lunar bases and space activities in the 21 st century, W. W. Mendell, ed., Lunar and Planetary Institute, Houston, Tex., 301-306.

Johnson, S. W. (1988). "Engineering for a 21st Century Lunar Observatory." Journal of Aerospace Engineering, 1, 35-51.

Johnson, S.W., G. J. Taylor, and J. P. Wetzel (1989). "Environmenta1 Effects on Lunar Astronomical Observatories." Second Symposium on Lunar Bases and Space Activities in the 21st Century, NASA-JSC, Houston, TX.

Nickle, N. L. (1971). "Surveyor III material analysis program." Proc. 2nd Lunar Sci. Conf., Vol. 3 (Supplement 2, Geochimica et Cosmochimica Acta, J. Geochem. Soc. and Meteorit. Soc.), MIT Press, Boston, Mass., 2683-2697.

Proceedings of the SMRM Degradation Study Workshop (1985). NASA Goddard Space Flight Center, Greenbelt, Md.

Scott, R. F., and Zuckerman, K. A. (1971). Examination of the Surveyor 3 Surface Sampler Scoop Returned by the Apollo 12 Mission. California Institute of Technology, Soil Mechanics Laboratory.

Sovie, R. J. (1988), "Power Systems for Production, Construction, Life Support and Operations in Space," Engineering, Construction and Operations in Space, S. W. Johnson, and J. P. Wetze1, editors, American Society of Civil Engineers, N.Y., N.Y., 928-939.

The Long Duration Exposure Facility (LDEF): Mission 1 Experiments (1984). Lenwood Clark, et al, editors, NASA SP-473, NASA,

Washington, D.C.

\section{ADDITIONAL SOURCES OF INFORMATION :}

Burns, J. O. and W. W. Mende11, ed. (1988). Future Astronomical Observatories on the Moon, NASA Conference Publication 2489.

Burns, J., Duric N., Johnson, S., and Taylor, G. J., editors (1988). Preliminary Study of a Lunar Far-Side Very Low Frequency Array, in preparation. 


\section{DISCUSSION}

Schember: What did you mean about the need for an improved clock?

Johnson: A suitable clock on the moon would be helpful for a radio astronomy experiment using lunar communications antennae in conjunction with ground based antennae. This experiment would be analogous to the radio astronomy experiment done with TDRS some time ago. The clock is not a major issue.

Burns: Dust may be a problem on the moon. The Apollo astronauts were literally covered with fine grain dust, presumably produced by "static cling." Calculations indicate that dust on the moon may migrate from light to dark areas. Depending on the magnitude of this process, a telescope behind a sunshade could be a target for dust contamination.

Johnson: Dust less than 20 microns constitutes about $25 \%$ of the lunar surface layer. We ultimately may find ways to stabilize the lunar surface layer. One possibility is through microwave processing. Until we can stabilize the dust, we must operate on the moon in ways that will mitigate transfer of dust to optics, thermal control surfaces, and mechanical parts. 


\section{Space Logistics: Launch Capabilities}

Randall B. Furnas

NASA Headquarters - Code MD

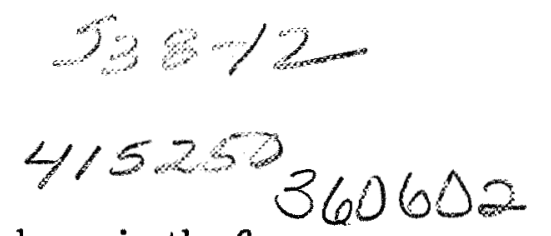

The current maximum launch capability for the United States are shown in the figure below. This is an optimistic maximum based on absolute maximum launch rates as well as lift capability. Actual average annual lift capability is likely closer to $800,000 \mathrm{lb}$.

\section{CURRENT LAUNCH VEHICLE FLEET \\ MAXIMUM CAPABILITY}

$\begin{array}{lccc}\text { YEHICLE } & \text { MAXLAUNCHRATE } & \text { LIFTCAPABILITY } & \begin{array}{c}\text { ANNUAL MAXIMUM } \\ \text { LIFTCAPABILITY }\end{array} \\ \text { NSTS } & 14 / \mathrm{YR} & 45,000 & 630,000 \\ \text { TITAN IV } & 10 / \mathrm{YR} & 38,000 & 380,000 \\ \text { ATLAS/CENTAUR } & 8 / \mathrm{YR} & 12,500 & 100,000 \\ \text { DELTA II } & 18 / \mathrm{NR} & 8,700 & 157,000 \\ & & & 1,267,000\end{array}$

- BASED ON 28.5 DEG., 160 MILE ORBIT

The predicted Earth-to-orbit requirements for the United States are shown in the next figure. The solid line represents the expanded NASA program including manned exploration. The dashed line indicates the projected requirements for the Space Defense Initiative.

Contrasting this chart with the previous chart indicates the strong National need for a major increase in Earth-to-orbit lift capability.

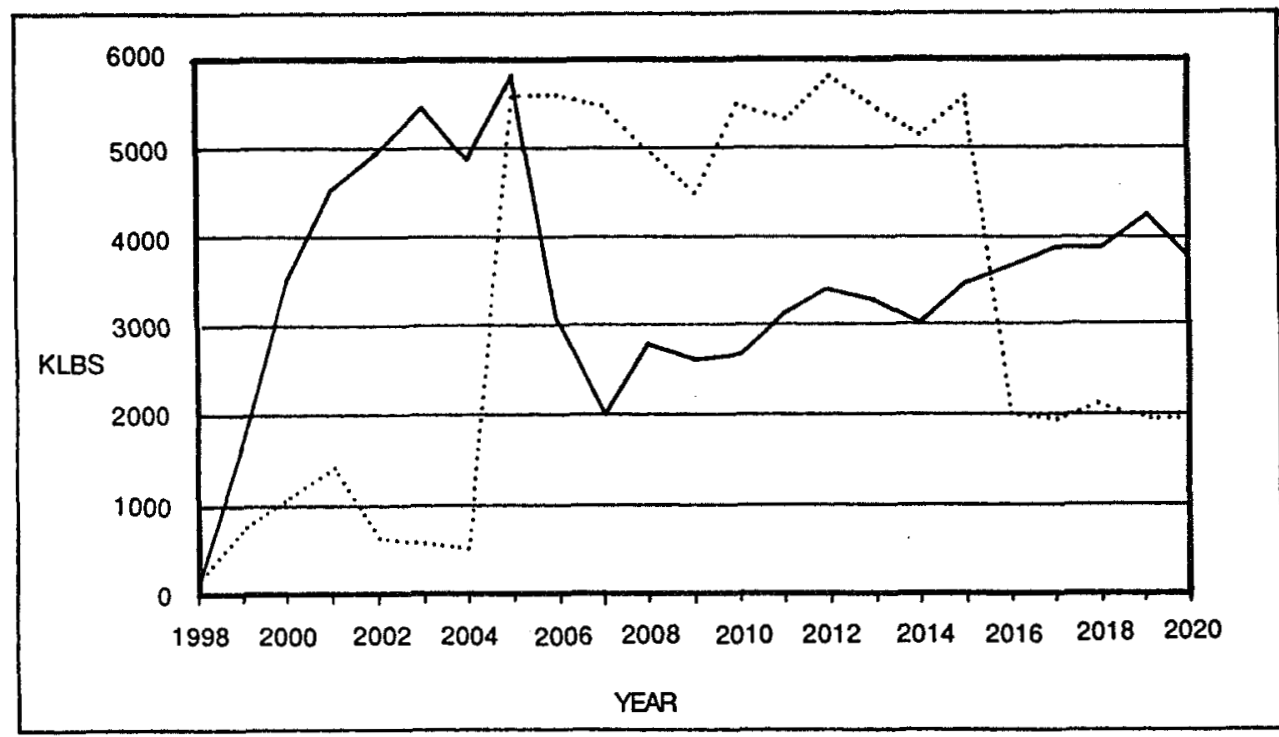

NATIONAL PLUSSDIO

NATIONAL PLUS EXPANDED NASA PROGRAM 
Approximate weights for planned payloads are shown in the following chart. Existing Payloads refers to spacecraft of the type that are currently being launched by the US. Existing Payload Growth refers to the expected evolution of those payloads to build in more capability and/or life. NASA Planetary Growth refers to anticipated growth in the unmanned planetary exploration spacecraft. NASA Space Station is a range showing a lift capability that would enhance the Space Station Freedom deployment, though the baseline deployment scenario remains the NSTS orbiter fleet. Finally, the last two ranges displayed on the chart represent specific SDI payload ranges that are predicted.

Current maximum lift capability in the US is defined by the grey band representing the NSTS Orbiter and Titan IV. This can be contrasted with the Soviet Energia on the top dashed line.

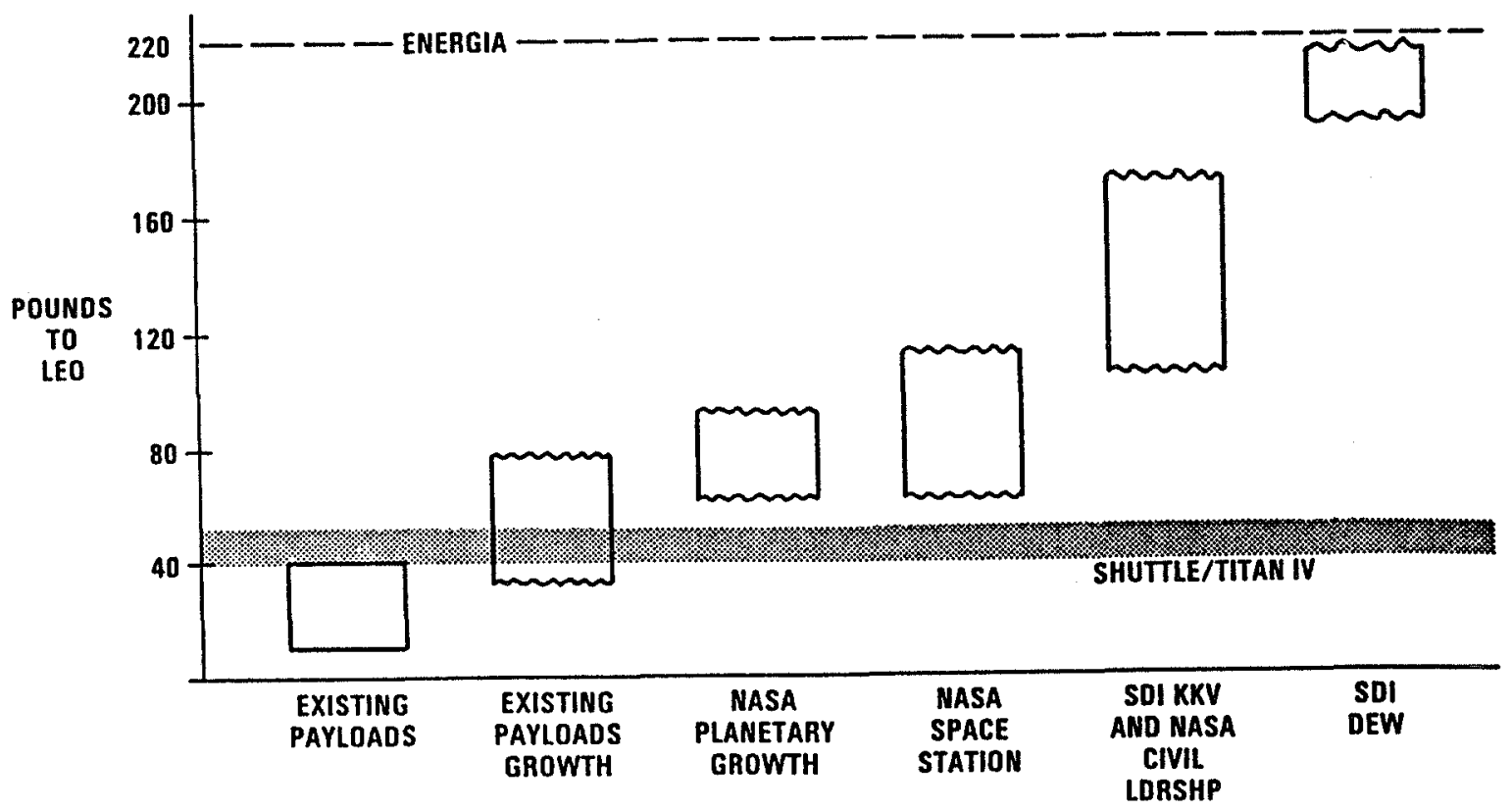

NASA is studying the following options to meet the need for a new heavy-lift capability by mid to late 1990 's:

- Shuttle-C for near term (include growth versions), and

- the Advanced Lauching System (ALS) for the long term.

The current baseline two-engine Shuttle-C has a $15 \times 82 \mathrm{ft}$ payload bay and an expected lift capability of $82,000 \mathrm{lb}$ to Low Earth Orbit. Several options are being considered which have expanded diameter payload bays. A three-engine Shuttle-C with an expected lift of $145,000 \mathrm{lb}$ to $\mathrm{LEO}$ is being evaluated as well.

The Advanced Launch System (ALS) is a potential joint development between the Air Force and NASA. This program is focused toward long-term launch requirements, specifically beyond the year 2000 . The basic approach is to develop a family of vehicles with the same high reliability as the Shuttle system, yet offering a much greater lift capability at a greatly reduced cost (per pound of payload). The ALS unmanned family of vehicles will provide a low end lift capability equivalent to Titan IV, and a high end lift capability greater than the Soviet Energia if requirements for such a high-end vehicle are defined. 


\section{ADVANCED LAUNCH SYSTEM}

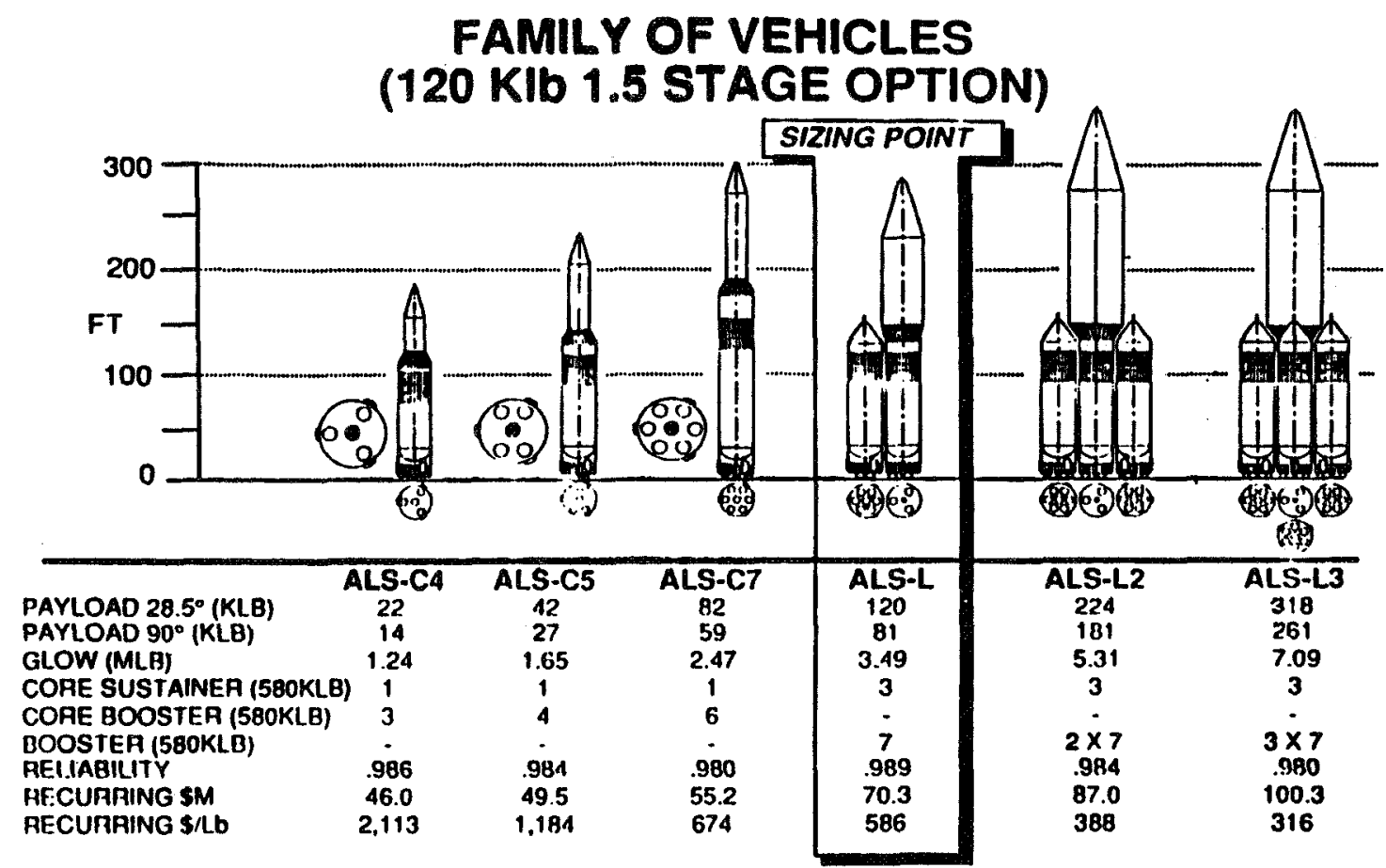

The proposed vehicle processing flow for ALS is shown conceptually on the next figure. A key to the ALS low cost will be minimum interface between the payload and the launch support system. The payload would be installed in a Cargo Integration Facility approximately five days before launch. The cargo is relatively inaccessible after installation. Payloads will need to be designed to be nearly self-sufficient for that five day period. This procedure contributes greatly to reduced launch operations costs.

\section{KEY ALS FACILITIES}

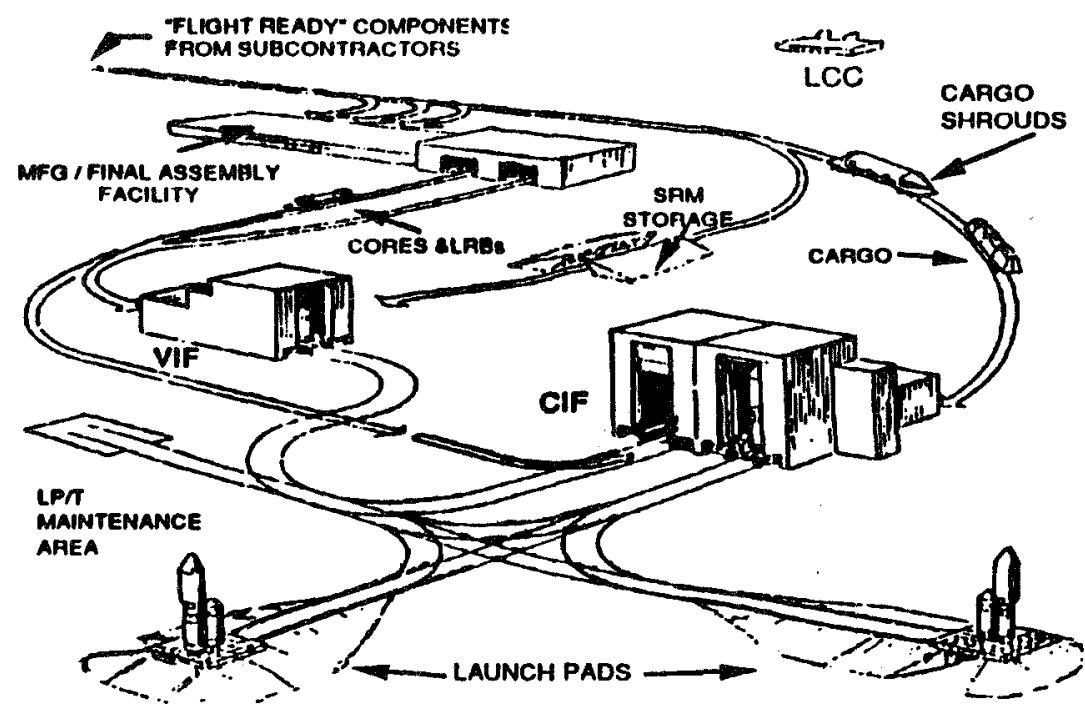

ALS Baseline Facillities Will Accommodate the Family 
As shown in the graph below, the ALS system plans to provide launch services at a cost per launch roughly equivalent to the Atlas II. The concept of a family of vehicles results in a band on this chart which can actually extend well to the right of the region displayed.

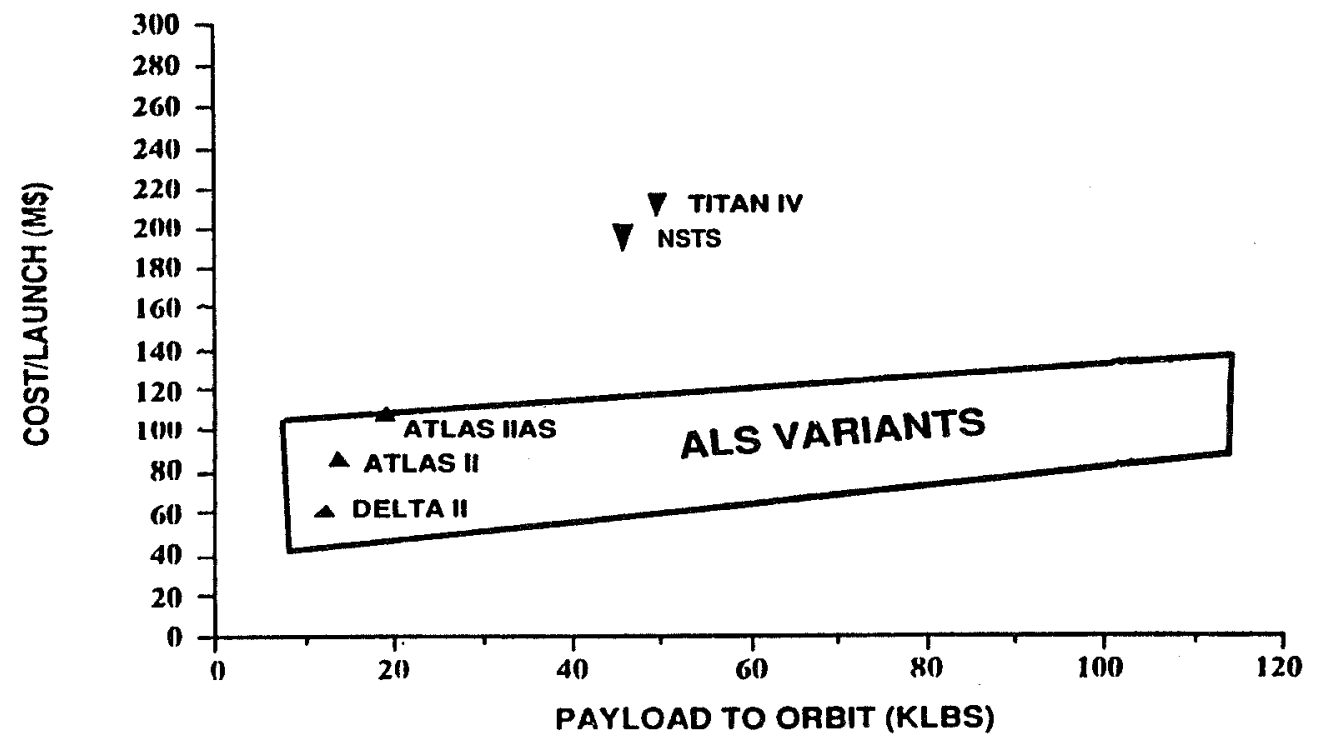

In conclusion, the planning of the next generation space telescope should not be constrained to the current launch vehicles. New vehicle designs will be driven by the needs of anticipated heavy users. 


\section{DISCUSSION}

Bely: What would be the capability of the largest ALS to HEO?

Turnas: This answer is entirely dependent upon the chosen upper stage. Also, the joint USAF/NASA program has not yet determined what the "largest" member of the family will be. At this early stage, you might calculate capability to HEO based on a booster of approximately $200 \mathrm{klb}$ lift combined with a Centaur (Titan IV version).

Mingworth: What is Energia's capability?

Bely: About $300 \mathrm{klb}$ to LEO.

Furnas: So ALS with $200-320 \mathrm{klb}$ to LEO is essentially as capable as Energia.

Thompson: Can you give us any timescales on ALS? This is important because it impacts the size and weight limits that would be available at the point where we need to fix the design of a large space telescope.

Turnas: This depends on several factors, one being the timescale and the way in which the lunar outpost program develops, and on when the Shuttle-C and ALS programs get their new starts. Both of these programs are in for new starts in 91-92. The baseline technology work is currently in progress.

mlingworth: Are there plans being developed for a higher performance upper stage to match the projected performance of ALS?

Iurnas: The ALS vehicle has no upper stage, as currently envisioned. The vehicle simply places the payload into a transfer orbit. Each payload will need to be assessed individually for available upper stages (e.g., Centaur) to determine combined ALS/upper stage performance.

Bisenhardt: A difference between GEO and HEO is the time required by the upper stage to achieve the orbit. With the Titan IV/Centaur, the time required to reach HEO pushes Centaur design on battery life and perhaps other issues.

Bely: What is the future of the OMV and OTV in the context of the ALS Program?

Inrnas: The future of OMV and OTV most likely rides with the future of the Lunar/Mars Initiative. These unfortunately are unknowns at this point due to the federal budget situation. The budget action that Congress takes in response to a National Space Council proposal for Lunar/Mars will be the best indicator of the future of both OMV and OTV (or STV). 


\section{On-orbit Assembly and Maintenance}

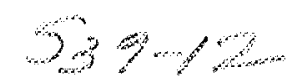

David R. Soderblom

Space Telescope Science Institute

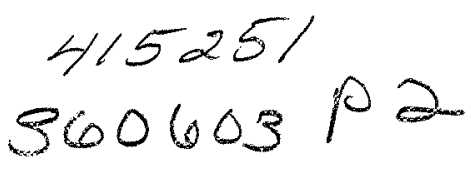

This subject is generally known as "Maintenance and Refurbishment," or "M\&R," and much of its origins lies in the design of the Hubble Space Telescope. HST is the first major NASA mission to incorporate $M \& R$ as an integral part of its design and construction. The basic concept is that $M \& R$ allows one to periodically replace worn or malfunctioning parts; to fix failures that compromise the success of the mission; to reboost the orbit to palliate atmospheric drag; to improve satellite performance through upgrades; and to replace the satellite's Science Instruments so as to keep it operating with up-todate devices. Thus $M \& R$ was to make a satellite more like a ground-based observatory in that one started with a telescope and an initial complement of instruments, then changed the instruments and support devices gradually over the years to take advantage of new technology.

In its original concept, $M \& R$ for HST meant bringing it back to the ground every five years for extensive refurbishment. This was soon. seen to be impractical: Where would the satellite be taken upon return? Could it be kept contamination-free? Could NASA afford two shuttle flights per refurbishment?

On-orbit maintenance is now the mode foreseen for HST's M\&R. The intervals at which it will be done are not clear. At first, two-year intervals were selected because of the anticipated rapid decline of the solar arrays and nickel-cadmium batteries. Improvements in both those areas have led to an estimate that we can wait five years before an $M \& R$ mission.

The motivation for $M \& R$ has been economic: by repairing an existing satellite perhaps much of its considerable cost can be saved, while still providing for advanced instrumentation as technology progresses. The desire to stretch the interval between M\&R missions has also been economic: shuttle flights (especially ones that require extensive training like an M\&R mission) cost at least $\$ 200$ million each. Even if that cost is ignored, flights are a scarce commodity for the foreseeable future. There is also a considerable cost in maintaining a reserve of parts to repair the satellite and the people with the knowledge to use those parts.

The future may vindicate HST M\&R, but it now appears that the best path to take is to build the quality in at the beginning and to place a satellite in the operationally best orbit you can afford, given whatever launch vehicle is available. If $M \& R$ is a real need, there is no entirely satisfactory compromise for your choice of orbit. In Low Earth Orbit, only about 24 work-hours total are available during one refurbishment flight, so that the range of activities that can be considered is limited. High Earth Orbit is much to be preferred for operational reasons, but is currently inaccessible for M\&R. The Space Station may be available for M\&R, but in an era when SS is being used as a stopover for a lunar base it will necessarily be a very dirty environment that is not suitable for ultraviolet telescopes such as HST. The original plans for SS called for a maintenance bay at the upper (clean) end, but that has been dropped. The obvious advantage of SS is the longer time available for work to be done. In the more distant future, the availability of an Orbital Transfer Vehicle could make much higher orbits accessible to M\&R. 
Working in space, in the absence of gravity, is very difficult. Maintenance work could be done much more easily on the Moon, especially if the telescope is near a lunar base. However, the costs would be extremely high.

In retrospect, $M \& R$ started with good intentions but on the whole will probably turn out to be unsatisfying. There are likely successes: At a cost of about $\$ 300$ to 400 million (including launch and other costs), the NICMOS instrument will provide dramatic new capabilities that could not have been otherwise achieved except at a much higher cost. On the other hand, the need for access to HST for M\&R has, as much as any other factor, forced the choice of a Low Earth Orbit, which will significantly complicate and compromise the science done by HST over its lifetime.

$M \& R$ has been pursued because of the very high costs of construction of complex satellites like HST. A more satisfactory solution in the long run is to bring those costs down because that means that more and different satellites can be built, with a higher overall return in scientific productivity. One key to reducing those costs is surely to drastically reduce the time needed to construct the satellite since most of the cost goes into labor. Reducing that time would also make undertaking projects like HST much more satisfying because one can hope to see the fruits of ones labors much sooner and with much less frustration. Thus I see the major impediment to long-term success in space astronomy as being administrative, not technological (as daunting as the technology may be). We need radically different concepts for the management of technology utilization at least as much as we need the technology itself if we are to succeed to the extent we all hope for in the coming years. 


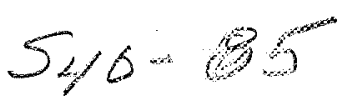

TECHNOLOGICAL SPIN-OFF FROM THE NEXT GENERATION $4 / 5252$

\author{
Virginia Trimble \\ $36060 \%$ CLOSE \\ Astronomy Program, University of Maryland, College Park MD 20742 \\ and \\ Department of Physics, University of California, Irvine CA 92717
}

ABSTRACT: The paper consists of a brief commercial announcement for (a) the Panel on Benefits to the Nation of Astronony and Astrophysics of the A\&A Survey Committee ("Bahcall Committee") and (b) for the idea that the potential for technological spin-off can be of use in building support for the Next Generation space telescope and should be kept in mind as the project is developed. The first point will cease to be relevant after June 1990, but the second will not.

Over the past decade, federal funding of astronomical research has lagged behind that of other sciences. The pure physical sciences in general have not done well in the NSF budget, but astronomy has done even less well than physics, chemistry, and mathematics. As a result, funds available both for the national observatories and for the individual grants programs have been essentially constant in real dollars since 1977, despite a roughly $50 \%$ increase in the number of American astronomers and in the number of pages published annually in the Astrophysical Journal, and a doubling of the numbers of observers using the national facilities. The projected NOAO budget for 1990 is actually almost 25\% below that for 1977 in deflated dollars.

Astronomy's failure to do better than barely holding its own in the funding race undoubtedly has several causes (though a shortage of scientifically interesting problems or of willing investigators does not seem to be among them). Our own lack of involvement in agressively selling what we have to offer probabiy is among the causes. As a result, one chapter of the report Astronomy and Astrophysics for the $90^{\prime} \mathrm{s}$ will address justifications for funding astronomy research in competition with other urgent national needs. The case will include astronomy's role in fostering public interest in science and public scientific literacy, its contributions to formal education and to the technologically-trained manpower pool, and its interactions with other, more obviously practical, sciences.

Technological spin-off may not be the most important reason that astronomy is worth funding and worth doing, but it is the reason that seems most likely to appeal to some of the organizations and individuals that will have to be persuaded. A few of the spin-offs are well known within 
the astronomical community (for instance, the origins of airport security systems in astronomical $\mathrm{X}$-ray detectors and the applications of radio astronomical ideas to the testing of dishes for communication purposes). The panel that is to write the AASC chapter on what astronomy is good for urgently needs additional specific, concrete examples of spin-off of this type, including software as well as hardware. Meeting participants included a large number of experts in the development of photon collectors, detectors, and processors, many of whom are bound to be aware of such spin-offs that are not currently known to the panel. Please, if you have any information or ideas along these lines, send them promptly to the present author, who is chairing the Panel on Benefits to the Nation of Astronomy and Astrophysics, which will write the relevant chapter.

In addition, obviously, the development of the Next Generation space telescope is going to require a great deal of support (both financial and moral!) from a very wide variety of funding sources as well as from the scientific community. Since the telescope, its cooling system, detectors, data transmission systems and many other items are somewhat beyond what can be easily done today, their development is bound to drive technologies that can be applied to other sciences, industry, and defense purposes. It is worth giving some thought, both now and in the future, to what these spin-offs are likely to be, since emphasizing them to the right audiences may help to make a 10-16 meter space telescope sound like part of the obvious, natural future of American science and technology. 


$$
\begin{aligned}
& \text { mor } \\
& 4 \times 2
\end{aligned}
$$

List of Participants 


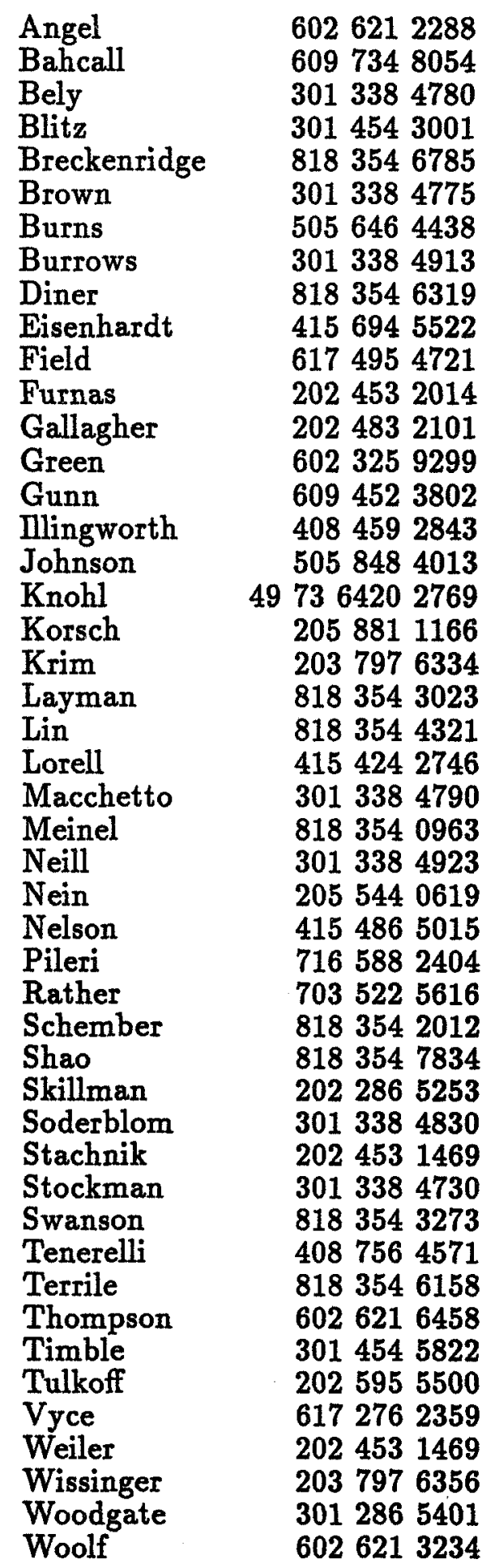




\section{PARTICIPANTS}

James Roger Angel Steward Observatory University of Arizona Tucson, AZ 85721

\section{John N. Bahcall}

School of Natural Sciences Inst. for Advanced Study Olden ln., Bldg.E

Princeton, NJ 08540

Pierre Y. Bely

Space Telescope Sc. Inst. 3700 San Martin Drive

Baltimore, MD 21218

Peter Bender Jila/Univ. of Colorado

Box 440

Boulder, CO 80309-0440

\section{Leo Blitz}

Astronomy Program University of Maryland College Park, MD 20742

Matthew Bobrowsky Space Telescope Sc. Inst. 3700 San Martin Drive

Baltimore, MD 21218

John F. Bolton

NASA Code 725.1

Goddard Space Flight Ctr.

Greenbelt, MD 20771

Howard E. Bond Space Telescope Sc. Inst. 3700 San Martin Drive Baltimore, MD 21218

\section{James B. Breckinridge}

Jet Propulsion Lab. 4800 Oak Grove Drive Pasadena, CA 91109
Robert A. Brown Space Telescope Sc. Inst. 3700 San Martin Drive Baltimore, MD 21218

Geoffrey Burbidge Ctr.for Astr. \& Space Sci. Univ. of Calif. at San Diego C-011, PO Box 109

La Jolla, CA 92038

\section{Jack Burns}

Dept of Astronomy New Mexico State Univ. Box 30001/Dept 4500 Lax Cruces, NM. 87003

Chris Burrows Space Telescope Sc. Inst. 3700 San Martin Drive Baltimore, MD 21218

Ivo Busko

Space Telescope Sc. Inst. 3700 San Martin Drive

Baltimore, MD 21218

Bruce Carney

Dept. of Physics and Astr. Univ. of North Carolina CB 3255 Phillips Hall Chapel Hill, NC 27599

\section{Stephane Charlot} Space Telescope Sc. Inst. 3700 San Martin Drive Baltimore, MD 21218

\section{James Cutts}

Jet Propulsion Laboratory 4800 Oak Grove Drive Pasadena, CA 91109
Laura Danly

Space Telescope Sc. Inst. 3700 San Martin Drive

Baltimore, MD 21218

\section{Lane Darnton}

Bldg $\mathrm{O} 1$

TRW

One Space Park

Redondo Beach, CA 90278

Roger Davies

University of Oxford

South Park Road

Oxford, OX13Q, UK

\section{Billy Davis}

PS02, Bldg 4200

Marshall Space Flight Ctr

Huntsville, AL 35812

Dominick Delavallee

Perkin Elmer Optical Gp.

100 Wooster Heights Rd

Danbury, CT 06810

David J. Diner

Mail Stop 169-237

Jet Propulsion Laboratory 4800 Oak Grove Dr.

Pasadena, CA 91109

Rodger E. Doxsey Space Telescope Sc. Inst. 3700 San Martin Drive

Baltimore, MD 21218

Peter Eisenhardt Mail Stop 245-6

NASA - Ames Research Ctr Moffett Field, CA 94035 
George Field

Center for Astrophysics

M.S. 51

60 Garden St

Cambridge, MA . 02138

Donald Finnegan

Jackson \& Tull

MD Trade Center 1 ,

Suite $640 \mathrm{~B}$

7500 Greenway Center Dr.

Greenbelt, MD 20770

\section{John Fitch}

Space Telescope Sc. Inst. 3700 San Martin Drive

Baltimore, MD 21218

Holland C. Ford

Space Telescope Sc. Inst. 3700 San Martin Drive

Baltimore, MD 21218

Chris Ftaclas

Perkin Elmer Optical Gp. 100 Wooster Heights Rd

Danbury, CT 06810

Randall B. Furnas

Code MD

NASA Headquarters

Washington, DC 20546

John S. Gallagher

AURA Inc.

Suite 701

1625 Massachusetts Ave,

Washington, DC 20036

Roberto Gilmozzi

Space Telescope Sc. Inst.

3700 San Martin Drive

Baltimore, MD 21218

Larry Goad

Litton Itek Optical Systems

10 Maquire Rd.

Lexington, MA 02173
Shireen Gonzaga

Space Telescope Sc. Inst.

3700 San Martin Drive

Baltimore, MD 21218

David Grant

JHU Applied Physics Lab.

Johns Hopkins Road

Laurel, MD 20707

Richard Green

Natl. Optical Astron. Obs. $950 \mathrm{~N}$. Cherry

Tucson, AZ 85726

Perry Greenfield

Space Telescope Sc. Inst.

3700 San Martin Drive

Baltimore, MD 21218

Richard E. Griffiths

Space Telescope Sc. Inst. 3700 San Martin Drive

Baltimore, MD 21218

James E. Gunn

Peyton Hall

Princeton University

Princeton NJ 08544

James Hagen

U.S. Naval Obs.

Massachussetts Av NW

Washington DC 20392

\section{Don Hall}

Inst. for Astronomy

2680 Woodlawn Dr.

Honolulu, HI 96822

George Hartig

Space Telescope Sc. Inst.

3700 San Martin Drive

Baltimore, MD 21218

Garth Illingworth

Lick Observatory, UCSC

Santa Cruz, CA 95064
Robert Jackson

Space Telescope Sc. Inst. 3700 San Martin Drive

Baltimore, MD 21218

William H. Jefferys

Dept of Astronomy

Univ. of Texas

Austin, TX 78712

Stewart Johnson

BDM International

1801 Randolph Rd, S.E.

Albuquerque, NM. 87106

Mark Johnston

Space Telescope Sc. Inst. 3700 San Martin Drive

Baltimore, MD 21218

Gess Jordyce

Jet Propulsion Lab.

4800 Oak Grove Drive

Pasadena, CA 91109

Mark Kahan

Optical Research Assoc.

945 Concord Av.

Framingham, MA 01701

John Keller

Kodak

921 Lincoln Av

Rochester, NY 146538117

Dieter Knohl

Produktbereichsleitung

Carl Zeiss

7082 Oberkochen

West Germany

Dietrich Korsch

Korsch Optics

10111 Bluff Drive

Huntsville, AL 35803 
Mike Krim

Perkin Elmer Optical Gp. 100 Wooster Heights Rd

Danbury, CT 06810

Tod R. Lauer

Princeton Univ. Obs.

Peyton Hall

Princeton, NJ 08544

William E. Layman

Mail Stop 198-326

Jet Propulsion Lab.

4800 Oak Grove Dr.

Pasadena, CA 91109

Kenneth Lorrell

Lockheed Missiles \& Space

O/92-30 B/205

3252 Hanover $\mathrm{St}$

Palo Alto, CA 94304

Richard Lin

Jet Propulsion Lab.

4800 Oak Grove Dr.

Pasadena, CA 91109

John MacKenty

Space Telescope Sc. Inst. 3700 San Martin Drive

Baltimore, MD 21218

Duccio Macchetto Space Telescope Sc. Inst. 3700 San Martin Drive

Baltimore, MD 21218

Tony Mallama

STX ST Systems Corp.

4400 Forbes Blvd.

Lanham, MD 20706-9008

Paul Manhart

Jet Propulsion Lab.

4800 Oak Grove Drive

Pasadena, CA 91109
Kevin McAloon

TRW Space Defence

Bldg. R1 Rm 2028

One Space Park

Redondo Beach, CA 90278

Austin McKenney

ITEK Optical

10 Maquire Rd.

Lexington, MA 02173

Aden Meinel

Mail Stop 186-134

Jet Propulsion Lab.

4800 Oak Grove Dr.

Pasadena, CA 91109

Glenn Miller

Space Telescope Sc. Inst. 3700 San Martin Drive

Baltimore, MD 21218

Joseph S. Miller

Lick Observatory

University of California

Santa Cruz, CA 95064

Warren Moos

Johns Hopkins Univ.

Homewood Campus

Baltimore, MD 21218

Max Nein

PS02, Bldg 4200

Marshall Space Flight Ctr

Huntsville, AL 35812

Jerry Nelson

Lawrence Berkeley Lab.

Bldg. 50, Rm.351

Berkeley, CA 94720

Colin A. Norman

Dept. of Physics and Astr.

Johns Hopkins Univ.

Baltimore MD 21218
Bland Norris

Perkin Elmer Optical Gp. 100 Wooster Heights Rd

Danbury, CT 06810

Robert West $O^{\prime}$ Connell

Univ. of Virginia

PO Box 3818

Charlottesville, VA 22903

William Oegerle

Space Telescope Sc. Inst. 3700 San Martin Drive

Baltimore, MD 21218

Goetz Oertel

AURA

1625 Massachusset Av

Washington, DC 20036

John F. Osantowski

Goddard Space Flight Ctr

Code 717

Greenbelt, MD 20771

Jean Oliver

TA01, NASA/MSFC

Huntsville, AL 35812

Nino Panagia

Space Telescope Sc. Inst. 3700 San Martin Drive

Baltimore, MD 21218

Francesco Paresce

Space Telescope Sc. Inst. 3700 San Martin Drive

Baltimore, MD 21218

Eugene Pawlik

Jet Propulsion Lab. 4800 Oak Grove Drive

Pasadena, CA 91109

Larry Petro

Space Telescope Sc. Inst. 3700 San Martin Drive

Baltimore, MD 21218 
Carl Pilcher

Office of Exploration

Code Z

NASA Headquarters

Washington, DC 20546

John Rather

Kaman Aerospace Corp.

1600 Wilson Blyd,

Suite 1200,

Arlington, VA 22209

Courtney Ray

JHU Applied Physics Lab.

Johns Hopkins Road

Laurel, MD 20707

Mark Rehfield

Hughes Aircraft

2000 E. El Segundo Blvd.

El Segundo, CA 90245

Guy Robinson

Jackson \& Tull

MD Trade Center 1,

Suite 640B

7500 Greenway Center Dr.

Greenbelt, MD 20770

Janet C. Rountree

AFE/Tech. Services Staff

Bolling Air Force Base

Washington, DC 20332

\section{Abhijit Saha}

Space Telescope Sc. Inst. 3700 San Martin Drive

Baltimore, MD 21218

Helene, Schember

Mail station 157-102

Jet Propulsion Lab.

4800 Oak Grove Drive

Pasadena, CA 91109
Francis H. Schiffer

Space Telescope Sc. Inst. 3700 San Martin Drive

Baltimore, MD 21218

Ethan J. Schreier

Space Telescope Sc. Inst.

3700 San Martin Drive

Baltimore, MD 21218

Amit Sen

Space Telescope Sc. Inst. 3700 San Martin Drive

Baltimore, MD 21218

Michael Shao

Jet Propulsion Lab.

BIdg 169, Rm 214

4800 Oak Grove Dr.

Pasadena, CA 91109

Michael Shara

Space Telescope Sc. Inst.

3700 San Martin Drive

Baltimore, MD 21218

David Skilmann

NASA Code 440

Goddard Space Flight Ctr.

Greenbelt, MD 20771

David Soderblom

Space Telescope Sc. Inst.

3700 San Martin Drive

Baltimore, MD 21218

Robert Stachnik

Code EZ

NASA Headquarters

Washington, DC 20546

Christopher Stevens

Jet Propulsion Lab.

4800 Oak Grove Drive

Pasadena, CA 91109
Hervey S. Stockman

Space Telescope Sc. Inst. 3700 San Martin Drive

Baltimore, MD 21218

Tom Strickwereda JHU Applied Physics Lab. Johns Hopkins Road

Laurel, MD 20707

\section{Paul Swanson}

Jet Propulsion Lab.

Mail stop 168-327

4800 Oak Grove Dr.

Pasadena, CA 91103

Richard Terrile

Jet Propulsion Lab.

4800 Oak Grove Dr.

Pasadena, CA 91103

Dominick Tenerelli

LMSC

P.O. Box 3504

Sunnyvale, CA 94088

\section{Lee Thienel}

Space Telescope Sc. Inst. 3700 San Martin Drive

Baltimore, MD 21218

Rodger I. Thompson

Steward Observatory

University of Arizona

Tucson, AZ 85721

Bruce Toth

Space Telescope Sc. Inst. 3700 San Martin Drive

Baltimore, MD 21218

Philipp Tulkoff

Swales \& Associates, Inc.

5050 Powder Mill Rd

Beltsville. MD 20705

Virginia Trimble

Astronomy Program

University of Maryland

College Park MD 20742 
Art Vaughan

Jet Propulsion Lab.

4800 Oak Grove Drive

Pasadena, CA 91109

Ray Villard

Space Telescope Sc. Inst. 3700 San Martin Drive

Baltimore, MD 21218

J. Richard Vyce

Litton Itek Optical Systems

10 Maquire Rd.

Lexington, MA 02173

Nolan R. Walborn

Space Telescope Sc. Inst.

3700 San Martin Drive

Baltimore, MD 21218

Steve Watson

USAF Inst. of Technology

Wright-Patterson AFB,

Ohio 45433

\section{Harold A. Weaver}

Space Telescope Sc. Inst.

3700 San Martin Drive

Baltimore, MD 21218

Edward J. Weiler

Astrophysics Division

Code EZ

NASA Headquarters

Washington, DC 20546

Richard L. White

Space Telescope Sc. Inst.

3700 San Martin Drive

Baltimore, MD 21218

\section{Allan Wissinger}

Perkin Elmer

761 Main Street

Norwork, CT 06859
Bruce Woodgate

LASP, NASA/GSFC

Code 681

Greenbelt, MD 20771

Neville J. Woolf

Steward Observatory

University of Arizona

Tucson, AZ 85721

Steve Zepf

Space Telescope Sc. Inst.

3700 San Martin Drive

Baltimore, MD 21218 